\section{SANDIA REPORT}

SAND97-0594 • UC-610

Unlimited Release

Printed March 1997

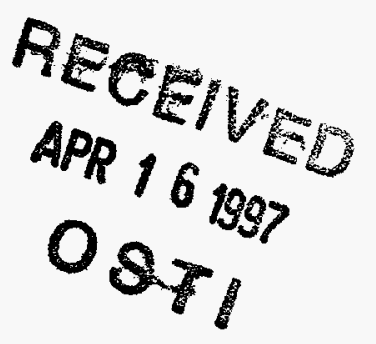

\title{
Code Manual for MACCS2: Volume 1, User's Guide
}

David I. Chanin, Mary L. Young

Prepared by

Sandia National Laboratories

Albuquerque, New Mexico 87185 and Livermore, California 94550

Sandia is a multiprogram laboratory operated by Sandia

Corporation, a Lockheed Martin Company, for the United States.

Department of Energy under Contract DE-AC04-94AL85000.

Approved for public release; distribution W Unithited.
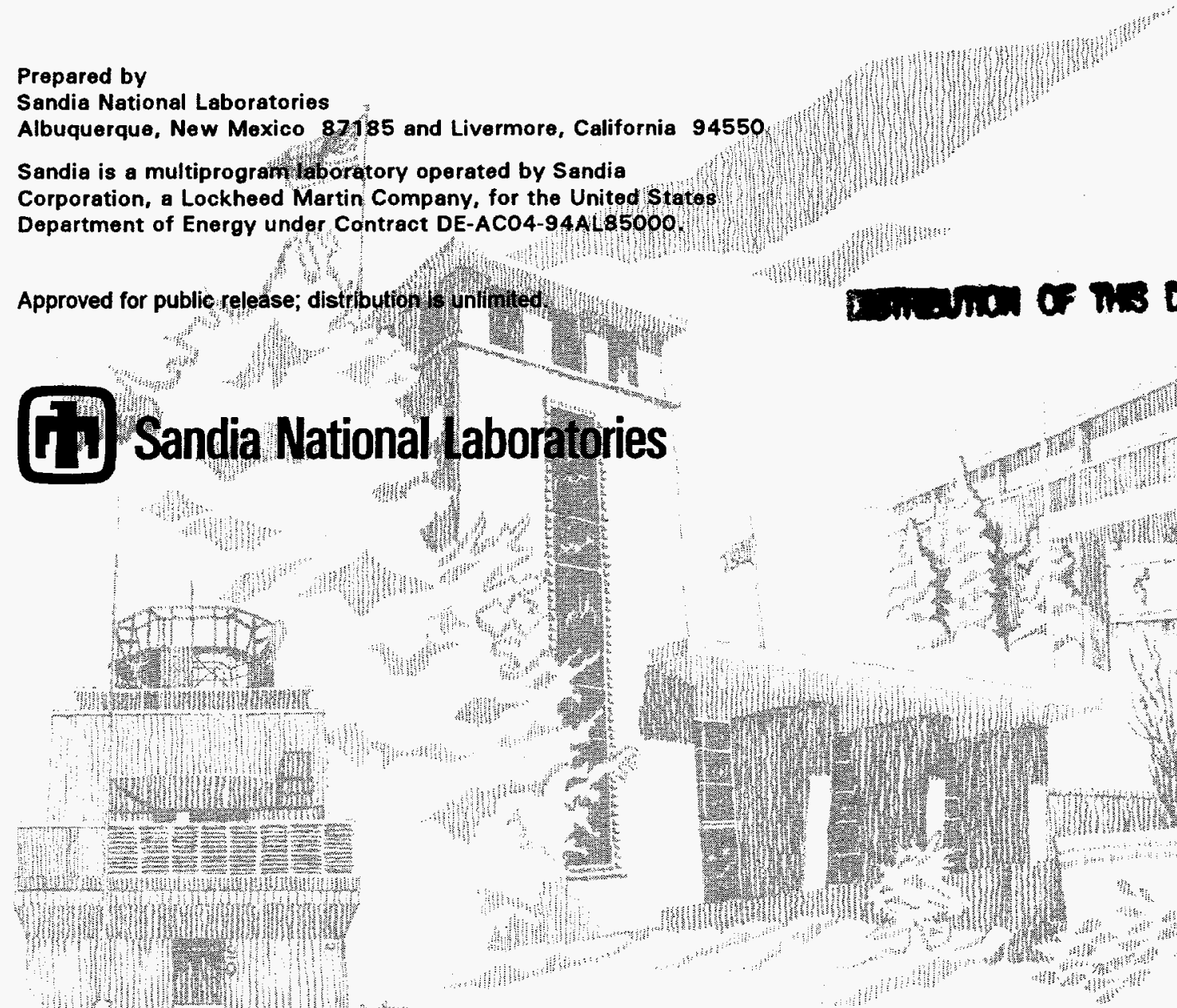
Issued by Sandia National Laboratories, operated for the United States Department of Energy by Sandia Corporation.

NOTICE: This report was prepared as an account of work sponsored by an agency of the United States Government. Neither the United States Government nor any agency thereof, nor any of their employees, nor any of their contractors, subcontractors, or their employees, makes any warranty, express or implied, or assumes any legal liability or responsibility for the accuracy, completeness, or usefulness of any information, apparatus, product, or process disclosed, or represents that its use would not infringe privately owned rights. Reference herein to any specific commercial product, process, or service by trade name, trademark, manufacturer, or otherwise, does not necessarily constitute or imply its endorsement, recommendation, or favoring by the United States Government, any agency thereof, or any of their contractors or subcontractors. The views and opinions expressed herein do not necessarily state or reflect those of the United States Government, any agency thereof, or any of their contractors.

Printed in the United States of America. This report has been reproduced directly from the best available copy.

Available to DOE and DOE contractors from

Office of Scientific and Technical Information

P.O. Box 62

Oak Ridge, TN 37831

Prices available from (615) 576-8401, FTS 626-8401

Available to the public from

National Technical Information Service

U.S. Department of Commerce

5285 Port Royal Rd

Springfield, VA 22161

NTIS price codes

Printed copy: A03

Microfiche copy: A01 


\section{DISCLAIMER}

Portions of this document may be illegible in electronic image products. Images are produced from the best available original document. 


\title{
Code Manual for MACCS2: Volume 1, User's Guide
}

\author{
David I. Chanin* and Mary L. Young \\ Sandia National Laboratories, Accident Analysis/Consequence Assessment Department \\ P.O. Box 5800, Albuquerque, NM 87185-0748 \\ *Albuquerque, NM
}

\begin{abstract}
This report describes the use of the MACCS2 code. The document is primarily a user's guide, though some model description information is included. MACCS2 represents a major enhancement of its predecessor MACCS, the MELCOR Accident Consequence Code System. MACCS, distributed by government code centers since 1990, was developed to evaluate the impacts of severe accidents at nuclear power plants on the surrounding public. The principal phenomena considered are atmospheric transport and deposition under time-variant meteorology, short- and long-term mitigative actions and exposure pathways, deterministic and stochastic health effects, and economic costs. No other U.S. code that is publicly available at present offers all these capabilities. MACCS2 was developed as a general-purpose tool applicable to diverse reactor and nonreactor facilities licensed by the Nuclear Regulatory Commission or operated by the Department of Energy or the Department of Defense. The MACCS2 package includes three primary enhancements: (1) a more flexible emergency-response model, (2) an expanded library of radionuclides, and (3) a semidynamic food-chain model. Other improvements are in the areas of phenomenological modeling and new output options. Initial installation of the code, written in FORTRAN 77, requires a 486 or higher IBM-compatible PC with 8 MB of RAM.
\end{abstract}




\section{Contents}

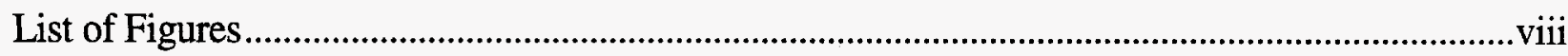

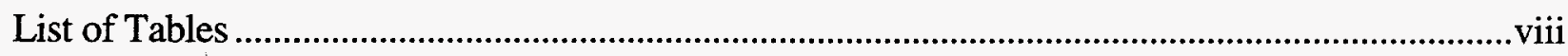

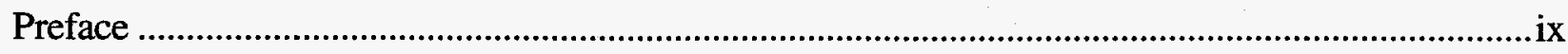

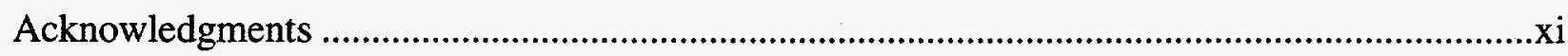

Conversion Factors and Abbreviations for Units of Measure ...........................................................xiii

Standard Prefixes for Powers of Ten .........................................................................................ii

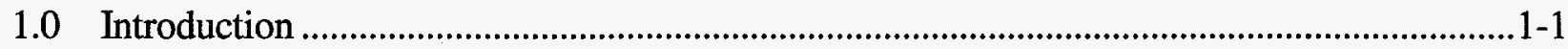

1.1 Historical Background ........................................................................................................1-1

1.2 Application of MACCS and the MACCS2 Package .......................................................1-4

1.2.1 PRA Applications ............................................................................................. 1-5

1.2.2 Research Studies .....................................................................................1-5

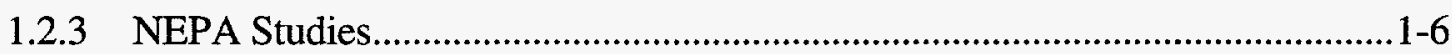

1.2.4 Authorization Basis Studies ...........................................................................1-7

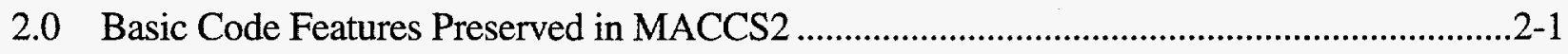

2.1 Overview of Code Structure............................................................................................2-1

2.1.1 Division of Calculations into "Modules" and "Phases" .......................................2-1

2.1.1.1 The ATMOS Module: Atmospheric Transport and Deposition.........2-2

2.1.1.2 The EARLY Module: Emergency Phase Calculations........................2-2

2.1.1.3 The CHRONC Module: Intermediate and Long-Term Phase

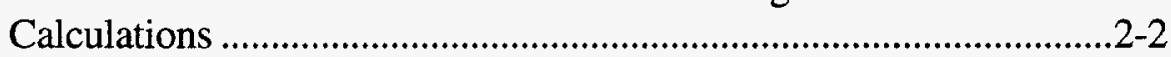

2.1.1.4 Intermediate Phase ....................................................................................2-2

2.1.1.5 Long-Term Phase ...............................................................................2-3

2.1.2 Distinction Between Acute and Lifetime Doses...................................................2-3

2.1.2.1 Acute Doses ........................................................................................2-3

2.1.2.2 Lifetime Doses.....................................................................................2-3

2.1.3 Polar-Coordinate Grid.....................................................................................2-3

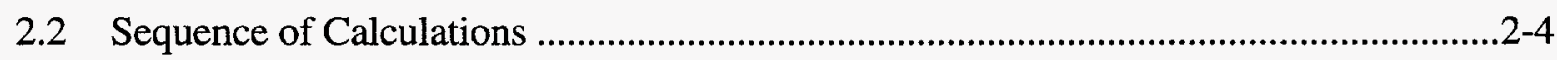

2.2.1 Input Processing and Validation .....................................................................2-4

2.2.1.1 Data Types Processed by the Code ........................................................2-6

2.2.1.2 Free-Format Input Processor .................................................................2-6

2.2.1.3 Error Checking of Input Data .........................................................2-7

2.2.1.4 Specification of Multiple Accident Scenarios .....................................2-8

2.2.2 Phenomenological Modeling ...........................................................................2-8

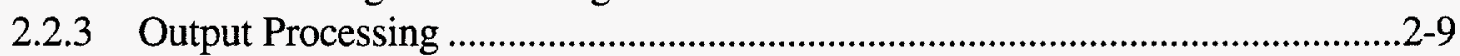

2.2.3.1 Presentation of Multiple Source Terms and Multiple Cohorts..........2-11

2.2.3.2 Interpretation of the One-Line Summaries ......................................... -13

2.2.3.3 Interpretation of the CCDF Tables...................................................2-17 
2.2.3.4 Interpretation of One-Line Summaries for Single Weather Trial Runs

2.2.3.5 Use of Interpolation ....................................................................2-19

3.0 Code Enhancements Implemented in MACCS2 ......................................................................

3.1 MACCS2 Preprocessors ................................................................................................. 3-1

3.1.1 Dose Conversion Factor Preprocessors …………...................................................

3.1.1.1 Three DCF Preprocessors..................................................................

3.1.1.2 DOSFAC2 …......................................................................

3.1.1.3 FGRDCF.....................................................................................

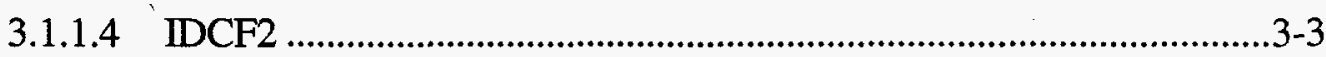

3.1.1.5 Choice of DCF Preprocessor...................................................................3-3

3.1.2 New Ingestion Pathway Preprocessor - COMIDA2 ................................................3-3

3.2 Enhancements to ATMOS ........................................................................................

3.2.1 Expansion of Radioactive Decay Chain Calculations ..........................................3-4

3.2.2 Increased Flexibility in Defining Initial Plume Dimensions.................................

3.2.3 New Lookup-Table Option for Dispersion Parameter Data..................................3-5

3.2.4 CCDFs of Atmospheric Dispersion and Deposition Results ...............................

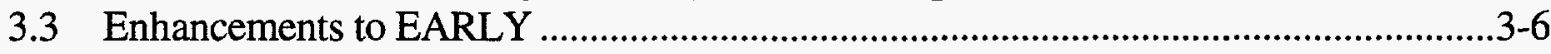

3.3.1 MACCS2 Emergency-Response Model .....................................................

3.3.2 Alternative Options for Modeling Evacuee Movement ........................................3-7

3.3.3 Direction-Dependent Doses................................................................................... 3-8

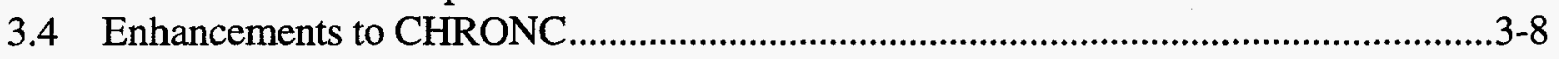

3.4.1 Specification of the Intermediate and Long-Term Phase Exposure Periods ......................................................................................................

3.4.2 Enhanced Food Pathway Modeling Capabilities...................................................

3.5 Minor Enhancements and Corrections to MACCS ......................................................

3.5.1 DDREFA Implementation Error........................................................................3-9

3.5.2 Type 3 Result (Number of People Exceeding Dose Threshold) .........................3-10

3.5.2.1 Incorrect Calculations when Ten Results Are Generated...................3-11

3.5.2.2 Inability to Report Small Values of the Dose Threshold.....................3-11

3.5.3 Incomplete Implementation of the Intermediate Phase .....................................3-12

3.5.3.1 Intermediate-Phase Health Effects and Collective Doses...................3-12

3.5.3.2 Lack of Interaction in MACCS Between Intermediate-Phase

Relocation and Farm Interdiction...................................................3-12

3.5.4 Summation of Early and Intermediate-Phase Costs ............................................ $3-13$

3.5.5 Dose Calculations for Groundshine Following Plume Passage ..........................3-13

3.5.6 Nonprintable Characters in Site Data File and FORTRAN Source Code ........3-14

3.5.7 Minor Changes to Input and Output ................................................................ $3-14$

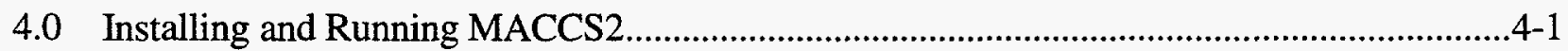

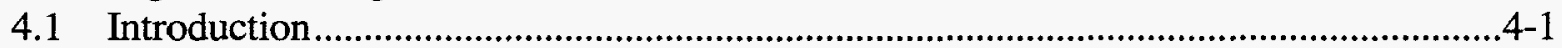

4.2 Hardware Requirements ................................................................................

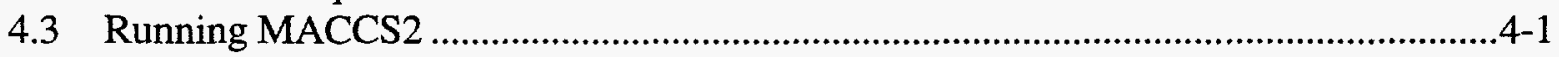

4.4 MACCS2 Sample Problems .....................................................................................4-3

4.4.1 Sample Problems A-C.....................................................................................4-3 
4.4.2 Sample Problem D ......................................................................................4-4

4.4.3 Sample Problem E..............................................................................................4-4

4.4.4 Sample Problem F............................................................................................4-4

4.5 Migration from MACCS to MACCS2 ............................................................................

4.5.1 MACCS ATMOS File ..........................................................................

4.5.2 MACCS EARLY File ............................................................................

4.5.2 MACCS CHRONC File ........................................................................... $4-8$

$5.0 \quad$ ATMOS Input File ............................................................................................................

5.1 Introduction to ATMOS ..........................................................................................

5.1.1 Format of Input Parameter Description Block......................................................5-2

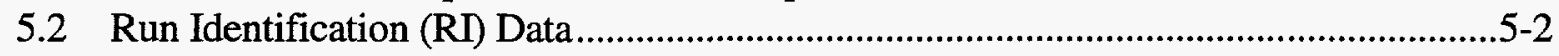

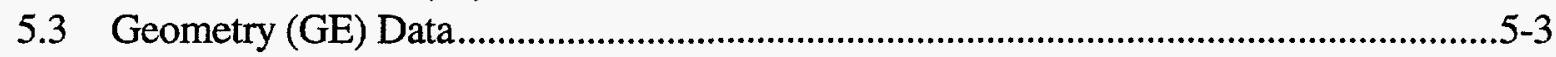

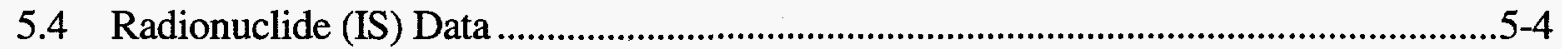

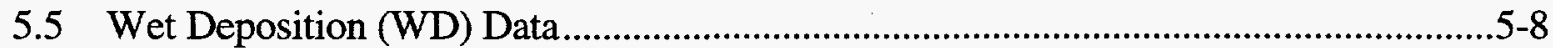

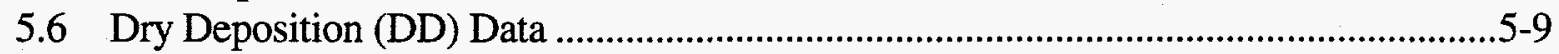

5.7 Dispersion Parameter (DP) Data ............................................................................ $5-10$

5.7.1 Definition of Power-Law Function Parameters................................................5-10

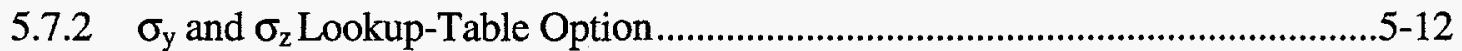

5.7.3 Scaling Factors for Dispersion.........................................................................

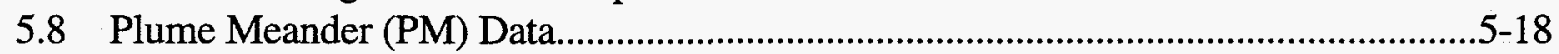

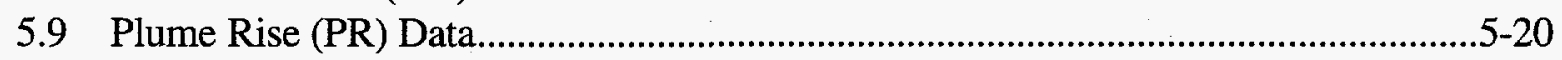

5.10 Wake Effects (WE) Data ............................................................................................

5.11 Release Description (RD) Data ............................................................................ $5-23$

5.12 Output Control (OC) Data ...........................................................................................

5.13 Meteorological Sampling (M1) Specification................................................................5-31

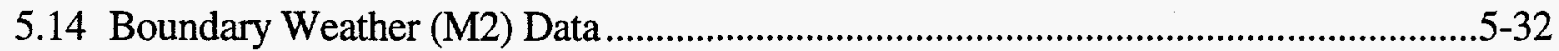

5.15 Fixed Start Time (M3) Data ...............................................................................

5.16 Meteorological Bin Sampling (M4) Data ..............................................................5-34

5.17 User-Supplied Weather Sequence (M5) Data..........................................................5-39

5.18 CCDFs of Atmospheric Results .....................................................................................5-40

6.0 EARLY Input File ..........................................................................................................6-1

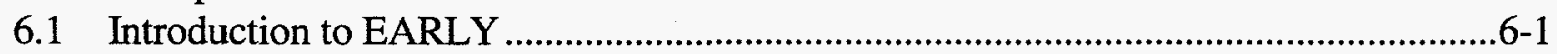

6.1.1 Overview of Dose Calculations ........................................................................6-1

6.1.2 Overview of New EARLY File Input Requirements Implemented in
MACCS2

6.2 Dose Conversion Factor Filename and Other Miscellaneous Input Data ........................6-4

6.2.1 Dose Conversion Factor Filename..................................................................6-4

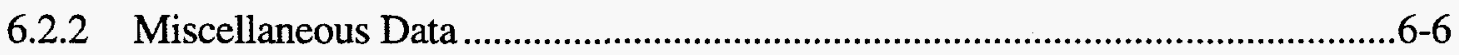

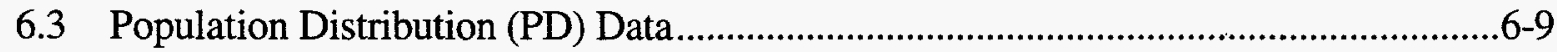

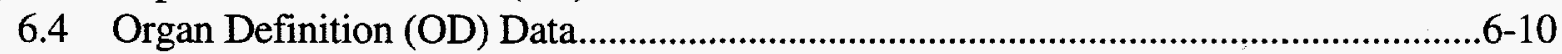

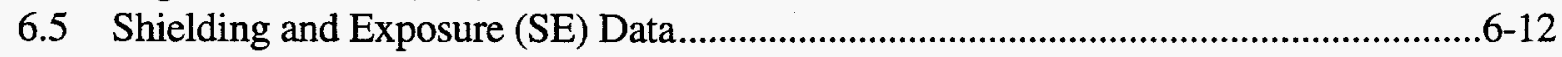

6.6 Evacuation Zone (EZ) Data..................................................................................6-14

6.6.1 User-Defined Emergency Response Scenarios ..................................................6-15

6.6.2 Early, Middle, and Late Phases of Evacuation ...................................................6-16 


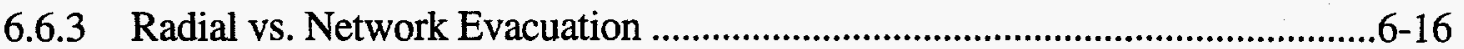

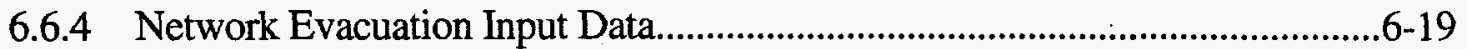

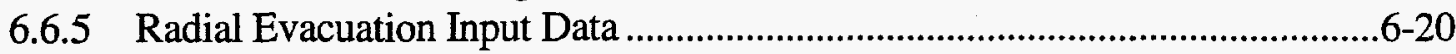

6.6.6 Processing of Evacuation Networks ................................................................6-20

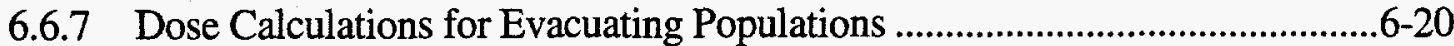

6.6.8 Evacuation Data Block Input Parameters ....................................................6-21

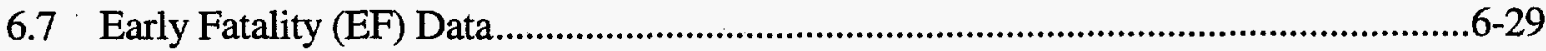

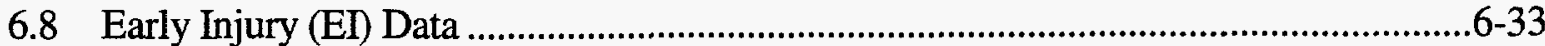

6.9 Latent Cancer (LC) Induction Model ..............................................................6-33

6.9.1 MAACS Linear Quadmatic Dose-Response Model ...................................6-35

6.9.2 MAACS Piecewise Linear Dose-Reponse Function.......................................6-36

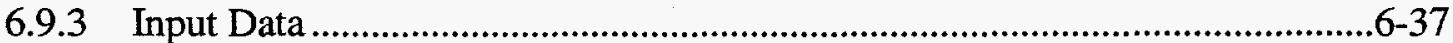

6.10 Generation of Consequence Distributions .............................................................6-40

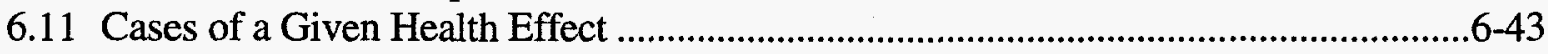

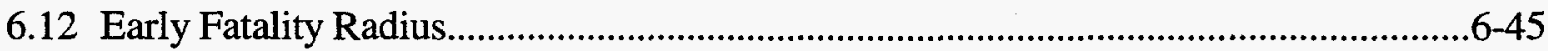

6.13 Population Exceeding a Dose Threshold ...........................................................6-45

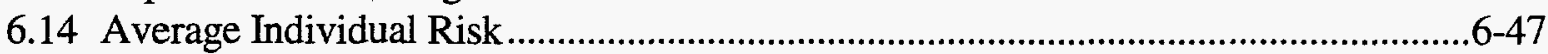

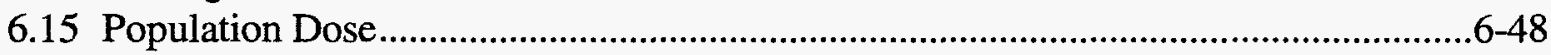

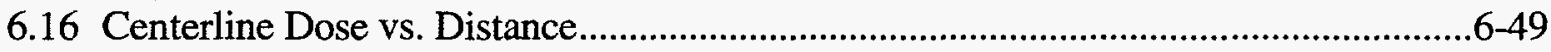

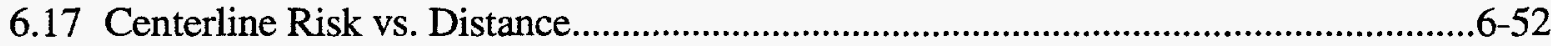

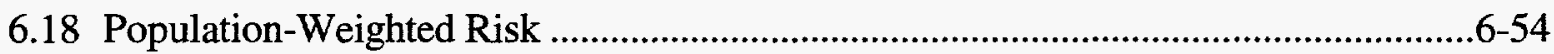

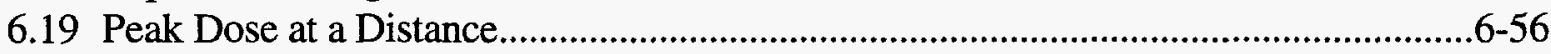

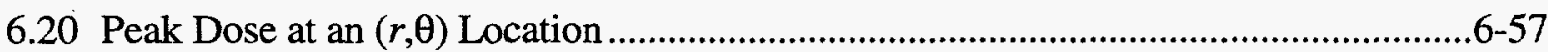

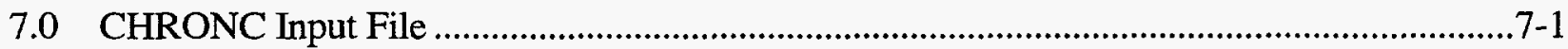

7.1 Overview of CHRONC Mitigative Action Models ..................................................7-2

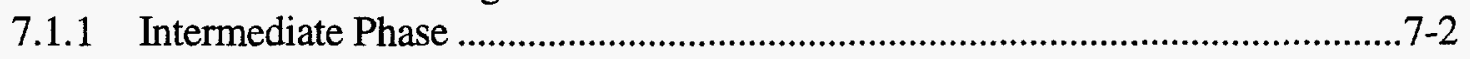

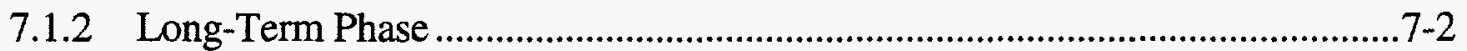

7.1.3 Division Between Farm and Population ....................................................... 7-3

7.1.4 Habitability Decision Making......................................................................... $7-3$

7.1.4.1 Land Immediately Habitable .........................................................

7.1.4.2 Land Habitable after Decontamination .......................................... $7-3$

7.1.4.3 Land Habitable after Decontamination and Interdiction .................... $7-4$

7.1.4.4 Limits on Interdiction Duration..................................................... $7-4$

7.1.4.5 Test for Cost Effectiveness......................................................... $7-4$

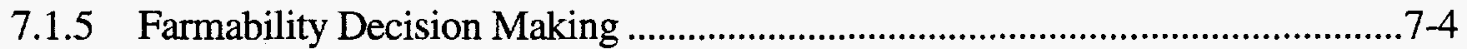

7.1.6 Primacy of Habitability over Farmability Decisions ..................................... 7-5

7.2 Problem Identification Data........................................................................... $7-5$

7.3 Emergency-Response Cost Data ....................................................................... $7-5$

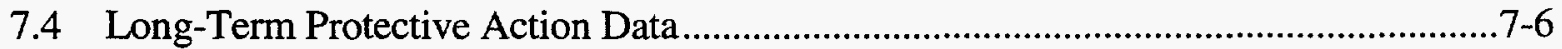

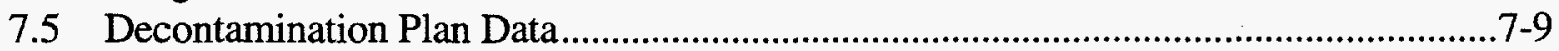

7.6 Interdiction Plan Cost Data................................................................................ 7-13

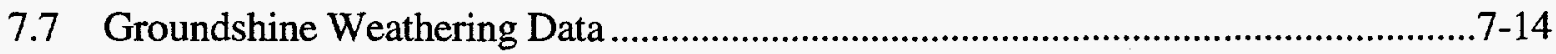

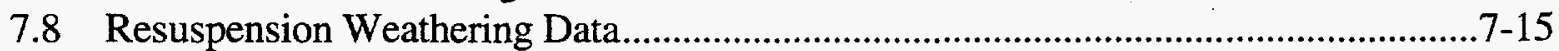

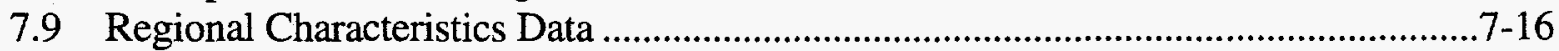


7.10 New COMIDA2-Based Food Ingestion Model .............................................................19

7.10.1 Modeling of Agricultural Countermeasures ....................................................... $7-19$

7.10.2 Input Variables for COMIDA2-Based Food Model............................................

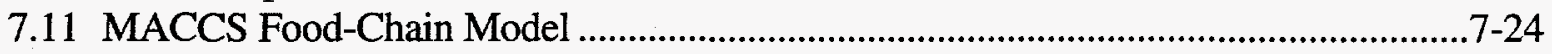

7.11.1 Ingestion Transfer Factors Data .................................................................24

7.11.2 Crop Share and Growing Season Data ............................................................ 7-36

7.11.3 Protective Action Guide for MACCS Food Pathway Model............................. 7-37

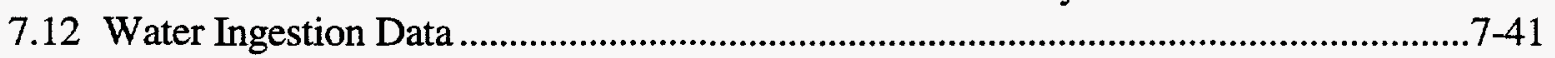

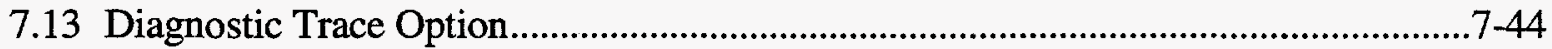

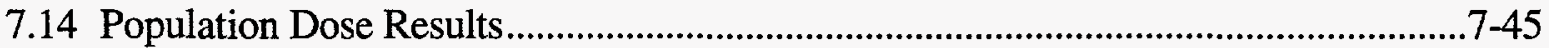

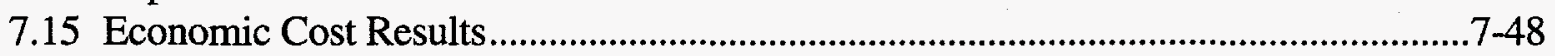

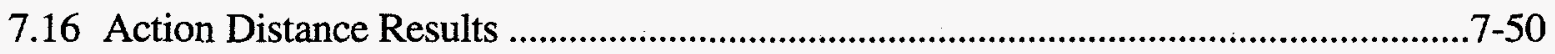

7.17 Impacted Area/Population Results ..............................................................................7-51

7.18 Maximum Individual Food Ingestion Dose at a Distance .............................................

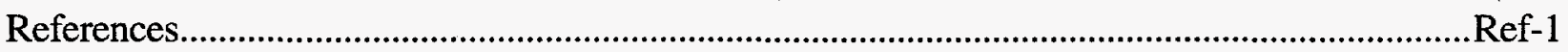

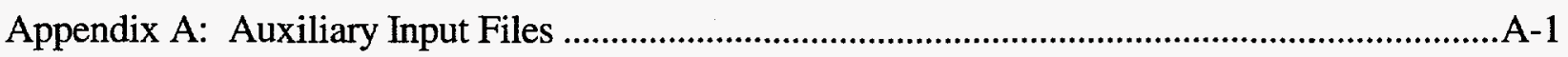

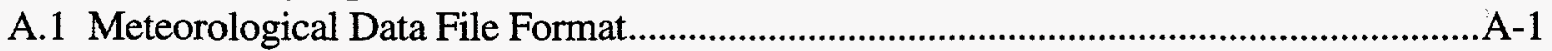

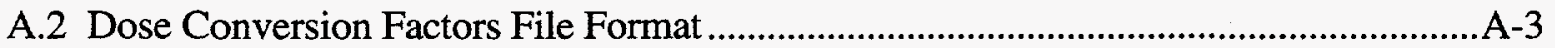

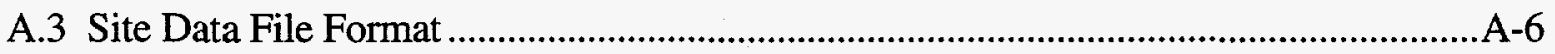

A.3.1 Geometry Data Block …………………………...............................................

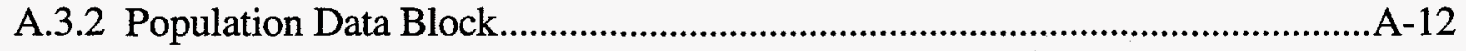

A.3.3 Land Fraction Data Block........................................................................13

A.3.4 Region Index Data Block ......................................................................13

A.3.5 Watershed Index Data Block..........................................................................

A.3.6 Crop and Season Share Data Block ………….......................................... 14

A.3.7 Watershed Definition Data Block .................................................................15

A.3.8 Regional Economic Data Block ……………….................................................15

Appendix B: READMAC2.TXT FILE..............................................................................

Appendix C: Sample Problem A LISTA_N.OUT Output Listing....................................................

Appendix D: Glossary of Input File Variables ............................................................................. 


\section{List of Figures}

2-1 A MACCS2 polar-coordinate grid with 3 radial divisions. The numbers included on the grid refer to the 16 compass directions........................................................... 2-5

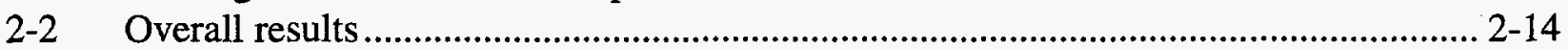

2-3 EARLY results for cohort 1: evacuation ............................................................... 2-15

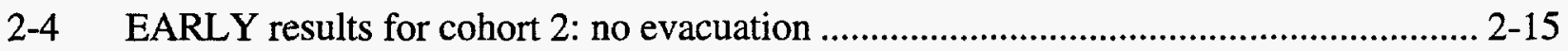

2-5 CHRONC results: cohort 3

2-6 CCDF tables for overall results and cohorts 1-3 ...................................................... 2-18

5-1 F-stability dispersion table-Tadmor-Gur formula (for 0.5 to $5 \mathrm{~km}$ )........................ 5-15

5-2 F-stability dispersion table-Briggs open country formula $(0.1$ to $10 \mathrm{~km})$.................. 5-16

5-3 F-stability dispersion table-Briggs urban formula $(0.1$ to $10 \mathrm{~km})$........................... 5-17

6-1 Radial evacuation network with DLTEVA values of 60,120 , and $240 \mathrm{~s}$ assigned to the three spatial intervals. ......................................................................................... 6-17

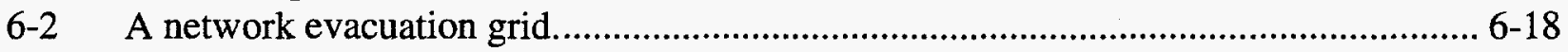

\section{List of Tables}

2-1 Phenomenological Models of ATMOS ...................................................................... 2-9

2-2 Phenomenological Models of EARLY …………................................................ 2-10

2-3 Phenomenological Models of CHRONC............................................................. 2-10

2-4 Results Available from ATMOS, EARLY, and CHRONC........................................ 2-12

3-1 MACCS2 vs. MACCS Sample Problem A Results for Total Weighted-Sum Overall Results-EARLY plus CHRONC ............................................................... 3-10

3-2 MACCS2 vs. MACCS Sample Problem A Results from EARLY AloneNonevacuating Cohort of EARLY.

4-1 Input Parameters that Must Be Added to MACCS ATMOS Input Files to Convert Them to MACCS2 Input Files......................................................................................... 4-6

4-2 Input Parameters that Must Be Added to MACCS EARLY Input Files to Convert Them to MACCS2 Input Files....................................................................................... 4-7

4-3 MACCS EARLY File Input Parameters That Are Not Processed by MACCS2 2............ 4-8

4-4 Input Parameters that Must Be Added to MACCS CHRONC Input Files to Convert Them to MACCS2 Input Files............................................................................... 4-9

5-1 Results Available from ATMOS in CCDF Form ...................................................... 5-41

6-1 Effective Acute Dose Reduction Factors (unitless) .................................................... 6-31 


\section{Preface}

This report describes the MACCS2 code; it is composed of two volumes. Volume 1 is based on the MACCS User's Guide, NUREG/CR-4691, Vol. 1 (Chanin et al. 1990). It is intended to allow experienced users of MACCS or other consequence codes to prepare input files and interpret code results.

The MACCS2 documentation does not duplicate information already contained in the MACCS Model Description, NUREG/CR-4691, Vol. 2 (Jow et al. 1990). It is thus essential that the MACCS Model Description be available to the reader.

The companion to this report, Volume 2, (Chanin and Young 1997-draft) describes the MACCS2 preprocessor codes COMDA2, FGRDCF, and IDCF2. COMIDA2 is a food pathway model that calculates food-chain doses per unit of deposited activity from multiple radionuclides. FGRDCF and IDCF2 generate dose conversion factors (DCFs) that can be used by MACCS2.

DOSFAC2, an additional DCF preprocessor provided in the MACCS2 package, is described in a separate draft document, the DOSFAC2 User's Guide (Young, Chanin, and Banjac 1997-draft). Sample files generated by these preprocessors for use by MACCS2 are provided as part of the distribution package. Operation of the MACCS2 sample problems requires that those files be installed on the host system, but all features of MACCS2 can be exercised without the need to actually run the preprocessor programs. The preprocessors are provided for those users who wish to generate DCF or food pathway data based on assumptions other than those used to generate the data in the DCF and COMIDA2 files provided with the MACCS2 sample problems.

The distribution package includes both FORTRAN source code and PC executables. To the greatest possible extent, MACCS2 and its preprocessors comply with American National Standards Institute standard FORTRAN, making only limited use of FORTRAN 90 extensions to the FORTRAN 77 language standard.

Installation of the complete software package requires a $486 \mathrm{PC}$ or higher processor, $8 \mathrm{MB}$ of RAM, and approximately $30 \mathrm{MB}$ of hard disk space. The executables provided were developed in a DOS environment. Additional software, such as proprietary memory managers for DOS, are not required, but some of these may facilitate the use of the code. Guidance is provided in regard to DOS memory managers and the installation of the package on other computer systems. 
THIS PAGE INTENTIONALLY LEFT BLANK 


\section{Acknowledgments}

The authors would like to acknowledge all of the individuals who have contributed to the development and refinement of the MACCS code. They include J.J. Gregory, H.-N. Jow, L.A. Miller, L.T. Ritchie, and J. L. Sprung of Sandia National Laboratories; J. D. Johnson and J. A. Rollstin of GRAM, Inc.; and J. Foster of Technadyne Engineering Consultants, Inc.

We would like to thank Kamiar Jamali of the U.S. Department of Energy and Christiana Lui and John Randall of the U.S. Nuclear Regulatory Commission for supporting the MACCS2 development effort.

A number of people made important contributions to this work: Dr. Eric Haskin and Ken Summa of the University of New Mexico carried out a productive independent verification of the code, and Mike Abbott and Art Rood of EG\&G Idaho, Inc. developed COMDA and supported the development of COMIDA2. We benefited greatly from the comments and feedback given by Jennifer Powell and Lih-Jenn Shyr of Sandia, the members of the MACCS2 Beta Test Group, and the International MACCS User's Group. We would particularly like to thank Jackie East of the Savannah River Site and Keith Eckerman of Oak Ridge National Laboratory for their comments and suggestions.

J. J. Jones and N. DeHerrera of Sandia provided invaluable administrative assistance during this project, and Ruth Haas of Tech Reps provided dedicated and thorough editorial review throughout the writing of this document. 
THIS PAGE INTENTIONALLY LEFT BLANK 


\section{Conversion Factors and Abbreviations for Units of Measure}

1 meter $(\mathrm{m})=$ one million microns $=3.281$ feet $(\mathrm{ft})$

1 square kilometer $\left(\mathrm{km}^{2}\right)=100$ hectares (ha) $=247.1$ acres $=0.3861$ square miles 1 cubic meter $\left(\mathrm{m}^{3}\right)=35.31$ cubic feet $\left(\mathrm{ft}^{3}\right)$

1 kilogram $(\mathrm{kg})=2.204$ pounds $(\mathrm{lb})$

1 curie $(\mathrm{Ci})=3.7 \times 10^{10}$ becquerels $(\mathrm{Bq})$

1 disintegration per minute $(\mathrm{dpm})=1 / 60$ becquerel

1 gray $(\mathrm{Gy})=100 \mathrm{rad}=1$ joule/kilogram

1 sievert $(\mathrm{Sv})=100 \mathrm{rem}$

\section{Standard Prefixes for Powers of Ten}

\begin{tabular}{lll} 
Prefix & Abbreviation & Power of Ten \\
\cline { 2 - 3 } tera & $\mathrm{T}$ & $10^{12}$ \\
giga & $\mathrm{G}$ & $10^{9}$ \\
mega & $\mathrm{M}$ & $10^{6}$ \\
kilo & $\mathrm{k}$ & $10^{3}$ \\
deci & $\mathrm{d}$ & $10^{-1}$ \\
centi & $\mathrm{c}$ & $10^{-2}$ \\
milli & $\mathrm{m}$ & $10^{-3}$ \\
micro & $\mu$ & $10^{-6}$ \\
nano & $\mathrm{n}$ & $10^{-9}$ \\
pico & $\mathrm{p}$ & $10^{-12}$ \\
femto & $\mathrm{f}$ & $10^{-15}$ \\
atto & $\mathrm{a}$ & $10^{-18}$
\end{tabular}


THIS PAGE INTENTIONALLY LEFT BLANK 


\subsection{Introduction}

\subsection{Historical Background}

The Reactor Safety Study (NRC 1975) presented the first comprehensive probabilistic risk assessment (PRA) of hypothetical nuclear power plant accidents. (This report is also commonly referred to as WASH-1400.) As part of that study, the CRAC code was developed to calculate the health and economic consequences of accidental releases of radioactive material to the atmosphere.

Prior to the Reactor Safety Study, the major focus of safety analyses for nuclear facilities was on evaluating the adequacy of facility siting and design in order to ensure that the public was adequately protected against potential accidents. These "authorization basis" analyses, as called for by 10 CFR 100 (Reactor Site Criteria), focus on the maximal off-site doses that could be faced by the population in the event of design basis accidents (DBAs).

DBAs are typically chosen to represent the design limits of the facility, for example, a largebreak loss-of-coolant accident (LOCA) in a commercial power reactor. The analysis of these accidents is documented in the facility's safety analysis report (SAR). Operation of commercial power plants requires the approval of SARs by the Nuclear Regulatory Commission (NRC). The analytical methods used to demonstrate compliance with the applicable requirements are very simple, and incorporate a high degree of conservatism.

Also, because these analyses are performed prior to the operation of the facility, information on equipment failure rates, accident precursors, etc., is not utilized. Operating experiences are an extremely valuable source of information on the risks posed by a facility as it is configured and operated from day to day, but this information is usually not considered in authorization basis studies.

As a result of this and other factors, there developed an interest in more realistic analysis methods so that an accurate picture could be obtained of the risks posed by the operation of these facilities. According to Kaplan and Garrick (1981), the estimation of accident risks requires consideration of both frequencies of accident occurrence as well as the resultant consequences of those accidents. For completeness, the entire spectrum of accidents, ranging from minor to severe, would need to be considered, and this was a major goal of the Reactor Safety Study.

Since its 1975 inception, probabilistic consequence modeling of postulated commercial reactor accidents has received widespread attention and application throughout the world, and a number of consequence models have been developed, both in the United States and abroad. CRAC2, released to the public by Sandia National Laboratories ( SNL) in 1982, incorporated major improvements over CRAC in the areas of weather sequence sampling and emergency-response modeling (Ritchie, Johnson, and Blond 1983; Ritchie et al. 1984). 
However, in using CRAC2 for diverse applications such as the Sandia Siting Study (Aldrich et al. 1982), it became apparent that the code did not offer sufficient flexibility for the performance of sensitivity studies and the evaluation of alternative parameter values for its models. In fact, because the purpose of CRAC was to perform the Reactor Safety Study calculations, with no other applications foreseen, many parameter values were fixed in CRAC and its successor CRAC2.

In order to perform parametric studies or site-specific analyses, it was necessary to repeatedly modify the FORTRAN source code of CRAC2 and maintain multiple versions of the software, a complication that resulted in extra costs and difficulties in quality assurance. In addition, CRAC2 was not portable across computer systems because the FORTRAN standard used at the time, FORTRAN 66 (American National Standards Institute 1966), encouraged a proliferation of language extensions with little or no consistency among the various compilers.

MACCS was developed to remedy these shortcomings. The goal of the MACCS development effort was to produce a portable code with a modular architecture and flexible database. These features were intended to facilitate the performance of site-specific calculations, evaluation of sensitivities and uncertainties, and the future incorporation of new phenomenological models. All MACCS coding was required to conform to American National Standards Institute (ANSI) standard FORTRAN 77 (American National Standards Institute 1978), a language designed to foster portability. Almost all of the MACCS model parameter values are defined by the user in order to facilitate the performance of uncertainty and sensitivity analyses.

The principal phenomena considered in MACCS are atmospheric transport, short-term and longterm mitigative actions and exposure pathways, deterministic and stochastic health effects, and economic costs. Among the U.S. consequence codes that are publicly available, MACCS is unique in its capability for modeling short-term and long-term mitigative actions, and their associated economic costs, in conjunction with modeling both deterministic and stochastic health effects.

The first version of MACCS released to the public, version 1.4, was distributed by Sandia National Laboratories beginning in 1987. Along with a draft user's guide and model description, it was distributed to over 40 recipients. MACCS 1.4 was used for the calculations of the Reactor Risk Reference Document (NRC 1987), a public first draft of the study now commonly referred to as NUREG-1150. (Subsequent references to this document will use NUREG-1150.)

MACCS 1.4 was also used to study the relative importance of individual radionuclides (Alpert et al. 1987) and the sensitivity and uncertainty of model predictions to variations in input parameters (Helton et al. 1989). This version was also used evaluate the potential economic costs of commercial reactor accidents ( for example, see Alonso and Gallego 1987; Alonso, Gallego, and Martin 1990; and Lonergan, Goble, and Cororaton 1990).

MACCS 1.4 had a major deficiency that impeded its convenient use, namely, it was composed of not a single FORTRAN code, but of five separate codes that had to be exercised in sequence, with the calculated results propagated from one code to the next through binary files on the host computer system. This was a cumbersome arrangement, but, despite this limitation, the code was 
used successfully outside of SNL for the K reactor probabilistic risk assessment (PRA) (O'Kula and Mendoza 1989) with a methodology based on that of the first-draft NUREG-1150. MACCS 1.4 was also used for Savannah River Site (SRS) K reactor analyses intended to fulfill both authorization basis purposes (Westinghouse Savannah River Company 1990) and those of a PRA (Amos et al. 1991). The use of MACCS to support a risk-based authorization basis for the K reactor was the first DOE application of this type. ${ }^{1}$

MACCS 1.5 remedied the cumbersome architecture by merging the five programs into one. During the course of that effort, an independent inspection of the code was conducted (Dobbe et al. 1990). Additional NRC-sponsored efforts in support of the code were those by Tveten (1990a,b; 1995).

In 1990, version 1.5.11 was made available to the public by the National Energy Software Center (NESC) of Argonne, IL and the Radiation Shielding Information Center (RSIC) of Oak Ridge, TN. A three-volume set of published manuals (Chanin et al. 1990; Jow et al. 1990; Rollstin et al. 1990) was issued as the code's documentation.

The Department of Energy (DOE) has had a long-standing interest in the evolution of MACCS. In order to assess the impact of the code upgrade from versions 1.4 to 1.5 on the calculated results, the DOE sponsored an investigation into the impact of the code changes (O'Kula and East 1990), and then requested an additional effort to define the DOE needs for enhancement of the code (O'Kula 1991).

MACCS 1.5.11 was distributed on 9-track magnetic tape, with the target machine being a Digital Equipment Corporation (DEC) VAX computer running the VMS operating system. Soon thereafter, the 386 processor came into use on IBM-PC compatibles and a FORTRAN 77 compiler for the PC platform became available. Because of the convenience offered by desktop computers, the code was converted to run on the PC (Jones, Dobbe, and Knudson 1991), but this version was not widely distributed, and the originators were not tasked with providing user support.

SNL was then tasked by the NRC to adapt the code to the PC platform, and to implement a revised cancer risk model based on BEIR V (National Academy of Sciences 1990). During the course of the effort, internal changes to the coding were made to make it aș portable as possible, given the limitations of ANSI FORTRAN 77. Also, a number of coding errors were corrected and a few additional minor enhancements were made to the code's models. The resultant code, MACCS 1.5.11.1, was readily installable on five different types of computer systems, with a diskette-based distribution package that included an executable for 386 or higher PC-compatibles (Chanin et al. 1993). ${ }^{2}$

\footnotetext{
${ }^{1}$ A similar approach, using MACCS and beta-test MACCS2, would later be adopted for riskbased authorization basis analyses by the Rocky Flats Plant.

${ }^{2}$ In the remainder of this report, for brevity, the simple term MACCS will often be used to refer to verson 1.5.11.1 of that code, the immediate predecessor of MACCS2. However, when the discussion pertains to only a specific version, the version number will always be specified.
} 
MACCS has come into wide use within the United States and abroad (Neymotin 1994) for the evaluation of commercial power plant safety, with over 170 copies distributed by the Energy Science and Technology Center (ESTSC) of Oak Ridge, TN. However, despite the code's intended use for nuclear power plants, a sizable fraction of the code's users have always been engaged in studies assessing the safety of DOE facilities, and the code has occasionally been used to assess Department of Defense (DoD) nuclear facilities as well.

When the successive versions of MACCS have been applied to DOE facilities, in almost every instance there has been a problem with the limited set of radionuclides selected for commercial reactor applications. In order to remedy this shortcoming, and implement a number of other changes that enhance the code's usefulness for all types of reactor and nonreactor facilities, the MACCS2 development effort was initiated at SNL in 1991. The purpose of this effort was to develop a generally applicable analysis tool for use in assessing potential accidents at a broad range of reactor and nonreactor nuclear facilities. MACCS2 development efforts have been sponsored by the DOE Office of Defense Programs and the NRC, with additional DOE-related work supported by Los Alamos National Laboratory (LANL) (Chanin 1992).

The MACCS2 enhancements were developed through cooperation and joint efforts with the technical staff at Brookhaven National Laboratory (BNL), Idaho National Engineering Laboratory (INEL), Los Alamos National Laboratory, Oak Ridge National Laboratory (ORNL), Rocky Flats Plant (RFP), Savannah River Site, and Hanford Reservation. Furthermore, it has benefited from ongoing NRC research, as well as NRC joint efforts with the European Commission (EC) in uncertainty analyses (Harper et al. 1995).

Over the course of the MACCS2 development process, a few coding errors "inherited" from MACCS have been corrected, as is discussed in Section 3. These are primarily (1) the incomplete implementation in all versions of MACCS of the coding for generation of the intermediate-phase results and (2) correction of the MACCS 1.5.11.1 implementation of the dose and dose rate reduction factor (DDREF) used to estimate cancer risks.

MACCS2 has been in limited-distribution beta test by a small set of DOE users since April 1993. Feedback from this core group of long-term MACCS/MACCS2 users has been of great value in the code development process, identifying errors and making suggestions on additional enhancements, which have been implemented from 1993 up to the present.

In addition to the ad hoc verification efforts of the beta-test group, the University of New Mexico (UNM) has completed a formal independent verification study of the code package which has included detailed hand calculations. The results of that effort are to be published in a formal report.

\subsection{Application of MACCS and the MACCS2 Package}

MACCS/MACCS2 allows the user to define values for a wide range of model parameters. In addition, MACCS2 allows the user to select among alternative modeling approaches with regard to emergency evacuation and radionuclide transfer through the food chain. This flexibility makes 
the code useful for diverse purposes. An overview of previous MACCS and MACCS2 applications is given in the following sections to illustrate possible uses of the code.

\subsubsection{PRA Applications}

PRAs are generally used to assess the relative risks posed by various types of operations and facilities, to understand the relative importance of the risk contributors, and to obtain insights on potential safety improvements. There is usually no pass-fail criterion that must be met to allow operations to continue. A major goal of PRAs is to gain insights that can be used to either minimize the chances (probability) of accidents or to minimize the impacts (consequences) of accidents that might occur.

For PRA applications, the radiological consequences are presented in the form of a complementary cumulative distribution function (CCDF). The CCDF produced by the code is based on the random sampling of a year of weather data. All other input parameters, however, are treated as point estimates. CCDFs are presented separately for each accident scenario modeled by MACCS2, and there is no provision for their summation by the code into a single overall CCDF representing a spectrum of potential accidents.

The foremost application of MACCS has been its use for the NUREG-1150 PRA-calculations (NRC 1987; 1991), assessing the risks posed by the operation of five commercial power reactors. The MACCS methodology developed by SNL for this study was subsequently applied, with some variations, to the following:

1. the previously discussed SRS K reactor PRA,

2. the Hanford $\mathrm{N}$ reactor (Camp et al. 1990),

3. the LaSalle commercial power plant (Payne 1992; Brown et al. 1993), and

4. the Grand Gulf commercial power plant (Brown et al. 1995).

\subsubsection{Research Studies}

The majority of the analysts using MACCS and beta-test versions of MACCS2 have been engaged in what can best be described as research studies. These studies have covered such topics as cost-benefit analyses of alternative interdiction criteria, the impact of proposed regulatory changes, and uncertainty/sensitivity studies of code output.

Helton et al. (1995a,b,c,d) investigated the sensitivity of MACCS consequence calculations to variations in values for imprecisely known input variables. MACCS early exposure, chronic exposure, and food pathway results were investigated. Input variables that had the greatest impact on code results were identified. The results of this effort were later applied to a joint $\mathrm{NRC}$ and European Commission effort to assess the uncertainty in the calculations of MACCS and the EC nuclear accident consequence assessment code, COSYMA (Commission of the European Communities 1991). This joint NRC/EC uncertainty study (Harper et al. 1995) elicited from phenomenological experts uncertainty distributions for the MACCS and COSYMA input parameters identified as having the greatest impact on the uncertainty in code output. Sandia, under NRC sponsorship, will use the elicited uncertainty distributions in a future study to assess the overall uncertainty of MACCS2 consequence calculations. 
MACCS was also included in an international collaborative effort to compare predictions obtained from seven consequence codes: ARANO (Finland), CONDOR (UK), COSYMA (EC), LENA (Sweden), MACCS (United States), MECA2 (Spain), and OSCAAR (Japan). (Nuclear Energy Agency of the Organization for Economic Cooperation and Development 1994). The study concluded that the spread in consequence analysis results obtained from the seven consequence codes was small compared with the overall uncertainty in consequence code predictions. The mean results obtained with the different codes typically varied by a factor of 2 although the spread in mean results was greater for a few of the tabulated consequence measures.

MACCS has also been used in a number of studies relating to commercial reactor regulatory issues. Several representative applications are as follows:

1. evaluation of proposed change in NRC guidance relating to population density in the emergency planning zone (EPZ) of commercial power reactors (Young 1994);

2. definition of a large release (Hanson, Davis and Mubayi 1994), per the NRC safety goal (NRC 1986, 1991); and

3. evaluation of the economic consequences of hypothetical severe accidents at commercial power plants (Mubayi, Sailor, and Anandalingam 1995).

\subsubsection{NEPA Studies}

When MACCS is used for National Environmental Policy Act (NEPA) studies such as environmental impact statements (EISs), the results of the calculations are typically used to compare the accident risks posed by various alternatives (see 40 CFR 1500-1508, Regulations for Implementing the Procedural Provisions of the National Environmental Policy Act; DOE 1992a, 1993, 1996e).

In contrast to EISs, for the smaller-scope NEPA analyses usually performed for environmental assessments (EAs), results from a MACCS analysis can be used to determine if a proposed action would result in significant impacts to the environment as defined in the pertinent regulations. For both EISs and EAs, however, NEPA imposes no fixed criterion by which to decide whether a given action is acceptable or unacceptable, allowing discretionary judgments to be made by the agency proposing to undertake the action.

MACCS has been widely used for DOE NEPA studies since the use of version 1.5.11 for the new production reactor (NPR) EIS (DOE 1991a). A number of large-scope EISs utilizing MACCS have recently been issued by the DOE:

1. tritium supply and recycling (DOE 1995b),

2. foreign research reactor fuel (DOE 1996a),

3. stockpile stewardship and management (DOE 1996b),

4 Pantex plant site-wide EIS (SWEIS) (DOE 1996c), and

5. storage and disposition of fissile materials (DOE 1996d). 
In addition, the beta-test MACCS2 was utilized for a large-scope EA pertaining to the Y-12 plant at Oak Ridge National Laboratory (DOE 1994a; Fisher and Lenox 1995). Further, MACCS is currently being utilized for the forthcoming LANL draft SWEIS and the forthcoming final version of the EIS on disposition of surplus highly enriched uranium (DOE 1995c). Finally, both MACCS and the beta-test MACCS2 have been utilized for the forthcoming Rocky Flats Environmental Technology Site (RFETS) draft SWEIS.

\subsubsection{Authorization Basis Studies}

In contrast to NEPA studies, in the authorization basis analyses performed for SARs, the analyst is interested in conservatively calculated, bounding dose estimates for well-defined DBA and beyond-DBA accident scenarios. The results of this analysis are used to determine if the safety basis of the facility is adequate for operation (DOE 1989, 1992b).

When MACCS2 is used for authorization basis studies, it is very important to carefully review the code's phenomenological models and input parameter values to ensure that they conform to applicable guidance and are appropriate for the accident scenario being modeled. The identification of deficiencies in these areas could bring into question the safety basis of a facility. If errors are later found in authorization basis calculations, an unreviewed safety question (USQ) could be raised (DOE 1991b), and continued operation of the facility would then require a demonstration that the facility's safety basis was adequate.

MACCS and MACCS2 have received limited use for DOE authorization basis studies. Two of the known applications are as follows:

1. MACCS 1.5.11.1 was used for the Rocky Flats Building 707 SAR (Stone and Webster 1991).

2. MACCS 1.5.11.1 was used for the Nevada Test Site (NTS) Device Assembly Facility (DAF) SAR (Raytheon Systems Services 1995).

Also notable is the use of beta-test versions of MACCS2 for detailed analyses focused on Rocky Flats (Peterson 1993a,b; 1994a,b; 1995). These analyses were performed for NEPA (such as EAs) as well as authorization basis studies. In addition, beta-test MACCS2 was used for studies relating to the SRS Defense Waste Processing Facility (DWPF) SAR (East 1996). 
THIS PAGE INTENTIONALLY LEFT BLANK 


\subsection{Basic Code Features Preserved in MACCS2}

This section presents an overview of the MACCS features that have been preserved unchanged in MACCS2. The present version of the code will be referred to as MACCS2, although the features discussed in this chapter apply to both MACCS and MACCS2.

\subsection{Overview of Code Structure}

The structure of MACCS2 is based on that of CRAC2 (Ritchie et al. 1984), as descended from the Reactor Safety Study (NRC 1975). Additional information on the technical background of reactor-based assessment codes can be found in the PRA Procedures Guide (American Nuclear Society and Institute of Electrical and Electronic Engineers 1983).

MACCS2 is used to estimate the radiological doses, health effects, and economic consequences that could result from postulated accidental releases of radioactive materials to the atmosphere. The specification of the release characteristics, designated a "source term," can consist of up to four Gaussian plumes (Systems Applications 1982), with these often referred to simply as "plumes."

The radioactive materials released are modeled as being dispersed in the atmosphere while being transported by the prevailing wind. During transport, whether or not there is precipitation, particulate material can be modeled as being deposited on the ground. If contamination levels exceed a user-specified criterion, mitigative actions can be triggered to limit radiation exposures. If mitigative actions are triggered, the economic costs of these actions are calculated and can be reported.

There are two aspects of the code's structure that are basic to understanding its calculations: (1) the calculations are divided into modules and phases and (2) the region surrounding the facility is divided into a polar-coordinate grid. These concepts are described in the following subsections.

\subsubsection{Division of Calculations into Modules and Phases}

MACCS2 is divided into three primary modules: ATMOS, EARLY, and CHRONC. The input data they require are described in Sections 5, 6, and 7, respectively. ${ }^{3}$

There is also another fundamental division in the code's calculations. This division is based on the sequence of societal responses that would follow the occurrence of an accident. These phases are defined by the Environmental Protection Agency (EPA) (1992) in its Protective Action

\footnotetext{
${ }^{3}$ As stated in Section 1.4, the original design of MACCS incorporated five separate FORTRAN programs executed in sequence. These were named ATMOS, EARLY, CHRONC, MERGER, and SUMMER. Functions of the former MERGER and SUMMER are now performed by the OUTPUT module. Results generated by ATMOS, EARLY, and CHRONC are written to binary files, which are then processed by OUTPUT in order to generate CCDFs.
} 
Guides, and referred to as the emergency, intermediate, and long-term phases. Because these concepts are basic to an understanding of the code, the relationships among the code's three modules and the three phases of exposure are summarized below.

\subsubsection{The ATMOS Module: Atmospheric Transport and Deposition}

ATMOS performs all of the calculations pertaining to atmospheric transport, dispersion, and deposition, as well as the radioactive decay that occurs prior to release and while the material is in the atmosphere. The results of the calculations are stored for use by EARLY and CHRONC. The downwind transport of up to four plumes can be modeled. A number of parameters are stored. In addition to the air and ground concentrations, ATMOS stores information on wind direction, arrival and departure times, and plume dimensions.

\subsubsection{The EARLY Module: Emergency-Phase Calculations}

EARLY performs all of the calculations pertaining to the emergency phase. The emergency phase begins, at each successive downwind distance point, when the first plume of the release arrives. The duration of the emergency phase is specified by the user, and it can range between 1 and 7 days. The exposure pathways considered during this period are cloudshine, groundshine, and resuspension inhalation. Mitigative actions that can be specified for the emergency phase include evacuation, sheltering, and dose-dependent relocation.

\subsubsection{The CHRONC Module: Intermediate- and Long-Term-Phase Calculations}

CHRONC performs all of the calculations pertaining to both the intermediate and long-term phases, as is summarized below. The mitigative action models that can be utilized for these periods are not fully described in the MACCS Model Description. For that reason, and because MACCS2 incorporates some changes to the corresponding models of MACCS, an expanded description of the CHRONC mitigative action models is presented in Section 7.1. This description supersedes the MACCS Model Description.

\subsubsection{Intermediate Phase}

The intermediate phase begins, at each successive downwind distance point, upon the conclusion of the emergency phase. The duration of the intermediate phase is specified by the user, and it can range between 0 and 1 year. The exposure pathways considered during this period are groundshine and resuspension inhalation. Potential doses from food and water ingestion during this period are not considered.

These models are implemented on the assumption that the radioactive plume has passed and the only exposure source is from ground-deposited material. It is for this reason that MACCS2 requires that the total duration of a radioactive release be limited to no more than 4 days. The only mitigative action that can be specified for the intermediate phase is dose-dependent relocation. If a user-specified dose criterion is exceeded, resident individuals are assumed to be relocated for the duration of the intermediate phase. 


\subsubsection{Long-Term Phase}

The long-term phase begins, at each successive downwind distance point, upon the conclusion of the intermediate phase. The exposure pathways considered during this period are groundshine, resuspension inhalation, and food and water ingestion.

The exposure pathways considered are those resulting from ground-deposited material. A number of protective measures can be modeled in the long-term phase in order to reduce doses to user-specified levels: decontamination, temporary interdiction, and condemnation. These mitigative actions are modeled separately for two land uses: residential and agricultural.

\subsubsection{Distinction Between Acute and Lifetime Doses}

Two types of doses are calculated by the code. They are referred to as "acute" and "lifetime doses" in this report. A brief introduction to the meaning of these terms is given below.

\subsubsection{Acute Doses}

Acute doses are calculated for the sole purpose of estimating the "deterministic" health effects that can result from high doses delivered at high dose rates. Such conditions may occur in the immediate vicinity of a nuclear power plant following hypothetical severe accidents where containment failure has been assumed to occur. Some examples of the health effects that are based on acute doses are as follows: early fatality, prodromal vomiting, and hypothyroidism. With only a few exceptions, typically focused on risks to on-site workers at DOE facilities, the need for calculation of acute doses has usually been limited to commercial power plant analyses.

For most DOE applications, where off-site doses fall well below the thresholds for inducing deterministic health effects, there is no need to calculate these doses, and the MACCS2 calculations can thereby be simplified to run at greater speed, benefiting also from a larger database of radionuclides that can be considered.

\subsubsection{Lifetime Doses}

Lifetime doses are the conventional measure of detriment used for radiological protection. These are the 50-year dose commitments to either specific tissues (e.g., red marrow and lungs), or a weighted sum of tissue doses defined by the International Commission on Radiological Protection (ICRP) (1991) and referred to as "effective dose." Lifetime doses may be used to calculate the stochastic health effect risk resulting from exposure to radiation. MACCS2 uses the calculated lifetime dose in cancer risk calculations.

\subsubsection{Polar-Coordinate Grid}

All of the calculations of MACCS2 are stored on the basis of a polar-coordinate spatial grid with a treatment that differs somewhat between (1) calculations of the emergency phase and (2) intermediate and long-term phases, as described later. The region potentially affected by a release is represented with an $(r, \theta)$ grid system centered on the location of the release. The 
radius, $r$, represents downwind distance. The angle, $\theta$, is the angular offset from north, going clockwise.

The user specifies the number of radial divisions as well as their endpoint distances. Up to 35 of these divisions may be defined, extending out to a maximum distance of $9999 \mathrm{~km}$. The angular divisions used to define the spatial grid are fixed in the code (they are not user defined) and correspond to the 16 points of the compass, each being 22.5 degrees wide. The 16 points of the compass are used in the United States to express wind direction. The compass sectors are referred to as the "coarse grid." Figure 2-1 provides an example of a MACCS2 spatial grid and the numbering system associated with the 16 compass directions.

Since the emergency-phase calculations utilize dose-response models for early fatalities and early injuries that can be highly nonlinear (again, as specified by the user), these calculations are performed on a finer grid basis than the calculations of the intermediate and long-term phases. For this reason, the calculations of the emergency phase are performed with the 16 compass sectors divided into 3,5, or 7 (as specified by the user) equal angular subdivisions. The subdivided compass sectors are referred to as the "fine grid."

The compass sectors are not subdivided into fine subdivisions for the intermediate and longterm phases because these calculations do not include estimation of the often highly nonlinear early fatality and early injury health effects, being limited to cancer and genetic effects. In contrast to the emergency phase, the calculations for these phases are performed using doses averaged over the full 22.5-degree compass sectors of the coarse grid.

\subsection{Sequence of Calculations}

MACCS2 calculations are performed in three phases: (1) input processing and validation, (2) phenomenological modeling, and (3) output processing. These are described in Sections 2.2.1 through 2.2.3.

\subsubsection{Input Processing and Validation}

The calculations begin with the processing of the primary user-supplied input. These input data are provided in three text files, named for the modules they supply: the ATMOS input file, EARLY input file, and CHRONC input file. Following the terminology used in the MACCS User's Guide, these three files are referred to generically as user input files. The variables defined in these files are described in Sections 5 through 7. The manner in which this input is processed is described in remainder of this section.

Error checking of the ATMOS, EARLY, and CHRONC input files is performed by the code so that errors are located and diagnosed before the phenomenological modeling phase is initiated. If any errors are detected, the program will sometimes validate as much of the subsequent input as possible to facilitate the debugging process. However, in many instances, the detection of an input error will cause an immediate termination of the program's execution. In all cases, when an error is detected in the code's input, the execution of the program will be terminated before the modeling calculations are performed. 


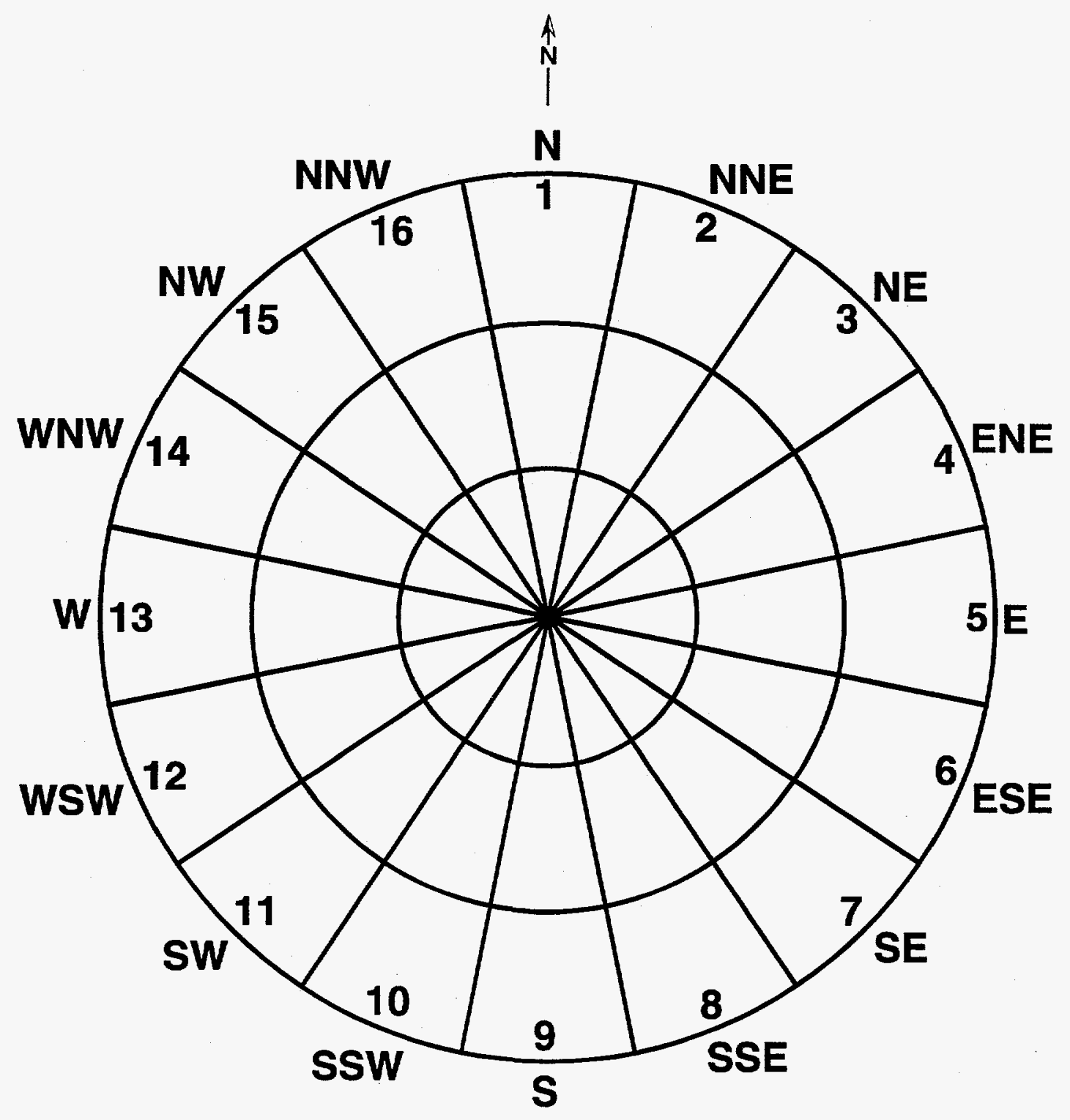

Figure 2-1. A MACCS2 polar-coordinate grid with 3 radial divisions. The numbers on the grid refer to the $\mathbf{1 6}$ compass directions. 


\subsubsection{Data Types Processed by the Code}

Data required by MACCS2 can be of four different types: logical, character, integer, or real. Logical values are represented as either .TRUE. or .FALSE. as defined in the American National Standards Institute (1978) FORTRAN 77 standard. Character values can be any ASCII string delimited by apostrophes ('). If the string has no embedded blanks, the apostrophes are optional provided that the string is not interpreted to be a data item of another type. Integer values can be preceded with an optional plus or minus sign but they cannot have a decimal point. Real values can be written either as an optionally signed number with a decimal point or else in exponential notation as defined in the ANSI standard.

The code first evaluates an item to determine if it is of type logical. If the item is not a logical data type, it is evaluated to determine if it is of type integer. If the item is neither a logical nor integer type, the item is evaluated to determine if it is a real variable. Items that are neither logical, integer, or real are considered to be of type character.

\subsubsection{Free-Format Input Processor}

The ATMOS, EARLY, and CHRONC input files are processed sequentially by the INPRE free-format input processor, which was developed specifically for MACCS. The INPRE processor allows comments to be freely interspersed with the data. The input file can thus be made essentially self-documenting. The INPRE free-field input processor reads each line of the text-based input file as a data record. Each line of the file is allowed a length of up to 100 characters.

The individual data records consist of the content of the text line up to a carriage return or the 100 th column. Each line of the file is either a comment record or a data record. Comment records, denoted by an asterisk (*) in column 1, are not processed by the INPRE processor. Data records are required to begin in column 1 with an 11-character record identifier consisting of 8 or 9 alpha-numeric identification characters that are usually followed by 2 or 3 numeric characters that are used for sequencing. An example of a record identifier is RIATNAM1001. The end of the input data file must be delimited by a period (.) in column 1 .

The input processor begins by sorting all the data records in the file according to the collating sequence of their record identifiers. If more than one data record has the same record identifier, the last data record encountered is used and all earlier data records with the same identifier are ignored. Data records may appear in any order. The ordering is important only for records sharing the same identifier.

The variables for which values are provided in the user input files consist of either a single value (scalar) or a set of values (array) that are all of the same type. The record identifier used for scalar values is always of the same form. The first two characters are a mnemonic for the data group to which the identifier belongs. For example, the mnemonic for the Run Identification data is RI. The next six characters of the record identifier are the name of the FORTRAN variable within the program that is used to store the value. The record identifier is always 001 . Only the first item found on the data record for a scalar quantity will be processed. Anything to 
the right of the data item will be ignored by the input processor. This allows the use of descriptive comments alongside the data item, as is illustrated by the sample problems.

Arrays are found in the user input files in two different forms. The first method is similar to the approach used for scalars. A starting record identifier is constructed the same way as above: a two-letter mnemonic, followed by the six-letter variable name, followed by 001 . More than one value can appear on the data record separated by blanks or a comma. Multiple blanks may be inserted between values; multiple commas between values are not allowed. You may place as many values on the card as will fit into the 100 columns. Successive cards with ascending sequence numbers are processed until all the required data items have been supplied. The record identifiers of the succeeding cards differ from the starting record identifier only in their last three digits.

The second method used for arrays is more structured. Several arrays of the same length whose values are related appear as columns across the page. The arrays can be of different type but they all share the same record identifier. The name of a program variable is not used to construct the record identifier since more than one variable receives its value from this data record. Instead of array values being read from left to right in rows, they are read from top to bottom in columns.

\subsubsection{Error Checking of Input Data}

The code checks for three different types of errors in the user input files:

1. mismatch between data type of the variable and data type of the input data,

2. data value outside the numerical limits required for the variable, and

3. specification of a data value inconsistent with another specified value.

The input processor will not convert data from one type to another. For example, if an integer value is to be supplied, as specified in later sections of this report, then an integer value must be supplied by the user. If a decimal point appears in what should be an integer value, an error flag will be set, and execution will be terminated upon completion of the input processing phase. Likewise, the absence of a decimal point in what should be a real value will be interpreted as an input error and cause the error flag to be set.

The code determines the validity of the numeric input parameters (both integer and real) by checking whether they fall within a specified range. The allowable range of each datum is listed in this report. Limits are also specified for lengths of character strings and these limits are also listed in this report. If a value is encountered that falls outside the required range, the error flag will be set and a diagnostic message issued to the output file. This message will list the minimum and maximum allowable values (or string lengths) for the item in order to facilitate the debugging process.

In addition to checking to see if a particular datum is of the proper type and within the allowable range, many user-supplied variables are further checked to determine if they are consistent with other data specifications. In all instances where these additional tests are performed, the applicable requirements are described in Sections 5 through 7 in close proximity to the 
description of the variable and the specification of its allowable range. If these constraints are violated, a diagnostic error message will be generated and further execution terminated.

\subsubsection{Specification of Multiple Accident Scenarios}

The code allows the consideration of multiple source terms and multiple emergency-response strategies in a single run of the code. Multiple source terms for ATMOS and multiple emergency-response strategies for EARLY can be specified by the addition of change records positioned at the end of the ATMOS and EARLY input files. Change records specify new values for previously defined source-term or emergency-response scenario variables. When these are encountered, the code's calculations are rerun based on the new values for variables defined in the set of change records and new output is generated for each specified source term and emergency-response scenario.

Sets of change records are input after a complete set of ATMOS or EARLY input values have been specified [the end of the base case is indicated by the period (.) in column 1]. The end of each set of change records is also specified by a period in column 1. ATMOS allows up to 59 sets of source term change records and EARLY allows up to two sets of emergency-response change records. A text field identifying the scenario must be defined for each source term and emergency-response scenario.

The change records can only be used to replace previously defined data items in the base-case input. The change-record processor simply replaces a previously defined data record with a new data record having the same record identifier. If a set of change records contains data records with identifiers that have not been defined in the base-case input, it is possible that the code will terminate execution abnormally or that spurious results will be generated. Also, if the user tries to redefine previously defined input variables that are not part of the source term or emergencyresponse data blocks, those revised data records will not be processed by the program. The source term data block variables are discussed in Section 5.11, and the emergency-response data block variables are discussed in Section 6.6.

\subsubsection{Phenomenological Modeling}

The second phase of the code execution is the phenomenological modeling phase. The sequence in which the phenomena are evaluated closely follows the temporal order of events that would occur in the real world in the event of a facility accident. The phenomenological models incorporated in the code are of a simple character, and mathematics beyond undergraduate-level calculus is not utilized. The incorporated models utilize empirical data, often have analytical solutions, and are computationally straightforward.

An overview of the sequence of the phenomenological modeling performed in each of the three code modules (ATMOS, EARLY, and CHRONC) is provided in Tables 2-1 through 2-3. For each of the major modeling areas, these tables indicate where the details of the modeling approach can be found. 
Table 2-1. Phenomenological Models of ATMOS

\begin{tabular}{||l|l||}
\hline \multicolumn{1}{|c|}{ Phenomenology } & \multicolumn{1}{c|}{ Location of Model Description } \\
\hline Source Term Specification & MACCS Model Description, Section 2.2 \\
\hline Weather Data & MACCS Model Description, Section 2.3 \\
\hline Risk-Dominant Plume & MACCS Model Description, Section 2.4 \\
\hline Initial Plume Dimensions & $\begin{array}{l}\text { Section } 5.10 \text { describes a change to MACCS Model } \\
\text { Description, Section 2.5 }\end{array}$ \\
\hline Representative Weather Point & MACCS Model Description, Section 2.6 \\
\hline Downwind Transport & MACCS Model Description, Section 2.7 \\
\hline Plume Rise & MACCS Model Description, Section 2.8 \\
\hline Dispersion-Gaussian Plume Model & MACCS Model Description, Section 2.9 \\
\hline Overview of Plume Depletion & MACCS Model Description, Section 2.10 \\
\hline Depletion by Radioactive Decay & $\begin{array}{l}\text { Section 3.2.1 describes a change to MACCS Model } \\
\text { Description, Section 2.10.1 }\end{array}$ \\
\hline Depletion by Dry Deposition & MACCS Model Description, Section 2.10 .2 \\
\hline Depletion by Wet Deposition & MACCS Model Description, Section 2.10 .3 \\
\hline Centerline Air and Ground Concentrations & MACCS Model Description, Section 2.11 \\
\hline
\end{tabular}

Of the three phenomenological modules in MACCS2, ATMOS is the only one that must always be exercised. The execution of the other modules (EARLY and CHRONC) may be inactivated by the specification of a control variable in the previous module's user input file. For example, if the last module to be exercised is EARLY, a control variable in the EARLY input file can be used to trigger the skipping of CHRONC. Similarly, if both EARLY and CHRONC are to be skipped, this can be achieved by setting a control variable in the ATMOS input file. If any module is inactivated in this way, there is no need to prepare its corresponding user input file.

\subsubsection{Output Processing}

The generation of results is controlled by user specifications in the ATMOS, EARLY, and CHRONC input files. There is no provision for specifying each accident's expected rate of occurrence (accident frequency). All consequence measures calculated by MACCS2 are conditional on the occurrence of a particular accident.

All of the results generated by MACCS2 are calculated in a manner that takes full account of any mitigative actions triggered by user input. These are all produced in CCDF form, even in cases when the code is utilizing only a single weather trial. A one-line summary of the CCDF for each of the requested results is written to the output file. For any subset of the results, the user can, in addition, instruct the code to print the entire CCDF table to the output file. 
Table 2-2. Phenomenological Models of EARLY

\begin{tabular}{|c|c|}
\hline Phenomenology & Location of Model Description \\
\hline Overview of Exposure Pathways & MACCS Model Description, Section 3.1 \\
\hline Off-Centerline Correction Factor & MACCS Model Description, Section 3.1.1 \\
\hline Cloudshine & MACCS Model Description, Section 3.2.2 \\
\hline Groundshine & MACCS Model Description, Section 3.1.3 \\
\hline Direct Inhalation & MACCS Model Description, Section 3.1.4 \\
\hline Resuspension Inhalation & MACCS Model Description, Section 3.1.5 \\
\hline Emergency-Phase Relocation Costs & MACCS Model Description, Section 4.1 \\
\hline Evacuation & $\begin{array}{l}\text { Section } 6.6 \text { describes revisions to MACCS Model } \\
\text { Description, Section 5.1.1 }\end{array}$ \\
\hline Sheltering & $\begin{array}{l}\text { Section } 6.6 \text { describes revisions to MACCS Model } \\
\text { Description, Section 5.1.2 }\end{array}$ \\
\hline Dose-Dependent Relocation & MACCS Model Description, Section 5.1.3 \\
\hline $\begin{array}{l}\text { Acute Health Effects-Early Fatality and Early Injury } \\
\text { Models }\end{array}$ & MACCS Model Description, Section 6.1 \\
\hline Cancer Health Effect Models & $\begin{array}{l}\text { Chanin et al. (1993) describes a revision to MACCS } \\
\text { Model Description, Section } 6.2\end{array}$ \\
\hline
\end{tabular}

Table 2-3. Phenomenological Models of CHRONC

\begin{tabular}{|l|l||}
\hline \multicolumn{1}{|c|}{ Phenomenology } & \multicolumn{1}{c|}{ Location of Model Description } \\
\hline Overview of Exposure Pathways & MACCS Model Description, Section 3.2 \\
\hline Off-Centerline Correction Factor & MACCS Model Description, Section 3.2 .1 \\
\hline Groundshine & MACCS Model Description, Section 3.2.2 \\
\hline Resuspension Inhalation & MACCS Model Description, Section 3.2.3 \\
\hline COMIDA2 Model for Food Ingestion & $\begin{array}{l}\text { Section 7.10 of this volume, and Chapter } 3 \text { of } \\
\text { Volume 2 of this report }\end{array}$ \\
\hline MACCS Model for Food Ingestion & $\begin{array}{l}\text { Section 7.11 of this volume, and MACCS Model } \\
\text { Description, Section 3.2.4 }\end{array}$ \\
\hline Water Ingestion & MACCS Model Description, Section 3.2.5 \\
\hline Mitigative Action Models & MACCS Model Description, Section 4.2 \\
\hline Economic Costs from Intermediate Phase & MACCS Model Description, Section 5.2 \\
\hline Economic Costs from Long-Term Phase & $\begin{array}{l}\text { Section 7.1 describes revisions to the MACCS Model } \\
\text { Description, Section 5.3 }\end{array}$ \\
\hline \hline
\end{tabular}


When more than one source term is specified, the results for each are presented on the output listing in the order of their appearance in the ATMOS input file. The OUTPUT module will print a description of all the results for each source term, as encountered in turn, before going on to the next source.

The various types of results that can be requested are referred to as Results $0-13$ and $A-B$. None of these outputs is generated by default. For each result requested, the user can define the region of interest (defined by its inner and outer boundaries), the health effect or organ of interest (as appropriate), as well as other parameters (as pertinent). The results available from each module are listed in Table 2-4.

When any of these results are requested, several numerical measures (or metrics) are written to the output file, representing a one-line summary of the CCDF. In addition to these one-line summaries, the code can also report the entire CCDF for a user-specified subset of the requested results. The appearance of the one-line summary is illustrated in Figures 2-2 through 2-4, while the appearance of the CCDF table is illustrated in Figure 2-5. Additional information on the interpretation of these outputs is given in the remainder of this section, and further details may be found in Section 6.10.

\subsubsection{Presentation of Multiple Source Terms and Multiple Cohorts}

When multiple source terms are defined, the treatment is quite straightforward, with the results from each presented successively in the output file in the order in which they were defined in the ATMOS input file. In that file, the user is required to provide a unique text identifier for each source term, and that identifier is echoed to the output file so that results from multiple source terms can be readily distinguished.

The treatment of multiple emergency-response scenarios differs somewhat from the treatment of multiple source terms, and is somewhat more complex. Three important points are crucial. First, the allowance for multiple emergency-response scenarios extends only to the EARLY module, and there is no capability offered for the variation of assumptions used by the CHRONC module.

Second, the code treats the results from each emergency-response scenario as a separate cohort for which results are reported separately in the output file. If CHRONC is exercised, then its results are also reported as a separate cohort, following the EARLY results.

Third, if the user has requested the generation of results for multiple cohorts (either by specifying multiple emergency-response scenarios, or by exercising the CHRONC module), the very first set of results presented is labeled the "overall results," and represents the combined results from the various cohorts that have been defined.

The most straightforward approach to describing this aspect of the code's output is to utilize output taken from one of the sample problems as an example. Sample problem A, based on the input data used for the NUREG-1150 calculations, will be used for that purpose. 
Table 2-4. Results Available from ATMOS, EARLY, and CHRONC

\begin{tabular}{|c|c|c|}
\hline \multicolumn{2}{|r|}{ Result Name } & \multirow{2}{*}{$\begin{array}{l}\text { Notes } \\
\text { Requested in ATMOS input file; see Section 5.18. Results only } \\
\text { available for the first source term of a multiple-source-term run. } \\
\text { They are provided as a block of eight results. User specifies the } \\
\text { radial distance index and the plume(s) of interest. }\end{array}$} \\
\hline Type 0: & $\begin{array}{l}\text { Atmospheric Results at Specified } \\
\text { Downwind Distances }\end{array}$ & \\
\hline Type 1: & Cases of Specified Health Effect & $\begin{array}{l}\text { Requested in EARLY input file; see Section } 6.11 \text {. If CHRONC } \\
\text { is exercised, it automatically generates corresponding outputs for } \\
\text { cancer health effects. }\end{array}$ \\
\hline Type 2: & Early Fatality Radius & $\begin{array}{l}\text { Requested in EARLY input file; see Section } 6.12 \text {. Only } \\
\text { generated by EARLY. }\end{array}$ \\
\hline Type 3: & $\begin{array}{l}\text { Population Exceeding Dose } \\
\text { Threshold }\end{array}$ & $\begin{array}{l}\text { Requested in EARLY input file; see Section } 6.13 \text {. Only } \\
\text { generated by EARLY. }\end{array}$ \\
\hline Type 4: & $\begin{array}{l}\text { Average Individual Risk of Health } \\
\text { Effects }\end{array}$ & $\begin{array}{l}\text { Requested in EARLY input file; see Section } 6.14 \text {. If CHRONC } \\
\text { is exercised, it automatically generates corresponding outputs for } \\
\text { cancer health effects. }\end{array}$ \\
\hline Type 5: & $\begin{array}{l}\text { Total Collective Dose from } \\
\text { Material Deposited Within Region }\end{array}$ & $\begin{array}{l}\text { Requested in EARLY input file; see Section } 6.15 \text {. If CHRONC } \\
\text { is exercised, it automatically generates corresponding outputs for } \\
\text { total collective dose from all pathways combined, including } \\
\text { ingestion. }\end{array}$ \\
\hline Type 6: & Centerline Dose versus Distance & $\begin{array}{l}\text { Requested in EARLY input file; see Section } 6.16 . \text { Result only } \\
\text { available when the straight-line plume option (IPLUME=1) is } \\
\text { being utilized. }\end{array}$ \\
\hline Type 7: & Centerline Risk versus Distance & $\begin{array}{l}\text { Requested in EARLY input file; see Section } 6.17 \text {. Result only } \\
\text { available when the straight-line plume option (IPLUME=1) is } \\
\text { being utilized. }\end{array}$ \\
\hline Type 8: & $\begin{array}{l}\text { Population-Weighted Safety Goal } \\
\text { Risk }\end{array}$ & $\begin{array}{l}\text { Requested in EARLY input file; see Section } 6.18 \text {. If CHRONC } \\
\text { is exercised, it automatically generates corresponding Type } 8 \\
\text { outputs for groundshine and resuspension. Ingestion is not } \\
\text { included in the Type } 8 \text { results. }\end{array}$ \\
\hline Type A: & $\begin{array}{l}\text { Maximum Observed Dose at a } \\
\text { Specified Distance Ring }(r) \\
\text { (Direction-Independent Dose) }\end{array}$ & $\begin{array}{l}\text { Requested in EARLY input file; see Section } 6.19 \text {. If CHRONC } \\
\text { is exercised, it automatically generates corresponding outputs for } \\
\text { groundshine and resuspension. Ingestion is not included in the } \\
\text { Type A results. }\end{array}$ \\
\hline Type B: & $\begin{array}{l}\text { Maximum Observed Dose at a } \\
\text { Specified }(r, \theta) \text { Location } \\
\text { (Direction-Dependent Dose) }\end{array}$ & $\begin{array}{l}\text { Requested in EARLY input file; see Section } 6.20 \text {. If CHRONC } \\
\text { is exercised, it automatically generates corresponding outputs for } \\
\text { groundshine and resuspension. Ingestion is not included in the } \\
\text { Type B results. }\end{array}$ \\
\hline Type 9: & $\begin{array}{l}\text { Breakdown of CHRONC } \\
\text { Population Dose by Pathway }\end{array}$ & $\begin{array}{l}\text { Requested in CHRONC input file; see Section } 7.14 \text {. The } \\
\text { reported doses are the result of contamination deposited within } \\
\text { the stated region; for the ingestion paths and decontamination } \\
\text { workers, doses may be incurred by individuals residing } \\
\text { elsewhere. }\end{array}$ \\
\hline
\end{tabular}


Table 2-4. Results Available from ATMOS, EARLY, and CHRONC (continued)

\begin{tabular}{|c|c|}
\hline Result Name & Notes \\
\hline $\begin{array}{l}\text { Type 10: Economic Costs of Mitigative } \\
\text { Actions }\end{array}$ & $\begin{array}{l}\text { Requested in CHRONC input file; see Section } 7.15 \text {. The } \\
\text { reported costs are the result of contamination deposited within } \\
\text { the stated region. }\end{array}$ \\
\hline $\begin{array}{l}\text { Type 11: Maximum Distance for the } \\
\text { Various Mitigative Actions }\end{array}$ & $\begin{array}{l}\text { Requested in CHRONC input file; see Section } 7.16 \text {. For each } \\
\text { weather simulation, the code reports the furthest distance at } \\
\text { which each of these actions is modeled as taking place. }\end{array}$ \\
\hline Type 12: Impacted Area/Population Results & $\begin{array}{l}\text { Requested in CHRONC input file; see Section } 7.17 \text {. For } \\
\text { mitigative actions affecting population, the number of affected } \\
\text { individuals within the stated region is reported. For agricultural } \\
\text { actions, the affected farmland area (hectares) is reported. }\end{array}$ \\
\hline $\begin{array}{l}\text { Type 13: Maximum Individual Dose from } \\
\text { COMIDA2 Food-Chain Model }\end{array}$ & $\begin{array}{l}\text { Requested in CHRONC input file; see Section } 7.18 \text {. Unavailable } \\
\text { when the MACCS food-chain model is utilized. Calculated using } \\
\text { the same individual food consumption rates used for triggering } \\
\text { mitigative actions for farming. }\end{array}$ \\
\hline
\end{tabular}

\subsubsection{Interpretation of the One-Line Summaries}

While NRC emergency planning requirements call for a high level of preparedness in the 10-mile emergency planning zone, it is possible that some population subgroups might remain behind, requiring steps by local authorities to locate them and provide any necessary assistance in leaving contaminated areas. In the 1987 public draft of NUREG-1150, it was assumed that $95 \%$ of the EPZ populace would evacuate upon receiving notification to do so, and that $5 \%$ of the EPZ populace would remain behind, with the remaining populace of contaminated areas subject to relocation after either 12 or $24 \mathrm{hr}$ of exposure, depending on the doses incurred.

Sample problem $A$ is based on the assumption that for the population within 10 miles of the plant there would be two emergency-response scenarios: evacuation at a specified time after the initiation of the accident and dose-dependent relocation. It thus incorporates two cohorts for the EARLY calculations. The CHRONC calculations represent a third and additional cohort. Sample problem A reports a large number of results; however, for simplicity, only the 50-mile cancer fatality results will be shown in the illustrative figures.

Figure 2-2 summarizes the cohorts included in the overall results. The two emergency-response scenarios (cohorts 1 and 2) are combined utilizing "fraction-of-the-people" weighting fractions of 


\section{Figure 2-2. Overall results.}

DATE AND TIME OF RUN = MACCS2 06/25/96 22:17:09 version 1.12, last modified 6/25/96: final test "ATMOS" DESCRIPTION = IN1A.INP, Sample Problem A--Using Table-Lookup Sigmas, ATMOS input

"EARLY" DESCRIPTION = IN2A.INP, Sample Problem A of NUREG/CR-4691, Vo1. 1, EARLY input

"CHRONC" DESCRIPTION = IN3A_O.INP, Sample Problem A, "Old" NUREG-1150 Food Model

SOURCE TERM 1 OF 2 :

SECOND DRAFT 1150, WORST CASE SOURCE TERM FOR EARLY FATALITIES

OVERALL RESULTS OBTAINED BY COMBINING 2 EMERGENCY RESPONSE COHORTS FROM "EARLY" WITH THE WEIGHTING FRACTIONS BELOW APPLIED TO THEM:

COHORT 1 = EVACUATION WITHIN 10 MILES, RELOCATION MODELS APPLY ELSEWHERE

FRACTION OF THE PEOPLE

$$
0.950
$$

COHORT 2 = NO EVACUATION, RELOCATION MODELS APPLY EVERYWHERE

AND THEN MERGING THE 2 RESULTS ABOVE WITH THE SINGLE SET OF RESULTS FROM "CHRONC" DESCRIBED BELOW:

COHORT 3 = IN3A_O.INP, Sample Problem A, "Old" NUREG-1150 Food Model

RESULTS WHICH ARE PRODUCED ONLY BY "EARLY" OR ONLY BY "CHRONC" ARE PRESENTED IN LATER SECTIONS.

PAGE 1

PROB

NONZERO

MEAN

Q0TH QUANTILES

$95 \mathrm{TH}$

$99 \mathrm{TH} \quad 99.5 \mathrm{TH}$

PEAK
CONS

PEAK PEAK

HEALTH EFFECTS CASES

CAN FAT/TOTAL

$0-80.5 \mathrm{~km} \quad 1.0000 \quad 1.72 \mathrm{E}+03 \quad 9.62 \mathrm{E}+02$

$4.07 \mathrm{E}+03$

$5.93 E+03$

$9.57 \mathrm{E}+03$

$1.11 \mathrm{E}+04$

$2.06 \mathrm{E}+04$

$2.85 E-04$ 


\section{Figure 2-3. EARLY results for cohort 1: evacuation.}

DATE AND TIME OF RUN = MACCS2 06/25/96 22:17:09 version 1.12, last modified 6/25/96: final test

"ATMOS" DESCRIPTION = IN1A. INP, Sample Problem A--Using Table-Lookup Sigmas, ATMOS input

"EARLY" DESCRIPTION = IN2A. INR, Sample Problem A of NUREG/CR-4691, Vo1. 1, EARLY input

SOURCE TERM 1 OF 2 :

SECOND DRAFT 1150, WORST CASE SOURCE TERM FOR EARLY FATALITIES

RESULTS FOR A SINGLE EMERGENCY RESPONSE COHORT WITHOUT ANY WEIGHTING FRACTIONS BEING APPLIED

COHORT 1 = EVACUATION WITHIN 10 MILES, RELOCATION MODELS APPLY ELSEWHERE

\begin{tabular}{|c|c|c|c|c|c|c|c|c|c|c|c|c|}
\hline $06 / 25 / 96 \quad 22: 17: 09$ & PAGE & 3 & PROB & & & QUANT & ILES & & & PEAK & PEAK & PEAK \\
\hline & & & NONZERO & MEAN & $50 \mathrm{TH}$ & $90 \mathrm{TH}$ & $95 \mathrm{TH}$ & $99 \mathrm{TH}$ & $99.5 \mathrm{TH}$ & CONS & PROB & TRIAL \\
\hline AN FAT/TOTAL & & $0-80.5 \mathrm{~km}$ & 1.0000 & $9.26 \mathrm{E}+02$ & $4.27 \mathrm{E}+02$ & $2.28 \mathrm{E}+03$ & $3.13 E+03$ & $7.11 \mathrm{E}+03$ & $8.79 E+03$ & $1.90 \mathrm{E}+04$ & $2.85 \mathrm{E}-0$ & 123 \\
\hline
\end{tabular}

Figure 2-4. EARLY results for cohort 2: no evacuation.

DATE AND TIME OF RUN = MACCS2 06/25/96 22:17:09 version 1.12, last modified 6/25/96: final test

"ATMOS" DESCRIPTION = IN1A.INP, Sample Problem A--Using Table-Lookup Sigmas, ATMOS input

"EARLY" DESCRIPTION = IN2A. INP, Sample Problem A of NUREG/CR-4691, Vol. 1, EARLY input

SOURCE TERM 1 OF 2

SECOND DRAFT 1150, WORST CASE SOURCE TERM FOR EARLY FATALITIES

RESULTS FOR A SINGLE EMERGENCY RESPONSE COHORT WITHOUT ANY WEIGHTING FRACTIONS BEING APPLIED

COHORT 2 = NO EVACUATION, RELOCATION MODELS APPLY EVERYWHERE

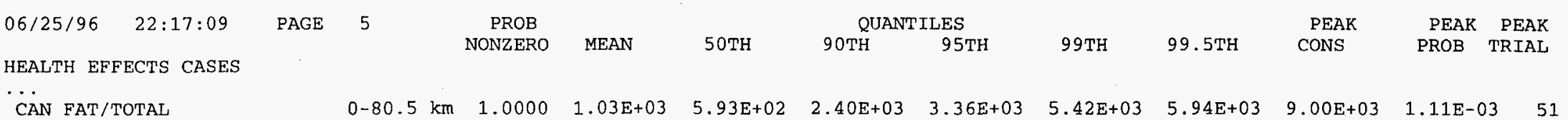


Figure 2-5. CHRONC results for cohort 3.

DATE AND TIME OF RUN = MACCS2 05/20/96 09:57:55 v. 1.11f, 4/16/96: new option BOUNDARY/CENTERPOINT "ATMOS" DESCRIPTION = IN1A. INP, Sample Problem A--Using Table-Lookup Sigmas, ATMOS input

"EARLY" DESCRIPTION = IN2A. INP, Sample Problem A of NUREG/CR-4691, Vo1. 1, EARLY input

"CHRONC" DESCRIPTION = IN3A_O.INP, Sample Problem A, "Old" NUREG-1150 Food Model

SOURCE TERM 1 OF 2 :

SECOND DRAFT 1150, WORST CASE SOURCE TERM FOR EARLY FATALITIES

RESULTS FROM THE "CHRONC" MODULE ALONE

COHORT 3 = IN3A_O.INP, Sample Problem A, "Old" NUREG-1150 Food Model

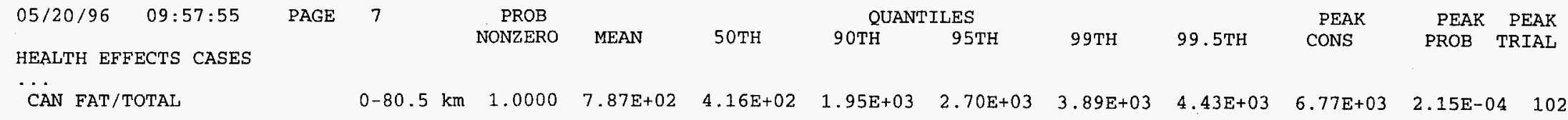


0.95 and 0.05 . Fraction-of-the-people weighting means that the respective consequence values from the two scenarios are weighted by the corresponding weighting fraction. ${ }^{4}$ In contrast, "fraction-of-the-people" weighting would indicate that the associated probabilities are to be scaled by the specified weighting fractions.

In the example of Figure 2-2, for each weather trial, and each wind direction, the overall result is calculated by summing two values: (1) $95 \%$ of the consequence value calculated for the evacuation cohort (cohort 1), and (2) $5 \%$ of the consequence value calculated for the relocation cohort (cohort 2).

The values of Figure 2-2 are discussed from left to right. The probability of a nonzero consequence was 1.0 , indicating that there were no weather conditions that resulted in a consequence estimate of 0 . The mean (expected value) was $1.72 \mathrm{E}+03$, the 50 th quantile (median) was $9.62 \mathrm{E}+02$, the 90 th quantile was $4.07 \mathrm{E}+03$, the 95 th quantile was $5.93 \mathrm{E}+03$, the 99th quantile was $9.57 \mathrm{E}+03$, and the 99.5 th quantile was $1.11 \mathrm{E}+04$. The observed peak value out of all weather conditions modeled was $2.06 \mathrm{E}+04$, with an associated probability of $2.85 \mathrm{E}-04$, resulting from weather trial number 123 .

A simple hand calculation can be performed to illustrate the relationship between the overall results and the results from the three cohorts given in Figures 2-3 through 2-5. This involves the mean consequence results from the respective cohorts. Of all the metrics presented, the mean is the only one that is additive. For cohort 1 , the mean cancer fatalities are shown as $9.26 \mathrm{E}+02$; for cohort 2 the mean cancer fatalities are shown as $1.03 \mathrm{E}+03$; and for cohort 3 the mean cancer fatalities are shown as $7.87 \mathrm{E}+02$. We have the following:

$$
1.72 \mathrm{E}+03=(0.95 \cdot 9.26 \mathrm{E}+02)+(0.05 \cdot 1.03 \mathrm{E}+03)+7.87 \mathrm{E}+02 .
$$

\subsubsection{Interpretation of the CCDF Tables}

The CCDF output for the three sample problem A cohorts and the overall results are presented in Figure 2-6. The CCDF for any of the MACCS2 results is represented as two arrays of floating point values, with the two arrays of the same length. These two arrays represent a series of (consequence, probability) doublets that indicate the spectrum of outcomes from lowconsequence/high-probability to high-consequence/low-probability. For additional discussion on representations of risk, see Kaplan and Garrick (1981).

The first array of the CCDF, listed in the second column of the output file, and denoted $P R O B>=X$ in the output file, represents the estimated probability of equaling or exceeding a specific consequence value, $X$. The second array of the CCDF represents the associated consequence values $\mathrm{X}$.

\footnotetext{
${ }^{4}$ The other alternatives are (1) fraction-of-the-time and (2) separate population distributions (SUMPOP), described in Sections 6.1.2, 6.6.7, and 6.10, and Section A.3 of Appendix A.
} 
Figure 2-6. CCDF tables for overall results and cohorts 1 to 3 .

MACCS2 06/25/96 22:17:09 version 1.12, last modified 6/25/96: final test PAGE 10

SOURCE TERM 1 OF 2 :

SECOND DRAFT 1150, WORST CASE SOURCE TERM FOR EARLY FATALITIES

RESULT NAME = HEALTH EFFECTS CASES

CAN FAT/TOTAL

PEOPLE FRACTION =

\begin{tabular}{|c|c|}
\hline $.00 E-04$ & $\begin{array}{l}\text { PRUB }>=X \\
1.00 E+00\end{array}$ \\
\hline $.00 E-04$ & $1.00 \mathrm{E}+00$ \\
\hline $3.00 E-04$ & $1.00 E+00$ \\
\hline $5.00 E-04$ & $1.00 \mathrm{E}+00$ \\
\hline $7.00 E-04$ & $1.00 \mathrm{E}+00$ \\
\hline $1.00 \mathrm{E}-03$ & $1.00 \mathrm{E}+00$ \\
\hline $2.00 E-03$ & $1.00 E+00$ \\
\hline $3.00 \mathrm{E}-03$ & $1.00 \mathrm{E}+00$ \\
\hline $5.00 E-03$ & $1.00 \mathrm{E}+00$ \\
\hline $7.00 E-03$ & $1.00 E+00$ \\
\hline $1.00 E-02$ & $1.00 \mathrm{E}+00$ \\
\hline 2.00E-02 & $1.00 \mathrm{E}+00$ \\
\hline 3.00E-02 & $1.00 E+00$ \\
\hline $5.00 \mathrm{E}-02$ & $1.00 E+00$ \\
\hline $7.00 E-02$ & $1.00 E+00$ \\
\hline $1.00 \mathrm{E}-01$ & $1.00 \mathrm{E}+00$ \\
\hline $2.00 E-01$ & $1.00 \mathrm{E}+00$ \\
\hline $3.00 E-01$ & $1.00 \mathrm{E}+00$ \\
\hline $5.00 \mathrm{E}-01$ & $1.00 E+00$ \\
\hline $7.00 \mathrm{E}-01$ & $1.00 \mathrm{E}+00$ \\
\hline 1. $.00 E+00$ & $1.00 \mathrm{E}+00$ \\
\hline $2.00 \mathrm{E}+00$ & $1.00 \mathrm{E}+00$ \\
\hline $3.00 \mathrm{E}+00$ & $1.00 E+00$ \\
\hline $5.00 \mathrm{E}+00$ & $1.00 E+00$ \\
\hline $7.00 E+00$ & $1.00 \mathrm{E}+00$ \\
\hline $1.00 \Xi+01$ & $1.00 \mathrm{E}+00$ \\
\hline $2.00 \mathrm{E}+01$ & $1.00 \mathrm{E}+00$ \\
\hline $3.00 E+01$ & 1. $.00 E+00$ \\
\hline $5.00 E+01$ & $1.00 \mathrm{E}+00$ \\
\hline $7.00 E+01$ & $1.00 E+00$ \\
\hline $1.00 \mathrm{E}+02$ & $9.99 E-01$ \\
\hline $2.00 E+02$ & $9.96 \mathrm{E}-01$ \\
\hline $3.00 \mathrm{E}+02$ & $9.53 \mathrm{E}-01$ \\
\hline $5.00 \mathrm{E}+02$ & $7.91 \mathrm{E}-01$ \\
\hline 7. $.00 \mathrm{E}+02$ & $6.38 \mathrm{E}-01$ \\
\hline $1.00 E+03$ & $4.85 \mathrm{E}-01$ \\
\hline $2.00 E+03$ & $2.40 \mathrm{E}-01$ \\
\hline $3.00 \mathrm{E}+03$ & $1.55 \mathrm{E}-01$ \\
\hline $5.00 \mathrm{E}+03$ & $7.47 \mathrm{E}-02$ \\
\hline $7.00 \mathrm{E}+03$ & $3.40 E-02$ \\
\hline $1.00 \mathrm{E}+04$ & $8.40 \mathrm{E}-03$ \\
\hline $2.00 E+04$ & $2.85 E-04$ \\
\hline $2.06 \mathrm{E}+04$ & $2.85 E-04$ \\
\hline N.D. & N.D. \\
\hline N.D. & N.D. \\
\hline N.D. & N.D. \\
\hline
\end{tabular}

0.9500

EMER. RESP. \# 1

$\begin{array}{cc}\mathrm{X} & \text { PROB }>=\mathrm{X} \\ 1.00 \mathrm{E}-04 & 1.00 \mathrm{E}+00\end{array}$

$2.00 \mathrm{E}-04 \quad 1.00 \mathrm{E}+00$

$3.00 \mathrm{E}-04 \quad 1.00 \mathrm{E}+00$

$5.00 \mathrm{E}-04 \quad 1.00 \mathrm{E}+00$

$7.00 \mathrm{E}-04 \quad 1.00 \mathrm{E}+00$

$1.00 \mathrm{E}-03 \quad 1.00 \mathrm{E}+00$

$2.00 \mathrm{E}-03 \quad 1.00 \mathrm{E}+00$

$3.00 \mathrm{E}-03 \quad 1.00 \mathrm{E}+00$

$5.00 \mathrm{E}-03 \quad 1.00 \mathrm{E}+00$

$7.00 \mathrm{E}-03 \quad 1.00 \mathrm{E}+00$

$1.00 \mathrm{E}-02 \quad 1.00 \mathrm{E}+00$

$2.00 \mathrm{E}-02 \quad 1.00 \mathrm{E}+00$

$3.00 \mathrm{E}-02 \quad 1.00 \mathrm{E}+00$

$5.00 \mathrm{E}-02 \quad 1.00 \mathrm{E}+00$

$7.00 \mathrm{E}-02 \quad 1.00 \mathrm{E}+00$

$1.00 \mathrm{E}-01 \quad 1.00 \mathrm{E}+00$

$2.00 \mathrm{E}-01 \quad 1.00 \mathrm{E}+00$

3.00E-01 1.00E+00

$5.00 \mathrm{E}-01 \quad 1.00 \mathrm{E}+00$

$7.00 \mathrm{E}-01 \quad 1.00 \mathrm{E}+00$

$1.00 \mathrm{E}+00 \quad 1.00 \mathrm{E}+00$

$2.00 \mathrm{E}+00 \quad 1.00 \mathrm{E}+00$

$3.00 E+00 \quad 1.00 E+00$

$5.00 \mathrm{E}+00 \quad 1.00 \mathrm{E}+00$

$7.00 \mathrm{E}+00 \quad 1.00 \mathrm{E}+00$

$1.00 E+01 \quad 1.00 E+00$

$2.00 \mathrm{E}+01 \quad 9.99 \mathrm{E}-01$

3. $00 \mathrm{E}+01 \quad 9.99 \mathrm{E}-01$

$5.00 \mathrm{E}+01 \quad 9.90 \mathrm{E}-01$

$7.00 E+01 \quad 9.71 E-01$

$1.00 \mathrm{E}+02 \quad 9.43 \mathrm{E}-01$

$2.00 \mathrm{E}+02 \quad 7.98 \mathrm{E}-01$

3. $00 \mathrm{E}+02 \quad 6.54 \mathrm{E}-01$

$5.00 \mathrm{E}+02 \quad 4.44 \mathrm{E}-01$

$7.00 \mathrm{E}+02 \quad 3.28 \mathrm{E}-01$

$1.00 \mathrm{E}+03 \quad 2.42 \mathrm{E}-01$

$2.00 \mathrm{E}+03 \quad 1.34 \mathrm{E}-01$

$3.00 \mathrm{E}+03 \quad 5.48 \mathrm{E}-02$

$5.00 E+03 \quad 1.73 E-02$

$7.00 \mathrm{E}+03 \cdot 1.05 \mathrm{E}-02$

$1.00 \mathrm{E}+04 \quad 3.27 \mathrm{E}-03$

$1.90 E+04 \quad 2.85 E-04$

II.

N.D.

N.D.

N.D.
$0-80.5 \mathrm{~km}$

0.0500

EMER. RESP. \# 2

$\begin{array}{cc}X & \text { PROB }>=X \\ .00 E-04 & 1.00 E+00\end{array}$

$1.00 \mathrm{E}-04 \quad 1.00 \mathrm{E}+00$

$2.00 \mathrm{E}-04 \quad 1.00 \mathrm{E}+00$

$3.00 E-04 \quad 1.00 E+00$

$5.00 \mathrm{E}-04 \quad 1.00 \mathrm{E}+00$

$7.00 \mathrm{E}-04 \quad 1.00 \mathrm{E}+00$

$1.00 \mathrm{E}-03 \quad 1.00 \mathrm{E}+00$

$2.00 \mathrm{E}-03 \quad 1.00 \mathrm{E}+00$

$3.00 \mathrm{E}-03 \quad 1.00 \mathrm{E}+00$

$5.00 \mathrm{E}-03 \quad 1.00 \mathrm{E}+00$

$7.00 \mathrm{E}-03 \quad 1.00 \mathrm{E}+00$

$1.00 E-02 \quad 1.00 E+00$

2.00E-02 $1.00 \mathrm{E}+00$

3.00E-02 1.00E+00

$5.00 \mathrm{E}-02 \quad 1.00 \mathrm{E}+00$

$7.00 \mathrm{E}-02 \quad 1.00 \mathrm{E}+00$

$1.00 \mathrm{E}-01 \quad 1.00 \mathrm{E}+00$

$2.00 E-01 \div 1.00 E+00$

3.00E-01 1.00E+00

5.00E-01 1.00E+00

$7.00 \mathrm{E}-01 \quad 1.00 \mathrm{E}+00$

$1.00 \mathrm{E}+00 \quad 1.00 \mathrm{E}+00$

$2.00 E+00 \quad 1.00 E+00$

$3.00 E+00 \quad 1.00 E+00$

$5.00 \mathrm{E}+00 \quad 1.00 \mathrm{E}+00$

$7.00 \mathrm{E}+00 \quad 1.00 \mathrm{E}+00$

$1.00 \mathrm{E}+01 \quad 1.00 \mathrm{E}+00$

$2.00 \mathrm{E}+01 \quad 1.00 \mathrm{E}+00$

3. $00 \mathrm{E}+01 \quad 1.00 \mathrm{E}+00$

$5.00 \mathrm{E}+01 \quad 1.00 \mathrm{E}+00$

$7.00 \mathrm{E}+01 \quad 9.98 \mathrm{E}-01$

$1.00 \mathrm{E}+02 \quad 9.91 \mathrm{E}-01$

$2.00 \mathrm{E}+02 \quad 8.87 \mathrm{E}-01$

$3.00 E+02 \quad 7.57 E-01$

$5.00 \mathrm{E}+02 \quad 5.79 \mathrm{E}-01$

$7.00 \mathrm{E}+02 \quad 4.34 \mathrm{E}-01$
$1.00 \mathrm{E}+03$

$1.00 \mathrm{E}+03 \quad 3.03 \mathrm{E}-01$

$2.00 E+03 \quad 1.40 E-01$

$3.00 \mathrm{E}+03 \quad 6.61 \mathrm{E}-02$

$5.00 E+03 \quad 1.86 E-02$

$7.00 \mathrm{E}+03 \quad 1.43 \mathrm{E}-03$

$9.00 \mathrm{E}+03 \quad 1.11 \mathrm{E}-03$

N.D.

N.D.

N.D.

N.D.

N.D.

N.D.

N.D.

N.D.

N.D.
CHRONC RESULTS

$X \quad P R O B>=X$

$1.00 E-04 \quad 1.00 E+00$

2.00E-04 $1.00 \mathrm{E}+00$

3.00E-04 1.00E+00

$5.00 \mathrm{E}-04$ 1.00E+00

$7.00 \mathrm{E}-04 \quad 1.00 \mathrm{E}+00$

$1.00 \mathrm{E}-03 \quad 1.00 \mathrm{E}+00$

$2.00 \mathrm{E}-03 \quad 1.00 \mathrm{E}+00$

$3.00 \mathrm{E}-03 \quad 1.00 \mathrm{E}+00$

$5.00 \mathrm{E}-03 \quad 1.00 \mathrm{E}+00$

$7.00 \mathrm{E}-03 \quad 1.00 \mathrm{E}+00$

$1.00 \mathrm{E}-02 \quad 1.00 \mathrm{E}+00$

$2.00 \mathrm{E}-02 \quad 1.00 \mathrm{E}+00$

3.00E-02 $1.00 \mathrm{E}+00$

5.00E-02 $1.00 \mathrm{E}+00$

$7.00 \mathrm{E}-02 \quad 1.00 \mathrm{E}+00$

$1.00 \mathrm{E}-01 \quad 1.00 \mathrm{E}+00$

$2.00 \mathrm{E}-01 \quad 1.00 \mathrm{E}+00$

3. $00 \mathrm{E}-01 \quad 1.00 \mathrm{E}+00$

5.00E-01 1.00E+00

$7.00 E-01 \quad 1.00 E+00$

$1.00 \mathrm{E}+00 \quad 1.00 \mathrm{E}+00$

$2.00 \mathrm{E}+00 \quad 1.00 \mathrm{E}+00$

$3.00 \mathrm{E}+00 \quad 1.00 \mathrm{E}+00$

$5.00 \mathrm{E}+00 \quad 1.00 \mathrm{E}+00$

$7.00 \mathrm{E}+00 \quad 1.00 \mathrm{E}+00$

$1.00 E+01 \quad 1.00 E+00$

$2.00 \mathrm{E}+01 \quad 1.00 \mathrm{E}+00$

$3.00 \mathrm{E}+01 \quad 1.00 \mathrm{E}+00$

$5.00 \mathrm{E}+01 \quad 1.00 \mathrm{E}+00$

7.00E+01 9.99E-01

$1.00 E+02 \quad 9.98 E-01$

2. $00 \mathrm{E}+02 \quad 8.41 \mathrm{E}-01$

$3.00 \mathrm{E}+02 \quad 6.51 \mathrm{E}-01$

$5.00 \mathrm{E}+02 \quad 4.30 \mathrm{E}-01$

$7.00 \mathrm{E}+02 \quad 3.24 \mathrm{E}-01$

$1.00 E+03 \quad 2.38 \mathrm{E}-01$

$2.00 \mathrm{E}+03 \quad 9.68 \mathrm{E}-02$

$3.00 \mathrm{E}+03 \quad 3.98 \mathrm{E}-02$

$5.00 \mathrm{E}+03 \quad 2.64 \mathrm{E}-03$

$6.77 E+03 \quad 2.15 E-04$

N.D. N.D.

N.D. N.D.

N.D. N.D.

N.D. N.D.

N.D. N.D.

N.D. N.D. 
Figure 2-2 shows that for the overall results, as previously discussed, the observed peak value from all weather conditions modeled was $2.06 \mathrm{E}+04$, with an associated probability of $2.85 \mathrm{E}-04$. An overall consequence value of $1.00 \mathrm{E}+03$ in Figure 2-6 is shown as being exceeded 4.85E-1 of the time (48.5\%), and this value is just slightly higher than the median value of $9.62 \mathrm{E}+02$ reported in the one-line summary, as expected.

Continuing on to the CCDF table (Figure 2-6) for cohort 1 (the evacuation case of EARLY), we see that a consequence magnitude of $7.00 \mathrm{E}+03$ is estimated to be equaled or exceeded only $1.05 \%$ of the time, which is very close, as expected, to the 99th quantile value of $7.11 \mathrm{E}+03$ shown in the one-line summary for this cohort (Figure 2-3).

\subsubsection{Interpretation of One-Line Summaries for Single Weather Trial Runs}

A CCDF is generated internally irrespective of whether weather sampling is utilized. If weather sampling is not utilized, depending on the user-specified option for input variable IPLUME (see Section 6.2.2), the code will generate a CCDF consisting of either 1 consequence/probability pair or 16 such pairs for the single weather sequence evaluated.

If IPLUME is assigned a value of 1 , all of the plume segments travel in the same direction and the modeling results (risk values) for each plume segment are rotated around the 16 compass sectors to obtain a set of results based on the probability of the wind blowing in each direction and the population distribution defined for that compass sector.

For IPLUME $=2$, each plume segment travels in the direction that the wind is blowing at the time of release and, as with IPLUME $=1$, the modeling results are rotated around the compass sectors to obtain a set of results based on the probability of the wind blowing in each direction and the population distribution defined for that compass sector.

For IPLUME $=3$, modeling results are not rotated around the compass sectors so that only one set of results is generated for each weather trial. Regardless of the defined wind rose or the population distribution, only mean consequence values will be listed for IPLUME $=3$ runs when weather sampling is not utilized.

\subsubsection{Use of Interpolation}

In conclusion, one more concept must be mentioned. This relates to the code's use of an interpolation algorithm to estimate the quantiles shown in the one-line summaries. The underlying data used to generate the quantiles shown in the one-line summaries are those shown in the CCDF tables, and interpolation is used to estimate the fixed quantiles of the one-line summaries.

The interpolation technique used to estimate the quantiles is log-linear (logarithmic on probability, linear on consequence). The calculation method unavoidably introduces some interpolation error in the quantile estimates, particularly for the higher quantile (e.g., the 99.5 th quantile results). For this reason, it may sometimes be found that a small change in input assumptions (such as variation of the initial random seed of the weather sampling algorithm) can 
yield differences of $10 \%$ or more in the higher quantile dose estimates. This behavior is inherent in the algorithm implemented in the code. 


\subsection{Code Enhancements Implemented in MACCS2}

The principal changes implemented in MACCS2 include the addition of code preprocessors (Section 3.1), enhancements to ATMOS (Section 3.2), enhancements to EARLY (Section 3.3), enhancements to CHRONC (Section 3.4), and additional minor enhancements and code corrections (Section 3.5). Appendix B describes the sequence of operations needed to convert MACCS user input files for use with MACCS2.

\subsection{MACCS2 Preprocessors}

The only preprocessor for MACCS developed prior to MACCS2 was the DOSFAC code. DOSFAC generated a file of dose conversion factors (DCF) required for MACCS dose calculations. DOSFAC was not publicly distributed because it was not designed to process userdefined parameter values through a user input file. All parameter values used in the DOSFAC calculations were fixed in the code.

The MACCS2 package includes a number of preprocessors that can be used to generate data files utilized in MACCS2 calculations. The MACCS2 package includes an enhanced version of DOSFAC, DOSFAC2, which processes user-defined parameter values provided in a user input file. Two additional DCF preprocessors and a new ingestion pathway model preprocessor are included in the code package. These preprocessors allow the code user to expand the list of radionuclides beyond the set provided in DOSFAC2 and used in the code's sample problems.

The MACCS2 distribution package includes data files generated by the new preprocessors and used in the code's sample problems. The user must run the preprocessors only if an analysis requires input data that differ from those included in the sample problem files of the distribution package.

An overview of the MACCS2 preprocessors is provided in the remainder of this section. Volume 2 of this report serves as a user's guide for the preprocessors distributed as part of the MACCS2 package.

\subsubsection{Dose Conversion Factor Preprocessors}

Dose conversion factors express the relationship between (1) environmental concentrations (or intakes) and (2) resultant human doses or dose rates. They are developed for specific exposure pathways, organs, and radionuclides. For example, the DCF for the skin dose from exposure to cesium-137 ground contamination is $2.75 \mathrm{E}-16 \mathrm{~Sv} / \mathrm{Bq}-\mathrm{s}-\mathrm{m}^{-2}$ (Eckerman and Ryman 1993). MACCS2 requires DCFs for the inhalation, ingestion, cloudshine, and groundshine exposure pathways for each organ for which doses are to be calculated, and for each radionuclide that is to be included in the scenario.

DCFs are provided to MACCS and MACCS2 through a data file read by the code. The DOSFAC code was developed to produce a MACCS input file of DCFs for the 60 radionuclides considered important for nuclear power plant analyses. Although MACCS was dimensioned to 
allow the simultaneous consideration of 150 radionuclides, calculations were limited by the availability of DCFs. ${ }^{5}$

As a practical matter, unless code users generated DCFs themselves, or obtained them from published sources, MACCS was limited to processing only the 60 radionuclides included in the DOSFAC-generated DCF file. Another factor that discouraged expansion of the MACCS radionuclide set by users was the code's limitation of decay chains to two members, with the branch fraction from parent to daughter fixed at $100 \%$. This limitation, for example, introduced a major difficulty for criticality source terms, where longer chains are needed.

\subsubsection{Three DCF Preprocessors}

The MACCS2 package contains three DCF preprocessors that can be used to generate MACCS2 DCF data files: FGRDCF, IDCF2, and DOSFAC2. Each of these preprocessors accesses different DCF databases and accepts user-defined input data.

\subsubsection{DOSFAC2}

DOSFAC2 allows the user to specify input parameters defining relative biological effectiveness, acute dose reduction factors, clearance class, and particle size. DOSFAC2 obtains DCFs for cloudshine and groundshine from a DOE (1988) database, referred to later by its report number, DOE/EH-0070. DCFs for exposure resulting from the inhalation or ingestion of radionuclides are generated from a 1987 DCF database provided by Keith Eckerman of Oak Ridge National Laboratory. The primary disadvantage of DOSFAC2 is that it is limited to generating DCFs for the original library of 60 radionuclides identified as important for commercial nuclear power plant analyses. This limitation is not a limitation of the code per se, but is due to the unavailability of inhalation dose commitments by time period for radionuclides other than the 60 considered important for commercial reactor accidents. The dose commitments by time period are used to generate acute inhalation DCFs.

\subsubsection{FGRDCF}

FGRDCF provides the user with access to the DCFs issued by the EPA in Federal Guidance Report (FGR) 11 (Eckerman, Wolbarst, and Richardson 1989) and FGR 12 (Eckerman and Ryman 1993). The FGRDCF preprocessor accesses inhalation and ingestion DCFs for over 600 radionuclides, and cloudshine and groundshine DCFs for 825 radionuclides (Radiation Shielding Information Center 1994). The DCFs provided by FGRDCF are sufficient for MACCS2 lifetime dose calculations. FGRDCF does not provide the DCFs required for MACCS2 acute dose calculations. However, for many applications, the analyst may be interested only in the calculation of the effective dose equivalent, for which an FGRDCF-generated file can be sufficient.

\footnotetext{
${ }^{5}$ The format of the DCF file utilized by MACCS2 is the same as that used by MACCS. This allows MACCS2 to be exercised using the DCF file that was distributed with MACCS. MACCS2 sample problem A (see Appendix C) makes use of the DOSDATA.INP file that was distributed with MACCS 1.5.11.1.
} 


\subsubsection{IDCF2}

IDCF 2 provides the capability of generating inhalation DCFs required for acute dose calculations that are not available from DOSFAC2 or FGRDCF. IDCF2 accesses immersion and groundshine DCFs for approximately 800 radionuclides from DOE/EH-0070. Inhalation and ingestion DCFs for approximately 500 radionuclides are calculated by the IDCF code developed by the Idaho National Engineering Laboratory for assessment of hypothetical accidents at fusion power reactors (Fetter 1988, 1991). IDCF calculates inhalation DCFs based on ICRP 30 models; however, its results sometimes differ from ICRP 30-based inhalation DCFs generated using other calculation methods (Rood and Abbott 1991).

\subsubsection{Choice of DCF Preprocessor}

For DOE applications, FGRDCF is the preferred source of DCFs because it accesses the DCF database issued by the EPA. The FGRDCF DCFs, however, because they only include 50-year dose commitments from inhalation, cannot be used to calculate acute health effect risks such as early fatality and prodromal vomiting. The calculation of those health effects requires a preprocessor that can generate dose commitments for incremental time periods as short as the days and weeks following inhalation intake.

The situation is different for NRC applications assessing the safety of commercial power plants. For those, there is often a need to model acute health effects resulting in early fatalities and early injuries. DOSFAC2 is recommended as a DCF source for reactor accident analyses because it allows the calculation of acute health effects. In addition, IDCF2 provides a source of DCFs required to calculate acute health effects that are not available from FGRDCF or DOSFAC2. It is possible that IDCF2 could be used to augment the set of 60 radionuclides considered by DOSFAC2, or to evaluate the need for such augmentation.

Additional details on FGRDCF and IDCF2 and the data files they generate are provided in Sections 4 and 5 of Volume 2 of this report. The DOSFAC2 preprocessor is described in the DOSFAC2 User's Guide (Young, Chanin, and Banjac 1997-draft).

\subsubsection{New Ingestion Pathway Preprocessor-COMIDA2}

MACCS incorporates a food ingestion pathway model for deposition to growing crops and root uptake that requires the user to supply unitless transfer coefficients for each radionuclide and crop type considered by the code. These transfer factors represent the fraction of material deposited on farmland that is eventually incorporated into edible portions of foodstuffs. The sample problems distributed with MACCS include input data for only six radionuclides: ${ }^{89} \mathrm{Sr}$, ${ }^{90} \mathrm{Sr},{ }^{131} \mathrm{I},{ }^{133} \mathrm{I},{ }^{134} \mathrm{Cs}$, and ${ }^{137} \mathrm{Cs}$. (These terms are written as Sr-89, Sr-90, I-131, I-133, Cs-134, and Cs-137 in the code.) The derivation of the corresponding input data required by the MACCS ingestion model is a very labor-intensive task, and is described in Sprung et al. (1990).

A major drawback of this model is that it stands somewhat alone among food-chain modeling approaches, deviating substantially from the predecessor models of the Reactor Safety Study. The MACCS food model is based on the original work of Ostmeyer (1986). This approach, making use of unitless transfer factors, is known to have been used for only two transportation-focused 
accident assessment codes: RADTRAN (Neuhauser and Kanipe 1996) and RISKIND (Yuan et al. 1996). As a result of this limited use, there is sparse literature to support the derivation of the requisite input parameters; all instances of such have been one of a kind, focused on transportation applications, as described in the three citations.

In contrast, the input parameters required by the new MACCS2 ingestion pathway model preprocessor, COMIDA2, are readily available in the literature. COMIDA2 is based on INEL's COMIDA code (Abbott and Rood 1993, 1994). COMIDA is a dynamic food-chain model that estimates yearly harvest concentrations for five human crop types and integrated concentrations for four animal products for the unit deposition of radionuclides that occur on any user-specified day of the year. COMIDA was developed to support accident analysis of fusion-power test facilities, and COMIDA's modeling capabilities are similar to those of the PATHWAY code (Whicker and Kirchner 1987; Whicker et al. 1990).

COMIDA2 generates a database of dose-to-source ratios in the units of dose to an organ per initial unit of deposition of a single deposited radionuclide, considering any daughter buildup after deposition. The COMIDA2-generated database provides dose-to-source ratios for up to nine accident times spaced throughout the year. This allows consideration of seasonality effects, which can have a marked influence on the food ingestion pathway. COMIDA2 is described in Section 3 of Volume 2 of this report.

\subsection{Enhancements to ATMOS}

ATMOS incorporates a number of enhancements that provide the user with greater flexibility than MACCS. These enhancements relate primarily to the following: radioactive decay, specification of initial plume dimensions, specification of dispersion parameters, and output capabilities. Overviews of these enhancements are provided in the following subsections. All of the input parameters utilized by ATMOS are described in Section 5 .

\subsubsection{Expansion of Radioactive Decay Chain Calculations}

MACCS models only simple parent-daughter decay (that is, two-member chains). MACCS2 incorporates decay-chain data for 825 radionuclides and models decay for decay chains with up to six generations. Decay in MACCS2 is calculated analytically using an iterative representation of the differential equations for first-order kinetics as described by Skrable et al. (1974) and Birchall (1986). The algorithm is the same as that used in the RSAC-5 code (Wenzel 1994).

\subsubsection{Increased Flexibility in Defining Initial Plume Dimensions}

The initial plume dimensions were calculated in MACCS based on the assumption that building wake effects determined the initial plume dimensions. The initial plume dimensions were calculated in the code based on the user input values for building height and width. $\sigma_{\mathrm{y}}$ was initialized to the building width divided by 4.3 , and $\sigma_{\mathrm{z}}$ was initialized to the building height divided by 2.15. MACCS2, in contrast, does not include a model to convert building height and width into initial plume dimensions. Instead, users now must consider building wake effects outside of the code, and specify the initial plume dimensions for $\sigma_{\mathrm{y}}$ and $\sigma_{\mathrm{z}}$. These values are 
specified separately for each plume of a multiple-plume release. Further, while previous betatest versions of MACCS2 did not allow plumes to overlap each other in time, the code now allows overlapping plumes. This change to MACCS2 thus allows the modeling, for example, of the HOTSPOT code's "stem and cap" model of initial cloud sizes that was used to represent explosive releases in the EIS for the Dual Axis Radiographic Hydrodynamic Test Facility (DOE 1995a).

When the source-term looping feature is utilized for consideration of multiple source terms in a single MACCS2 run, the initial dimensions of each plume of each source term can be specified independently. In contrast, MACCS utilized a single specification of initial plume dimensions (based on the user-specified building height and width) to initialize all of the released plumes; there was no provision to reset the building dimensions for the successive source terms of a multiple source-term run. Guidance on the specification of initial $\sigma_{\mathrm{y}}$ and $\sigma_{\mathrm{z}}$ values as a function of building dimensions, and a description of the format of this new input data are provided in Section 5.10.

\subsubsection{New Lookup-Table Option for Dispersion Parameter Data}

ATMOS has been modified to include a new code feature allowing a lookup-table scheme to be used as an alternative to the power-law functions for the $\sigma_{\mathrm{y}}$ and $\sigma_{\mathrm{z}}$ dispersion parameters. The $\sigma_{\mathrm{y}}$ and $\sigma_{\mathrm{z}}$ parameters represent plume expansion in the vertical and horizontal directions in the Gaussian plume model implemented in the code. The power-law function and the lookup-table options are described in Sections 5.7.1 and 5.7.2. The new option allows the user to provide a table of $\sigma_{\mathrm{y}}$ and $\sigma_{\mathrm{z}}$ values versus downwind distance in the ATMOS input file.

$\sigma_{\mathrm{y}}$ and $\sigma_{\mathrm{z}}$ lookup tables for three dispersion parameterization schemes are included in the MACCS2 package. The dispersion parameterization schemes included are the Tadmor-Gur (for 0.5 to $5 \mathrm{~km}$ ) curves as presented in Dobbins (1979), as well as the two sets of Briggs curves for open country and urban terrain as presented in Hanna, Briggs, and Hosker (1982). For additional information on the use of the lookup-table option, see Section 5.7.2.

The motivation for implementing this new feature was to allow the use of parameterizations other than the simple power law. Also, if tracer experiments are available for a site, it might be possible to process such data into the tables of horizontal and vertical standard deviations $\left(\sigma_{y}\right.$ and $\sigma_{z}$ ) required by this new feature of MACCS2.

\subsubsection{CCDFs of Atmospheric Dispersion and Deposition Results}

No versions of MACCS had the capability to generate complementary cumulative distribution functions of the atmospheric modeling parameters calculated by ATMOS. The only capability offered by MACCS to report the results of the atmospheric modeling was the debug print option in ATMOS that printed a page of formatted output for each plume and each weather trial. For sample problem A, with two source terms each having two plumes, and 135 weather trials, turning on this option would result in 540 pages of output written to the output file. 
In MACCS2, if requested by the user, ATMOS will now generate CCDFs of ten atmospheric modeling parameters for user-specified distances and plume segments. Air and ground concentrations are reported for a user-specified radionuclide. In addition to the results produced for the single specified radionuclide, the total radioactivity on the ground from all radionuclides is also reported.

\subsection{Enhancements to EARLY}

The enhancements to EARLY include the implementation of a more flexible evacuation model and the capability to calculate direction-dependent doses. The more flexible evacuation model was added to enable the code to model the characteristics of an evacuation that could occur as a result of an accident at a large DOE reservation. The new approach to evacuee movement enables the code to model variable evacuation speeds and simplifies the modeling of evacuee movement with respect to the plume.

The direction-dependent dose option allows the calculation of 99.5th percentile directiondependent dose as defined in Reg. Guide 1.145 (NRC 1983) and Snell and Jubach (1981). As called for by Reg. Guide 1.145 and DOE Order 6430.1A (DOE 1989), MACCS2 allows the user to select from the larger of (1) the 95th percentile dose calculated for direction-independent meteorology and (2) the 99.5 th percentile dose at a user-specified distance and direction.

Direction-dependent doses can be of great interest at facilities where the distance to the site boundary varies significantly with direction from the release point, or where authorization basis calculations make use of the $95 \%$ direction-independent dose and the $99.5 \%$ direction-dependent dose as stipulated in DOE Order 6430.1A.

\subsubsection{MACCS2 Emergency-Response Model}

The MACCS evacuation model could only model a straight-line evacuation path away from a release point and a constant evacuation speed. In 1992, under the sponsorship of Los Alamos National Laboratory, the coding of MACCS 1.5.11.1 was modified to improve its flexibility (Chanin 1992). The three principal enhancements include the capabilities to model the following: (1) nonradial evacuation paths; (2) evacuation travel speeds that vary with time; and (3) up to three population distributions, with each cohort following its own distinct pattern of emergency response. Additional enhancements included the options to: (1) model sheltering prior to evacuation, (2) implement different evacuation delay times at each distance, and (3) model sheltering/evacuation triggered by plume arrival.

These new features allow the user to model the following: (1) evacuation travel on road networks, (2) traffic jams causing slowdowns in travel speed, and (3) distinct population subgroups (e.g., guards and health physics teams) responding to the accident. Any evacuation scenario modeled with MACCS can also be modeled with MACCS2. Information describing the MACCS2 evacuation model is provided in Section 6.6. 


\subsubsection{Alternative Options for Modeling Evacuee Movement}

The MACCS and MACCS2 evacuation models move evacuees in discrete increments rather than in a smooth fashion over their predefined travel path. Evacuees "jump" from point to point after the elapse of a delay time based on the travel distance and their travel speed. The successive points at which they are located are always the centerpoints of the polar-grid elements that they traverse. This simplification is inherent in the design of the code.

Since the earliest versions of MACCS, evacuees have been modeled as leaving one element (their origin) and entering their destination when a sufficient time has elapsed for them to travel the distance required for them to cross the boundary line that separates the origin element from the destination element.

MACCS2 provides the user with the option of modeling evacuee movement using:

1. the MACCS approach in which movement occurs when an individual crosses the boundary line between the two elements, or

2. a new approach developed specifically for MACCS2 where an individual is presumed to enter the destination element when he or she has traveled a sufficient distance to reach the centerpoint of the destination.

The advantages of the new CENTERPOINT option are that:

1. The evacuee movement algorithm for the CENTERPOINT option can better model scenarios where a change in evacuation speed occurs. The BOUNDARY option could not adequately handle scenarios involving variable evacuation speeds.

2. The CENTERPOINT evacuee movement option is consistent with the plume dispersion model, which is based on the movement of the plume from element centerpoint to centerpoint. The CENTERPOINT option thus simplifies the modeling of evacuee movement with respect to the movement of the plume.

The use of the BOUNDARY option is restricted to cases where the evacuation speed is defined to be constant. If the user attempts to perform a calculation with a time-variant evacuation speed in conjunction with the BOUNDARY option, an input error will be diagnosed, and further execution inhibited. The results of the MACCS sample problems A-C may thus be replicated using the MACCS2 BOUNDARY option because those cases utilize an evacuation speed that is constant over time.

The CENTERPOINT option may be implemented for cases where evacuation speed is constant, as well as where it is time-variant. Although the CENTERPOINT approach has certain conceptual advantages over the BOUNDARY approach, it was deemed imprudent to simply substitute the new approach for the old. A major reason for preserving the prior model was the desirability of being able to replicate MACCS analyses with MACCS2. If the BOUNDARY approach had been deleted from MACCS2, the replication of MACCS results from sample problems A-C with MACCS2 would not be possible. 


\subsubsection{Direction-Dependent Doses}

Both MACCS and MACCS2 provide the user with the option of calculating plume-centerline dose and risk values (Type 6 and 7 results). The plume-centerline dose is calculated independently of wind direction and represents the maximum dose or risk to an individual directly under the plume. Because the plume-centerline dose and risk calculations are calculated independently of wind direction, they are not appropriate for analyses for which nonradial evacuation is being modeled. In order to remedy this deficiency, MACCS2 has a new output option, the Type B result, which provides a maximum direction-dependent dose for a userspecified direction and downwind distance.

The Type B result of MACCS2 may be used to obtain site boundary doses for directiondependent meteorology. The results are for direct exposure only; i.e., doses resulting from groundshine, cloudshine, inhalation, and skin deposition exposures. The generation of the Type $B$ results is controlled by user input in the EARLY input file and these results are reported separately for each of up to three emergency-response scenarios. If CHRONC is exercised, it will automatically generate the corresponding Type B results in the same manner as is done for the Type $1,4,5$, and 8 results.

\subsection{Enhancements to CHRONC}

The enhancements to the CHRONC module include modifications to the user input required to specify the duration of the intermediate and long-term phases and the capability to process data from the COMIDA2 food pathway model.

\subsubsection{Specification of the Intermediate and Long-Term Phase Exposure Periods}

In MACCS the user defined the end of the intermediate phase by specifying the CHRONC input variable TMIPND. TMIPND was defined as the number of seconds following plume arrival at which the intermediate phase ended. The long-term phase period was defined as beginning at TMIPND, and extended into time for one million years.

In MACCS2, the duration of the intermediate and long-term phases is specified by the user and is not dependent on the definition of other input parameters; i.e., the user simply defines a duration for the intermediate phase and for the long-term phase-MACCS2 variables DUR_INTPHAS and EXPTIM respectively. The maximum exposure period the user can define for the long-term phase is 317 years.

\subsubsection{Enhanced Food Pathway Modeling Capabilities}

MACCS2 allows the user to choose either the COMIDA2 or MACCS food ingestion model. Some of the advantages of COMIDA2 over the MACCS food model are as follows:

1. uptake into the edible portion of plants is modeled as a function of plant growth, and thus seasonality effects are considered; 
2. parameter values required by the code are widely available in the literature, facilitating further augmentation of the list; and

3. radioactive decay chains with lengths of up to four generations can be modeled, with branch ratios fixed at $100 \%$ (MACCS is limited to one- or two-member chains).

Section 7.10 describes the implementation of the MACCS2 input parameters required for operation of the COMIDA2 food model. Additional details on COMIDA2 are provided in Section 3 of Volume 2 of this report.

\subsection{Minor Enhancements and Corrections to MACCS}

MACCS2 corrects three coding errors present in MACCS 1.5.11.1, as described in Sections 3.5.1 through 3.5.3. Section 3.5.4 describes an improvement in the code's reporting of economic cost measures. Section 3.5.5 discusses changes to the way the code calculates groundshine doses after passage of a plume. Section 3.4.6 reviews an additional error-checking capability. Finally, Section 3.5.7 reviews two minor changes relating to MACCS input and output. Minor modifications that improve the code's ability to detect and diagnose errors in the user input files are not described because their correction should have no impact on any prior calculations.

\subsubsection{DDREFA Implementation Error}

A coding error was made in the MACCS 1.5.11.1 implementation of the dose-dependent reduction factor (input variable DDREFA) that is used to modify the cancer risk factor in order to distinguish between low and high dose exposures incurred during the emergency phase (Abrahamson et al. 1991; Chanin et al. 1993). The error affected only the application of the dose-dependent reduction factor in the EARLY module. The reduction factor was correctly applied in the CHRONC dose calculations.

The test that was newly implemented in the MACCS 1.5.11.1 EARLY module to adjust cancer risk estimates between low-dose and high-dose regimes incorrectly based the dose test on the dose calculated to occur at the rightmost (or outermost) fine-grid element of the compass sector. This dose test should be based on the doses calculated for each fine-grid element.

Because the doses in the outermost fine-grid elements are almost always lower than those in the more central fine-grid elements, MACCS was incorrectly using DDREFA for much more of the population than was warranted. As a result, if DDREFA is greater than 1 , the cancer predictions generated by the EARLY module of MACCS will be underestimated. If, however, DDREFA is 1 , the coding error has no effect.

The magnitude of the error will depend on the magnitude of the doses calculated by EARLY. If all the calculated doses fall below a specified threshold (input variable DDTHRE, Section 6.9), the error will have no effect. As an example of the potential impact of the error, Table 3-1 
Table 3-1. MACCS2 vs. MACCS Sample Problem A Results for Total Weighted-Sum Overall ResultsEARLY plus CHRONC

\begin{tabular}{|l|c|c|c|c|c|c|}
\hline & \multicolumn{3}{|c|}{ MACCS2 } & \multicolumn{3}{c|}{ MACCS 1.5.11.1 } \\
\cline { 2 - 7 } & Mean & 50 th & 95 th & Mean & 50 th & 95 th \\
\hline $\begin{array}{l}0-1609 \mathrm{~km} \\
\text { Total Cancer Fatalities }\end{array}$ & $1.35 \mathrm{E}+4$ & $7.90 \mathrm{E}+3$ & $4.29 \mathrm{E}+4$ & $1.24 \mathrm{E}+4$ & $6.93 \mathrm{E}+3$ & $4.04 \mathrm{E}+4$ \\
\hline $\begin{array}{l}0-16 \mathrm{~km} \text { Safety Goal } \\
\text { Cancer Risk }\end{array}$ & $4.53 \mathrm{E}-3$ & $1.11 \mathrm{E}-3$ & $1.92 \mathrm{E}-2$ & $2.96 \mathrm{E}-3$ & $7.31 \mathrm{E}-4$ & $1.36 \mathrm{E}-2$ \\
\hline $\begin{array}{l}\text { 0-1609 km Population } \\
\text { Dose (Sv-effective) }\end{array}$ & $2.92 \mathrm{E}+5$ & $1.63 \mathrm{E}+5$ & $1.01 \mathrm{E}+6$ & $2.94 \mathrm{E}+5$ & $1.67 \mathrm{E}+5$ & $9.90 \mathrm{E}+5$ \\
\hline
\end{tabular}

presents the overall results of the weighted sum (EARLY plus CHRONC) estimates of three important consequence measures for source term 1 of sample problem $\mathrm{A}$.

The two results shown represent the combined results from EARLY and CHRONC and are for identical input assumptions and an identical DCF file. Minor differences (of about $1 \%$ ) in the dose algorithms implemented by MACCS and MACCS2 are apparent in the results listed for total population dose. The total cancers and cancer risk values calculated by MACCS, however, are somewhat less than the total cancers and cancer risk values calculated by MACCS 2 as a result of this coding error.

Because the DDREFA error has no effect on CHRONC results, and only affects the results generated by EARLY, an additional comparison is presented in Table 3-2. This comparison shows the impact of the DDREFA error on results generated by EARLY for the same source term as before, but with consequence values reported for the nonevacuating cohort of EARLY. This set of results from sample problem A yields the highest individual doses as calculated by EARLY, and is thus most sensitive to the coding change. For this most-affected cohort, exposures are subject to mitigation using the 0.5 and $0.25 \mathrm{~Sv}$ relocation criteria.

The results of Table 3-2 show a small decrease (1-2\%) in the $0-1609 \mathrm{~km}$ population dose that is due to the revised (and more accurate) algorithm for groundshine after passage of a cloud. The $0-16 \mathrm{~km}$ safety goal cancer risk results show a bigger change (48-89\%) than is seen for the 01609 total cancer fatalities (29-39\%) because doses are higher within the close-in region, and the impact of the DDREFA error is largest when doses are high.

\subsubsection{Type 3 Result (Number of People Exceeding Dose Threshold)}

When the user requests the code to generate the Type 3 results, information can be obtained on the number of people whose emergency phase doses exceed various user-specified dose thresholds. These results can be useful, for example, as an adjunct to the code's reporting of the number of cases of health effects (the Type 1 results). 
Table 3-2. MACCS2 vs. MACCS Sample Problem A Results from EARLY Alone-Nonevacuating Cohort of EARLY

\begin{tabular}{||l|c|c|c|c|c|c||}
\hline & \multicolumn{3}{|c|}{ MACCS2 } & \multicolumn{3}{c||}{ MACCS 1.5.11.1 } \\
\cline { 2 - 7 } & Mean & 50 th & 95 th & Mean & 50 th & 95 th \\
\hline $\begin{array}{l}\text { 0-1609 km } \\
\text { Total Cancer Fatalities }\end{array}$ & $1.58 \mathrm{E}+3$ & $1.16 \mathrm{E}+3$ & $3.74 \mathrm{E}+3$ & $1.14 \mathrm{E}+3$ & $8.97 \mathrm{E}+2$ & $2.69 \mathrm{E}+3$ \\
\hline $\begin{array}{l}\text { 0-16 km Safety Goal } \\
\text { Cancer Risk }\end{array}$ & $5.59 \mathrm{E}-3$ & $2.07 \mathrm{E}-3$ & $2.10 \mathrm{E}-2$ & $3.14 \mathrm{E}-3$ & $1.39 \mathrm{E}-3$ & $1.11 \mathrm{E}-2$ \\
\hline $\begin{array}{l}\text { 0-1609 km Population } \\
\text { Dose (Sv-effective) }\end{array}$ & $3.12 \mathrm{E}+4$ & $2.41 \mathrm{E}+4$ & $7.48 \mathrm{E}+4$ & $3.18 \mathrm{E}+4$ & $2.44 \mathrm{E}+4$ & $7.60 \mathrm{E}+4$ \\
\hline
\end{tabular}

MACCS 1.5.11.1 incorporated a coding error affecting situations where the user requested the generation of ten of the Type 3 results (the maximum number allowed). Also, when that code echoed the user-specified dose criterion to the output file, small values were erroneously reported as zero. These two problems, and the resultant fixes incorporated into MACCS2, are summarized below.

\subsubsection{Incorrect Calculations when Ten Results Are Generated}

MACCS and MACCS2 both allow the user to request the generation of ten of the Type 3 results (number of people exceeding a dose threshold, see Section 6.13). Due to a coding error, MACCS 1.5.11.1 generates erroneous results or fails to execute if NUM3 is assigned a value of 10. The code, however, operates properly if NUM3 is limited to values between 0 and 9 . The impact of the error can vary across different computer systems because the storage area corrupted by the error can occur in different locations. In some cases, ten results have been generated without error because the corrupted storage location was not utilized in the calculations.

If prior analyses using MACCS have utilized EARLY input files with NUM3 values of 10, the results of those calculations may be in error. In order to determine if results are erroneous, it is recommended that the number of Type 3 results be reduced to nine, and the calculations be rerun with MACCS. If there is no change in the code outputs, the code error has had no effect on the results. The subject coding error has been corrected in MACCS2.

\subsubsection{Inability to Report Small Values of the Dose Threshold}

In addition to the correction described above, a minor enhancement has been made to the functionality of the Type 3 result. If the user of MACCS specifies dose thresholds less than $0.0005 \mathrm{~Sv}(50 \mathrm{mrem})$, the output file summary of results will identify all of the corresponding dose thresholds as being $0.000 \mathrm{~Sv}$, because of that code's use of a fixed-format edit descriptor.

In order to avoid this problem, MACCS2 utilizes an edit descriptor that automatically switches between fixed and exponential formats to write the dose threshold to the output file, allowing the meaningful reporting of dose thresholds specified to have extremely small values. 


\subsubsection{Incomplete Implementation of the Intermediate Phase}

The predecessor codes to MACCS (CRAC and CRAC2) incorporated the capability to model only two postaccident periods: the emergency phase and the long-term phase. When MACCS was first developed, these phases were augmented to include a third, the intermediate phase, which could, at the option of the user, be defined to occur in-between the emergency phase and the long-term phase. The intermediate phase was anticipated to be useful because the Protective Action Guides (EPA 1992) refer to an intermediate period during which detailed assessments could be made of contamination levels, and the planning for long-term interdiction efforts would be accomplished.

The intermediate-phase model, however, was not implemented in NUREG-1150 and was never fully implemented in MACCS. None of the formal verification activities performed for MACCS investigated this feature of the code. MACCS2 fully implements the intermediate-phase calculations and models mitigative actions based on the intermediate-phase results.

\subsubsection{Intermediate-Phase Health Effects and Collective Doses}

MACCS correctly provided intermediate-phase Type 6 and 7 results (centerline dose and risk) but intermediate-phase Type 1 and 5 results (cases of health effects and collective dose) were always reported as 0 . It was found that the implementation of the intermediate phase did not correctly store the intermediate-phase doses for later use. This error was corrected in MACCS2.

\subsubsection{Lack of Interaction in MACCS Between Intermediate-Phase Relocation and Farm Interdiction}

The MACCS mitigative action model for the long-term phase is based on the assumption that agricultural production can only take place in locations suitable for habitation. The reverse condition is not applied: that is, habitation can take place in locations exceeding the criteria for agricultural production.

The problem with the MACCS mitigative action model as it pertains to the intermediate phase is that the doses were not utilized in any way in the code's determination of whether land was habitable, and therefore whether agricultural production would occur if the criteria for agriculture were satisfied. It was only the habitability tests for the long-term phase that governed whether this land was suitable for farming activities.

In MACCS2, the user can specify the existence of both an intermediate and a long-term phase, and the code allows the duration of each to be specified as 1 year. If this is done, the code will model a 1-year intermediate phase followed by a 1-year long-term phase. In making its decision as to whether land is suitable for agriculture in the first year after deposition, MACCS and betatest versions of MACCS2 prior to 1996 have utilized the results of the habitability test from the long-term phase.

In the example at hand, this means that the decision on the first year's farming will depend on whether the land is suitable for habitation in the second year, and this is clearly of doubtful validity. At least for this example, where the intermediate phase includes within its range all of 
the first year of potential farming, it would be more logical to utilize the habitability test of the intermediate phase (rather than the long-term phase) in deciding whether the agricultural land was habitable.

MACCS2 has been modified to ensure that intermediate-phase mitigative actions are based upon intermediate-phase results. Section 7 describes the revised mitigative action models of the intermediate and long-term phases, and how the mitigative action models triggered by habitability criteria interact with the mitigative action models for agricultural production.

Section 7 also describes the code's assessment of the corresponding economic costs and the usersupplied input parameters that control the operation of those models.

\subsubsection{Summation of Early and Intermediate-Phase Costs}

All versions of MACCS report the Type 10 result, evacuation and relocation costs. This code output is calculated as the sum of (1) per-diem expenses for evacuees and (2) the per-diem costs for individuals relocated for the duration of the CHRONC intermediate phase. MACCS thus was summing the aggregate economic costs from two phases of the calculations (emergency phase and intermediate phase), and there was no provision to allow reporting the two cost components separately.

In MACCS2, what was previously reported as evacuation and relocation cost is now reported as two separate results: emergency-phase costs and intermediate-phase costs. This code enhancement requires no action on the part of the user since the Type 10 results are automatically produced as a set, reporting the total costs as well as the breakdown of costs by category.

\subsubsection{Dose Calculations for Groundshine Following Plume Passage}

MACCS2 implements a more accurate approach for the calculation of doses from groundshine following plume passage than was implemented in MACCS. MACCS calculates these doses in the emergency phase by interpolating and extrapolating groundshine doses based on 8-hr and 1-week groundshine DCFs using a logarithmic interpolation scheme. The resultant errors can be quite sizable for short-lived radionuclides with half-lives of a few hours or less.

MACCS2 does not utilize 8-hr and 1-week groundshine DCFs. It performs an exact numerical integration of the EARLY dose for groundshine following plume passage for ten time periods of successively increasing duration spanning EARLY's maximum exposure period of 1 week. MACCS2 linearly interpolates between the ten tabulated values to obtain the appropriate DCF for the exposure period. The resultant tables of DCF data for the initial deposit of a single radionuclide are generated in a way that takes account of all radioactive progeny that the user 
included in the list of radionuclides being considered (as well as any implicit daughters defined in the DCF file). ${ }^{6}$

Linear interpolation is less accurate, everything else being equal, than logarithmic interpolation because the dose typically decreases exponentially. Nevertheless, because ten periods of increasing duration are defined, the MACCS2 method for calculating doses from groundshine following plume passage in EARLY is much more accurate than the MACCS approach, particularly when short-lived radionuclides are important contributors to groundshine dose.

\subsubsection{Nonprintable Characters in Site Data File and FORTRAN Source Code}

When code users have used text editors to edit the population counts in the Site Data file, there have been several occurrences where the code diagnoses an input conversion error and aborts execution. It has proved very difficult for users to identify and remedy the problem. A possible explanation for these problems is the inadvertent inclusion of nonprintable ASCII characters or control codes in the file.

In order to facilitate user debugging of Site Data files, MACCS2 tests for and diagnoses the location of any embedded nonprintable characters in the Site Data file. If any unusual ASCII characters are encountered during the echoing of the Site Data file to the output file, a diagnostic message is issued to the console and further processing is inhibited.

In such events, the output file will contain a series of messages that identify the location of the unusual ASCII characters on the line in which they appear. For further help in remedying the problem, or preventing its occurrence, the decimal ASCII codes causing the problem are also printed. In the context of MACCS2, unusual ASCII characters are defined as those with control codes less than 32 or greater than 126 . None of the Site Data files currently used in the sample problems contain unusual ASCII characters.

In conjunction with the implementation of this change to MACCS2, there was a related modification to the MACCS2 FORTRAN source code. ANSI FORTRAN 77 does not specify how a compiler is to treat nonprintable characters embedded in the FORTRAN source code, allowing a processor-dependent treatment of special ASCII codes such as TAB. The source code for MACCS was distributed in a form that included the TAB. All nonprintable characters have been deleted from the MACCS2 FORTRAN source code in order to maximize the portability of the code.

\subsubsection{Minor Changes to Input and Output}

The column of results previously used to report the 99.9th quantile was changed in MACCS2 so that it now shows the 99.5 th quantile. This change was made to allow examination of the $\chi / Q$

\footnotetext{
${ }^{6}$ An implicit daughter is defined here as a short-lived daughter product assumed to be in secular equilibrium with its parent. A well-known example is the ${ }^{137} \mathrm{Cs} \rightarrow{ }^{137 \mathrm{~m}} \mathrm{Ba}$ decay chain. For economy, the decay from parent to daughter is not explicitly modeled; rather the ${ }^{137} \mathrm{Cs} \mathrm{DCF}$ represents the parent as well as the daughter.
} 
and maximally exposed individual doses according to the methodology described in NRC Reg. Guide 1.145 (NRC 1983).

The variable DLBCST defined in the CHRONC input file specifies the cost of labor (dollars/person-year) for personnel involved in decontamination. In MACCS, the user was allowed to specify a value of 0 for this parameter. However, DLBCST is used in the denominator of an expression and thus the specification of a zero value leads to a floating-point exception due to a divide-by-zero. The minimum allowable value for this parameter in MACCS2 has been changed to 1.0 (that is, one dollar per person-year). 
THIS PAGE INTENTIONALLY LEFT BLANK 


\subsection{Installing and Running MACCS2}

\subsection{Introduction}

Installation instructions and system requirements for the MACCS2 package are provided in the READMAC2.TXT file listed in Appendix B of this document and distributed with the MACCS2 package ZIP files. This file provides additional information not included in this section. It is recommended that the user review this file before exercising MACCS2.

Fourteen sample problems are included in the MACCS2 package. These may be exercised to ensure that the code has been correctly installed. The sample problems also provide example applications of various features of the code and are briefly described in this section.

\subsection{Hardware Requirements}

MACCS2 requires an IBM-compatible/486 or Pentium PC with $8 \mathrm{MB}$ of RAM and approximately $30 \mathrm{MB}$ of free disk space for installation of the entire software package. The use of MACCS2 on non-PC platforms will require a recompilation of the source code. The READMAC2.TXT file included in the code package provides information for users who need to exercise MACCS2 on a non-PC platform.

When MACCS2 is installed on other computers, the numeric output values by and large should be identical or very close to identical. The only significant deviation might lie in the probabilities of nonzero and peak value consequences since the precision of arithmetic and the handling of underflow can vary on different computer systems.

\subsection{Running MACCS2}

A MACCS2 run is generated by calling the RUNMAX2.BAT file. Six filenames may be specified along with the RUNMAX2.BAT filename. MACCS2 sample problem A, for example, is executed as follows:

\section{RUNMAX2 IN1A IN2A IN3A METSUR SURSIT LISTA}

Unless specified as null parameter (indicated by the use of " "), each of the first five filenames specified must be a valid pathname to a previously created file to which the user has read access. The input sequence of the six filenames and a brief description of the files is as follows:

1. ATMOS User Input File: IN1A This parameter is always required and a null parameter is not allowed. 
2. EARLY User Input File: IN2A

This parameter can be omitted if the user is exercising only the ATMOS module (ENDAT1 set to .TRUE. on the ATMOS input); in that case, the parameter is given as double quotation marks ("").

3. CHRONC User Input File: IN3A

This parameter can be omitted if the user is skipping the CHRONC module (if ENDAT1 was set to .TRUE. on the ATMOS input or ENDAT2 was set to.TRUE. on the EARLY input); in that case, the parameter is given as double quotation marks ("").

4. Meteorological Data File: METSUR

Name of a weather data file with a year of hourly recordings. This file is described in Appendix A.1 of this volume. The format and processing of the file are unchanged from MACCS. The parameter may be omitted if a single weather sequence is defined on the ATMOS user input file (METCOD $=3$ or 4$)$; in that case, the parameter is given as double quotation marks ("').

5. Site Data File: SURSIT

Name of a Site Data file for the surrounding region. This file is described in Section A.3 of Appendix A of this volume. MACCS2 will successfully process Site Data files generated for use with MACCS. Previously generated MACCS site files are thus forward-compatible with MACCS2. The parameter may be omitted if a uniform population density is defined on the EARLY user input file (POPFLG = .TRUE.); in that case, the parameter is given as double quotation marks ("').

6. List Output File: LISTA

Name of the file on which to write the list output of the run. An existing file with this filename and the .OUT extension will be overwritten. If this argument is omitted, the results will be written to the system default filename for FORTRAN unit 6; a filename must be specified here, and it must be a valid pathname to a file that the user can write upon. Six arguments must be supplied. No default filenames are provided. If any of the input files are not required for the analysis, a null argument is specified as double quotation marks ("'). The minimum filenames that must be specified are the ATMOS input and list output filenames. With the files provided, sample problem $\mathrm{C}$ is executed as follows:

\section{RUNMAX2 IN1C IN2C IN3C "" "" TEMPC}

The EARLY and CHRONC modules require a dose conversion factor file. The DCF filename is specified in the EARLY input file (see EARLY DCF_FLEE input parameter described in Section 6.2.2). The format for the DCF file is presented in Section A.2 of Appendix A.

The MACCS2 ZIP files include a .BAT file, RUNEM.BAT, that executes all of the sample problems. This .BAT file is executed by simply typing RUNEM on the DOS command line when in the MACCS2 subdirectory. 


\subsection{MACCS2 Sample Problems}

The MACCS2 ZIP files contain both the input and output files associated with the 14 sample problems. The input files may be identified by the .INP extension and the output files may be identified by the .OUT extension. The ATMOS input data, EARLY input data, CHRONC input data, Meteorology Data, Site Data, and output filenames used in a MACCS2 run are listed in the first few lines of the output file. The output file echoes the contents of the ATMOS, EARLY, CHRONC, and Site Data input files.

All of the sample problems exercise the new Type 0 and Type A results. Sample problem F exercises the new Type B results.

\subsubsection{Sample Problems A-C}

Sample problems A-C of MACCS2 represent a close-to-exact translation of sample problems A$\mathrm{C}$ distributed with MACCS 1.5.11.1. The three sample problems distributed with versions of MACCS prior to MACCS2 are analyses of commercial nuclear reactor accident scenarios. These sample problems, denoted with either an A, B, or C suffix to the input filename, are focused on a severe accident at the Surry commercial reactor, as evaluated in NUREG- 1150.

Two versions of sample problem A are provided. Sample problem A_O implements the MACCS food pathway model. Sample problem A_N implements the new COMIDA2 food pathway model. In addition, the ATMOS input files for both MACCS2 versions of sample problem $A$ use the new lookup-table option for assigning values to the $\sigma_{\mathrm{y}}$ and $\sigma_{\mathrm{z}}$ dispersion parameters.

Sample problem A illustrates how MACCS can automatically loop on source terms and emergency-response assumptions in a single run of the code. The weather category bin sampling method is used in this problem to estimate the distribution of consequences based on the uncertainty in predicting weather conditions at the time of the accident. Automatic looping is illustrated as follows: the ATMOS user input file for sample problem A causes results to be calculated for two hypothetical source terms, and the EARLY user input file specifies two different emergency-resonse scenarios (95\% evacuation and 5\% nonevacuation). An additional sheltering case is presented for illustration. There is no provision in MACCS or MACCS2 for looping on the CHRONC module and only a single set of assumptions is used to determine the modeling in the long-term chronic exposure phase of the calculations.

Sample problem B illustrates how MACCS2 can be used to examine one of the weather sequences that was selected in the weather sampling used for sample problem A. This type of examination is usually done when some type of unusual result is noticed on the output listing and the user wishes to determine if the calculations are being performed properly. The output listing of sample problem A shows that the peak value of economic cost from the first source term was 271 billion dollars and it was obtained from the calculation of weather trial 2 . By going back to the first part of the listing, we can see that this trial began at day $=157$ and hour $=10$ on the Surry weather data file. 
For sample problem B, the ATMOS user input file of sample problem A has been modified to run only the first source term and to calculate results for only the single weather sequence that led to the peak economic cost as described earlier. We have turned on the diagnostic print options in all three of the user input files by changing the values of the following input parameters from 0 to 1: IDEBUG in ATMOS, IPRINT in EARLY, and KSWTCH in CHRONC. A large amount of print output results.

In sample problem $\mathrm{C}$, the code is set up to run the source term of sample problem $\mathrm{B}$ with constant meteorology; D-stability, $5 \mathrm{~m} / \mathrm{s}$ wind speed, no rain. A uniform population distribution of 50 people $/ \mathrm{km}^{2}$ is used for the calculations and therefore there is no need for a Site Data file. Since wind direction is not defined for constant meteorology, the value of IPLUME is changed from 2 to 1 . In this run, the diagnostic print statements of sample problem $B$ have been turned off and only the standard presentation of results from the OUTPUT module appears on the output listing.

\subsubsection{Sample Problem D}

Sample problem D is focused on an accident considered in the F-Canyon plutonium solutions EIS (DOE 1994b). The F-Canyon facility at the Savannah River Site was previously used to reprocess spent fuel and target assemblies from DOE production reactors. As a result of the reconfiguration of the weapons complex, reprocessing activities at SRS were temporarily suspended but solutions containing plutonium remained present in the F-Canyon. The accidental release of plutonium at this facility could present a health risk to on-site workers and the public.

The EIS for the F-Canyon includes analyses of both on-site worker and public doses that could result from a total collapse of the facility. The EIS assumed that a seismically induced total collapse of F-Canyon could result in a release of $92.1 \mathrm{Ci}$ of material to the atmosphere. The analysis completed for the EIS estimated that this source term would yield an uninvolved worker dose of $21.8 \mathrm{rem}$ (at $640 \mathrm{~m}$ ) and an off-site dose of $0.447 \mathrm{rem}$ (at the 8-km site boundary).

Six versions of sample problem $D$ are provided that implement six different dispersion parameterization schemes. The D set of sample problems illustrates the impact of varying user input values for the dispersion parameter.

Two sample problems utilize the Briggs curves (Hanna, Briggs, and Hosker 1982).

LISD_BRO.OUT was generated using the lookup-table option with the Briggs open country curves, and LISD_BRU.OUT was generated using the lookup-table option with the Briggs urban curves.

Two sample problems utilize the Tadmor and Gur (1969) curves, as corrected by Dobbins (1979). LISD_TG1.OUT was generated using the lookup-table option to implement the Tadmor-Gur power-law function for the 0.5- to 5-km distance range, and LISD_TG2.OUT was generated using the lookup-table option to implement the Tadmor-Gur power-law function for the 5- to 50-km range. 
Finally, two sample problems utilize different parameterizations of the "Julich" system, developed for Kernforschungszentrum Karlsruhe in Germany. LISD_JU1.OUT was generated using the power-law function to implement the Julich system for a release at a height of $50 \mathrm{~m}$ (Vogt 1977), and LISD_JU2.OUT was generated using an alternative power-law parameterization implementing the Julich system for the same 50-m release height (Panitz 1989).

\subsubsection{Sample Problem E}

Sample problem E illustrates a comparison of the two food-chain models-the "old" MACCS food-chain model and the "new" COMIDA2-based food-chain model. This case illustrates the source-term looping feature by defining eight unit-release source terms for single radionuclides. The weather conditions are held constant at D-stability, with a $5 \mathrm{~m} / \mathrm{s}$ wind speed.

\subsubsection{Sample Problem F}

Sample problem $\mathrm{F}$ illustrates a comparison of the weather-category sampling algorithm (METCOD=2, see Section 5.13) with the stratified random sampling algorithm (METCOD=5; see Section 5.13), which has been set up to sample every hour of the year. This comparison illustrates an approach that can be used to evaluate the adequacy of the weather-category sampling algorithm for a site-specific calculation.

\subsection{Migration from MACCS to MACCS2}

This section provides instructions for converting MACCS ATMOS, EARLY, and CHRONC files into MACCS2 input files. The format and input requirements for MACCS Site, DCF, and Meteorology files are identical to MACCS2 and thus no modifications to these files are required. This section includes tables that list the new MACCS2 input variables that must be added to the MACCS ATMOS, EARLY, and CHRONC input files. A glossary of MACCS2 ATMOS, EARLY, and CHRONC input file variables and the location of the variable descriptions is provided in Appendix D.

MACCS2 radionuclide names are specified using uppercase letters for the first letter in the radionuclide symbol and lowercase for the second letter. The second letter in all radionuclide names must be changed from uppercase to lowercase in the MACCS input files. The "M" metastable designation must also be changed to lowercase. It is necessary, for example, to change TC-99M to Tc-99m. All organ names specified in the EARLY and CHRONC input files must be prefixed by an 'A-' or an 'L-' (see Sections 6.2.1 and 6.4).

MACCS input parameters that are not required by MACCS2 are not processed. With the exception of the PARENT data provided in the MACCS ATMOS input file, including obsolete MACCS parameters will not result in an error in MACCS2 calculations.

\subsubsection{MACCS ATMOS File}

Table 4-1 lists the new MACCS2 ATMOS input parameters that must be added to the MACCS ATMOS input file. The primary difference between the MACCS and MACCS2 ATMOS files is 
Table 4-1. Input Parameters that Must Be Added to MACCS ATMOS Input Files to Convert Them to MACCS2 Input Files

\begin{tabular}{||l|c|c|l||}
\hline $\begin{array}{c}\text { MACCS2 FORTRAN } \\
\text { Variable Name } \\
\text { (ATMOS File Record } \\
\text { Identifier) }\end{array}$ & $\begin{array}{c}\text { ATMOS Input } \\
\text { File Data Block }\end{array}$ & $\begin{array}{c}\text { Location of Description in } \\
\text { MACCS2 User's Guide }\end{array}$ & $\begin{array}{c}\text { Function of Input Parameter } \\
\text { Value }\end{array}$ \\
\hline $\begin{array}{l}\text { NUMSTB } \\
\text { (ISNUMSTB) }\end{array}$ & Radionuclide & 5.4 & $\begin{array}{l}\text { Defines the number of pseudo- } \\
\text { stable nuclides }\end{array}$ \\
\hline $\begin{array}{l}\text { NAMSTB } \\
\text { (ISNAMSTB) }\end{array}$ & Radionuclide & 5.4 & $\begin{array}{l}\text { Identifies pseudostable } \\
\text { radionuclides }\end{array}$ \\
\hline $\begin{array}{l}\text { SIGYINIT } \\
\text { (SIGYINIT) }\end{array}$ & Release & 5.10 & Defines initial size of $\sigma_{\mathrm{y}}$ \\
\hline $\begin{array}{l}\text { SIGZINIT } \\
\text { (SIGZINIT) }\end{array}$ & Release & 5.10 & Defines initial size of $\sigma_{\mathrm{z}}$ \\
\hline $\begin{array}{l}\text { APLFRC } \\
\text { (RDAPLFRC) }\end{array}$ & Release & 5.11 & $\begin{array}{l}\text { Specifies how release fractions are } \\
\text { applied to ingrowth decay } \\
\text { products }\end{array}$ \\
\hline $\begin{array}{l}\text { NUM0 } \\
\text { (TYPEONUMBER) }\end{array}$ & 5.18 & $\begin{array}{l}\text { Specifies number of Type 0 } \\
\text { outputs requested }\end{array}$ \\
\hline
\end{tabular}

${ }^{a}$ This column provides the name of the data block that contains the new parameters in the MACCS2 sample problems.

the format and input requirements for the definition of the radionuclide inventory. MACCS2 automatically calculates six generations of radioactive decay and ingrowth for each radionuclide specified in the inventory unless pseudostable radionuclides are specified by the user.

Parent-daughter relationships and radiological decay rates are no longer specified in the ATMOS file. The PARENT column in the Radionuclide Group Data section (ISOTPGRP record identifier) must be deleted. The data contained in the HAFLIF column of the ISOTPGRP records will not be processed by the code. The WEBUILDW record indentifier is also not processed by MACCS2. A value of WEBUILDH must be defined for each plume released.

\subsubsection{MACCS EARLY File}

Table 4-2 lists the new MACCS2 EARLY input parameters that must be added to the MACCS EARLY input file. Table 4-3 lists the MACCS EARLY file input parameters that are not processed by MACCS2. 
Table 4-2. Input Parameters that Must Be Added to MACCS EARLY Input Files to Convert Them to MACCS2 Input Files

\begin{tabular}{|c|c|c|c|}
\hline $\begin{array}{l}\text { MACCS2 FORTRAN } \\
\text { Variable Name } \\
\text { (EARLY File Record } \\
\text { Identifier) }\end{array}$ & $\begin{array}{l}\text { EARLY Input File } \\
\text { Data Block }^{\mathrm{a}}\end{array}$ & $\begin{array}{l}\text { Location of Description } \\
\text { in MACCS2 User's } \\
\text { Guide }\end{array}$ & $\begin{array}{l}\text { Data Provided by Input } \\
\text { Parameter Value }\end{array}$ \\
\hline $\begin{array}{l}\text { DCF_FILE } \\
\text { (DCF_FILE) }\end{array}$ & Miscellaneous & 6.2 .1 & DCF filename and location \\
\hline $\begin{array}{l}\text { ORGNAM } \\
\text { (MIORGDEF) }\end{array}$ & Miscellaneous & 6.4 & Defined organs \\
\hline $\begin{array}{l}\text { ORGFLG } \\
\text { (MIORGDEF) }\end{array}$ & Miscellaneous & 6.4 & $\begin{array}{l}\text { Flag indicating whether an organ } \\
\text { is to be used in the calculations }\end{array}$ \\
\hline $\begin{array}{l}\text { TRAVELPOINT } \\
\text { (TRAVELPOINT) }\end{array}$ & $\begin{array}{l}\text { Evacuation } \\
\text { Zone }\end{array}$ & 6.6 .8 & Evacuee movement option \\
\hline $\begin{array}{l}\text { EVATYP } \\
\text { (EZEVATYP) }\end{array}$ & $\begin{array}{l}\text { Evacuation } \\
\text { Zone }\end{array}$ & 6.6 .8 & Type of evacuation \\
\hline $\begin{array}{l}\text { DURBEG } \\
\text { (EZDURBEG) }\end{array}$ & $\begin{array}{l}\text { Evacuation } \\
\text { Zone }\end{array}$ & 6.6 .8 & $\begin{array}{l}\text { Duration of beginning of } \\
\text { evacation phase }\end{array}$ \\
\hline $\begin{array}{l}\text { DURMID } \\
\text { (EZDURMID) }\end{array}$ & $\begin{array}{l}\text { Evacuation } \\
\text { Zone }\end{array}$ & 6.6 .8 & $\begin{array}{l}\text { Duration of middle of evacuation } \\
\text { phase }\end{array}$ \\
\hline $\begin{array}{l}\text { REFPNT } \\
\text { (EZREFPNT) }\end{array}$ & $\begin{array}{l}\text { Evacuation } \\
\text { Zone }\end{array}$ & 6.6 .8 & $\begin{array}{l}\text { Reference time point for } \\
\text { beginning of evacuation and } \\
\text { sheltering }\end{array}$ \\
\hline $\begin{array}{l}\text { NUMEVA } \\
\text { (EZNUMEVA) }\end{array}$ & $\begin{array}{l}\text { Evacuation } \\
\text { Zone }\end{array}$ & 6.6 .8 & $\begin{array}{l}\text { Outer boundary of } \\
\text { evacuation/sheltering region }\end{array}$ \\
\hline $\begin{array}{l}\text { DLTSHL } \\
\text { (EZDLTSHL) }\end{array}$ & $\begin{array}{l}\text { Evacuation } \\
\text { Zone }\end{array}$ & 6.6 .8 & $\begin{array}{l}\text { Delay to take shelter for each } \\
\text { interval }\end{array}$ \\
\hline $\begin{array}{l}\text { DLTEVA } \\
\text { (EZDLTEVA) }\end{array}$ & $\begin{array}{l}\text { Evacuation } \\
\text { Zone }\end{array}$ & 6.6 .8 & Delay to evacuation for each ring \\
\hline $\begin{array}{l}\text { NUMA } \\
\text { (TYPEANUMBER) }\end{array}$ & Result A Options & 6.19 & Number of Type A results \\
\hline $\begin{array}{l}\text { NUMB } \\
\text { (TYPEBNUMBER) }\end{array}$ & Result B Options & 6.20 & Number of Type B results \\
\hline
\end{tabular}

a This column provides the name of the data block that contains the new parameters in the MACCS2 sample problems. 
Table 4-3. MACCS EARLY File Input Parameters that Are Not Processed by MACCS2

\begin{tabular}{|l|l||}
\hline $\begin{array}{c}\text { MACCS EARLY File } \\
\text { Record Identifier }\end{array}$ & \multicolumn{1}{c|}{$\begin{array}{c}\text { MACCS } \\
\text { EARLY Input File Data Block }\end{array}$} \\
\hline ODNUMORG & Organ Definition \\
\hline ODORGNAM & Organ Definition \\
\hline EZINIEVA & Evacuation Zone \\
\hline EZLASEVA & Evacuation Zone \\
\hline EZEDELAY & Evacuation Zone \\
\hline SRTTOSH1 & Shelter and Relocation Zone \\
\hline SRSHELT1 & Shelter and Relocation Zone \\
\hline SRLASHE2 & Shelter and Relocation Zone \\
\hline SRTTOSH2 & Shelter and Relocation Zone \\
\hline SRSHELT2 & Shelter and Relocation Zone \\
\hline $\begin{array}{l}\text { TYPE3OUT } \\
\text { (Dose Flag) }\end{array}$ & Dose flag switch indicates whether the dose is an acute or lifetime dose \\
\hline
\end{tabular}

The ESPEED parameter must be defined for the three phases of evacuation modeled in MACCS2. The MACCS2 ORGFLG input parameter (see Section 6.4) must be set to .TRUE. for all organs used in the analysis.

\subsubsection{MACCS CHRONC File}

Table 4-4 lists the new MACCS2 CHRONC input parameters that must be added to the MACCS CHRONC input file. TMIPND is the only MACCS CHRONC input parameter that is not processed by MACCS2 when the MACCS food model is implemented. 
Table 4-4. Input Parameters that Must Be Added to MACCS CHRONC Input Files to Convert Them to MACCS2 Input Files

\begin{tabular}{|l|l|c|l||}
\hline $\begin{array}{c}\text { MACCS2 FORTRAN } \\
\text { Variable Name } \\
\text { (CHRONC File } \\
\text { Record Identifier) }\end{array}$ & \multicolumn{1}{|c||}{$\begin{array}{c}\text { CHRONC Input File } \\
\text { Data Block }^{\mathrm{a}}\end{array}$} & $\begin{array}{c}\text { Location of } \\
\text { Description } \\
\text { MACCS2 } \\
\text { User's Guide }\end{array}$ & \multicolumn{1}{|c||}{ Function of Input Parameter Value } \\
\hline $\begin{array}{l}\text { FDPATH } \\
\text { (CHFDPATH) }\end{array}$ & Miscellaneous & 7.10 .2 & $\begin{array}{l}\text { Specifies whether the MACCS or } \\
\text { COMIDA2 food pathway model is to be } \\
\text { applied }\end{array}$ \\
\hline $\begin{array}{l}\text { DUR_INTPHAS } \\
\text { (DUR_INTPHAS) }\end{array}$ & $\begin{array}{l}\text { Long-term Protective } \\
\text { Action }\end{array}$ & 7.4 & Duration of intermediate phase \\
\hline $\begin{array}{l}\text { EXPTIM } \\
\text { (CHEXPTIM) }\end{array}$ & $\begin{array}{l}\text { Long-term Protective } \\
\text { Action }\end{array}$ & 7.4 & $\begin{array}{l}\text { Maximum exposure time for CHRONC } \\
\text { calculations }\end{array}$ \\
\hline
\end{tabular}

${ }^{a}$ This column provides the name of the data block that contains the new parameters in the MACCS2 sample problems. 
THIS PAGE INTENTIONALLY LEFT BLANK 


\subsection{ATMOS Input File}

\subsection{Introduction to ATMOS}

ATMOS calculates the dispersion and deposition of material released to the atmosphere as a function of downwind distance. It utilizes a Gaussian plume model with Pasquill-Gifford dispersion parameters (Turner 1970). The phenomena that ATMOS treats are (1) building wake effects, (2) buoyant plume rise, (3) plume dispersion during transport, (4) wet and dry deposition, and (5) radioactive decay and ingrowth. A detailed discussion of the atmospheric dispersion and deposition models implemented in MACCS2 is provided in Chapter 2 of the MACCS Model Description.

At the midpoint of each spatial interval along the transport path, air and ground concentrations for all the radionuclides are calculated as well as miscellaneous information about plume size, height, and transport timing. These data are stored in common blocks which are used later by the EARLY and CHRONC modules of MACCS2.

Transport and deposition in ATMOS are treated with a one-dimensional model. Concentration values are calculated only for the plume centerline. There is no calculation in ATMOS of off-centerline concentrations. The adjustment for off-axis location is handled in the EARLY and CHRONC modules.

MACCS incorporated a database limited to 60 radionuclides. In any single MACCS2 run, the user can define a list of up to 150 radionuclides (including all of the daughter products) which are to be considered in the analysis. All of the selected radionuclides must be present in the decaychain database included in the MACCS2 package and in the selected DCF file used for code execution.

In order to allow the user more flexibility in selecting the radionuclides to be considered, a new feature has been added to MACCS2, namely, the definition of a list of radionuclides that are to be excluded from the calculations. These radionuclides are designated as "pseudostable" and defined in the ATMOS input file. The pseudostable radionuclides are treated by MACCS2 as if they were not radioactive. They do not contribute to doses and they produce no radioactive progeny. The definition of pseudostable radionuclides was motivated by the following.

In the very first beta-test version of MACCS2, version 1.00 , the user was required to include in the calculations all progeny down to six generations. In the code's sample problems, this meant that several chains of actinides six generations long needed to be included, despite the fact that progeny beyond two or three generations are negligible contributors to dose. This resulted in a lack of economy in the calculations, since daughter products far down the chains generally do not contribute to the total dose.

The decay-chain processor considers radioactive decay over a maximum of six generations. Six generations means that the sequential list of radionuclides in a chain is limited to six members. For species that have progeny spanning more than six generations $\left(e . g .{ }^{253} \mathrm{Cf}\right)$, the dose due to 
progeny beyond six generations is ignored. At each step of decay in the chain, branching is allowed with up to three different daughter products, using branch ratios.

The decay-chain database was obtained from the Radiation Shielding Information Center (1994). It is present in the distribution package as file INDEXR.DAT. If radionuclides are defined to be pseudostable, the specified pseudostable radionuclides are not used in the calculations and there is no need to include their progeny in the calculations. The DOS FORTRAN program, CHAIN.EXE, provides decay chains for user input radionuclides. CHAIN.EXE is included in the MACCS2 program package.

Several different options for specifying weather conditions are available to the user. These include two weather sampling options - category bin sampling and strictly random sampling - as well as three different methods of specifying a single weather trial: (1) constant weather conditions, (2) fixed start time in the weather file, and (3) user-supplied 120-hr weather sequence.

It is up to the user to specify the various parameters needed for these calculations. There are no default values. All of this information is supplied through the user input file to ATMOS and all of the input parameters are described in this chapter.

\subsubsection{Format of Input Parameter Description Block}

The conventions used in processing the ATMOS input file are identical to the conventions followed in processing the EARLY and CHRONC input files. Section 2.2.1.2 provides an overview of the MACCS/MACCS2 free-format input processor.

Each file line containing user input data must begin with a record identifier. Record identifiers must begin in column 1 and consist of 11 characters. Record identifiers usually begin with a two-character mnemonic for the data group to which the identifier belongs, followed by the variable name and three numeric characters that are used for sequencing. The two-character data group mnemonics are included in parentheses in the remaining headings of this section. Sample data records are provided for each input parameter.

Each ATMOS input parameter is described in a stylized block of text that presents the following information: (1) the FORTRAN variable name used in the code, (2) the type of the data item (integer, real, logical, or character), (3) an indication of whether only a single value (scalar) or multiple values (array) are required, (4) minimum and maximum allowable values (or lengths), (5) a statement describing the variable, and (6) an example of the variable's use.

\subsection{Run Identification (RI) Data}

In order to identify the computer run that is being performed, the user is required to supply a text field that will be printed on all of the list output produced for this run by MACCS2. All of the MACCS2 programs obtain the current date and time from the computer operating system so it is not necessary to include information of that type. In addition to this text field, a text field 
describing the source term is supplied separately in the release description data block that defines the source term.

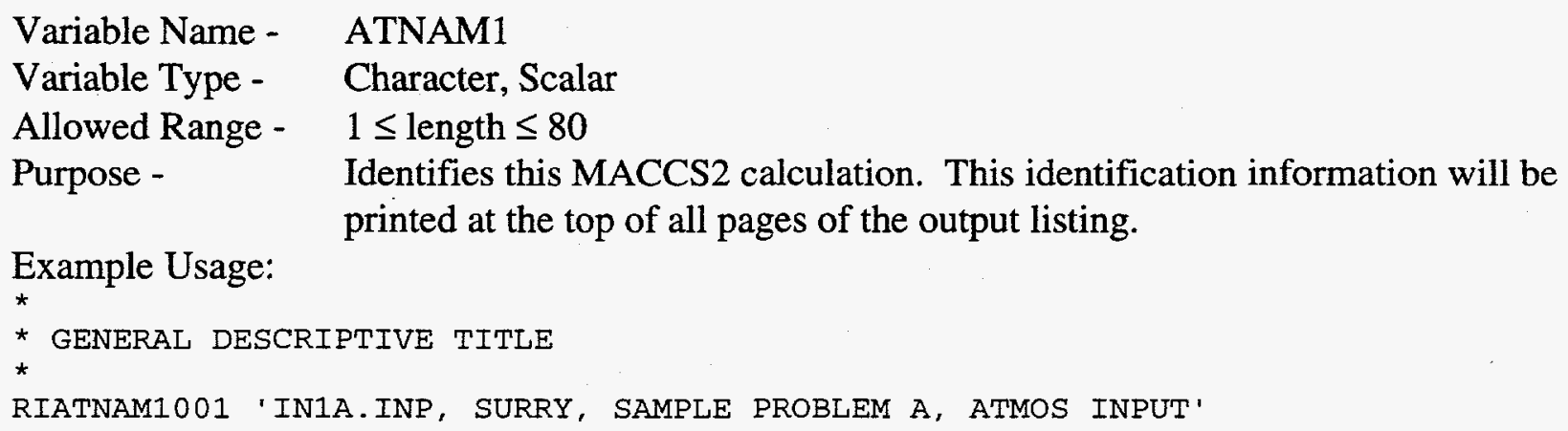

Note: The asterisk in column 1 denotes a comment.

\subsection{Geometry (GE) Data}

A polar-grid coordinate system is used by MACCS2 to represent the region surrounding the facility. The facility itself is always located at the centerpoint of the coordinate system $(r=0)$. The data in this section define the grid spacing between spatial elements in the radial direction. All of the consequence calculations performed by MACCS2 are stored on the basis of the radial spacing defined here.

For example, air and ground concentrations are calculated to be representative of the entire length of the spatial element (not its centerpoint). The values of $\sigma_{y}$ and $\sigma_{z}$ representative of the spatial interval are average values, as follows,

$$
\sigma_{\text {average }}=0.5\left(\sigma_{\text {initial }}+\sigma_{\text {final }}\right)
$$

where $\sigma_{\text {initial }}$ is the plume size upon entering the spatial interval and $\sigma_{\text {final }}$ is the plume size upon leaving the spatial interval. Deposition processes are similarly averaged over the length of the spatial interval, as described in the MACCS Model Description, pages 2-18 through 2-24.

\footnotetext{
Variable Name: NUMRAD

Variable Type: Integer, Scalar

Allowed Range: $\quad 2 \leq$ value $\leq 35$

Purpose:

Number of radial spatial intervals defined in the model. This quantity defines the polar coordinate spatial grid that will be used by all three of the program modules: ATMOS, EARLY, and CHRONC. If a Site Data file is being used, the value supplied here must match exactly the value supplied on that file as variable NSPDTS.
} 
Example Usage:

$\star$

* Number of radial spatial elements

GENUMRAD001 26

Variable Name: SPAEND

Variable Type: Real, Array

Allowed Range: $\quad 0.05 \leq$ value $\leq 9999$. (kilometers)

Purpose:

Distance in kilometers to the endpoints of the spatial intervals. If a Site Data file is being used, the values supplied here must be within $10 \%$ of the corresponding parameter values supplied on that file for the array SPDSTS. The spacing between adjacent spatial intervals is now required to be at least 0.1 $\mathrm{km}$.

Note: The Gaussian plume dispersion parameterizations commonly available (see Section 5.7) are likely to be of limited value at distances less than $0.5 \mathrm{~km}$ because of building wake effects. If close-in assessments are required, the user is cautioned to examine the CCDFs of the atmospheric dispersion parameters (Section 5.18) and verify the adequacy of the results.

Example Usage:

*

* spatial endpoint distances in kilometers

*

GESPAENDO01

GESPAEND002

0.16

3.22

11.27

0.52

4.02

1.21

4.83

16.09

20.92

GESPAENDO 04

40.23

48.28

64.37

160.93

241.14

321.87

$\begin{array}{ll}1.61 & 2.13 \\ 5.63 & 8.05 \\ 25.75 & 32.19 \\ 80.47 & 112.65 \\ 563.27 & 804.67\end{array}$

GESPAEND006

1609.34

Note: Elements in the array must be separated by blanks or a comma. The number of items per card is left to the user's discretion. The sequence numbers of the record identifiers must be in ascending order.

\subsection{Radionuclide (IS) Data}

This section defines the physical characteristics of the radionuclides that are to be modeled. They are divided into groups that share similar physical and chemical characteristics. The radioactive decay data (half-life and decay schemes) are provided to MACCS2 by the file INDEXR.DAT. Decay chain data may be obtained by exercising the CHAIN.EXE program included in the MACCS2 program package. 

Variable Name:
NUMISO
Variable Type:
Integer, Scalar
Allowed Range:
Purpose:
$1 \leq$ value $\leq 150$
Purpo:
Number of radionuclides defined in the model. Several other input parameters use this value to determine the number of values that must be supplied. Each defined radionuclide must be present in the decay-chain definition file, INDEXR.DAT. Also, the dose conversion factor file specified for EARLY (see Section 6.2), must include data for each of the radionuclides defined here.

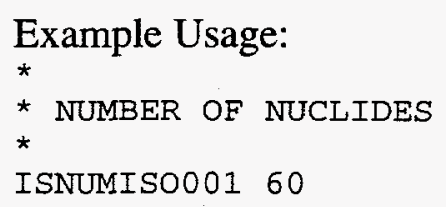

Variable Name:

MAXGRP

Variable Type:

Integer, Scalar

Allowed Range:

$1 \leq$ value $\leq 10$

Purpose:

Number of chemical element groups defined in the model. Each radionuclide will be assigned to a chemical element group, e.g., noble gases. The wet and dry deposition characteristics of a radionuclide, as well as its release fraction, depend on the number of the element group (IGROUP) to which it is assigned.

Example Usage:

*

* NUMBER OF ELEMENT GROUPS

ISMAXGRPO01 9

Variable Name: WETDEP

Variable Type: Logical, Array

Allowed Value: $\quad$.TRUE. or .FALSE.

Purpose:

Logical flag for each of the element groups that indicates whether they are subject to wet deposition. The user must supply MAXGRP number of values in column 1 of the data block.

Variable Name: DRYDEP

Variable Type: Logical, Array

Allowed Value: $\quad$.TRUE. or .FALSE.

Purpose:

Logical flag for each of the radionuclide groups that indicates whether they are subject to dry deposition. The user must supply MAXGRP number of values in column 2 of the data block. 


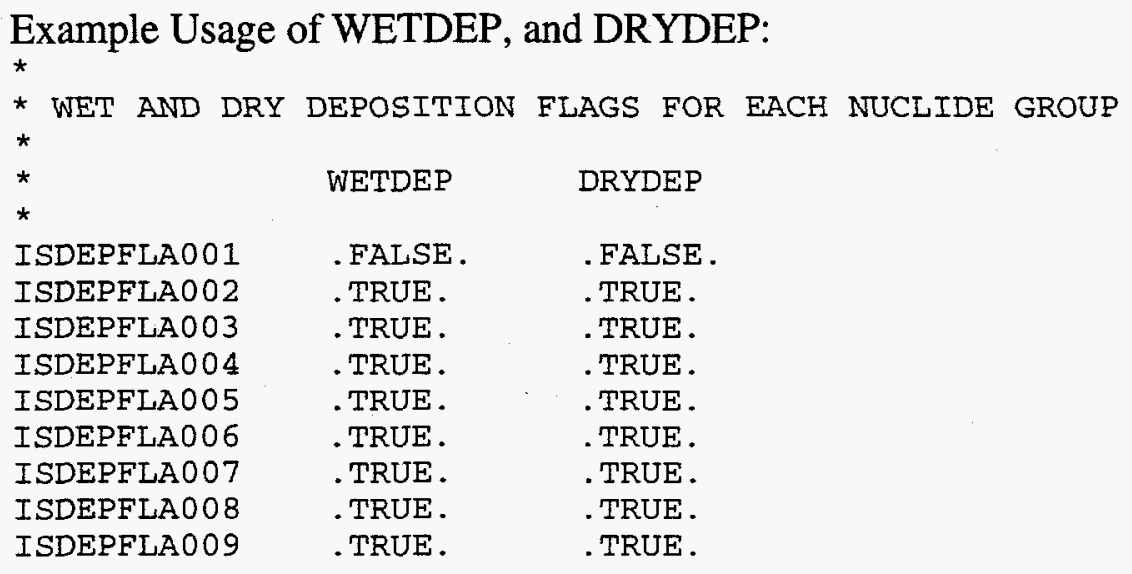

In contrast to MACCS, MACCS2 obtains decay chains directly from a database file. As a result, the ATMOS input file no longer contains information on parent-daughter relationships or radiological decay rates. Those data are taken from the file INDEXR.DAT, supplied by the Radiation Shielding Information Center as part of the FGR-DOSE/DLC-167 data package.

When MACCS2 is executed, it attempts to open a file named INDEXR.DAT in the current directory. If the file is not present, the code will not execute. There is no provision for the user to specify an alternative directory (or filename) for this information as is possible for the dose conversion factor file. If special applications require switching between alternative versions of the decay-chain database, the user would have to modify the RUNMAX2.BAT file in order to copy the various files into a file in the local directory named INDEXR.DAT.

Since the decay-chain database INDEXR.DAT defines the parent-daughter relationships, the input variables PARENT and HAFLIF are not used in MACCS2. As a result, the ISOTPGRP data block in MACCS2 consists of two columns of data, whereas the corresponding data block in MACCS has four columns of data defining the radionuclide list and the chemical group assignment of each radionuclide.

Note that the spelling of radionuclide names in MACCS and MACCS2 input files is casesensitive, that is, an uppercase letter is different from a lowercase letter. In the dose conversion factor database provided with prior versions of MACCS, radionuclide names were specified using all uppercase (capital) letters. In the MACCS2 databases, only the first letters of the radionuclide names are capitalized.

Each decay chain considered by MACCS2 is limited to a maximum of six generations. There is no transfer of material between the separate chains. The code's list output file lists the chains that are being used in the calculations. If an input error occurs because a daughter product is omitted, a message on the output listing will identify the missing radionuclide(s) and further execution will be inhibited. If error messages are generated by the omission of daughter products, the user should determine if the missing daughters are needed in the calculations. If they are not needed, calculational expense may be reduced by specifying the missing daughters to be pseudostable. 
MACCS2 requires approximately twice as much execution time as MACCS version 1.5.11.1. The increased run time of MACCS2 is due almost entirely to the number of calculations performed in the decay-chain processor's integration of daughter ingrowth and decay. Thus, the most expedient means of reducing run time is to keep the radionuclide set limited to those species thought to be important contributors to risk.

Variable Name: NUMSTB

Variable Type: $\quad$ Integer, Scalar

Allowed Range: $\quad 0 \leq$ value $\leq 150$

Purpose: Defines the number of pseudostable radionuclides.

Variable Name: NAMSTB

Variable Type: $\quad$ Character, Array

Allowed Length: $\quad 3 \leq$ length $\leq 8$

Purpose:

Defines the list of pseudostable radionuclides. Pseudostable radionuclides do not contribute to doses. Progeny of pseudostable radionuclides should not be included on the list of radionuclides, NUCNAM. The user must supply NUMSTB values in column 1 of the data block.

Example Usage:

$\star$

* NUMBER OF PSEUDOSTABLE RADIONUCLIDES (USED TO TRUNCATE THE DECAY CHAINS)

ISNUMSTB001 11

* LIST OF PSEUDOSTABLE RADIONUCLIDES

*

* NAMSTB

ISNAMSTB001 I-129

ISNAMSTBO02 Xe-131m

ISNAMSTBO0 $3 \mathrm{Xe}-133 \mathrm{~m}$

ISNAMSTB004 Xe-1351m

ISNAMSTBO0 $\mathrm{CS}-135$

ISNAMSTBO06 $\mathrm{Sm}-147$

ISNAMSTB007 U-234

ISNAMSTB008 U-235

ISNAMSTBO09 U-236

ISNAMSTB010 U-237

ISNAMSTB011 Np-237

\begin{tabular}{|c|c|c|c|}
\hline (DAUGHTER & OF & $\mathrm{Te}-129$ AND & $\mathrm{Te}-129 \mathrm{~m})$ \\
\hline (DAUGHTER & OF & $I-131)$ & \\
\hline (DAUGHTER & OF & $I-133)$ & \\
\hline (DAUGHTER & OF & $I-135)$ & \\
\hline (DAUGHTER & OF & $\mathrm{Xe}-135 \mathrm{AND}$ & $\mathrm{Xe}-135 \mathrm{~m})$ \\
\hline (DAUGHTER & OF & Pm-147) & \\
\hline (DAUGHTER & OF & Pu-238) & \\
\hline (DAUGHTER & OF & $P u-239)$ & \\
\hline (DAUGHTER & OF & $\mathrm{Pu}-240)$ & \\
\hline (DAUGHTER & OF & Pu-241) & \\
\hline (DAUGHTER & $O F$ & Am-241) & \\
\hline
\end{tabular}

Variable Name: NUCNAM

Variable Type: $\quad$ Character, Array

Allowed Range: $\quad 3 \leq$ length $\leq 8$

Purpose:

Name of the radionuclide, e.g.. Co-58. The user must supply NUMISO values for this variable in column 1 of the data block. 


\begin{tabular}{|c|c|c|}
\hline Variable Name: & \multicolumn{2}{|l|}{ IGROUP } \\
\hline Variable Type: & \multicolumn{2}{|c|}{ INTEGER, Array } \\
\hline Allowed Range: & \multicolumn{2}{|c|}{$1 \leq$ value $\leq$ MAXGRP } \\
\hline Purpose: & \multicolumn{2}{|c|}{$\begin{array}{l}\text { The radionuclides should be gro } \\
\text { properties. Both the deposition } \\
\text { radionuclide depend on the eler } \\
\text { members of an element group } \\
\text { release fraction. The user must } \\
\text { column } 2 \text { of the data block. }\end{array}$} \\
\hline \multicolumn{3}{|l|}{$\begin{array}{l}\text { Example Usage: } \\
\text { * }\end{array}$} \\
\hline $\begin{array}{l}\star \\
\star \\
\star\end{array}$ & NUCNAM & IGROUP \\
\hline ISOTPGRP001 & Co-58 & 6 \\
\hline ISOTPGRP002 & Co- 60 & 6 \\
\hline ISOTPGRP003 & $\mathrm{Kr}-85$ & 1 \\
\hline ISOTPGRP004 & $\mathrm{Kr}-85 \mathrm{M}$ & 1 \\
\hline ISOTPGRP005 & $\mathrm{Kr}-87$ & 1 \\
\hline ISOTPGRP006 & $\mathrm{K} r-88$ & 1 \\
\hline ISOTPGRP007 & $\mathrm{Rb}-86$ & 3 \\
\hline ISOTPGRP008 & Sr-89 & 5 \\
\hline
\end{tabular}

\subsection{Wet Deposition (WD) Data}

The washout model predicts how much material is deposited on the ground by rainfall. Washout is a function of both rain duration and rain intensity. The fraction remaining after wet deposition is:

$$
\exp \left(-\mathrm{CWASH} 1 \cdot \text { rain duration • rain intensity }{ }^{\mathrm{CWASH} 2}\right) \text {, }
$$

where rain duration is in seconds and rain intensity is in millimeters per hour (Brenk and Vogt 1981). 


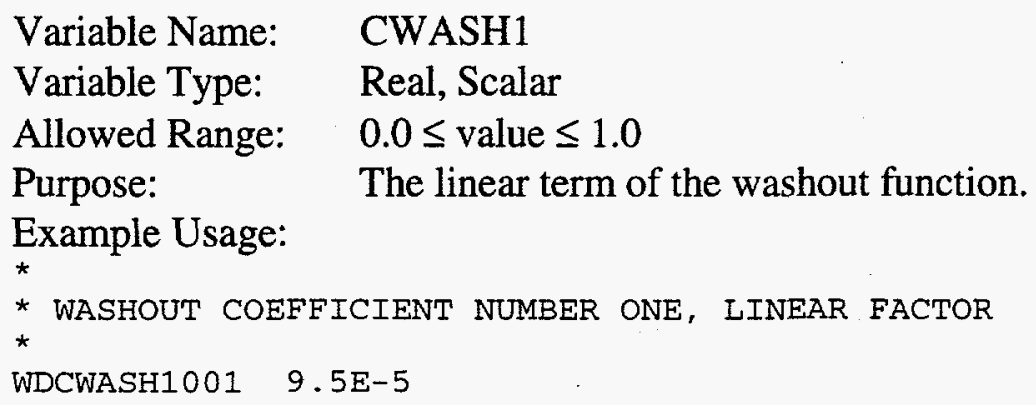

Variable Name: CWASH2

Variable Type: $\quad$ Real, Scalar

Allowed Range: $\quad 0.0 \leq$ value $\leq 1.0$

Purpose: The exponential term of the washout function.

Example Usage:

*

* WASHOUT COEFFICIENT NUMBER TWO, EXPONENTIAL FACTOR

WDCWASH2001 0.8

\subsection{Dry Deposition (DD) Data}

Dry deposition is modeled using the source depletion method. This method makes use of the simplifying assumption that deposition onto the ground does not affect the vertical distribution of the material. That is, the plume always maintains a Gaussian distribution.

The concentration of material at any single point on the ground is the product of the integrated ground-level air concentration times the deposition velocity. The material in each element group can be distributed among several particle size groups, with each element group having a different distribution of material among the particle size groups. The particle size distribution of each element group is specified in the release description data. All of the plume segments will have the same distribution of particle sizes at the time of their release, but this distribution can change with time as the plumes travel downwind.

Variable Name: NPSGRP

Variable Type: Integer, Scalar

Allowed Range: $\quad 1 \leq$ value $\leq 10$

Purpose:

The number of particle size groups that will be used for the dry deposition model. A deposition velocity must be specified for each representative particle size group. 
Example Usage:

* NUMBer of particle size groups

$*$

DDNPSGRPO01 3

Variable Name: VDEPOS

Variable Type: $\quad$ Real, Array

Allowed Range: $\quad 0.0 \leq$ value $\leq 10.0$ (meters/second)

Purpose:

The representative dry deposition velocities of the particle size groups. The user must supply NPSGRP values for this variable on one or more cards.

Example Usage:

$\begin{array}{llll}\text { DDVDEPOS001 } & 0.001 & 0.01 & 0.02\end{array}$

\subsection{Dispersion Parameter (DP) Data}

The Gaussian plume model of atmospheric dispersion uses spatially dependent dispersion parameters, $\sigma_{\mathrm{y}}$ and $\sigma_{\mathrm{z}}$. These can be supplied in two different ways: as power-law functions or in the form of precalculated tables for a lookup-table algorithm.

The power-law function approach is described in Section 5.7.1. The lookup-table approach is described in Section 5.7.2. The user's choice as to which of the two models is to be used can be controlled by the ATMOS input variable NUM_DIST (described in Section 5.7.1). If this data record is not found, or if an input value of 0 is specified, the code will perform its calculations using the power-law approach described in the next section. If, however, a data record containing this record identifier is found, the code will process a set of tables defining $\sigma_{y}$ and $\sigma_{z}$ as functions of distance, and use those data values for the dispersion calculations, thus bypassing the power-law functions completely.

\subsubsection{Definition of Power-Law Function Parameters}

The MACCS2 power-law functions for $\sigma_{\mathrm{y}}$ and $\sigma_{\mathrm{z}}$ are functions of the form:

$$
\begin{aligned}
& \sigma_{\mathrm{y}}=\mathrm{CYSIGA} \cdot \mathrm{X}^{\mathrm{CYSIGB}}, \text { and } \\
& \sigma_{\mathrm{z}}=\mathrm{CZSIGA} \cdot \mathrm{X}^{\mathrm{CZSIGB}},
\end{aligned}
$$

where $\sigma_{y}, \sigma_{z}$, and $X$ (the downwind distance from the source), are all in meters. There are numerous parameterizations available in the literature. The user must ensure that the categorization scheme used to define the stability classes for the meteorology is consistent with the dispersion parameterization defined by the following variables. Input parameter values for three parameterization schemes are presented in the Example Usage section that follows. 
Variable Name:

CYSIGA

Variable Type:

Real, Array

Allowed Range:

$1 . E-6 \leq$ value $\leq 10.0$

Purpose:

The linear term of the expression for $\sigma_{\mathrm{y}}$. The user must supply six values of CYSIGA, one for each of the six Pasquill-Gifford stability classes (classes A through F).

Variable Name:

CYSIGB

Variable Type:

Real, Array

Allowed Range:

1. E- $6 \leq$ value $\leq 10.0$

Purpose:

The exponential term of the expression for $\sigma_{\mathrm{y}}$. The user must supply six values of CYSIGB, one for each of the six Pasquill-Gifford stability classes (classes A through F).

Variable Name:

CZSIGA

Variable Type:

Real, Array

Allowed Range:

1.E- $6 \leq$ value $\leq 10.0$

Purpose:

The linear term of the expression for $\sigma_{z}$. The user must supply six values of CZSIGA, one for each of the six Pasquill-Gifford stability classes (classes A through F).

Variable Name:

\section{CZSIGB}

Variable Type:

Real, Array

Allowed Range:

Purpose:

1.E- $6 \leq$ value $\leq 10.0$

The exponential term of the expression for $\sigma_{z}$. The user must supply six values of CZSIGB, one for each of the six Pasquill-Gifford stability classes (classes A through F).

Example Usage:

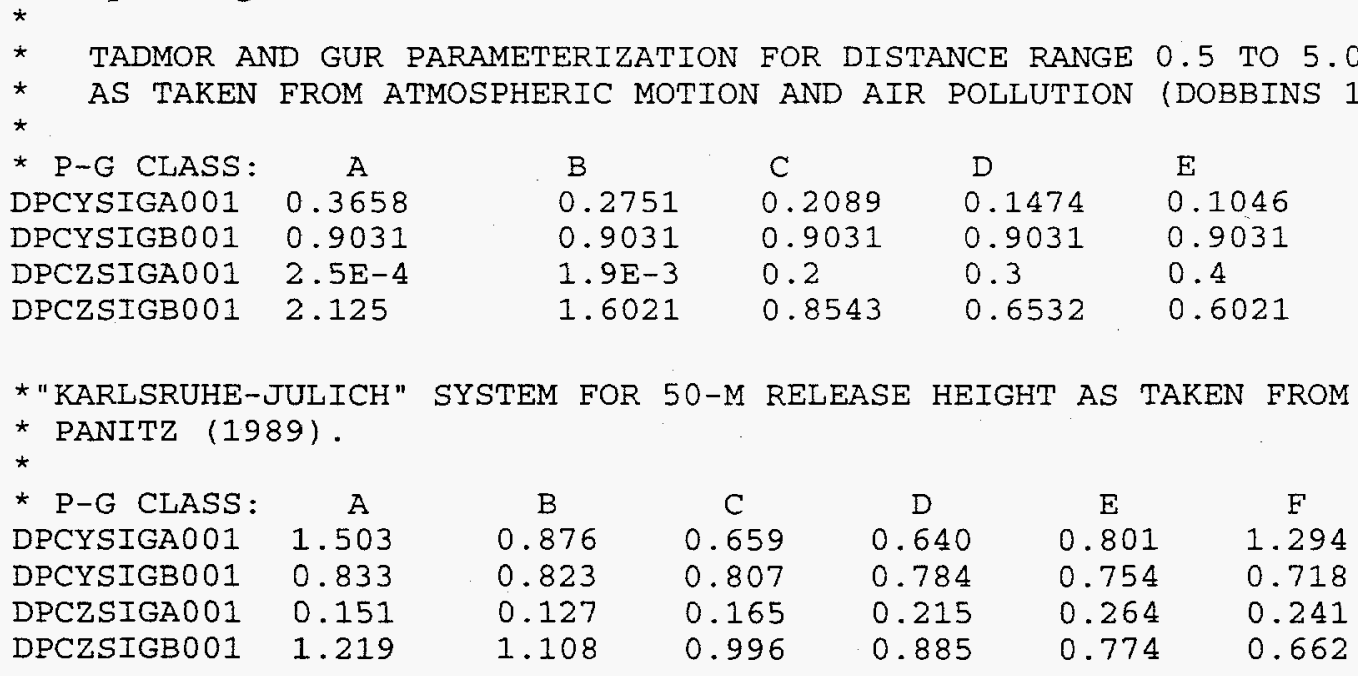




* "ST. LOUIS" SYSTEM FOR URBAN AREAS AS TAKEN FROM VOGT $(1977)$.
*(NOTE: THE REFERENCE PROVIDED DATA ONLY FOR CLASSES B THROUGH E.
*
$\begin{array}{cccccccc}\text { * P-G CLASS : } & \text { A } & \text { B } & \text { C } & \text { D } & \text { E } & \text { F } \\ \text { DPCYSIGA001 } & 1.7000 & 1.7000 & 1.4400 & 0.9100 & 1.0200 & 1.0200 \\ \text { DPCYSIGB001 } & 0.7170 & 0.7170 & 0.7100 & 0.7290 & 0.6480 & 0.6480 \\ \text { DPCZSIGA001 } & 0.0790 & 0.0790 & 0.1310 & 0.9100 & 1.9300 & 1.9300 \\ \text { DPCZSIGB001 } & 1.2000 & 1.2000 & 1.0460 & 0.7020 & 0.4650 & 0.4650\end{array}$

\subsection{2 $\sigma_{\mathrm{y}}$ and $\sigma_{\mathrm{z}}$ Lookup-Table Option}

The lookup-table option allows the user to bypass the power-law functions for $\sigma_{\mathrm{y}}$ and $\sigma_{\mathrm{z}}$. This code option utilizes an interpolation algorithm that avoids the numerical instabilities observed with cubic spline fits. The calculational approach utilizes the Hermite cubic approach of SUBROUTINE PCHEZ, as obtained from Kahaner, Moler, and Nash (1989). This new lookuptable algorithm can be used to implement alternative dispersion parameterizations or to utilize fits to site-specific tracer data.

Invocation of the lookup-table feature is triggered by the inclusion of a card with a record identifier of NUM_DIST on the ATMOS input file. Allowable values for NUM_DIST, if such a card is present, are either 0 (signifying that no tables are being provided), or integers between 3 and 50 , inclusive.

Specifying a value of 0 is equivalent to deleting the data record and the code will then proceed to use the power-law model for calculating the $\sigma_{\mathrm{y}}$ and $\sigma_{\mathrm{z}}$ dispersion parameters, functioning exactly the same as its predecessors. Specifying values of 1 or 2 for NUM_DIST will result in the diagnosis of an input error.

When NUM_DIST is given a value between 3 and 50, MACCS2 will not process the data records with record identifiers CYSIGYA, CYSIGB, etc., instead obtaining dispersion parameter data from the input file tables with record identifiers A-STB/DIS01 through F-STB/DIS50. However, irrespective of which option is utilized, the data records DPYSCALE and DPZSCALE, defining linear scaling factors for $\sigma_{\mathrm{y}}$ and $\sigma_{\mathrm{z}}$, must be supplied.

The format of that file is intended to be self-explanatory. Figures 5-1 through 5-3 list portions of the $\sigma_{y}$ and $\sigma_{z}$ input tables developed to represent the Tadmor-Gur $(0.5-5-\mathrm{km})$ curves as presented in Dobbins (1979) and the Briggs curves for open country and urban terrain as presented in Hanna, Briggs, and Hosker (1982).

This new feature was implemented to allow the use of parameterizations other than the simple power law (e.g., the Briggs formulas). Also, if tracer experiments are available for a site, it might be possible to process such data into the tables of horizontal and vertical standard deviations $\left(\sigma_{\mathrm{y}}\right.$ and $\left.\sigma_{\mathrm{z}}\right)$ required by this new feature of MACCS2. 


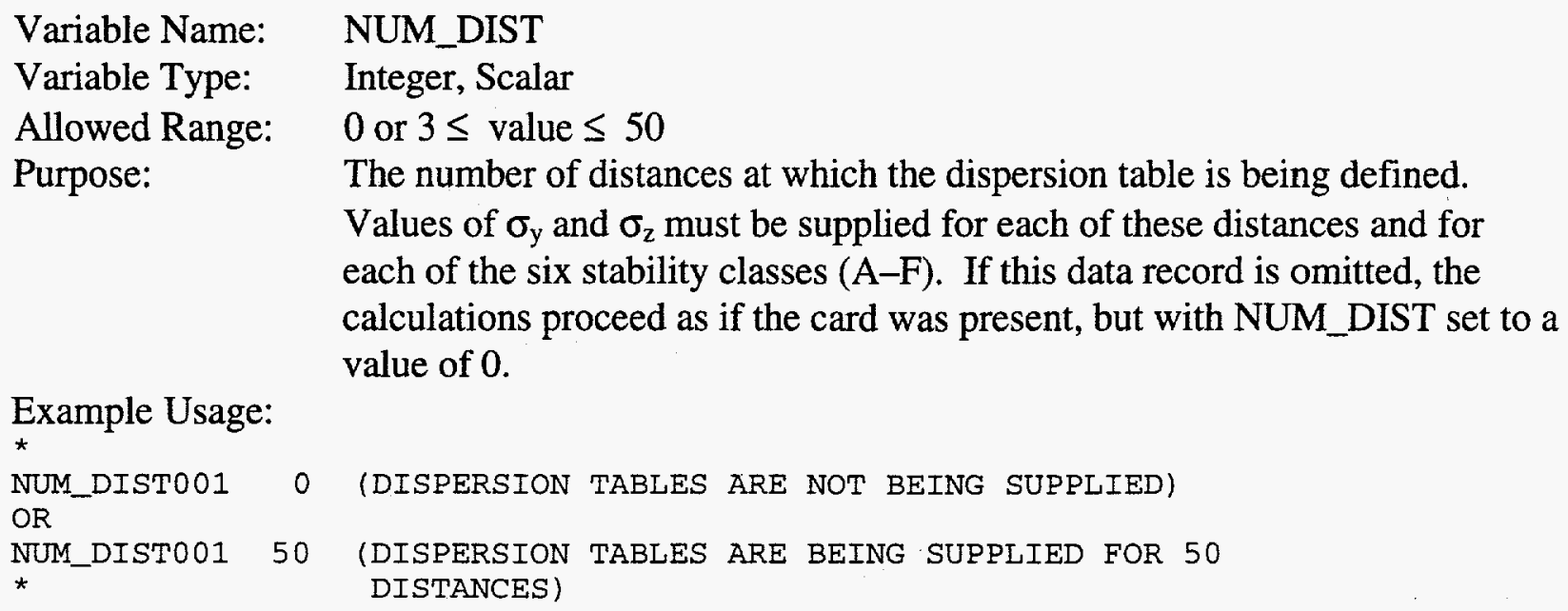

Variable Name: DISTANCE

Variable Type: Real, Array

Allowed Range: $\quad 1.0 \leq$ value $\leq 1 . E+8$ (meters)

Purpose:

The downwind distances at which $\sigma_{\mathrm{y}}$ and $\sigma_{\mathrm{z}}$ values are being defined. This record is only processed when NUM_DIST is defined to have a value of at least 3. The user must supply NUN_DIST values of DISTANCE. The values specified must be monotonically increasing, and an error will be diagnosed if successive values are decreasing or identical. These values are organized into six different data-block tables, with each table corresponding to one of the stability classes A through F. The values of DISTANCE are specified in column 1 of the data blocks.

\footnotetext{
Variable Name: $\quad$ SIGMA_Y

Variable Type: $\quad$ Real, Array

Allowed Range: $\quad 1.0 \mathrm{E}-6 \leq$ value $\leq 1 . \mathrm{E}+20$ (meters)

Purpose:

Defines the user-specified tables of $\sigma_{y}$ as a function of distance. One table is provided for each of the six stability classes. The user must supply NUM_DIST values of SIGMA_Y. The values specified must be monotonically increasing, and an error will be diagnosed if successive values are decreasing or identical. These values are organized into six different data-block tables, with each table corresponding to one of the stability classes A through F. The values of SIGMA_Y are specified in column 2 of the data blocks.
} 
Variable Name:

SIGMA_Z

Variable Type:

Real, Array

Allowed Range:

$1.0 \mathrm{E}-6 \leq$ value $\leq 1 . \mathrm{E}+20$ (meters)

Purpose:

Defines the user-specified tables of $\sigma_{z}$ as a function of distance. One table is provided for each of the six stability classes. The user must supply NUM_DIST values of SIGMA_Z. The values specified must be monotonically increasing, and an error will be diagnosed if successive values are decreasing or identical. These values are organized into six different data-block tables, with each table corresponding to one of the stability classes A through F. The values of SIGMA_Z are specified in column 3 of the data blocks.

Examples of the appearance of DISTANCE, SIGMA_Y, and SIGMA_Z for F-stability are shown in Figures 5-1 through 5-3. Figure 5-1 illustrates $\sigma_{\mathrm{y}}$ and $\sigma_{\mathrm{z}}$ values as functions of distance when the Tadmor-Gur formula (for 0.5 to $5 \mathrm{~km}$ ) is utilized; this is the formula used to generate the ATMOS input file for sample problem A (input file IN1A.INP included in the distribution package). Figure 5-2 illustrates the Briggs formula values for open country $(0.1$ to $10 \mathrm{~km})$; this is the formula used for sample problem D, subcase BRO (input file IN1D_BRO.INP included in the distribution package). Finally, Figure 5-3 illustrates the Briggs formula values for urban areas $(0.1$ to $10 \mathrm{~km})$; this is the formula used for sample problem D, subcase BRU (input file IN1D_BRU.INP included in the distribution package).

\subsubsection{Scaling Factors for Dispersion}

In order to allow for situations where the user wishes to apply across-the-board linear scaling factors to $\sigma_{\mathrm{y}}$ and $\sigma_{\mathrm{z}}$, the input parameters YSCALE and ZSCALE are provided. These scale factors are applied under both the power-law and lookup-table options. When specified to have values other than 1 , these scaling factors are used to adjust the values of $\sigma_{y}$ and $\sigma_{2}$ that have been defined under either of those options. The same scaling factor is applied for each of the six stability classes.

Variable Name: $\quad$ YSCALE

Variable Type: Real, Scalar

Allowed Range: $\quad 0.01 \leq$ value $\leq 100.0$ (unitless)

Purpose:

A linear scaling factor that is applied to the formula to calculate $\sigma_{\mathrm{y}}$. This parameter is a convenient method for adjusting all the linear factors (CYSIGA) by a constant multiplicative factor.

Example Usage:

* Linear scaling factor for sigma-y function, Normally 1

DPYSCALE001 1. 
Figure 5-1. F-stability dispersion table-Tadmor-Gur formula (for 0.5 to $5 \mathrm{~km}$ ).

* F-Stabi1ity
F-STB/DIS01
F-STB/DIS02
F-STB/DIS03
F-STB/DIS04
F-STB/DIS05
F-STB/DIS06
F-STB/DIS07
F-STB/DIS08
F-STB/DIS09
F-STB/DIS10
F-STB/DIS11
F-STB/DIS12
F-STB/DIS13
F-STB/DIS14
F-STB/DIS15
F-STB/DIS16
F-STB/DIS17
F-STB/DIS18
F-STB/DIS19
F-STB/DIS20
F-STB/DIS21
F-STB/DIS22
F-STB/DIS23
F-STB/DIS24
F-STB/DIS25
F-STB/DIS26
F-STB/DIS27
F-STB/DIS28
F-STB/DIS29
F-STB/DIS30
F-STB/DIS31
F-STB/DIS32
F-STB/DIS33
F-STB/DIS34
F-STB/DIS35
F-STB/DIS36
F-STB/DIS37
F-STB/DIS38
F-STB/DIS39
F-STB/DIS40
F-STB/DIS41
F-STB/DIS42
F-STB/DIS43
F-STB/DIS44
F-STB/DIS45
F-STB/DIS 6
F-STB/DIS47
F-STB/DIS48
F-STB/DIS 99
F-STB/DIS50

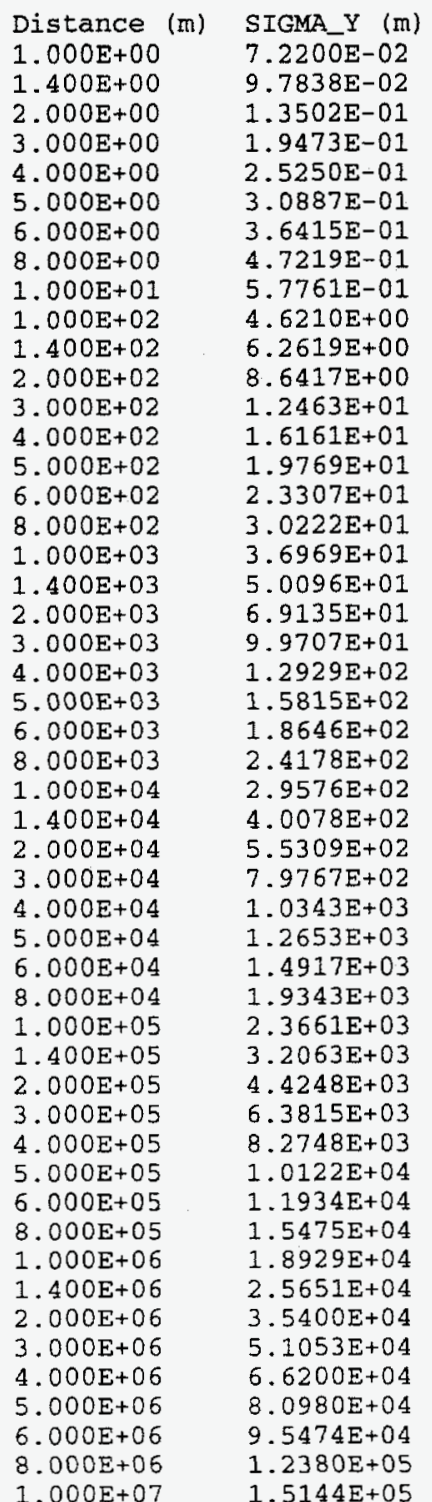

SIGMA_Z (m) 2. $0000 \mathrm{E}-01$ 2. 4491E-01 3. $0356 \mathrm{E}-01$ 3. $8749 \mathrm{E}-01$ 4. $6076 \mathrm{E}-01$ $5.2700 \mathrm{E}-01$ 5. $8814 \mathrm{E}-01$ $6.9934 \mathrm{E}-01$ 7. $9989 \mathrm{E}-01$ 3. $1991 \mathrm{E}+00$ 3. $9174 \mathrm{E}+00$ $4.8557 \mathrm{E}+00$ 6. $1981 \mathrm{E}+00$ 7. $3700 \mathrm{E}+00$ 8. $4297 \mathrm{E}+00$ $9.4076 \mathrm{E}+00$ 1. $1186 \mathrm{E}+01$ 1. $2795 \mathrm{E}+01$ 1. $5667 \mathrm{E}+01$ 1. $9420 \mathrm{E}+01$ 2. $4789 \mathrm{E}+01$

2. $9476 \mathrm{E}+01$ 3. $3714 \mathrm{E}+01$ 3. $7625 \mathrm{E}+01$ 4. $4739 \mathrm{E}+01$ 5. $1172 \mathrm{E}+01$ 6. $2661 E+01$ 7. $7669 \mathrm{E}+01$ 9. $9142 E+01$ 1. $1789 \mathrm{E}+02$ 1. $3484 \mathrm{E}+02$ 1. $5048 \mathrm{E}+02$ 1. $7893 E+02$ 2. $0466 E+02$ 2. $5061 E+02$ 3. $1063 E+02$ 3. $9651 \mathrm{E}+02$ 4. $7149 \mathrm{E}+02$ $5.3927 E+02$ 6. $0183 \mathrm{E}+02$ $7.1563 \mathrm{E}+02$ 8. $1852 \mathrm{E}+02$ 1. $0023 \mathrm{E}+03$ 1. $2424 \mathrm{E}+03$ 1. $5858 \mathrm{E}+03$ 1. $8857 \mathrm{E}+03$ $2.1568 \mathrm{E}+03$ $2.4070 \mathrm{E}+03$ $2.8621 \mathrm{E}+03$ $3.2736 \mathrm{E}+03$
Tadmor/Gur $(0.5-5 \mathrm{~km})$ Tadmor/Gur $(0.5-5 \mathrm{~km})$ Tadmor/Gur $(0.5-5 \mathrm{~km})$ Tadmor/Gur $(0.5-5 \mathrm{~km})$ Tadmor/Gur $(0.5-5 \mathrm{~km})$ Tadmor/Gur $(0.5-5 \mathrm{~km})$ Tadmor/Gur $(0.5-5 . \mathrm{km})$ Tadmor/Gur $(0.5-5 \mathrm{~km})$ Tadmor/Gur $10.5-5 \mathrm{~km}$ ) Tadmor/Gur $(0.5-5 \mathrm{~km})$ Tadmor/Gur $(0.5-5 \mathrm{~km})$ Tadmor/Gur (0.5-5 km) Tadmor/Gur (0.5-5 km) Tadmor/Gur $(0.5-5 \mathrm{~km})$ Tadmor/Gur (0.5-5 km) Tadmor/Gur (0.5-5 km) Tadmor/Gur $(0.5-5 \mathrm{~km})$ Tadmor/Gur $(0.5-5 \mathrm{~km})$ Tadmor/Gur $(0.5-5 \mathrm{~km})$ Tadmor/Gur $(0.5-5 \mathrm{~km})$ Tadmor/Gur $(0.5-5 \mathrm{~km})$ Tadmor/Gur $(0.5-5 \mathrm{~km})$ Tadmor/Gur $(0.5-5 \mathrm{~km})$ Tadmor/Gur $(0.5-5 \mathrm{~km})$ Tadmor/Gur $(0.5-5 \mathrm{~km})$ Tadmor/Gur $(0.5-5 \mathrm{~km})$ Tadmor/Gur $(0.5-5 \mathrm{~km})$ Tadmor/Gur $(0.5-5 \mathrm{~km})$ Tadmor/Gur $(0.5-5 \mathrm{~km})$ Tadmor/Gur $(0.5-5 \mathrm{~km})$ Tadmor/Gur $(0.5-5 \mathrm{~km})$ Tadmor/Gur $(0.5-5 \mathrm{~km})$ Tadmor/Gur (0.5-5 km) Tadmor/Gur (0.5-5 km) Tadmor/Gur (0.5-5 km) Tadmor/Gur (0.5-5 km) Tadmor/Gur (0.5-5 km) Tadmor/Gur $(0.5-5 \mathrm{~km})$ Tadmor/Gur (0.5-5 km) Tadmor/Gur (0.5-5 km) Tadmor/GuI $(0.5-5 \mathrm{~km})$ Tadmor/Gur (0.5-5 km) Tadmor/Gur (0.5-5 km) Tadmor/Gur $(0.5-5 \mathrm{~km})$ Tadmor/Gur $(0.5-5 \mathrm{~km})$ Tadmor/Gur $(0.5-5 \mathrm{~km})$ Tadmor/Gur $(0.5-5 \mathrm{~km})$ Tadmor/Gur (0.5-5 km) Tadmor/Gur $(0.5-5 \mathrm{~km})$ Tadmor/Gur $(0.5-5 \mathrm{~km})$ 
Figure 5-2. F-stability dispersion table-Briggs open country formula $(0.1$ to $10 \mathrm{~km})$.

* F-stability
F-STB/DIS01
F-STB/DIS02
F-STB/DIS03
F-STB/DIS04
F-STB/DIS05
F-STB/DIS06
F-STB/DIS07
F-STB/DIS08
F-STB/DIS09
F-STB/DIS10
F-STB/DIS11
F-STB/DIS12
F-STB/DIS13
F-STB/DIS14
F-STB/DIS15
F-STB/DIS16
F-STB/DIS17
F-STB/DIS18
F-STB/DIS19
F-STB/DIS20
F-STB/DIS21
F-STB/DIS22
F-STB/DIS23
F-STB/DIS24
F-STB/DIS25
F-STB/DIS26
F-STB/DIS27
F-STB/DIS28
F-STB/DIS29
F-STB/DIS30
F-STB/DIS31
F-STB/DIS32
F-STB/DIS33
F-STB/DIS34
F-STB/DIS35
F-STB/DIS36
F-STB/DIS37
F-STB/DIS38
F-STB/DIS39
F-STB/DIS40
F-STB/DIS41
F-STB/DIS42
F-STB/DIS43
F-STB/DIS44
F-STB/DIS45
F-STB/DIS46
F-STB/DIS47 /DIS48
F-STB/DIS49

* F-stability

Distance (m)

1. $000 \mathrm{E}+00$

1. $400 \mathrm{E}+00$

2. $000 E+00$

3. $000 \mathrm{E}+00$

4. $000 E+00$

5. $000 \mathrm{E}+00$

6. $000 \mathrm{E}+00$

8. $000 E+00$

$1.000 \mathrm{E}+01$

1. $000 \mathrm{E}+02$

1. $400 \mathrm{E}+02$

2. $000 \mathrm{E}+02$

3. $000 \mathrm{E}+02$

$4.000 \mathrm{E}+02$

$5.000 \mathrm{E}+02$

$6.000 \mathrm{E}+02$

$8.000 \mathrm{E}+02$

$1.000 \mathrm{E}+03$

1. $400 \mathrm{E}+03$

2. $000 \mathrm{E}+03$

3. $000 \mathrm{E}+03$

$4.000 \mathrm{E}+03$

5. $000 \mathrm{E}+03$

$6.000 \mathrm{E}+03$

8. $000 \mathrm{E}+03$

1. $000 \mathrm{E}+04$

1. $400 \mathrm{E}+04$

2. $000 E+04$

3. $000 \mathrm{E}+04$

$4.000 E+04$

5. $000 \mathrm{E}+04$

$6.000 \mathrm{E}+04$

8. $000 \mathrm{E}+04$

1. $000 \mathrm{E}+05$

1. $400 E+05$

2. $000 \mathrm{E}+05$

3. $000 \mathrm{E}+05$

4. $000 \mathrm{E}+05$

5. $000 E+05$

6. $000 E+05$

$8.000 E+05$

1. $000 \mathrm{E}+06$

I. $400 \mathrm{E}+06$

2. $000 E+06$

$3.000 \mathrm{E}+06$

$4.000 E+06$

$5.000 \mathrm{E}+06$

6. $000 \mathrm{E}+06$

8. $000 E+06$

1. $000 \mathrm{E}+07$
SIGMA Y (m) 3. $9998 \mathrm{E}-02$

5. 5996E-02

7. $9992 \mathrm{E}-02$

1. $1998 \mathrm{E}-01$

1. $5997 \mathrm{E}-01$

1. $9995 \mathrm{E}-01$

2.3993E-01

3. $1987 \mathrm{E}-01$

3. $9980 \mathrm{E}-01$

3. $9801 \mathrm{E}+00$

5. $5612 \mathrm{E}+00$

7. $9212 \mathrm{E}+00$

1. $1824 \mathrm{E}+01$

1. $5689 \mathrm{E}+01$

1. $9518 \mathrm{E}+01$

2. $3311 \mathrm{E}+01$

$3.0792 \mathrm{E}+01$

3. $8139 \mathrm{E}+01$

5. $2449 \mathrm{E}+01$

$7.3030 \mathrm{E}+01$

1. $0525 \mathrm{E}+02$

1. $3522 \mathrm{E}+02$

1. $6330 \mathrm{E}+02$

1. $8974 \mathrm{E}+02$

2. $3851 \mathrm{E}+02$

2. $8284 \mathrm{E}+02$

3. $6148 \mathrm{E}+02$

4. $6188 \mathrm{E}+02$

$6.0000 E+02$

7. $1554 \mathrm{E}+02$

8. $1650 \mathrm{E}+02$

$9.0711 \mathrm{E}+02$

1. $0667 \mathrm{E}+03$

1. $2060 \mathrm{E}+03$

1. $4459 \mathrm{E}+03$

1. $7457 \mathrm{E}+03$

$2.1553 E+03$

2. $4988 \mathrm{E}+03$

2. $8006 \mathrm{E}+03$

$3.0729 \mathrm{E}+03$

$3.5556 \mathrm{E}+03$

3. $9801 E+03$

$4.7161 \mathrm{E}+03$

5. $6428 E+03$

$6.9167 \mathrm{E}+03$

$7.9900 \mathrm{E}+03$

$8.9353 E+03$

$9.7898 \mathrm{E}+03$

1. $1307 \mathrm{E}+04$

1. $2643 \mathrm{E}+04$
SIGMA_Z (m)

1. 5995E-02

2. $2391 E-02$

$3.1981 \mathrm{E}-02$

$4.7957 \mathrm{E}-02$

$6.3923 E-02$

$7.9880 \mathrm{E}-02$

$9.5828 \mathrm{E}-02$

1. $2769 \mathrm{E}-01$.

1. $5952 \mathrm{E}-01$

1. $5534 \mathrm{E}+00$

2. $1497 \mathrm{E}+00$

$3.0189 \mathrm{E}+00$

$4.4037 E+00$

5. $7143 \mathrm{E}+00$

6. $9565 E+00$

8. $1356 \mathrm{E}+00$

1. $0323 E+01$

1. $2308 E+01$

1. $5775 \mathrm{E}+01$

2. $0000 \mathrm{E}+01$

2. $5263 E+01$

2. $9091 \mathrm{E}+01$

$3.2000 E+01$

$3.4286 \mathrm{E}+01$

$3.7647 \mathrm{E}+01$

4. $0000 \mathrm{E}+01$

$4.3077 \mathrm{E}+01$

$4.5714 \mathrm{E}+0 \mathrm{I}$

4. $8000 \mathrm{E}+01$

4. $9231 E+01$

$5.0000 \mathrm{E}+01$

$5.0526 \mathrm{E}+01$

$5.1200 \mathrm{E}+01$

5. $1613 \mathrm{E}+01$

5. $2093 \mathrm{E}+01$

5. $2459 \mathrm{E}+01$

$5.2747 \mathrm{E}+01$

5. $2893 \mathrm{E}+01$

$5.2980 \mathrm{E}+01$

5. $3039 \mathrm{E}+01$

5. $3112 \mathrm{E}+01$

5. $3156 \mathrm{E}+01$

5. $3207 \mathrm{E}+01$

5. $3245 E+01$

5. $3274 E+01$

5. $3289 E+01$

5. $3298 E+01$

5. $3304 \mathrm{E}+01$

5. $3311 E+01$

5. $3316 \mathrm{E}+01$
Briggs Open Country $10.1-10 \mathrm{~km}$ Briggs Open Country $(0.1-10 \mathrm{~km})$ Briggs Open Country $(0.1-10 \mathrm{~km})$ Briggs Open Country $(0.1-10 \mathrm{~km})$ Briggs Open Country $(0.1-10 \mathrm{~km})$ Briggs Open Country $(0.1-10 \mathrm{~km})$ Briggs Open Country $(0.1-10 \mathrm{~km})$ Briggs Open Country $(0.1-10 \mathrm{~km})$ Briggs open Country $(0.1-10 \mathrm{~km})$ Briggs open Country $(0.1-10 \mathrm{~km})$ Briggs Open Country $(0.1-10 \mathrm{~km})$ Briggs Open Country $(0.1-10 \mathrm{~km})$ Briggs Open Country $(0.1-10 \mathrm{~km})$ Briggs Open Country $(0.1-10 \mathrm{~km})$ Briggs Open Country $(0.1-10 \mathrm{~km})$ Briggs Open Country $(0.1-10 \mathrm{~km})$ Briggs Open Country $(0.1-10 \mathrm{~km})$ Briggs Open Country (0.1-10 km) Briggs Open Country $(0.1-10 \mathrm{~km})$ Briggs Open Country $(0.1-10 \mathrm{~km})$ Briggs Open Country $(0.1-10 \mathrm{~km})$ Briggs Open Country $(0.1-10 \mathrm{~km})$ Briggs Open Country $(0.1-10 \mathrm{~km})$ Briggs open Country $(0.1-10 \mathrm{~km})$ Briggs Open Country $(0.1-10 \mathrm{~km})$ Briggs Open Country $(0.1-10 \mathrm{~km})$ Briggs open Country $(0.1-10 \mathrm{~km})$ Briggs open Country $(0.1-10 \mathrm{~km})$ Briggs Open Country $(0.1-10 \mathrm{~km})$ Briggs open Country $(0.1-10 \mathrm{~km})$ Briggs Open Country $(0.1-10 \mathrm{~km})$ Briggs Open Country $(0.1-10 \mathrm{~km})$ Briggs open Country $(0.1-10 \mathrm{~km})$ Briggs Open Country $(0.1-10 \mathrm{~km})$ Briggs Open Country (0.1-10 km) Briggs open Country $(0.1-10 \mathrm{~km})$ Briggs open country $(0.1-10 \mathrm{~km})$ Briggs open Country $(0.1-10 \mathrm{~km})$ Briggs open Country $(0.1-10 \mathrm{~km})$ Briggs Open Country (0.1-10 km) Briggs Open Country (0.1-10 km) Briggs open Country (0.1-10 km) Briggs Open Country $(0.1-10 \mathrm{~km})$ Briggs Open Country $(0.1-10 \mathrm{~km})$ Briggs Open Country $(0.1-10 \mathrm{~km})$ Briggs Open Country $(0.1-10 \mathrm{~km})$ Briggs Open Country $(0.1-10 \mathrm{~km})$ Briggs Open Country $(0.1-10 \mathrm{~km})$ Briggs Open Country $(0.1-10 \mathrm{~km})$ Briggs open country $(0.1-10 \mathrm{~km})$ 
Figure 5-3. F-stability dispersion table—Briggs urban formula (0.1 to $10 \mathrm{~km})$.

*F-Stability
F-STB/DIS01
F-STB/DISO2
F-STB/DIS03
F-STB/DIS04
F-STB/DIS05
F-STB/DIS06
F-STB/DIS07
F-STB/DISO8
F-STB/DISO9
F-STB/DIS10
F-STB/DIS11
F-STB/DIS12
F-STB/DIS13
F-STB/DIS14
F-STB/DIS15
F-STB/DIS16
F-STB/DIS17
F-STB/DIS18
F-STB/DIS19
F-STB/DIS20
F-STB/DIS21
F-STB/DIS22
F-STB/DIS23
F-STB/DIS24
F-STB/DIS25
F-STB/DIS26
F-STB/DIS27
F-STB/DIS28
F-STB/DIS29
F-STB/DIS30
F-STB/DIS31
F-STB/DIS32
F-STB/DIS33
F-STB/DIS34
F-STB/DIS35
F-STB/DIS36
F-STB/DIS37
F-STB/DIS38
F-STB/DIS39
F-STB/DIS40
F-STB/DIS41
F-STB/DIS42
F-STB/DIS43
F-STB/DIS44
F-STB/DIS45
F-STB/DIS46
F-STB/DIS47
F-STB/DIS48
F-STB/DIS49
F-STB/DIS50

\begin{tabular}{|c|c|c|}
\hline Distance & (m) SIGMA & (m) SIGMA_Z \\
\hline $1.000 E+00$ & $1.0998 \mathrm{E}-01$ & $7.9994 \mathrm{E}-02$ \\
\hline $1.400 \mathrm{E}+00$ & $1.5396 \mathrm{E}-01$ & $1.1199 \mathrm{E}-01$ \\
\hline $2.000 E+00$ & $2.1991 \mathrm{E}-01$ & $1.5998 \mathrm{E}-01$ \\
\hline $3.000 \mathrm{E}+00$ & $3.2980 \mathrm{E}-01$ & $2.3995 \mathrm{E}-01$ \\
\hline $4.000 E+00$ & $4.3965 E-01$ & $3.1990 \mathrm{E}-01$ \\
\hline $5.000 \mathrm{E}+00$ & $5.4945 E-01$ & $3.9985 \mathrm{E}-01$ \\
\hline $6.000 E+00$ & $6.5921 \mathrm{E}-01$ & $4.7978 \mathrm{E}-01$ \\
\hline $8.000 E+00$ & $8.7860 E-01$ & $6.3962 \mathrm{E}-01$ \\
\hline $1.000 \mathrm{E}+01$ & $1.0978 \mathrm{E}+00$ & $7.9940 \mathrm{E}-01$ \\
\hline $1.000 E+02$ & $1.0786 \mathrm{E}+01$ & $7.9407 \mathrm{E}+00$ \\
\hline $1.400 E+02$ & $1.4986 \mathrm{E}+01$ & $1.1084 E+01$ \\
\hline $2.000 \mathrm{E}+02$ & $2.1170 \mathrm{E}+01$ & $1.5765 E+01$ \\
\hline $3.000 \mathrm{E}+02$ & $3.1182 \mathrm{E}+01$ & $2.3478 \mathrm{E}+01$ \\
\hline $4.000 E+02$ & $4.0853 E+01$ & $3.1081 E+01$ \\
\hline $5.000 E+02$ & $5.0208 \mathrm{E}+01$ & $3.8579 E+01$ \\
\hline $6.000 \mathrm{E}+02$ & $5.9270 \mathrm{E}+01$ & $4.5976 E+01$ \\
\hline $8.000 E+02$ & $7.6594 \mathrm{E}+01$ & $6.0474 E+01$ \\
\hline $1.000 E+03$ & $9.2967 E+01$ & $7.4600 \mathrm{E}+01$ \\
\hline $1.400 \mathrm{E}+03$ & $1.2330 E+02$ & $1.0182 \mathrm{E}+02$ \\
\hline $2.000 \mathrm{E}+03$ & $1.6398 \mathrm{E}+02$ & $1.4033 E+02$ \\
\hline $3.000 \mathrm{E}+03$ & $2.2249 E+02$ & $1.9931 E+02$ \\
\hline $4.000 \mathrm{E}+03$ & $2.7288 \mathrm{E}+02$ & $2.5298 E+02$ \\
\hline $5.000 \mathrm{E}+03$ & $3.1754 E+02$ & $3.0237 E+02$ \\
\hline $6.000 E+03$ & $3.5794 E+02$ & 3. $4823 E+02$ \\
\hline $8.000 \mathrm{E}+03$ & $4.2940 \mathrm{E}+02$ & $4.3149 E+02$ \\
\hline $1.000 \mathrm{E}+04$ & $4.9193 E+02$ & $5.0596 \mathrm{E}+02$ \\
\hline $1.400 \mathrm{E}+04$ & $5.9944 E+02$ & $6.3612 \mathrm{E}+02$ \\
\hline $2.000 E+04$ & $7.3333 E+02$ & $8.0000 E+02$ \\
\hline $3.000 \mathrm{E}+04$ & $9.1526 E+02$ & $1.0234 \mathrm{E}+03$ \\
\hline $4.000 E+04$ & $1.0672 E \div 03$ & $1.2095 \mathrm{E}+03$ \\
\hline $5.000 \mathrm{E}+04$ & $1.2002 E+03$ & $1.3720 \mathrm{E}+03$ \\
\hline $6.000 E+04$ & $1.3200 E+03$ & $1.5179 E+03$ \\
\hline $8.000 \mathrm{E}+04$ & $1.5319 \mathrm{E}+03$ & $1.7750 \mathrm{E}+03$ \\
\hline $1.000 \mathrm{E}+05$ & $1.7179 \mathrm{E}+03$ & $2.0000 \mathrm{E}+03$ \\
\hline 1. $400 \mathrm{E}+05$ & $2.0398 E+03$ & $2.3878 E+03$ \\
\hline $2.000 E+05$ & $2.4444 E+03$ & $2.8737 E+03$ \\
\hline $3.000 \mathrm{E}+05$ & $3.0000 E+03$ & $3.5386 E+03$ \\
\hline $4.000 \mathrm{E}+05$ & $3.4677 \mathrm{E}+03$ & $4.0972 \mathrm{E}+03$ \\
\hline $5.000 \mathrm{E}+05$ & $3.8794 E+03$ & $4.5883 E+03$ \\
\hline $6.000 E+05$ & $4.2514 \mathrm{E}+03$ & $5.0318 E+03$ \\
\hline $8.000 E+05$ & $4.9117 \mathrm{E}+03$ & $5.8182 E+03$ \\
\hline $1.000 \mathrm{E}+06$ & $5.4931 E+03$ & $6.5103 E+03$ \\
\hline $1.400 \mathrm{E}+06$ & $6.5019 \mathrm{E}+03$ & $7.7104 E+03$ \\
\hline $2.000 E+06$ & $7.7733 E+03$ & $9.2222 E+03$ \\
\hline $3.000 \mathrm{E}+06$ & $9.5223 E+03$ & $1.1301 \mathrm{E}+04$ \\
\hline $4.000 E+06$ & $1.0997 \mathrm{E}+04$ & $1.3053 E+04$ \\
\hline $5.000 E+06$ & $1.2295 E+04$ & $1.4596 \mathrm{E}+04$ \\
\hline $6.000 E+06$ & $1.3469 \mathrm{E}+04$ & $1.5991 \mathrm{E} \div 04$ \\
\hline $8.000 E+06$ & $1.5554 \mathrm{E}+04$ & I. $8468 \mathrm{E}+04$ \\
\hline $1.000 \mathrm{E}+07$ & $1.7390 E+04$ & $2.0649 E+04$ \\
\hline
\end{tabular}

(m)

Briggs Urban $(0.1-10 \mathrm{~km})$ Briggs Urban $(0.1-10 \mathrm{~km})$ Briggs Urban $(0.1-10 \mathrm{~km})$ Briggs Urban $(0.1-10 \mathrm{~km})$ Briggs Urban $(0.1-10 \mathrm{~km})$ Briggs Urban $(0.1-10 \mathrm{~km})$ Briggs Urban $(0.1-10 \mathrm{~km})$ Briggs Urban $(0.1-10 \mathrm{~km})$ Briggs Urban $(0.1-10 \mathrm{~km})$ Briggs Urban $(0.1-10 \mathrm{~km})$ Briggs Urban $(0.1-10 \mathrm{~km})$ Briggs Urban $(0.1-10 \mathrm{~km})$ Briggs Urban $(0.1-10 \mathrm{~km})$ Briggs Urban $(0.1-10 \mathrm{~km})$ Briggs Urban $(0.1-10 \mathrm{~km})$ Briggs Urban $(0.1-10 \mathrm{~km})$ Briggs Urban $(0.1-10 \mathrm{~km})$ Briggs Urban $(0.1-10 \mathrm{~km})$ Briggs Urban $(0.1-10 \mathrm{~km})$ Briggs Urban $(0.1-10 \mathrm{~km})$ Briggs Urban $(0.1-10 \mathrm{~km})$ Briggs Urban $(0.1-10 \mathrm{~km})$ Briggs Urban $(0.1-10 \mathrm{~km})$ Briggs Urban $(0.1-10 \mathrm{~km})$ Briggs Urban $(0.1-10 \mathrm{~km})$ Briggs Urban (0.1-10 km) Briggs Urban $(0.1-10 \mathrm{~km})$ Briggs Urban $(0.1-10 \mathrm{~km})$ Briggs Urban $(0.1-10 \mathrm{~km})$ Briggs Urban $(0.1-10 \mathrm{~km})$ Briggs Urban $(0.1-10 \mathrm{~km})$ Briggs Urban $(0.1-10 \mathrm{~km})$ Briggs Urban $(0.1-10 \mathrm{~km})$ Briggs Urban $(0.1-10 \mathrm{~km})$ Briggs Urban $(0.1-10 \mathrm{~km})$ Briggs Urban $(0.1-10 \mathrm{~km})$ Briggs Urban $(0.1-10 \mathrm{~km})$ Briggs Urban $(0.1-10 \mathrm{~km})$ Briggs Urban $(0.1-10 \mathrm{~km})$ Briggs Urban $(0.1-10 \mathrm{~km})$ Briggs Urban $(0.1-10 \mathrm{~km})$ Briggs Urban $(0.1-10 \mathrm{~km})$ Briggs Urban $(0.1-10 \mathrm{~km}$ ) Briggs Urban $(0.1-10 \mathrm{~km}$ ) Briggs Urban $(0.1-10 \mathrm{~km})$ Briggs Urban $(0.1-10 \mathrm{~km}$ ) Briggs Urban $(0.1-10 \mathrm{~km})$ Briggs Urban $(0.1-10 \mathrm{~km})$ Briggs Urban $(0.1-10 \mathrm{~km})$ Briggs Urban $(0.1-10 \mathrm{~km})$ 


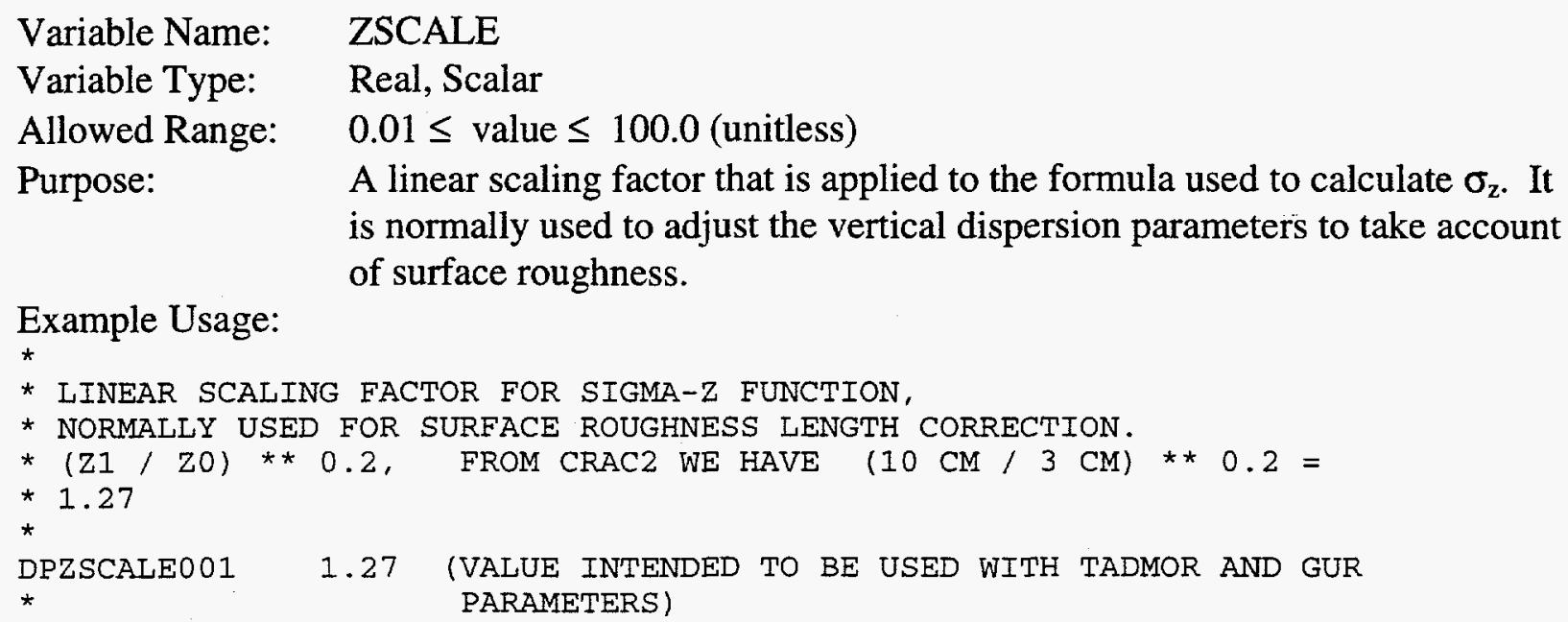

\subsection{Plume Meander (PM) Data}

In order to account for the effect of meander during transport of the plume, an expansion factor, EXPFAC, is calculated which serves to widen the plumes in the cross-wind direction. It acts as a linear factor on $\sigma_{\mathrm{y}}$ during the calculation of $\chi / \mathrm{Q}$, but it does not affect the rate of growth of $\sigma_{\mathrm{y}}$. A two-part function is used. The expansion factors used for different plume segments are independent of each other. If the release duration of the plume segment is less than or equal to BRKPNT, then the following formula will be used,

$$
\text { EXPFAC }=[\text { MAX (plume segment release duration, TIMBAS }) / \text { TIMBAS }^{\text {XPFAC1 }} .
$$

If the plume segment duration exceeds BRKPNT, then a different factor is used for the exponent of the function,

$$
\mathrm{EXPFAC}=\left[\mathrm{MAX} \text { (plume segment release duration, TIMBAS) } / \mathrm{TIMBAS}^{\mathrm{XPFAC} 2} .\right.
$$

In both expressions, the duration of the plume segment should be limited to $10 \mathrm{hr}$ because the formula is not intended for use outside of that range. For that reason, a nonfatal warning is printed on the output listing if the user specifies a release duration exceeding $10 \mathrm{hr}$.

If the user does not wish to use the plume meander model, this can be accomplished by setting TIMBAS to the duration of the plume (PLUDUR). However, this approach to turning off the meander model can only be used if there is a single plume, or if all plumes have the same duration.

Variable Name: $\quad$ TIMBAS

Variable Type: Real, Scalar

Allowed Range: $\quad 60.0 \leq$ value $\leq 86400.0$ (seconds) 
Purpose: $\quad$ The time base associated with the parameterization of the plume meander

Example Usage: adjustment factor (seconds).

* TIME BASE FOR EXPANSION FACTOR (SECONDS)

PMTIMBASO01 600. (10 MIN)

Variable Name: BRKPNT

Variable Type: $\quad$ Real, Scalar

Allowed Range: $\quad 60.0 \leq$ value $\leq 86400.0$ (seconds)

Purpose:

The time breakpoint in the formula used to calculate the plume meander expansion factor (seconds). If the release duration is less than or equal to this value, the first formula is used. If the release duration exceeds this value, the second formula is used.

Example Usage:

*

* BREAKPOINT FOR FORMULA CHANGE (SECONDS)

PMBRKPNTO01 3600.

Variable Name: $\quad$ XPFAC1

Variable Type: Real, Scalar

Allowed Range: $\quad 0.01 \leq$ valúe $\leq 1.0$ (unitless)

Purpose:

The exponential factor used in calculating the plume meander expansion

Example Usage:

factor for releases having durations that are less than or equal to BRKPNT.

*

* EXPONENTIAL EXPANSION FACTOR NUMBER 1

$\star$

PMXPFAC1001 0.2

Variable Name: $\quad$ XPFAC2

Variable Type: $\quad$ Real, Scalar

Allowed Range: $\quad 0.01 \leq$ value $\leq 1.0$ (unitless)

Purpose:

The exponential factor used in calculating the plume meander expansion

Example Usage:

factor for releases having durations that are greater than BRKPNT.

*

* exponential expansion factor NUMBer 2

PMXPFAC2001 0.25 


\subsection{Plume Rise (PR) Data}

A plume rise model based on the recommendations of Briggs $(1975,1984)$ is incorporated into MACCS. There are three basic components of the model: (1) entrainment of buoyant plumes in a building wake, (2) plume rise under unstable and neutral conditions (classes A to D), and (3) plume rise under stable conditions (classes $\mathrm{E}$ to $\mathrm{F}$ ).

These component models are described in the MACCS Model Description. The individual numeric coefficients utilized by these models are fixed in the code with no provision for their convenient modification by the user. While it is not possible for the user to vary the individual coefficients utilized by the three components of the plume rise model, it is possible to modify their end results by the specification of linear scaling factors that are described in this section.

\section{Variable Name: SCLCRW}

Variable Type: $\quad$ Real, Scalar

Allowed Range: $\quad 0.001 \leq$ value $\leq 1.0 \mathrm{E} 6$

Purpose: $\quad$ Linear scaling factor on the critical wind speed used in determining if buoyant plumes will be trapped in the turbulent wake of the facility building complex. Parameter values less than unity make plume rise less likely to occur because plume liftoff occurs only if the ambient wind speed at the time of release is less than the calculated critical wind speed.

MACCS2 implements the plume rise entrainment model recommended by Hall and Waters (1986), as described in the MACCS Model Description, page 2-6, where the critical wind speed is a function of the height of the building and the thermal power of the plume (ATMOS input variables PLHEAT and BUILDH). This model is based on wind tunnel experiments for buildings of a size associated with commercial reactors.

If there is a need to ignore the effect of building wake entrainment, for example, for a pointsource release, or from a small building, this can be achieved by setting SCLCRW to its maximum allowable value. When that is done, the calculation of the height of the plume is based strictly on the Briggs plume rise formulas, irrespective of building height and the corresponding critical wind speed.

Example Usage:

* SCALing factor for entrainMent of buoyant plume

*

PRSCLCRWO01 1.

Variable Name: SCLADP

Variable Type: Real, Scalar

Allowed Range: $\quad 0.01 \leq$ value $\leq 100.0$ (unitless) 
Purpose: Linear scaling factor on the plume rise formula used to determine the amount of plume rise that will occur when unstable or neutral atmospheric conditions occur (classes A through D).

Example Usage:

* SCALING FACTOR FOR THE A-D STABILITY PLUME RISE FORMULA

PRSCLADPO01 1.

Variable Name: SCLEFP

Variable Type: $\quad$ Real, Scalar

Allowed Range: $\quad 0.01 \leq$ value $\leq 100.0$ (unitless)

Purpose: Linear scaling factor on the plume rise formula used to determine the amount of plume rise that will occur when atmospheric conditions are stable (classes $\mathrm{E}$ and $\mathrm{F}$ ).

Example Usage:

$\star$

* SCALING FACTOR FOR THE E-F STABILITY PLUME RISE FORMULA

$\star$

PRSCLEFP001 1.

\subsection{Wake Effects (WE) Data}

The initial size of the plume is determined by the width and height of the building wake. In MACCS, the initial plume dimensions were initialized to fixed fractions of the user-specified building dimensions. $\sigma_{\mathrm{y}}$ was initialized to the building width divided by 4.3 , and $\sigma_{\mathrm{z}}$ was initialized to the building height divided by 2.15 .

The height of the building wake is also used to determine if the plume is entrained in the turbulent region surrounding the building. Consequently, in addition to determining the initial plume size, the wake height is used to determine whether buoyant plume rise will occur ( see Section 5.9).

The dispersion of a plume of material released in the wake of a large building is subject to a large degree of uncertainty. For that reason, MACCS2 should not be used for estimating doses at distances less than $0.5 \mathrm{~km}$ from laboratory or industrial-scale facilities. It has long been recognized that the turbulent eddies in the lee of an obstacle are very difficult to model. A good example is the data of Ramsdell (1990), who showed that, in contrast to standard Gaussian plume formulas in which concentrations are inversely proportional to wind speed, air concentrations in a building's wake can increase with increased wind speed.

The earliest formulation for building wake effects in current use is the "area source" model attributed to Fuquay by Gifford (1960). In that formulation, for a ground-level source and receptor, 


$$
\chi / Q=1 /\left(\pi \cdot u \cdot \sigma_{\mathrm{y}} \cdot \sigma_{\mathrm{z}}+c \cdot A\right)
$$

where $A$ is the cross-sectional area of the building normal to the wind, and $c$ is an empirical factor judged intuitively to be between 0.5 and 2.0. Later work (Gifford 1976) shows that a value of 0.5 for $c$ is supported by empirical data and is an appropriate conservative value.

In CRAC2, the initial plume standard deviations are

$$
\begin{aligned}
& \sigma_{\mathrm{y}}=W / 3 \\
& \sigma_{\mathrm{z}}=H / 2.15,
\end{aligned}
$$

where $W$ is building width and $H$ is building height, while in MACCS the corresponding formulas are

$$
\begin{aligned}
& \sigma_{y}=W / 4.3 \\
& \sigma_{z}=H / 2.15 .
\end{aligned}
$$

A very similar model is recommended by Jones (1983):

$$
\begin{aligned}
& \sigma_{y}=W / 3 \\
& \sigma_{z}=H / 3
\end{aligned}
$$

with the additional guidance that the building releases should be adjusted to occur at an effective release height of $H / 3$.

Variable Name: $\quad$ BULLDH

Variable Type: $\quad$ Real, Scalar

Allowed Range: $\quad 1.0 \leq$ value $\leq 1000.0$ (meters)

Purpose: $\quad$ Defines the height of the facility building. This value is used in the evaluation of whether a buoyant plume is entrained in the turbulent wake of the building. The building height, in contrast to MACCS, is no longer utilized to define the initial value of $\sigma_{z}$ for the plume.

Example Usage:

*

* BUILDING HEIGHT (METERS) THIS IS NOW USED ONLY FOR PLUME RISE

* ENTRAINMENT.

WEBUILDHO01 50. SURRY

Variable Name: $\quad$ SIGYINIT

Variable Type: $\quad$ Real, Scalar

Allowed Range: $\quad 0.1 \leq$ value $\leq 1000.0$ (meters)

Purpose: $\quad$ Defines the initial value of $\sigma_{\mathrm{y}}$ for each of the plumes released.

Example Usage: 


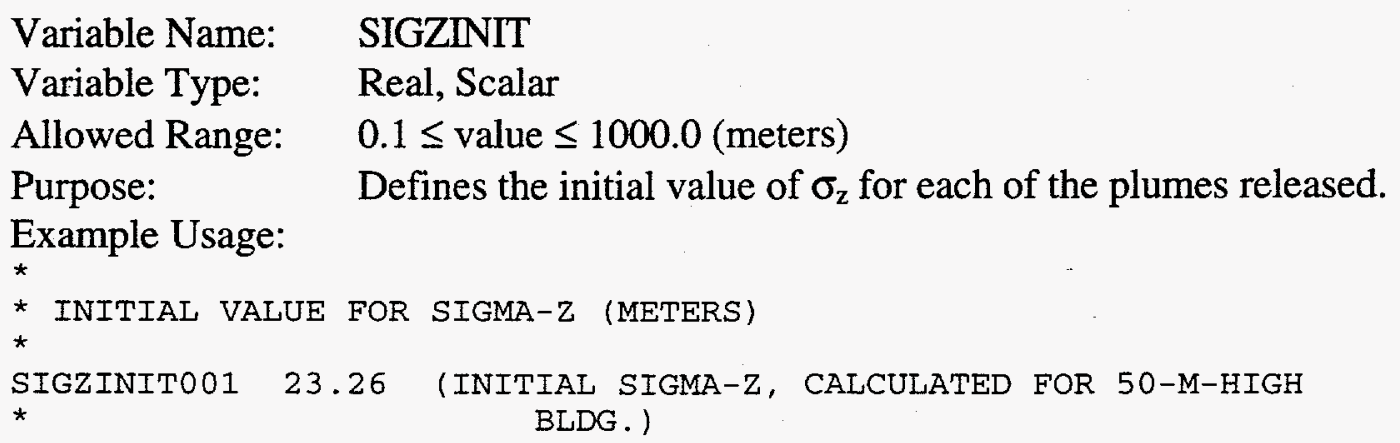

\subsection{Release Description (RD) Data}

ATMOS can handle multiple plume segments in order to treat the release of a source term that has a composition that varies with time. The plume segments that comprise a release can be separated by a time gap, can directly follow the preceding segment, or they can overlap. Different release heights, heat contents, release durations, and initial values for $\sigma_{y}$ and $\sigma_{z}$ may be assigned to each plume. Only one particle size distribution may be assigned to each chemical element group.

MACCS2 incorporates the capability for calculating the consequences from up to 60 different source terms in a single run of the code. This is accomplished by appending change records to the ATMOS input file. The first source term is defined in the main body of the ATMOS input file. Up to 59 additional source terms can be defined through change record sets positioned at the end of the file.

The delimiter used to separate the change record sets is a period (.) in column 1, which also signifies the end of the file. All of the MACCS2 user input files must thus end with a period in column 1. The sample ATMOS input file listed in Appendix D.1 of the MACCS User's Guide is an example illustrating the use of change records for a PRA application of the code.

The purpose of the change record processing in ATMOS is solely to allow modification of the previously specified release description data. If data items from another data block appear in the change records, they will be ignored. Each set of change records must include a new value of ATNAM2, a text field describing the source term. Also, each set of change records must specify a change in at least one of the numeric input variables described in this data block. 
Variable Name: ATNAM2

Variable Type: Character, Scalar

Allowed Range: $\quad 1 \leq$ length $\leq 80$

Purpose:

Example Usage:

Identifies the name of the source term being studied. This name will be printed on all pages of the OUTPUT listing. A unique name must be specified for each source term.

*

* SPECIFIC DESCRIPTIVE TEXT DESCRIBING THIS PARTICULAR SOURCE

* TERM

RDATNAM2001 'HYPOTHETICAL SOURCE TERM NUMBER 1'

Variable Name: OALARM

Variable Type: Real, Scalar

Allowed Range: $\quad 0.0 \leq$ value $\leq 604800.0 \mathrm{~s}$ (1 week)

Purpose: Defines the time at which notification is given to off-site emergency

response officials to initiate protective measures for the surrounding population. This time is a function of the accident sequence. It is measured

Example Usage:

from accident initiation (scram time) and is given in units of seconds.

*

* TIME AFTER ACCIDENT INITIATION THAT OFF-SITE ALARM IS INITIATED

RDOALARM001 17280.

Variable Name: NUMREL

Variable Type: Integer, Scalar

Allowed Range: $\quad 1 \leq$ value $\leq 4$

Purpose: Defines the number of plumes that will be released.

Note: If the multiple source term feature is being used, it is not possible to provide a larger value for NUMREL in the change records than the value that was defined previously. That is, the values of NUMREL specified on change records cannot be larger than the value of NUMREL specified in the base-case data.

Example Usage:

$\star$

* Number of pLUME sEgments that are REIEASED

RDNUMRELO01 2

Variable Name: MAXRIS

Variable Type: Integer, Scalar

Allowed Range: $1 \leq$ value $\leq$ NUMREL 
Purpose: $\quad$ Specifies which plume segment is to be considered risk dominant. The selection of this plume is usually based on its potential for causing early fatalities. Release of the risk-dominant plume always begins at the selected meteorological start time of the weather sequence.

Example Usage:

*

* SELECTION OF RISK-DOMINANT PLUME SEGMENT

$\star$

RDMAXRIS001 1

Variable Name: REFTIM

Variable Type: Real, Array

Allowed Range: $\quad 0.0 \leq$ value $\leq 1.0$

Purpose: $\quad$ Specifies the representative time point of each plume segment $(0.0=$ leading edge, $0.5=$ midpoint, $1.0=$ trailing edge). The characteristics of a plume are uniform along its length. This parameter allows the user to locate the contents of the plume in a so-called bucket of material situated at some point within the plume's length. The radioactive decay, dry deposition, and dispersion calculations are all performed as if the entire contents of the plume segment are located at this point. The user must supply NUMREL values of REFTIM, one for each plume segment.

The choice of this parameter will have no impact on the wet deposition calculations since those are performed as if the entire contents of the plume are uniformly distributed along its length.

Example Usage:

$\star$

* REPRESENTATIVE TIME POINT FOR DISPERSION AND RADIOACTIVE DECAY

*

RDREFTIMO01 0.0 .5 (CORRESPONDING TO HEAD AND MIDPOINT WEATHER)

Variable Name: $\quad$ PLHEAT

Variable Type: Real, Array

Allowed Range: $\quad 0.0 \leq$ value $\leq 1$. E10 (watts)

Purpose: $\quad$ Specifies the rate of release of sensible heat in each plume segment. This quantity should be calculated as the amount of sensible heat in the plume segment divided by the duration of the plume segment. The value specified here is used to determine the amount of buoyant plume rise that will occur. The user must supply NUMREL values of PLHEAT, one for each plume segment.

Example Usage:

$\star$

* heat content of the release segments (Watts)

* A VALUE SPECIFIED FOR EACH OF THE RELEASE SEGMENTS

RDPLHEAT001 $6.37 E+06 \quad 3.43 E+06$ 
Variable Name: PLHITE

Variable Type: Real, Array

Allowed Range: $\quad 0.0 \leq$ value $\leq 1000.0$ (meters)

Purpose:

Example Usage:

Specifies the height above ground level at which each plume segment is released. The user must supply NUMREL values of PLHITE, one for each plume segment.

*

* HEIGHT OF THE PLUME SEGMENTS AT RELEASE (METERS)

* A VAlUE SPECIfied FOR EACH OF THE RELEASE SEGMENTS

RDPLHITE001 30. 30.

Variable Name: PLUDUR

Variable Type: $\quad$ Real, Array

Allowed Range: $\quad 60.0 \leq$ value $\leq 86400.0$ (seconds) 1 day

Purpose:

Specifies the duration in seconds of each plume segment. The user must supply NUMREL values of PLUDUR, one for each plume segment. In contrast to MACCS, where multiple plume segments were not allowed to overlap each other in time, MACCS2 allows the specification of overlapping plumes.

Example Usage:

$\star$

* DURATION OF THE PLUME SEGMENTS (SECONDS)

* a VAlue sPeCified For EACH of THE RELEASE segments

$\star$

RDPLUDUR001 9000. 12588.

Variable Name: PDELAY

Variable Type: Real, Array

Allowed Range: $\quad 0.0 \leq$ value $\leq 345600.0$ (seconds) (4 days)

Purpose: $\quad$ Specifies the start time of each plume segment in seconds from the time of accident initiation, e.g., reactor scram. The user must supply NUMREL values for PDELAY, one for each plume segment. In contrast to MACCS, where multiple plume segments were not allowed to overlap each other in time, MACCS2 allows the specification of overlapping plumes.

Example Usage:

* TIME OF RELEASE FOR EACH PLUME (SECONDS FROM SCRAM)

* A VALUE SPECIFIED FOR EACH OF THE RELEASE SEGMENTS

RDPDELAYO01 17280. 26280. 
Variable Name:

Variable Type:

Allowed Range:

Purpose:
PSDIST

Real, Array

$0.0 \leq$ value $\leq 1.0$

Defines the fraction of the released material allocated to each of the particle size (deposition velocity) bins. All of the plume segments must use the same distribution of material among these bins.

The user must specify one data record for each of the chemical element groups. The number of chemical element groups was defined as variable MAXGRP in Section 5.4. On each of these cards, the user must supply an allocation fraction to be associated with each of the particle size groups. The number of particle size groups was defined as the input variable NPSGRP in Section 5.6.

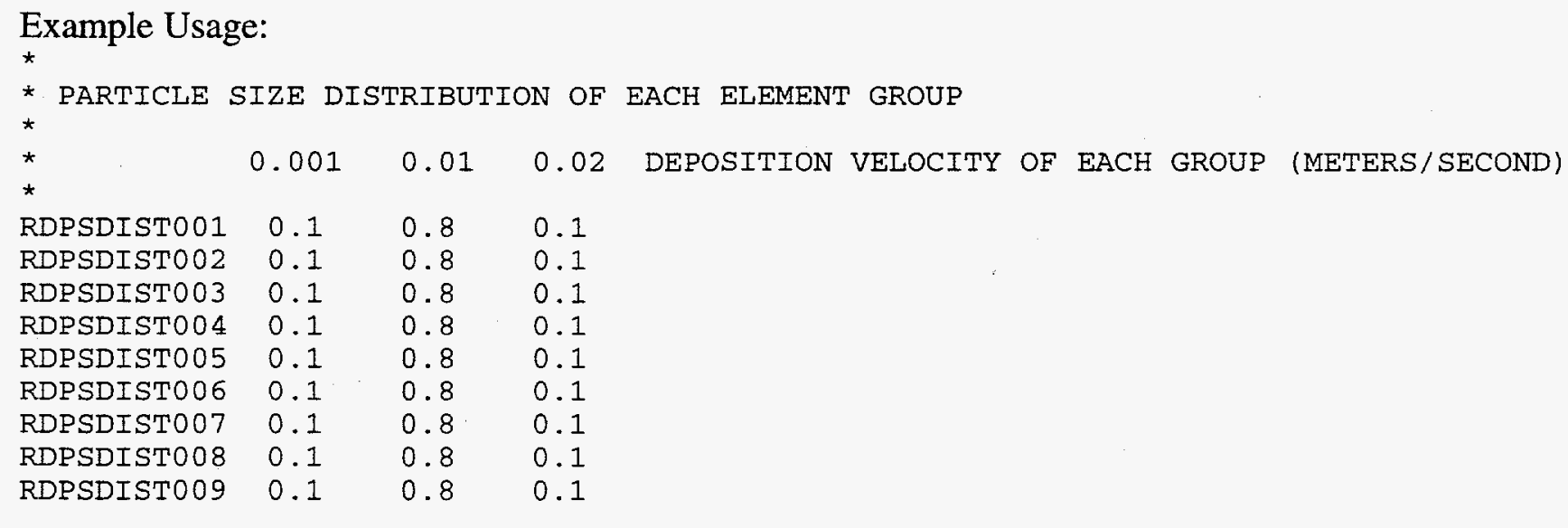

Variable Name: CORINV

Variable Type: $\quad$ Real, Array

Allowed Range: $\quad 0.0 \leq$ value $\leq 1 . \mathrm{E} 20$ (becquerels)

Purpose:

Defines the inventory of each radionuclide present in the facility at the time of accident initiation. The inventory is given in units of becquerels

(disintegrations/second). All of the radionuclides that have been previously defined in the list of radionuclides (NUCNAM array in Section 5.4) must be listed here. The radionuclide names and their respective inventories may appear in any order.

Example Usage:

\begin{tabular}{lll}
$*$ & NUCNAM & CORINV (BECQUERELS ) \\
$\star$ & & \\
RDCORINV001 & $\mathrm{CO}-58$ & $3.223 \mathrm{E}+16$ \\
RDCORINV002 & $\mathrm{CO}-60$ & $2.465 \mathrm{E}+16$ \\
RDCORINV003 & $\mathrm{Kr}-85$ & $2.475 \mathrm{E}+16$ \\
RDCORINV004 & $\mathrm{Kr}-85 \mathrm{~m}$ & $1.159 \mathrm{E}+18$ \\
\hline
\end{tabular}


Variable Name:

CORSCA

Variable Type:

Real, Scalar

Allowed Range:

$2.7 \mathrm{E}-10 \leq$ value $\leq 1.0 \mathrm{E} 12$

Purpose:

This is a linear scaling factor that can be used to adjust the inventory of all the radionuclides defined in the model. It is preferable to obtain new sets of inventory values when studying reactors of varying power levels but this is not always possible.

When facility-specific inventories are not available, a representative inventory may be obtained by linear scaling of the inventory of a similar reactor having a different thermal power level. The scale factor used can be chosen to be the ratio of the two reactors' thermal power levels.

The parameter CORSCA may also be used to convert the facility inventory from one set of units to another during the input processing phase in order to avoid the tedium of manually converting a set of data from one set of units to another. For example, to convert from curies to becquerels, use a value of $3.7 \mathrm{E} 10$ for CORSCA.

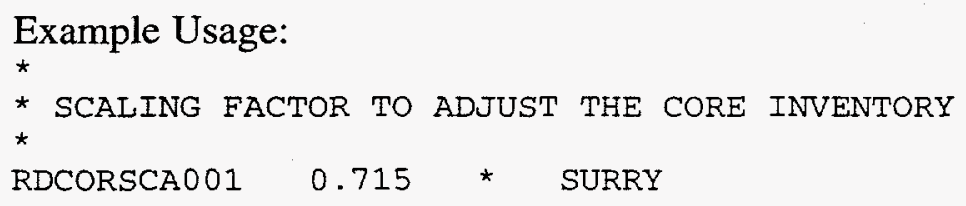

Variable Name: RELFRC

Variable Type: $\quad$ Real, Array

Allowed Range: $\quad 0.0 \leq$ value $\leq 1.0$

Purpose: Defines the release fractions for each of the plume segments. One card is supplied for each plume and it contains as many values as there are element groups. All components of an element group are released from the facility in the same fraction.

Variable Name: APLFRC

Variable Type: Character, Scalar

Allowed Value: $\quad$ 'PARENT' or 'PROGENY'

Purpose:

Specifies how release fractions are applied to ingrowth decay products produced after accident initiation. PARENT indicates that the code is to handle the release fractions in the same way that they are fixed in previous versions of MACCS. That is, daughter ingrowth products are released in the same proportion as their parent.

The new option, PROGENY, indicates that the release fraction applied to daughter ingrowth products is defined by the release fraction for the isotope group to which the daughter is assigned (variable ISOGRP). 


\section{Example Usage:}

\begin{tabular}{|c|c|c|c|c|c|c|c|c|c|c|}
\hline RDAPLFRC001 & PAREN' & (APE & & RELEASE & E FRACI & IONS THE & SAME AS & PRIOR & VERSIONS) & \\
\hline * & $\mathrm{Xe} / \mathrm{Kr}$ & I & & $\mathrm{Cs}$ & $\mathrm{Te}$ & $\mathrm{Sr}$ & $\mathrm{Ru}$ & La & $\mathrm{Ce}$ & $\mathrm{Ba}$ \\
\hline $\begin{array}{l}\text { RDRELFRCO } 1 \\
\text { RDRELFRC } 002\end{array}$ & $\begin{array}{l}\text { 7. } 0 \mathrm{E}-1 \\
2.9 \mathrm{E}-1\end{array}$ & $\begin{array}{l}4.7 E-1 \\
1.0 E-2\end{array}$ & & $\begin{array}{l}7 E-1 \\
0 E-3\end{array}$ & $\begin{array}{l}.4 E-1 \\
3 E-3\end{array}$ & $\begin{array}{l}3.0 E-1 \\
2.0 E-3\end{array}$ & $\begin{array}{l}5.0 E-3 \\
4 . E-04\end{array}$ & $\begin{array}{l}\text { 3. } 0 \mathrm{E}-2 \\
1.0 \mathrm{E}-4\end{array}$ & $\begin{array}{l}4.5 E-2 \\
9.6 E-4\end{array}$ & $\begin{array}{l}2.2 E-1 \\
2.3 E-3\end{array}$ \\
\hline
\end{tabular}

\subsection{Output Control (OC) Data}

The user has the option of looking at tables of dispersion data for all of the trials that are performed. This information includes air and ground concentrations, $\sigma_{y}$ and $\sigma_{z}$ values, arrival time, and time overhead for each plume segment at each spatial interval. These data are written to the list output file.

Variable Name: $\quad$ ENDAT1

Variable Type: Logical, Scalar

Allowed Value: $\quad$.TRUE. or .FALSE.

Purpose:

Control flag that allows the user to execute only the ATMOS module. A value of .TRUE. tells the code that EARLY and CHRONC will not be run. When this is done, the user input files for EARLY and CHRONC and a site data file need not be supplied. If the user wishes to skip execution of the CHRONC module, that can be accomplished through the input variable ENDAT2 on the EARLY input file.

Example Usage:

$\star$

* FLAG INDICATING THAT ONLy ATMOS IS TO BE RUN

*

OCENDAT1001 .FALSE. (SET TO .TRUE. IF YOU WANT TO SKIP EARLY AND CHRONC)

Variable Name: IDEBUG

Variable Type: Integer, Scalar

Allowed Range: $\quad 0 \leq$ value $\leq 8$

Purpose:

Specifies the quantity of debug output to be printed. (Note: If DEBUG is assigned a value greater than 1, the ATMOS input parameter NUMO must also be assigned a value greater than 1.) For normal runs, IDEBUG should be set to 0 (i.e., no debug output is printed). If IDEBUG is set to 1 or 2 , a print of the atmospheric transport results described below will be generated for each weather trial and each plume segment. If IDEBUG is set to a value of 3 or more, the hourly meteorological data used for each weather trial will also be printed.

The debug output for atmospheric transport prints the results described below, based on conventional nomenclature for Gaussian plume models (Jow et al. 1990). 
NUCNAM = name of the radionuclide for which results are being presented,

DISTANCE $=$ distance to the center of the spatial interval $(\mathrm{m})$,

GL AIRCON = centerline ground-level integrated air concentration from this plume segment averaged over the spatial interval's length $\left(\mathrm{Bq}-\mathrm{s} / \mathrm{m}^{3}\right)$,

GRNCON = centerline ground concentration after passage of this plume averaged over the spatial interval's length $\left(\mathrm{Bq} / \mathrm{m}^{2}\right)$,

GL $\chi / Q=$ centerline ground-level $\chi / Q$; the ratio of air concentration $(\chi)$ to source strength $(Q)$, averaged over the interval's length $\left(\mathrm{s} / \mathrm{m}^{3}\right)$,

WETREM = fraction of material remaining in the plume segment after wet deposition over the spatial interval's length (unitless),

DRYREM = fraction of material remaining in the plume segment after dry deposition over the spatial interval's length (unitless),

REMINV = adjusted source strength of the plume upon entering each spatial interval after adjustment for losses in the previous intervals due to radioactive decay and wet and dry deposition $(\mathrm{Bq})$,

PLSIGY = horizontal dispersion parameter $\sigma_{\mathrm{y}}$ averaged over the spatial interval's length $(\mathrm{m})$,

PLSIGZ = vertical dispersion parameter $\sigma_{z}$ averaged over the spatial interval's length (m),

WEATHER = indices to the first and last hours of the weather sequence used for determining atmospheric conditions during transport across each spatial interval,

HTFCTR = ratio of the centerline ground-level air concentration $(z=0)$ to the plume centerline air concentration $(z=H)$, (unitless),

AVGHIT = average height $(H)$ of the plume as it traversed the spatial interval $(\mathrm{m})$,

TIMCEN = time after accident initiation at which the leading edge of the plume arrived at the center of the spatial interval (s),

TIMOVH = duration for which the plume was overhead at the centerpoint of the spatial interval (s).

Example Usage:

OCIDEBUG001 1

(REQUEST A TRACE OF ATMOSPHERIC DISPERSION) 


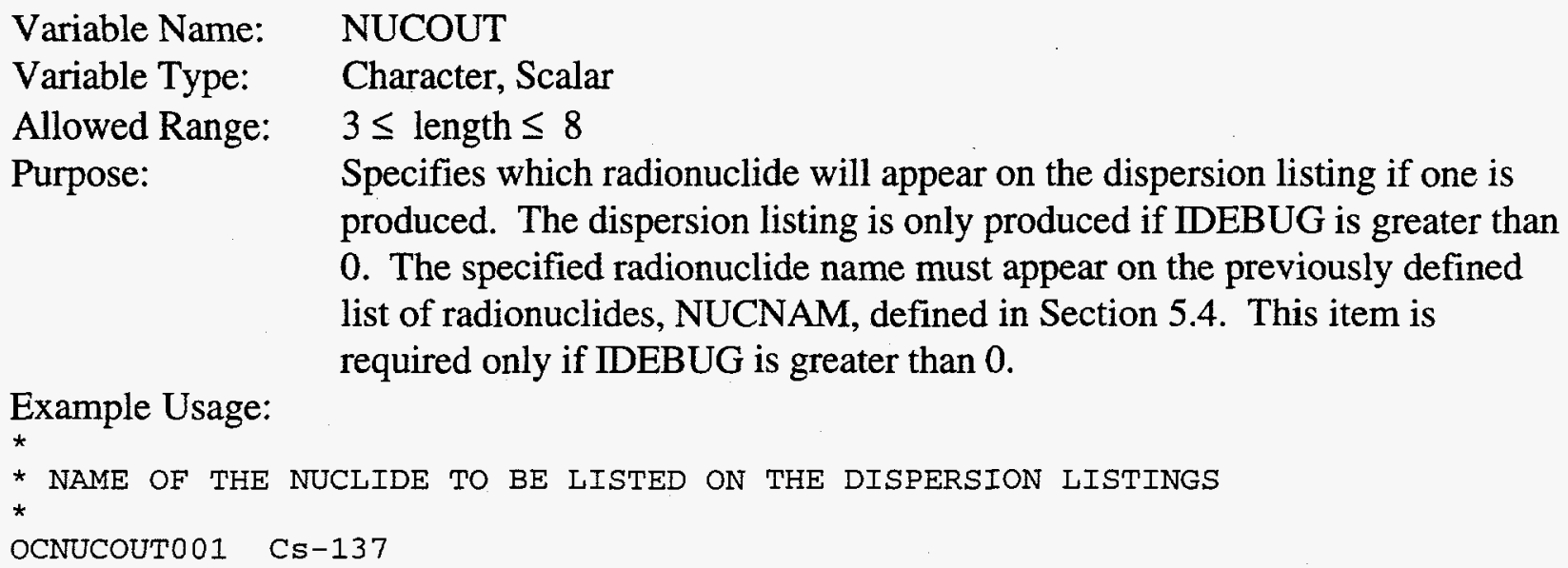

\subsection{Meteorological Sampling (M1) Specification}

There are five options available to the user for specifying the weather data that will be used by ATMOS. The code can be used to run either a single weather sequence or multiple weather sequences, as described in the following paragraphs.

If a single weather sequence is desired, there are three ways to specify the weather. The user can (1) specify data for $120 \mathrm{hr}$ of weather on the ATMOS input file, (2) specify a starting day and hour in the weather data file for the weather sequence, or (3) specify constant weather conditions.

For the specified starting day and hour option, the program will obtain $120 \mathrm{hr}$ of weather data from the weather file beginning at the specified date and time. A file of hourly weather data covering a period of 1 year $(8760 \mathrm{hr})$ is required if the fixed start time or either of the weather sampling options are to be used. The format of this file is described in Section A.1 of Appendix A.

The two methods of weather sampling are (1) a modified version of the weather bin sampling method used by CRAC2 (Ritchie et al. 1984) and (2) a stratified purely random sampling approach. The weather bin sampling method sorts weather sequences into categories and assigns a probability to each category according to the initial conditions (wind speed and stability class) and the occurrence of rain (intensity and distance). Because the rain bins depend on rain intensity as well as the downwind distance at which rain occurs, the user is required to supply parameters defining the rain weather bins as part of the ATMOS input file. The definitions of the other, nonrain weather bins, that is, those defined by initial stability class and wind speed, are fixed in the code. A description of the MACCS2 weather sampling algorithm can be found in the MACCS Model Description (Jow et al. 1990).

The stratified random sampling method allows the user to sample weather from each day of the year after division of each day into one, two, three or four equal time periods. Each weather sequence selected is considered to have the same probability of occurrence, that is, 


$$
P=\frac{1}{\text { total selected samples }} \text {. }
$$

Because of the flexibility that ATMOS affords in the specification of the geometric grid, it is necessary to guard against the possibility of running out of weather data. It is possible that 120 hr of weather may not suffice to carry all the plume segments out to the last spatial interval. Also, the user may wish to specify the occurrence of rain in the outermost spatial intervals in order to prevent radioactive material from escaping consideration. For these reasons, the user must specify a set of boundary weather conditions.
Variable Name:
METCOD
Variable Type:
Integer, Scalar
Allowed Range:
$1 \leq$ value $\leq 5$
Purpose:
Meteorological sampling option code:
1 - fixed start time in the weather file (day,hour),
2 - weather bin sampling,
$3-120 \mathrm{hr}$ of weather supplied by the user,
4 - constant weather conditions (use boundary weather),
5 - stratified random sampling from equally spaced intervals.

.Example Usage:

* METEOROLOGICAL SAMPLING OPTION CODE:

M1METCOD001 2

\subsection{Boundary Weather (M2) Data}

Boundary weather data are required for all possible values of METCOD. This data block specifies the weather conditions that will be used if $120 \mathrm{hr}$ of recorded weather data do not transport the last plume through the limiting spatial interval for measured weather, LIMSPA. The boundary weather data are also used to predict the behavior of the plume at all spatial intervals beyond LIMSPA.

For the case of constant weather, METCOD $=4$, the boundary weather data in this section define the constant weather conditions that will be used. In this case, the boundary weather is used throughout the atmospheric calculations and the value of LIMSPA is ignored by the program.

Variable Name: LIMSPA

Variable Type: Integer, Scalar

Allowed Range: $\quad 0 \leq$ value $\leq$ NUMRAD

Purpose:

This is the limiting spatial interval for use of recorded weather data. All spatial intervals beyond this interval will use the boundary weather conditions specified below. 
Boundary weather conditions are applied to all spatial intervals if a value of 0 is specified for this parameter. If $M E T C O D=4$, the value of LIMSPA is ignored.

Example Usage:

* LASt spatial interval for measured weather

M2LIMSPAO01 25

Variable Name: $\quad$ BNDMXH

Variable Type: Real, Scalar

Allowed Range: $\quad 1 . \mathrm{E} 2 \leq$ value $\leq 1$.E4 (meters)

Purpose:

This is the mixing layer height that will be used for the boundary weather conditions.

Example Usage:

* BOUNDARY WEATHER MIXING LAYER HEIGHT

M2BNDMXH001 1000. (METERS)

Variable Name: IBDSTB

Variable Type: Integer, Scalar

Allowed Range: $\quad 1 \leq$ value $\leq 6$

Purpose:

This is the stability class that will be used for the boundary weather conditions. The integers 1 through 6 represent Pasquill-Gifford stability

Example Usage: classes A through $F$, respectively.

* BoUNdary WeAther STABILITy CLASS INDEX

*

M2IBDSTB001 1 (A-STABILITY)

Variable Name: $\quad$ BNDRAN

Variable Type: $\quad$ Real, Scalar

Allowed Range: $\quad 0.0 \leq$ value $\leq 99.0$ (millimeters / hour)

Purpose:

This is the rain rate that will be used for the boundary weather conditions.

Example Usage:

* boundary weather RAin RAte

M2BNDRAN001 $0 . \quad$ (0 MILLIMETERS $/$ HOUR $=$ NO RAIN $)$ 
Variable Name:

BNDWND

Variable Type:

Real, Scalar

Allowed Range:

$0.5 \leq$ value $\leq 30.0$ (meters / second)

Purpose -

This is the wind speed that will be used for the boundary weather conditions.

Example Usage:

*

* boUNdaRY WEATHER WIND SPEED

M2BNDWND001 $0.5 \quad$ (METERS/SECOND)

\subsection{Fixed Start Time (M3) Data}

The data in this section must be supplied for all values of METCOD except METCOD=2 (meteorological bin sampling) and METCOD=5 (stratified random sampling). The data are needed for all of these option choices because the food pathway calculations of the CHRONC module depend on the day the accident occurs. If the user has chosen METCOD $=1$, the values of ISTRDY and ISTRHR specify the starting day and hour in the weather file of the single weather trial that will be performed.

Variable Name: ISTRDY

Variable Type: Integer, Scalar

Allowed Range: $\quad 1 \leq$ value $\leq 365$

Purpose: $\quad$ This is the day in the year on which the weather sequence is to begin.

Example Usage:

* START daY of the weATHER SEQUENCE

M3ISTRDY001 152

Variable Name: ISTRHR

Variable Type: Integer, Scalar

Allowed Range: $\quad 1 \leq$ value $\leq 24$

Purpose:

Example Usage:

This is the hour of day on which the weather sequence is to begin.

* start hour of the weather sequence

M3ISTRHR001

17

\subsection{Meteorological Bin Sampling (M4) Data}

The data in this section must be supplied if the user chooses METCOD=2. This sampling method requires that the meteorological data be sorted into a set of weather bins. The bins are defined to represent rain conditions in different distance intervals downwind from the accident site together with 16 bins for initial conditions (stability class and wind speed). 
Definition of the rain intensities and distance intervals that define the rain bins is the responsibility of the user. The user must specify either two or three rain intensities that are used as breakpoints in the categorization of rain rate (NRINTN). A rain intensity of 0 is not allowed. For example, if the user specifies two rain intensity breakpoints of $1 \mathrm{~mm} / \mathrm{hr}$ and $4 \mathrm{~mm} / \mathrm{hr}$, the following three rain intensity bins, where $x$ is the rain intensity, will result:

1. $0 \mathrm{~mm} / \mathrm{hr}<x \leq 1 \mathrm{~mm} / \mathrm{hr}$,

2. $1 \mathrm{~mm} / \mathrm{hr}<x \leq 4 \mathrm{~mm} / \mathrm{hr}$,

3. $4 \mathrm{~mm} / \mathrm{hr}<x$.

The concept of rain distance intervals used in MACCS2 is similar to that used by CRAC2 but MACCS2 requires that the user specify the rain distances to be used in the weather categorization. Let us suppose that the user specifies $2,4,8$, and $16 \mathrm{~km}$ as four distance intervals (i.e., NRNINT $=4$. Then these values define the following four rain distance intervals:

1. $0 \mathrm{~km} \leq$ distance of first rain occurrence $\leq 2 \mathrm{~km}$,

2. $2 \mathrm{~km}<$ distance of first rain occurrence $\leq 4 \mathrm{~km}$,

3. $4 \mathrm{~km}<$ distance of first rain occurrence $\leq 8 \mathrm{~km}$,

4. $8 \mathrm{~km}<$ distance of first rain occurrence $\leq 16 \mathrm{~km}$.

The total number of weather bins, $\mathrm{N}$, is determined by the expression $\mathrm{N}=\mathrm{NRNINT} \cdot(\mathrm{NRINTN}+1)+16$, where NRNINT is the number of distance intervals and NRINTN is the number of rain intensity breakpoints. Thus, the total number of defined bins can range from 28 to 40 , depending on the values supplied by the user.

The 16 initial condition weather bins are fixed in the code as follows:

\section{METBIN STABILITY WIND SPEED (u)}

$\begin{array}{lll}1 & \mathrm{~A} / \mathrm{B} & 0 \mathrm{~m} / \mathrm{s}<\mathrm{u} \leq 3 \mathrm{~m} / \mathrm{s} \\ 2 & \mathrm{~A} / \mathrm{B} & 3 \mathrm{~m} / \mathrm{s}<\mathrm{u} \\ 3 & \mathrm{C} / \mathrm{D} & 0 \mathrm{~m} / \mathrm{s}<\mathrm{u} \leq 1 \mathrm{~m} / \mathrm{s} \\ 4 & \mathrm{C} / \mathrm{D} & 1 \mathrm{~m} / \mathrm{s}<\mathrm{u} \leq 2 \mathrm{~m} / \mathrm{s} \\ 5 & \mathrm{C} / \mathrm{D} & 2 \mathrm{~m} / \mathrm{s}<\mathrm{u} \leq 3 \mathrm{~m} / \mathrm{s} \\ 6 & \mathrm{C} / \mathrm{D} & 3 \mathrm{~m} / \mathrm{s}<\mathrm{u} \leq 5 \mathrm{~m} / \mathrm{s} \\ 7 & \mathrm{C} / \mathrm{D} & 5 \mathrm{~m} / \mathrm{s}<\mathrm{u} \leq 7 \mathrm{~m} / \mathrm{s} \\ 8 & \mathrm{C} / \mathrm{D} & 7 \mathrm{~m} / \mathrm{s}<\mathrm{u} \\ 9 & \mathrm{E} & 0 \mathrm{~m} / \mathrm{s}<\mathrm{u} \leq 1 \mathrm{~m} / \mathrm{s} \\ 10 & \mathrm{E} & 1 \mathrm{~m} / \mathrm{s}<\mathrm{u} \leq 2 \mathrm{~m} / \mathrm{s} \\ 11 & \mathrm{E} & 2 \mathrm{~m} / \mathrm{s}<\mathrm{u} \leq 3 \mathrm{~m} / \mathrm{s} \\ 12 & \mathrm{E} & 3 \mathrm{~m} / \mathrm{s}<\mathrm{u} \\ 13 & \mathrm{~F} & 0 \mathrm{~m} / \mathrm{s}<\mathrm{u} \leq 1 \mathrm{~m} / \mathrm{s} \\ 14 & \mathrm{~F} & 1 \mathrm{~m} / \mathrm{s}<\mathrm{u} \leq 2 \mathrm{~m} / \mathrm{s} \\ 15 & \mathrm{~F} & 2 \mathrm{~m} / \mathrm{s}<\mathrm{u} \leq 3 \mathrm{~m} / \mathrm{s} \\ 16 & \mathrm{~F} & 3 \mathrm{~m} / \mathrm{s}<\mathrm{u}\end{array}$


The user controls how many weather sequences are chosen from each weather bin by the choice of a value for NSMPLS, defined later in this section. This can be done in two different ways: either (1) request that the same number of weather sequences is to be chosen from each bin and specify the number of sequences, NSMPLS, to be selected ( $1 \leq$ NSMPLS $\leq 10)$; or (2) specify a nonuniform sampling from the categories (NSMPLS $=0$ ) as defined below.

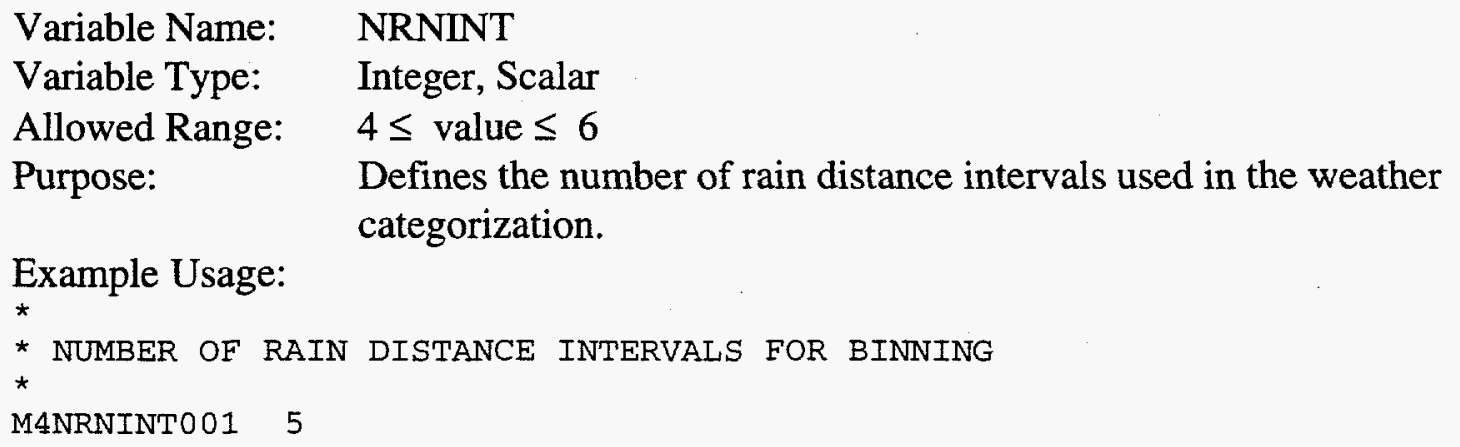

Variable Name: $\quad$ NRNINT

Variable Type: $\quad$ Integer, Scalar

Allowed Range: $\quad 4 \leq$ value $\leq 6$

Purpose: $\quad$ Defines the number of rain distance intervals used in the weather categorization.

Example Usage:

* nUMBer of rain Distance intervals for biNNING

M4NRNINT001 5

Variable Name: RNDSTS

Variable Type: $\quad$ Real, Array

Allowed Range: $\quad 0.001 \leq$ value $\leq 99.9$ (kilometers)

Purpose:

Defines the rain distance interval endpoints to be used for the weather categorization. These distance values must lie within $10 \%$ of the spatial interval endpoint distances (variable SPAEND in Section 5.3), i.e., $0.9 \cdot \operatorname{SPAEND}(i) \leq \operatorname{RNDSTS}(j) \leq 1.1 \cdot \operatorname{SPAEND}(i)$, for each $j$ and some value of $i$. The user must supply NRNINT unique values in ascending order.

Example Usage:

* ENDPOINTS OF THE RAIN DISTANCE INTERVALS (KILOMETERS)

* NOTE: MUST BE CHOSEN TO MATCH THE SPATIAL ENDPOINT DISTANCES

* SPECIFIED FOR THE ARRAY SPAEND (10\% ERROR IS ALLOWED).

$\begin{array}{lllllll}\text { MARNDSTSO01 } & 3.22 & 5.63 & 11.27 & 20.92 & 32.19 & \mathrm{KM}\end{array}$

Variable Name: NRINTN

Variable Type: . Integer, Scalar

Allowed Range: $\quad 2 \leq$ value $\leq 3$

Purpose: $\quad$ Defines the number of rain intensity breakpoints to be used for the weather categorization.

Example Usage:

* NUMBER of RAIN INTENSITY BREAKPOINTS

*

M4NRINTNOO1 3 


$\begin{array}{ll}\text { Variable Name: } & \text { RNRATE } \\ \text { Variable Type: } & \text { Integer, Array } \\ \text { Allowed Range: } & 0.001 \leq \text { value } \leq 100.0 \text { (millimeters/hour) }\end{array}$

Purpose:

Example Usage:

Defines the rain intensity breakpoints. The user must supply NRINTN different values in ascending order.

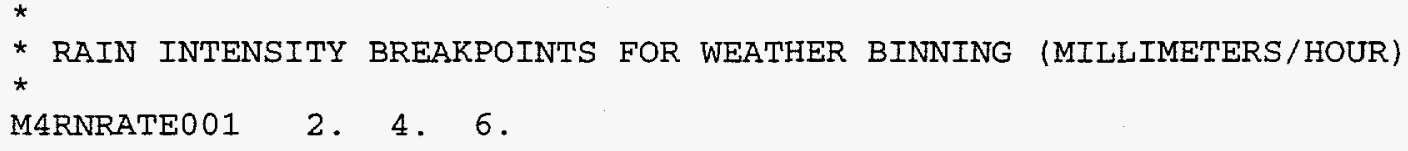

Variable Name: $\quad$ NSMPLS

Variable Type: Integer, Scalar

Allowed Range: $\quad 0 \leq$ value $\leq 24$

Purpose:

Example Usage:

Defines the number of weather sequences to be chosen from each of the individual weather-category bins (when METCOD=2), or from each day of the year (when METCOD=5).

When METCOD=2, it is advisable to set a value of at least 4; the more samples that are taken, the more robust is the sampling. If the user supplies a value of 0 for NSMPLS, and METCOD=2, the variables NSBINS, INDXBN, and INWGHT allow the user to specify how many samples are to be chosen from each of the weather-category bins.

When METCOD=5, setting NSMPLS=24 will have the code sample every hour of the year, a capability that can provide insights into the adequacy of the operation of the weather binning algorithm. In using the METCOD=5 option, it is advisable to select values for NSMPLS that are divisors of 24 because then each interval is composed of a whole number of hours. That is, NSMPLS should be set to one of the following: 1, 2, 3, 4, 6, 8, 12, or 24 . Test calculations by the code developers indicate that taking stratified random samples from each 6-hr interval of the year yields results close to those obtained from sampling all $8760 \mathrm{hr}$ of the year. There may thus be diminishing returns from taking more samples, since calculation time is proportional to the number of samples.

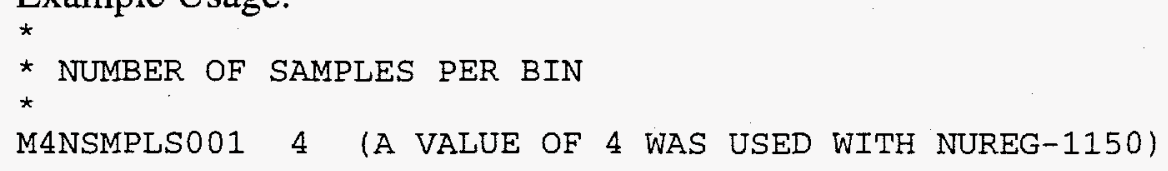


Variable Name: IRSEED

Variable Type: Integer, Scalar

Allowed Range: $\quad 0 \leq$ value $\leq 255$

Purpose: Defines the initial seed of the random number generator. Changes to this value will cause different weather sequences to be selected.

The random number generator of MACCS2 is included in the FORTRAN source code and therefore runs made on different types of computers should select identical sets of weather sequences.

Example Usage:

* initial SEED for RANDOM NUMBer genERATOR

M4IRSEED001 79

Note: The cards in this section are needed only if NSMPLS $=0$.

Variable Name: $\quad$ NSBINS

Variable Type: Integer, Scalar

Allowed Range: $\quad 1 \leq$ value $\leq \mathrm{N}$, total number of weather bins

Purpose: $\quad$ Defines the number of bins from which weather sequences are to be chosen.

Sampling a subset of all the weather bins allows the effects of weather type to be examined.

Example Usage:

* NUMBer of bins to be SAMPLED (WHEN NSMPLS $=0$ )

M4NSBINS001 6

Variable Name: INDXBN

Variable Type: Integer, Array

Allowed Range: $\quad 1 \leq$ value $\leq \mathrm{N}$, total number of weather bins

Purpose:

Defines the list of bins from which weather sequences are to be selected.

The user must supply NSBINS values in column 1 of the data block.

In order to find the index number to a rain bin, please refer to the page of the output listing with the title METEOROLOGICAL BIN SUMMARY.

Variable Name: INWGHT

Variable Type: $\quad$ Integer, Array

Allowed Range: $1 \leq$ value $\leq 8760$

Purpose:

Defines the number of weather sequences which the user would like to be selected from the specified weather bin. If the requested number of 
Example Usage:

sequences cannot be found, the code will select all of the sequences in the specified bin. The user must supply NSBINS values in column 2 of the data block.

\begin{tabular}{lc}
$*$ & \\
$*$ & BIN-NUMBER \\
$*$ & INDXBN \\
M4SMPLDF001 & 3 \\
M4SMPLDF002 & 4 \\
M4SMPLDF003 & 5 \\
M4SMPLDF004 & 6 \\
M4SMPLDF005 & 7 \\
M4SMPLDF006 & 8 \\
\hline
\end{tabular}

SAMPLE-SIZE

INWGHT

8

16

12

4

4

4

\subsection{User-Supplied Weather Sequence (M5) Data}

The data in this section must be supplied if the user chooses METCOD=3. There must be one data record for each hour of weather in the sequence. The five arrays in this section are supplied in a block of data as columns.

Variable Name:

HRMXHT

Variable Type:

Real, Array

Allowed Range:

$1 . \mathrm{E} 2 \leq$ length $\leq 1 . \mathrm{E} 4$ (meters)

Purpose:

These are the mixing layer heights that will be used for the single trial. They are given in units of meters. The user must supply 120 values of ISTAB in column 1 of the data block.

Note: The atmospheric dispersion model currently being used cannot accommodate a mixing layer height that varies with time during a weather sequence. The single value of mixing height that will be used in the atmospheric model is the largest value in the following set of values: the 120 values supplied here, and the boundary weather mixing layer height, BNDMXH.

Variable Name:

IHRSTB

Variable Type:

Integer, Array

Allowed Range:

$1 \leq$ value $\leq 6$

Purpose:

Defines the stability classes that will be used for the single trial. The integers 1 through 6 represent the Pasquill-Gifford stability classes A through F. The user must supply 120 values of ISTAB in column 2 of the data block corresponding to each of the $120 \mathrm{hr}$ in the weather sequence.

Variable Name:

HRRAIN

Variable Type:

Real, Array 
Allowed Range: $\quad 0.0 \leq$ value $\leq 99.0$ (millimeters/hour)

Purpose: $\quad$ Defines the rain rates that will be used for the single trial. They are given in units of millimeters per hour. The user must supply 120 values of RNMM in column 3 of the data block.

Variable Name: HRWNDV

Variable Type: $\quad$ Real, Array

Allowed Range: $\quad 0.5 \leq$ value $\leq 30.0$ (millimeters/second)

Purpose:

Defines the wind speeds that will be used for the single trial. They are given in units of meters per second. The user must supply 120 values of WINDSP in column 4 of the data block.

Variable Name: $\quad$ IHRDIR

Variable Type: Integer, Array

Allowed Range: $\quad 1 \leq$ value $\leq 16$

Purpose:

Defines the wind directions that will be used for the single trial. They are given as integers corresponding to the wind directions north through north northwest. The user must supply 120 values of IHRDIR in column 5 of the data block.

Example Usage:

*

* 120 HR OF WEATHER EXPLICITLY SPECIFIED BY THE USER

* (THESE DATA ARE PROCESSED ONLY IF METCOD IS SET TO 3)

$\star$

$\star$

M5METDAT001 1000 .

M5METDAT002 1000 .

M5METDAT003 1000 .

$\begin{array}{cc}\text { IHRSTB } & \text { HRRAIN } \\ 4 . & 0 . \\ 3 . & 0 . \\ 4 . & 0 .\end{array}$

HRWNDV
$\begin{aligned} & 3.4 \\ & 4.6 \\ & 3.8\end{aligned}$

IHRDIR
2
4
3

AND LIKEWISE FOR A TOTAL OF 120 DATA RECORDS

M5METDAT120 1000

5.4

8

\subsection{CCDFs of Atmospheric Results}

In MACCS, complementary cumulative distribution functions could be produced by the EARLY and CHRONC modules. However, there was no capability for generating CCDFs of the atmospheric modeling parameters calculated by ATMOS. In MACCS2, if requested by the user, the ATMOS module will now generate CCDFs of ten atmospheric modeling parameters for userspecified distances and plume segments. When the multiple source term looping capability of ATMOS is utilized (i.e., multiple source terms are defined in a single ATMOS input file), these CCDFs may be produced only for the first defined source term.

Air and ground concentrations are reported for the radionuclide specified for the variable NUCOUT. In addition to the results produced for the single specified radionuclide, the total 
radioactivity on the ground (from all radionuclides) is reported as well. Within a single run of the code, there is no provision in MACCS2 for generating CCDFs of air and ground concentrations for multiple individual radionuclides. If such output is needed, separate MACCS2 runs would be required to produce results for each radionuclide. With NUCOUT given a value of Cs-137, for example, the ten consequence measures produced are listed in Table 5-1.

Table 5-1. Results Available from ATMOS in CCDF Form

\begin{tabular}{||ll|l||}
\hline (1) & $\begin{array}{l}\text { Selected Radionuclide Centerline Air } \\
\text { Concentration }\end{array}$ & (becquerel-seconds/cubic meter) \\
\hline (2) & $\begin{array}{l}\text { Selected Radionuclide Ground-Level Air } \\
\text { Concentration }\end{array}$ & (becquerel-seconds/cubic meter) \\
\hline (3) & $\begin{array}{l}\text { Selected Radionuclide Centerline Ground } \\
\text { Concentration }\end{array}$ & (becquerels/square meter) \\
\hline (4) & Total Centerline Ground Concentration & (becquerels/square meter) \\
\hline (5) & Ground-Level $\chi / Q$ Dispersion Factor & (seconds/cubic meter) \\
\hline (6) & $\begin{array}{l}\text { Selected Radionuclide Adjusted Source } \\
\text { Strength, } Q^{\mathrm{a}}\end{array}$ & (becquerels) \\
\hline (7) & Plume $\sigma_{\mathrm{y}}$, Crosswind Size & (meters) \\
\hline (8) & Plume $\sigma_{\mathrm{z}}$, Vertical Size & (meters) \\
\hline (9) & Plume Centerline Height & (meters) \\
\hline (10) & Plume Arrival Time at Centerpoint & (seconds) \\
\hline
\end{tabular}

${ }^{\text {a }}$ In Gaussian plume equations, $Q$ is commonly used to represent the amount released. When material is deposited on the ground during transport, the effective source strength for downwind distances is diminshed. This is treated in MACCS through the definition of an "effective" source strength, which is diminished by the deposition and radioactive decay that occur over each spatial interval.

The generation of these consequence measures is requested through definition of the following variables in the ATMOS input file.

Variable Name: NUM0

Variable Type: Integer, Scalar

Allowed Range: $\quad 0 \leq$ value $\leq 35$

Purpose: $\quad$ Specifies how many results of this type are being requested. For each result requested, a block of ten consequence measures is produced in the list output file.

Note: If DEBUG is assigned a value greater than 1, NUMO must also be assigned a value greater than 1 . 
Variable Name: INDREL

Variable Type: Integer, Scalar

Allowed Range: $\quad 1 \leq$ value $\leq$ NUMREL

Purpose:

Specifies the index of the plume segment for which results are to be generated.

Variable Name: INDRAD

Variable Type: Integer, Scalar

Allowed Range: $\quad 1 \leq$ value $\leq$ NUMRAD

Purpose:

Specifies the index of the spatial distance for which results are to be generated.

Note: In order to obtain the entire CCDF tables for the ten ATMOS results described in this section, append the character string ' $C C D F$ ' to the line requesting the result as the third item on the data record, immediately following INDRAD, as illustrated below. The $C C D F$ tables will be printed in the output file. If requested, these tables will be printed as blocks of ten pages each, one page of output for each $C C D F$.

Example Usage:

*

* NUMO

TYPEONUMBER 2

* INDREL

TYPEOOUTO01

TYPEOOUT002

1

INDRAD

9

$10 \mathrm{CCDF}$ 


\subsection{EARLY Input File}

\subsection{Introduction to EARLY}

The EARLY module models the time period immediately following a radioactive release. This period is commonly referred to as the emergency phase. It may extend up to 1 week after the arrival of the first plume at any downwind spatial interval. The subsequent intermediate and long-term periods are treated by CHRONC.

In the EARLY module the user may specify emergency response scenarios that include evacuation, sheltering, and dose-dependent relocation. The EARLY module has the capability for combining results from up to three different emergency response scenarios. This is accomplished by appending change records to the EARLY input file. The first emergencyresponse scenario is defined in the main body of the EARLY input file. Up to two additional emergency-response scenarios can be defined through change record sets positioned at the end of the file. The delimiter used to separate the change record sets is a period (.) in column 1 that is also used to signify the end of a MACCS2 user input file. All of the MACCS2 user input files must thus end with a period in column 1.

The purpose of the change-record processing in EARLY is solely to allow modification of the previously specified emergency response scenario data. Any records appearing in the change record sets must have been previously defined in either the evacuation zone data or the shelter and relocation data. If data items from another data block appear in the change records, they will be ignored. Each set of change records must include a new value of EANAM2, a text field describing the emergency response scenario. Also, each set of change records must produce a change in at least one of the numeric input variables described in the evacuation zone data or in the shelter and relocation data.

Results are output for each of the user-defined emergency-response scenarios and for a weighted sum of the emergency-response scenarios. Scenarios may be combined by assigning time fractions (frequencies of occurrence) or population fractions (fraction of the population engaging in the specified behavior) to each scenario or by a simple summation of the results for each emergency-response scenario (when a unique population distribution is defined for each emergency-response scenario).

CCDFs calculated for emergency-response scenarios combined based on time fractions are a function of the probability for each meteorological trial/wind direction multiplied by the time fraction applied to the emergency-response scenario. Emergency-response scenarios combined using population fractions are a function of the consequence calculated for each meteorological trial/wind direction multiplied by the fraction of people assigned to the scenario. The approach selected (fraction of people or fraction of time) will affect the shape of the CCDF but will not affect the mean results.

For results that are calculated by both EARLY and CHRONC, such as population dose and cancer cases, the OUTPUT module automatically adds the value of the consequence calculated 
by CHRONC to the value of the same consequence measure produced by EARLY in order to generate the overall combined results. If more than one EARLY emergency response scenario is being run, these results are combined according to the weighting fractions supplied by the user in the EARLY input file, and the weighted sum is then combined with the CHRONC result to produce the overall result. Whenever results are combined by the code, the listing produced by the OUTPUT module will present the overall combination of results as well as each of the components from which it is constructed.

\subsubsection{Overview of Dose Calculations}

The calculation of radiation doses from early exposure considers five pathways: (1) direct external exposure to radioactive material in the plume (cloudshine), (2) exposure from inhalation of radionuclides in the cloud (cloud inhalation), (3) exposure to radioactive material deposited on the ground (groundshine), (4) inhalation of resuspended material (resuspension inhalation), and (5) skin dose from material deposited on the skin.

Two kinds of doses are calculated: (1) acute doses used for calculating early fatalities and injuries and (2) lifetime dose commitment used for calculating cancers resulting from the early exposure. The accumulation of radiation doses from early exposure is strongly dependent on the assumed emergency response, that is, evacuation, sheltering, or early relocation. Cloudshine and cloud inhalation exposures are limited to the time of cloud passage. Groundshine and resuspension inhalation doses for early exposure are limited to the duration of the emergency phase.

In general, the dose equation for an early exposure pathway in MACCS2 in a given spatial element is the product of the following quantities: radionuclide concentration, dose conversion factor, duration of exposure, and shielding factor. The quantities used in the dose equations depend on the exposure pathway. For example, for the cloud inhalation exposure pathway, these quantities are the ground-level air concentration at a spatial element, inhalation dose conversion factor, duration of exposure, and inhalation shielding factor.

The dose conversion factors for all exposure pathways are provided in the same format as the DOSDATA.INP file distributed with MACCS. The duration of exposure depends on the exposure pathway and the emergency response at a spatial element and is calculated based on the user-supplied data. The shielding factor is a unitless quantity used to reduce the radiation dose as a result of shielding protection provided by a given protective action for a given exposure pathway. Shielding factors for the various exposure pathways (cloudshine, inhalation, groundshine, and skin dose) and for three different groups of people (evacuees, people doing normal activity, and people taking shelter) are specified by the user.

The MACCS 2 evacuation model incorporates a delay time before public movement, followed by evacuation radially away from the facility at an effective radial constant speed. Different shielding factors and breathing rates can be used while people await evacuation (normal activity) or are being evacuated (evacuees). 
The EARLY module of MACCS2 accumulates the radiation doses for the evacuating people by adding the doses they received before they started moving and the doses received during evacuation out to a distance at which they are assumed to avoid further exposure.

Evacuees receive no additional emergency phase radiation doses after they are modeled as moving out of the evacuation zone. The CHRONC module habitability criterion determines if evacuees are moved back to their element of origin during the intermediate or long-term phase. Any additional radiation doses to the evacuees are calculated in the CHRONC module.

Before the evacuating people start moving, they are assumed to be carrying out normal activities. Shielding factors (cloudshine, groundshine, inhalation, and skin) for normal activity apply to them during this period of time. After they start moving, they become evacuees and the shielding factors for evacuees apply to them during evacuation.

The MACCS2 plume transport model assigns the plume a finite length calculated by using the assumed release duration and wind speed during the release. To simplify the treatment, the length of the cloud is assumed to remain constant following the release (i.e., the front and back of the plume travel at the same speed), and the concentration of radioactive material is assumed to be uniform over the length of the cloud. The radial position of evacuating persons, while stationary and while in transit, is compared with the positions of the front and back of the plume as a function of time to determine the period of exposure to airborne radionuclides.

\subsubsection{Overview of New EARLY File Input Requirements Implemented in MACCS2}

The MACCS input variable ESPEED is utilized by MACCS2 but three values must now be supplied instead of the single value used by MACCS. The three evacuation speeds thus defined are used for the initial, middle, and late phases of the evacuation.

The input variable WTNAME, which in MACCS determines whether fraction-of-the-people or fraction-of-the-time weighting is to be used in combining results, is now allowed to take on three possible values: PEOPLE, TIME, or SUMPOP. The new SUMPOP option indicates that results are to be combined by simple unweighted summation.

If SUMPOP is specified, the weighting fraction input variable, WTFRAC, will not be processed. Furthermore, if SUMPOP is specified, the user must define in the Site Data file a separate population distribution for each of the up to three emergency response scenarios. The option of a uniform population density (POPFLG='UNIFORM') is not allowed in these cases.

The population distribution used for CHRONC is obtained by summing the population distributions defined for EARLY. For example, if a given spatial element on the grid was defined to have 12,20 , and 30 individuals, respectively, for the three different emergencyresponse scenarios, the long-term calculations performed by the CHRONC module would be based on 62 individuals residing at that location.

The shielding and exposure factors for cloudshine, groundshine, and inhalation (CSFACT, SKPFAC, GSHFAC, PROTIN, and BRRATE) may now be redefined for each emergencyresponse scenario. MACCS utilized a single set of these factors for both EARLY and CHRONC. 
The CHRONC module, if exercised, will use numeric values from the last emergency-response scenario that was defined in the EARLY input file.

It is up to the user to specify the various parameters needed for these calculations. There are no default values. In addition to specifying the characteristics of the model, the user has complete control over the output produced by EARLY and must explicitly specify which results are to be produced. All of this information is supplied through the input file for EARLY and all of the input parameters are described in this chapter.

\subsection{Dose Conversion Factor Filename and Other Miscellaneous Input Data}

\subsubsection{Dose Conversion Factor Filename}

The MACCS2 user has a great deal of flexibility in the selection of a dose conversion factor file. The MACCS2 package includes a number of sample DCF files. Section 3.1 provides an overview of the MACCS2 DCF preprocessors that can be used to generate DCF files. MACCS2 can utilize the following types of DCF files:

1. DOSFAC files (e.g., the DOSDATA.INP file distributed with MACCS 1.5.11.1),

2. DOSFAC2 files,

3. IDCF2 files, and

4. FGRDCF files.

In addition, data may be combined from these files into a single DCF file. All DCF files must contain a list of organs. MACCS2 will process a list of up to 20 organs when Types 1 - 3 DCF files (DOSFAC, DOSFAC2, and IDCF2) are utilized. However, these types of DCF files must list a minimum organ set, which can appear in any order on the DCF file. The required organ set is as follows:

EDEWBODY
RED MARR
BONE SUR
BREAST
LUNGS
THYROID
LOWER LI
BLAD WAL
LIVER
THYROIDH

Organs not included in this list are not used in MACCS2 calculations when Types 1-3 DCF files are utilized. The list of organs that can be included in MACCS2 calculations is fixed in the code and is discussed in Section 6.4. 
When a COMIDA2-generated input file is used in a MACCS2 run, MACCS2 must use the same DCF file used to generate the COMIDA2 input file. The first two header records of the DCF files used by MACCS2 and COMIDA2 are read and compared to ensure that the two sets of calculations used the same DCF file.

When COMIDA2 and MACCS2 are exercised using a DCF file generated by FGRDCF, those two programs utilize an abbreviated list of nine organs that is fixed in MACCS2 and cannot be modified by the user. COMIDA2 and MACCS2 detect the use of an FGRDCF file by examining the first seven characters of the DCF file's header record.

If the DCF file was generated by FGRDCF, the first of the two header records will contain the letters FGRDCF. When COMIDA2 and MACCS2 determine that an FGRDCF file is being used, as specified by the variable DCF_FILE, the list of available organs is set to the following:
L-GONADS
L-BREAST
L-LUNGS
L-RED MARR
L-BONE SUR
L-THYROID
L-REMAINDER
L-EFFECTIVE
L-SKIN(FGR)

with those names based on the nomenclature of FGR 11 and 12. The L- prefix indicates a 50year committed dose. The A-prefix indicates an acute organ dose. The organ L-SKIN(FGR) is based on the skin dose of FGR 12, and it differs substantially from the acute skin dose used in MACCS2 to calculate acute health effects from material deposited on the skin.

\section{Variable Name: DCF_FILE}

Variable Type: Character, Scalar

Allowed Range: $\quad 1 \leq$ length $\leq 40$

Purpose:

Identifies the DCF file to be used for the MACCS2 calculations. This filename can include a directory path; the file need not be in the current directory.

If the CHRONC module is to utilize the COMIDA2 food-chain model via a COMIDA2 binary data file of food ingestion parameters, the DCF file used in the MACCS2 calculations needs to be the same file that was used by COMIDA2.

Example Usage:

DCF_FILE001 'C:\DOSFAC2 \DOSDATA.INP' 


\subsubsection{Miscellaneous Data}

In addition to the specification of a DCF file, the user must supply information to identify the run, define the histogram approximating the crosswind Gaussian distribution, and also supply information describing the handling of the wind rose. It is possible to specify a single wind rose that will override the wind roses that were calculated in ATMOS for each of the weathercategory sampling bins. The user must also specify how to treat changes in wind direction.

$\begin{array}{ll}\begin{array}{l}\text { Variable Name: } \\ \text { Variable Type: }\end{array} & \begin{array}{l}\text { EANAM1 } \\ \text { Character, Scalar }\end{array} \\ \begin{array}{l}\text { Allowed Range: } \\ \text { Purpose: }\end{array} & \begin{array}{l}1 \leq \text { length } \leq 80 \\ \text { Identifies a name describing the EARLY calculations. } \\ \text { pages of the OUTPUT listing. A name describing the par } \\ \text { response assumption will be requested in addition to this }\end{array} \\ \begin{array}{l}\text { Example Usage: } \\ \text { * GENERAL DESCRIPTIVE INFORMATION FOR THE "EARLY" INPUT FILE } \\ \text { * INANAM1001 'IN2A. INP, SURRY, SAMPLE PROBLEM A, EARLY INPUT' }\end{array}\end{array}$

Variable Name: ENDAT2

Variable Type: $\quad$ Logical, Scalar

Allowed Value: $\quad$.TRUE. or .FALSE.

Purpose: $\quad$ Control flag that allows the user to execute only the ATMOS and EARLY modules while skipping execution of the CHRONC module. If CHRONC is to be skipped, there is no need for the user to specify a CHRONC input file. A value of .TRUE. causes MACCS2 to skip the CHRONC module. A value of .FALSE. causes the CHRONC module to be executed.

Example Usage:

$\star$

* FLAG TO INDICATE THIS IS THE LAST PROGRAM IN THE SERIES TO BE RUN

MIENDAT2001 .FALSE. (SET THIS VALUE TO .TRUE. TO SKIP CHRONC)

Variable Name: $\quad$ IPLUME

Variable Type: Integer, Scalar

Allowed Range: $1 \leq$ value $\leq 3$

Purpose:

Dispersion model option code:

1 - Straight-line dispersion model:

All plume segments travel in the same direction. Each set of modeling results is rotated around the 16 compass directions (population sectors) to yield 16 sets of results for each weather trial. 
2 - Wind-shift plume dispersion model with rotation:

Each plume segment in the release travels in the direction that the wind is blowing at the time that its representative time point (REFTIM in Section 5.11) leaves the facility. Each set of modeling results is rotated around the 16 compass directions (population sectors) to yield 16 sets of results for each weather trial.

3 - Wind-shift dispersion model without rotation:

Each plume segment in the release travels in the direction that the wind is blowing at the time that its representative time point (REFTIM in Section 5.11) leaves the facility. No rotation of the wind shift pattern is performed. Each weather trial yields one set of results.

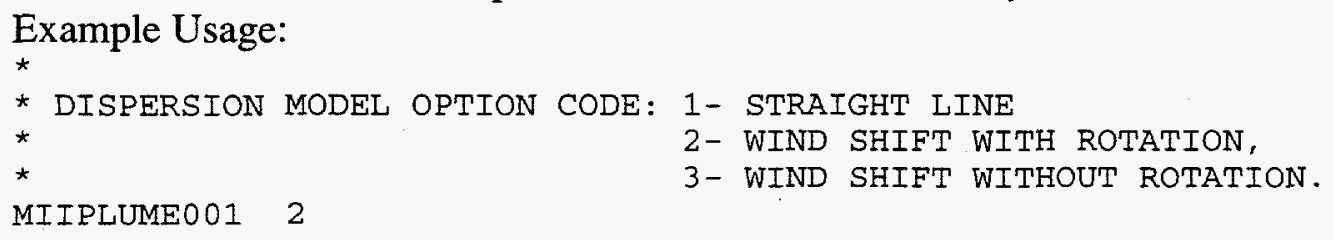

Variable Name: NUMFIN

Variable Type: Integer, Scalar

Allowed Range: 3,5 , or 7

Purpose:

Number of fine-grid subdivisions used by the model. A step function is used to approximate the Gaussian distribution of the plume in the crosswind direction. Each of the 22.5-degree sectors is subdivided into NUMFIN finegrid elements, with doses and risks being uniform in each element.

NUMFIN is only used in the calculations performed by EARLY; it is not

Example Usage: utilized in any respect in the calculations performed by CHRONC.

* NUMBER OF FINE-GRID SUBDIVISIONS USED BY THE MODEL

MINUMFINOO1 7 (3，5 OR 7 ALLOWED)

Variable Name: IPRINT

Variable Type: Integer, Scalar

Allowed Range: $\quad 0 \leq$ value $\leq 10$

Purpose:

Specifies the quantity of debug output that is desired. The higher the value, the more output will be printed. Debug output is written to FORTRAN unit 6. Normal runs should specify a value of 0 . The choice of any other value should be made only by people familiar with the code for detailed examination of single weather sequences.

For various values of IPRINT, the code will print out intermediate results on the list output file. These are described below. 
IPRINT $\geq 1$ : skin dose conversion factors, centerline doses for all organs (if IPLUME=1), Gaussian histogram and cloudshine correction factors, return code values (RETCOD).

IPRINT $\geq 2$ : final groundshine dose rate for each organ, each plume segment.

IPRINT $\geq 4$ : total acute dose for organs 2 and 3, early fatality, early injury, and cancer risk values for each spatial element.

IPRINT $\geq 8$ : acute dose to organs 2 and 3 after completion of subroutine RELZON, acute dose to organs 2 and 3 after completion of subroutine ESTAT.

Example Usage:

* LEVEL OF DEBUG OUTPUT REQUIRED, NORMAL RUNS SHOULD SPECIFY ZERO

MIIPRINT001 0

Variable Name: RISCAT

Variable Type: Logical, Scalar

Allowed Value: $\quad$.TRUE. or .FALSE.

Purpose:

If the option of weather-category bin sampling was chosen by the user in the ATMOS input file (METCOD=2), the display of results produced by the OUTPUT module can show the relative contribution of each of the weathercategory bins to the mean consequence value.

Example Usage:

MIRISCATO01 .FALSE. (DO NOT PRINT OUT THE RISK CONTRIBUTION TABLES)

Variable Name: OVRRID

Variable Type: $\quad$ Logical, Scalar

Allowed Value: $\quad$.TRUE. or .FALSE.

Purpose: $\quad$ Specifies whether the wind rose probabilities are to be supplied by the user. If the weather sampling option was chosen in ATMOS (METCOD=2), wind roses for each weather sampling bin have been passed down from ATMOS. Those wind roses will be used if OVRRID $=$.FALSE. If no wind rose is available, a uniform wind rose will be used, that is, $\mathrm{P}=0.0625$ in each direction.

Example Usage:

$\star$

* FLAG INDICATING IF WIND ROSES FROM ATMOS ARE TO BE OVERRIDDEN

MIOVRRIDO01 .FALSE. (USE THE WIND ROSE CALCULATED FOR EACH BIN) 
Variable Name: WINROS

Variable Type: $\quad$ Real, Array

Allowed Range: $\quad 0.0 \leq$ value $\leq 1.0$

Purpose:

This input parameter is only required if the OVRRID input parameter is set to .TRUE. The values input for this parameter are the probabilities of the wind blowing from the site into each of the 16 compass sectors (rotating clockwise from $\mathrm{N}$ to $\mathrm{NNW}$ ).

The sum of these values must be between 0.95 and 1.05 . The user must supply 16 values in rows on one or more data records.

Example Usage:

*

* SITING STUDY WIND ROSE FOR THE PEACH BOTTOM SITE

$\begin{array}{lllll}\text { MIWINROSO01 } & 8.521 \mathrm{E}-02 & 6.360 \mathrm{E}-02 & 4.605 \mathrm{E}-02 & 5.189 \mathrm{E}-02 \\ \text { MIWINROS002 } & 6.869 \mathrm{E}-02 & 9.493 \mathrm{E}-02 & 1.145 \mathrm{E}-01 & 1.090 \mathrm{E}-01 \\ \text { MIWINROS003 } & 6.019 \mathrm{E}-02 & 4.326 \mathrm{E}-02 & 3.148 \mathrm{E}-02 & 3.238 \mathrm{E}-02 \\ \text { MIWINROS004 } & 3.383 \mathrm{E}-02 & 4.625 \mathrm{E}-02 & 5.446 \mathrm{E}-02 & 6.424 \mathrm{E}-02\end{array}$

\subsection{Population Distribution (PD) Data}

The user must supply information to define the polar-coordinate population distribution surrounding the site. This information can be supplied from the Site Data file or a uniform population distribution can be specified by the user. The format of the Site Data file is described in Section A.3 of Appendix A.

Note: Whatever values are supplied here will be used by both the EARLY and the CHRONC modules in defining the characteristics of the region surrounding the site. There is no possibility of having EARLY and CHRONC use different population distributions.

Variable Name: POPFLG

Variable Type: Character, Scalar

Allowed Range: $\quad 4 \leq$ length $\leq 7$

Purpose:

Specifies whether the population is to be defined by the Site Data file or if it is to be uniform. The value specified must be either FILE or UNIFORM. If a value of UNIFORM is supplied, the program will not attempt to read the Site Data file.

Example Usage:

*

* POPUlation DISTRIBUtion FLAG, SPECIFy 'UNIFORM' OR 'FILE'

PDPOPFLG001 UNIFORM 
Variable Name: IBEGIN

Variable Type: Integer, Scalar

Allowed Range: $\quad 1 \leq$ value $\leq$ NUMRAD

Purpose:

Specifies the spatial interval at which the population begins. Inside of this region there are no people. This allows the modeling of an exclusion zone. This value is only required if POPFLG='UNIFORM'.

Example Usage:

* First spatial interval is an exclusion zone

PDIBEGINO01 2

Variable Name: POPDEN

Variable Type: $\quad$ Real, Scalar

Allowed Range: $\quad 0.0 \leq$ value $\leq 1 . \mathrm{E} 6\left(\right.$ people $\left./ \mathrm{km}^{2}\right)$

Purpose:

Specifies the uniform population density of the region. This value is only required if POPFLG='UNIFORM'.

Example Usage:

PDPOPDENO01 100.0 (100 PEOPLE PER SQUARE KILOMETER)

\subsection{Organ Definition (OD) Data}

The organs referenced in the MACCS2 EARLY and CHRONC input files must be assigned a .TRUE. value for the ORGFLG variable defined in this section.

MACCS2 can calculate two kinds of doses: lifetime dose and effective acute dose. Lifetime dose is utilized to determine the need for mitigative actions and for calculating the cancer induction and population dose results. It represents the 50-year dose commitment. Effective acute doses are used for calculating the acute health effects in the EARLY module. The acute health effects are early fatalities and early injuries. A full description of the meaning of effective acute dose can be found in Section 6.7.

The list of organs for which dosimetry data are available is presented below.

\begin{tabular}{lll} 
Organ-Name & Acute & \multicolumn{2}{l}{ Lifetime } \\
\cline { 2 - 3 } 'SKIN' & YES & NO \\
'LUNGS' & YES & YES \\
'RED MARR' & YES & YES (red bone marrow) \\
'SMALL IN' & YES & YES (small intestine) \\
'LOWER LI' & YES & YES (lower large intestine) \\
'STOMACH' & YES & YES \\
'THYROID' & NO & YES \\
'THYROIDH' & YES & YES (pseudothyroid just for health effects) \\
'EDEWBODY' & NO & YES (effective whole-body dose equivalent)
\end{tabular}




$\begin{array}{lccl}\text { 'BONE SUR' } & \text { NO } & \text { YES } & \text { (bone surface) } \\ \text { 'BREAST' } & \text { NO } & \text { YES } & \\ \text { 'OVARIES' } & \text { NO } & \text { YES } & \\ \text { 'TESTES' } & \text { NO } & \text { YES } & \end{array}$

In MACCS2, the two types of doses are distinguished by an L- or A- prefix to the organ name. The acute doses were constructed using effective acute dose reduction factors. A description of acute dose calculations is provided in the MACCS Model Description and in Section 6.7.

Within the EARLY input file, organ names must be specified as they appear in the Example Usage section following the ORGNAM and ORGFLG variable descriptions. Organ names must be specified in the EARLY and CHRONC input files as they appear in this list. Since the organ list is fixed in the code, the MACCS EARLY input file variables NUMORG and ORGNAM are no longer processed.

In applications that do not require the calculation of acute health effects, the code's run time can be reduced somewhat by defining only organs that will be actually used in the analysis. However, testing of this feature revealed that the reduction of the organ list to L-EDEWBODY and omission of all health effect calculations reduced the run time of sample problem A by only $28 \%$.

If a DCF file generated by FGRDCF is being used for the calculations, the list of available organs is set automatically to the nine organs of FGR 11 and 12. If the header record of that file begins with the characters FGRDCF, the data below, ORGNAM and ORGFLG, are not processed by MACCS2 and the list of organs is fixed to the following:
L-GONADS
L-BREAST
L-LUNGS
L-RED MARR
L-BONE SUR
L-THYROID
L-REMAINDER
L-EFFECTIVE
L-SKIN(FGR)

Variable Name: ORGNAM

Variable Type: $\quad$ Character, Array

Allowed Length: $3 \leq$ length $\leq 10$

Purpose:

Defines the list of organs to be included in the calculations. This list of organs must be specified in exactly the same order as shown in the example that follows. If the list is specified in a different order, an input error will be diagnosed and further execution inhibited. A- indicates an acute dose and Lindicates a 50-year dose commitment. 
Variable Name:

ORGFLG

Variable Type:

Logical, Array

Allowed Values:

.TRUE. or .FALSE.

Purpose:

Specifies whether each organ on the list is to be used in the calculations. At present, deleting organs from the list yields only minimal decreases in the code's execution time.

Example Usage:

$\star$

*

MIORGDEF001

MIORGDEF002

MIORGDEF003

MIORGDEF004

MIORGDEF005

MIORGDEF006

MIORGDEF007

MIORGDEF008

MIORGDEF009

MIORGDEF010

MIORGDEF 011

MIORGDEF 012

MIORGDEF013

MIORGDEF 014

MIORGDEF 015

MIORGDEF 016
ORGNAM

'A-SKIN'

'A-RED MARR'

' A-LUUNGS '

' A-THYROIDH '

' A-STOMACH '

'A-LOWER LI'

' L-EDEWBODY'

' L-RED MARR'

'L-BONE SUR'

' L-BREAST'

'L-LUNGS'

'L-THYROID'

' L-LOWER LI'

' L-BLAD WAL'

' L-IIVER'

' L-THYROIDH '
ORGFLG

. TRUE.

. TRUE.

. TRUE.

. TRUE.

. TRUE.

. FALSE.

. TRUE.

. TRUE.

. TRUE.

. TRUE.

. TRUE.

. FALSE.

. TRUE.

. TRUE.

. FALSE.

. TRUE.

Note: If the COMIDA2-based food-chain model is being utilized, the doses L-EDEWBODY and L-THYROID must be assigned values of .TRUE.

\subsection{Shielding and Exposure (SE) Data}

This section defines the shielding factors for exposure to cloudshine, groundshine, inhalation, and deposition to skin for three types of activities (normal activity, evacuation, and sheltering). A breathing rate is also specified for each type of activity. In addition, the resuspension parameters to be used for the emergency phase time period (EARLY), the resuspension coefficient, and resuspension half-life are also defined.

Some of the parameter values defined in this section are used in both the EARLY and the CHRONC modules; these are (1) the normal activity groundshine and (2) inhalation protection factors as well as the (3) normal activity breathing rate. The CHRONC module does not calculate the effects resulting from direct exposure to the radioactive cloud, therefore it makes no use of the cloudshine and deposition to skin shielding factors. The long-term resuspension parameters are defined on the basis of data supplied in the CHRONC input file, and the emergency-phase resuspension parameters defined here are not used in the CHRONC module. 
Variable Name: CSFACT

Variable Type: Real, Array

Allowed Range: $\quad 0.0 \leq$ value $\leq 1.0$ (unitless)

Purpose:

Cloudshine shielding factor for the three types of activity.

Example Usage:

* THREE VALUES OF EACH PROTECTION FACTOR ARE SUPPLIED,

* ONE FOR EACH TYPE OF ACTIVITY. THE FACTORS ARE LISTED

* IN THE FOLLOWING ORDER: EVACUATION, NORMAL ACTIVITY, SHELTERING

$\star$

* CLOUd SHIELDING FACTOR

SECSFACTO01 1 . $0.75 \quad 0.6$

Variable Name: $\quad$ PROTIN

Variable Type: $\quad$ Real, Array

Allowed Range: $\quad 0.0 \leq$ value $\leq 1.0$ (unitless)

Purpose:

Inhalation protection factor for the three types of activity.

Example Usage:

* PROTECTION FACTOR FOR INHALATION

$\begin{array}{lllll} & \text { SEPROTINOO } & 1 . & 0.41 & .33\end{array}$

Variable Name: BRRATE

Variable Type: $\quad$ Real, Array

Allowed Range: $\quad 0.0 \leq$ value $\leq 1.0$ (cubic meters per second)

Purpose:

Breathing rates for the three types of activity.

Example Usage:

*

* BREATHING RATE (CUBIC METERS PER SECOND)

$\begin{array}{llll}\text { SEBRRATEO01 } & 0.000266 & 0.000266 & 0.000266\end{array}$

Variable Name: SKPFAC

Variable Type: Real, Array

Allowed Range: $\quad 0.0 \leq$ value $\leq 1.0$ (unitless)

Purpose:

Skin protection factors for the three types of activity.

Example Usage:

* SKIN PROTECTION FACTOR

$\star$

$\begin{array}{llll}\text { SESKPFACO01 } & 1.0 & 0.41 & 0.33\end{array}$

Variable Name: $\quad$ GSHFAC

Variable Type: $\quad$ Real, Array

Allowed Range: $\quad 0.0 \leq$ value $\leq 1.0$ (unitless) 
Purpose: Groundshine shielding factors for the three types of activity.

Example Usage:

* GROUND SHIELDING FACTOR

SEGSHFACO01 $0.5 \quad 0.33 \quad 0.2$

Variable Name: RESCON

Variable Type: Real, Scalar

Allowed Range: $\quad 0.0 \leq$ value $\leq 1.0$ (seconds/meter)

Purpose:

Initial value for emergency-phase resuspension concentration factor. The weathering half-life associated with RESCON is defined below as RESHAF. The resuspension model used is as follows:

air concentration $=$ ground concentration $\bullet \operatorname{RESCON} \bullet \exp (-t \bullet 0.693 /$ RESHAF)

where air concentration is the instantaneous air concentration resulting from resuspension of material deposited by a single plume segment at $t$ seconds following the departure of that plume segment $\left(\mathrm{Bq} / \mathrm{m}^{3}\right)$; ground concentration is the final ground concentration following the passage of the plume segment $\left(\mathrm{Bq} / \mathrm{m}^{2}\right)$; RESCON is the initial value of the resuspension coefficient $\left(\mathrm{m}^{-1}\right)$; $t$ is the time after passage of the plume (s); and RESHAF is the resuspension coefficient half-life (s).

\section{Example Usage:}

* RESUSPENSION INHALATION MODEL CONCENTRATION COEFFICIENT (SECONDS/METER)

SERESCONOO1 1.E-6 (RESUSPENSION IS TURNED ON)

Variable Name: RESHAF

Variable Type: Real, Scalar

Allowed Range: $\quad 1.0 \leq$ value $\leq 1 . E 10$ (seconds)

Purpose: $\quad$ Emergency phase resuspension concentration coefficient weathering half-life.

Example Usage:

* RESUSPENSION CONCENTRATION COEFFICIENT HALF-LIFE (SECONDS)

SERESHAF001 1.E10 (CONSTANT RESUSPENSION FACTOR)

\subsection{Evacuation Zone (EZ) Data}

This section describes the input parameters that control the dose calculations in the regions where sheltering and/or evacuation are defined to occur. MACCS2 is structured to allow the analyst a 
great deal of flexibility in defining emergency response strategies. This is achieved within a framework that is conceptually simpler than the MACCS model. For most sites, it will probably be unnecessary to utilize all the features of the new model. However, availability of the MACCS2 modeling features will facilitate performance of sensitivity studies, etc. to evaluate alternative assumptions for emergency response.

\subsubsection{Emergency-Response Scenarios}

MACCS2 as well as MACCS allows the user to specify up to three emergency-response scenarios. The population data associated with each of these scenarios may be defined in either of two ways: (1) with a single population data block (as with MACCS) or (2) a separate population data block for each of the emergency-response scenarios. If multiple population distributions are defined, the user is then unable to define fraction-of-the-people or fraction-ofthe-time weighting factors for summation of the overall results. In that case, the various results are simply added in reporting the CCDF summary tables generated by the code.

As was the case with MACCS, evacuation and sheltering actions may be modeled in the region surrounding the release point. Outside the evacuation and sheltering region, dose-dependent relocation actions may take place in exactly the same manner as with the previous code using the so-called hot-spot and normal relocation dose criteria.

The user may no longer define a sheltering region per se since evacuation and sheltering actions have been merged. However, this merging of the two actions has been accomplished in a manner that allows emulation of the MACCS model's features. In the new code's modeling of the evacuation and sheltering region, the user explicitly defines the reference time to be used for initiation of the action as being either the alarm time (input variable OALARM) associated with the source term or the arrival time of the first plume reaching the downwind location. In the region beyond the evacuation and sheltering region, that is, the so-called relocation zone, the reference time point for actions remains the plume arrival time, and no change to the MACCS model has been made.

The evacuation and sheltering region is user specified to extend a given radial distance (i.e., radial spatial interval). For each radial distance in this region, the user specifies a sheltering period (which may have a zero duration) that occurs prior to the initiation of the evacuation. During the sheltering phase, shielding factors appropriate for sheltered activity are used to calculate doses for the individuals in contaminated areas.

By allowing the delay times to vary for each ring of the polar grid, a staged evacuation may be modeled in which the evacuation delay time increases as a function of distance. If desired, each emergency response scenario, of the three allowed, may utilize its own set of shielding factors.

At the termination of the sheltering phase, the resident individuals start their travel out of the region. In each of the scenarios chosen, the user defines whether evacuation is to follow radial paths (as in MACCS) or follow complex paths defined as a network. 
The actions defined for each evacuation scenario are wholly independent of the other evacuation scenarios defined by the user. That is, a radial evacuation may be used for the first scenario, a network evacuation could be defined for the second scenario, and the third could have neither evacuation nor sheltering taking place, with the entire region subject only to dose-dependent relocation actions. The results from these scenarios in the overall CCDF are combined using either (1) a single population distribution modified by weighting fractions, or (2) the simple summation of results from separate population data blocks.

\subsubsection{Early, Middle, and Late Phases of Evacuation}

In the new model, the user divides the evacuation period into three phases: early, middle, and late. For each of the three phases, the user defines the travel speed of the evacuees during that period. For situations where only one or two speeds are defined, the duration of the first and/or second evacuation phase may be set to 0 .

For both types of evacuation (i.e., radial and network) the implementation of the travel speed model is identical. Three travel speeds are defined in the EARLY input file. As individuals traverse the path defined for them, their speed of travel can vary due to transitions from one phase to the next. If these transitions occur while an individual is traversing a spatial element, the code uses linear interpolation to determine the traversal time for that element.

In order to allow all individuals to leave the evacuation region, the late phase is considered to last as long as is necessary for all evacuating individuals to leave the region. Thus, the user specifies the duration of the early and middle evacuation periods but not for the late phase.

The initial evacuation period begins when the first individuals begin their travel out of the region and lasts for the user-specified duration, DURBEG. After that period has elapsed, the middlephase evacuation period takes place for the user-specified duration DURMID. Finally, the remainder of time necessary for all individuals to complete their travel is considered the latephase evacuation period.

\subsubsection{Radial vs. Network Evacuation}

MACCS2 allows the user a choice between two evacuation network models: radial and network. Figures 6-1 and 6-2 provide illustrations of these evacuations. For both radial and excavation models, sheltering has been integrated into the model, with sheltering (if defined) always preceding evacuation. The internal implementation of the radial and network models is largely identical, since a radial evacuation can be described as a network evacuation constrained to have all evacuation paths in the radial directions, representing a subset of the network functionality.

For this reason, and ease of description, the functionality of these models will be explained by first describing the user-specified input parameters that define the operation of the network evacuation model. This will be followed by an explanation of how MACCS2 creates an evacuation network to represent the radial evacuation case. Finally, the approach used by MACCS2 to calculate resultant doses to residents of the sheltering/evacuation region will be described. 


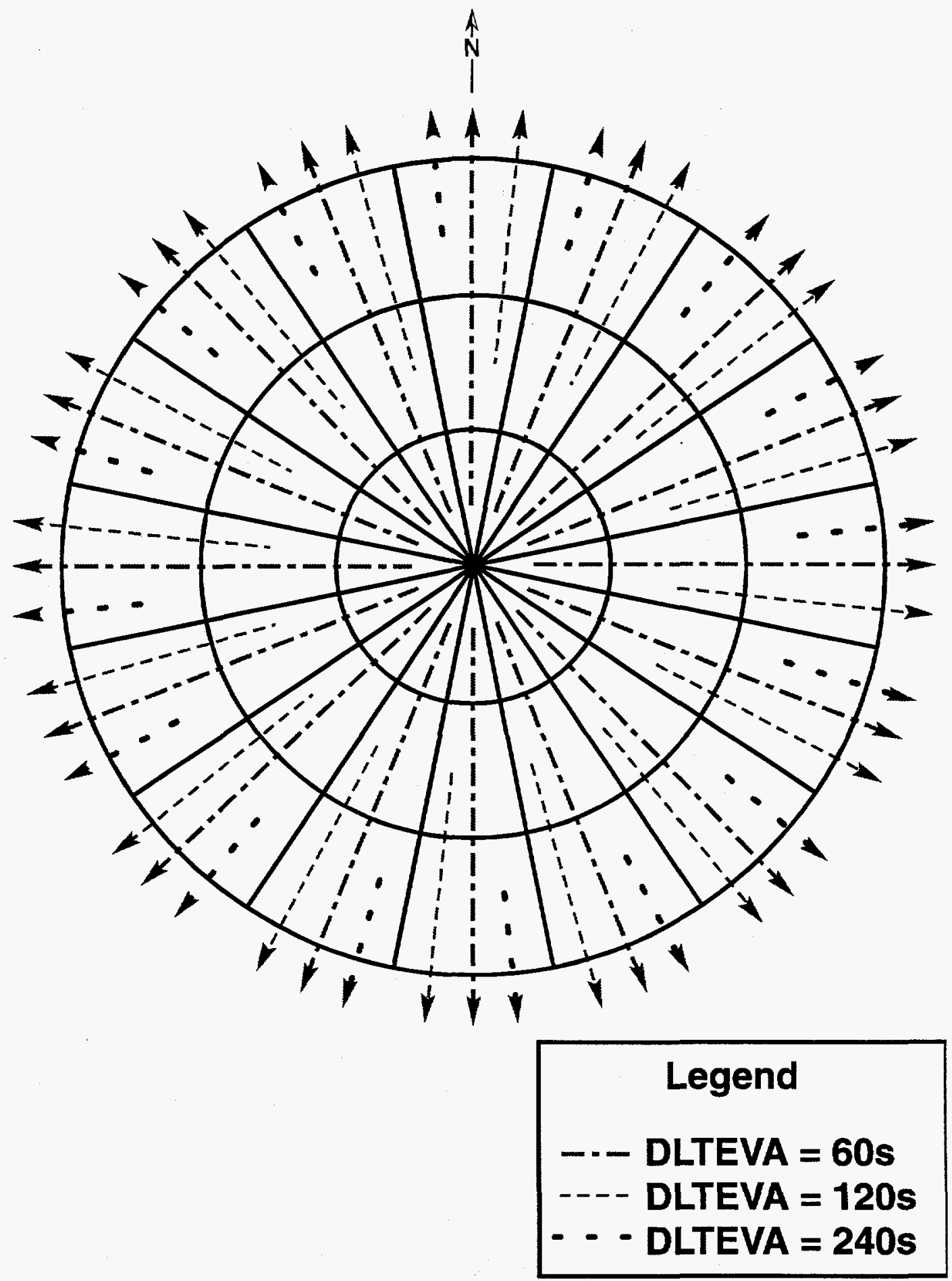

Figure 6-1. Radial evacuation paths with DLTEVA values of 60, 120, and $240 \mathrm{~s}$ assigned to the three spatial intervals. The DLTEVA input variable defines the duration of the sheltering period before the beginning of evacuation and is described in this section. 


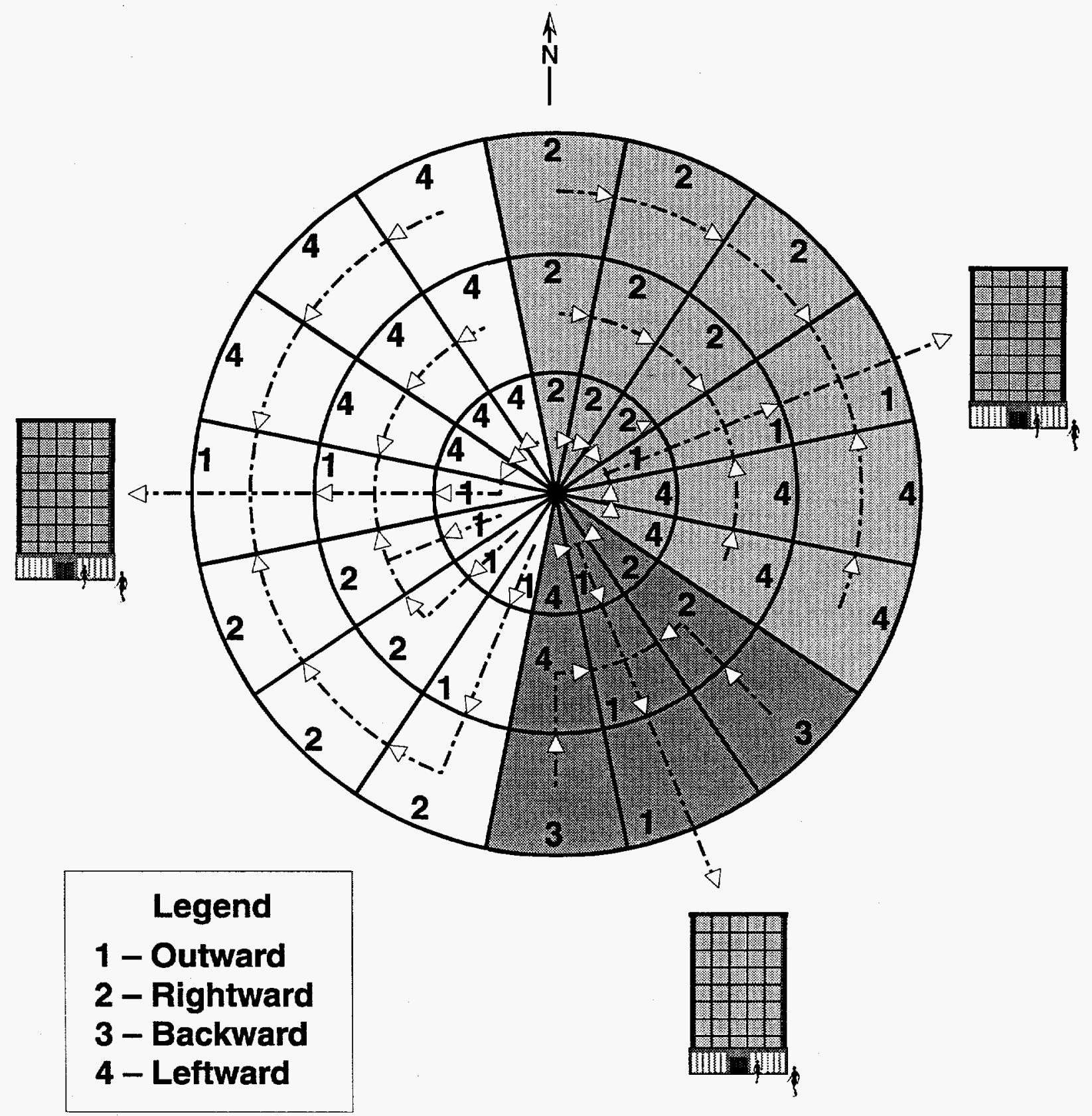

Figure 6-2. A network evacuation grid. The numbers contained in each spatial element represent the value that would be assigned to the IDIREC input variable for the definition of evacuee movement in that spatial element. The IDIREC input variable is defined in this section and the example usage provided for this variable describes the network represented in this figure. 


\subsubsection{Network Evacuation Input Data}

The region in which evacuation and sheltering can occur is circular, extending from the grid centerpoint out to a user-specified distance. A number of parameters are supplied by the user to define the operation of the model when the network evacuation model is specified.

LASMOV: Defines the furthest ring in which evacuees will incur exposures. When evacuees exit this ring and proceed to the next outermost ring, no further exposures are incurred.

NUMEVA: Defines the number of concentric rings in which evacuation and/or sheltering can occur for the resident population.

REFPNT: Control flag indicating which of two time points is to be the reference time point for initiation of sheltering and evacuation. If ALARM is specified, the initiation of sheltering and evacuation actions will occur at the off-site alarm time (ATMOS input variable OALARM). If ARRIVAL is specified, the initiation of sheltering and evacuation actions will occur at the time when the first plume arrives at the centerpoint of the spatial interval, with the centerpoint of the spatial interval defined as the point midway between the inner and outer circular arcs that bound the spatial interval.

ESPEED(3): An array of three values that define evacuation travel speeds $(\mathrm{m} / \mathrm{s})$ for the three phases of evacuation: early, middle, and late.

DURBEG: Defines the duration (seconds) of the early phase of evacuation. This period begins when the sheltering period has elapsed. During this period, evacuees travel at the rate specified by ESPEED(1).

DURMID: Defines the duration (seconds) of the middle phase of evacuation. This period begins when the early-phase evacuation period has elapsed. During this period, evacuees travel at the rate specified by $\operatorname{ESPEED}(2)$. When this period has elapsed, evacuees travel at the rate specified by $\operatorname{ESPEED}(3)$.

DLTSHL(IRAD): For each distance ring in the shelter/evacuate region, this variable defines the delay to take shelter (seconds) for resident individuals. When DLTSHL is specified as 0, sheltering will occur with no delay (that is, immediately) at that distance. Prior to the initiation of sheltering, individuals may (if plume arrival has occurred) receive exposures calculated by using the shielding factors for normal activity.

DLTEVA(IRAD): For each distance ring in the shelter/evacuate region, this variable defines the duration of the sheltering period (seconds) that is to occur before evacuation begins for residents. When DLTEVA is specified as 0, evacuation will occur with no additional delay (that is, there is no shelter period at that distance).

If DLTSHL is specified to be 0 at a particular distance, sheltering begins at either of two times: off-site alarm time or plume arrival (as specified by the input variable REFPNT). If DLTSHL is specified to be 0 and REFPNT='ARRIVAL', individuals at this distance will incur no exposure 
utilizing normal activity shielding factors since they are assumed to take shelter immediately upon plume arrival.

If DLTSHL is nonzero at a particular distance, sheltering begins at DLTSHL seconds after either the off-site alarm time or plume arrival. The duration of their shelter period is always DLTEVA.

At the conclusion of the sheltering period, the resident individuals begin their evacuation travel. During the sheltering period, and prior to the initiation of evacuation, individuals may (if plume arrival has occurred) receive exposures calculated by utilizing the sheltering shielding factors. At the conclusion of the sheltering period, the individuals begin their evacuation travel.

IDIREC(IRAD, JANG): A two-dimensional array defining exit directions for each element within the sheltering/evacuation region (IRAD and JANG are the indices for the spatial interval and the wind direction respectively). Allowable values are 1 through 4: 1 signifies radially outward travel, 2 signifies clockwise travel, 3 signifies radially inward travel, and 4 signifies counterclockwise travel.

\subsubsection{Radial Evacuation Input Data}

When radial evacuation is specified (EVATYP='RADIAL'), with only a single exception the user must supply all of the data required for specification of network evacuation. The single input parameter not required in this case is IDIREC. A network evacuation path is constructed internally by the code by setting all values for IDIREC to 1 , signifying that all travel is to be in a radially outward direction.

\subsubsection{Processing of Evacuation Networks}

In preparation for network evacuation calculations, the code tests the network to ensure that it is valid. A valid network cannot contain any closed cycles. That is, it cannot contain a path in which evacuees would travel in an infinite loop. Radially inward travel is not allowed for the distance ring closest to the release point. Elements at the outer boundary of the region are not required to have a destination direction away from the release point though at least one boundary element must offer an outbound path.

For convenient performance of the calculations, the network is then reduced to an internally stored sequence of linear paths. This is done by testing each spatial element within the region to determine if it is a root node. A root node is a node that is not the travel destination of any other node in the network. Each network will include some number, $n$, of root nodes. Each root node is the starting point of a linear path through the network that culminates in an exit from the network. Each of the $n$ linear paths is a sequence of nodes (spatial elements).

\subsubsection{Dose Calculations for Evacuating Populations}

In preparation for the dose calculations, the travel time in each spatial element is calculated. The travel time for each node is a simple function of travel speed and distance. The travel distance varies depending on the direction of travel within the node and its size. For radially outward or inward travel and the TRAVELPOINT = 'BOUNDARY' option, the travel distance for a node is 
the difference between the radii to the spatial element's inner and outer boundaries. For the TRAVELPOINT = 'CENTERPOINT' option, the travel distance for a node is the radial distance between the centerpoints of contiguous spatial elements. When individuals initiate their travel, they are assumed to be located at the centerpoint of the spatial element and therefore their travel distance through the element is only half of the travel distance calculated for the spatial element for evacuees who begin their travel elsewhere.

For clockwise or counterclockwise travel, the travel distance is the length of the circular arc between a node and its destination node, with these nodes lying directly under the radii corresponding to the 16 compass points, and at a distance from the grid centerpoint midway between the inner and outer boundary rings surrounding the node. For example, consider a node located within a circular ring having an inner boundary of $2 \mathrm{~km}$ and an outer boundary of $3 \mathrm{~km}$. The node is considered to be located at a distance of $2.5 \mathrm{~km}$ from the grid centerpoint. The distance, $D$, to the neighboring node in the clockwise or counterclockwise travel direction is then $D=2 \pi r / 16$, and, with $r=2.5 \mathrm{~km}, D=0.98 \mathrm{~km}$. In this manner, travel times are calculated for each node in the network and for the travel speeds assigned to each evacuation phase.

Doses to individuals moving within the evacuation network are calculated by tracing the travel paths that begin at each of the root nodes, in turn. This is done as follows.

Each root node is the starting point of a path that terminates when individuals on that path exit the network by reaching a radial distance greater than LASMOV. The calculations for a particular root node begin with a calculation of the dose received by residents of the starting point of the path, the root node itself. These individuals will traverse all of the nodes in that path before exiting the network. Their doses are calculated by modeling their transport from element to element. If the evacuation speed changes during traversal of a node, the change in speed is fully accounted for.

When the residents of the root node (the first node on the path) exit the network, their dose calculations are complete and they receive no further exposures in EARLY. The next step in the calculations is to repeat the calculations for the residents of the second node (spatial element) on the path (the destination node of the root node), accumulating their doses until they leave the network, and so on, until the dose calculations are completed for all of the nodes on the path associated with this root node.

Upon the completion of dose calculations for all of the nodes on the first linear path, the paths for the remaining root nodes are traversed in turn. When the paths for all residents of all of the paths have been traversed, and all of the associated doses calculated, the dose calculations are complete.

\subsubsection{Evacuation Data Block Input Parameters}

Variable Name:

EANAM2

Variable Type:

Character, Scalar

Allowed Range:

$1 \leq$ length $\leq 80$ 
Purpose: $\quad$ Identifies the name of the emergency response scenario being studied. This name will be printed on all pages of the output listing. A unique name must be specified for each emergency response.

Example Usage:

EZEANAM2001 'EVACUATION WITHIN 10 MILES, RELOCATION ELSEWHERE'

Variable Name: WTNAME

Variable Type:

Character, Scalar

Allowed Value:

'PEOPLE' or 'TIME' or 'SUMPOP'

Purpose:

Defines the type of weighting to be used in generating the overall weighted sum of results. If PEOPLE or TIME are selected, the code's behavior is unchanged from the NRC version of MACCS. If the SUMPOP option is selected, the value of WTFRAC is not processed and the overall weighted sum is generated by simple summation. Also, the selection of SUMPOP indicates that the Site Data file will include a separate population distribution for each emergency response cohort.

Selection of SUMPOP requires that the population be defined via a Site Data file. An input error will thus result if the user specifies both WTNAME='SUMPOP' and POPFLG='UNIFORM' in a single run of the code.

Example Usage:

* DEFINE THE TYPE OF WEIGHTING TO BE USED

EZWTNAME001 'PEOPLE'

Variable Name: WTFRAC

Variable Type: $\quad$ Real, Scalar

Allowed Range: $\quad 0.0 \leq$ value $\leq 1.0$

Purpose:

Weighting fraction to be applied to the results from this emergency response scenario. This value is used by the OUTPUT module in combining results for the overall weighted sum. The weighting can be done for either fractionof-the-people or fraction-of-the-time as determined by the input variable WTNAME.

Example Usage:

EZWTFRAC001 0.9

(95\% OF THE PEOPLE WITHIN 10 MILES EVACUATE)
Variable Name:
EVATYP
Variable Type:
Character, Scalar
Allowed Value:
'RADIAL' or 'NETWORK' 
Purpose: $\quad$ Defines whether a radial or network evacuation is to be modeled. If the radial option is chosen, the code automatically generates a network path where all individuals travel radially outward.

If the network option is chosen, the user is required to supply data for variable IDIREC.

Furthermore, the selection of network evacuation is inconsistent with the straight-line plume (i.e., IPLUME=1) dispersion option. When a network evacuation is specified, the user must specify a value of 2 or 3 for IPLUME. Thus, the centerline dose and risk results (Type 6 and Type 7) are unavailable when the network evacuation option is selected.

Example Usage:

*

EZEVATYPOOI 'NETWORK'

Variable Name: TRAVELPONT

Variable Type: Character, Scalar

Allowed Value: 'BOUNDARY' or 'CENTERPOINT'

Purpose: $\quad$ Defines the option as to whether evacuees are presumed to move from a spatial element when they cross the boundary dividing the two elements ('BOUNDARY') or when they reach the centerpoint of the destination element ('CENTERPOINT').

If the 'BOUNDARY' option is chosen, the user is not allowed to specify a set of evacuation speeds (ESPEED) that vary. It is only with the 'CENTERPOINT' option that the user is allowed to specify values of ESPEED that differ.

Example Usage:

*

TRAVELPOINT 'BOUNDARY'

Variable Name: ESPEED

Variable Type: Real, Array

Allowed Range: $\quad 1.0 \mathrm{E}-6 \leq$ value $\leq 1.0 \mathrm{E}+6$ (meters/second)

Purpose: Defines the travel speed of evacuees during the three phases of the evacuation: initial, middle, and late.

The initial evacuation phase begins when the first individual begins to travel out of the region. The durations of the initial and middle phases of the evacuation are defined by input variables DURBEG and DURMID. As stated above, if TRAVELPOINT='BOUNDARY' all three values of ESPEED must be identical.

The late phase of the evacuation extends as long as necessary for all individuals to complete their travel. The user must supply three values for this parameter. 


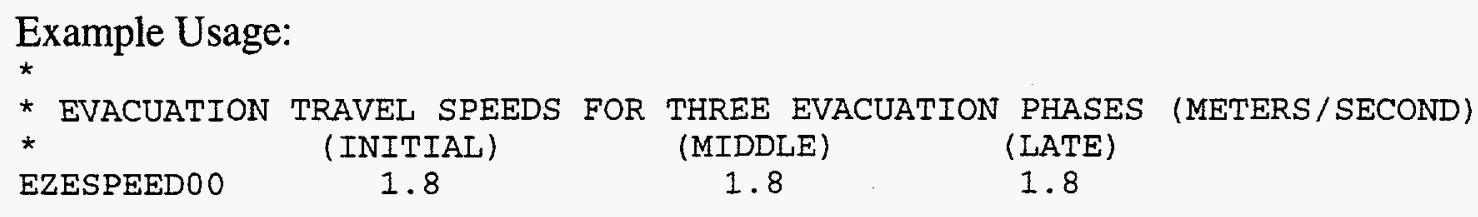

Variable Name: REFPNT

Variable Type: Character, Scalar

Allowed Value: $\quad$ 'ALARM' or 'ARRIVAL'

Purpose: $\quad$ Defines the reference time point for actions in the evacuation and sheltering zone. If ALARM is chosen, the reference time point for these actions is the off-site alarm time (OALARM) as defined in the ATMOS source term definition. If ARRIVAL is chosen, the reference time for evacuation and sheltering actions is the arrival of the first plume at the spatial element.

Example Usage:

* REFERENCE TIME POINT FOR EVACUATION AND SHELTERING

$\star$

EZREFPNT001 'ARRIVAL'

Variable Name: DURBEG

Variable Type: Real, Scalar

Allowed Value: $\quad 0.0 \leq$ value $\leq 86400.0$ (seconds)

Purpose: Defines the duration of the initial phase (beginning) of evacuation. This phase starts when the first individual in the shelter and evacuation region begins travel from the region. The speed used during this phase of the evacuation is the first value of array ESPEED.

Example Usage:

$\star$

* DURATION OF the INITIAI pHASE OF tHE EVACUATION

EZDURBEG001 86400.0

Variable Name: DURMID

Variable Type: Real, Scalar

Allowed Value: $\quad 0.0 \leq$ value $\leq 86400.0$ (seconds)

Purpose: Defines the duration of the middle phase of evacuation. This phase begins at the termination of the initial phase of the evacuation from the region. The evacuation speed used is the second value of ESPEED. After the middle phase has elapsed, evacuees travel at the speed defined by the third value of ESPEED.

Example Usage:

* DURATion OF the middie pHase of the eVAcuation

EZDURMID001 $\quad 86400.0$ 
Variable Name: NUMEVA

Variable Type: Integer, Scalar

Allowed Range: $1 \leq$ value $\leq$ LASMOV

Purpose: Defines the number of radial spatial elements (i.e., contiguous rings) comprising the sheltering and evacuation region. For each ring of the region, values of DLTSHL and DLTEVA must be supplied.

Note: A clarification of the difference between LASMOV and NUMEVA is necessary. LASMOV defines the outer boundary of the region that shelterees and evacuees traverse. In contrast, NUMEVA defines the region where individuals are subject to sheltering and/or evacuation. It is possible to assign the same value to both parameters; however, there is no requirement that this be the case. An example of LASMOV being larger than NUMEVA is the NUREG-1150 evacuation assumption, under which the residents of a 10mile EPZ travel 20 miles.

Example Usage:

*

* NUMBER OF RINGS IN THE SHELTER AND EVACUATION REGION

EZNUMEVA001 12 (REGION EXTENDS TO A 10-MILE RADIUS)

Variable Name: DLTSHL

Variable Type: $\quad$ Real, Array

Allowed Range: $\quad 0.0 \leq$ value $\leq 86400.0$ (seconds)

Purpose: $\quad$ Defines the delay that elapses from the reference time point REFPNT to when individuals take shelter. The user must supply NUMEVA values for

Example Usage: this parameter, one for each ring in the shelter/evacuate region.

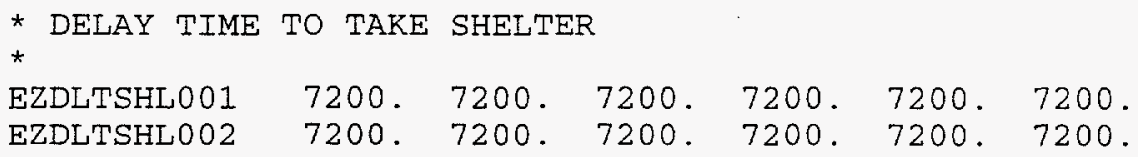

Variable Name: DLTEVA

Variable Type: $\quad$ Real, Array

Allowed Range: $\quad 0.0 \leq$ value $\leq 86400.0$ (seconds)

Purpose:

Defines the delay that elapses from the beginning of the shelter period to when individuals begin their evacuation. The user must supply NUMEVA

Example Usage: values for this parameter, one for each ring in the shelter/evacuate region.

* DELAY TIME TO BEGIN EVACUATION

*

$\begin{array}{lllllll}\text { EZDLTEVA001 } & 0 . & 0 . & 0 . & 0 . & 0 . & 0 . \\ \text { EZDLTEVA002 } & 0 . & 0 . & 0 . & 0 . & 0 . & 0 .\end{array}$


Variable Name: IDIREC

Variable Type: Integer, Array

Allowed Value: $\quad 1 \leq$ value $\leq 4$

Purpose:

Defines the destination direction of every spatial element in the evacuation and sheltering region. The destination direction is defined in terms of a coordinate system in which all individuals are facing away from the centerpoint of the polar grid. A value of 1 indicates forward travel (radially outward); 2 indicates travel to the right (clockwise travel); 3 indicates travel backward toward the centerpoint; and 4 indicates leftward (or counterclockwise) travel.

For every ring in the evacuation and sheltering region (i.e., at intervals extending out to LASMOV), an input data record must be supplied. Each of those LASMOV cards contains 16 values, corresponding to the compass points $\mathrm{N}$ through NNW. The network path defined below is illustrated in Figure 6-2.

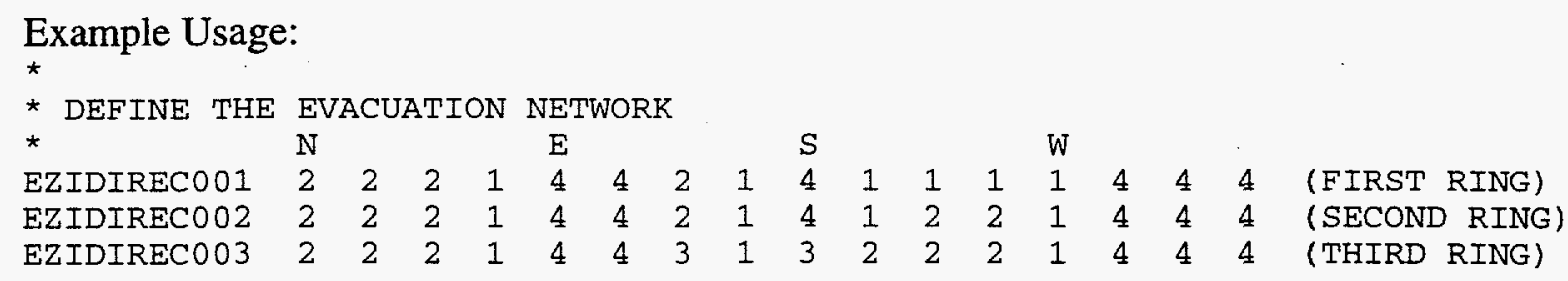

Variable Name: LASMOV

Variable Type: Integer, Scalar

Allowed Range: $\quad 0 \leq$ value $\leq($ NUMRAD - 1$)$

Purpose: The outermost spatial interval of the evacuation movement zone. This is the distance after which evacuees are assumed to disappear from the early health effects model and receive no further dose.

If the user specifies a value of 0 , there will be no evacuation and there is no need to supply data for EVATYP, TRAVELPOINT, ESPEED, REFPNT, DURBEG, DURMID, NUMEVA, DLTSHL, DLTEVA, or IDIREC.

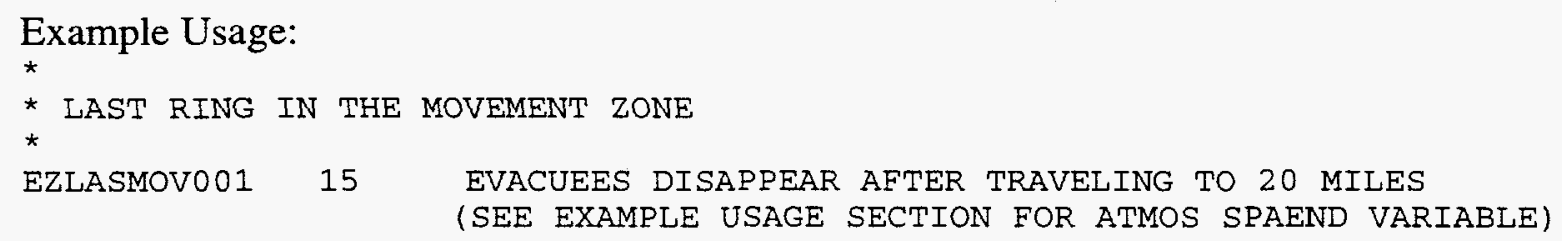


Variable Name:

ENDEMP

Variable Type:

Real, Scalar

Allowed Range:

$86400.0 \leq$ value $\leq 604800.0$ (seconds after plume arrival)

Purpose:

Defines the duration of the emergency-phase period. EARLY only

calculates doses that would be received during the emergency-phase time period. Doses at each spatial interval are cut off at ENDEMP seconds after the arrival of the first plume segment to reach the interval. This cutoff applies to all individuals, no matter where they are located. Any subsequent doses will be calculated by CHRONC.

ENDEMP is also the time evacuees and shelterees are kept away from their homes if there is any contamination in the coarse-grid element in which they reside.

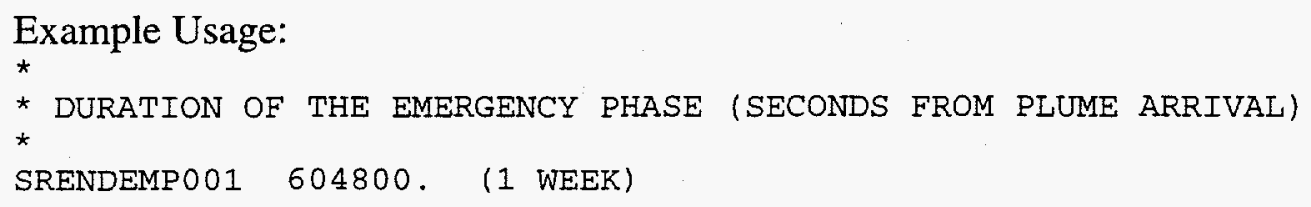

Variable Name: $\quad$ CRIORG

Variable Type: Character, Scalar

Allowed Range: $\quad 2 \leq$ length $\leq 10$

Purpose:

Defines the critical organ for relocation decisions during the emergencyphase period considered by EARLY. In order to determine whether people can remain in the relocation zone, the total committed dose to the critical organ of an individual who remained in place for the entire emergency phase is calculated. The critical organ must be found on the list of organs, ORGNAM, defined in Section 6.4.

Example Usage:

* CRITICAL ORGAN FOR RELOCATION

SRCRIORG001 'L-EFFECTIVE'

Variable Name: TIMHOT

Variable Type: $\quad$ Real, Scalar

Allowed Range: $\quad 0.0 \leq$ value $\leq$ ENDEMP (seconds after plume arrival)

Purpose:

Defines the hot-spot relocation action time. Hot-spot relocation can only occur for individuals residing outside of the emergency-response zone. That is, doses to people awaiting evacuation or protected in shelters will not be affected by the hot-spot relocation model.

If the total lifetime dose commitment for any individual in a coarse-grid element in the relocation region exceeds DOSHOT sieverts to the critical organ, CRIORG, for someone remaining there 
for the entire emergency phase (ENDEMP), people in that element are relocated at TIMHOT seconds after the arrival of the first plume at that distance.

For the purpose of evaluating the need for hot-spot and normal relocation, the total dose commitment is the dose that would be received by an individual who remained in place for the entire emergency-phase period while engaging in normal activity. The pathways used for calculating the total dose commitment are cloudshine, groundshine, direct inhalation, and resuspension inhalation. Any individuals relocated due to hot spots are removed from the problem for the duration of the emergency phase and receive no additional dose during this phase.

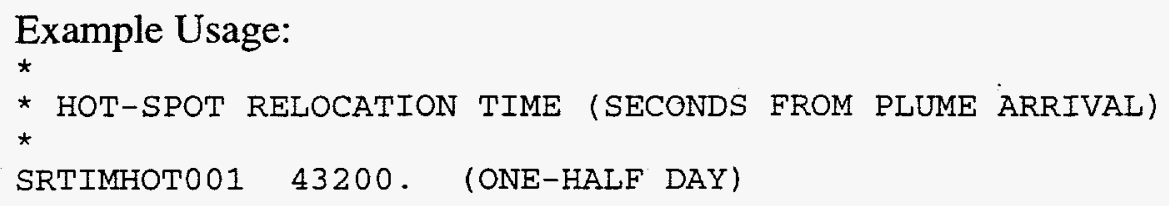

Variable Name: TIMNRM

Variable Type: Real, Scalar

Allowed Range: $\quad$ TIMHOT $\leq$ value $\leq$ ENDEMP (seconds after plume arrival)

Purpose:

Defines the normal relocation action time. Normal relocation can only occur for individuals residing outside of the emergency-response zone. That is, doses to people awaiting evacuation or protected in shelters will not be affected by the normal relocation model.

If the total dose commitment to any individual in a coarse-grid element in the relocation region exceeds DOSNRM sieverts to the critical organ, CRIORG, for someone remaining there for the entire emergency phase (ENDEMP), people in that element are relocated at TIMNRM seconds after the arrival of the first plume at that distance. Any individuals subject to normal relocation are removed from contaminated areas for the duration of the emergency phase and receive no additional dose during this phase.

For the purpose of evaluating the need for hot-spot and normal relocation, the total dose commitment is the dose that would be received by an individual who remained in place for the entire emergency phase period while engaging in normal activity. The pathways used for calculating the total dose commitment are cloudshine, groundshine, direct inhalation, and resuspension inhalation.

Example Usage:

* NORMAL RELOCATION TIME (SECONDS FROM PLUME ARRIVAL)

*

SRTIMNRMO01 86400. (1 DAY)

Variable Name: DOSHOT

Variable Type: Real, Scalar

Allowed Range: $\quad 0.0 \leq$ value $\leq 1 . \mathrm{E} 10$ ( sieverts) 
Purpose: Defines the hot-spot relocation dose threshold. If the total dose commitment to individuals outside of the evacuation and sheltering zones who remained stationary for the entire emergency phase period would exceed DOSHOT, those people are relocated (removed) at the hot-spot relocation time (TIMHOT).

Example Usage:

* HOT-SPOT RELOCATION DOSE THRESHOLD (SIEVERTS)

SRDOSHOTO01 $0.5 \quad$ (50 REM)

Variable Name: DOSNRM

Variable Type: Real, Scalar

Allowed Range: $\quad 0.0 \leq$ value $\leq$ DOSHOT (sieverts)

Purpose:

Defines the normal relocation dose threshold. If the total dose commitment to individuals outside of the evacuation and sheltering zones who remained stationary for the entire emergency phase period exceeds DOSNRM, those people are relocated (removed) at the normal relocation time TIMNRM.

Example Usage:

* NORMAL RELOCATION DOSE THRESHOLD (SIEVERTS)

SRDOSNRMO01 $0.25 \quad$ (25 REM)

\subsection{Early Fatality (EF) Data}

The individual risk of prompt fatality is modeled in MACCS2 using a two-parameter Weibull function here called a hazard function (Evans, Moeller, and Cooper 1985). The hazard function is used to sum the cumulative risk from a number of potential types of damage as follows:

$$
\text { RISK }=1-\exp \left[-\sum_{i} H_{i}\right]
$$

where $H=0.693\left(\frac{\text { DOSE }}{\text { EFFACA }}\right)^{\mathrm{EFACB}}$

DOSE = effective acute dose to the target organ (described later),

EFFACA $=$ the alpha $\left(\mathrm{LD}_{50}\right)$ parameter in the hazard function (Evans, Moeller, and Cooper, 1985, p. II-8), and

EFFACB = the beta or exponential parameter in the hazard function that defines the steepness of the dose-response function.

A dose threshold is incorporated into the early fatality model in the following manner. If the dose to any of the target organs is below the user-specified threshold (see EFFTHR below), then the hazard $(H)$ from that organ is set to 0 . 
When radioactive material is inhaled and retained in the respiratory system, an individual may continue to receive a radiation dose for long periods of time after the material was inhaled. Depending on particle size and chemical form, clearance mechanisms may remove the material from the body or transport it from the respiratory system to other organs of the body. For this reason, it does not make sense to refer to radiation dose without specifying the time period of interest. The concept of lifetime dose commitment is widely used in radiation protection (Eckerman, Wolbarst, and Richardson 1989).

As applied in MACCS, lifetime dose refers to the dose received over the 50-year period following inhalation by a standard reference man who is 30 years old. This lifetime dose is used to determine the need for mitigative actions and to calculate cancers and population dose.

Implementation of the Evans, Moeller, and Cooper (1985) early health effects models requires a calculation method that takes account of dose protraction for radioactive material inhaled and retained in the respiratory system. MACCS2 applies dose reduction factors to protracted doses that contribute to early health effects. Dose reduction factors are derived from $\mathrm{LD}_{50}$ or $\mathrm{D}_{50}$ values that apply to a sequential set of time periods of fixed length. In addition, for the calculation of early fatalities and injuries in MACCS2, a new measure of dose was defined in order to reduce the computational demands of the calculations. Throughout this document it will be referred to as effective acute dose.

The effective acute dose, $D_{e}$, is that dose which if delivered entirely in 1 day, would induce the same health effects as an actual dose delivered over many days. Thus,

$$
H=\ln 2\left(\frac{D_{e}}{\alpha_{1}}\right)^{\beta}=\ln 2\left(\sum_{t} \frac{D_{t}}{\alpha_{t}}\right)^{\beta},
$$

where

$D_{e}=C \cdot F_{e}$

$D_{t}=C \bullet F_{t}$

$C=$ the amount of material inhaled from the plume,

$F_{e}=$ the effective acute dose conversion factor,

$F_{t}=$ the dose conversion factor for the actual dose, $D_{t}$, delivered in time period $t$.

$\alpha_{t}=$ the $\mathrm{LD}_{50}$ or $\mathrm{D}_{50}$ for time period $t$.

$\alpha_{1}=$ the $\mathrm{LD}_{50}$ or $\mathrm{D}_{50}$ for time period of 1 day.

Substitution now yields

$$
F_{e}=\left(\sum_{t} \frac{\alpha_{1}}{\alpha_{t}}\right) F_{t}
$$

Effective acute dose conversion factors are supplied only for the organs used for calculating the early health effects. The acute dose commitment period from inhaled and internally deposited radionuclides is 1 year. The other organs on the dose conversion file have been given effective acute dose conversion factors of -1.0 , which prevents their inadvertent use since any resulting 
doses would be negative. The default dose reduction factors (i.e., $\left.\alpha_{1} / \alpha_{t}\right)$ applied in the effective acute dose calculations are shown in Table 6-1.

Table 6-1. Effective Acute Dose Reduction Factors (unitless)

\begin{tabular}{||l|c|c|c|c|c|c||}
\hline & \multicolumn{7}{|c||}{ Time Period after Exposure (Days) } \\
\hline & $\mathbf{0 - 1}$ & $\mathbf{1 - 7}$ & $\mathbf{7 - 1 4}$ & $\mathbf{1 4 - 3 0}$ & $\mathbf{3 0 - 2 0 0}$ & $\mathbf{2 0 0 - 3 6 5}$ \\
\hline & \multicolumn{7}{|c|}{ Effective Acute Dose Reduction Factors $\left(\alpha_{\mathbf{1}} / \alpha_{t}\right)$} \\
\hline RED MARR & 1.0 & 0.5 & 0.5 & 0.25 & & \\
\hline LUNGS & 1.0 & 0.0625 & 0.0625 & 0.027 & 0.027 & 0.0109 \\
\hline THYROID & 1.0 & 0.2 & 0.2 & 0.2 & & \\
\hline STOMACH & 1.0 & 0.37 & & & & \\
\hline LOWER LI & 1.0 & 0.43 & & & & \\
\hline SMALL IN & 1.0 & 0.43 & & & & \\
\hline
\end{tabular}

The MACCS2 DCF preprocessor, DOSFAC2, allows the user to modify the acute dose reduction factors. In the DCF preprocessor IDCF2, the acute dose reduction factors are fixed in the code and may not be modified through a user input file.

For bone marrow death (with supportive treatment), Evans, Moellen, and Cooper (1985) show $\mathrm{LD}_{50}$ values of $4.5 \mathrm{~Gy}$ for the 0 - to 1-day time period, $9 \mathrm{~Gy}$ for the 1- to 14-day time period, and $18 \mathrm{~Gy}$ for the 14- to 30-day time period. Instead of calculating three different red marrow doses and applying the three different values of $\mathrm{LD}_{50}$ to calculate risk, we calculate a single red marrow dose using the effective acute dose conversion factor.

So, according to the above table, effective acute red marrow dose is $100 \%$ of the first day's dose, $50 \%$ of the next 13 days' dose, and $25 \%$ of the next 16 days' dose. This acute dose is then used in the risk equation with the $\mathrm{LD}_{50}$ for the 0 - to 1-day time period to obtain the hazard to bone marrow.

The dose protraction effect described here is applied only to the internal exposure resulting from inhalation. Dose from the direct exposure pathways in EARLY (groundshine and cloudshine) are summed with no reduction factor being applied even if the exposure lasted more than 1 day. The current implementation of the early health effects models does not distinguish among cloudshine, groundshine, and the 0- to 1-day inhalation dose commitment. They are considered in this model to be equally effective in causing damage.

The error introduced by attributing all the direct exposure dose to the first day is small and is in the direction of overestimating risk. In most cases, emergency plans should ensure that no individuals are permitted to remain on contaminated ground for much longer than a day if dose levels are high enough to pose a risk of early health effects.

Resuspension inhalation is another area where the importance of the pathway is overestimated somewhat. The inhalation dose from resuspended material is treated identically to the inhalation 
dose from direct inhalation of the plume. That is, it is treated as though all the resuspended material was inhaled at the beginning of the exposure period.

Information in this section is used only to control the calculation of individual risk. Results to be processed by OUTPUT (e.g., total cases of early fatality, average individual risk of early fatality, and centerline risk vs. distance of early fatality) are described in later sections of this volume. In order for the code to produce early fatality results, the early fatality model must be defined in this section.

Variable Name: $\quad$ NUMEFA

Variable Type: Integer, Scalar

Allowed Range: $\quad 0 \leq$ value $\leq 5$

Purpose: $\quad$ The number of early fatality effects to be included in the total risk of early fatality. A value of zero means that the early fatality model will not be used.

Example Usage:

*

* nUmber of early fatality efFects

EFNUMEFAOO1 3

Note: The remaining parameters in this section are required only if NUMEFA is greater than 0.

Variable Name: ORGNAM

Variable Type: $\quad$ Character, Array

Allowed Range: $\quad 2 \leq$ length $\leq 10$

Purpose:

The name of the target organ for each early fatality effect. The target organ must be one of the organs listed in the organ definition data. The user must supply NUMEFA values in column 1 of the data block.

Variable Name:

\section{EFFACA}

Variable Type:

Real, Array

Allowed Range:

$1.0 \leq$ value $\leq 100.0$

Purpose:

The alpha factor $\left(\mathrm{LD}_{50}\right)$ in the hazard function for the target organ. The user must supply NUMEFA values in column 2 of the data block.

Variable Name:

EFFACB

Variable Type:

Real, Array

Allowed Range:

$1.0 \leq$ value $\leq 100.0$

Purpose:

The beta factor (shape parameter) in the hazard function for the target organ. The user must supply NUMEFA values in column 3 of the data block. 
$\begin{array}{ll}\text { Variable Name: } & \text { EFFTHR } \\ \text { Variable Type: } & \text { Real, Array } \\ \text { Allowed Range: } & 0.0 \leq \text { value } \leq 100.0\end{array}$

Purpose:

The threshold dose associated with this target organ. If the acute dose to this organ falls below threshold, it will not contribute to the risk of fatality. The user must supply NUMEFA values in column 4 of the data block.

Example Usage of EFFACA and EFFACB:

$\begin{array}{lcccc}* & & & \\ * & \text { ORGNAM } & \text { EFFACA } & \text { EFFACB } & \text { EFFTHR } \\ * & & & & \\ \text { EFATAGRP001 } & \text { 'A-RED MARR' } & 3.8 & 5.0 & 1.5 \\ \text { EFATAGRP002 } & \text { 'A-LUNGS' } & 10.0 & 7.0 & 5.0 \\ \text { EFATAGRP003 } & \text { 'A-LOWER LI' } & 15.0 & 10.0 & 8.0\end{array}$

\subsection{Early Injury (EI) Data}

The individual risk of each type of early injury is modeled in MACCS2 using an approach analogous to that used for early fatality risk (Evans, Moller and Cooper 1985). The early injury risk model differs from the early fatality model in that instead of summing the damage from more than one organ, only a single organ is used. The early injury risk function is as follows:

$$
\begin{aligned}
& \text { RISK }=1-e^{-H} \text {, } \\
& H=0.693\left(\frac{\text { DOSE }}{\text { EIFACA }}\right)^{\mathrm{EIFACB}} \\
& \text { DOSE = effective acute dose (described in Section 6.7) to the target organ, } \\
& \text { EIFACA }=\text { the alpha }\left(D_{50}\right) \text { parameter in the hazard function (Evans, Moller and } \\
& \text { Cooper 1985, p. II-8), and } \\
& \text { EIFACB }=\text { the beta or exponential parameter in the hazard function that defines the } \\
& \text { steepness of the dose-response function. }
\end{aligned}
$$

A dose threshold is incorporated into the early injury model in the following manner. If the dose to any of the target organs is below the user-specified threshold (see EITHRE in the following discussion), then the risk of this type of early injury is set to 0 .

In addition to the values described above, the user must specify the fraction of the population that is susceptible to the injury, EISUSC. Information in this section is used only to control the calculation of individual risk. Results to be processed by OUTPUT( e.g., total cases of a given injury, average individual risk of a given injury, and centerline risk vs. distance of a given injury) are described in later sections. In order for the code to produce early injury results, the injuries must be defined in this section. 
Variable Name: NUMEIN

Variable Type: Integer, Scalar

Allowed Range: $\quad 0 \leq$ value $\leq 10$

Purpose:

The number of different types of early injuries that will be calculated. A value of 0 means that no early injuries will be calculated.

Example Usage:

*

* NUMBER of EARLY INJURY EFFECTS

$\star$

EINUMEINOO1 7

Note: The remaining parameters in this section are required only if NUMEIN is greater than 0. The six arrays are arranged as rows of data in a single data block.

Variable Name: EINAME

Variable Type: $\quad$ Character, Array

Allowed Range: $1 \leq$ length $\leq 16$

Purpose:

The name of each type of early injury. The user may specify any name. Apostrophes are mandatory if there are any embedded blanks. The user must supply NUMEIN values in column 1 of the data block.

Variable Name:

ORGNAM

Variable Type:

Character, Array

Allowed Range:

$2 \leq$ length $\leq 10$

Purpose:

The name of the target organ for each type of early injury. The target organ must be one of the organs listed in the organ definition data. The user must supply NUMEIN values in column 2 of the data block.

Variable Name: $\quad$ EISUSC

Variable Type: $\quad$ Real, Array

Allowed Range: $\quad 0.0 \leq$ value $\leq 1.0$

Purpose:

The fraction of the population that is susceptible to the early injury. The user must supply NUMEIN values in column 3 of the data block.

Variable Name: $\quad$ EITHRE

Variable Type: $\quad$ Real, array

Allowed Range: $\quad 0.0 \leq$ value $\leq 1000.0$

Purpose:

The threshold dose below which the risk of the injury is 0 . The user must supply NUMEIN values in column 4 of the data block. 
Variable Name: EIFACA

Variable Type: Real, Array

Allowed Range: $\quad 1.0 \leq$ value $\leq 1000.0$

Purpose:

The alpha factor $\left(D_{50}\right)$ in the hazard function for the injury. The user must supply NUMEIN values in column 5 of the data block.

$\begin{array}{ll}\text { Variable Name: } & \text { EIFACB } \\ \text { Variable Type: } & \text { Real, Array } \\ \text { Allowed Range: } & 1.0 \leq \text { length } \leq 100.0 \\ \text { Purpose: } & \text { The beta (shape) factor in the hazard function for the injury. The user must } \\ & \text { supply NUMEIN values in column } 6 \text { of the data block. }\end{array}$

Example Usage of EINAME, EISUSC, EITHRE, EIFACA and EFFACB:

\begin{tabular}{|c|c|c|c|c|c|c|}
\hline * & EINAME & ORGNAM & EISUSC & EITHRE & EIFACA & EIFACB \\
\hline EINJUGRP001 & 'PRODROMAL VOMIT' & 'A-STOMACH' & 1. & .5 & 2. & 3. \\
\hline EINJUGRPO 02 & ' DIARRHEA' & 'A-STOMACH' & 1. & 1. & 3. & 2.5 \\
\hline EINJUGRP003 & ' PNEUMONITIS ' & 'A-IUNGS' & 1. & 5 . & 10. & 7. \\
\hline EINJUGRP004 & ' SKIN ERYTHEMA' & 'A-SKIN' & 1. & 3. & 6 & 5 . \\
\hline EINJUGRPO0 5 & ' TRANSEPIDERMAL' & 'A-SKIN' & 1. & 10 . & 20. & 5. \\
\hline EINUUGRP006 & 'THYROIDITIS' & 'A-THYROIDH' & 1. & 40 . & 240 . & 2 . \\
\hline EINUUGRP007 & 'HYPOTHYROIDISM' & 'A-THYROIDH' & 1. & 2. & 60. & 1.3 \\
\hline
\end{tabular}

\subsection{Latent Cancer (LC) Induction Model}

The cancer risk model included in the first public release version of MACCS was based on a linear-quadratic risk model (see MACCS Model Description). In response to recommendations presented in an NRC-sponsored reassessment of cancer risk models published in 1991 and referred to as LMF-132 (Abrahamson et al. 1991), the cancer risk model was updated in MACCS version 1.5.11.1 (Chanin et al. 1993) to include a piecewise linear risk function. The capability to exercise the linear-quadratic risk model was retained in MACCS 1.5.11.1 and MACCS2 although it is no longer recommended for the calculation of cancer induction risk.

\subsubsection{MACCS Linear-Quadratic Dose-Response Model}

The linear-quadratic risk model assumes that risk, $R$, increases linearly with increases in dose, $D$, at low doses and that risk increases quadratically with respect to dose at higher dose levels. The linear-quadratic dose-response function is of the form (International Commission on Radiological Protection 1991):

$$
R(D)=D \bullet(a+\beta D) \text {. }
$$

The implementation of the linear-quadratic dose-response model in MACCS 1.5 was based on the recommendations of NRC-sponsored work (Evans 1990) and is described as follows. In the period modeled by EARLY, a linear-quadratic dose-response relationship is used if the dose to the target organ is less than a user-specified limit (MACCS ACTHRE input parameter). 
The dose-response function used in this case was of the form:

$$
R(D)=D O S E \bullet C F R I S K \bullet(D O S E F A+D O S E \bullet D O S E F B) \bullet A C S U S C,
$$

where

DOSE $=50$-year lifetime dose commitment to the target organ,

CFRISK = lifetime risk factor for cancer injury,

DOSEFA $=$ the linear factor, $\alpha$, of the dose dependence,

DOSEFB $=$ the quadratic factor, $\beta$, of the dose dependence, and

ACSUSC $=$ fraction of the population susceptible to the latent cancer.

If dose to the target organ is greater than the dose limit ACTHRE, the upper-bound linear doseresponse relationship is used:

$$
R(D)=\mathrm{DOSE} \bullet \mathrm{CFRISK} \bullet(\mathrm{DOSEFA}+\mathrm{ACTHRE} \cdot \mathrm{DOSEFB}) \cdot \mathrm{ACSUSC}
$$

During the long-term phase modeled by CHRONC, it is assumed that exposure of the population will be limited to low levels by mitigative actions and therefore the quadratic term of the linear-quadratic cancer risk equation is ignored. Cancer risk is calculated using only the linear term, $R(D)=\alpha D$, of the linear-quadratic dose response function. Cancer risk from all pathways (groundshine, resuspension inhalation, and ingestion) is modeled with the linear dose-response function given below:

$$
R(D)=\mathrm{DOSE} \cdot \mathrm{CFRISK} \cdot \mathrm{DOSEFA} \cdot \mathrm{ACSUSC}
$$

where the meaning of DOSE and CFRISK depends on whether individual risk (groundshine and resuspension) or collective risk (ingestion and decontamination worker's doses) is being calculated.

The linear-quadratic model is not implemented in the MACCS2 sample problems. The quadratic form of the dose-response relationship is deactivated by assigning a 0 to the MACCS2 ACTHRE input parameter.

\subsubsection{MACCS Piecewise Linear Dose-Response Function}

In response to recommendations presented in LMF-132, the MACCS cancer risk model was updated in MACCS version 1.5.11.1 to include the capability to model a piecewise linear cancer risk function of the form:

$$
\begin{array}{ll}
R(D)=\alpha \cdot \frac{D}{\text { DDREF }} & D<0.2 \text { Gy or } 0.1 \text { Gy per hour } \\
R(D)=\alpha \cdot D & D>0.2 \text { Gy or } 0.1 \text { Gy per hour }
\end{array}
$$


DDREF is defined as a "dose and dose rate reduction factor." The LMF-132 report states that the DDREF is to be applied "when the total dose is less than 0.2 Gray, and for higher doses when the dose rate is less than 0.1 Gray per hour. " This guidance for the application of the DDREF is identical to the recommendations provided in ICRP 60. The DDREF is given a value of 2 in LMF-132 for central estimates of most cancer types. In contrast, for central estimates of breast and thyroid cancers the DDREF is assigned a value of 1 in LMF-132.

The user defines the lifetime dose commitment, MACCS2 input parameter DDTHRE, below which the DDREF is applied to cancer risk calculations for emergency-phase exposures. MACCS2 applies the DDREF to all of the dose calculations in the CHRONC module because exposures should always be less than 0.1 Gy per hour after the end of the emergency phase.

\subsubsection{Input Data}

In order for the code to produce cancer results, the cancers must be defined in this section. Sample problem A provides an example of the calculation of cancer risk based on doses to individual organs. An example of the implementation of ICRP 60 cancer risk factors based on the effective dose equivalent is provided in the MACCS2 series of sample problems denoted D.

Risk of latent cancer injury is calculated separately for each cancer syndrome. The risk factors for cancer injury are calculated using the same equations that are used to calculate cancer fatalities except that CIRISK is used instead of CFRISK.

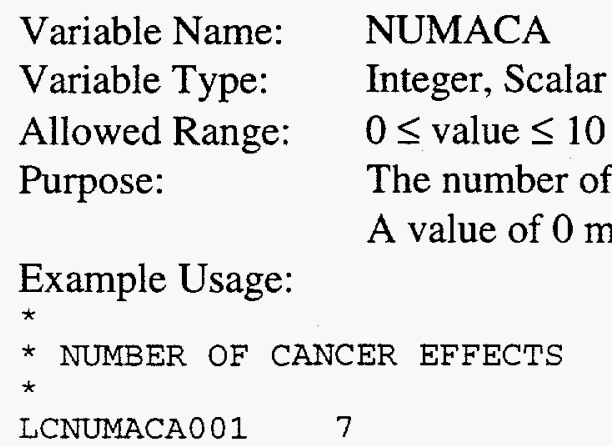

Note: The remaining parameters in this section are required only if NUMACA is greater than 0 .
Variable Name:
ACTHRE
Variable Type:
Real, Scalar
Allowed Range:
$0.0 \leq$ value $\leq 10.0$ ( sieverts) 
Purpose: $\quad$ The lower dose limit for the linear dose-response relationship. For doses less than ACTHRE, the linear-quadratic relationship is used. This parameter should be assigned a value of 0 to deactivate the linear-quadratic risk model.

Note: The parameter ACTHRE is used only in the EARLY module. It is not used in the CHRONC module.

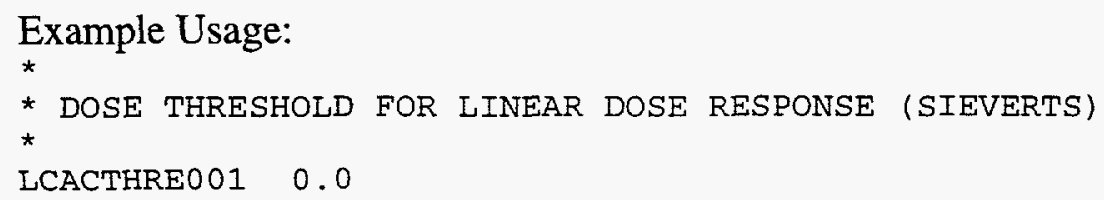

Variable Name: DDTHRE

Variable Type: Real, Scalar

Allowed Range: $\quad 0.0 \leq$ value $\leq 1.0$ (sieverts)

Purpose: $\quad$ The threshold dose for applying the dose-dependent reduction factor

DDREFA. If the lifetime dose commitment incurred during the EARLY

exposure period is less than DDTHRE, the risk of cancer from irradiation of that organ is reduced by a factor of DDREFA.

Example Usage:

* DOSE THRESHOLD FOR APPLYING THE DOSE-DEPENDENT REDUCTION FACTOR

$\star$

LCDDTHREO01 0.2

Note: The next eight variables are supplied in a block of data as columns. An example of their use follows.

Variable Name: ACNAME

Variable Type: $\quad$ Character, Array

Allowed Range: $\quad 1 \leq$ length $\leq 10$

Purpose: $\quad$ The name of each type of latent cancer effect. The user may specify any name. Apostrophes are mandatory if there are any embedded blanks. The user must supply NUMACA values in column 1 of the data block.

Variable Name: ORGNAM

Variable Type: Character, Array

Allowed Range: $\quad 2 \leq$ length $\leq 10$

Purpose:

The name of the target organ for each type of latent cancer effect. The target organ must be one of the organs listed in the organ definition data. The user must supply NUMACA values in column 2 of the data block. 
Variable Name:

ACSUSC

Variable Type:

Real, Array

Allowed Range:

$0.0 \leq$ value $\leq 1.0$

Purpose:

The fraction of the population that is susceptible to the latent cancer. The user must supply NUMACA values in column 3 of the data block.

Variable Name:

DOSEFA

Variable Type:

Real, Array

Allowed Range:

$0.0 \leq$ value $\leq 2.0$

Purpose:

Defines the linear factor, $\alpha$, of the dose dependence in the cancer risk model.

The user must supply NUMACA values in column 4 of the data block.

Variable Name:

DOSEFB

Variable Type:

Real, array

Allowed Range:

$0.0 \leq$ value $\leq 2.0$

Purpose:

Defines the quadratic factor, $\beta$, of the dose dependence in the cancer risk model which is used only by the EARLY module and not by the CHRONC module. Within EARLY, if the dose to the target organ is below ACTHRE, this parameter defines the quadratic term of the cancer dose-response function. The user must supply NUMACA values in column 5 of the data block. This parameter should be assigned a value of 0 to deactivate the linear-quadratic risk model.

Variable Name:

CFRISK

Variable Type:

Real, array

Allowed Range:

$0.0 \leq$ value $\leq 1.0$

Purpose:

Lifetime risk factor for cancer death. For calculating risk to individuals, this parameter has the units risk/sievert, while for calculating collective risk from ingestion and decontamination exposures, it has the units

cases/person-sievert. The user must supply NUMACA values in column 6 of the data block.

Variable Name:

CIRISK

Variable Type:

Real, array

Allowed Range:

$0.0 \leq$ value $\leq 1.0$

Purpose:

Lifetime risk factor for cancer injury. For calculating risk to individuals, this parameter has the units risk/sievert, while for calculating collective risk from ingestion and decontamination exposures, it has the units cases/person-sievert. The user must supply NUMACA values in column 7 of the data block. 
Variable Name: DDREFA

Variable Type: $\quad$ Real, array

Allowed Range: $\quad 1.0 \leq$ value $\leq 10.0$

Purpose:

Dose-dependent reduction factor. If the lifetime dose commitment incurred during the EARLY exposure period is less than DDTHRE, the risk of cancer from irradiation of that organ is reduced by a factor of DDREFA. The user must supply NUMACA values in column 8 of the data block.

Example use of ACNAME, ACSUSC, DOSEFA, DOSEFB, CFRISK, CIRISK, and DDREFA based on EPA (1994) (Estimating Radiogenic Risks: EPA 402-R-93-076):

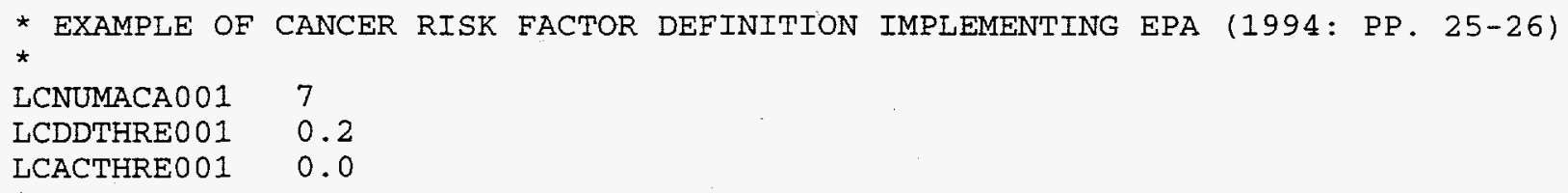

$\begin{array}{llll}\text { 'Bladder } & \text { 'L-BLAD WAL' } & 1.0 & 1.0 \\ \text { 'Bone Sur' } & \text { 'L-BONE SUR' } & 1.0 & 1.0 \\ \text { 'Breast' } & \text { 'L-BREAST' } & 1.0 & 1.0 \\ \text { 'Colon' } & \text { 'L-LOWER LI' } & 1.0 & 1.0 \\ \text { 'Leukemia' } & \text { 'L-RED MARR' } & 1.0 & 1.0 \\ \text { 'Liver' } & \text { 'L-LIVER' } & 1.0 & 1.0 \\ \text { 'Lung' } & \text { 'L-LUNGS' } & 1.0 & 1.0 \\ \text { 'Stom./Eso.' 'L-STOMACH' } & 1.0 & 1.0 \\ \text { 'Thyroid' } & \text { 'L-THYROID' } & 1.0 & 1.0 \\ \text { 'Remainder' } & \text { 'L-EFFECTIVE' } & 1.0 & 1.0\end{array}$

\section{0}

0.0

4. $97 \mathrm{E}-3$

1. $90 \mathrm{E}-4$

$0.04 .62 \mathrm{E}-3$

$0.0 \quad 1.96 \mathrm{E}-2$

$0.09 .91 \mathrm{E}-3$

$0.03 .00 \mathrm{E}-3$

$0.0 \quad 1.43 \mathrm{E}-2$

$0.0 \quad 1.07 E-2$

$0.0 \quad 6.40 \mathrm{E}-4$

$0.02 .92 \mathrm{E}-2$
$9.94 \mathrm{E}-3$

$2.71 \mathrm{E}-4$

9. $24 \mathrm{E}-3$

3. $56 \mathrm{E}-2$

1. $00 \mathrm{E}-2$

3. $16 \mathrm{E}-3$

1. $51 \mathrm{E}-2$

1. $18 \mathrm{E}-2$

6. $40 \mathrm{E}-3$

1. $41 \mathrm{E}-1$
2.0

2.0

1.0

2.0

2.0

2.0

2.0

2.0

2.0

2.0

Note: In regard to the above risk coefficients, the relative biological effectiveness (RBE) for alpha radiation for $R E D M A R R$ dose is defined to be 1; for all other organs, the alpha radiation $R B E$ is defined to be 20 (EPA 1994).

Example use of ACNAME, ACSUSC, DOSEFA, DOSEFB, CFRISK, CIRISK, and DDREFA based on ICRP 60 (International Commission on Radiological Protection 1991):

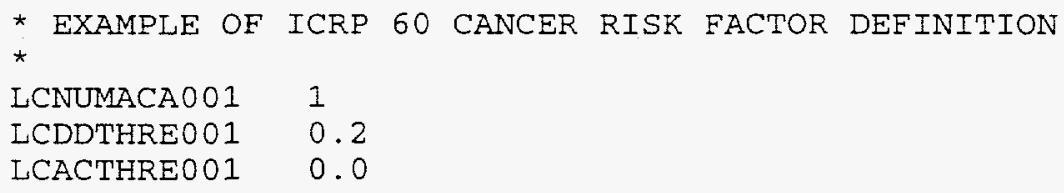

DDREFA

\subsection{Generation of Consequence Distributions}

Under the control of parameters supplied by the user on the EARLY and CHRONC input files, the EARLY and CHRONC modules can calculate a variety of different consequence measures to portray the impact of a facility accident on the surrounding region. The user has total control 
over the results that will be produced. By choosing appropriate values in the user input files, the user can ensure that the code does not perform unnecessary calculations. This affords a great deal of flexibility but it also requires that the user anticipate which results will be of interest. If any are omitted, it is necessary to correct the user input and rerun the program.

In this regard, please remember that a result can only be produced if the model needed for its calculation has been previously defined in the appropriate section. If any results pertaining to health effects are requested, risk factors for that model must have been supplied in the sections entitled Early Fatality (EF), Early Injury (EI), and Latent Cancer (LC).

EARLY can produce ten different types of results. These are described in the next ten sections. Some of these types of results can also be calculated by CHRONC, but some cannot. For instance, both EARLY and CHRONC calculate cancer cases and population dose, but EARLY alone calculates early fatalities, and CHRONC alone calculates economic costs.

If the user requests EARLY to produce a result that can also be produced by CHRONC, the code will ensure that it will be produced by both EARLY and CHRONC. Whenever a result can be produced by both modules, this will be indicated in the following ten sections.

Neither EARLY nor CHRONC generate complementary cumulative distribution functions of the results that they calculate. As EARLY and CHRONC generate the requested consequence measures, those numbers are written to binary files for later processing into CCDFs.

CCDFs are generated by the OUTPUT module of MACCS2. It reads the binary files of consequence measures and automatically combines the results in a predetermined way. The user has no direct control over the OUTPUT module other than through the EARLY and CHRONC data blocks that control the generation of consequence measures.

The CCDF is an estimate of the distribution of consequence magnitudes. The variability of consequence values in MACCS2 CCDFs is due solely to the uncertainty of the weather conditions existing at the time of the accident.

If a consequence measure was calculated by both EARLY and CHRONC, the output module will present those results and their CCDFs separately for EARLY and CHRONC. Also, the output module will sum the results of EARLY and CHRONC and provide the CCDFs of their sums. The contribution of up to three sets of results generated by EARLY can be combined according to the weighting fractions described in Sections 6.1 and 6.6. The weighted sum of all consequence measures, calculated by summation of results from separate runs of EARLY, is presented at the beginning of each section of the listing produced by OUTPUT. Following the overall weighted sum, the results from each of its components are presented.

The following material describes the format of the listing produced by the OUTPUT module. 
At the top of each page is printed the date and time of the run and a page number. The numbering of pages begins with the first page produced by the OUTPUT module. The leftmost 38 columns of the page provide the name of the consequence measure. These names are split into two parts: the general and the specific. For example, we have

\section{"HEALTH EFFECTS CASES"}

as the general name and

$$
\text { "ERL FAT/TOTAL } \quad 0-1609 \mathrm{KM}
$$

as the specific part of the name. The consequence measure being presented on this line is the number of early fatality cases expected to occur in the circular region which begins at the release point and extends outward to a distance of $1609 \mathrm{~km}$.

Going across the page from left to right, there are ten columns of numeric data which provide a summary of the CCDF generated by the OUTPUT module. These are described as follows:

PROB NON-ZERO Conditional probability of having a nonzero consequence estimate, conditional on the occurrence of the release under consideration.

MEAN

The average (expected) consequence over all weather trials. This is calculated by taking the sum of all the products [(consequence value) $\times$ (associated probability of that value)] for each weather trial.

50TH QUANTILE The median of the estimated distribution function.

90TH QUANTILE Based on the estimated distribution function, there is a $10 \%$ chance this consequence magnitude will be exceeded.

95TH QUANTILE Based on the estimated distribution function, there is a 5\% chance this consequence magnitude will be exceeded.

99TH QUANTILE Based on the estimated distribution function, there is a $1 \%$ chance this consequence magnitude will be exceeded.

99.5TH QUANTILE Based on the estimated distribution function, there is a $0.5 \%$ chance this consequence magnitude will be exceeded.

PEAK CONS

The largest consequence magnitude obtained from the full set of weather trials which were examined.

PEAK PROB

The probability associated with the largest consequence magnitude.

PEAK TRIAL

In the series of weather samples, the sequence number of the weather trial that gave rise to the largest consequence magnitude. By going back to the ATMOS output listing, the user can determine the start time (day and hour) of this weather sequence. 


\subsection{Cases of a Given Health Effect}

EARLY can calculate the number of health effect cases that will occur within a range of distances. The risk models for these health effects must have been previously defined in the proper section.

Note: Any cancer results requested here are automatically produced by CHRONC so that the results can be combined by OUTPUT. In the results calculated by CHRONC, the cancer values include direct exposure cancers in the population residing in the region as well as the indirect exposure cancers resulting from both (1) consumption of food and water produced in the region and (2) exposure of decontamination workers working in the region.

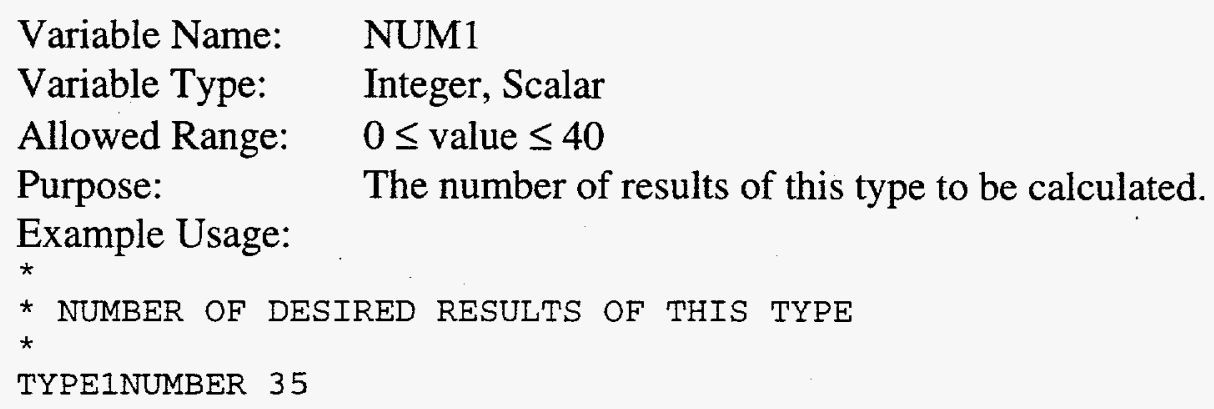

Note: The remaining parameters in this section are required only if NUM1 is greater than 0.

Variable Name: NAME

Variable Type: Character, Array

Allowed Range: $\quad 10 \leq$ length $\leq 24$

Purpose:

Defines the name of each Type 1 result for which the number of cases will be calculated. Depending on the value of NAME, six different types of results may be produced. They are listed below along with examples of their specification for input.

cases of early fatality: cases of a given early injury: cases of a given cancer death: total cases of cancer death: cases of a given cancer injury: total cases of cancer injury:
'ERL FAT/TOTAL'

'ERL INJ/name of an injury'

'CAN FAT/name of a cancer'

'CAN FAT/TOTAL'

'CAN INJ/name of a cancer'

'CAN INJ/TOTAL'

Variable Name: IIDIS1

Variable Type: Integer, Array 
Allowed Range: $\quad 1 \leq$ value $\leq$ NUMRAD

Purpose:

Defines the inner spatial interval of the region of interest for this result. The user must supply NUM1 values in column 2 of the data block.

Variable Name: I2DIS1

Variable Type: $\quad$ Integer, Array

Allowed Range: $\quad$ I1DIS1 $\leq$ value $\leq$ NUMRAD

Purpose:

Defines the outer spatial interval of the region of interest for this result. The user must supply NUM1 values in column 3 of the data block.

Note: In order to obtain the CCDF tables of a consequence measure requested in this section, append the character string ' $C C D F^{\prime}$ to the line requesting that result as the fourth item on the data record. The CCDF tables will be printed on the list output file.

Example Usage:

*

* NUMber of DEsiRED REsULts of this tyPE

*

TYPE1NUMBER 35

$\star$

TYPE1OUT001

TYPE1OUT003 'ERI INJ/DIARRHEA'

TYPEIOUTOO4 'ERL INJ/PNEUMONITIS'

TYPE1OUT005 'ERL INJ/THYROIDITIS'

TYPE1OUT006 'ERL INJ/HYPOTHYROIDISM'

TYPE1OUT007 'ERL INJ/SKIN ERYTHEMA'

TYPE1OUT008 'ERL INJ/TRANSEPIDERMAL'

TYPE1OUTO09 'CAN FAT/TOTAL'

TYPE1OUT010 'CAN FAT/LUNG'

TYPEIOUT011 'CAN FAT/THYROID'

TYPE1OUT012 'CAN FAT/BREAST'

TYPE1OUT013 'CAN FAT/GI'

TYPEIOUT014 'CAN FAT/LEUKEMIA'

TYPE1OUT015 'CAN FAT/BONE'

TYPE1OUT016 'CAN FAT/OTHER'

TYPE1OUT017 'CAN INJ/TOTAL'

TYPE1OUT018 'ERL FAT/TOTAL'

TYPE1OUT019 'ERL INJ/PRODROMAL VOMIT'

TYPE1OUT020 'ERL INJ/DIARRHEA'

TYPE1OUT021 'ERL INJ/PNEUMONITIS'

TYPE1OUT022 'ERL INJ/THYROIDITIS'

TYPE1OUT023 'ERL INJ/HYPOTHYROIDISM'

TYPE1OUT024 'ERL INJ/SKIN ERYTHEMA'

TYPE1OUTO25 'ERL INJ/TRANSEPIDERMAL'

TYPE1OUTO26 'CAN FAT/TOTAL'

TYPE1OUT027 'ERL FAT/TOTAL'

TYPE1OUTO28 'ERL INJ/PRODROMAL VOMIT'

TYPE1OUTO29 'ERL INJ/DIARRHEA'

TYPE1OUT030 'ERL INJ/PNEUMONITIS'

TYPE1OUT031 'ERL INJ/THYROIDITIS'

TYPE1OUTO32 'ERL INJ/HYPOTHYROIDISM'

TYPE1OUT033 'ERL INJ/SKIN ERYTHEMA'

TYPE1OUTO 34 'ERI INJ/TRANSEPIDERMAL '

TYPEIOUT035 'CAN FAT/TOTAL'

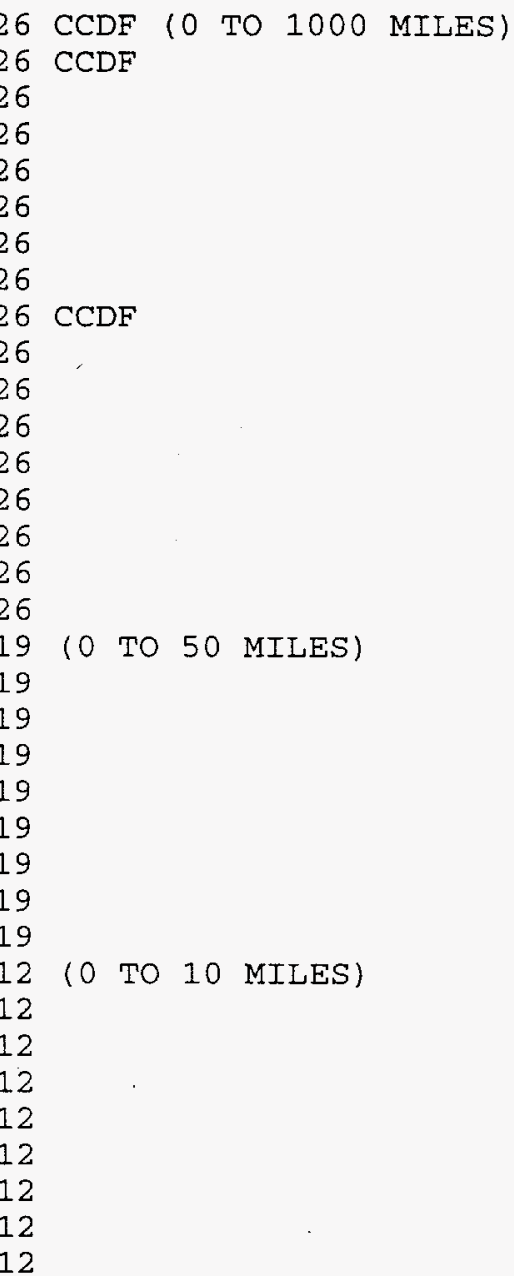




\subsection{Early Fatality Radius}

It may be of interest to know the greatest distance at which a specified level of early fatality risk is exceeded. The user can obtain information about the size of the region in which early fatalities are predicted to occur by setting the variable RISTNR (defined below) to 0 .

Variable Name: NUM2

Variable Type: $\quad$ Integer, Scalar

Allowed Range: $\quad 0 \leq$ value $\leq 10$

Purpose: $\quad$ The number of results of this type to be calculated.

Example Usage:

* NUMber of desiRed RESULts of this tyPE

*

TYPE2NUMBER 3

Variable Name: RISTHR

Variable Type: $\quad$ Real, Scalar

Allowed Range: $\quad 0.0 \leq$ value $\leq 1.0$

Purpose: $\quad$ Defines the risk threshold used for calculating the fatality radius (reported in kilometers). The user must supply NUM2 values in column 1 of the data block. This is the only array in the data block.

Note: In order to obtain the CCDF tables of a consequence measure requested in this section, append the character string ' $C C D F^{\prime}$ to the line requesting that result as the second item on the data record. The CCDF tables will be printed on the list output file.

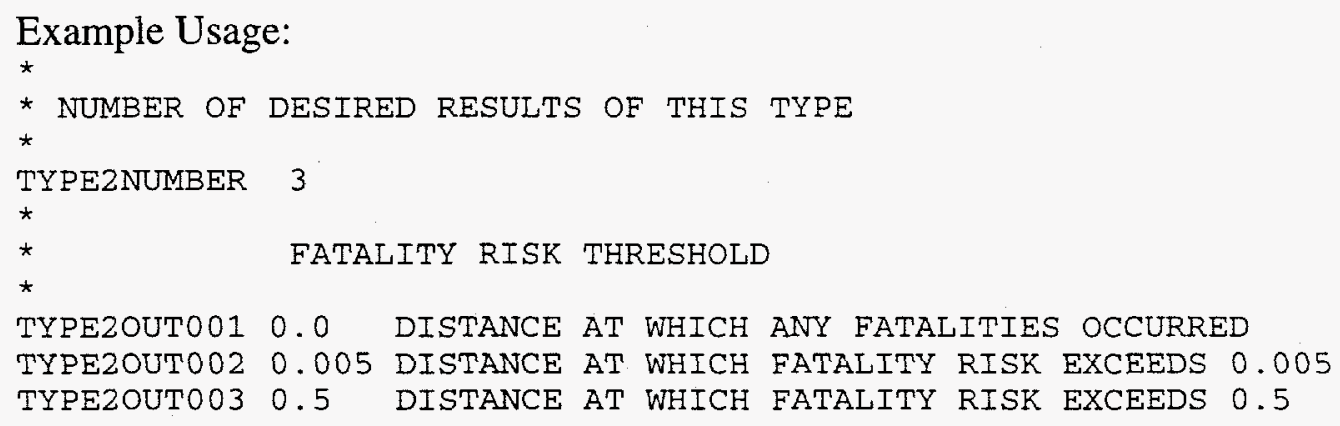

\subsection{Population Exceeding a Dose Threshold}

Within the EARLY module alone, it may be of interest to know how many people received doses exceeding certain levels. This information can be obtained by requesting the production of the result described below. It is important to remember that this consequence measure is calculated 
solely on the basis of the dose calculations performed in the EARLY module. There is no provision for examining an analogous result for the CHRONC module, or for determining the number of people whose total dose from both EARLY and CHRONC exceeded a certain level.

The user can specify two type of doses to be used for the comparison: acute and lifetime. The acute dose is used by the code to calculate early fatality and early injury health effects (see Section 6.7). The lifetime dose is used to calculate cancer fatality and cancer injury health effects (see Section 6.9). The lifetime dose represents the 50-year dose commitment to a reference adult that results from exposure during the emergency phase (EARLY) time period.

Variable Name: NUM3

Variable Type: Integer, Scalar

Allowed Range: $\quad 0 \leq$ value $\leq 10$

Purpose:

The number of results of this type to be calculated.

Variable Name: NAME

Variable Type: Character, Scalar

Allowed Range: $\quad 2 \leq$ length $\leq 10$

Purpose:

Defines the name of the organ to which the dose threshold applies. This organ must be found on the list of organs, ORGNAM. The user must supply NUM3 values in column 1 of the data block.

Variable Name: $\quad$ DOSTH3

Variable Type: Real, Array

Allowed Range: $\quad 0.0 \leq$ value $\leq 1000.0$ (sieverts)

Purpose:

Defines the dose threshold that will be used for counting the population.

The user must supply NUM3 values in column 2 of the data block.

Note: In order to obtain the CCDF tables of a consequence measure requested in this section, append the character string ' $C C D F^{\prime}$ to the line requesting that result as the fourth item on the data record. The CCDF tables will be printed on the list output file.

Example Usage:

TYPE3NUMBER 3

$\star$

* organ NAME dose thrEshold (SIEVERTS)

TYPE3OUT001 'A-RED MARR' 1.5

TYPE3OUT002 'A-LUNGS' 5.0

TYPE3OUT003 'L-EDEWBODY' 0.05 


\subsection{Average Individual Risk}

Average individual risk is obtained by taking the sum of the risk values in all sectors at a given distance and dividing it by the number of sectors.

Note: Any cancer results requested here are automatically produced by CHRONC so that the results can be combined by OUTPUT. When this result is produced by CHRONC, it is only a measure of the risk from direct exposure to people at that distance. Direct exposure includes the cloudshine, groundshine, and inhalation dose to the people living around the facility. The risk presented in this result does not include doses from ingestion of food and water by the region's population or doses to decontamination workers working in the region.

$\begin{array}{ll}\text { Variable Name: } & \text { NUM4 } \\ \text { Variable Type: } & \text { Integer, Scalar } \\ \text { Allowed Range: } & 0 \leq \text { value } \leq 20 \\ \text { Purpose: } & \text { The number of results of this type to be calculated. }\end{array}$

Variable Name: I1DIS4

Variable Type: Integer, Array

Allowed Range: $1 \leq$ value $\leq$ NUMRAD

Purpose: $\quad$ Radial spatial interval of the distance of interest. The user must supply NUM4 values in column 1 of the data block.

Variable Name: NAME

Variable Type: Character, Array

Allowed Range: $\quad 10 \leq$ length $\leq 24$

Purpose: $\quad$ Name of the health effect risk. Average individual risk may be calculated for five types of health effects.

risk of early fatality: 'ERL FAT/TOTAL'

risk of a given early injury: 'ERL INJ/name of an injury'

risk of a given cancer death: 'CAN FAT/name of a cancer'

risk of cancer death: 'CAN FAT/TOTAL'

risk of a given cancer injury: 'CAN INJ/name of a cancer'

The user must supply NUM4 values of NAME in column 2 of the data block.

Note: In order to obtain the CCDF tables of a consequence measure requested in this section, append the character string 'CCDF' to the line requesting that result as the third item on the data record. The CCDF tables will be printed on the output file. 


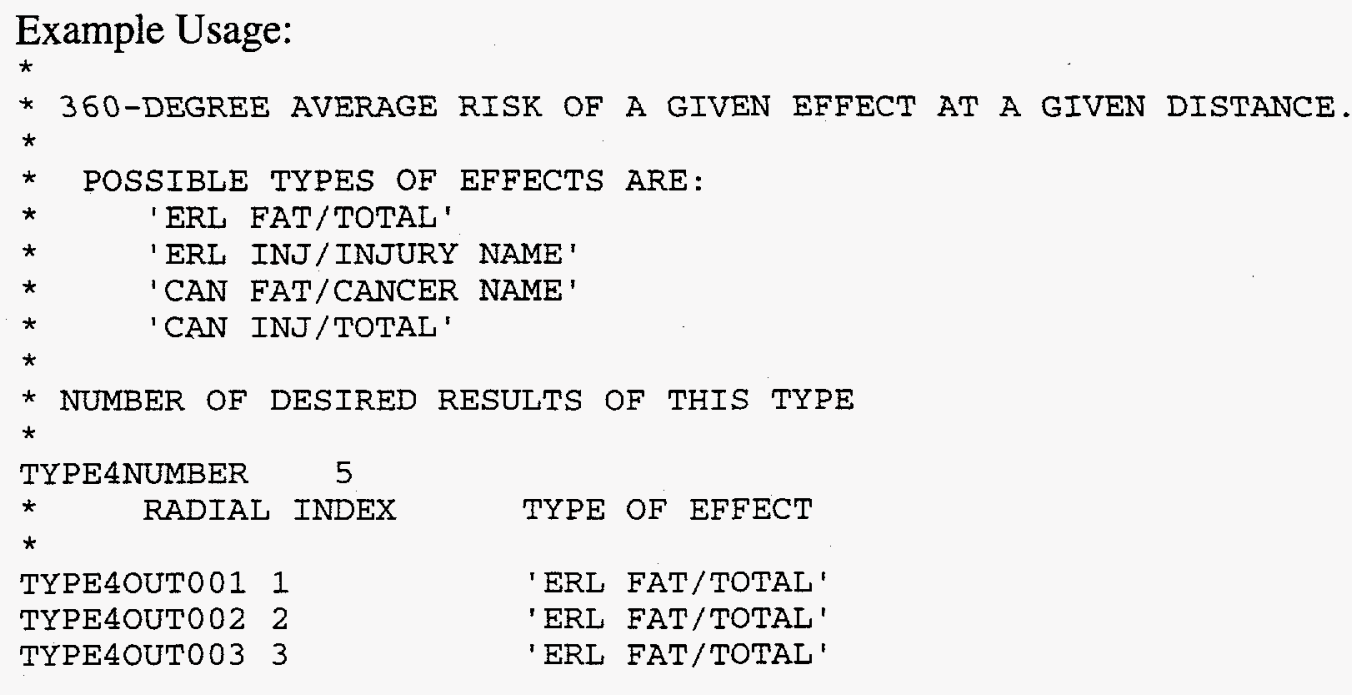

\subsection{Population Dose}

The total long-term population dose to a given organ resulting from the contamination of a specified region can be calculated. The user must supply the name of the target organ as well as the inner and outer spatial intervals of the region of interest. If only the EARLY module is being run, this result reflects only the pathways considered by EARLY. If both EARLY and CHRONC are being run, the population dose from all pathways is included in the calculation. The CHRONC pathways include both (1) food and water ingestion doses resulting from material deposited in the region and (2) doses to decontamination workers working in the region. On the output listing, population dose is presented in units of person-sieverts.

Variable Name: NUM5

Variable Type: $\quad$ Integer, Scalar

Allowed Range: $\quad 0 \leq$ value $\leq 10$

Purpose: $\quad$ The number of results of this type to be calculated.

Variable Name: NAME

Variable Type: $\quad$ Character, Scalar

Allowed Range: $\quad 2 \leq$ length $\leq 10$

Purpose:

Defines the name of the organ to which the population dose applies. This organ must be found on the list of organs, ORGNAM. The user must supply NUM5 values in column 1 of the data block.

Variable Name: I1DIS5

Variable Type: Integer, Array

Allowed Range: $1 \leq$ value $\leq$ NUMRAD 
Purpose: $\quad$ Defines the inner spatial interval of the region of interest for this result. The user must supply NUM5 values in column 2 of the data block.

Variable Name: I2DIS5

Variable Type: Integer, Array

Allowed Range: $\quad$ I1DIS5 $\leq$ value $\leq$ NUMRAD

Purpose:

Defines the outer spatial interval of the region of interest for this result. The user must supply NUM5 values in column 3 of the data block.

Note: In order to obtain the CCDF tables of a consequence measure requested in this section, append the character string 'CCDF' to the line requesting that result as the fourth item on the data record. The CCDF tables will be printed on the list output file.

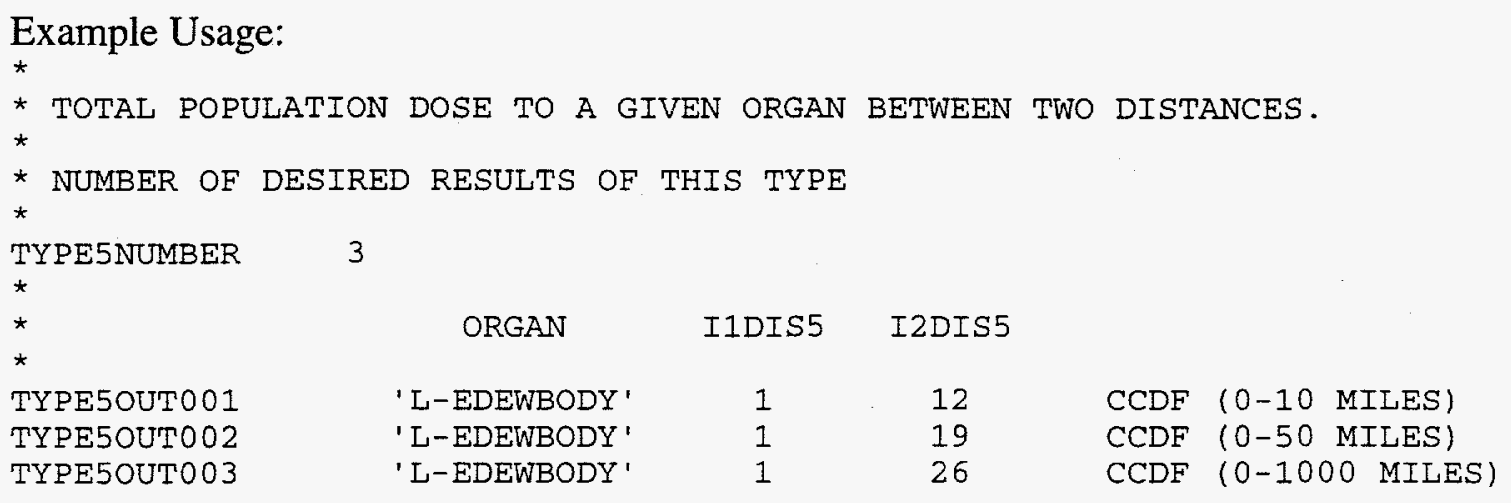

\subsection{Centerline Dose vs. Distance}

If the straight-line plume model was chosen (IPLUME=1), the code can keep track of the centerline dose between a range of distances for the various pathways. The centerline dose at each distance is treated as a separate result and OUTPUT will generate a set of results for each of the radial spatial intervals within the specified range. The user must supply a value for the pathway name in all cases. The centerline dose vs. distance results may be obtained for the following pathways.

\begin{tabular}{|c|c|}
\hline PATHNM & DESCRIPTION \\
\hline CLD & cloudshine dose (EARLY only) \\
\hline GRD & groundshine dose (EARLY and CHRONC) \\
\hline $\mathrm{INH} A C U$ & dose from inhalation of the passing plume (EARLY only) \\
\hline INH LIF & dose from inhalation of the passing plume (EARLY only) \\
\hline TOT ACU & total dose from all direct exposure pathways (EARLY and CHRONC) \\
\hline TOT LIF & total dose from all direct exposure pathways (EARLY and CHRONC) \\
\hline RES LIF & $\begin{array}{l}\text { dose from inhalation of resuspended material after plume passage } \\
\text { (EARLY and CHRONC) }\end{array}$ \\
\hline RES ACU & $\begin{array}{l}\text { dose from inhalation of resuspended material after plume passage } \\
\text { (EARLY and CHRONC) }\end{array}$ \\
\hline
\end{tabular}


Depending on the exposure pathways specified, this result will be calculated by EARLY and/or CHRONC. If both EARLY and CHRONC are being run, then CHRONC will automatically produce all of the results for the pathways it considers. If pathways 'GRD', 'RES LIF', or 'TOT LIF' are chosen, those results are automatically produced by CHRONC so that the results can be combined by OUTPUT. The other pathway values are only produced by EARLY.

The user must specify the organ doses and exposure pathways for which Type 6 results are to be output. In prior versions of MACCS the acute dose and the lifetime dose were specified by requesting RES ACU or RES LIF and INH ACU or INH LIF, for resuspension and direct inhalation, respectively. In MACCS2, the distinction between acute and lifetime doses is made via the organ name prefixes A- or L-. The ACU and LIF references in the pathway name are ignored in MACCS2. The new code does not distinguish between RES ACU and RES LIF; both flags indicate resuspension dose. Likewise, INH ACU and INH LIF both refer to direct inhalation from the cloud.

To illustrate, consider the following fragment from an EARLY input file for prior versions of MACCS which request Type 6 results for (1) the total acute dose to red marrow, (2) the total lifetime effective dose from all pathways, (3) the acute dose from resuspension to red marrow, and (4) the lifetime effective dose from resuspension.

$\begin{array}{lllll}\text { TYPE6OUT001 } & \text { 'RED MARR' } & \text { 'TOT ACU' } & 1 & 19 \\ \text { TYPE6OUT002 } & \text { 'EDEWBODY' } & \text { 'TOT LIF' } & 1 & 26 \\ \text { TYPE6OUT003 } & \text { 'RED MARR' } & \text { 'RES ACU' } & 1 & 19 \\ \text { TYPE6OUT004 } & \text { 'EDEWBODY' } & \text { 'RES LIF' } & 1 & 26\end{array}$

In a MACCS2 input file this fragment could be rewritten with minimal change as

$\begin{array}{lllll}\text { TYPE6OUT001 } & \text { 'A-RED MARR' } & \text { 'TOT ACU' } & 1 & 19 \\ \text { TYPE6OUT002 } & \text { 'L-EDEWBODY' } & \text { 'TOT LIF' } & 1 & 26 \\ \text { TYPE6OUT003 } & \text { 'A-RED MARR' } & \text { 'RES ACU' } & 1 & 19 \\ \text { TYPE6OUT004 } & \text { 'L-EDEWBODY' } & \text { 'RES LIF' } & 1 & 26\end{array}$

The A- and L-prefixes of the organ names control whether an acute or lifetime dose is output for the requested pathway. In MACCS2, unlike MACCS, the pathway name controls only the exposure pathway for which the centerline dose is output.

The specification of a LIF pathway with an A- prefixed organ or an ACU pathway with an Lprefixed organ will not result in a calculational error or an error message and termination of code calculations. Since MACCS2 ignores the ACU and LIF components of the pathway code, equivalent numerical output results would be produced if the above-listed fragment is rewritten as

$\begin{array}{lllll}\text { TYPE6OUT001 } & \text { 'A-RED MARR' } & \text { 'TOT LIF' } & 1 & 19 \\ \text { TYPE6OUT002 } & \text { 'L-EDEWBODY' } & \text { 'TOT ACU' } & 1 & 26 \\ \text { TYPE6OUT003 } & \text { 'A-RED MARR' } & \text { 'RES LIF' } & 1 & 19 \\ \text { TYPE6OUT004 } & \text { 'L-EDEWBODY' } & \text { 'RES ACU' } & 1 & 26\end{array}$


The descriptive titles for these results on the list output file will match the pathway names specified in the EARLY input file. That is, if misleading titles are requested as above, the same misleading titles will be output as follows:

\section{CENTERLINE DOSE AT SOME DISTANCES (SV)}

$\begin{array}{lll}\text { A-RED MARR } & \text { TOT LIF } & 0-0.2 \mathrm{KM} \\ \text { L-EDEWBODY } & \text { TOT LIF } & 0-0.2 \mathrm{KM} \\ \text { A-RED MARR } & \text { RES LIF } & 0-0.2 \mathrm{KM} \\ \text { L-EDEWBODY } & \text { RES ACU } & 0-0.2 \mathrm{KM}\end{array}$

The interpretations of the four centerline dose results above are (1) total dose from all pathways to red marrow using the dose reduction factors for acute health effects, (2) total effective dose from all pathways using a 50-year commitment period for inhalation, (3) resuspension dose to red marrow using the dose reduction factors for acute health effects, and (4) EDE from resuspension using a 50-year commitment period for inhalation. In future versions of MACCS2, the definition of pathway names will be revised.

An alternative method for examining centerline dose from EARLY alone is to set the output control variable, IPRINT, to a value greater than 0 . If this is done, a listing of dose vs. distance for all the organs will be printed on the list output. Since this is written for each weather trial, it is recommended that this be done only for single weather trial runs.

Variable Name: NUM6

Variable Type: $\quad$ Integer, Scalar

Allowed Range: $\quad 0 \leq$ value $\leq 10$

Purpose: $\quad$ The number of results of this type to be calculated. This result is only available when straight-line plume dispersion (IPLUME=1) and radial evacuation (EVATYP='RADIAL') are being utilized. If IPLUME $>1$ or EVATYP='NETWORK', an input error will be diagnosed and execution terminated.

Note: Unless IPLUME=1, you must specify a value of 0.

The direct exposure pathways are groundshine, cloudshine, plume inhalation, and inhalation of resuspended material. The pathway name is ignored when 'A-SKIN' is specified as the target organ because direct dry deposition is the only exposure pathway considered for skin.

Variable Name: ORGNAM

Variable Type: Character, Scalar

Allowed Range: $\quad 2 \leq$ length $\leq 10$ 
Purpose: Defines the names of the organs for which centerline doses are to be reported. These organs must be found on the list of organs, ORGNAM. The user must supply NUM6 values in column 1 of the data block.

Variable Name:

PATHNM

Variable Type:

Character, Scalar

Allowed Range:

$3 \leq$ length $\leq 7$

Purpose:

Defines the names of the pathways for which centerline doses are to be reported. The name of each pathway must be on the list of pathways. The user must supply NUM6 values in column 2 of the data block.

Variable Name: I1DIS6

Variable Type: Integer, Array

Allowed Range: $\quad 1 \leq$ value $\leq$ NUMRAD

Purpose:

Defines the inner spatial interval of the region of interest for this result. The user must supply NUM6 values in column 3 of the data block.

Variable Name: I2DIS6

Variable Type: Integer, Array

Allowed Range: $\quad$ I1DIS6 $\leq$ value $\leq$ NUMRAD

Purpose:

Defines the outer spatial interval of the region of interest for this result. The user must supply NUM6 values in column 4 of the data block.

Note: In order to obtain the CCDF tables of a consequence measure requested in this section, append the character string ' $C C D F^{\prime}$ to the line requesting that result as the fifth item on the data record. The CCDF tables will be printed on the list output file.

Example Usage:

* NUMBer of desired RESUlts of this type

TYPE6NUMBER 3

* ORGNAM PATHNM IIDIS6 I2DIS6

*

TYPE60UT001

TYPE6OUT002

TYPE6OUT003

ORGNAM

' A-RED MARR'

'A-LUNGS'

' L-EDEWBODY '
' TOT ACU'

'TOT ACU

'TOT LIF'

$\begin{array}{ll}1 & 19 \\ 1 & 19 \\ 1 & 26\end{array}$

(0-50 MILES)

(0-50 MILES)

(0-1000 MILES)

\subsection{Centerline Risk vs. Distance}

If the straight-line plume model was chosen (IPLUME=1), the code can keep track of centerline risk between two specified spatial intervals for the various types of health effects. Centerline risk 
is calculated for hypothetical individuals located directly under the path of the plume who are exposed to the Gaussian peak of the air and ground concentrations. The centerline risk at each distance in the region is treated as a separate result and OUTPUT will generate a distribution of the consequence measure for each of the spatial intervals within the specified range.

Note: Any cancer results requested here are automatically produced by CHRONC so that the results can be combined by OUTPUT. When this result is produced by CHRONC, it is a measure only of the risk from direct exposure to people at those distances. Direct exposure includes the cloudshine, groundshine, and inhalation dose to the resident population in the specified region. The risk presented in this result does not include societal doses from ingestion of any food and water contaminated as a result of the accident or doses to decontamination workers working in the contaminated area.

Variable Name: NUM7

Variable Type: Integer, Scalar

Allowed Range: $\quad 0 \leq$ value $\leq 10$

Purpose:

The number of results of this type to be calculated. This result is available only when straight-line plume dispersion (IPLUME=1) and radial evacuation (EVATYP='RADIAL') are being utilized. If IPLUME $>1$ or EVATYP='NETWORK', an input error will be diagnosed and execution terminated.

Note: Unless IPLUME=1, you must specify a value of 0 .

Variable Name: NAME

Variable Type: Character, Array

Allowed Range: $\quad 10 \leq$ length $\leq 24$

Purpose: $\quad$ Defines the option for results of Type 7 , centerline risk of a given type of health effect. Depending on the value of NAME, six different types of results may be produced. They are listed below along with examples of their use. The user must supply NUM7 values in column 1 of the data block.

risk of early fatality: 'ERL FAT/TOTAL'

risk of a given early injury: 'ERL INJ/name of an injury'

risk of a given cancer death: 'CAN FAT/name of a cancer'

risk of cancer death: 'CAN FAT/TOTAL'

risk of a given cancer injury: 'CAN INJ/name of a cancer'

risk of cancer injury: 'CAN INJ/TOTAL'

Variable Name: I1DIS7

Variable Type: Integer, Array

Allowed Range: $1 \leq$ value $\leq$ NUMRAD 
Purpose:

Defines the inner spatial interval of the region of interest for this result. The user must supply NUM7 values in column 2 of the data block.

Variable Name: I2DIS7

Variable Type: Integer, Array

Allowed Range: $\quad$ I1DIS7 $\leq$ value $\leq$ NUMRAD

Purpose:

Defines the outer spatial interval of the region of interest for this result. The user must supply NUM7 values in column 3 of the data block.

Note: In order to obtain the CCDF tables of a consequence measure requested in this section, append the character string ' $C C D F^{\prime}$ to the line requesting that result as the fourth item on the data record. The CCDF tables will be printed on the list output file.

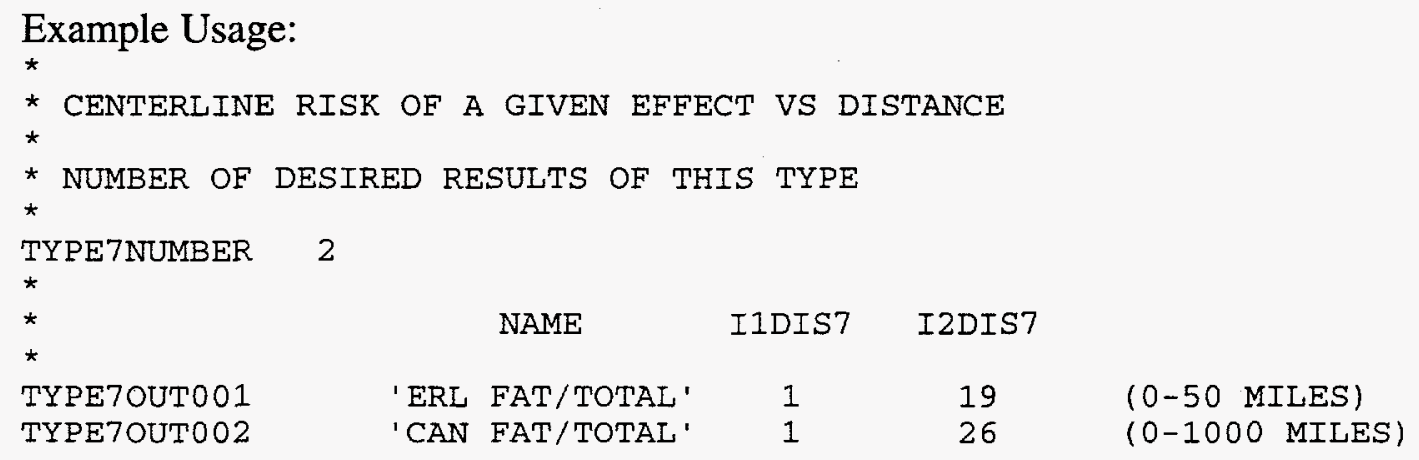

\subsection{Population-Weighted Risk}

The population-weighted health effect risk is obtained by calculating the cases of a health effect in a certain region and then dividing by the total population in the region. It takes account of both the population distribution and the wind rose.

Note: Any cancer results requested here are automatically produced by CHRONC so that the results can be combined by OUTPUT. When this result is produced by CHRONC, it is a measure only of the risk from direct exposure to the resident population in the specified region. The direct exposure pathways are cloudshine, groundshine, direct inhalation, and resuspension inhalation.

The population-weighted risk in CHRONC is calculated by estimating the expected cancer cases and dividing that value by the current population in the region. The risk presented in this result does not include the societal pathways of (1) ingestion of contaminated food and water or (2) doses to decontamination workers working in the area.

Variable Name: NUM8

Variable Type: Integer, Scalar 
Allowed Range:

Purpose:

Variable Type:

Allowed Range:
Variable Name:

Purpose:

$0 \leq$ value $\leq 20$

The number of results of this type to be calculated.

NAME

Character, Array

$10 \leq$ length $\leq 24$

Defines the names of the Type 8 results to be calculated: populationweighted risk of a given type of health effect. Depending on the value of NAME, six different types of results may be produced. These six options are listed below along with examples of their use. The user must supply NUM8 values in column one of the data block.

risk of early fatality: 'ERL FAT/TOTAL' risk of a given early injury: 'ERL INJ/name of an injury' risk of a given cancer death: 'CAN FAT/name of a cancer' risk of cancer death: 'CAN FAT/TOTAL' risk of a given cancer injury: 'CAN INJ/name of a cancer' risk of cancer injury: 'CAN INJ/TOTAL'

Variable Name: I1DIS8

Variable Type: Integer, Array

Allowed Range: $1 \leq$ value $\leq$ NUMRAD

Purpose:

Defines the inner spatial interval of the region of interest for this result. The user must supply NUM8 values in column 2 of the data block.

Variable Name: I2DIS8

Variable Type: Integer, Array

Allowed Range: $1 \leq$ value $\leq$ NUMRAD

Purpose:

Defines the outer spatial interval of the region of interest for this result. The user must supply NUM8 values in column 3 of the data block.

Note: In order to obtain the CCDF tables of a consequence measure requested in this section, append the character string ' $C C D F^{\prime}$ to the line requesting that result as the fourth item on the data record. The CCDF tables will be printed on the list output file.

Example Usage:

$\star$

* NUMBER of DESIRED RESULtS of this type

*

*

*

*

TYPE8OUT001 'ERL FAT/TOTAL' 1 TYPE8OUTOO2 


\subsection{Peak Dose at a Distance}

The Type A result, peak dose at a distance, was developed as a result of the addition of the network evacuation option. Type 6 results, centerline dose vs. distance, can be output only when the straight-line plume option without rotation (IPLUME=1) is selected. However, the network evacuation model may not be utilized when IPLUME is set to 1 . The Type A result was added to allow the reporting of individual doses for network evacuation scenarios. This result is based on the total dose at each distance over a selected range of distances in a manner analogous to the Type 6 results. The implementation of this result differs from Type 6 results in that there is no capability for reporting a breakdown of individual doses by pathway.

This result is requested in the EARLY input file and produced for each emergency response scenario. There is no dependence on population data. The dose is reported for phantom individuals assumed to be present at all locations. If the CHRONC module is exercised, this result is then automatically produced by CHRONC as well.

The overall weighted sum of results represents the combination of doses calculated by EARLY and CHRONC. Caution should be taken with the use of the overall results obtained by combining the emergency response cohorts because peak doses for the various cohorts may occur at different angular locations. Summation of these values is most likely meaningless if multiple plume segments are allowed to travel in different directions (i.e., when NUMREL is greater than 1 and IPLUME is equal to 2 or 3 ).

Variable Name: NUMA

Variable Type: Integer, Scalar

Allowed Range: $\quad 1 \leq$ value $\leq 10$

Purpose: Defines the number of results of this type being requested.

Variable Name: NAME

Variable Type: Character, Array

Allowed Range: $\quad 1 \leq$ length $\leq 10$

Purpose: Defines the name of the organ for this dose measure.

Variable Name: IIDISA

Variable Type: Integer, Array

Allowed Range: $1 \leq$ value $\leq$ NUMRAD

Purpose:

Defines the inner boundary of the region for which this result is to be calculated.

Variable Name: I2DISA

Variable Type: Integer, Array 
Allowed Range: $\quad 1 \leq$ value $\leq$ NUMRAD

Purpose: $\quad$ Defines the outer boundary of the region for which this result is to be calculated.

Example Usage:

* PEAK DOSE FOUND on tHe sPatial GRID

*

TYPEANUMBER

$\star$

*

TYPEAOUTO01

TYPEAOUT002

2

NAME IIDISA I2DISA

'L-EFFECTIVE' 1126

'A-RED MARR' 1126

$(0-1609 \mathrm{KM})$

$(0-1609 \mathrm{KM})$

\subsection{Peak Dose at an $(r, \theta)$ Location}

The Type B result provides the peak dose at a user-specified $(r, \theta)$ location. Type 6 and A results differ from Type $B$ results in that they are limited to specifying the centerline dose under the plume. Type B results report individual doses at locations other than directly under the plume(s). Type B results also differ from Type 6 results in that there is no capability for reporting individual doses by pathway for Type B results.

Although the Type B result is only requested on the EARLY file, if CHRONC is being exercised, the Type B results will be generated by CHRONC as well as by EARLY, and the results obtained presented both separately and in combination. The Type B results correspond to the total dose estimated to be incurred by a representative phantom individual assumed to reside at a particular $(r, \theta)$ location on the spatial grid. User input population data have no bearing on the generation of this consequence measure. Only direct exposure is considered in the generation of this result. Exposures resulting from the ingestion of contaminated food and water are not included. Also, the generation of this result takes full account of any mitigative action models activated by exceedance of user-specified dose thresholds.

The location for which this result is to be calculated is specified as a radial index (which can range from 1 to NUMRAD) and an angular index (which can range from 1 to 16). Following the convention used in other parts of the code, an angular index of 1 "points" to an individual assumed to be located north of the release point, with successive directions rotating clockwise around the compass. An angular index of 16 points to the NNW direction.

If the controlling card (record identifier NUMBOUT001) is omitted, the calculations proceed on the assumption that the Type $B$ results are not being requested.

Variable Name: NUMB

Variable Type: Integer, Scalar

Allowed Range: $\quad 0 \leq$ value $\leq 32$

Purpose: $\quad$ Defines the number of results of this type being requested. 
Variable Name: NAME

Variable Type: Character, Array

Allowed Range: $\quad 1 \leq$ length $\leq 10$

Purpose: Defines the name of the organ for this dose measure.

Variable Name: IRAD_B

Variable Type: Integer, Array

Allowed Range: $1 \leq$ value $\leq$ NUMRAD

Purpose:

Defines the inner boundary of the region for which this result is to be calculated.

Variable Name: IANG_B

Variable Type: Integer, Array

Allowed Range: $\quad 1 \leq$ value $\leq 16$

Purpose:

Defines the angular index to the location at which this result is to be calculated.

Example Usage:

* PEAK DOSE FOUND ON THE SPATIAL GRID

* TYPEBNUMBER 2

*

NAME IRAD_B IANG_B

$\begin{array}{lllll}\text { 'L-EFFECTIVE' } & 1 & 1 & (0-1609 \mathrm{KM}) \\ \text { 'A-RED MARR' } & 1 & 1 & (0-1609 \mathrm{KM})\end{array}$




\subsection{CHRONC Input File}

The CHRONC module simulates the events that occur following the emergency-phase time period modeled by EARLY. Various long-term protective actions may be taken during this period to limit radiation doses to acceptable levels. The parameters defining these protective actions are under user control, and all of them are described in this chapter.

CHRONC calculates the individual health effects that result from both (1) direct exposure to contaminated ground and from inhalation of resuspended materials as well as (2) indirect health effects caused by the consumption of contaminated food and water by individuals who could reside both on and off of the computational grid. CHRONC also calculates the economic costs of the long-term protective actions as well as the cost of the emergency response actions that were modeled in the EARLY module.

It is up to the user to specify the various parameters needed for these calculations. There are no default values for the parameters described in this section. In addition to specifying the characteristics of the model, the user has complete control over the output produced by CHRONC and must explicitly specify which results are to be produced. All of this information is supplied through the CHRONC input file, and all of the input parameters are described in this chapter.

Four long-term exposure pathways are modeled in MACCS2 to predict the long-term radiation exposures from accidental radiological releases: groundshine, resuspension inhalation, ingestion of contaminated food, and ingestion of contaminated drinking water. The models utilized in predicting the doses from these four pathways are described individually in the following sections. The dose from each of the long-term pathways is evaluated for each spatial element surrounding the accident site. For the intermediate phase, only the groundshine and resuspension inhalation exposure pathways are considered.

MACCS2 incorporates two options for the user regarding food ingestion models: (1) the food ingestion model of MACCS and (2) the new COMIDA-based food ingestion model. A brief discussion of the differences between these two models is provided in Section 3.1.2 of this document. The MACCS food ingestion model is based on the simple principle that the long-term dose produced by any radionuclide to an organ via a pathway is the product of (1) the ground concentration of the radionuclide, (2) the integrated transfer factor for the radionuclide to human intake for the pathway, and (3) the ingestion dose conversion factor. There are a number of limitations of the MACCS food ingestion model. A main drawback of this model is that the integrated transfer factors for food pathway radionuclides not included in the MACCS sample problems must be derived by the user external to the code. The calculational procedures are difficult and error prone. In contrast, the COMIDA2-based ingestion model is based on a preprocessor that can be exercised by the user, with consideration of site-specific data, if such data are available.

The radiation dose for the exposure pathways of the intermediate and long-term phases is calculated for each of the coarse spatial elements using the initial ground concentration under the 
plume centerline calculated by the ATMOS module. Similar to the early exposure pathways, MACCS2 uses an off-centerline correction factor and the ground concentration under the plume centerline to estimate the initial ground concentration at the off-centerline region of various spatial elements. In contrast to EARLY, however, which utilizes a Gaussian histogram subdividing each 22.5-degree compass sector, the CHRONC calculations do not utilize the Gaussian histogram subdivisions in any respect.

For all of the CHRONC calculations, relating to both direct exposure (groundshine and resuspension as well as food and water ingestion) the Gaussian is averaged over the entire 22.5degree compass sector to yield a single off-centerline geometric adjustment factor. A description of the intermediate and long-term phase off-centerline correction factor is provided in Section 3.2.1 of the MACCS Model Description.

\subsection{Overview of CHRONC Mitigative Action Models}

CHRONC incorporates calculations for two distinct periods of time, the intermediate phase and the long-term phase, as follows.

\subsubsection{Intermediate Phase}

The mitigative action model for the intermediate phase is very simple. If the intermediate-phase dose criterion is satisfied, the resident population is assumed to be present and subject to radiation exposure from groundshine and resuspension for the entire intermediate phase. If the intermediate-phase exposure leads to doses in excess of DSCRTI, then the population is assumed to be relocated to uncontaminated areas for the entire intermediate phase, with a corresponding per-capita economic cost defined by the user through the input variable POPCST (see Section 7.6).

The user can configure the calculations with an intermediate phase having a duration as short as zero (essentially, no intermediate phase, and a long-term phase beginning immediately upon conclusion of the emergency phase), or as long as 1 year. The calculations of food and water ingestion doses are based on the ground contamination levels estimated to be present at the beginning of the intermediate phase.

\subsubsection{Long-Term Phase}

The mitigative action models for the long-term exposure phase implemented in MACCS2 differ slightly from the corresponding models implemented in MACCS. The reason for this is that the decisions on mitigative action in the long-term phase are based on two sets of independent actions: (1) decisions relating to whether land at a specific location and time is suitable for human habitation, "habitability," and (2) decisions relating to whether land at a specific location and time is suitable for agricultural production, "farmability."

Since the COMIDA2-based food-chain model of MACCS2 differs markedly from the MACCS food-chain model, it is necessary to provide a description of how both of these food-chain models interact with the long-term mitigative actions triggered by habitability considerations. As 
a start, several concepts fundamental to MACCS2 that are unchanged from MACCS are discussed: (1) division between farm and population, (2) habitability decision making, (3) farmability decision making, and (4) primacy of habitability over farmability decisions. These are discussed in Sections 7.1.3 through 7.1.6.

\subsubsection{Division Between Farm and Population}

The long-term phase incorporates a fundamental distinction between the consequences associated with (1) agricultural uses of land (denoted AREA-DEPENDENT on the code output), and (2) habitation of land by humans (denoted POP.-DEPENDENT on the code output).

\subsubsection{Habitability Decision Making}

Habitability decision making can result in three possible outcomes: (1) land is immediately habitable, (2) land will be habitable after decontamination, and (3) land will be habitable after a combination of decontamination and interdiction. The parameters controlling the choice of these outcomes are described in Sections 7.4 through 7.6.

\subsubsection{Land Immediately Habitable}

The first step in the decision making regarding habitability is a determination of whether, in the absence of any mitigative actions, the land is suitable for habitation during the long-term exposure period. This is done by comparing (1) the projected individual dose calculated by the code for the long-term dose projection period (input variable TMPACT, described in Section 7.4) against (2) the user-specified dose criterion for long-term exposure (input variable DSCRLT, described in Section 7.4). If the projected individual dose does not exceed DSCRLT, then the land is considered immediately habitable, and no further tests regarding habitability are made.

\subsubsection{Land Habitable after Decontamination}

If the land is not immediately habitable, a progressive series of actions are evaluated, beginning with decontamination for the various user-defined decontamination levels. The influence of decontamination is twofold: (1) the decontamination factor (DF) (input variable DSRFCT, described in Section 7.5) is a linear scaling factor by which the doses are reduced, and (2) weathering and radioactive decay occurring during the decontamination period also reduce doses.

The influence of these two factors is considered to be independent. That is, if the user specifies a DF of 3, and a decontamination period of 1 year, the doses to habitants at the conclusion of decontamination will generally be less than one-third of the doses that would be incurred in the absence of decontamination because both decontamination and weathering and decay would ordinarily serve to reduce subsequent doses. Continuing this example, if weathering and decay resulted in a twofold reduction in the level of contamination at the end of the 1-year period, the combined effect of decontamination with a DF of 3 and weathering and decay would be to reduce subsequent doses by a factor of six. 


\subsubsection{Land Habitable after Decontamination and Interdiction}

If the maximum-level decontamination effort is insufficient to allow habitability at the conclusion of the decontamination period, then the dose-reduction impacts of decontamination in combination with temporary interdiction are evaluated to determine if the habitability criterion is satisfied at the conclusion of the decontamination and interdiction periods. As above, the DF obtained from decontamination is considered to be independent of the dose reduction obtained from weathering and decay. The maximum interdiction period considered by the code is 30 years.

The effect of weathering and decay over the interdiction period is calculated with an interpolation technique that utilizes precalculated doses for predefined interdiction periods of 1,5 , and 30 years, as fixed in the code. These three predefined interdiction periods all begin at the conclusion of the maximum-level decontamination effort, which is a user-specified parameter that can range up to 1 year.

\subsubsection{Limits on Interdiction Duration}

For the two separate land uses, farm and population, mitigative actions are chosen that will yield the lowest-cost approach to satisfying the applicable criteria. For farms, the criteria define acceptable levels of foodstuff contamination, while for populations, the criteria define acceptable levels of radiation exposures to resident individuals. The maximum duration of temporary farmland interdiction that can be selected by the code is 8 years, while the corresponding maximum duration for interdiction of population is 30 years. If the maximum-duration interdiction of either land use is insufficient to satisfy the respective criteria for use, then that land use is assumed to be permanently interdicted, or condemned.

\subsubsection{Test for Cost Effectiveness}

As described above, the code evaluates potential mitigative actions for both farm and population in order to determine if it is possible to satisfy the applicable criteria for acceptable exposures. If either of these criteria, for farm or land, cannot be satisfied after the maximum-duration interdiction, then that land use is permanently interdicted, or condemned. However, the use of land for farm or population can also be condemned if the total cost involved in restoring it to use would exceed the user-specified value of the property. If this is done, the use of land for either farm or population or both can be condemned. When a land use is condemned for either reason (i.e., the dose criteria cannot be satisfied, or the cost of reclamation exceeds the property's value), the code calculates the corresponding long-term food and population exposures as zero, and assesses an economic cost for the condemnation of the property.

\subsubsection{Farmability Decision Making}

The decision on whether land is suitable for farming is based on prior evaluations of its suitability for human habitation. The farmland interdiction models differ slightly, depending on which of the two food-chain models is being utilized. For a description of the interdiction model utilized for the COMIDA2-based food-chain model of MACCS2, see Section 7.10.2. For a 
description of the interdiction model utilized for the MACCS food-chain model, see Section 7.11.3.

\subsubsection{Primacy of Habitability over Farmability Decisions}

For both food-chain model options, farm production is allowed to occur only when the environmental contamination levels are sufficiently low to allow habitation. However, the converse condition is allowed. That is, if land is not suitable for farming, the existence of that condition imposes no constraint on its habitability.

\subsection{Problem Identification Data}

The problem identification data block is used to identify the CHRONC input file and the calculations that are being performed.

Variable Name:

CHNAME

Variable Type:

Character, Scalar

Allowed Range:

$1 \leq$ length $\leq 80$

Purpose:

Describes this particular CHRONC input file. Choose a text string that briefly describes the input data file, the assumptions and the model choices of this particular application of the CHRONC module.

This text will be printed on all of the MACCS2 list output as an aid in identifying the results. It is not necessary to include the date of the run since that information is automatically included.

Example Usage:

CHCHNAMEO01 'SURRY CHRONC INPUT FOR FINAL NUREG-1150 CALCULATIONS'

\subsection{Emergency-Response Cost Data}

The emergency-response cost data block is used to define the compensation costs for people who are subject to the emergency actions of evacuation, sheltering, or relocation. Evacuation is solely under the control of models defined in the EARLY module. Relocation of individuals can occur either during the EARLY emergency phase or during the CHRONC intermediate-phase period. In the presentation of economic cost results (see Section 7.15), the costs associated with the emergency phase (i.e., evacuation and short-term relocation) are reported separately from the costs associated with the intermediate phase (i.e., per-diem costs for relocation for the duration of the intermediate phase).

Variable Name: $\quad$ EVACST

Variable Type: Real, Scalar

Allowed Range: $\quad 0.0 \leq$ value $\leq 1000.0$ (dollars/person-day) 
Purpose: Defines the daily cost of compensation for evacuees and short-term relocatees who are removed from their homes as a result of radiation exposure during the emergency-phase period. This value could include the following components: food, housing, transportation, and lost income.

Example Usage:

CHEVACSTO01 27.00

Variable Name: RELCST

Variable Type: $\quad$ Real, Scalar

Allowed Range: $\quad 0.0 \leq$ value $\leq 1000.0$ (dollars/person-day)

Purpose:

Defines the daily cost of compensation for individuals removed from their homes due to intermediate-phase relocation modeled by CHRONC. The costs should include the following components: food, housing, transportation, lost income, and replacement of lost personal property.

Example Usage:

CHRELCST001 $\quad 27.00$

\subsection{Long-Term Protective Action Data}

The long-term protective action data block defines the intermediate and long-term action time periods as well as the maximum doses that people are allowed to receive during these periods. The maximum allowable doses defined here are used to determine the need for relocation, decontamination, interdiction, or condemnation.

In all versions of MACCS, the exposure period considered by CHRONC was fixed within the code to a value of one million years. That is, the code calculated both individual and collective doses assuming that the population remained present for the entire million-year exposure period. The principal reason for this was to be consistent with the assumptions used in the CRAC2 code (Ritchie et al. 1984).

MACCS2 allows the user to define the long-term exposure period through the TMPACT input variable. It is suggested that 30 years is a reasonable value since that is the value used by the EPA in its Superfund guidance (EPA 1991).

Variable Name: DUR_INTPHAS

Variable Type: Real, Scalar

Allowed Range: $\quad 0.0 \leq$ value $\leq 3.1536 \mathrm{E}+7$ (seconds) ( 1 year)

Purpose: Defines the duration of the intermediate-phase period. This period follows the emergency-phase period, EARLY variable ENDEMP. The value of ENDEMP is defined in the EARLY input file and passed to CHRONC. 
The MACCS input variable TMIPND is no longer utilized to define the intermediate-phase period. Previously, this phase was defined as ending at TMIPND seconds after arrival of the plume at a spatial interval. TMIPND is no longer processed from the CHRONC input file. Within the code however, the variable TMIPND remains, and it is calculated as follows:

$$
\text { TMIPND = DUR_INTPHAS + ENDEMP }
$$

Example Usage:

*

DUR_INTPHAS 604800. ( 7 DAYS, NO INTERMEDIATE PHASE)

Variable Name: TMPACT

Variable Type: $\quad$ Real, Scalar

Allowed Range: $\quad$ TMIPND $\leq$ value $\leq 1 . E+10$ (seconds)

Purpose: $\quad$ Defines the long-term dose projection period. Protective actions such as decontamination, or decontamination followed by interdiction are evaluated to determine if the exposure of an individual during this period can be reduced so that it does not exceed the long-term phase allowable dose (DSCRLT) to the long-term phase critical organ (CRTOCR).

If temporary interdiction is required, individuals are returned to their homes at a time when it is estimated that they will receive a dose of exactly DSCRLT to the critical organ over an exposure period of this duration.

Once individuals are returned to their home, their long-term doses are integrated over an exposure period specified by input variable EXPTIM.

Example Usage:

CHTMPACT001 1.58E8 (5 YEARS)

Variable Name: DSCRTI

Variable Type: $\quad$ Real, Scalar

Allowed Range: $\quad 0.0 \leq$ value $\leq 1 . E+5$ (sieverts)

Purpose: Defines the intermediate-phase dose criterion. This is the maximum allowable direct exposure dose commitment to the long-term critical organ (CRTOCR) during the intermediate-phase period (TMIPND). The direct exposure pathways considered in this evaluation are groundshine and resuspension inhalation.

If the intermediate-phase dose criterion (DSCRTI) is exceeded for an individual remaining at some location for the entire intermediate-phase period, the resident population there is relocated for the entire intermediate-phase period. 
Example Usage:

CHDSCRTIO01 1.0E5 (NO INTERMEDIATE-PHASE RELOCATION)

Variable Name: DSCRLT

Variable Type: Real, Scalar

Allowed Range: $\quad 1 . E-20 \leq$ value $\leq 1 . E+5$ (sieverts)

Purpose:

Defines the long-term phase dose criterion. This is the maximum allowable direct exposure dose commitment to the long-term critical organ (CRTOCR) during the long-term phase action period (TMPACT).

Direct exposure in the CHRONC module is defined as the sum of the groundshine and resuspension doses. The user-specified value of DSCRLT determines whether land is considered suitable for habitation during the long-term phase period. This parameter affects the food ingestion pathway indirectly since the code does not model agricultural production in locations (and time periods) where the land is uninhabitable.

If this dose criterion is exceeded in any spatial element during the long-term action period, mitigative actions such as decontamination or decontamination followed by temporary interdiction are employed to limit the dose to the critical organ so that the allowable dose level is not exceeded in that spatial grid element. The long-term critical organ is defined below in this data block.

If it is not possible to reduce doses to this level in a cost-effective manner, the property is condemned and the resident population is permanently relocated.

Example Usage:

CHDSCRLTO01 $0.04^{\circ}$ (2 REM IN FIRST YEAR, 0.5 REM PER YEAR FOR YEARS 2-5)

Variable Name: EXPTIM

Variable Type: $\quad$ Real, Scalar

Allowed Range: $\quad 0.0 \leq$ value $\leq 1 . E 10$ (seconds) (a period of from 0 to 317 years)

Purpose: $\quad$ Specifies the duration of the long-term exposure period considered by CHRONC.

If a zero is assigned to the EXPTIM parameter, then no long-term phase is defined, and only exposures incurred during the intermediate phase are assessed by CHRONC.

The CHRONC exposure period is not measured from the start of the long-term phase. The period begins when people are allowed to return to their property. For example, if the code utilizes 5 years of interdiction (referenced to the beginning of the long-term phase) at a specific location to satisfy the dose criterion, and EXPTIM is set to 30 years, the doses to resident population will be calculated for an exposure period that begins at $t=5$ years, and ends at $t=35$ years. 
The accrual of doses from food and water ingestion is not affected by the user-specified value of EXPTIM. This accrual is not strictly associated with the population surrounding the facility. The ingestion of contaminated food and water is assumed to result in doses to an unspecified population.

Example Usage:

* CHEXPTIM001 9.45E8 (30 YEARS PER EPA STANDARD DEFAULT EXPOSURE FACTORS)

Variable Name: CRTOCR

Variable Type: Character, Scalar

Allowed Range: $\quad 2 \leq$ length $\leq 10$

Purpose: $\quad$ Defines the long-term phase critical organ. If the total direct exposure dose commitment to this organ in a grid element would exceed the dose criteria in either the intermediate phase period (TMIPND) or the long-term phase period (TMPACT), protective actions would be taken to limit that dose to acceptable levels.

Example Usage:

CHCRTOCRO01

'L-EFFECTIVE'

\subsection{Decontamination Plan Data}

The decontamination plan data block defines the decontamination actions that may be taken during the long-term period to reduce doses to acceptable levels. These data define the decontamination strategies that are possible, their effectiveness, and their cost. Each decontamination level represents an alternative strategy that would reduce the projected long-term groundshine and resuspension doses by a factor called the "dose reduction factor. " Up to three levels of decontamination can be defined.

The objective of decontamination is to reduce projected doses below the long-term dose criterion in a cost-effective manner. If the maximum decontamination level is insufficient to restore an area to immediate habitability, a period of temporary interdiction following the maximum decontamination level is considered in order to allow for dose reduction through radioactive decay and weathering. If the property cannot be made habitable within 30 years or if the cost of reclaiming the habitability of the property exceeds the cost of condemning it, the property will be condemned and permanently withdrawn from use.

Decontamination cost is divided into two categories and these two types of cost are calculated separately. Farm-dependent decontamination cost represents the cost of farmland decontamination in a grid element. Farm-dependent decontamination cost is a function of the area of the grid element devoted to agriculture. Population-dependent decontamination represents the cost of nonfarmland decontamination. Population-dependent decontamination cost is a function 
of the population residing in the grid element. The strategy of decontamination within a grid element is independent of the type of area being decontaminated.

For a given decontamination level, the same decontamination time and effectiveness apply to both farmland and nonfarmland, but the two costs are unique and are maintained independently for each type of decontamination. Owing to the requirement that the recovery of property must be cost-effective, it is possible, in a given element, that decontamination of nonfarmland is not performed, but farmland is instead condemned.

Decontamination of a grid element serves to reduce the contamination level in that element by the dose reduction factor associated with the decontamination effort being applied. Everything else being equal, a decontamination factor of 10 will cause the integrated dose over any exposure period to be reduced by a factor of 10 .

During the decontamination period, which is assumed to begin at the end of the intermediatephase time period (beginning of the long-term phase period), the population from areas that will be decontaminated is assumed to be relocated to uncontaminated areas, and the associated cost from loss of use is calculated in the same manner as temporary interdiction.

While engaged in cleanup efforts, decontamination workers are assumed to wear respiratory protection devices. Therefore, they accumulate only groundshine doses. These doses and the cancer fatalities that they induce contribute to the aggregated doses and cases of cancer fatalities tabulated in the MACCS2 output.

Decontamination reduces direct exposure doses (both groundshine and resuspension) caused by contamination of land and buildings. Many decontamination processes (e.g., plowing, fire-hosing) reduce groundshine and resuspension doses by washing surface contamination down into the ground. Since these processes may not move contamination out of the root zone, the WASH-1400-based economic cost model of MACCS2 assumes that farmland decontamination reduces direct exposure doses to farmers without reducing uptake of radioactivity by root systems. Thus, decontamination of farmland does not reduce the ingestion doses produced by consumption of crops that are contaminated by root uptake.

$\begin{array}{ll}\text { Variable Name: } & \text { LVLDEC } \\ \text { Variable Type: } & \text { Integer, Scalar } \\ \text { Allowed Range: } & 1 \leq \text { value } \leq 3 \\ \text { Purpose: } & \text { Defines the number of decontamination levels that can be utilized. } \\ \text { Example Usage: } & \\ \star & \\ \text { CHLVLDEC } 001 & 2\end{array}$

Variable Name: TIMDEC

Variable Type: Real, Array

Allowed Range: $\quad 1 . E-6 \leq$ value $\leq 3.15 \mathrm{E} 7$ (seconds) (1 year) 
Purpose: $\quad$ Defines the time required for completion of each of the decontamination levels. The user must define a decontamination time for each of the LVLDEC decontamination levels. Decontamination begins at the end of the intermediate phase (TMIPND). The values must be monotonically

Example Usage: increasing.

CHTIMDEC001 $5.184 \mathrm{E} 6 \quad 1.0368 \mathrm{E} 7 \quad(60,120$ DAYS $)$

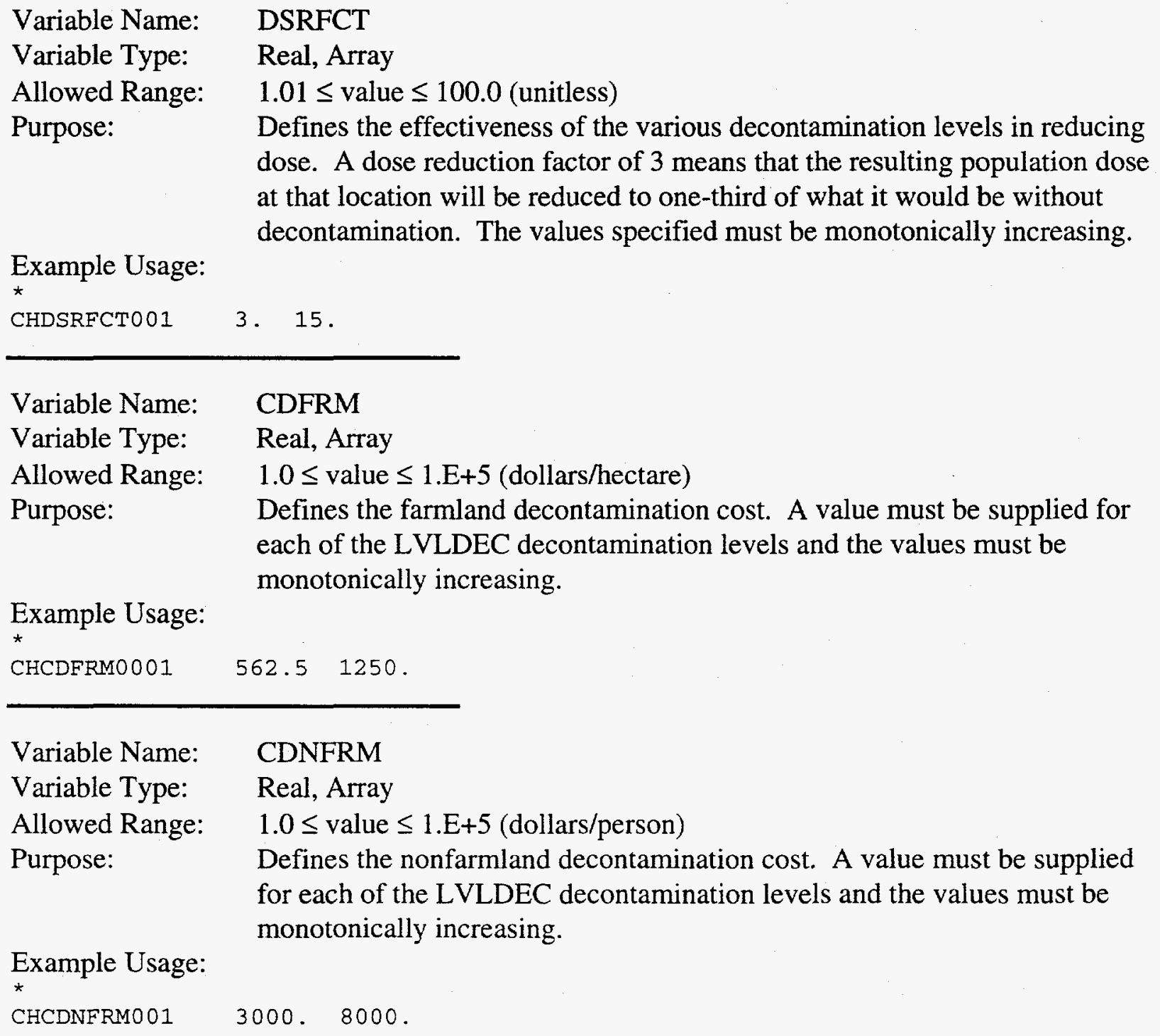

Note: The remaining parameters in this section are used only to calculate the dose received from decontamination activities. 
Variable Name: FRFDL

Variable Type: Real, Array

Allowed Range: $\quad 0.0 \leq$ value $\leq 1.0$ (unitless)

Purpose:

Defines the fraction of the farmland decontamination cost that is due to labor. A value must be supplied for each of the LVLDEC decontamination levels.

Example Usage:

CHFRFDL0001 $\quad .3 \quad .35$

Variable Name: FRNFDL

Variable Type: $\quad$ Real, Array

Allowed Range: $\quad 0.0 \leq$ value $\leq 1.0$ (unitless)

Purpose:

Defines the fraction of the nonfarmland decontamination cost that is due to labor. A value must be supplied for each of the LVLDEC decontamination levels.

Example Usage:

CHFRNFDL001 $\quad .7 \quad .5$

Variable Name: $\quad$ TFWKF

Variable Type: $\quad$ Real, Array

Allowed Range: $\quad 0.0 \leq$ value $\leq 1.0$ (unitless)

Purpose: $\quad$ Defines the fraction of the decontamination period (TIMDEC) that a farmland decontamination worker spends in the contaminated area. A value must be supplied for each of the LVLDEC decontamination levels.

Example Usage:

CHTFWKF0001 $.10 \quad .33$

Variable Name: TFWKNF

Variable Type: $\quad$ Real, Array

Allowed Range: $\quad 0.0 \leq$ value $\leq 1.0$ (unitless)

Purpose:

Defines the fraction of the decontamination period (ITMDEC) that a nonfarmland decontamination worker spends in the contaminated area during the decontamination period. A value must be supplied for each of the LVLDEC decontamination levels.

Example Usage:

$\begin{array}{lll}\text { CHTFWKNFOO1 } \quad .33 & .33\end{array}$ 
Variable Name:

DLBCST

Variable Type:

Real, Scalar

Allowed Range:

$1.0 \leq$ value $\leq 1 . E+6$ (dollars/man-year)

Purpose:

Defines the labor cost of a decontamination worker.

Example Usage:

CHDLBCSTO01

35000 .

\subsection{Interdiction Plan Cost Data}

The interdiction plan cost data block defines the parameters needed to calculate the cost of interdiction. The data supplied here are combined with data in the Site Data file and the regional characteristics data in the course of the calculations.

The model used in MACCS2 for assessing the cost of interdiction is based on the model described in WASH-1400, Appendix 6. It is currently used to calculate the economic cost of loss of use during both decontamination and temporary interdiction periods.

Variable Name: DPRATE

Variable Type: Real, Scalar

Allowed Range: $\quad 0.0 \leq$ value $\leq 1.0$ (per year)

Purpose:

Defines the depreciation rate that applies to property improvements during a period of interdiction. This depreciation rate is intended to account for the loss of value of buildings and other structures resulting from a lack of habitation and maintenance.

Example Usage:

CHDPRATEO 01

Variable Name: DSRATE

Variable Type: Real, Scalar

Allowed Range: $\quad 0.0 \leq$ value $\leq 1.0$ (per year)

Purpose:

Defines the expected rate of return from land, buildings, equipment, etc. For example, the inflation-adjusted real mortgage rate for land and buildings

could be used.

Example Usage:

*

CHDSRATE001

.12

Variable Name: $\quad$ POPCST

Variable Type: Real, Scalar

Allowed Range: $\quad 1 . \mathrm{E}-6 \leq$ value $\leq 1 . \mathrm{E}+6$ (dollars/person) 
Purpose: $\quad$ Defines the per capita removal cost for temporary or permanent relocation of population and businesses in a region rendered uninhabitable during the long-term phase time period. This cost is assessed if any of the following actions are required: decontamination alone, decontamination followed by interdiction, or condemnation. This value should be derived in a way that takes account of both personal and corporate income losses for a transitional Example Usage: period as well as moving expenses.

CHPOPCSTO01 5000 .

\subsection{Groundshine Weathering Data}

The groundshine weathering definition data block defines the groundshine weathering equation from Gale, Miller, and Fisher (1964). The groundshine weathering relationship is defined as

$$
\begin{aligned}
G W(t)= & G W C O E F(1) \bullet \exp [-\ln (2) \bullet t / T G W H L F(1)]+\ldots+ \\
& G W C O E F(n) \bullet \exp [-\ln (2) \bullet t / T G W H L F(n)]
\end{aligned}
$$

where $G W(t)$ represents the groundshine weathering at time $t$, given the weathering coefficients, GWCOEF, and the weathering half-lives, TGWHLF. The user must specify the number of terms, $n$, in the relationship and the values for the weathering coefficients and weathering halflives.

Variable Name: NGWTRM

Variable Type: Integer, Scalar

Allowed Range: $\quad 1 \leq$ value $\leq 2$

Purpose: Defines the number of terms in the groundshine weathering relationship.

Example Usage:

CHNGWTRMO01 2

Variable Name: GWCOEF

Variable Type: $\quad$ Real, Array

Allowed Range: $\quad 1.0 \mathrm{E}-20 \leq$ value $\leq 1.0$ (unitless)

Purpose: $\quad$ Defines the array of NGWTRM coefficients in the groundshine weathering equation.

Example Usage:

$\begin{array}{lll}\text { CHGWCOEFO01 } & 0.5 & 0.5\end{array}$ 
Variable Name: TGWHLF

Variable Type: Real, Array

Allowed Range: $\quad 1 . E-6 \leq$ value $\leq 1 . E+12$ (seconds)

Purpose:

Defines the array of NGWTRM half-lives in the groundshine weathering equation.

Example Usage:

CHTGWHLFOO1 $\quad 1.6 \mathrm{E} 7 \quad 2.8 \mathrm{E} 9$

\subsection{Resuspension Weathering Data}

The resuspension weathering definition data block defines the resuspension weathering equation. The resuspension weathering relationship is defined as:

$$
\begin{aligned}
R W(t)= & R W C O E F(1) \bullet \exp [-\ln (2) \bullet t / T R W H L F(1)]+\ldots+ \\
& R W C O E F(n) \bullet \exp [-\ln (2) \bullet t / T R W H L F(n)]
\end{aligned}
$$

where $R W(t)$ represents the resuspension weathering at time $t$, given the weathering coefficients, RWCOEF, and the weathering half-lives, TRWHLF. The user must specify the number of terms, $n$, in the relationship and the values for the weathering coefficients and weathering half-lives.

The user must specify the number of terms, $n$, in the relationship and the values for the weathering coefficients and weathering half-lives.

Variable Name: NRWTRM

Variable Type: Integer, Scalar

Allowed Range: $\quad 1 \leq$ value $\leq 3$

Purpose:

Defines the number of terms in the resuspension weathering relationship.

Example Usage:

CHNRWTRM001

Variable Name: RWCOEF

Variable Type: Real, Array

Allowed Range: $\quad 1.0 \mathrm{E}-20 \leq$ value $\leq 1.0$ (per meter)

Purpose: Defines the array of NRWTRM coefficients in the resuspension weathering equation.

Example Usage:

CHRWCOEF001 $1.0 \mathrm{E}-5 \quad 1.0 \mathrm{E}-7 \quad 1.0 \mathrm{E}-9$

Variable Name: TRWHLF

Variable Type: Real, Array

Allowed Range: $\quad 1 . E-6 \leq$ value $\leq 1 . E+12$ (seconds) 
Purpose: $\quad$ Defines the array of NRWTRM half-lives in the resuspension weathering equation.

Example Usage:

CHTRWHLF001 1.6E7 1.6E8 1.6E9 (6 MONTHS, 5 YEARS, 50 YEARS)

\subsection{Regional Characteristics Data}

The regional characteristics data block defines the aggregate economic and agricultural characteristics of the area surrounding the accident site.

If mitigative actions are necessary to restrict exposure to allowable levels, these data are used to evaluate the cost-effectiveness of those actions. For instance, if the average property values supplied here indicate that reclaiming a piece of land will cost more than the cost of condemning it, the land will be condemned instead of reclaimed.

Once a set of actions at a certain distance is decided upon, these actions may be "rotated" around the circle if the user has chosen the option of IPLUME=1 or IPLUME=2 as defined in Section 6.2.2. In some of these wind directions, there could be an extremely valuable property. It is implicit in our calculation method that the cost-effectiveness test cannot take account of this nonhomogeneity of the valuation field because of the need for performing a rotation of the chosen actions around the circle.

The choice of actions is always determined by the aggregate characteristics of the region, but the calculation of the resulting consequence measures can be done in two different ways, depending on the value of the input variable POPFLG, which is defined in Section 6.3.

When a uniform population distribution is being used (POPFLG='UNIFORM'), all of the data in this section are used for the calculation of the CHRONC consequence measures. However, if the user requests that a Site Data file be utilized (POPFLG='FILE'), the first four parameters defined in this section are not actually used in the calculations though they all must be supplied. The values corresponding to these four parameters are obtained from the Site Data file for each spatial element on the computational grid.

Variable Name: FRACLD

Variable Type: Real, Scalar

Allowed Range: $\quad 1 . E-6 \leq$ value $\leq 1.0$ (unitless)

Purpose: Defines the fraction of the site region covered by land. The remainder is assumed to be water.

Note: If a Site Data file is being used ( $P O P F L G=' F I L E^{\prime}$ ), the value of this input parameter is not used by the code; nevertheless a valid parameter value must be supplied. 
Example Usage:

*

CHFRACLD001 0.95

Variable Name: FRCFRM

Variable Type: Real, Scalar

Allowed Range: $\quad 1 . E-6 \leq$ value $\leq 1.0$ (unitless)

Purpose:

Defines the average fraction of land in the region devoted to farm production. The land area under cultivation is as follows:

cultivated area $=$ total area $\bullet$ FRACLD $\bullet$ FRCFRM.

Note: If a Site Data file is being used (POPFLG $=^{\prime} F I L E^{\prime}$ ), the value of this input parameter is not used by the code; nevertheless a valid parameter value must be supplied.

Example Usage:

CHFRCFRMO01 $0.382 \quad$ VIRGINIA

Variable Name: $\quad$ FRMPRD

Variable Type: $\quad$ Real, Scalar

Allowed Range: $\quad 0.0 \leq$ value $\leq 1 . E+5$ (dollars/hectare)

Purpose: Defines the value of the average annual farm production (gross sales) in the region.

Note: If a Site Data file is being used (POPFLG=FILE), the value of this input parameter is not used by the code; nevertheless a valid parameter value must be supplied.

Example Usage:

CHFRMPRD001 $371.0 \quad$ VIRGINIA

Variable Name: DPFRCT

Variable Type: $\quad$ Real, Scalar

Allowed Range: $\quad 0.0 \leq$ value $\leq 1.0$ (unitless)

Purpose: $\quad$ Defines the fraction of annual farm production (gross sales) in the region resulting from dairy production.

Note: If a Site Data file is being used (POPFLG=FILE), the value of this input parameter is not used by the code; nevertheless a valid parameter value must be supplied.

Example Usage:

CHDPFRCT001 0.198 VIRGINIA 
$\begin{array}{ll}\text { Variable Name: } & \text { VALWF } \\ \text { Variable Type: } & \text { Real, Scalar } \\ \text { Allowed Range: } & 1 . E-6 \leq \text { value } \leq 1 . E+6 \text { (dollars/hectare) }\end{array}$

Purpose: Defines the value of farm wealth in the region. This value should include both publicly and privately owned grazing lands, farmland, farm buildings, and nonrecoverable farm machinery, as well as any publicly owned infrastructure serving the farm industry.

Example Usage:

*

CHVALWF0001 $2613.0 \quad$ SURRY

Variable Name: FRFIM

Variable Type: $\quad$ Real, Scalar

Allowed Range: $\quad 0.0 \leq$ value $\leq 1.0$ (unitless)

Purpose: $\quad$ Defines the fraction of farm wealth in the region due to improvements. This value includes farm buildings, and nonrecoverable machinery, as well as any infrastructure such as silos or irrigation, which is devoted exclusively to the support of farming.

Example Usage:

CHFRFIM0001

0.25 SURRY

Variable Name: VALWNF

Variable Type: Real, Scalar

Allowed Range: $\quad 1 . E-6 \leq$ value $\leq 1 . E+6$ (dollars/person)

Purpose: Defines the value of the nonfarm wealth in the region. Nonfarm wealth includes all public and private property not associated with farming that would be unusable if the region was rendered either temporarily or permanently uninhabitable. This value should include the cost of land, buildings, infrastructure, and the cost of any nonrecoverable equipment or machinery.

Example Usage:

CHVALWNF001 84000. VIRGINIA

Variable Name: FRNFIM

Variable Type: Real, scalar

Allowed Range: $\quad 0.0 \leq$ value $\leq 1.0$ (unitless)

Purpose:

Defines the fraction of nonfarm wealth in the region due to improvements. This value includes buildings and infrastructure such as roads and utilities, as well as any nonrecoverable equipment or machinery.

Example Usage:

CHFRNFIM001

0.8 


\subsection{New COMIDA2-Based Food Ingestion Model}

If the user wishes to use the newly implemented COMIDA2-based food ingestion model, this is accomplished by setting FDPATH='NEW'. When this is done, MACCS2 utilizes a binary data file written by COMIDA2, the .BIN file, which contains the results of the COMIDA2 food-chain modeling calculations. The MACCS2 user chooses which .BIN file to use with the input variable BIN_FILE.

The farmland interdiction and cost model implemented in conjunction with the COMIDA-based food chain model was developed with the intention that it be as close as possible to the farmland interdiction and cost model of WASH-1400, as preserved in the present version of MACCS2.

\subsubsection{Modeling of Agricultural Countermeasures}

Aside from the specification of the name of the binary file, only three input variables are used in the CHRONC input file to control the operation of the COMIDA2-based food-chain model. These are DOSEMILK, DOSEOTHR, and DOSELONG-loosely equivalent to the input parameters PSCMILK, PSCOTH, and GCMAXR of the MACCS food-chain model, which define the farmland interdiction criteria.

DOSEMILK and DOSEOTHR define the maximum allowable food ingestion dose from milk crops and nonmilk crops respectively during the year of the accident. DOSELONG defines the maximum allowable long-term annual dose to an individual from ingestion of the combination of milk and nonmilk crops.

The MACCS food model input variables PSCMILK, PSCOTH, and GCMAXR are specified in terms of maximum allowable ground concentrations for each food radionuclide. The new variables DOSEMIK, DOSEOTHR, and DOSELONG are specified in terms of maximum individual dose resulting from all food pathway radionuclides combined.

LASTACUM, a COMIDA2 input parameter, specifies the duration of the ingestion dose period. MACCS2 calculates the accumulated societal dose starting with the year that the land satisfies the human consumption criteria DOSEMILK and DOSEOTHR, with the exposure period ending in the LASTACUM year after the accident. The allowable range for the LASTACUM variable is from 1 to 50 years. The value of the LASTACUM variable is defined in the COMIDA2 user input file. It is communicated to MACCS2 as part of the header information on the .BIN file written by COMIDA2.

Consistent with the MACCS interdiction model, the maximum farmland interdiction period considered by MACCS2 is 8 years. Land that cannot be returned to production after 8 years is condemned. MACCS2 will also condemn land that cannot be returned to production within LASTACUM years when the COMIDA2 model is used and the LASTACUM input variable is assigned a value less than 9 .

To preserve the functionality of the MACCS food-chain model, agricultural countermeasures are always subordinate to the code's evaluation of habitability. That is, if land is uninhabitable 
because projected groundshine and resuspension doses exceed the long-term dose criterion DSCRLT, agricultural production at that location is not allowed.

Furthermore, if the resident population is never allowed to return, either because of a failure to meet the dose criterion, or because the costs of allowing the population to return would exceed the value of the property, then the farmland is also assumed to be condemned, irrespective of the projected doses from food ingestion. If the farmland is condemned, none of the additional tests described below are performed. The costs associated with the condemnation of the farmland are based on the value of the property, input variable VALWF.

If the habitability dose criterion specified by the user is met, the following tests are performed by the code to determine the level of interdiction of agricultural production. The second year following the accident, year 2, is the first year for which the long-term ingestion dose criterion (DOSELONG) is evaluated. Milk and crop disposal during the year of the accident are triggered if the habitability criterion (DSCRLT) is exceeded in the year of the accident or if the DOSELONG criterion is exceeded in year 2 following the accident. If neither DSCRLT or DOSELONG lead to the triggering of milk and nonmilk crop disposal, then the individual criteria for the first year's milk (DOSEMILK) and the first year's nonmilk crops (DOSEOTHR) are examined separately to find if either is exceeded. The disposal of milk and/or nonmilk crops is triggered if the projected ingestion dose for these two categories exceeds the DOSEMILK and/or DOSEOTHR criteria. The first-year dose criteria are evaluated using the consumption rates specified by the COMIDA2 input variable CONSUM_RATES.

Milk and crop disposal costs are calculated only for the year of the accident. Beginning in the second year after the accident, acceptability of food production is evaluated by comparing the projected individual dose for year 2 with DOSELONG.

Note: For the purposes of this section, and MACCS2, "years" are defined as successive periods with a duration of 365.25 days, each beginning on the anniversary of the fallout event. COMIDA (and COMIDA2), however, both utilize "years" with durations of exactly 365 days.

If the projected individual dose for the second year does not exceed the dose criterion DOSELONG, the production of that year's agricultural production and the production from all subsequent years is allowed. Implicit in the model is the assumption that the food doses resulting from successive years of production do not increase with time. Also, just as with the MACCS food-chain model, the long-term interdiction of farmland applies to all crop categories, and there is no provision for long-term interdiction of a subset of the crops.

If the projected individual dose from the second year of agricultural production exceeds the dose criterion, the projected doses from up to eight successive annual periods are each examined in order to determine if production can be resumed within the first 9 years after the accident.

If the projected doses in each of the years 2 through 9 exceed DOSELONG, no further tests are performed and the farmland is treated as if it were condemned. When farmland is condemned, 
the associated cost is the market value of the farmland, VALWF, with the dollar values for market value reported on the output listing as FARM DEPENDENT CONDEMNATION COST.

If the projected doses for one of the years examined satisfy the long-term dose criterion, agricultural production is assumed to resume in that year. In that case, societal ingestion doses are assessed for the period ending with the LASTACUM'th year.

For example, if DOSELONG is satisfied in year 3, and LASTACUM has a value of 10, societal doses are accrued for the period denoted as years 3-10, an exposure period with a duration of 8 years. Since agricultural production was not allowed in the first and second years, economic costs for 2 years of interdiction are assessed using the WASH-1400 economic cost model for farmland. The WASH-1400 model calculates the cost of temporary interdiction as the depreciation of the farm's land and improvements (see variables VALWF and FRFIM), and as specified by the depreciation rates for land and improvements (see input variables DSRATE and DPRATE).

\section{First-Year Crop Disposal Costs}

The economic cost of milk and/or nonmilk crop disposal during year 1 is modeled as the economic costs assessed for the loss of sales. The two crop disposal cost calculations, however, consistent with CRAC2, are treated differently, as follows.

If the disposal of the first year's milk production is triggered because the dose criterion DOSEMILK is exceeded, milk disposal costs are assessed as 0.25 of annual milk sales (see variables FRMPRD and DPFRCT). The rationale for the reduction in milk disposal costs by the application of the 0.25 adjustment factor was based on the assumption that cows would be taken off pasture and fed uncontaminated feed, allowing dairy production to resume after one-quarter of a year. In order to maintain consistency with the MACCS food-chain model, the application of the fixed 0.25 adjustment factor on milk disposal costs has also been implemented for the COMIDA2 food-chain model implemented in MACCS2.

It is noted that the model implemented in MACCS and MACCS2 for crop disposal costs, and the 0.25 fudge factor for lost dairy sales, is not based on WASH-1400 because WASH-1400 did not account for crop disposal costs. CRAC2, in contrast to WASH-1400, did implement separate milk and nonmilk crop disposal costs, providing the technical basis for the MACCS model to assess such costs. CRAC2, however, in assessing the costs of milk and nonmilk crop disposal, assessed a milk disposal cost based on the full year's dairy sales, and not the 0.25 of dairy sales used in the MACCS cost model.

The technical basis for the 0.25 adjustment factor for milk disposal is not mentioned in the MACCS Model Description, but it was based on the assumption that dairy cows spend only a fraction of the year on pasture and obtain most of their food from stored feed. The 0.25 adjustment factor is preserved in MACCS2 in order to maintain consistency with MACCS.

\subsubsection{Input Variables for COMIDA2-Based Food Model}

This section describes the specification of MACCS2 input parameters that are used to control the code's operation when the COMIDA2-based food-chain model is being used. Very few input 
variables are defined on the CHRONC input file and appear below. The majority of the input parameters of interest are defined on the COMIDA2 input files, described in Section 3 of Volume 2 of this report.

Variable Name: $\quad$ FDPATH

Variable Type: Character, Scalar

Allowed Value: 'NEW' or 'OLD'

Purpose: $\quad$ Specifies whether the "old" food-chain model of MACCS, or the "new" COMIDA2-based food model of MACCS2 is to be used in the calculations. If the value NEW is specified, the remaining data records in this section must be supplied. If the value OLD is specified, the remaining data records in this section are not processed.

Note: If NEW is specified, the user must ensure that lifetime dose ( $L$-) calculations for two organs, effective and thyroid, have been requested in the organ definition data (see Section 6.4).

Example Usage:

$\star$

CHFDPATH0O1 'NEW'

Variable Name: BIN_FILE

Variable Type: $\quad$ Character, Scalar

Allowed Range: $\quad 1 \leq$ length $\leq 40$

Purpose:

Identifies the COMIDA2 binary to be used for the MACCS2 calculations. This filename can include a directory path; the file need not be in the current directory. It must, however, be a valid filename on the host computer.

The DCF file that was used in the COMIDA2 calculations needs to be the same file that is currently being used in MACCS2. If MACCS2 detects a discrepancy by comparing the two-line header of the DCF files, an error will be diagnosed and MACCS2 execution terminated.

Example Usage:

* NAME OF THE COMIDA2 BINARY OUTPUT FILE

BIN_FILE001 'C: \COMIDA2 \COM_A.BIN'

Variable Name: $\quad$ DOSEMILK

Variable Type: Real, Array

Allowed Range: $\quad 0.0 \leq$ value $\leq 1 . \mathrm{E} 10$ (sieverts)

Purpose: $\quad$ Defines the maximum allowable food ingestion dose from milk crops during the year of the accident. This variable is intended to fulfill a purpose similar 
to that served by the variable PSCMILK of the MACCS food-chain model. The dose limit for effective dose is obtained from column 1 of the data record; the dose limit for thyroid dose is obtained from column 2 .

Variable Name: DOSEOTHR

Variable Type: Real, Array

Allowed Range: $\quad 0.0 \leq$ value $\leq 1 . E 10$ (sieverts)

Purpose:

Defines the maximum allowable food ingestion dose from nonmilk crops during the year of the accident. This variable is intended to fulfill a purpose similar to that served by the variable PSCOTHR of the MACCS food-chain model. The dose limit for effective dose is obtained from column 1 of the data record; the dose limit for thyroid dose is obtained from column 2 .

Example Usage:

$\star$

* DOSE LIMITS TRIGGERING FIRST YEAR CROP DISPOSAL OF THE SEPARATE

* MILK AND NONMILK COMPONENTS OF THE DIET, CORRESPONDING IN PURPOSE,

* AS MUCH AS POSSIBLE, TO THE MACCS 1.5 INPUT VARIABLES PSCMLK AND PSCOTH

$\star$

*

DOSEMILKO01

DOSEOTHRO01

L-EFFECTIVE

0.005

0.005
L-THYROID

0.015

0.015

Variable Name: DOSELONG

Variable Type: Real, Array

Allowed Range: $\quad 0.0 \leq$ value $\leq 1 . E 10$ (sieverts)

Purpose:

Defines the maximum allowable "long-term" annual dose to an individual from ingestion of the combination of milk and nonmilk crops when the COMIDA2-based food model is being used. These parameter values are used for determining if agricultural production is suitable for consumption in years subsequent to the year of the accident (i.e., from years 2 to LASTACUM).

If not acceptable, production is interdicted for up to LASTACUM-1 years, until such time as the dose criterion is satisfied. If LASTACUM-1 years of interdiction is insufficient, then the farmland is considered condemned. The dose limit for effective dose is obtained from column 1 of the data record; the dose limit for thyroid dose is obtained from column 2.

Example Usage:

*

* ANNUAL DOSE LIMITS FOR THE SUBSEQUENT YEAR'S ( $I . E .$, AFTER THE FIRST YEAR)

* INTERDICTION OF BOTH THE MILK AND NONMILK (COMBINED) COMPONENTS OF THE DIET

$\star$

* L-EFFECTIVE L-THYROID

$\begin{array}{ccc}\star \text { DOSELONGOO1 } & 0.005 & 0.015\end{array}$ 


\subsection{MACCS Food-Chain Model}

If the user wishes to use the unitless transfer factor food-chain model of MACCS, this is accomplished by setting FDPATH='OLD'. When that model is selected, MACCS2 performs food and water ingestion calculations in exactly the same manner as MACCS.

When FDPATH='OLD', the input parameters described in Sections 7.11.1 to 7.11.3 must be supplied, and the allowable values for those parameters are the same as for MACCS. If the COMIDA2-based ingestion model is being utilized, none of the input parameters in Sections 7.11.1 through 7.11.3 must be supplied, except for the variable FDPATH.

In the following text, the predecessor food-chain model will be referred to as the "MACCS" model and the new COMIDA2-based food-chain model will be referred to as the "MACCS2" model.

\section{Variable Name: FDPATH \\ Variable Type: Character, Scalar \\ Allowed Value: $\quad$ 'NEW' or 'OLD'}

Purpose:

Specifies whether the MACCS food-chain model or the new COMIDA2based MACCS2 food-chain model is to be used in the calculations.

Example Usage:

CHFDPATHOO1 'OLD' (USING MACCS FOOD-CHAIN MODEL)

\subsubsection{Ingestion Transfer Factors Data}

When radioactive material is deposited on land, some fraction of this material may make its way through the food chain and ultimately be consumed by man in the form of contaminated food or drinking water. The ingestion pathway is modeled in MACCS as a series of transfer processes that the material must undergo between the time of deposition and the consumption of the contaminated food products by humans; these processes decrease the amount of material passed on to the next step.

To calculate the population dose resulting from the accumulated contamination of an area, it is necessary to know the efficiency of the entire food chain taken as a whole in transferring material from the ground to man. MACCS calculates the overall efficiency of the two ingestion pathways (food and water) by multiplication of all of the individual transfer factors which are described below, yielding an overall weight sum representing the effectiveness of the pathway in transferring material from the ground to human consumption. 
Variable Name: NFICRP

Variable Type: Integer, Scalar

Allowed Range: $\quad 1 \leq$ value $\leq 10$

Purpose:

Defines the number of crop categories that will be used by the food pathway model.

Variable Name: NAMCRP

Variable Type: Character, Array

Allowed Range: $\quad 6 \leq$ length $\leq 20$

Purpose: Defines the name of a crop category used in the food pathway model. The user must supply NFICRP names in column 1 of the data block.

Note: Within the code, there is a distinction between two types of crops: those harvested at the end of the growing season, and those harvested continuously over the entire growing season. The first seven letters of the crop names supplied here are used to distinguish between these two types of crops.

If a crop's name begins with 'PASTURE', it is harvested continuously; if it doesn't, it is harvested at the end of the growing season.

Variable Name: FRCTCH

Variable Type: Real, Array

Allowed Range: $\quad 0.0 \leq$ value $\leq 1$. (unitless)

Purpose:

Specifies the fraction of the edible portion of the harvested crop that is consumed by humans. The user must supply NFICRP values for this variable in column 2 of the data block.

Variable Name: FRCTCM

Variable Type: Real, Array

Allowed Range: $\quad 0.0 \leq$ value $\leq 1$. (unitless)

Purpose:

Specifies the fraction of the edible portion of the harvested crop that is consumed by milk-producing animals. The user must supply NFICRP values for this variable in column 3 of the data block.

Variable Name:

FRCTCB

Variable Type:

Real, Array

Allowed Range:

$0.0 \leq$ value $\leq 1$. (unitless)

Purpose:

Specifies the fraction of the edible portion of the harvested crop that is consumed by meat-producing animals. The user must supply NFICRP values for this variable in column 4 of the data block.

Example Usage:

CHNFICRPOO1

7 (UP TO 10 ALLOWED) 


\begin{tabular}{|c|c|c|c|c|}
\hline $\begin{array}{l}\text { * } \\
\text { * }\end{array}$ & & CON OF CROP & $\begin{array}{l}\text { CONSUMED BY } \\
\text { DAIRY }\end{array}$ & MEAT \\
\hline & CROP NAME & MAN & ANIMALS & ANIMALS \\
\hline * & NAMCRP & FRCTCH & FRCTCM & FRCTCB \\
\hline CHCRPTBL001 & 'PASTURE' & 0.0 & 0.1 & 0.9 \\
\hline CHCRPTBLOO2 & 'STORED FORAGE' & 0.0 & 0.13 & 0.87 \\
\hline CHCRPTBLOO3 & 'GRAINS' & 0.35 & 0.04 & 0.61 \\
\hline CHCRPTBL004 & 'GRN LEAFY VEG.' & 1.0 & 0.0 & 0.0 \\
\hline CHCRPTBLOO 5 & 'OTHER FOOD CROPS' & 1.0 & 0.0 & 0.0 \\
\hline CHCRPTBLOO 6 & 'LEGUMES AND SEEDS' & 0.24 & 0.046 & 0.714 \\
\hline CHCRPTBL007 & 'ROOTS AND TUBERS' & 1.0 & 0.0 & 0.0 \\
\hline
\end{tabular}

Variable Name: NFIISO

Variable Type: Integer, Scalar

Allowed Range: $\quad$ NUMWPI $\leq$ value $\leq 10$

Purpose: $\quad$ Defines the number of radionuclides for which data will be specified for the food ingestion pathway.

Note: There is no provision for accounting for the food ingestion dose resulting from radioactive daughter products of the food pathway radionuclides defined in NAMIPI.

Variable Name: NAMIPI

Variable Type: Character, Array

Allowed Range: $\quad 3 \leq$ length $\leq 8$

Purpose: $\quad$ Defines the name of a radionuclide used in the food pathway model. The user must supply NFIISO radionuclide names in column 1 of the data block. This list must include all of the radionuclides that were specified for the drinking water pathway. The drinking water pathway radionuclides must appear first in this data list and in the same order as they occurred in the radionuclide list for the drinking water pathway, variable NAMWPI.

Variable Name: DCYPMH

Variable Type: Real, Array

Allowed Range: $\quad 0.0 \leq$ value $\leq 1.0$ (unitless)

Purpose:

Transfer factor describing the processing losses and radioactive decay that occur between the production and the consumption of milk products.

Specifically, DCYPMH is the ratio of the amount of a radionuclide in milk products at the time of consumption to the amount of that radionuclide in the milk at the time of its production (milking).

The values given here will be multiplied by the values given for TFMLK in order to define the efficiency of the milk-to-man food pathway. The user must supply NFIISO values for this variable in column 2 of the data block. 
Variable Name:

DCYPBH

Variable Type:

Real, Array

Allowed Range:

$0.0 \leq$ value $\leq 1.0$ (unitless)

Purpose:

Transfer factor describing the processing losses and radioactive decay that occur between the production and the consumption of meat products.

Specifically, DCYPBH is the ratio of the amount of a radionuclide in meat products at the time of consumption to the amount of that radionuclide in the meat at the time of its production (slaughter).

The values given here will be multiplied by the values given for TFBF in order to define the efficiency of the meat-to-man pathway. The user must supply NFISO names in column 3 of the data block.

Variable Name: TFMLK

Variable Type: $\quad$ Real, Array

Allowed Range: $\quad 0.0 \leq$ value $\leq 1.0$ (unitless)

Purpose:

Transfer factor describing how much of the material ingested by milkproducing animals ends up in milk products consumed by humans.

Specifically, TFMLK is the ratio of the amount of a radionuclide in fresh milk to the amount of the radionuclide consumed by milk-producing animals. It takes account of biological transport within the animal as well as excretion and radioactive decay.

This factor defines the fraction of the radioactive material ingested by an animal that is transferred to the milk produced by an animal. The values given here will be multiplied by the values given for DCYPMH in order to define the efficiency of the milk-to-man food pathway. The user must supply NFIISO values for this variable in column 4 of the data block.

Variable Name: $\quad$ TFBF

Variable Type: $\quad$ Real, Array

Allowed Range: $\quad 0.0 \leq$ value $\leq 1.0$ (unitless)

Purpose: $\quad$ Transfer factor describing how much of the material ingested by meatproducing animals ends up in meat products consumed by humans.

Specifically, TFBF is the ratio of the amount of a radionuclide in edible meat at the time of slaughter to the amount of the radionuclide that was consumed by the meat-producing animals. It takes account of biological transport within the animals as well as excretion and radioactive decay.

This factor defines the fraction of the radioactive material ingested by the animal that is transferred to the meat products produced from the animal. The values given here will be multiplied by the values given for DCYBH to define the efficiency of the meat-to-man food pathway. The user must supply NFIISO values for this variable in column 5 of the data block. 


\begin{tabular}{|c|c|c|c|c|}
\hline \\
\hline \\
\hline \\
\hline & & & & \\
\hline \multicolumn{5}{|c|}{$\begin{array}{l}\text { Example Usage: } \\
\text { * } \\
\text { * NUMBER OF NUCLIDES IN THE CHRONC FOOD INGESTION MODEL } \\
\text { CHNFIISOOOI } 6 \text { (UP TO } 10 \text { ALLOWED, DAUGHTER BUILDUP IS NOT TREATED) } \\
\text { * NUCLIDES THAT WERE DEFINED IN THE WATER PATHWAY DATA ABOVE MUST } \\
\text { * BE A SUBSET OF THE CHRONC INGESTION PATHWAY NUCLIDES. THE WATER } \\
\text { * PATHWA NUCLIDES MUST BE LISTED FIRST IN THIS DATA BLOCK AND IN } \\
\text { * THE SAME ORDER AS THEY WERE LISTED IN THE WATER PATHWAY DATA } \\
\text { * BLOCK } \\
\text { * }\end{array}$} \\
\hline & INGESTION & PROCESSING AND DECAY & (BQ I & INGESTED) ] \\
\hline \\
\hline & & & & \\
\hline ISODEF001 & & & & \\
\hline IISODEF & & 1.0 & 0.022 & 0.00022 \\
\hline & & & & \\
\hline DEr & & & & \\
\hline & & & & \\
\hline & $I-133$ & & & \\
\hline
\end{tabular}

Variable Name: TCROOT

Variable Type: Real, Array

Allowed Range: $\quad 0.0 \leq$ value $\leq 1.0$ (unitless)

Purpose: Defines the transfer factor for the long-term transfer of radionuclides from soil to edible crops.

Specifically, TCROOT is the ratio of the amount of a radionuclide ultimately taken up from soil into edible portions of a crop to the amount of that radionuclide that was deposited onto the soil.

The long-term transfer of radionuclides from soil to plants occurs principally by uptake (sorption) by plant root systems. Transfer to plant surfaces by rainsplash and by deposition of materials resuspended from surface soil can also contribute to this pathway. Ingestion of contaminated soil by animals grazing on pastureland may also contribute to the overall dose.

The values of TCROOT supplied in the data block should be calculated by integrating the overall uptake rate over the period from $t=0$ to infinity. In other words, TCROOT incorporates the total uptake over all time after the initial deposition. Since radionuclides are removed from the soil compartment not only by root uptake but also by radioactive decay, percolation, and irreversible chemical binding, all of these processes should be incorporated into the derivation of TCROOT. Values of TCROOT must be specified for each crop category for all radionuclides treated by the food pathway model (i.e., for all possible radionuclide/crop combinations).

The annual rate at which the material is made unavailable by these processes is specified by the input variable QROOT, defined at the end of this section. The required data are entered in a block as a two-dimensional array. The first column of the block repeats the list of food pathway radionuclides. This list must be ordered exactly the same as the original definition of the variable NAMIPI. 
All the other columns of the data block present values of TCROOT for one crop category and all food radionuclides. The order of the columns in going from left to right is assumed to be the same as is specified by NAMCRP as described earlier.

Example Usage:

*

*

*

CHTCROOTO01

CHTCROOTOO2

CHTCROOTO03

CHTCROOTOO4

CHTCROOTO05

CHTCROOT006

\begin{tabular}{lll} 
RADIONUCLIDE & \multicolumn{2}{c}{$\begin{array}{c}\text { STORED } \\
\text { PASTURE }\end{array}$} \\
FORAGE \\
NAMISO & CROOT & TCROOT \\
Sr-89 & $4.1 E-4$ & $1.3 \mathrm{E}-3$ \\
Sr-90 & $2.6 \mathrm{E}-2$ & $9.0 \mathrm{E}-2$ \\
CS-134 & $1.3 \mathrm{E}-3$ & $7.1 \mathrm{E}-4$ \\
CS-137 & $6.9 \mathrm{E}-3$ & $1.5 \mathrm{E}-3$ \\
I-131 & $1.6 \mathrm{E}-4$ & 0.0 \\
I-133 & $1.7 \mathrm{E}-6$ & 0.0
\end{tabular}

$\begin{array}{ll} & \begin{array}{l}\text { GREEN } \\ \text { LEAFY }\end{array} \\ \text { GRAINS } & \text { VEG. } \\ & \\ \text { TCROOT } & \text { TCROOT } \\ 4.3 E-5 & 1.7 E-4 \\ 3.3 E-3 & 1.3 E-2 \\ 3.5 E-5 & 1.4 E-5 \\ 7.6 E-5 & 3.0 E-5 \\ 0.0 & 0.0 \\ 0.0 & 0.0\end{array}$

OTHER
FOOD
CROPS
TCROOT
$8.6 \mathrm{E}-6$
$6.6 \mathrm{E}-4$
$1.1 \mathrm{E}-4$
$2.3 \mathrm{E}-4$
0.0
0.0

LEGUMES
AND
SEEDS
TCROOT
$3.7 E-4$
$2.8 E-2$
$9.3 E-5$
$2.0 \mathrm{E}-4$
0.0
0.0

ROOTS AND

TUBERS

TCROOT

$1.1 \mathrm{E}-4$

$8.4 \mathrm{E}-3$

$5.6 \mathrm{E}-5$

1. $2 \mathrm{E}-4$

0.0

0.0

Variable Name: $\quad$ DCYPCH

Variable Type: $\quad$ Real, Array

Allowed Range: $\quad 0.0 \leq$ value $\leq 1.0$ (unitless)

Purpose:

This factor characterizes the loss due to radioactive decay occurring between the time of harvest and the time of the crop's consumption by humans.

Specifically, DCYPCH is the ratio between the amount of a radionuclide present in a crop at the time of its consumption and the amount of that radionuclide in the crop at the time of harvest after taking account of losses due to radioactive decay. The data supplied here are used in calculating ingestion dose arising from both direct deposition onto growing crops and the long-term uptake processes of subsequent growing seasons.

This factor is applied only to crops that are directly consumed by humans (e.g., grains, vegetables, and legumes). Any values supplied for crops not consumed by humans will have no impact on the calculations. The values given here are multiplied by the corresponding values of FPLSCH, which characterizes processing losses, to obtain the overall transfer factor for this part of the food chain. Values of DCYPCH must be specified for each crop category for all radionuclides treated by the food pathway model (i.e., for all possible radionuclide/crop combinations).

The required data are entered in a block as a two-dimensional array. The first column of the block repeats the list of food pathway radionuclides. This list must be ordered exactly the same as the original definition of the variable NAMIPI. All the other columns of the data block present values of DCYPCH for one crop category and all food radionuclides. The order of the columns in going from left to right is assumed to be the same as previously specified for NAMCRP. 
Example Usage:

*

$\star$

$\star$

$\star$

$\star$

*

CHDCYPCHOO1

CHDCYPCHOO2

CHDCYPCHOO3

CHDCYPCHOO 4

CHDCYPCHOO5

CHDCYPCHO 06

\begin{tabular}{|c|c|c|c|c|c|c|c|}
\hline & & STORED & $\begin{array}{l}\text { GREEN } \\
\text { LEAFY }\end{array}$ & & $\begin{array}{l}\text { OTHER } \\
\text { FOOD }\end{array}$ & $\begin{array}{l}\text { LEGUMES } \\
\text { AND }\end{array}$ & $\begin{array}{c}\text { ROOTS } \\
\text { AND }\end{array}$ \\
\hline NUCLIDE & PASTURE & FORAGE & GRAINS & VEG. & CROPS & SEEDS & TUBERS \\
\hline NAMISO & DCYPCH & DCYPCH & DCYPCH & DCYPCH & DCYPCH & DCYPCH & DCYPCH \\
\hline$S r-89$ & 0.0 & 0.0 & 0.18 & 0.67 & 0.21 & 0.18 & 0.18 \\
\hline $\mathrm{Sr}-90$ & 0.0 & 0.0 & 0.99 & 1.0 & 0.99 & 0.99 & 0.99 \\
\hline $\mathrm{Cs}-134$ & 0.0 & 0.0 & 0.84 & 0.96 & 0.85 & 0.84 & 0.84 \\
\hline $\mathrm{Cs}-137$ & 0.0 & 0.0 & 0.99 & 1.0 & 0.99 & 0.99 & 0.99 \\
\hline$I-131$ & 0.0 & 0.0 & 0.0099 & 0.21 & 0.024 & 0.0099 & 0.0099 \\
\hline$I-133$ & 0.0 & 0.0 & 0.0 & 0.0 & 0.0 & 0.0 & 0.0 \\
\hline
\end{tabular}

Variable Name: $\quad$ DCYPCM

Variable Type: Real, Array

Allowed Range: $\quad 0.0 \leq$ value $\leq 1.0$ (unitless)

Purpose:

This factor characterizes the loss due to radioactive decay occurring between the time of harvest and the time of the crop's consumption by meat-producing animals.

Specifically, DCYPCM is the ratio of the amount of a radionuclide in the crop at the time of its consumption to the amount of that radionuclide in the crop at the time of harvest. The data supplied here are used in calculating ingestion dose arising from both direct deposition onto growing crops and the long-term uptake processes of subsequent growing seasons.

This factor is applied only to crops that are directly consumed by milk-producing animals (e.g., pasture and forage). Any values supplied for crops not consumed by milk-producing animals will have no impact on the calculations.

For pasture crops, harvest and consumption are simultaneous and so DCYPCM should be set to 1.0 for the pasture crop categories. Values of DCYPCM must be specified for each crop category for all radionuclides treated by the food pathway model (i.e., for all possible radionuclide-crop combinations).

The required data are entered in a block as a two-dimensional array. The first column of the block repeats the list of food pathway radionuclides. This list must be ordered exactly the same as the original definition of the variable NAMIPI. All the other columns of the data block present values of DCYPCM for one crop category and all food radionuclides. The order of the columns in going from left to right is assumed to be the same as previously specified for NAMCRP. 
Example Usage:

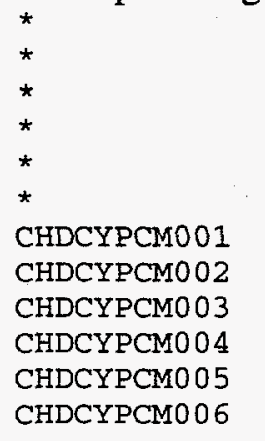

\begin{tabular}{|c|c|c|c|c|c|c|c|}
\hline \multirow{3}{*}{ NUCLIDE } & \multirow[b]{3}{*}{ PASTURE } & \multirow{3}{*}{$\begin{array}{l}\text { STORED } \\
\text { FORAGE }\end{array}$} & \multicolumn{2}{|l|}{ GREEN } & OTHER & \multirow{2}{*}{$\begin{array}{c}\text { LEGUMES } \\
\text { AND }\end{array}$} & \multirow{2}{*}{$\begin{array}{c}\text { ROOTS } \\
\text { AND }\end{array}$} \\
\hline & & & LEAFY & & FOOD & & \\
\hline & & & GRAINS & VEG. & CROPS & SEEDS & TUBERS \\
\hline NAMISO & DCYPCH & DCYPCH & DCYPCH & DCYPCH & DCYPCH & DCYPCH & DCYPCH \\
\hline $\mathrm{Sr}-89$ & 1.0 & 0.37 & 0.20 & 0.0 & 0.0 & 0.20 & 0.0 \\
\hline$S r-90$ & 1.0 & 0.99 & 0.99 & 0.0 & 0.0 & 0.99 & 0.0 \\
\hline $\mathrm{Cs}-134$ & 1.0 & 0.92 & 0.85 & 0.0 & 0.0 & 0.85 & 0.0 \\
\hline $\mathrm{Cs}-137$ & 1.0 & 0.99 & 0.99 & 0.0 & 0.0 & 0.99 & 0.0 \\
\hline$I-131$ & 1.0 & 0.063 & 0.032 & 0.0 & 0.0 & 0.032 & 0.0 \\
\hline$I-133$ & 1.0 & 0.0068 & 0.0034 & 0.0 & 0.0 & 0.0034 & 0.0 \\
\hline
\end{tabular}

Variable Name: DCYPCB

Variable Type: Real, Array

Allowed Range: $\quad 0.0 \leq$ value $\leq 1.0$ (unitless)

Purpose:

This factor specifies the retention of material after radioactive decay occurs between the time of harvest and the time of consumption by meat-producing animals.

Specifically, DCYPCB is the ratio of the amount of a radionuclide in the crop at the time of its consumption to the amount of that radionuclide in the crop at the time of harvest. This factor is applied only to crops that are directly consumed by meat-producing animals (e.g. pasture and forage). Any values supplied for crops not consumed by meat-producing animals will have no impact on the calculations.

Values of DCYPCB must be specified for each crop category for all radionuclides treated by the food pathway model (i.e., for all possible radionuclide-crop combinations). The required data are entered in a block as a two-dimensional array. The first column of the block repeats the list of food pathway radionuclides. This list must be ordered exactly the same as the original definition of the variable NAMIPI.

All the other columns of the data block present values of DCYPCB for one crop category and all food radionuclides. The order of the columns in going from left to right is assumed to be the same as previously specified for NAMCRP.

Example Usage:

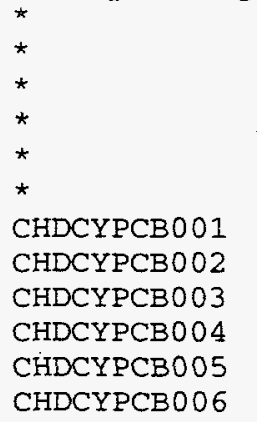

$\begin{array}{llll} & & \text { GREEN } \\ \text { NUCLIDE } & \text { PASTURE } & \begin{array}{l}\text { GORAGE } \\ \text { LEAFY }\end{array} & \text { GRAINS } \\ \text { NAMISO } & \text { DCYPCH } & \text { DCYPCH } & \text { DCYPCH } \\ \text { Sr-89 } & 1.0 & 0.37 & 0.20 \\ \text { S }-90 & 1.0 & 0.99 & 0.99 \\ \text { CS-134 } & 1.0 & 0.92 & 0.85 \\ \text { CS-137 } & 1.0 & 0.99 & 0.99 \\ I-131 & 1.0 & 0.063 & 0.032 \\ \text { I-133 } & 1.0 & 0.0068 & 0.0034\end{array}$

\begin{tabular}{|c|c|}
\hline & OTHER \\
\hline & FOOD \\
\hline VEG. & CROPS \\
\hline DCYPCH & DCYPCH \\
\hline 0.0 & 0.0 \\
\hline 0.0 & 0.0 \\
\hline 0.0 & 0.0 \\
\hline 0.0 & 0.0 \\
\hline 0.0 & 0.0 \\
\hline 0.0 & 0.0 \\
\hline
\end{tabular}

LEGUMES
AND
SEEDS
DCYPCH
0.20
0.99
0.85
0.99
0.032
0.0034

ROOTS AND TUBERS

DCYPCH

0.0

0.0

0.0

0.0

0.0

0.0 
Variable Name: $\quad$ FPLSCH

Variable Type: $\quad$ Real, Array

Allowed Range: $\quad 0.0 \leq$ value $\leq 1.0$ (unitless)

Purpose:

This factor specifies the fraction of material retained in the harvested crop after accounting for the losses resulting from its preparation for consumption by humans.

Specifically, FPLSCH is the ratio of the amount of a radionuclide in the crop after it has been processed for human consumption to the amount of that radionuclide in the crop before processing. The retention factor reflects the fraction of radioactive material in the edible portion of the plant that is retained after washing, peeling, or cooking. The values supplied here apply only to crops that are directly consumed by humans. Any values specified for crops such as pasture which are supplied here will have no impact on the calculations.

Values of FPLSCH must be specified for each crop category for all radionuclides treated by the food pathway model (i.e., for all possible radionuclide-crop combinations). The values are multiplied with the corresponding values of DCYPCH in order to obtain the overall transfer factor for this part of the food chain.

The data are entered in a block as a two-dimensional array. The first column of the block repeats the list of food pathway radionuclides. This list must be ordered exactly the same as the original definition of the variable NAMIPI. All the other columns present values of FPLSCH for one crop category and all food radionuclides. The order of the columns in going from left to right is assumed to be the same as previously specified for NAMCRP.

Example Usage:

$\star$

$\star$

$\star$

CHFPLSCHOO1

CHFPLSCHOO2

CHFPLSCHOO3

CHFPLSCHOO 4

CHFPLSCHO0 5

CHFPLSCH0O6

$\begin{array}{cccc} & & & \text { GREEN } \\ \text { NUCLIDE } & \text { PASTURE } & \text { FORAGE } & \begin{array}{l}\text { LEAFY } \\ \text { GRAINS }\end{array} \\ \text { NAMISO } & \text { DCYPCH } & \text { DCYPCH } & \text { DCYPCH } \\ \text { Sr-89 } & 0.0 & 0.0 & 0.25 \\ \text { Sr-90 } & 0.0 & 0.0 & 0.25 \\ \text { Cs-134 } & 0.0 & 0.0 & 0.25 \\ \text { Cs-137 } & 0.0 & 0.0 & 0.25 \\ \text { I-131 } & 0.0 & 0.0 & 0.33 \\ \text { I-133 } & 0.0 & 0.0 & 0.33\end{array}$

$\begin{array}{cc} & \text { OTHER } \\ \text { VEG. } & \text { COOD } \\ & \text { CROPS } \\ \text { DCYPCH } & \text { DCYPCH } \\ 0.5 & 0.71 \\ 0.5 & 0.71 \\ 0.5 & 0.71 \\ 0.5 & 0.71 \\ 0.5 & 0.71 \\ 0.5 & 0.71\end{array}$

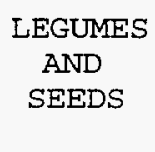

DCYPCH

0.8

0.8

0.8

0.8

0.8

0.8

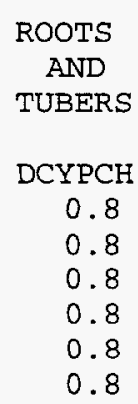

When an accident occurs during the growing season, part of the radioactive material deposited on farmland will be retained on plant surfaces and the remainder will fall on the ground. Between the time of deposition and the time of harvest, radioactive material can be lost from plant surfaces due to weathering, radioactive decay, translocation to interior portions of the plant, and the harvesting process.

The fraction of radioactive material that is removed from the air due to dry and wet deposition that ends up in edible portions of the harvested plant is here called the growing crop retention factor. Specifically, this factor is defined to be the ratio between the amount of a radionuclide 
present in the crop at harvest and the total amount of material initially deposited onto the land used for producing that crop.

For all crops except pasture, harvesting occurs at the end of the growing season. The harvesting of pasture differs from the other crops in that it is a continuous process. In MACCS it is assumed that grazing takes exactly one growing season to harvest the year's entire production of the pasture crop. The numerical integration used in the pasture dose calculations is therefore different from that used for the other crop categories. The type of integration performed by the code is determined by the name given to the crop categories. Crop names beginning with PASTURE are treated differently from the rest.

For crops where the edible portion of the plant is exposed to the environment, weathering losses over the period from deposition to harvesting will decrease the amount of radionuclides retained as a function of time. That is, the longer the time between deposition and harvesting, the lower the resultant dose.

Crops such as grains and legumes, which have the edible portions internal to the plant, may show the opposite behavior, with material being absorbed into the plant over time. Since this is a slow process and data on the translocation rate are hard to obtain, the user should try to define an average retention factor appropriate for deposition onto crops that is independent of time in the growing season, and not use the weathering model for these types of crops. The situation is further complicated by the fact that the available data suggest that total retention for grains is greatest when the deposition occurs near the middle of the growing season rather than at the end of it.

Both types of crops are modeled with a weathering equation that can have up to three exponential terms, each with a different weathering rate. For the types of crops not subject to weathering losses (that is, grains and legumes), a very long half-life can be specified for the weathering rate as a way of replacing the weathering function with a constant transfer fraction.

In MACCS the removal of radioactivity from plant surfaces by weathering is treated as a sum of terms that have the following form:

$$
\mathrm{CTCOEF} \cdot \exp (-\lambda \cdot \mathrm{t})
$$

where CTCOEF represents the fraction of material deposited on a cultivated field that is removed by weathering with a decay rate of $\lambda=0.693$ / CTHALF.

CTCOEF equals the product of two quantities: the interception fraction and the availability fraction. The interception fraction is the fraction of material deposited onto a field that is intercepted by crop surfaces and the availability fraction is the fraction of material deposited onto crop surfaces that is weathered away with the half-life CTHALF.

Because translocation from the plant surface to interior portions influences the retention of radioactivity for grains, legumes, and root crops, the weathering model just described is not appropriate for these crop categories, but as discussed in the next paragraph, the model can be 
modified through user-defined input parameters to in effect yield a transfer function that is constant with respect to time.

For the sample data presented in this section, the efficiency of grains, legumes, and root crops in transferring radioactivity was not derived in the same way as the data were derived for the other types of crops. For the grain, legume, and "other" categories, an empirical transfer factor derived from fallout studies was used in which the empirical factor represents the combined effects of interception, weathering, translocation to seeds, and root uptake.

Since long-term uptake is treated separately from the growing-season pathway, this may cause the long-term uptake dose from grains and legumes to be double counted in the first growing season. However, since root uptake in a single season is typically small relative to the contamination resulting from direct deposition, the potential impact of such a double counting is unlikely to be significant.

For convenience, this empirical factor is input as a value of CTCOEF. Since this empirical value includes the effects of weathering, the exponential part of the weathering decay expression associated with this empirical value for CTCOEF is reduced to unity by setting CTHALF to the maximum allowable value, $3.15 \mathrm{E} 13 \mathrm{~s}$.

For all food pathway radionuclides, values of CTCOEF and CTHALF are required for each term in the weathering expression for each crop category. Unnecessary weathering terms are eliminated by setting CTCOEF equal to 0 and CTHALF equal to unity for those terms.

\section{Variable Name: NTTRM}

Variable Type: Integer, Scalar

Allowed Range: $\quad 1 \leq$ value $\leq 3$

Purpose: Defines the number of terms used in the growing crop retention model.

Example Usage:

* NUMBER OF TERMS IN THE GROWING CROP RETENTION MODEL

*

CHNTRTRMO01 2

Variable Name: CTCOEF

Variable Type: $\quad$ Real, Array

Allowed Range: $\quad 0.0 \leq$ value $\leq 1.0$ (unitless)

Purpose: $\quad$ For crops with exposed edible portions, defines the product of the interception fraction and the availability fraction for each term in the weathering model. For plants with edible portions internal to the plant, defines the combined transfer fraction for interception, weathering, and translocation to seeds averaged over an entire growing season.

For each term of the weathering equation (and there can be up to three of them), values of CTCOEF must be specified for each crop category for all radionuclides treated by the food 
pathway model. The required data are entered in up to three data blocks for each of the possible terms of the weathering equation, with each of the data blocks being arranged in the same manner as the variables described earlier (FPLSCH, DCYPCH, etc.).

The values of CTCOEF entered in different data blocks are distinguished from each other by the ninth character in the record identifier. For variable CTCOEF, the record identifier has the form CHCTCOEFNMM where $\mathrm{N}$ is 1 for the first weathering term, 2 for the second weathering term, and 3 for the third weathering term, and MM varies from 01 for the first food pathway radionuclide to $\mathrm{NN}$ for the last food pathway radionuclide.

The data format for CTCOEF is identical to that used for the subsequent variable CTHALF. There should be a one-to-one correspondence between the data supplied for these two variables.

Example Usage:

*

$\star$

$\star$

CHCTCOEF 101

CHCTCOEF 102

CHCTCOEF 103

CHCTCOEF 104

CHCTCOEF10 5

CHCTCOEF106

$\star$

* TERM 2

CHCTCOEF201

CHCTCOEF 202

CHCTCOEF203

CHCTCOEF204

CHCTCOEF 205

CHCTCOEF206

$\begin{array}{lll} & & \\ \text { RADIONUCLIDE } & \text { PASTURE } & \text { FTORED } \\ \text { Fr-89 } & 0.3 & 0.2 \\ \text { Sr-90 } & 0.3 & 0.2 \\ \text { Cs-134 } & 0.3 & 0.2 \\ \text { Cs-137 } & 0.3 & 0.2 \\ I-131 & 0.3 & 0.2 \\ I-133 & 0.3 & 0.2 \\ & & \\ \text { Sr-89 } & & \\ \text { Sr-90 } & 0.076 & 0.05 \\ \text { Cs-134 } & 0.076 & 0.05 \\ \text { Cs-137 } & 0.076 & 0.05 \\ I-131 & 0.076 & 0.05 \\ I-133 & 0.076 & 0.05 \\ & 0.076 & 0.05\end{array}$

$\begin{array}{ll} & \text { GREEN } \\ \text { LEAFY } \\ \text { VEANS } & \text { VEG. } \\ 0.01 & 0.24 \\ 0.01 & 0.24 \\ 0.05 & 0.24 \\ 0.05 & 0.24 \\ 0.0 & 0.24 \\ 0.0 & 0.24 \\ & \\ 0.0 & 0.06 \\ 0.0 & 0.06 \\ 0.0 & 0.06 \\ 0.0 & 0.06 \\ 0.0 & 0.06 \\ 0.0 & 0.06\end{array}$

OTHER
FOOD
CROPS

LEGUMES
AND
SEEDS

ROOTS

SEEDS

TUBERS

$0.2 \quad 0.005$

$0.2 \quad 0.005$

0.0006

$0.2 \quad 0.01$

$0.2 \quad 0.01$

0.2

0.0

$\begin{array}{lll}0.2 & 0.0 & 0.0\end{array}$

0.0006

0.025

0.025

0.0

\subsection{5 \\ 0.05 \\ 0.05 \\ 0.05 \\ 0.05 \\ 0.05}

0.0

0.0

0.0

0.0

0.0

0.0

0.0

0.0

0.0

0.0

0.0

0.0

Variable Name:

CTHALF

Variable Type:

Real, Array

Allowed Range:

$1 . \leq$ value $\leq 1$. E14 (seconds)

Purpose:

Half-life values for the various terms of the weathering model. The values supplied for CTHALF in this data block must be properly paired with the corresponding values of CTCOEF defined in the previous data block.

For each term of the weathering equation (there can be up to three), values of CTHALF must be specified for each crop category for all radionuclides treated by the food pathway model. The required data are entered in up to three data blocks for each of the possible terms of the weathering equation, with each of the data blocks being arranged in the same manner as the variables described earlier (FPLSCH, DCYPCH, etc.).

The values of CTHALF entered in different data blocks are distinguished from each other by the ninth character in the record identifier. For variable CTHALF, the record identifier has the form CHCTHALFNMM where $\mathrm{N}$ is 1 for the first weathering term, 2 for the second weathering term, 
and 3 for the third weathering term, and MM varies from 01 for the first food pathway radionuclide to $\mathrm{NN}$ for the last food pathway radionuclide. The data format for CTHALF is identical to that used for the preceding variable CTCOEF. There should be a one-to-one correspondence between the data supplied for these two variables.

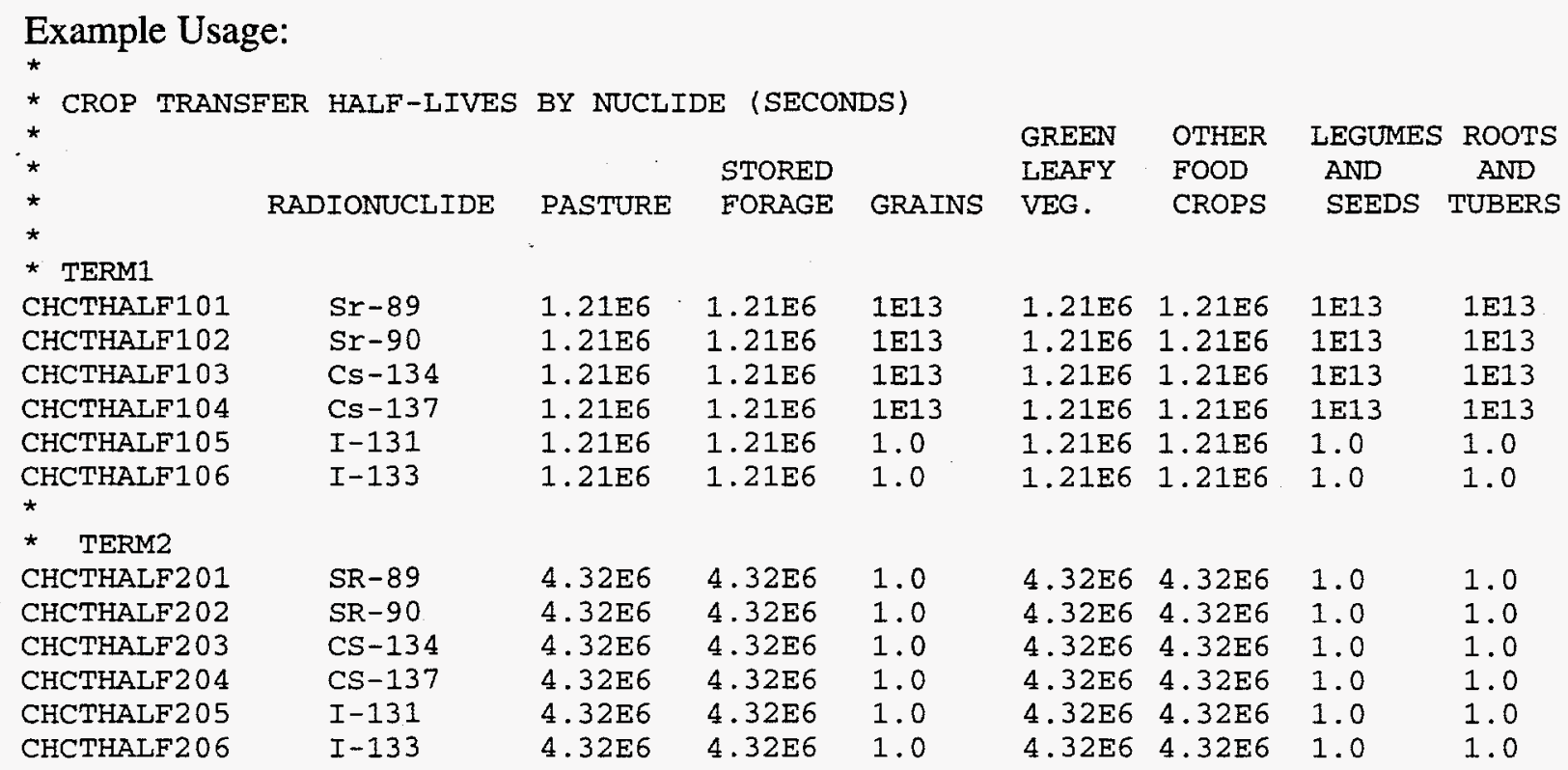

\subsubsection{Crop Share and Growing Season Data}

In the previous section, the user has defined the characteristics of a number of different crop categories with regard to their efficiency in transferring radioactive material to man through the food chain. If MACCS2 is being used without a Site Data file (see Section 6.3), the data in this section are used to supply the information necessary to calculate the doses resulting from the food ingestion pathway.

Note: When FDPATH='NEW', the data in this section must always be supplied. If the user is supplying a Site Data file (POPFLG = 'FILE' in Section 6.3), then the data supplied here will have no impact on the calculations because they will be superseded by the corresponding data on that file.

Variable Name: NAMCRP

Variable Type: $\quad$ Character, Array

Allowed Range: $\quad 6 \leq$ length $\leq 20$

Purpose:

Specifies the crop categories for which this set of growing season and farm fraction data will apply. The user must supply NFICRP crop category names in column 1 of the data block. 
Note: The list of crop names specified here must be identical to the list of names specified in the previous section.

Variable Name: TGSBEG

Variable Type: Real, Array

Allowed Range: $\quad 1.0 \leq$ value $\leq 365.0$

Purpose: Defines the start of the growing season for the named crop category in terms of the Julian day (January 1 is day 1). The user must supply NFICRP values in column 2 of the data block.

\begin{abstract}
Variable Name: TGSEND
Variable Type: Real, Array

Allowed Range: $\quad$ TGSBEG $\leq$ value $\leq 365.0$

Purpose:

Defines the end of the growing season for the named crop category in terms of the Julian day. The user must supply NFICRP values in column 3 of the data block.
\end{abstract}

\begin{tabular}{|c|c|c|c|c|}
\hline \multirow{4}{*}{$\begin{array}{l}\text { Variable Name: } \\
\text { Variable Type: } \\
\text { Allowed Range: } \\
\text { Purpose: }\end{array}$} & \multicolumn{4}{|l|}{ FRCTFL } \\
\hline & \multicolumn{4}{|l|}{ Real, Array } \\
\hline & \multicolumn{4}{|c|}{$0.0 \leq$ value $\leq 1.0$} \\
\hline & \multicolumn{4}{|c|}{$\begin{array}{l}\text { Defines the fraction of cultivated farmland that is used } \\
\text { crop category. The user must supply NFICRP values in } \\
\text { block. }\end{array}$} \\
\hline \multicolumn{5}{|l|}{$\begin{array}{l}\text { Example Usage: } \\
\star\end{array}$} \\
\hline * & NAMCRP & TGSBEG & TGSEND & FRCTFL \\
\hline CHCRPRGNO01' & 'PASTURE' & 90 . & 270 & 0.41 \\
\hline CHCRPRGNOO2 ' & 'STORED FORAGE' & 150. & 240 . & 0.13 \\
\hline CHCRPRGNO03 & ' GRAINS' & 150. & 240 . & 0.21 \\
\hline CHCRPRGNO04' & 'GRN LEAFY VEGETABLES' & 150. & 240 . & 0.002 \\
\hline CHCRPRGNO05 ' & 'OTHER FOOD CROPS' & 150. & 240 . & 0.004 \\
\hline CHCRPRGN006 & 'LEGUMES AND SEEDS' & 150. & 240 . & 0.15 \\
\hline CHCRPRGNO07' & 'ROOTS AND TUBERS' & 150 . & 240 . & 0.003 \\
\hline
\end{tabular}

\title{
7.11.3 Protective Action Guide for MACCS Food Pathway Model
}

In the event of an accident at a nuclear facility, it is likely that an assessment of the accident's impact on agricultural production in the surrounding region will be performed. Based on a projection of dose to an individual consuming locally produced food products, the local authorities will determine if these food products are safe to eat. If the food product is judged to be unsafe, two kinds of actions can be taken: disposal of already growing crops and long-term restriction of agricultural production in subsequent growing seasons. 
The MACCS food-chain model divides agricultural activities into four components representing two sets of binary pairs:

\section{MILK DIRECT-DEPOSITION CROP DIRECT-DEPOSITION MILK ROOT-UPTAKE CROP ROOT-UPTAKE}

This terminology is defined as follows. MILK refers to both fresh milk as well as to dairy products such as cheese and butter. CROP refers to all other foodstuffs. DIRECT-

DEPOSITION refers to doses that result when an accident occurs during the growing season and the doses are incurred in the single annual period following the accident. If an accident occurs outside of the growing season, the code does not evaluate the need for disposal of growing crops, and the corresponding doses from the milk and crop pathways will be reported as zero. In contrast, ROOT-UPTAKE refers to food doses that result irrespective of whether the accident occurs during the growing season, and these are calculated for an integration period with an endpoint of $t=$ infinity. These two periods may overlap. That is, for accidents that occur during the growing season, doses may be incurred from both the direct-deposition and root-uptake components of the MACCS food-chain model.

The stringency (degree of protection) for both types of mitigative actions is specified by the user through input parameters described in this section. All of these parameters are specified in terms of allowable ground concentration and will be referred to as "action guides."

For an accident that occurs during the growing season, there are two types of actions that can occur: disposal of milk and its products and disposal of crops other than milk (nonmilk crops). All agricultural production is divided between these two categories. The action guides used for determining if these two actions are necessary are the input variables PSCMLK and PSCOTH, which are defined below.

For high levels of contamination, it may be necessary to restrict agricultural production for a number of years after the accident. In order to determine if this is necessary, a single set of values for allowable ground concentration (variable GCMAXR) is used. During the long-term interdiction period, either all crops (both milk and nonmilk) can be produced or no crops can be produced.

For accidents that occur outside of the growing season, MACCS2 will, in all cases, evaluate only the long-term action guide (GCMAXR). Outside of the growing season, the growing season action guides PSCMLK and PSCOTH are never evaluated since crop disposal can only occur during the growing season.

For accidents that occur during the growing season, however, both types of criteria (growing season and long-term) may be evaluated by the code. It is up to the user to determine whether these two types of evaluation are performed either independently of each other (uncoupled) or in such a way that the exceedance of one type of criteria will automatically force the exceedance of 
the other criteria (coupled). The choice of these two options is defined by the user-specified input parameter, COUPLD, described below.

When the two types of criteria are chosen to be evaluated independently, there are no interactions between the growing season pathway and the long-term pathway. The resultant doses and economic costs from these two components of the food model are calculated in a completely independent manner.

If the user chooses the option of a coupled evaluation, the following rules define the interactions between two types of actions. Whenever the long-term criteria (GCMAXR) are exceeded, the disposal of any growing crops (both milk and nonmilk) will automatically be triggered. Alternatively, whenever both milk and nonmilk crop disposal are called for as a result of exceeding both the PSCMLK and PSCOTH criteria, the code will automatically impose at least 1 year of long-term farmland interdiction.

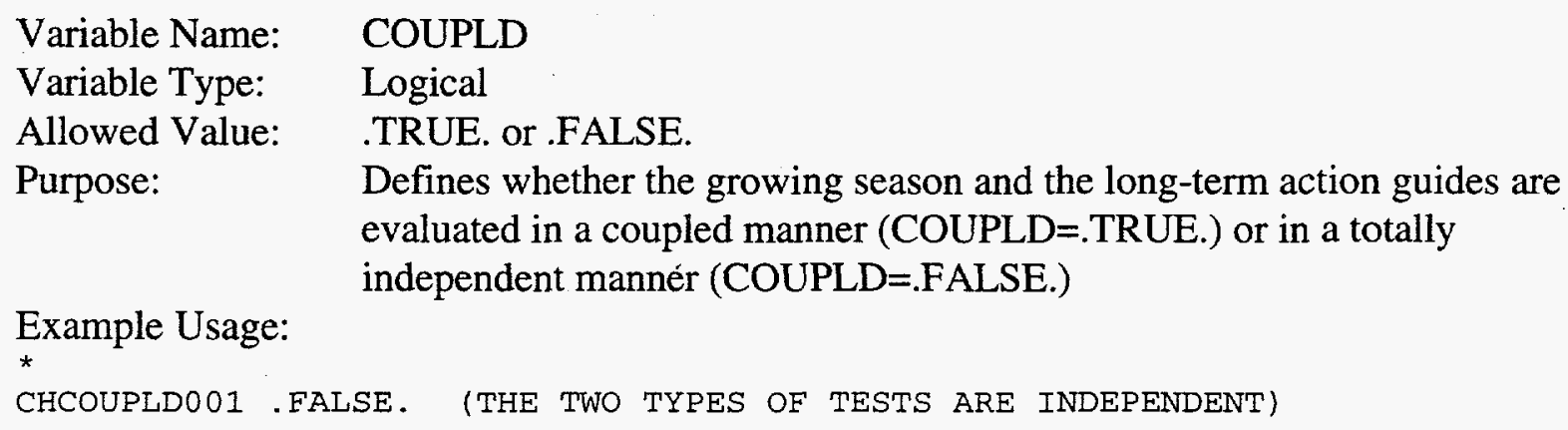

Variable Name: NAMIPI

Variable Type: $\quad$ Character, Array

Allowed Range: $\quad 3 \leq$ length $\leq 8$

Purpose: $\quad$ Defines the names of the ingestion model radionuclides for which growing season protective action guides will be specified. The user must supply all of the NFIISO names in column 1 of the data block in the same order as they were originally defined for variable NAMIPI in the previous section.

\footnotetext{
Variable Name: $\quad$ PSCMLK

Variable Type: $\quad$ Real, Array

Allowed Range: $\quad 1 . E-6 \leq$ value $\leq 1 . E 20$ (becquerels/square meter)

Purpose: $\quad$ Defines the growing season protective action guide (i.e., maximum permissible surface concentration), for milk and milk products for the named radionuclide. The user must define NFIISO protective action guide values in column 2 of the data block.
} 
Variable Name: Variable Type:

Allowed Range: Purpose:

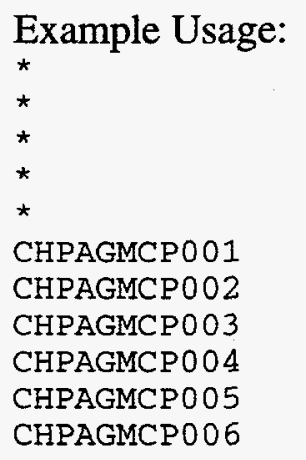

\section{PSCOTH}

Real, Array

1.E-6 $\leq$ value $\leq 1 . E 20$ (becquerels/square meter)

Defines the growing season protective action guide, maximum permissible surface concentration, for nonmilk crops and their products for the named radionuclide. The user must define NFIISO protective action guide values in column 3 of the data block.

\begin{tabular}{|c|c|c|}
\hline & & $\begin{array}{l}\text { OTHER CROPS } \\
\text { AND PRODUCTS }\end{array}$ \\
\hline NAMIPI & PSCMLK & PSCOTH \\
\hline$S r-89$ & $2.2 \mathrm{E} 07$ & 2. $2 \mathrm{E07}$ \\
\hline Sr-90 & $2.4 \mathrm{E} 05$ & $2.4 \mathrm{E} 05$ \\
\hline $\mathrm{Cs}-134$ & $2.2 \mathrm{E} 05$ & $2.2 \mathrm{E} 05$ \\
\hline $\mathrm{Cs}-137$ & $2.7 \mathrm{E} 05$ & $2.7 \mathrm{E} 05$ \\
\hline$I-131$ & 1. $3 \mathrm{E} 06$ & 8.0EO6 \\
\hline$I-133$ & $1.0 \mathrm{E} 10$ & 1. OE20 \\
\hline
\end{tabular}

The long-term uptake fractions for root uptake and soil ingestion by animals have been previously defined by variable TCROOT. These uptake fractions are integrated over all time, that is, from $t=0$ to $t=$ infinity. MACCS allows the user to define a model for the temporary interdiction of the long-term uptake pathway if certain ground contamination levels are exceeded at the time of the accident. If this model is activated, and temporary interdiction of long-term uptake is needed, the period of temporary interdiction is the shortest number of whole years that allows the long-term criteria to be met. The longest allowed period of long-term interdiction is 8 years. If 8 years of weathering and radioactive decay are insufficient, the farmland is condemned and permanently removed from production.

The data defining this interdiction model are given below. They consist of two parts: the criteria to be met for each ingestion radionuclide and a rate constant for the decrease of the radionuclide's availability over the temporary interdiction period. Values for these parameters must be supplied in the same order as originally used to define the list of radionuclides in the ingestion model, the initial definition of NAMIPI.

Variable Name: NAMIPI

Variable Type: $\quad$ Character, Array

Allowed Range: $\quad 3 \leq$ length $\leq 8$

Purpose:

Defines the name of the food ingestion radionuclide whose maximum permissible surface concentration for long-term production and weathering depletion rate are being defined in the adjacent two columns. The user must name the NFIISO food ingestion radionuclides in column 1 of the data block in the same order as they were originally defined. 
Variable Name:

Variable Type:

Allowed Range:

Purpose:

Variable Name:

Variable Type:

Allowed Range:

Purpose:
GCMAXR

Real, Array

$1 . \mathrm{E}-6 \leq$ value $\leq 1 . \mathrm{E} 35$ (becquerels/square meter)

Defines the protective action guide(i.e., maximum permissible surface concentration) for long-term crop production for the named radionuclide. The user must supply NFIISO values for this parameter in column 2 of the data block.

\section{QROOT}

Real, Array

$0.0 \leq$ value $\leq 1$.E+35 (per year)

Defines the rate constant for the decrease in availability of the named radionuclide. The following processes should be considered in the specification of the value for this parameter:

1. radioactive decay,

2. irreversible chemical binding to the soil,

3. percolation downward into the soil,

4. uptake into plants or ingestion by animals.

The value assigned to QROOT is used to determine how effective temporary interdiction of the long-term agricultural pathway will be in reducing ingestion doses. For example, if the depletion rate is 0.5 per year (i.e., $50 \%$ /annum), a year of temporary interdiction will cause the integrated dose to be $\operatorname{EXP}(-0.5)$ of what it would be if there were no interdiction.

The user must define NFIISO values for this parameter in column 3 of the data block.

Example Usage:

*

CHPAGLTSO01

CHPAGLTSOO2

CHPAGLTSO0 3

CHPAGLTSO04

CHPAGLTSO0 5

CHPAGLTSO06

$\begin{array}{lll}\text { NAMIPI } & \text { GCMAXR } & \text { QROOT } \\ \text { Sr-89 } & 1.8 \mathrm{E} 08 & 4.9 \\ \text { Sr-90 } & 3.7 \text { E04 } & 0.065 \\ \text { Cs }-134 & 4.1 \text { E06 } & 0.59 \\ \text { Cs-137 } & 1.8 E 06 & 0.28 \\ I-131 & 1.0 \text { E20 } & 32.0 \\ I-133 & 1.0 \text { E20 } & 290.0\end{array}$

\subsection{Water Ingestion Data}

When radioactive material is deposited on the ground, it is expected that some fraction of this material will make its way into drinking water consumed by humans (Helton, Muller, and Bayer, 1985). MACCS2 models this uptake into drinking water as two separate paths: (1) deposition of 
material directly onto freshwater bodies and (2) deposition of material onto land with subsequent washoff into freshwater bodies.

The water ingestion model assumes that the area surrounding the site is divided into two categories: water and land. The radioactive material deposited on a spatial element is apportioned between water and land according to the fraction of the region covered by land (see variable FRACLD in the previous section).

For coastal sites, where both fresh water and ocean water need to be treated, it is recommended that the user supply a Site Data file where the limitation of having only one kind of watershed can be overcome. A Site Data file can define up to four types of watersheds. One of those watersheds (e.g., ocean) can be defined to have a 0 uptake fraction.

The user is required to supply three numeric parameters in the following section. They define the behavior of the single watershed that is utilized if a uniform population density has been specified.

Of the material deposited directly onto water or transferred from land to water, the fraction represented by WINGF determines how much of that material will eventually be consumed by humans. There is no adjustment of WINGF within the code to account for radioactive decay.

Of the material that has been deposited on land, some fraction makes its way through runoff into the freshwater supply over a relatively short period after deposition. This fraction is specified by the value of WSHRTI.

The remainder of the material deposited on land is assumed to be washed off to the freshwater supply at a constant fractional rate over the time from $t=0$ to $t=$ infinity. The rate at which this subsequent washoff occurs is specified by the value of the rate constant WSHRTA.

The code uses the values of the two variables, WSHRTI and WSHRTA, and evaluates the integral of the washoff fraction in a way that takes account of radioactive decay for the material deposited on land surfaces. The model is described by Helton, Muller, and Bayer (1985). The evaluation of this integral produces a numeric value, $F$, which represents the fraction of material falling on land that will be eventually transferred to surface water bodies that supply drinking water.

With this value of $F$, we can now calculate the uptake fraction for material deposited on solid ground. It is simply WINGF• $F$. The input variable WINGF is the ratio between the total amount of a radionuclide consumed via the drinking water pathway (i.e., by the entire population of the region surrounding the facility) and the amount entering potable surface-water bodies. Typically, WINGF would be derived by the MACCS 2 user from a model for radionuclide movement in the surface-water system in the surrounding region.

The models used to determine WINGF can vary in complexity from the very simple (e.g., a single uniformly mixed cell) to the very complex (e.g., three-dimensional fluid transport with temporal and spatial variability). The value of WINGF supplied with the sample problems has been derived in a very simple manner. 
Note: The data in this section describing the water ingestion factors must be supplied on the CHRONC input file, but they are used in the calculations only if the user has chosen the option of a uniform population density surrounding the site (in Section 6.3, $\left.P O P F L G={ }^{\prime} U N I F O R M^{\prime}\right)$.

\section{Variable Name: NUMWPI \\ Variable Type: Integer, Scalar \\ Allowed Range: $\quad 1 \leq$ value $\leq 10$}

Purpose:

Defines the number of radionuclides to be considered in the drinking water pathway. If the MACCS food-chain model is being utilized, the drinking water radionuclides must be a subset of the food ingestion radionuclides (defined by NFIISO and NAMIPI as described in Section 7.11.1).

\section{Variable Name: $\quad$ NAMWPI}

Variable Type: Character, Array

Allowed Range: $\quad 2 \leq$ length $\leq 10$

Purpose: Defines the name of a radionuclide used in the drinking water pathway. The user must supply NUMWPI radionuclide names in column 1 of the data block.

Note: For the purpose of calculating water ingestion doses, there is no provision for modeling the buildup of any radioactive daughter products that result from decay. There is no provision for accounting for the water ingestion dose resulting from radioactive daughter products of the water pathway radionuclides defined in NAMWPI.

Variable Name:

Variable Type:

Allowed Range:

Purpose:

\section{WSHFRI}

Real, Array

$0.0 \leq$ value $\leq 1.0$ (unitless)

Defines the initial washoff fraction for the specified radionuclide. This is the fraction of material deposited on land that is easily washed off into the watershed drainage system immediately following the deposition of that radionuclide. The user must supply NUMWPI values for this variable in column 2 of the data block.

Variable Name:

WSHRTA

Variable Type:

Allowed Range:

Real, Array

Purpose:
$0.0 \leq$ value $\leq 1.0$ (per year)

Defines the annual washoff rate for the specified radionuclide. This is the rate at which material deposited on land is washed off into the watershed drainage system following the initial deposition. The user must supply NUMWPI values for this variable in column 3 of the data block. 
Variable Name: WINGF

Variable Type: Real, Array

Allowed Range: $\quad 0.0 \leq$ value $\leq 1.0$ (unitless)

Purpose:

Defines the water ingestion factor for the radionuclide specified in column 1 of the data block. This factor specifies the fraction of the radioactivity washed into the drainage system of the watershed that is ultimately consumed by humans. The user must supply NUMWPI values for this variable in column 4 of the data block.

Note: If a site file is being used (POPFLG=FILE), the value of WINGF is not used by the code; nevertheless a valid parameter value must be supplied.

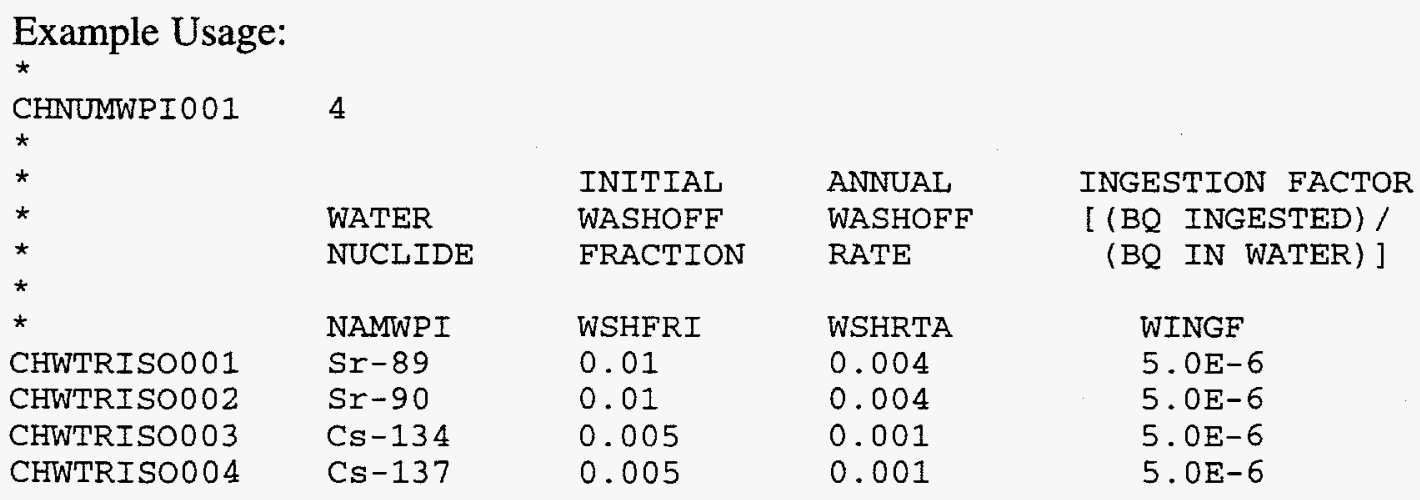

\subsection{Diagnostic Trace Option}

The diagnostic options data block allows the user to print a detailed listing of values for intermediate variables used in CHRONC calculations. The output for this option is written to the output file. Most of the variables output are CHRONC internal FORTRAN variables. The user will need to examine the definitions of these variables provided in the MACCS2 FORTRAN coding in order to fully understand the output listing generated with this option.

Variable Name: KSWTCH

Variable Type: Integer, Scalar

Allowed Range: $\quad 0 \leq$ value $\leq 1$

Purpose:

Defines the set of special diagnostics to be printed by CHRONC. This variable is used to enable the generation and printing of intermediate calculations on the output listing. It is recommended that the user set the value of each of these options to 0 for normal calculations. Specification of any value greater than 0 will generate a large amount of printed output. Therefore, the trace output option should be used only for single weather trial runs. 
Example Usage:

CHKSWTCHOO1 1 (TURNS ON DEBUG PRINT STATEMENTS FOR CHRONC)

\subsection{Population Dose Results}

The CHRONC module calculates the long-term population dose broken down by pathway for a list of organs defined by the user through the EARLY input file (see Section 6.4). The option to examine this breakdown of long-term population doses for organs selected from the set of available organs is controlled by the user as defined in this section. The breakdown of long-term population dose results has no corresponding result in the EARLY module. The EARLY module produces only one consequence measure relating to population dose, the Type 5 result, total population dose.

No long-term population dose results for a defined cancer organ are produced on the output listing unless the user specifically requests them. Each request for the breakdown of the long-term population dose to an organ produces the block of either 12 or 15 dose results (depending on which food model is being used) identified below. All of the dose results are reported in person-sieverts although the units are listed simply as sieverts (Sv) in the output file.

TOTAL LONG-TERM PATHWAYS DOSE-total long-term population dose from groundshine and resuspension, from the consumption of contaminated food, from the ingestion of contaminated surface water, and from decontamination work.

LONG-TERM DIRECT EXPOSURE PATHWAYS-total long-term population dose to resident population from groundshine and resuspension.

TOTAL INGESTION PATHWAYS DOSE-total long-term population dose from the consumption of contaminated dairy products, contaminated nondairy products, and contaminated water.

LONG-TERM GROUNDSHINE DOSE-total long-term population dose received by resident population from groundshine.

LONG-TERM RESUSPENSION DOSE-total long-term population dose received by resident population from resuspension.

POP.-DEPENDENT DECONTAMINATION DOSE-total long-term population dose received from groundshine by workers performing "population dependent" (nonfarm) decontamination (decontamination workers receive no inhalation dose).

FARM-DEPENDENT DECONTAMINATION DOSE-total long-term population dose received from groundshine by workers performing "farm dependent" (farmland) decontamination (decontamination workers receive no inhalation dose). 
WATER INGESTION DOSE-total long-term population dose from ingestion of contaminated surface water.

If the MACCS food-chain model is being utilized, the following food pathway results are reported:

MILK GROWING SEASON DOSE-total long-term population dose resulting from consumption of milk and dairy products contaminated as a result of deposition onto crops during the growing season.

CROP GROWING SEASON DOSE-total long-term population dose resulting from consumption of nonmilk food products contaminated as a result of deposition onto crops during the growing season.

MILK LONG-TERM DOSE-total long-term population dose resulting from consumption of milk and dairy products contaminated by long-term uptake in the period following the accident.

CROP LONG-TERM DOSE-total long-term population dose resulting from consumption of nondairy crops and their products contaminated by long-term uptake in the period following the accident.

If the COMIDA2 food-chain model is being utilized, the following food pathway results are reported:

INGESTION OF GRAINS-total long-term population dose resulting from consumption of grains by humans.

INGESTION OF LEAF VEG-total long-term population dose resulting from consumption of leafy vegetables by humans.

INGESTION OF ROOT CROPS-total long-term population dose resulting from consumption of root crops by humans.

INGESTION OF FRUITS-total long-term population dose resulting from consumption of fruits by humans.

INGESTION OF LEGUMES-total long-term population dose resulting from consumption of legumes by humans.

INGESTION OF BEEF-total long-term population dose resulting from consumption of beef by humans.

INGESTION OF MILK-total long-term population dose resulting from consumption of milk by humans.

INGESTION OF POULTRY - total long-term population dose resulting from consumption of poultry by humans. 
INGESTION OF OTHER MEAT CROPS-total long-term population dose resulting from consumption of other meat crops by humans.

$\begin{array}{ll}\text { Variable Name: } & \text { NXUM9 } \\ \text { Variable Type: } & \text { Integer, Scalar } \\ \text { Allowed Range: } & 0 \leq \text { value } \leq 10 \\ \text { Purpose: } & \begin{array}{l}\text { Specifies the number of long-term population dose result blocks to be } \\ \text { printed. }\end{array}\end{array}$

Variable Name: $\quad$ ORGNAM

Variable Type: $\quad$ Character, Scalar

Allowed Range: $\quad 2 \leq$ length $\leq 10$

Purpose: Defines the name of the organ for which the long-term dose breakdown is to be reported. The user must supply NXUM9 organ names in column 1 of the data block.

Variable Name: $\quad$ IX1DS9

Variable Type: Integer, Scalar

Allowed Range: $\quad 1 \leq$ value $\leq$ NUMRAD

Purpose:

Defines the inner spatial interval of the region of interest for this result

block. The user must supply NXUM9 values in column 2 of the data block.

Variable Name: IX2DS9

Variable Type: Integer, Scalar

Allowed Range: $\quad$ IX1DS9 $\leq$ value $\leq$ NUMRAD

Purpose:

Defines the outer spatial interval of the region of interest for this result

block. The user must supply NXUM9 values in column 3 of the data block.

Note: The region of interest, as specified earlier, is used to determine the size of the potentially contaminated area being evaluated. In the context of this consequence measure, the population dose within a region is the population dose that occurs as a result of material deposited within the region. For the direct exposure pathways of groundshine and resuspension, the dose is received by the resident population, but for the indirect exposure pathways of ingestion and decontamination, the dose could be received by individuals who reside elsewhere.

In order to obtain the CCDF tables of a consequence measure requested in this section, append the character string ' $C C D F^{\prime}$ ' to the line requesting that result as the fourth item on the data card. The CCDF tables will be printed on the output file (unit 6). 


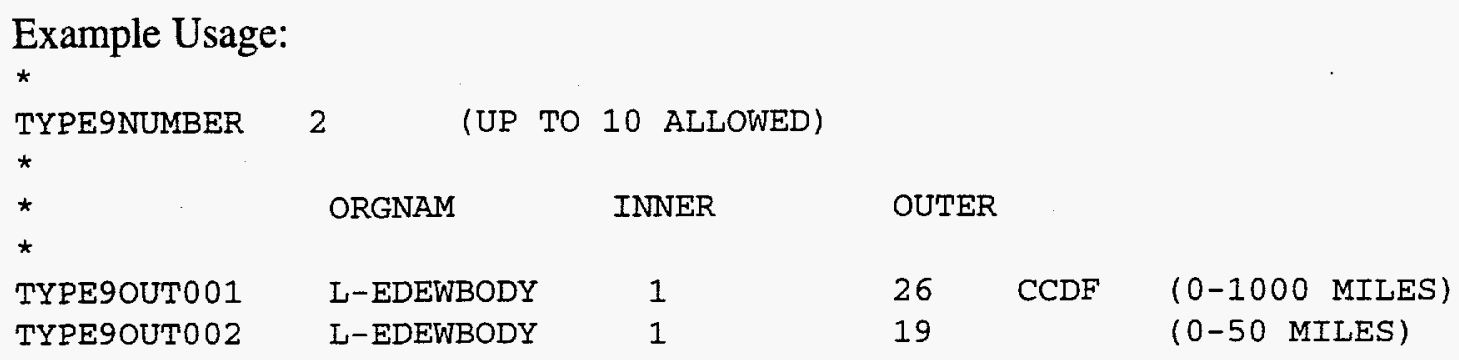

\subsection{Economic Cost Results}

The CHRONC module calculates the economic costs of all the long-term protective actions as well as the cost of the emergency response actions that were modeled by EARLY. The option to print these economic results is controlled by the user.

No economic costs are printed unless the user specifically requests them. Each request for economic results produces the block of 13 economic results described below. All of the economic cost measures are reported in dollars.

TOTAL ECONOMIC COSTS-the sum of population- and farm-dependent costs.

POP.-DEPENDENT COSTS-the sum of population-dependent decontamination, interdiction, and condemnation costs.

FARM-DEPENDENT COSTS-the sum of farm-dependent decontamination, interdiction, and condemnation costs as well as milk and crop disposal costs.

POP.-DEPENDENT DECONTAMINATION COST—nonfarm property (i.e., property associated with resident population) decontamination cost.

FARM-DEPENDENT DECONTAMINATION COST_farm property decontamination cost.

POP.-DEPENDENT INTERDICTION COST-depreciation and deterioration of nonfarm property during the period it cannot be used during both decontamination and interdiction plus the cost of population removal (see POPCST in Section 7.6).

FARM-DEPENDENT INTERDICTION COST-depreciation and deterioration of farm property during the period it cannot be used during both decontamination and interdiction.

POP.-DEPENDENT CONDEMNATION COST-compensation paid for permanent loss of nonfarm property plus the cost of population removal.

FARM-DEPENDENT CONDEMNATION COST - compensation paid for permanent loss of farm property because it could not be returned to production within 8 years of the accident.

EMERGENCY PHASE COSTS-per-diem costs to compensate people for being away from home due to evacuation and relocation during the emergency phase. 
Note: When more than one emergency-response scenario is being evaluated by the EARLY module, the presentation of evacuation and relocation cost is calculated on the basis of the scenario that was defined last in sequence on the EARLY input file.

INTERMEDIATE PHASE COSTS—per-diem costs to compensate people for being away from home due to relocation for the duration of the intermediate phase if DSCRTI is exceeded.

MILK DISPOSAL COSTS-compensation for lost milk sales during a quarter of a year if the first year's crops require disposal. This cost is incurred if the accident occurs during the growing season and any of the following conditions are found:

1. the growing-season milk action guide is exceeded, or

2. any decontamination actions are required, or

3. (for MACCS food model only) if COUPLD=.TRUE. and any long-term interdiction is required.

CROP DISPOSAL COSTS-compensation for lost nonmilk crop sales during a full year. This cost is incurred if the accident occurs during the growing season and any of the following conditions are found:

1. the growing-season nonmilk action guide is exceeded, or

2. any decontamination actions are required, or

3. (for MACCS food model only) if COUPLD=.TRUE. and any long-term interdiction is required.

Variable Name: NXUM10

Variable Type: Integer, Scalar

Allowed Range: $\quad 0 \leq$ value $\leq 10$

Purpose: Specifies the number of economic result blocks to be printed.

Variable Name: I1DS10

Variable Type: Integer, Scalar

Allowed Range: $\quad 1 \leq$ value $\leq$ NUMRAD

Purpose:

Defines the inner spatial interval of the region of interest for this result block. The user must supply NXUM10 values in column 1 of the data block.

Variable Name: I2DS10

Variable Type: Integer, Scalar

Allowed Range: $\quad$ I1DS10 $\leq$ value $\leq$ NUMRAD

Purpose:

Defines the outer spatial interval of the region of interest for this result block. The user must supply NXUM10 values in column 2 of the data block. 
Note: In order to obtain the CCDF tables of a consequence measure requested in this section, append the character string ' $C C D F^{\prime}$ ' to the line requesting that result as the third item on the data record. The CCDF tables will be printed on the output file.

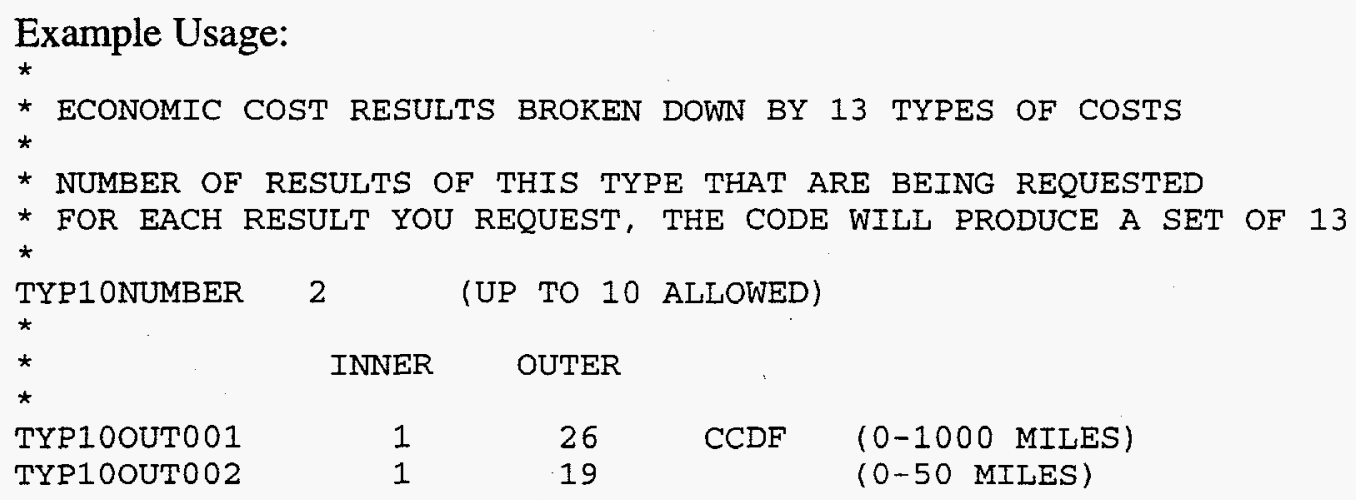

\subsection{Action Distance Results}

The long-term protective actions that result from the calculations of the CHRONC module depend on the data supplied by the user. Associated with the long-term actions of decontamination, interdiction, and crop disposal are the maximum distances to which these actions are implemented. The user must specify whether these maximum action distance results are to be printed.

The option to print or not print these long-term action distances is controlled by a flag specified by the user. The flag value.TRUE. will produce the eight maximum action distance results that are described below. Each result is identified by the result name used on the output file together with a description of the result. All of the distances are reported in kilometers.

FARM-DEPENDENT DECONTAMINATION DIST.-maximum distance at which farmland decontamination is required.

POP.-DEPENDENT DECONTAMINATION DIST.-maximum distance at which nonfarmland decontamination is required.

FARM-DEPENDENT INTERDICTION DIST.-maximum distance at which farmland decontamination or interdiction is required.

POP.-DEPENDENT INTERDICTION DIST.-maximum distance at which nonfarmland decontamination or interdiction is required.

FARM-DEPENDENT CONDEMNATION DIST.-maximum distance at which farmland condemnation is required.

POP.-DEPENDENT CONDEMNATION DIST.-maximum distance at which nonfarmland condemnation is required. 
MILK DISPOSAL DIST.-maximum distance at which the loss of 3 months of milk and dairy products sales is required.

CROP DISPOSAL DIST.-maximum distance at which the loss of 1 year of nonmilk crop sales is required.

The flag value .FALSE. will eliminate the maximum action distance results from the output.

Variable Name: FLAG11

Variable Type: Logical, Scalar

Allowed Value: $\quad$.TRUE. or .FALSE.

Purpose: $\quad$ Specifies the print flag for the maximum action distance results.

Note: In order to obtain the CCDF tables of a consequence measure requested in this section, append the character string ' $C C D F^{\prime}$ 'to the line requesting that result as the second item on the data record. The CCDF tables will be printed on the output file.

Example Usage:

*

TYP1IFLAG11 . TRUE.

\subsection{Impacted Area/Population Results}

The long-term protective actions that result from the calculations of the CHRONC module depend on the data supplied by the user. Associated with the long-term actions of decontamination, interdiction, condemnation, and crop disposal are the farm areas and populations that are affected by these actions. The option to print these impacted area/population results is controlled by the user.

No impacted farm-area/population results are printed unless the user specifically requests them. Each request for impacted farm-area/population results produces the block of eight results identified below. All farm-area results are reported in hectares and all population results are reported as number of individuals.

FARM DECONTAMINATION (HECTARES)—area within which farmland decontamination was required.

POP. DECONTAMINATION (INDIVIDUALS) - population of areas that required decontamination of nonfarm property.

FARM INTERDICTION (HECTARES)—farmland area which required either decontamination or interdiction.

POP. INTERDICTION (INDIVIDUALS)—population of areas that required either decontamination or interdiction of nonfarm property. 
FARM CONDEMNATION (HECTARES)-area within which farmland condemnation was required.

POP. CONDEMNATION (INDIVIDUALS)—population of areas that required condemnation of nonfarm property.

MILK DISPOSAL AREA (HECTARES)—affected area requiring the loss of milk and dairy products sales for 3 months.

CROP DISPOSAL AREA (HECTARES)—affected area requiring the loss of nonmilk crop sales for a year.

Variable Name: NUM12

Variable Type: Integer, Scalar

Allowed Range: $\quad 0 \leq$ value $\leq 10$

Purpose: $\quad$ Specifies the number of impacted farm-area/population result blocks to be printed.

Variable Name: I1DS12

Variable Type: Integer, Scalar

Allowed Range: $\quad 1 \leq$ value $\leq$ NUMRAD

Purpose: Defines the inner spatial interval of the region of interest for this result block. The user must supply NUM12 values in column 1 of the data block.

Variable Name: I2DS12

Variable Type: Integer, Scalar

Allowed Range: $\quad$ I1DS12 $\leq$ value $\leq$ NUMRAD

Purpose: $\quad$ Defines the outer spatial interval of the region of interest for this result block. The user must supply NUM12 values in column 2 of the data block.

Note: In order to obtain the CCDF tables of a consequence measure requested in this section, append the character string ' $C C D F$ ' to the line requesting that result as the third item on the data record. The CCDF tables will be printed on the output file.

Example Usage:

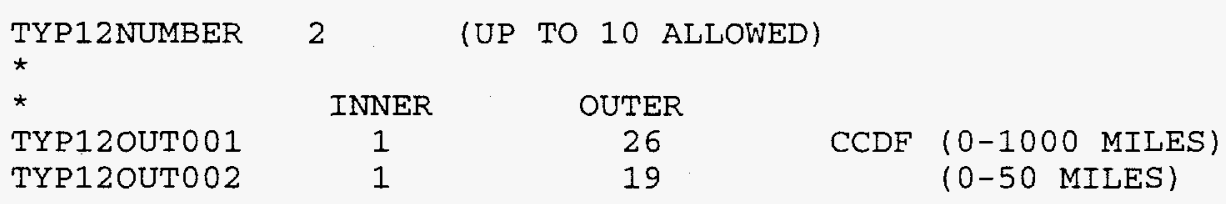




\subsection{Maximum Individual Food Ingestion Dose at a Distance}

This result is available only when the COMIDA2-based food model option is specified (FDPATH='NEW'). If requested, MACCS2 will report statistics on the maximum food ingestion dose calculated for the 16 wind directions within a user-specified spatial interval, for effective dose or thyroid dose. No other organs are available for this result.

The maximum dose is the dose calculated using the food consumption rates specified in the COMIDA2 input file for a representative individual. The projected doses in years 1 through 9 are examined in turn, and the maximum value found is used in generating this result.

Variable Name: NUM13

Variable Type: Integer, Scalar

Allowed Range: $\quad 0 \leq$ value $\leq 20$

Purpose: $\quad$ Specifies the number of maximum dose from food ingestion result blocks to be calculated.

Variable Name: IRAD13

Variable Type: Integer, Scalar

Allowed Range: $\quad 1 \leq$ value $\leq$ NUMRAD

Purpose:

Defines the spatial interval of the distance of interest for this result block.

The user must supply NUM13 values in column 1 of the data block.

Variable Name: ORGN13

Variable Type: Character, Scalar

Allowed Value: 'EFFECTIVE' or 'THYROID'

Purpose: Defines the organ to be used for each requested result. The user must supply NUM13 values in column 2 of the data block.

Note: In order to obtain the CCDF tables of a consequence measure requested in this section, append the character string ' $C C D F^{\prime}$ to the line requesting that result as the third item on the data record. The CCDF tables will be printed on the list output file.

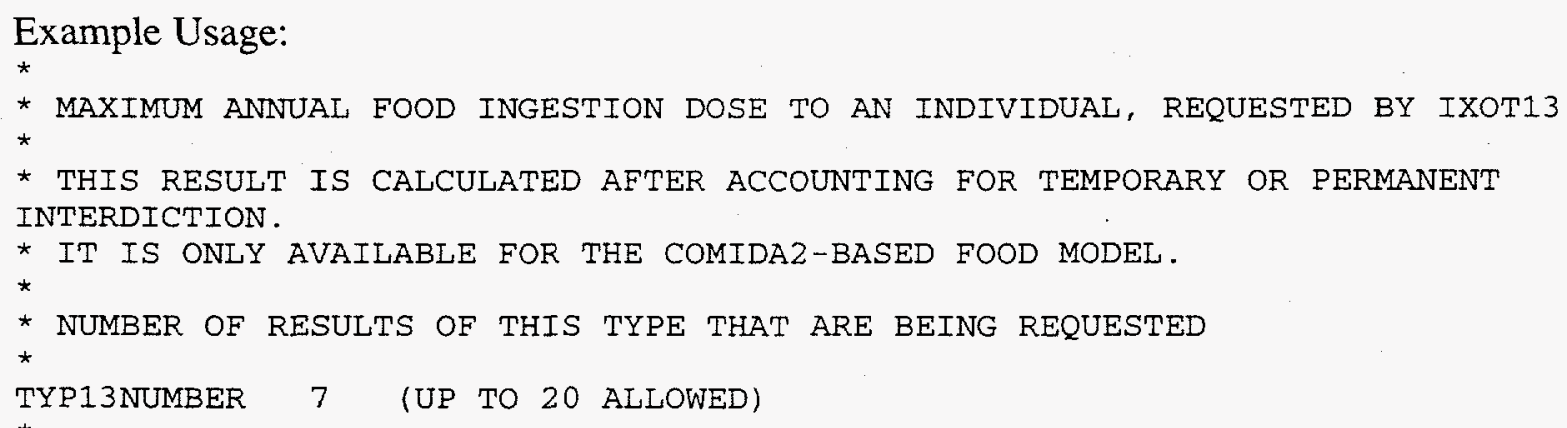




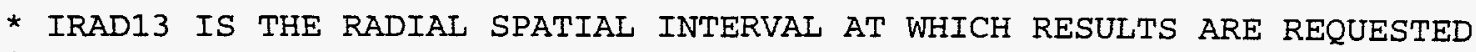

* ORGN13 IS THE NAME OF THE ORGAN FOR WHICH RESULTS ARE REQUESTED

* (ALLOWABLE VALUES For ORGN13 ARE 'EFFECTIVE' OR 'THYROID')

$\star$

TYP130UT001

TYP130UT002

TYP13OUT003

TYP130UT004

TYP130UT005

TYP130UT006

TYP130UT007

$\operatorname{IRAD13}$

1

2

3

4

5

1

2
ORGN13

EFFECTIVE

EFFECTIVE

EFFECTIVE

EFFECTIVE

EFFECTIVE

THYROID

THYROID 


\section{References}

Abbott, M. L., and A. S. Rood (1993), COMIDA: A Radionuclide Food-Chain Model for Acute Fallout Deposition, EGG-GEO-10367, available from OSTI, Idaho National Engineering Laboratory, Idaho Falls, ID.

Abbott, M. L., and A. S. Rood (1994), "COMIDA: A Radionuclide Food-Chain Model," Health Physics, Vol. 66, No. 1, pp. 17-29.

Abrahamson, S., M. A. Bender, B. B. Boecker, E. S. Gilbert, and B. R. Scott (1991), Health Effects Models for Nuclear Power Plant Accident Consequence Analysis, Modifications of Models Resulting from Recent Reports of Health Effects of Ionizing Radiation, Low LET Radiation, Part II: Scientific Bases for Health Effects Models, NUREG/CR-4214, Rev. 1, Part II, Addendum 1, LMF-132, Inhalation Toxicology Research Institute, Albuquerque, NM.

Aldrich, D. C., J. L. Sprung, D. J. Alpert, K. Diegert, R. M. Ostmeyer, L. T. Ritchie, and D. R. Strip (1982), Technical Guidance for Siting Criteria Development, NUREG/CR-2239, SAND81-1539, Sandia National Laboratories, Albuquerque, NM.

Alonso, A., and E. Gallego (1987), Cost-Effectiveness Analysis of Countermeasures Using Accident Consequence Assessment Models, CTN-36-87, Universidad Politécnica de Madrid, Madrid, Spain.

Alonso, A., E. Gallego, and J. E. Martin (1990), The Modeling of Off-Site Economic Consequences of Nuclear Accidents, CTN-29-90, Universidad Politécnica de Madrid, Madrid, Spain.

Alpert, D. J., D. I. Chanin, and L. T. Ritchie (1987), "Relative Importance of Individual Elements to LWR Accident Consequence Estimates Using Equal Release Fractions," Nuclear Safety, Vol. 28, No. 1, pp. 77-86.

American Nuclear Society and Institute of Electrical and Electronic Engineers (1983), PRA Procedures Guide, NUREG/CR-2300, Nuclear Regulatory Commission, Washington, DC.

Amos, C. N., W. H. Baker, M. D. Brandyberry, D. P. Kearnaghan, K. R. O'Kula, J. J. Weingardt, and N. D. Woody (1991), Savannah River Site Reactor Safety Assessment, WSRC-RP91-041-DRAFT, Westinghouse Savannah River Company, Aiken, SC.

American National Standards Institute (1966), FORTRAN, ANSI X3.9-1966, New York.

American National Standards Institute (1978), Programming Language FORTRAN, ANSI X3.91978, ISO 1539-1980(E), New York. 
Birchall, A. (1986), "A Microcomputer Algorithm for Solving Compartmental Models Involving Radionuclide Transformations," Health Physics, Vol. 50, No. 3 (March), pp. 389-397.

Briggs, G. A. (1975), "Plume Rise Predictions," in Lectures on Air Pollution and Environmental Impact Analysis, Workshop proceedings, American Meteorological Association, Boston, MA.

Briggs, G. A. (1984), "Plume Rise and Buoyancy Effects," in Atmospheric Science and Power Production, DOE/TIC-27601, Department of Energy, Washington, DC.

Brown, T. D., A. C. Payne, Jr., L. A. Miller, J. D. Johnson, D. I. Chanin, A. W. Shiver, S. J. Higgins, and T. T. Sype (1993), Integrated Risk Assessment for the LaSalle Unit 2 Nuclear Power Plant, NUREG/CR-5305, SAND90-2765, Vol. 2, Part 2, Sandia National Laboratories, Albuquerque, NM.

Brown, T. D., L. N. Kmetyk, D. Whitehead, J. Forester, L. Miller, and J. Johnson (1995), Evaluation of Potential Severe Accidents During Low Power and Shutdown Operations at Grand Gulf, Unit 1, NUREG/CR-6143, SAND93-2440, Vol. 6, Part 1, Sandia National Laboratories, Albuquerque, NM.

Bureau of the Census (1994), Statistical Abstract of the United States: 1994, Government Printing Office, Washington, DC.

Camp, A. L., D. M. Kunsman, L. A. Miller, J. L. Sprung, T. A. Wheeler, and G. D. Wyss (1990), Level III Probabilistic Risk Assessment for N Reactor, WHC-MR-0045, SAND89-2102, Westinghouse Hanford Company, Richland, WA.

Chanin, D. I. (1992), A New Emergency Response Model for MACCS, LA-SUB-94-67, DE94017051, available from NTIS, Los Alamos National Laboratory, Los Alamos, NM.

Chanin, D. I., and M. L. Young (1997), Code Manual for MACCS2. Vol. 2, Pre-Processor Codes COMIDA2, FGRDCF, IDCF2, to appear as SAND report, Sandia National Laboratories, Albuquerque, NM.

Chanin, D. I., J. L. Sprung, L. T. Ritchie, and H.-N. Jow (1990), MELCOR Accident Consequence Code System (MACCS), User's Guide, NUREG/CR-4691, SAND86-1562, Vol. 1, Sandia National Laboratories, Albuquerque, NM.

Chanin, D., J. Rollstin, J. Foster, and L. Miller (1993), MACCS Version 1.5.11.1: A Maintenance Release of the Code, NUREG/CR-6059, SAND92-2146, Sandia National Laboratories, Albuquerque, NM.

Commission of the European Communities (1991), COSYMA: A New Programme Package for Accident Consequence Assessment, EUR 13028, Commission of the European Communities, Luxembourg. 
Dobbe, C. A., E. R. Carlson, N. H. Marshall, E. S. Marwil, and J. E. Tolli (1990), Quality Assurance and Verification of the MACCS Code, Version 1.5, NUREG/CR-5376, Idaho National Engineering Laboratory, Idaho Falls, ID.

Dobbins, R. A. (1979), Atmospheric Motion and Air Pollution, John Wiley \& Sons, New York.

DOE (1988), External Dose-Rate Conversion Factors for Calculation of Dose to the Public, DOE/EH 0070, DE88 014691, Washington, DC.

DOE (1989), General Design Criteria, Order 6430.1A, issued April 6, 1989, Department of Energy, Washington, DC.

DOE (1991a), Draft Environmental Impact Statement for the Siting, Construction, and Operation of New Production Reactor Capacity, DOE/EIS-0144D, Washington, DC.

DOE (1991b), Unreviewed Safety Questions, Order 5480.21, issued December 24, 1991, Department of Energy, Washington, DC.

DOE (1992a), "National Environmental Policy Act Implementing Procedures: Final Rule, 10 CFR 1021," Federal Register, Vol. 57, pp. 15122-15159, April 24, 1992.

DOE (1992b), Safety Analysis Reports, Order 5480.23, issued April 30, 1992, Department of Energy, Washington, DC.

DOE (1993), Guidelines for Preparation of Environmental Impact Statements and Environmental Assessments, no doc. number, issued May 27, 1993, NEPA Oversight Office, Department of Energy, Washington, DC.

DOE (1994a), F-Canyon Plutonium Solutions Environmental Impact Statement, DOE/EIS-0219, Department of Energy, Aiken, SC.

DOE (1994b), Environmental Assessment for the Proposed Interim Storage of Highly Enriched Uranium Above the Maximum Historical Storage Level at the Y-12 Plant, Oak Ridge, TN, DOE/EA-0929, Department of Energy, Oak Ridge, TN.

DOE (1995a), Dual Axis Radiographic Hydrodynamic Test Facility Final Environmental Impact Statement, DOE/EIS-0228, Department of Energy, Albuquerque, NM.

DOE (1995b), Final Programmatic Environmental Impact Statement for Tritium Supply and Recycling, DOE/EIS-0161, Department of Energy, Washington, DC.

DOE (1995c), Disposition of Surplus Highly Enriched Uranium Draft Environmental Impact Statement, DOE/EIS-0240-D, Department of Energy, Washington, DC.

DOE (1996a), Final Environmental Impact Statement on a Proposed Nuclear Weapons Nonproliferation Policy Concerning Foreign Research Reactor Spent Nuclear Fuel, DOE/EIS-0218F, Department of Energy, Washington, DC. 
DOE (1996b), Draft Programmatic Environmental Impact Statement for Stockpile Stewardship and Management, DOE/EIS-0236, Department of Energy, Washington, DC.

DOE (1996c), Draft Environmental Impact Statement for the Continued Operation of the Pantex Plant and Associated Storage of Nuclear Weapon Components, DOE/EIS-0225D, Department of Energy, Washington, DC.

DOE (1996d), Storage and Disposition of Weapons-Usable Fissile Materials Draft Programmatic Environmental Impact Statement, DOE/EIS-0229-D, Department of Energy, Washington, DC.

DOE (1996e), "National Environmental Policy Act Implementing Procedures: Proposed Rule, 10 CFR 1021," Federal Register, Vol. 61, No. 34, p. 6413 et seq., February 20, 1996.

East, J. M. (1996), Benchmark of MACCS2 Against MACCS Using DWPF SAR Chapter 9 Integrated Risk Analyses, WSRC-RP-94-61, Westinghouse Savannah River Company, Aiken, SC.

Eckerman, K. F., and J. C. Ryman (1993), External Exposure to Radionuclides in Air, Water, and Soil, Federal Guidance Report 12, EPA 402-R-93-081, Environmental Protection Agency, Washington, DC.

Eckerman, K. F., A. B. Wolbarst, and A. C. B. Richardson (1989), Limiting Values for Radionuclide Intake and Air Concentration and Dose Conversion Factors for Inhalation, Submersion, and Ingestion, Federal Guidance Report 11, EPA 520/1-88-020, second printing with corrections, Environmental Protection Agency, Washington, DC.

EPA (Environmental Protection Agency) (1991), Human Health Evaluation Manual, Supplemental Guidance: Standard Default Exposure Factors, OSWER Directive 9285.603, PB91-921314, Washington, DC.

EPA (Environmental Protection Agency) (1992), Manual of Protective Action Guides and Protective Actions for Nuclear Incidents, EPA 400-R-92-001, PB92-164763, Washington, DC.

EPA (Environmental Protection Agency) (1994), Estimating Radiogenic Cancer Risks, EPA 402-R-93-076, Washington, DC.

Evans, J. S. (1990), Health Effects Models for Nuclear Power Plant Accident Consequence Analysis, Part I, Introduction, Integration, and Summary, NUREG/CR-4214, SAND85-7185, Rev. 1, Part I, Sandia National Laboratories, Albuquerque, NM.

Evans, J. S., D. W. Moeller, and D. W. Cooper (1985), Health Effects Model for Nuclear Power Plant Accident Consequence Analysis, Parts I and II, NUREG/CR-4214, SAND85-7185, Sandia National Laboratories, Albuquerque, NM. 
Fetter, S. (1988), Internal Dose Conversion Factors for 19 Target Organs and 9 Irradiation Times and External Dose-Rate Conversion Factors for 21 Target Organs for 259 Radionuclides Produced in Potential Fusion Materials, EGG-FSP-8036, Idaho National Engineering Laboratory, Idaho Falls, ID.

Fetter, S. (1991), Internal Dose Conversion Factors for 19 Target Organs and 9 Irradiation Times and External Dose-Rate Conversion Factors for 21 Target Organs for 144 Radionuclides, EGG-FSP-9875, Idaho National Engineering Laboratory, Idaho Falls, ID.

Fisher, S. E., and K. E. Lenox (1995), An Assessment of the Radiological Doses Resulting from Accidental Uranium Aerosol Releases and Fission Product Releases from a Postulated Criticality Accident at the Oak Ridge Y-12 Plant, ORNL/TM-12782, Oak Ridge Y-12 Plant, Oak Ridge, TN.

Gale, H. J., D. L. O. Miller, and E. M. R. Fisher (1964), "Weathering of Caesium-137 in Soil," Nature, Vol. 201, pp. 257-261.

Gifford, F. J. (1960), "Atmospheric Dispersion Calculations Using the Generalized Gaussian Plume Model," Nuclear Safety, Vol. 2, No. 2, pp. 56-59.

Gifford, F. J. (1976), "Turbulent Diffusion-Typing Schemes: A Review," Nuclear Safety, Vol. 1, No. 1 , pp. $68-86$.

Hall, D. J., and R. A. Waters (1986), Further Experiments on a Buoyant Emission from a Building, LR 567 (PA), Warren Spring Laboratory, Stevenage, England.

Hanna, S. R., G. A. Briggs, and R. P. Hosker, Jr. (1982), Handbook on Atmospheric Diffusion, DOE/TIC-11223, Department of Energy, Washington, DC.

Hanson, A. L., R. E. Davis, and V. Mubayi (1994), Calculations in Support of a Potential Definition of a Large Release, NUREG/CR-6094, Brookhaven National Laboratory, Upton, NY.

Harper, F. T., S. C. Hora, M. L. Young, L. A. Miller, C. H. Lui, M. D. McKay, J. C. Helton, L. H. J. Goossens, R. M. Cooke, J. Päsler-Sauer, B. Kraan, and J. A. Jones (1995), Probabilistic Accident Consequence Uncertainty Analysis: Dispersion and Deposition Uncertainty Assessment, Vols. 1-3, NUREG/CR-6244, EUR 15855EN, Commission of the European Communities, Brussels-Luxembourg.

Helton, J. C., A. B. Muller, and A. Bayer (1985), "Contamination of Surface Water Bodies After Reactor Accidents by Erosion of Atmospherically Deposited Radionuclides," Health Physics, Vol. 48, No. 6, p. 757.

Helton, J. C., et al. (1989), "An Uncertainty/Sensitivity Analysis of MACCS Code Predictions for Severe Nuclear Reactor Accident Consequences," SAND88-1688c, Proceedings: PSA '89-International Topical Meeting on Probability, Reliability and Safety Assessment, Sandia National Laboratories, Albuquerque, NM. 
Helton, J. C., J. D. Johnson, J. A. Rollstin, A. W. Shiver, and J. L. Sprung (1995a), Uncertainty and Sensitivity Analysis of Chronic Exposure Results with the MACCS Reactor Accident Consequence Model, NUREG/CR-6134, SAND93-2370, Sandia National Laboratories, Albuquerque, NM.

Helton, J. C., J. D. Johnson, M. D. McKay, A. W. Shiver, and J. L. Sprung (1995b), Uncertainty and Sensitivity Analysis of Early Exposure Results with the MACCS Reactor Accident Consequence Model, NUREG/CR-6135, SAND93-2371, Sandia National Laboratories, Albuquerque, NM.

Helton, J. C., J. D. Johnson, J. A. Rollstin, A. W. Shiver, and J. L. Sprung (1995c), Uncertainty and Sensitivity Analysis of Food Exposure Results with the MACCS Reactor Accident Consequence Model, NUREG/CR-6136, SAND93-2372, Sandia National Laboratories, Albuquerque, NM.

Helton, J. C., J. D. Johnson, and J. L. Sprung (1995d), "Robustness of an Uncertainty and Sensitivity Analysis of Early Exposure Results with the MACCS Reactor Accident Consequence Model," Reliability Engineering and System Safety, Vol. 48, No. 2, p. 129.

International Commission on Radiological Protection (1977), Recommendations of the International Commission on Radiological Protection, Vol. 1, No. 3, ICRP 26, Pergamon Press, New York.

International Commission on Radiological Protection (1989), "Age-dependent Doses to Members of the Public from Intake of Radionuclides: Part 1," Annals of the ICRP, ICRP Publication 56, Vol. 20, No. 2, Pergamon Press, New York.

International Commission on Radiological Protection (1991), "1990 Recommendations of the International Commission on Radiological Protection," Annals of the ICRP, ICRP Publication 60, Vol. 21, Nos. 1-3, Pergamon Press, New York.

Jones, J. A. (1983), Models to Allow for the Effects of Coastal Sites, Plume Rise and Buildings on Dispersion of Radionuclides and Guidance on the Value of Deposition Velocity and Washout Coefficients, NRPB-R157, National Radiological Protection Board, England.

Jones, K. R., C. A. Dobbe, and D. L. Knudson (1991), INEL Personal Computer Version of MACCS 1.5, NUREG/CR-5667, Idaho National Engineering Laboratory, Idaho Falls, ID.

Jow, H.-N., J. L. Sprung, J. A. Rollstin, L. T. Ritchie, and D. I. Chanin (1990), MELCOR Accident Consequence Code System (MACCS), Model Description, NUREG/CR-4691, SAND86-1562, Vol. 2, Sandia National Laboratories, Albuquerque, NM.

Kahaner, D., C. Moler, and S. Nash (1989) Numerical Methods and Software, Prentice Hall, Englewood Cliffs, NJ.

Kaplan, S., and B. J. Garrick (1981), "On the Quantitative Definition of Risk," Risk Analysis, Vol. 1, No. 1, pp. 11-27. 
Lonergan, S. C., R. L. Goble, and C. Cororaton (1990), "Estimating the Financial Consequences of a Severe Reactor Accident in Canada," Energy, Vol. 15, No. 6, pp. 507-522.

Mubayi, V., V. Sailor, and G. Anandalingam (1995), Cost-Benefit Considerations in Regulatory Analysis, NUREG/CR-6349, Brookhaven National Laboratory, Upton, NY.

National Academy of Sciences (1990), Health Effects of Exposure to Low Levels of Ionizing Radiation: BEIR V, National Research Council, National Academy Press, Washington, DC.

Neuhauser, K. S., and F. L. Kanipe (1996), RADTRAN 4: Vol. 2, Technical Manual, SAND892370, Sandia National Laboratories, Albuquerque, NM.

Neymotin, L. (1994), Comparisons of MACCS Users Calculations for the International Comparison Exercise on Probabilistic Consequence Assessment Codes, NUREG/CR6053, Brookhaven National Laboratory, Upton, NY.

NRC (1975), Reactor Safety Study: Calculation of Reactor Accident Consequences, WASH1400, NUREG 75/014, Appendix 6, Nuclear Regulatory Commission, Washington, DC.

NRC (1983), Atmospheric Dispersion Models for Potential Accident Consequence Assessment at Nuclear Power Plants, Reg. Guide 1.145, Rev. 1, Nuclear Regulatory Commission, Washington, DC.

NRC (1986), "Safety Goals for the Operations of Nuclear Power Plants; Policy Statement," Federal Register, Vol. 51, No. 149, August 4, 1986, Nuclear Regulatory Commission, Washington, DC

NRC (1987), Reactor Risk Reference Document, NUREG-1150 (public draft for comment), Nuclear Regulatory Commission, Washington, DC.

NRC (1990), Formulation of a Large Release Definition and Supporting Rationale, SECY-90405, Policy Issue (Notation Vote), December 14, 1990, Nuclear Regulatory Commission, Washington, DC.

NRC (1991), Severe Accident Risks: An Assessment for Five Commercial Nuclear Power Plants, NUREG-1150, Nuclear Regulatory Commission, Washington, DC.

Nuclear Energy Agency of the Organization for Economic Cooperation and Development (NEA OECD), and the Commission of the European Communities (1994), Probabilistic Accident Consequence Assessment Codes: Second International Comparison Overview Report, Paris, France.

O'Kula, K. R. (1991), "Recommendations for MACCS 1.5 Improvements: Modifications to Improve Applicability to DOE Facilities," SRL-PRA-910018, Letter Report to Ken Murphy of DOE/DP dated January 15, 1991, Westinghouse Savannah River Company, Aiken, SC. 
O'Kula, K. R., and J. M. East (1990), Comparison of MACCS 1.4 and 1.5 With Basis Data from the Savannah River SID, WSRC-RP-90-1322, Rev. 0, Westinghouse Savannah River Company, Aiken, SC.

O'Kula, K. R., and Z. T. Mendoza (1989), SRP PRA of Reactor Operation-Level 3 Phase 1 OffSite Consequence Analysis, DPST-88-1043, Savannah River Laboratory, Aiken, SC [UCNI report].

Ostmeyer, R. M. (1986), An Approach to Estimating Food Ingestion Exposures for Nuclear Waste Transportation Accidents, SAND85-1722, Sandia National Laboratories, Albuquerque, NM.

Panitz, H.-J. (1989), Accident Consequence Assessments with Different Atmospheric Dispersion Models, A Benchmark Study, KfK 4445, Kerforschungszentrum, Karlsruhe, Germany.

Payne, A. C., Jr. (1992), Analysis of the LaSalle Unit 2 Nuclear Power Plant: Risk Methods Integration and Evaluation Program (RMIEP), NUREG/CR-4832, SAND92-0537, Vol. 1, Sandia National Laboratories, Albuquerque, NM.

Peterson, V. L. (1993a), Population Distribution and Building Specifications Relevant to Atmospheric Modeling for Rocky Flats Plant, 1993, NSTR-016-93, Rocky Flats Plant, Golden, $\mathrm{CO}$ [report not for public distribution: may contain UCNI].

Peterson, V. L. (1993b), Reference Computations of Public Dose and Cancer Risk from Airborne Releases of Plutonium, RFP-4910, Rocky Flats Plant, Golden, CO.

Peterson, V. L. (1994a), Verification and Validation of the MELCOR Accident Consequence Code System (MACCS), NSTR-017-94, Rev. 0, Rocky Flats Plant, Golden, CO.

Peterson, V. L. (1994b), Tools and Methodology for Collocated-Worker Consequence Assessments, RFP-4911, Rev. 0, Rocky Flats Plant, Golden, CO.

Peterson, V. L. (1995), Reference Computations of Public Dose and Cancer Risk from Airborne Releases of Uranium and Class W Plutonium, RFP-4965, Rev. 0, Rocky Flats Plant, Golden, CO.

Radiation Shielding Information Center (1994), FGR-DOSE: Dose Coefficients from Federal Guidance Reports 11 and 12, DLC-167, Oak Ridge National Laboratory, Oak Ridge, TN.

Ramsdell, J. V., Jr. (1990), "Diffusion in Building Wakes for Ground-Level Releases," Atmospheric Environment, Vol. 24B, No. 3, pp. 377-388.

Raytheon Systems Services (1995), Draft Safety Analysis Report for the Device Assembly Facility at the Nevada Test Site, DAF-SAR-001-193-5394C, M. H. Chew \& Associates, Livermore, CA [UCNI report]. 
Ritchie, L. T., J. D. Johnson, and R. M. Blond (1983), Calculations of Reactor Accident Consequences Version 2 CRAC2: Computer Code, User's Guide, NUREG/CR-2326, SAND81-1994, Sandia National Laboratories, Albuquerque, NM.

Ritchie, L. T., D. J. Alpert, R. P. Burke, J. D. Johnson, R. M. Ostmeyer, D. C. Aldrich, and R. M. Blond (1984), CRAC2 Model Description, NUREG/CR-2552, SAND82-0342, Sandia National Laboratories, Albuquerque, NM.

Rollstin, J.A., D. I. Chanin, and H.-N. Jow (1990), MELCOR Accident Consequence Code System (MACCS), Programmer's Reference Manual, NUREG/CR-4691, SAND86-1562, Sandia National Laboratories, Albuquerque, NM.

Rood, A. S., and M. L. Abbott (1991), A Comparison of Dose and Dose-Rate Conversion Factors from the Soviet Union, United Kingdom, U.S. Department of Energy, and the Idaho National Engineering Laboratory Fusion Safety Program, EGG-FSP-9865, Idaho National Engineering Laboratory, Idaho Falls, ID.

Runkle, G. E., and R. M. Ostmeyer (1985), An Assessment of Dosimetry Data for Accidental Radionuclide Releases from Nuclear Reactors, NUREG/CR-4185, SAND85-0283, Sandia National Laboratories, Albuquerque, NM.

Science Applications International Corporation (SAIC) (1993), U.S. Department of Energy Defense Programs Safety Survey Report-Vol. I: Main Report, DOE/DP-70056-H1, D. Pinkston, ed., SAIC, Germantown, MD.

Skrable, K., C. French, G. Chabot, and A. Major (1974), "A General Equation for the Kinetics of Linear First Order Phenomena and Suggested Applications," Health Physics, Vol. 27 (July), pp. 155-157.

Snell, W. G., and R. W. Jubach (1981), Technical Basis for Regulatory Guide 1.145: Atmospheric Dispersion Models for Potential Accident Consequence Assessments at Nuclear Power Plants, NUREG/CR-2260, NUS Corp.

Sprung, J. L., J. A. Rollstin, J. C. Helton, and H.-N. Jow (1990), Evaluation of Severe Accident Risks: Quantification of Major Input Parameters, MACCS Input, NUREG/CR-4551, SAND86-1309, Vol. 2, Rev. 1, Part 7, Sandia National Laboratories, Albuquerque, NM.

Stone and Webster (1991), FSAR Review Team Report on Rocky Flats Plant Building 707, no doc. number, Stone \& Webster Engineering Corp., Boston, MA [UCNI report].

Systems Applications, Inc. (1982), Survey of Plume Models for Atmospheric Application, EPRI EA-2243, Electric Power Research Institute, Palo Alto, CA.

Tadmor, J., and Y. Gur (1969), "Analytical Expressions for the Vertical and Lateral Dispersion Coefficients in Atmospheric Diffusion," Atmospheric Environment, Vol. 3, pp. 688-689. 
Turner, D. B. (1970), Workbook of Atmospheric Dispersion Estimates, PSH-999-AP-26, Department of Health, Education, and Welfare, Washington, DC.

Tveten, U. (1990a), Radionuclide Behavior in the Environment, NUREG/CR-5304, SAND907116, Institutt for Energiteknikk, Kjeller, Norway.

Tveten, U. (1990b), Review of the Chronic Exposure Pathway Models in MACCS and Several Other Well-Known Probabilistic Risk Assessment Models, NUREG/CR-5377, Institutt for Energiteknikk, Kjeller, Norway.

Tveten, U. (1995), "Use of Post-Chernobyl Data from Norway to Validate the Long-Term Exposure Models of the Accident Consequence Code MACCS," Nuclear Technology, Vol. 65, No. 3, p. 322.

Vogt, K.-J. (1977), "Empirical Investigations of the Diffusion of Waste Air Plumes in the Atmosphere," Nuclear Technology, Vol. 34, pp. 43-57.

Wenzel, D. R. (1994), The Radiological Safety Analysis Computer Program (RSAC-5) User's Manual, WINCO-1123, Rev. 1, Idaho National Engineering Laboratory, Idaho Falls, ID.

Westinghouse Savannah River Company (1990), Reactor Operation Safety Information Document, WSRC-RP-89-820, Westinghouse Savannah River Company, Aiken, SC.

Whicker, F. W., and T. B. Kirchner (1987), "PATHWAY: A Dynamic Food-Chain Model to Predict Radionuclide Ingestion After Fallout Deposition," Health Physics, Vol. 52, No. 6, pp. 717-737.

Whicker, F. W., T. B. Kirchner, D. D. Breshears, and M. D. Otis (1990), "Estimation of Radionuclide Ingestion: The PATHWAY Food-Chain Model," Health Physics, Vol. 59, pp. 645-657.

Young, M. (1994), Evaluation of Population Density and Distribution Criteria in Nuclear Power Plant Siting, SAND93-0848, Sandia National Laboratories, Albuquerque, NM.

Young, M., D. Chanin, and V. Banjac (1997), DOSFAC2: A Code That Calculates Dose Factors for MACCS, in press, to appear as a NUREG/CR and SAND report, Sandia National Laboratories, Albuquerque, NM.

Yuan, Y. C., S. Y. Chen, B. M. Biwer, and D. J. LePoire (1995), RISKIND—A Computer Program for Calculating Radiological Consequences and Health Risks from Transportation of Spent Nuclear Fuel, ANL/EAD-1, Argonne National Laboratory, Argonne, IL. 


\section{Appendix A: Auxiliary Input Files}

\section{A.1 Meteorological Data File Format}

For METCOD values of 1,2 or 5 (see Section 5.13), a meteorological data file must be provided by the user. The file consists of 1 year of hourly recordings (8760) of the wind direction, wind speed, atmospheric stability, and accumulated precipitation. Generally, the data are taken from either the facility site or from a nearby weather station.

The meteorological data file used in MACCS2 is a formatted text file. The format of this file is identical to that used with MACCS and similar to that used in the CRAC2 code.

There are a total of 8763 records in the meteorological data file. The first two records contain identification information. Up to 80 characters may be used on each line. This header information is printed on the output listing. If an additional identification record is added at the beginning of the file, CRAC2 meteorological data files may be used with MACCS2. Records 3 through 8762 contain hourly meteorological observations, one hour per line. The hourly meteorological data is input as integers. The format of the information is as follows:

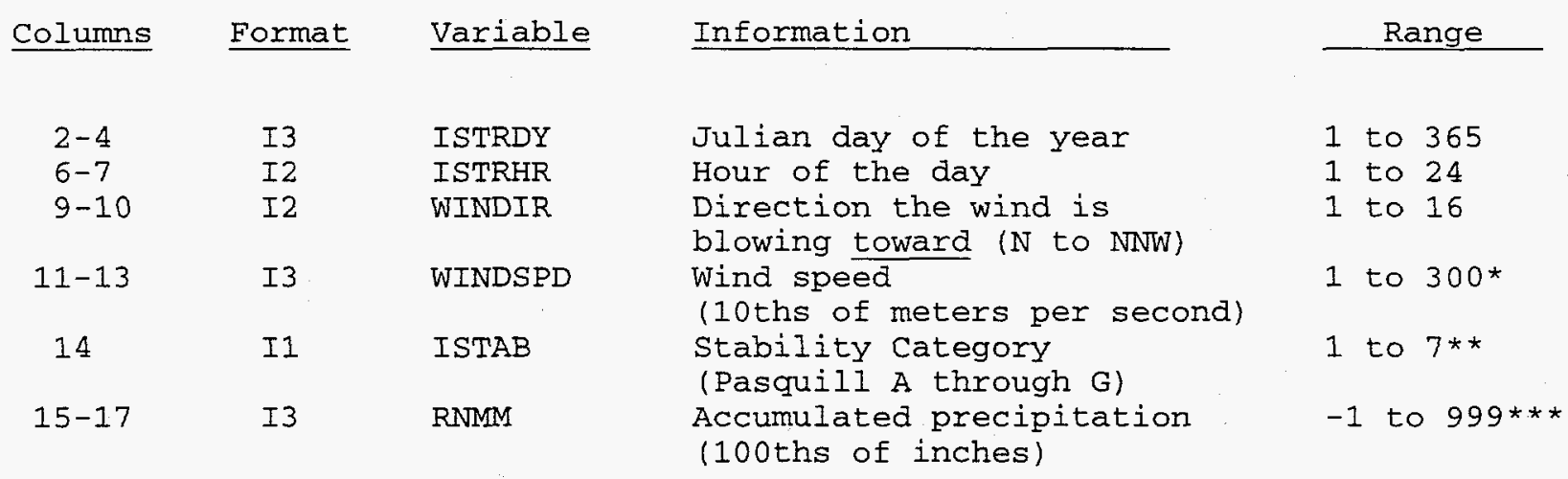

*Values between 1 and 4 are automatically changed to $5(0.5 \mathrm{~m} / \mathrm{s})$.

**A value of 7 is automatically changed to 6 by the code.

$* * *$ Some meteorological data files use -1 to indicate a trace of precipitation during the hour. MACCS2 assumes these values to be 0 .

The 8763 rd record contains a table of eight values of mixing layer height. Two values of mixing height are supplied for each of the four seasons of the year. The first of these two values corresponds to the morning mixing height and the second to the afternoon height. In the current implementation, the larger of these two values and the value of the boundary weather mixing height is used by the code.

In its present form, that atmospheric model implemented in MACCS2 does not allow a change in mixing layer to occur during transport of the plume. Mixing layer height is assumed to be 
constant and therefore only a single value is used by the code. Only seasonal variability can be considered for this parameter. The start day of each weather sequence determines the season in which that sequence lies.

Note: The following data are supplied in units of hundreds of meters and as real numbers.

\begin{tabular}{|c|c|c|}
\hline Columns & Format & Variable \\
\hline $1-10$ & F10.0 & $\operatorname{HEIGHT}(1,1)$ \\
\hline $11-20$ & F10.0 & $\operatorname{HEIGHT}(2,1)$ \\
\hline $21-30$ & F10.0 & $\operatorname{HEIGHT}(3,1)$ \\
\hline $31-40$ & F10.0 & $\operatorname{HEIGHT}(4,1)$ \\
\hline $41-50$ & F10.0 & $\operatorname{HEIGHT}(1,2)$ \\
\hline $51-60$ & F10.0 & $\operatorname{HEIGHT}(2,2)$ \\
\hline $61-70$ & F10.0 & $\operatorname{HEIGHT}(3,2)$ \\
\hline $71-80$ & F10.0 & $\operatorname{HEIGHT}(4,2)$ \\
\hline
\end{tabular}

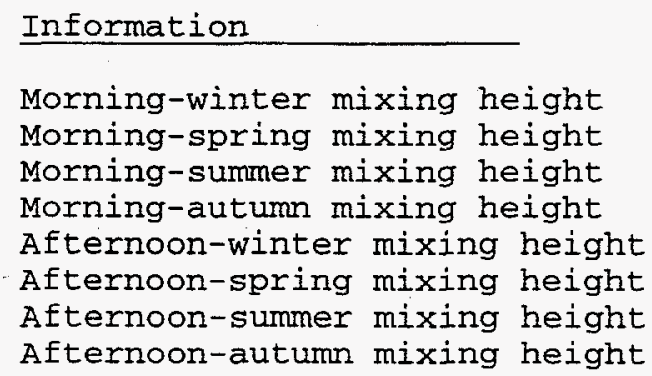

\begin{tabular}{l} 
Range \\
\hline 0. to 999. \\
0. to 999. \\
0. to 999. \\
0. to 999. \\
0. to 999. \\
0. to 999. \\
0. to 999. \\
0. to 999.
\end{tabular}

A sample set of records from the meteorological data file is provided in Figure A-1, which shows the first and last ten records of the file. For example, the weather conditions for day one, hour one (record number three) are: wind direction-blowing toward the SE, wind speed of $2.6 \mathrm{~m} / \mathrm{s}$, stability 4 (category D), and accumulated precipitation of 0.07 inches $(1.8 \mathrm{~mm})$ in the hour. The winter, spring, summer, and autumn mixing heights for neutral and unstable conditions (record 8763 ) are all $1200 \mathrm{~m}$.

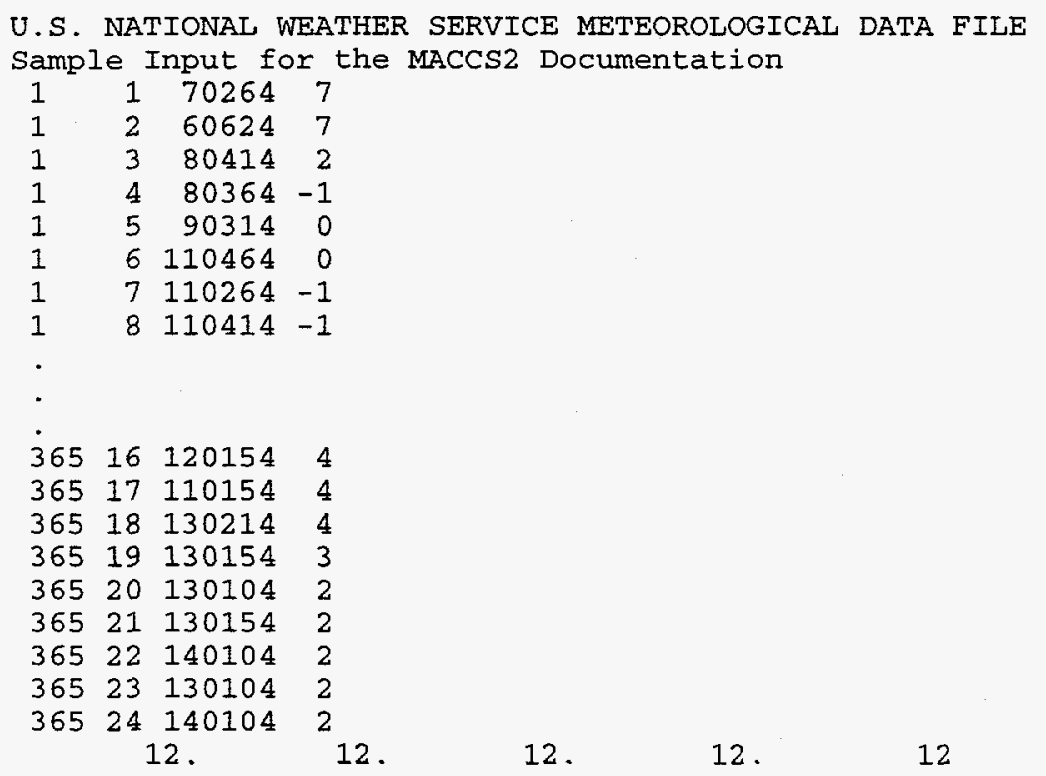

Figure A-1. The first and last ten records from a MACCS/MACCS2 meterological file. 


\section{A.2 Dose Conversion Factors File Format}

A dose conversion factor (DCF) file is required for both the EARLY and CHRONC modules. The format of this file is identical to that of MACCS, allowing both codes to be used with identical DCF data for purposes of code comparison.

In contrast to MACCS, however, where the name of the DCF file is specified in the batch file controlling execution, MACCS2 requires the user to specify a DCF filename as input variable DCF_FILE defined in Section 6.2.1. Also, in contrast to MACCS, which utilizes the DCF file's values for 8-hr and 7-day groundshine dose, MACCS2 does not process those columns of data, and they may be omitted.

MACCS2 utilizes the following data from the DCF file:

1. cloudshine dose-rate factor $\left[\mathrm{Sv} /\left(\mathrm{Bq}-\mathrm{s} / \mathrm{m}^{3}\right)\right]$,

2. groundshine dose-rate factor $\left[\mathrm{Sv} /\left(\mathrm{Bq}-\mathrm{s} / \mathrm{m}^{2}\right)\right]$,

3. "acute" short-term inhalation doses $(\mathrm{Sv} / \mathrm{Bq})$ used for calculation of deterministic health effects,

4. "lifetime" 50-year committed inhalation doses ( $\mathrm{Sv} / \mathrm{Bq})$ used for calculation of individual and societal doses and stochastic health effects, and

5. 50-year committed ingestion doses $(\mathrm{Sv} / \mathrm{Bq})$ used for calculation of individual and societal doses and stochastic health effects from food and water ingestion.

If an organ is not considered in the deterministic health effects models, no internal dose for early exposure from inhalation is given, or needed; the values on the file are set to -1.0 .

The DCF data file used in MACCS2 is a formatted file. An example of a MACCS2 DCF file is provided in Figure A-2. The first two records contain identification information. Up to 80 characters may be used on each line. This header information is printed on the output listing. The next line gives the number of organs included in the file. The format for this line is I10. The organ names are specified next, one name per line. The spelling must be the same as that used in all other program data files. Next the number of radionuclides included in the file is given. The format is again I10.

The names of the radionuclides are given on the following lines, one name per line. The spelling of the radionuclide names must again be the same as in all other program modules. Following the list of radionuclides, there are two lines in the dosimetry data file that are not read but aid user understanding of the file. On the next line is the name of the first radionuclide followed on the next lines by the organ names and the associated dosimetry data. The format of the dosimetry data is as follows: 


\begin{tabular}{lll} 
Column & \multicolumn{1}{c}{ Variable } & Format \\
\cline { 1 - 1 } $1-10$ & FILORG & A10 \\
$11-20$ & CDCF & F10.0 \\
$21-30$ & IGDCF $(I, J, 1)$ & F10.0 \\
$31-40$ & IGDCF $(I, J, 2)$ & F10.0 \\
$41-50$ & GRDCF & F10.0 \\
$51-60$ & $\operatorname{IDCF}(I, J, 1)$ & F10.0 \\
& & \\
$61-70$ & IDCF $(I, J, 2)$ & F10.0 \\
$71-80$ & DFING & F10.0
\end{tabular}

Identification

Name of the Organ

Cloudshine Dose-Rate Factor

Not Applicable-Not Used by MACCS2

Not Applicable-Not Used by MACCS2

Groundshine Dose-Rate Factor

Inhalation Dose Factor Used Only

with the Deterministic Health

Effects Models

50-year Inhalation Dose Commitment

50-year Inhalation Dose Commitment 
MACCS2 DCF File

Sample DCF File For MACCS2 Documentation

17 ORGANS DEFINED IN THIS FILE:

STOMACH

SMALI IN

LUNGS

RED MARR

THYROID

LOWER LI

BONE SUR

BREAST

TESTES

OVARIES

EDEWBODY

THYROIDH

ADRENALS

BLAD WAI

KIDNEYS

LIVER

PANCREAS

2 NUCLIDES DEFINED IN THIS FILE:

CO- 58

$\mathrm{CO}-60$

CLOUDSHINE GROUND GROUND GROUND INHALED INHALED INGESTION SHINE 8HR SHINE 7DAY SHINE RATE ACUTE CHRONIC

$\mathrm{CO}-58$

STOMACH

SMAIL IN

LUNGS

RED MARR

THYROID

LOWER II

BONE SUR

BREAST

TESTES

OVARIES

EDEWBODY

THYROIDH

ADRENALS

BLAD WAI

KIDNEYS

IIVER

PANCREAS

CO-6O

STOMACH

SMAII IN

LUNGS

RED MARR

THYROID

LOWER LI

BONE SUR.

BREAST

TESTES

OVARIES

EDEWBODY

THYROIDH

ADRENALS

BLAD WAL

KIDNEYS

IIVER

PANCREAS

$3.520 \mathrm{E}-14 \quad 1.979 \mathrm{E}-11 \quad 4.023 \mathrm{E}-10 \quad 6.881 \mathrm{E}-16 \quad 1.558 \mathrm{E}-10 \quad 1.394 \mathrm{E}-09 \quad 3.853 \mathrm{E}-10$

$3.203 \mathrm{E}-14 \quad 1.796 \mathrm{E}-11 \quad 3.652 \mathrm{E}-10 \quad 6.247 \mathrm{E}-16 \quad 3.307 \mathrm{E}-10 \quad 7.495 \mathrm{E}-10 \quad 1.130 \mathrm{E}-09$

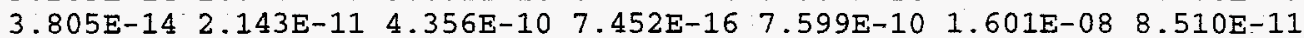

$3.869 \mathrm{E}-142.179 \mathrm{E}-11 \quad 4.430 \mathrm{E}-10 \quad 7.579 \mathrm{E}-16 \quad 1.577 \mathrm{E}-10 \quad 9.228 \mathrm{E}-10 \quad 2.601 \mathrm{E}-10$

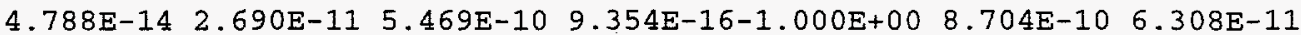

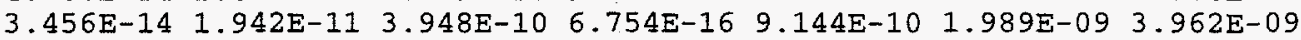

$\begin{array}{llllll}4.249 \mathrm{E}-14 & 2.389 \mathrm{E}-11 & 4.857 \mathrm{E}-10 & 8.308 \mathrm{E}-16-1.000 \mathrm{E}+00 & 6.926 \mathrm{E}-10 & 1.252 \mathrm{E}-10\end{array}$ $4.566 \mathrm{E}-142.571 \mathrm{E}-11 \quad 5.228 \mathrm{E}-10 \quad 8.942 \mathrm{E}-16-1.000 \mathrm{E}+00 \quad 9.367 \mathrm{E}-10 \quad 1.788 \mathrm{E}-10$ $5.074 \mathrm{E}-14 \quad 2.845 \mathrm{E}-11 \quad 5.784 \mathrm{E}-10 \quad 9.894 \mathrm{E}-16-1.000 \mathrm{E}+00 \quad 1.060 \mathrm{E}-10 \quad 1.614 \mathrm{E}-10$

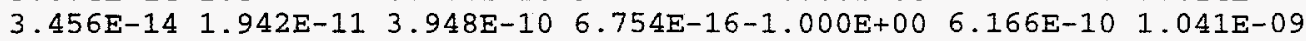
$4.398 \mathrm{E}-142.459 \mathrm{E}-11 \quad 5.000 \mathrm{E}-10 \quad 8.553 \mathrm{E}-16-1.000 \mathrm{E}+00 \quad 3.088 \mathrm{E}-09 \quad 8.206 \mathrm{E}-10$ $\begin{array}{lllllll}4.788 \mathrm{E}-14 & 2.690 \mathrm{E}-11 & 5.469 \mathrm{E}-10 & 9.354 \mathrm{E}-16 & 6.142 \mathrm{E}-11 & 8.704 \mathrm{E}-10 & 6.308 \mathrm{E}-11\end{array}$ $3.742 \mathrm{E}-142.097 \mathrm{E}-11 \quad 4.264 \mathrm{E}-10 \quad 7.293 \mathrm{E}-16-1.000 \mathrm{E}+00 \quad 1.601 \mathrm{E}-09 \quad 1.509 \mathrm{E}-10$ $3.583 \mathrm{E}-142.015 \mathrm{E}-11 \quad 4.097 \mathrm{E}-10 \quad 7.008 \mathrm{E}-16-1.000 \mathrm{E}+00.2 .387 \mathrm{E}-10 \quad 3.643 \mathrm{E}-10$ $3.742 \mathrm{E}-142.106 \mathrm{E}-11 \quad 4.282 \mathrm{E}-10 \quad 7.325 \mathrm{E}-16-1.000 \mathrm{E}+00 \quad 7.523 \mathrm{E}-10 \quad 2.094 \mathrm{E}-10$ $\begin{array}{llllll}3.583 \mathrm{E}-14 & 2.006 \mathrm{E}-11 & 4.078 \mathrm{E}-10 & 6.976 \mathrm{E}-16-1.000 \mathrm{E}+00 & 1.637 \mathrm{E}-09 & 2.456 \mathrm{E}-10\end{array}$ $3.079 \mathrm{E}-14 \quad 1.723 \mathrm{E}-11 \quad 3.504 \mathrm{E}-10 \quad 5.993 \mathrm{E}-16-1.000 \mathrm{E}+00 \quad 1.675 \mathrm{E}-09 \quad 1.997 \mathrm{E}-10$

$9.132 \mathrm{E}-14 \quad 4.602 \mathrm{E}-11 \quad 9.655 \mathrm{E}-10 \quad 1.598 \mathrm{E}-15 \quad 3.840 \mathrm{E}-10 \quad 2.726 \mathrm{E}-08 \quad 1.611 \mathrm{E}-09$ $8.530 \mathrm{E}-14 \quad 4.301 \mathrm{E}-11 \quad 9.022 \mathrm{E}-10 \quad 1.494 \mathrm{E}-15 \quad 8.077 \mathrm{E}-10 \quad 7.046 \mathrm{E}-09 \quad 3.591 \mathrm{E}-09$ $9.862 \mathrm{E}-14 \quad 4.986 \mathrm{E}-11 \quad 1.046 \mathrm{E}-09 \quad 1.731 \mathrm{E}-15 \quad 3.511 \mathrm{E}-09 \quad 3.448 \mathrm{E}-07 \quad 8.768 \mathrm{E}-10$ $9.957 \mathrm{E}-14 \quad 5.032 \mathrm{E}-11 \quad 1.055 \mathrm{E}-09 \quad 1.747 \mathrm{E}-15 \quad 3.986 \mathrm{E}-10 \quad 1.718 \mathrm{E}-08 \quad 1.311 \mathrm{E}-09$

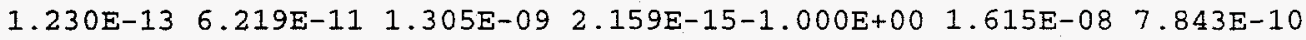
$9.069 \mathrm{E}-14 \quad 4.575 \mathrm{E}-11 \quad 9.597 \mathrm{E}-10 \quad 1.589 \mathrm{E}-15 \quad 2.386 \mathrm{E}-09 \quad 7.916 \mathrm{E}-09 \quad 1.113 \mathrm{E}-08$ $1.056 \mathrm{E}-13.5 .333 \mathrm{E}-11 \quad 1.119 \mathrm{E}-09 \quad 1.852 \mathrm{E}-15-1.000 \mathrm{E}+00 \quad 1.353 \mathrm{E}-08 \quad 9.415 \mathrm{E}-10$ $1.164 \mathrm{E}-13 \quad 5.872 \mathrm{E}-11$ 1.232E-09 2.039E-15-1.000E+00 1.843E-08 1.100E-09

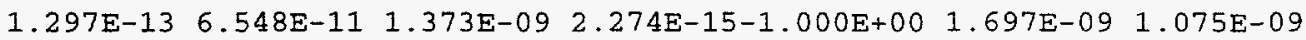
8.879E-14 4.484E-11 $9.406 \mathrm{E}-10 \quad 1.557 \mathrm{E}-15-1.000 \mathrm{E}+00 \quad 4.753 \mathrm{E}-09 \quad 3.187 \mathrm{E}-09$

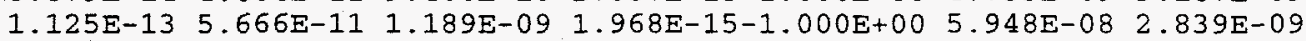
$\begin{array}{lllllll}1.230 E-13 & 6.219 \mathrm{E}-11 & 1.305 \mathrm{E}-09 & 2.159 \mathrm{E}-15 & 1.465 \mathrm{E}-10 & 1.615 \mathrm{E}-08 & 7.843 \mathrm{E}-10\end{array}$ $9.418 \mathrm{E}-14 \quad 4.749 \mathrm{E}-11 \quad 9.961 \mathrm{E}-10 \quad 1.649 \mathrm{E}-15-1.000 \mathrm{E}+00 \quad 2.990 \mathrm{E}-08 \quad 1.586 \mathrm{E}-09$ $9.354 \mathrm{E}-14 \quad 4.721 \mathrm{E}-11 \quad 9.904 \mathrm{E}-10 \quad 1.639 \mathrm{E}-15-1.000 \mathrm{E}+00 \quad 2.949 \mathrm{E}-09 \quad 1.771 \mathrm{E}-09$ $9.830 \mathrm{E}-14 \quad 4.959 \mathrm{E}-11 \quad 1.040 \mathrm{E}-09 \quad 1.722 \mathrm{E}-15-1.000 \mathrm{E}+00 \quad 1.557 \mathrm{E}-08 \quad 1.355 \mathrm{E}-09$ $9.323 \mathrm{E}-14 \quad 4.703 \mathrm{E}-11 \quad 9.865 \mathrm{E}-10 \quad 1.633 \mathrm{E}-15-1.000 \mathrm{E}+00 \quad 3.352 \mathrm{E}-08 \quad 2.326 \mathrm{E}-09$ $8.086 \mathrm{E}-14 \quad 4.073 \mathrm{E}-11 \quad 8.544 \mathrm{E}-10 \quad 1.414 \mathrm{E}-15-1.000 \mathrm{E}+00 \quad 3.179 \mathrm{E}-08 \quad 1.290 \mathrm{E}-09$

Figure A-2. Sample MACCS2 DCF file. 


\section{A.3 Site Data File Format}

In the Site Data file, the user specifies the population distribution and land use information for the region surrounding the site. Contained in the Site Data file are the geometry data used for the site (spatial intervals), population distribution, fraction of the area that is land, watershed data for the liquid pathways model, information on agricultural land use and growing seasons, and regional economic information. An example of a Site Data file is provided in NUREG/CR-4691, Volume 1, Appendix D.2.

The user specifies in the EARLY input file whether a Site Data file is to be used (see variable POPFLG in Section 6.3). If a Site Data file is not being used, the population density applied in the EARLY and CHRONC modules is specified in the EARLY input file. It is not possible for the user to supply differing population data for the two modules.

The Site Data file used in MACCS2 is a formatted file. The data must appear exactly as described below and in exactly the same order. In contrast to the input files for MACCS2, which are processed by a free-format input processor, the Site Data file is processed with fixed format FORTRAN READ statements.

The use of fixed format READ statements requires that the user exercise special attention to line up the data items in their proper fields. Any numeric items specified in exponential format (e.g., 1.E-6) must be right-justified in the field because trailing blanks are processed as zeroes in the FORTRAN READ statements.

In contrast to the ATMOS, EARLY, and CHRONC user input files, where every value is validated by the code to ensure that it lies within a range of allowable values, the validation performed on the Site Data is only partially complete. Some of the input parameters on this file are rigorously checked to ensure that they fall within the allowed range, while other values are not checked at all.

It is recommended that the user exercise scrupulous care in constructing a Site Data file. It is very important that all items appear in their proper fields and that all numeric values lie within the range of acceptable values. Failure to conform to these requirements may lead to the generation of spurious results.

When code users edited the population counts in the Site Data file using text editors, there were several occurrences where the code diagnosed an input conversion error and aborted execution, and it has proved very difficult to identify and remedy the problem. A possible explanation for these problems is the inadvertent inclusion of nonprintable ASCII characters or control codes in the file. A likely culprit appears to be the TAB character (ASCII code decimal 9). Control codes such as the TAB can be treated differently by different text editors, or their handling can vary, depending on user-specified editor settings.

In order to aid users in identifying the cause of these problems, and maximize the portability of the Site Data file across computer platforms, MACCS2 incorporates a test for nonprintable 
characters in the site data file. If any such characters are found to be present, their location on the input data line is indicated, and further execution is terminated.

The ANSI standard for FORTRAN 77 does not specify how the TAB and other control codes should be handled if they are encountered during formatted input operations. The set of processor characters handled by standard FORTRAN 77 is limited to the blank, numeric digits, upper case letters, and the following twelve symbols: '+-*/(),::=\$. However, it seems logical to extend this set to include all of the printable characters that can be generated by a IBM PC-ATcompatible keyboard; that is, the characters with decimal ASCII control codes between 32 (blank) and $126(\sim)$. As a result, the definition of allowable characters in the site data file is that it can be allowed to contain all of the characters having decimal ASCII control codes between 32 and 126 , inclusive.

A sample MACCS2 site data file is listed in Figure A-3. The first two records of the site data file contain identification information. Up to 80 characters may be used on each line. This header information is printed on the output listing.

Following the descriptive text fields, there are six data records that specify the amount of data that is being supplied on the file. The values defined on these data records must be consistent with the MACCS2 model definition data defined by the ATMOS, EARLY, and CHRONC input files. The value of the Site Data file input variable and the corresponding ATMOS, EARLY, and CHRONC variable must be identical. If any inconsistencies are detected, execution of the program will be terminated.

The data are input as integers and the format is as follows:

\begin{tabular}{|c|c|c|c|c|c|c|}
\hline Line & Columns & $\begin{array}{l}\text { Fortran } \\
\text { Format } \\
\text { Descriptor }\end{array}$ & $\begin{array}{l}\text { Site-File } \\
\text { Variable }\end{array}$ & Identification & $\begin{array}{l}\text { MACCS } 2 \\
\text { Variable }\end{array}$ & $\begin{array}{l}\text { Allowed } \\
\text { Range }\end{array}$ \\
\hline 3 & $1-4$ & I4 & NSPDTS & No. of spatial intervals & NUMRAD & $2-35$ \\
\hline 4 & $1-4$ & I4 & NWNDIR & No. of wind directions & NUMCOR & $16-16$ \\
\hline 5 & $1-4$ & I4 & NCPGZN & No. of food crops & NFICRP & $1-10$ \\
\hline 6 & $1-4$ & I4 & NWPISO & water radionuclides & NUMWPI & $1-10$ \\
\hline 7 & $1-4$ & I4 & NWTRSH & watersheds & NUMWPA & $1-4$ \\
\hline 8 & $1-4$ & I4 & NECRGN & No. of economic regions & N.A. & $1-99$ \\
\hline
\end{tabular}

Eight blocks of site data follow the introductory block described above. Each of these data blocks is introduced by a separator line that identifies the block of data to follow. The first line of each data block must be the separator for that block. The first character (column 1) of the separator line is ignored and the following 22 characters must match the identification field for that specific data block. The eight blocks of data are identified by the following character separators: 
SPATIAL DISTANCES

POPULATION

LAND FRACTION

REGION INDEX

WATERSHED INDEX

CROP SEASON AND SHARE

WATERSHED DEFINITION

REGIONAL ECONOMIC DATA

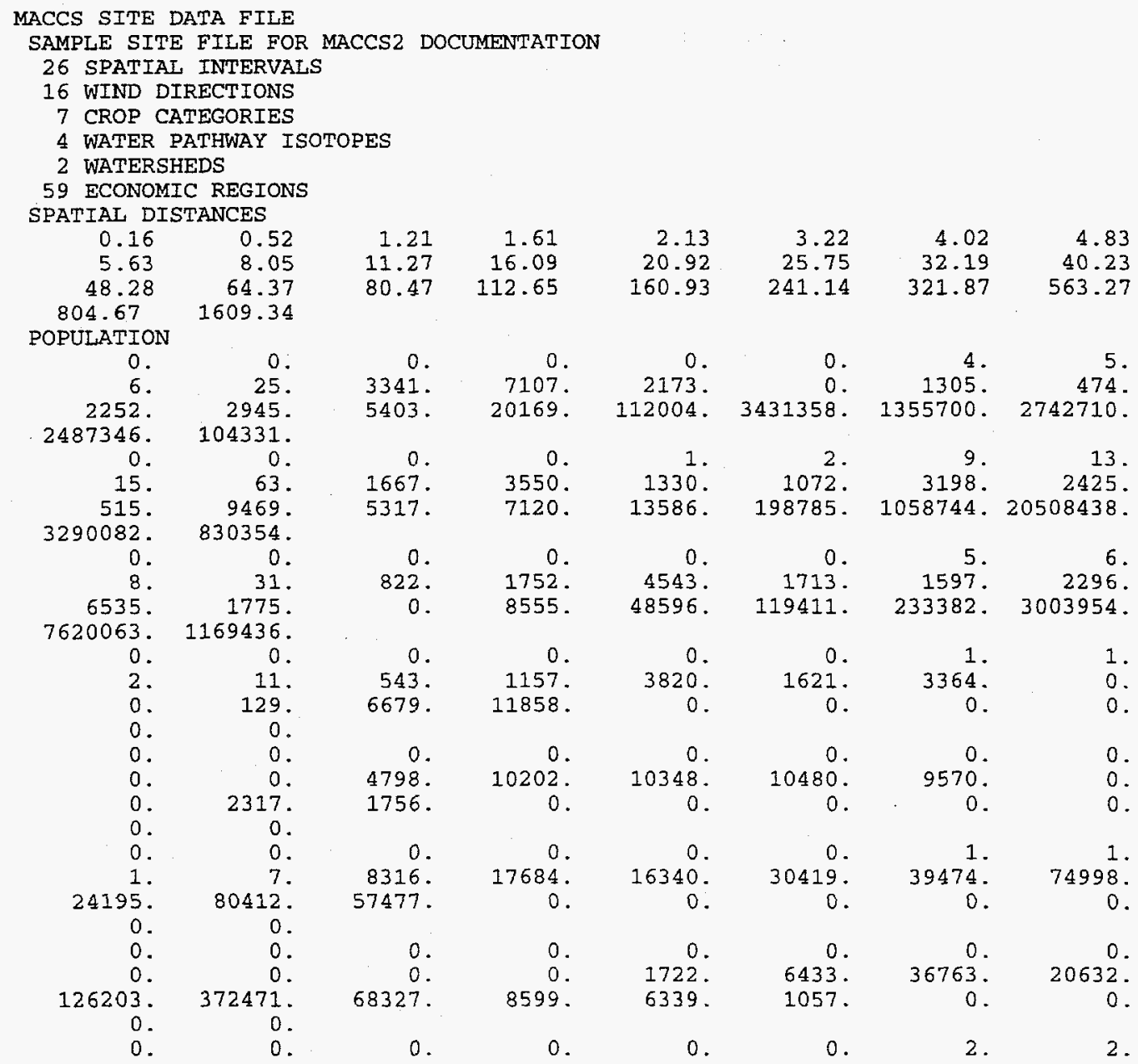

Figure A-3. MACCS/MACCS2 sample Site Data file (continued on following page). 




Figure A-3. MACCS/MACCS2 sample Site Data file (continued on following page). 


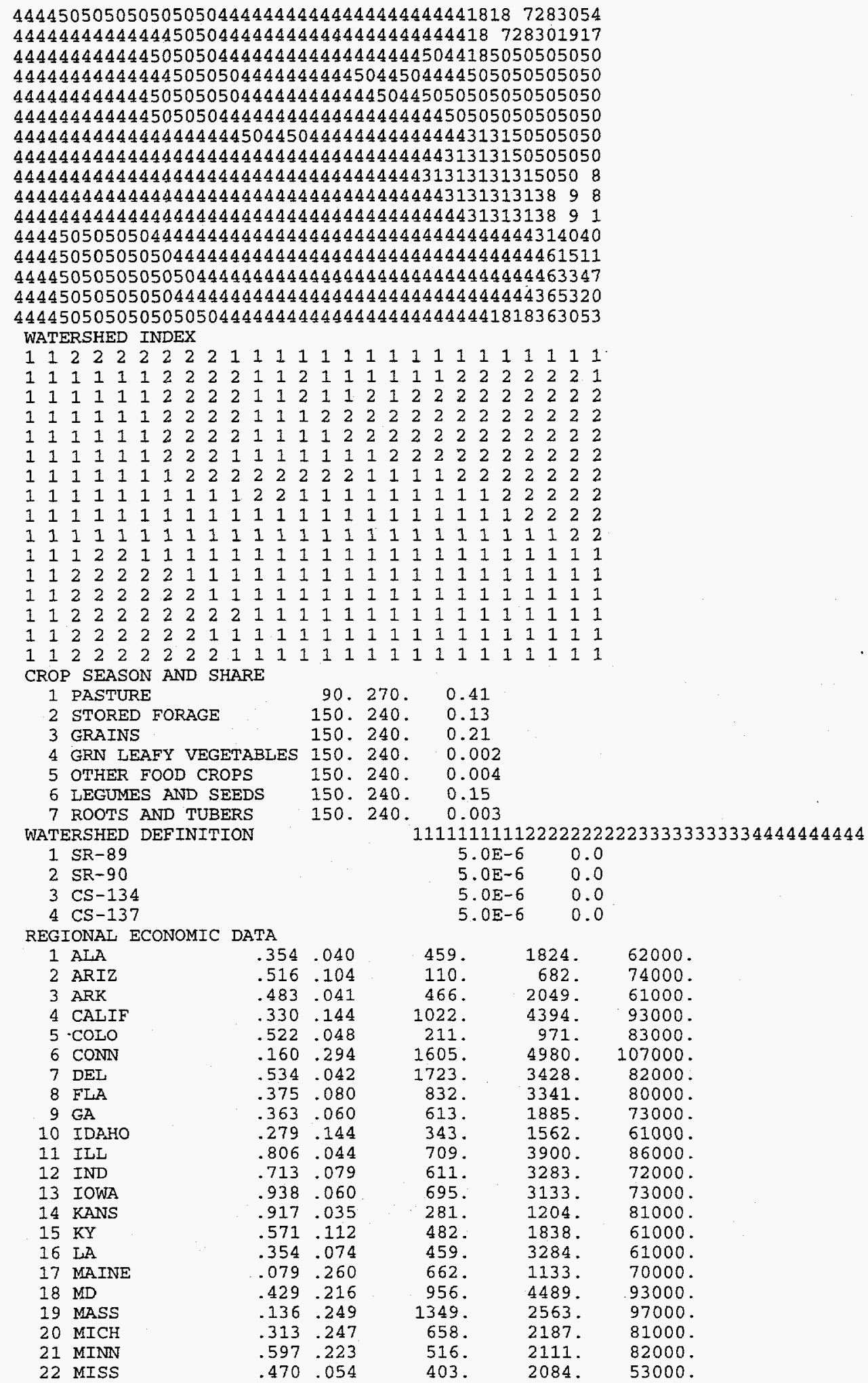

Figure A-3. MACCS/MACCS2 sample Site Data file (continued on following page). 


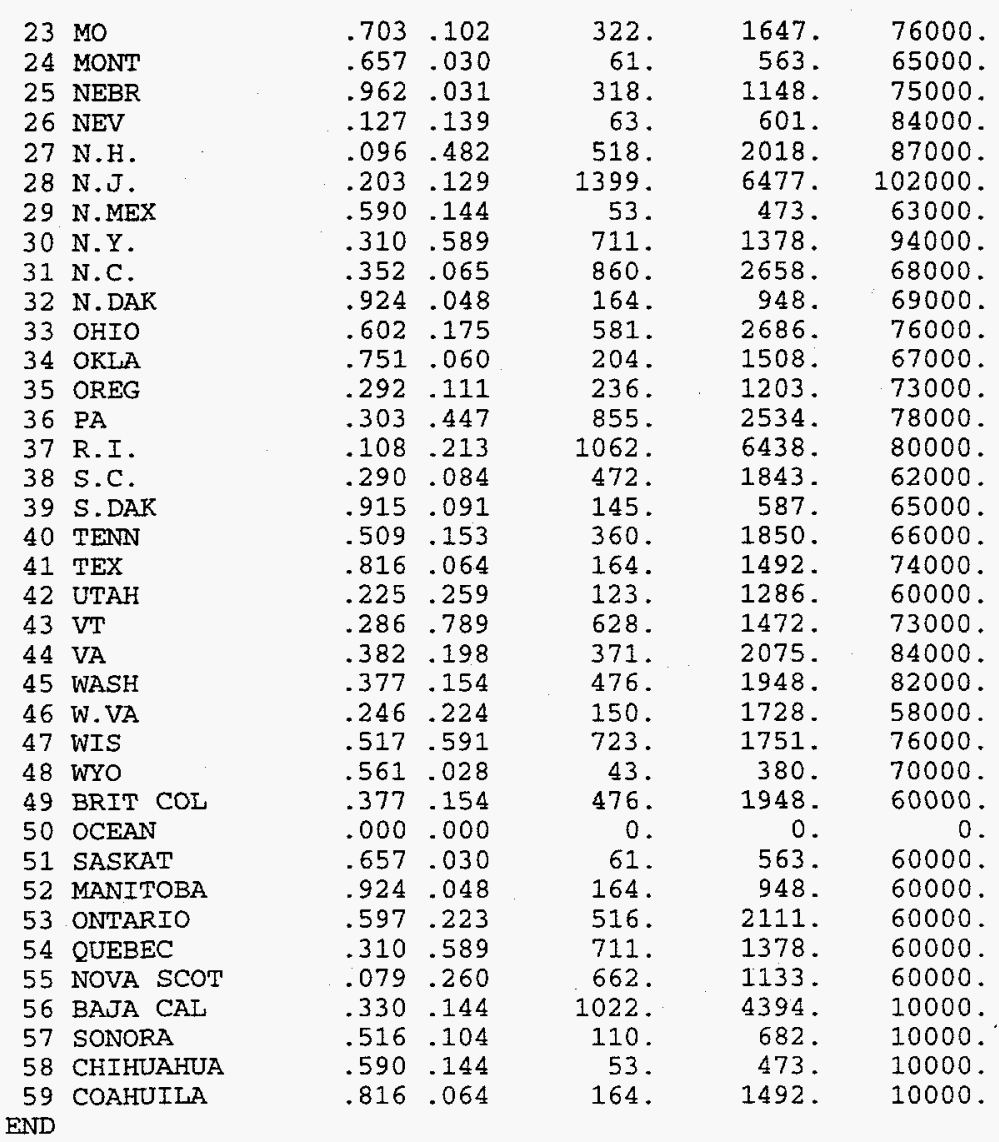

Figure A-3. MACCS/MACCS2 sample Site Data file.

\section{A.3.1 Spatial Distances Data Block}

The spatial distance data define the spatial grid for which the population and land use data are specified. The data define the distance in kilometers to the endpoints of the spatial intervals. The areas between the spatial interval endpoints within each of the 16 wind direction sectors are referred to as spatial elements. This grid definition must agree with the grid defined in the ATMOS input file (see the variable SPAEND in Section 5.3). A relative error of $10 \%$ in the endpoint distances is allowed. For larger discrepancies in the geometry data, the error flag will be set and execution will terminate upon completion of the Site Data file input processing.

The first line of the spatial distance data block contains the 22-character separator beginning with SPATIAL DISTANCES in column 2. Next, the endpoint distances in kilometers are specified, eight values per line, using the format described below. As many lines as are needed to define the spatial distances are used. The miminum spacing between adjacent spatial intervals is 0.1 $\mathrm{km}$.

A maximum of eight interval endpoints may be input per line within ten column intervals; i.e., the first interval endpoint would be input in columns 1-10, the second interval endpoint would be input in columns 11-20, etc. The data are read as a real numbers with two decimal places. The format used to process the data is as follows: 


\begin{tabular}{|c|c|c|c|c|c|}
\hline Columns & $\begin{array}{l}\text { Fortran } \\
\text { Format } \\
\text { Descriptor }\end{array}$ & $\begin{array}{l}\text { Site-File } \\
\text { Variable }\end{array}$ & Identification & $\begin{array}{l}\text { MACCS2 } \\
\text { Variable }\end{array}$ & $\begin{array}{c}\text { Allowed } \\
\text { Range }\end{array}$ \\
\hline $1-80$ & $8 \mathrm{E} 10.2$ & $\overline{\text { SPDSTS }}$ & $\begin{array}{l}\text { Spatial interval } \\
\text { endpoints }(\mathrm{km})\end{array}$ & $\overline{\text { SPAEND }}$ & $0.001-999.0$ \\
\hline
\end{tabular}

\section{A.3.2 Population Data Block}

The population data for each element in the spatial grid is defined here. The first line of the data block contains the 22-character separator beginning with POPULATION in column 2. Next, the number of people in each element is given for the first wind sector. (The first wind sector is assumed to be centered on north.) The population data are specified, eight values per line, using the format described below. As many lines as needed to cover all the spatial elements in the sector are used. Proceeding in a clockwise rotation, the population data for the second (NNE) and subsequent sectors follow. Data for all 16 wind directions (sectors) must be provided. Data for each sector begin on a new line.

The population data are input as a real number although no fractional numbers are stored. The population data for up to eight spatial elements may be defined per line and the data are input per ten column intervals; i.e., the population in the first element would be input in columns $1-10$, the population in the second element would be input in columns 11-20, etc. The format used to process the data is as follows:

$\begin{array}{llll}\begin{array}{l}\text { Fortran } \\ \text { Format } \\ \text { Columns }\end{array} & \begin{array}{l}\text { Site-File } \\ \text { DE10.0 }\end{array} \frac{\text { Variable }}{\text { POPDAT }} & \frac{\text { Identification }}{\text { Population data }} & \begin{array}{c}\text { Allowed } \\ \text { Range }\end{array} \\ 0.0-1 . E 9\end{array}$

If the user specifies that fraction of the people or fraction of the time weighting is to be performed (see variable WTNAME in Section 6.6.8, the new code functions exactly the same as MACCS 1.5.11.1. In cases where the SUMPOP option is selected, the processing and interpretation of population data are treated in a different manner, as described below.

For each emergency response scenario (of the up to three allowed) defined in the EARLY input file, a distinct population distribution must be supplied in the Site Data file. In MACCS 1.5.11.1, the population data block is delimited with the header record POPULATION (see Figure B.3.1 in Appendix B) beginning at column 2, followed by the population data.

When the SUMPOP option is selected, the header cards for each population data block are POPULATION1, POPULATION2, and POPULATION3, corresponding to the respective emergency-response scenarios, all beginning at column 2. An error will result if the number of population data blocks does not equal the number of emergency-response scenarios defined in the EARLY input file. 


\section{A.3.3 Land Fraction Data Block}

The fraction of each spatial element that is land (as opposed to lakes, oceans, etc.) must be defined. The first line of the data block contains the 22-character separator beginning with LAND FRACTION in column 2. Next, the fraction of area that is land in each radial spatial interval of the first sector is given. All values must be between 0 and 1 . A value of 0 means the element has no land, a value of 1 means the element is all land. The land fraction data are specified, 16 values per line, with the format described below. As many lines as needed to define all the spatial intervals in the sector are used. The land fraction data for the second and subsequent sectors follow in a clockwise rotation. Data for all 16 wind direction sectors must be provided. The data for each sector begin on a new line.

The land fraction data are read as a real number and land fractions are input every five columns. The format used to process the data is as follows:

\begin{tabular}{|c|c|c|c|c|}
\hline & $\begin{array}{l}\text { Fortran } \\
\text { Format }\end{array}$ & & & \\
\hline Columns & $\frac{\text { Descriptor }}{1655}$ & Variable & Identification & Allowed Range \\
\hline & $16 \mathrm{~F} 5.2$ & $\overline{\text { FRCLND }}$ & Land Fraction & $0.0-1.0$ \\
\hline
\end{tabular}

\section{A.3.4 Region Index Data Block}

In this data block the user assigns a user-defined economic region to each of the spatial intervals. The economic regions are defined in the Regional Economic data block. Section A.3.8 contains a description of the types of economic data that can be defined for each economic region.

The first line in the Region Index data block contains the 22-character separator beginning with REGION INDEX in column 2. The next line contains two-digit integers associating a region index with each of the spatial elements in the first sector. The data are specified with the format defined below. All of the region indices for one sector are input on one line. The region indices for the second and subsequent sectors are on the following lines, a new line for each sector in a clockwise rotation. A total of 16 lines are required. For example, a region index of 09 means that economic data for region number nine will be used for the spatial element.

The format used to process the data is as follows:

\begin{tabular}{|c|c|c|c|c|}
\hline & $\begin{array}{l}\text { Fortran } \\
\text { Format }\end{array}$ & Site-File & & \\
\hline$\frac{\text { Columns }}{1-80}$ & $\frac{\text { Descriptor }}{40 \text { I2 }}$ & $\frac{\text { Variable }}{\text { INDREG }}$ & $\frac{\text { Identification }}{\text { Region Index }}$ & $\frac{\text { Allowed Range }}{1-\text { NECRGN }}$ \\
\hline
\end{tabular}

\section{A.3.5 Watershed Index Data Block}

Each of the spatial intervals in the grid must be associated with one of the watershed classes. The definition of the watershed classes is provided in Section A.3.7. The watershed identification data block begins with the 22-character separator beginning with WATERSHED INDEX in column 2. The next line contains two-digit integers associating a watershed type with 
each of the spatial elements in the first sector. The data are specified with the format defined below.

All of the watershed type indices for one wind sector will fit on a single line. The watershed indices for the second and subsequent sectors are on the following lines, a new line for each sector in a clockwise rotation. A total of 16 lines are required. For example, a watershed index of 1 means that the water ingestion factor for watershed type 1 will be used for all material deposited on that spatial element. A watershed index of 2 means that the water ingestion factor for watershed type 2 will be used for all material deposited on that spatial element.

The format used to process the data is as follows:

\begin{tabular}{|c|c|c|c|c|}
\hline Columns & $\begin{array}{l}\text { Fortran } \\
\text { Format } \\
\text { Descriptor }\end{array}$ & $\begin{array}{l}\text { Site-File } \\
\text { Variable }\end{array}$ & Identification & Allowed Range \\
\hline $1-80$ & $40 I 2$ & INDWTR & Watershed Index & 1-NWTR.SH \\
\hline
\end{tabular}

\section{A.3.6 Crop and Season Share Data Block}

The length of the growing season and the average fraction of the farmland area at the site devoted to each crop type must be specified. These fractions need not sum to exactly 1 , but their sum should not exceed a value of 1 . If these values sum to a value less than 1 , that sum indicates the fraction of farmland in production in an average year (some fraction of farmland may be fallow). Data must be given for each of the crop categories. The data block begins with the separator CROP SEASON AND SHARE in column 2.

The format used to process the data is as follows:

\begin{tabular}{|c|c|c|c|c|}
\hline Columns & $\begin{array}{l}\text { Fortran } \\
\text { Format } \\
\text { Descriptor }\end{array}$ & $\begin{array}{l}\text { Site-File } \\
\text { Variable } \\
\end{array}$ & Identification & Allowed Range \\
\hline $1-4$ & I4 & $I$ & Crop Index & 1-NCPGZN \\
\hline $6-25$ & $\mathrm{~A} 20$ & CROP & Crop Name & Not Applicable \\
\hline $26-30$ & F5. 0 & GBEG & $\begin{array}{l}\text { Day of the Year } \\
\text { the Growing } \\
\text { Season Begins }\end{array}$ & 1. 0-GEND \\
\hline $31-35$ & F5. 0 & GEND & $\begin{array}{l}\text { Day of the Year } \\
\text { the Growing } \\
\text { Season Ends }\end{array}$ & GBEG-365.0 \\
\hline $36-45$ & F10.0 & FRCLCP & $\begin{array}{l}\text { Fraction of the } \\
\text { Site-Averaged } \\
\text { Farmland Devoted } \\
\text { to this Crop }\end{array}$ & $0.0-1.0$ \\
\hline
\end{tabular}




\section{A.3.7 Watershed Definition Data Block}

The data block begins with the 22-character separator beginning with WATERSHED DEFINITION in column 2. For each of the radionuclides considered in the liquid pathways model, an initial washoff fraction and an annual washoff rate $\left(\right.$ year $\left.^{-1}\right)$ must be specified together with an ingestion factor (Bq ingested/Bq in water) for each of the NUMWPA (NWTRSH) watershed classes.

The format used to process the data is as follows:

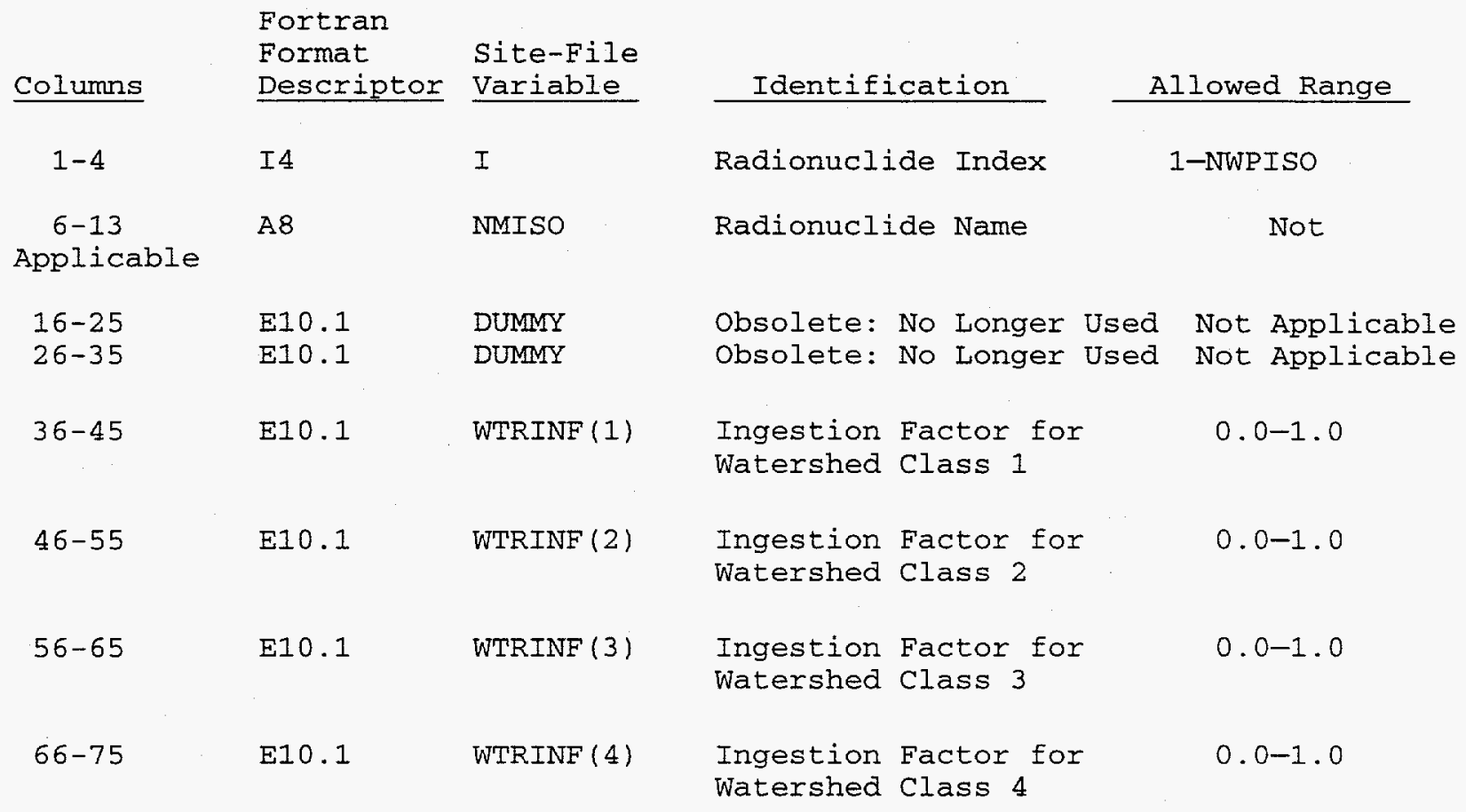

\section{A.3.8 Regional Economic Data Block}

Economic data must be specified for each of the economic regions. The data block begins with the separator REGIONAL ECONOMIC DATA in column 2. An economic region is typically identified with an existing county, a state, or country to provide an indication of the source of the data or the type of geographical area it is intended to represent. The economic regions defined in this section are identified with spatial elements in the Region Index data block.

The format used to process this data is as follows:

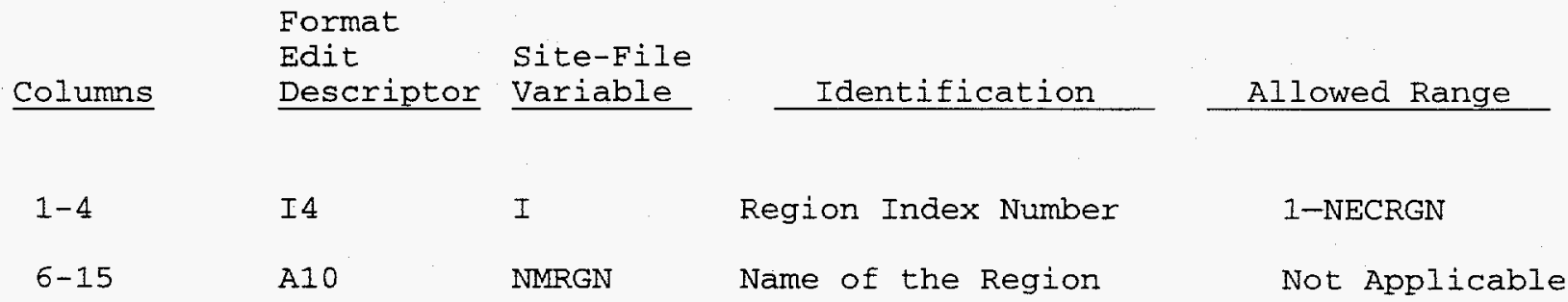




\begin{tabular}{|c|c|c|c|c|}
\hline $21-25$ & F5. 3 & FRMFRC & $\begin{array}{l}\text { Fraction of Land Devoted } \\
\text { to Farming in Region }\end{array}$ & $0.0-1.0$ \\
\hline $26-30$ & F5. 3 & $\mathrm{DPF}$ & $\begin{array}{l}\text { Fraction of Farm Sales } \\
\text { Resulting from Dairy } \\
\text { Production in Region }\end{array}$ & $0.0-1.0$ \\
\hline $31-40$ & F10.1 & ASFP & $\begin{array}{l}\text { Total Annual Farm Sales } \\
\text { for the Region } \\
\text { (dollars/hectare) }\end{array}$ & $0.0-1 . \mathrm{E9}$ \\
\hline $41-50$ & F10.1 & VFRM & $\begin{array}{l}\text { Farmland Property Value } \\
\text { for the Region } \\
\text { (dollars/hectare) }\end{array}$ & $0.0-1 . \mathrm{E9}$ \\
\hline $51-60$ & F10.1 & VNFRM & $\begin{array}{l}\text { Nonfarm Property Value } \\
\text { for the Region } \\
\text { (dollars/person) }\end{array}$ & $0.0-1 . \mathrm{Eg}$ \\
\hline
\end{tabular}




\section{Appendix B: READMAC2.TXT FILE}

MACCS2 VERSION 1.12

\section{OVERVIEW}

The complete MACCS2 package includes the MACCS2 code and four preprocessors. The package is distributed as six ZIP files:

MAC2ZIPA.ZIP

MAC2ZIPB.ZIP

COMIDA2.ZIP

FGRDCF

IDCF2.ZIP

DOSFAC2

MAC2ZIPA.ZIP and MAC2ZIPB.ZIP contain the MACCS2 code and the files required to exercise the 14 MACCS2 sample problems. The preprocessors are not required to exercise the code. The preprocessors may be used to create input files containing dose conversion factor (DCF) or food pathway data not contained in the sample problem files.

\section{HARDWARE AND SOFTWARE REQUIREMENTS}

IBM-compatible 486 or Pentium PC with $8 \mathrm{MB}$ of RAM

The MACCS2 package ZIP files require approximately the following disk space when decompressed:

MAC2ZIPA.ZIP $6 \mathrm{MB}$

MAC2ZIPB.ZIP $5 \mathrm{MB}$

COMIDA2.ZIP $7 \mathrm{MB}$

FGRDCF $3 \mathrm{MB}$

IDCF2.ZIP $5 \mathrm{MB}$

DOSFAC2 $5 \mathrm{MB}$

The files may be decompressed using PKZIP version 2.04g (or higher). Norton Utilities can also be used to decompress the archives. The MACCS2 software requires Microsoft DOS 3.3 or higher (or a DOS-compatible operating system).

The MACCS2.EXE file included in this package was compiled using the 486 instruction set option. This option reduces the MACCS2 computer run time but 
requires that the code be exercised on a 486 or Pentium CPU.

The provided executables include the Phar-Lap DOS extender, and, as a result, successful operation of the MACCS2 software does not require the use of any memory managers such as Microsoft EMM386.EXE or HIMEM.SYS. In fact, the use of the Microsoft memory managers sometimes results in conflicts with the Phar-Lap DOS extender used by Lahey FORTRAN to access extended memory.

The Phar-Lap DOS-extender executables of the MACCS2 software package do not run in conventional memory. For that reason, the amount of free conventional memory is less important for the installation of MACCS2 than the amount of EMS or XMS available. Approximately $6 \mathrm{MB}$ of extended memory (XMS) or $6 \mathrm{MB}$ of expanded memory (EMS) must be available for MACCS2 execution. When the MACCS2.EXE file is invoked, a pop-up screen will appear indicating how much memory the DOS extender can access. If it shows that $5860 \mathrm{kB}$ or more is available, there is sufficient RAM available to execute MACCS2 and all of the preprocessors.

The use of a Microsoft disk cache may interfere with the successful operation of MACCS2. Default settings for the Microsoft SMARTDRV disk cache may allocate one-half of the available RAM for the disk cache. A disk cache size of $512 \mathrm{kB}$ of RAM is sufficient for most operations. A $512-\mathrm{kB}$ disk cache can be specified for SMARTDRV by invoking it on the AUTOEXEC.BAT file as,

\section{SMARTDRV 512}

MACCS2 can be tested on systems started up with a "clean boot" disk if it is suspected that memory managers or memory-resident software is interfering with the successful operation of MACCS2. A clean boot disk would include a set of startup files, CONFIG.SYS and AUTOEXEC.BAT, which contain no references to the HIMEM.SYS and EMM386.EXE drivers from Microsoft. For MACCS2 sessions, the substitute startup files might need to be used instead of the startup files that are set up by the WINDOWS installation process.

No conflicts have ever been encountered when using the Quarterdeck Office Systems QEMM memory manager software. However, sometimes the use of third-party memory managers like QEMM can result in problems running WINDOWS.

INSTALLATION INSTRUCTIONS FOR MACCS2

Create a MACCS2 subdirectory. The MACCS2 subdirectory can be created using the following command:

MD MACCS2 
Copy MAC2ZIPA.ZIP and MAC2ZIPB.ZIP into the MACCS2 subdirectory.

Decompress using Norton Utilities or PKZIP (version 2.04g). Using PKZIP the files may be decompressed using the PKUNZIP command.

The MAC2ZIPA.ZIP and MAC2ZIPB.ZIP files contains 14 sample problems. All of the files required to run the sample problems are included in these two ZIP files. The MACCS2 output from the 14 sample problem runs is provided in the .OUT files on the distribution disk. The contents of the ATMOS, EARLY, and CHRONC input files are echoed in the .OUT files generated for each MACCS2 run. A description of the sample problems is provided in Section 4.4 of the code manual for MACCS2: Volume 1, User's Guide.

On a Pentium $133 \mathrm{MHz}$ PC, the complete set of MACCS2 sample problems, performed using the provided RUNEM.BAT, required approximately $2.5 \mathrm{hrs}$ to execute. The last sample problem executed by RUNEM.BAT, LISTF_RND, required 60 percent of the total run time. To execute the MACCS2 sample problems, type the following command:

\section{RUNEM}

which will regenerate the .OUT files in the MACCS2 directory, overwriting the provided set of output files.

A single MACCS2 run is initiated by calling the RUNMAX2.BAT file and specifying six MACCS2 input file names. On the command line, the user must specify the ATMOS, EARLY, CHRONC, meteorological, and Site Data file names. Sample problem A_O, for example, may be initiated with the following command line:

\section{RUNMAX2 IN1A IN2A IN3A_O METSUR SURSIT LISTA_O}

IN1A is the ATMOS input file

IN2A is the EARLY input file

IN3A_O is the CHRONC input file

METSUR is the meteorological data input file

SURSIT contains site-specific data

LISTA_O is the name of the output file to which the MACCS2 output is to be written

Double quotes "" must be specified as a place holder when a null argument is being supplied.

\section{DOSE CONVERSION FACTOR FILES}

The MAC2ZIPB.ZIP file contains seven DCF files:

DOSDATA.INP - Created by DOSFAC2 and contains the 60 radionuclides considered important to commercial reactor accidents.

DOSD825.INP - Created by FGRDCF and contains all of the radionuclides accessed by FGRDCF. 
DOSD60.INP - Created by FGRDCF and contains the 60 radionuclides contained in DOSDATA.INP.

IDCF2_1.INP, IDCF2_2.INP, IDCF2_3.INP - Combined, these files contain all of the radionuclides that can be accessed by IDCF2.

IDCF2_4.INP - Created by IDCF2 and contains the 60 radionuclides contained in DOSDATA.INP.

The external DCFs produced by IDCF2 are obtained from DOE/EH-0070. A number of radionuclides listed in the IDCF2-produced files are assigned values of 0 for external DCFs for which Federal Guidance Report 12 lists DCF values greater than 0 (provided in the FGRDCF produced files).

\section{INSTALLATION INSTRUCTIONS FOR MACCS2 PREPROCESSORS}

Create subdirectories for each preprocessor to be installed on the system. Decompress the preprocessor ZIP files into the subdirectories.

The MACCS2 DCF input file name is specified by the user in the EARLY input file as the DCF_FILE input parameter. The COMIDA2-generated MACCS2 input file name is user specified in the CHRONC input file as the BIN_FILE input parameter. The user must specify the drive and directory containing the DCF and COMIDA2 MACCS2 input files if the files are not contained in MACCS2 subdirectory.

If COMIDA2-generated MACCS2 input files are used in MACCS2 analyses, the DCF file specified in the EARLY input file must be the same DCF file used to generate the COMIDA2 MACCS2 input file.

The input files required for the FGRDCF and DOSFAC2 preprocessor sample problems can be identified by the .SEL extension. The FGRDCF and DOSFAC2 preprocessors may be exercised using the following commands:

FGRDCF: RUNFGR "filename".SEL

DOSFAC2: DOSFACPC "filename".SEL

The MACCS2 DCF input files created by these two preprocessors are "filename".inp.

All of the DOSFAC2 sample problems can also be run by simply using the RUNEM.BAT file included in the DOSFAC2.ZIP archive.

The IDCF2 sample problem input file, TEST.INP, may be run with the command:

\section{RUNIDCF2 TEST}

The name of the MACCS2 DCF file produced by IDCF2 is fixed in the FORTRAN coding as DOSDATAX.INP. 
The input files for the COMIDA2 sample problems can be identified by the INP extension. (The DOSDATA.INP file included in the COMIDA2 archive is not a COMIDA2 sample problem. It is a DCF file required by the COMIDA2 sample problems.) The COMIDA2 sample problems may be run using the RUNEM.BAT file or by using the following command:

RUNCOM2 "filename".INP

\section{EXECUTION IN THE DOS WINDOW OF WINDOWS 3.1 AND WINDOWS_95}

The MACCS2 software package was developed in a DOS environment. MACCS2 version 1.12 was compiled with Lahey FORTRAN F77L-EM/32 Version 5.20 and can be successfully executed in the DOS window of Microsoft WINDOWS 3.1 and WINDOWS 95.

No information is available on the use of MACCS2 in the IBM OS/2 multitasking environment. However, if MACCS2 is successfully installed and made operational in multitasking environments other than Microsoft WINDOWS, please inform the code developers so that the information can be shared with other code users.

\section{CODE COMPILATION UNDER LAHEY FORTRAN}

The MACCS2.EXE file provided in this archive was generated using Lahey FORTRAN. To recompile MACCS2 using Lahey FORTRAN, execute the DOS command

\section{LAHEY MACCS2}

which will recompile (and use machine-dependent routines in MXXLAHEY.FOR), creating an executable named MACCS2.EXE.

The MACCS2.EXE included in this distribution package was compiled with the following options of Lahey FORTRAN F77L/EM32, version 5.20:

\begin{tabular}{|c|c|c|}
\hline OPTI & ION DESCRIPTION & ON DESCRIPTION \\
\hline /no & - Standard FORTRAN 77 IMPLICIT & /L - Line-number traceback table \\
\hline $\ln 2$ & - Generate 387 constants and code & - Protect constant arguments \\
\hline 14 & - Optimize for 80486 processor & / Q1 - Limit NDP stack entries \\
\hline $\ln 7$ & - Optimize interstatement & /nQ2 - No protected-mode RPC \\
\hline$/ \mathrm{nA} 2$ & - No allocatable array checking & /nQ3 - No real-mode RPC \\
\hline / B & - Check array subscript bounds & InR - No remembering local items \\
\hline$/ \mathrm{nC}$ & - Ignore nonstandard usage & - Create filename.SLD for SOLD \\
\hline$/ \mathrm{nC} 1$ & - INTEGER constants 4 bytes & - INTEGER $* 4$, LOGICAL $* 4$ default \\
\hline$/ \mathrm{D}$ & - DIRECT files without headers & - Not VAX interpretation \\
\hline$/ \mathrm{nH}$ & - No hardcopy source listing & - Display warning messages \\
\hline /I & - Check subprogram Interfaces & - No Xref listing \\
\hline $\operatorname{lnK}$ & - Generate $80 \times 87$ code & - Production optimiZations \\
\hline
\end{tabular}


and it was linked with the following linker statement:

386 link \%1,MXXLAHEY -pack -checksum -stack 300000 -symbols -stub runb

\section{CODE COMPILATION UNDER MICROSOFT POWERSTATION FORTRAN}

MACCS2 version 1.12 can also be recompiled using the Microsoft Powerstation FORTRAN compiler version 1.0a. If MACCS2 is recompiled with the Microsoft compiler, COMIDA2 will also require recompilation because the binary file formats used by the two languages are incompatible.

Under Microsoft FORTRAN, MACCS2 can be recompiled using the MICROSOF.BAT file enclosed as part of this archive, by executing the DOS command

\section{MICROSOF MACCS2}

which will recompile the program and its machine-dependent routines (found in the file MXXMICRO.FOR), and then LINK the program into an executable named MACCS2.EXE. The MICROSOF.BAT file in the MACCS2 archive is different from the file of the same name provided in the COMIDA2 archive. The reason for this is that MACCS2 can be exercised (while COMIDA2 cannot) using the full run-time error checking features of Microsoft FORTRAN.

\section{COMPILING FOR NON-PC SYSTEMS}

The MACCS2 package includes a file of machine-dependent subroutines that must be modified for non-PC environments. These subroutines are provided in MXXLAHEY.FOR and MXXMICRO.FOR for code complilation under Lahey FORTRAN and Microsoft Powerstation FORTRAN respectively. The subroutines include the coding required for VAX/VMS, IBM S/6000, SUN UNIX workstations, and CRAY UNICOS systems. The user who intends to exercise MACCS2 on a non-PC platform must delete the comment characters for the coding required by the platform to be used and comment out the coding required by the PC platform. 


\section{Appendix C: Sample Problem A LISTA_N.OUT Output Listing}

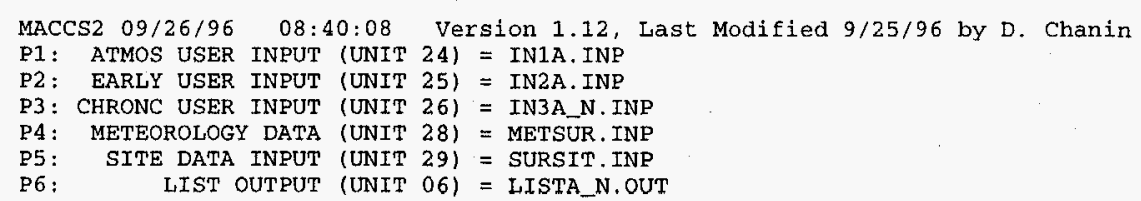

USER INPUT IS READ FROM UNIT 24

RECORD IDENTIFIER FIELDS 11 CHARACTERS LONG ARE EXPECTED.

THE MAXIMUM NUMBER OF IDENTIFIER RECORDS THAT MAY BE SAVED AS THE BASE CASE IS 1000.

RECORD

NUMBER

RECORD

* GENERAL DESCRIPtive titLE DESCRIBING this "ATMOS" INPUT

1 RIATNAM1001 'IN1A. INP, Sample Problem A--Using Table-Lookup Sigmas, ATMOS input: - GEOMETRY DATA BLOCK, LOADED BY INPGEO, STORED IN /GEOM/

* Number of radial Spatial ELEMENTS

2 GENUMRADOO1 2

* SURRY

3 GESPAENDOO 1

GESPAENDO02

GESPAENDO04

$\begin{array}{r}.16 \\ 3.22 \\ \hline\end{array}$

.52
4.02
15.09

4.02
16.09

1.21
4.83
20.92

GESPAEND005

160.93

241.14

321.87

1.61
5.63
25.75

8.05

32.19
112.65

GESPAENDO0 1609.3

* NUCLIDE DATA BLOCK, LOADED BY INPISO, STORED IN /ISOGRP/, /ISONAM/

* Number of pseudo-stable nuclides (used to truncate the decay chains)

ISNUMSTBO0

- List of pseudo-stable nuclides

10 ISNAMSTBO01

ISNAMSTBO02

ISNAMSTBO03

ISNAMSTB004

I-129 (daughter of $\mathrm{Te}-129$ and $\mathrm{Te}-129 \mathrm{~m}$ )

ISNAMSTB005

$\mathrm{Xe}-131$

(daughter of $\mathrm{Te}-129$

(daughter of I-133)

ter of I-135)

ISNAMSTB007

ISNAMSTB008

ISNAMSTB009

ISNAMSTB010

Cs -135

(daughter of $\mathrm{Xe}-135$ )

$\begin{array}{ll}\mathrm{Sm}-147 & \text { (daughter of } \mathrm{Pm}-147 \text { ) } \\ \mathrm{U}-234 & \text { (daughter of } \mathrm{Pu}-238 \text { ) }\end{array}$

$\mathrm{U}-235$ (daughter of $\mathrm{Pu}-239$ )

$\mathrm{U}-236$ (daughter of $\mathrm{Pu}-240$ )

Np-237 (daughter of $A m-241$ )

Np-237 (daughter of Am-241) 


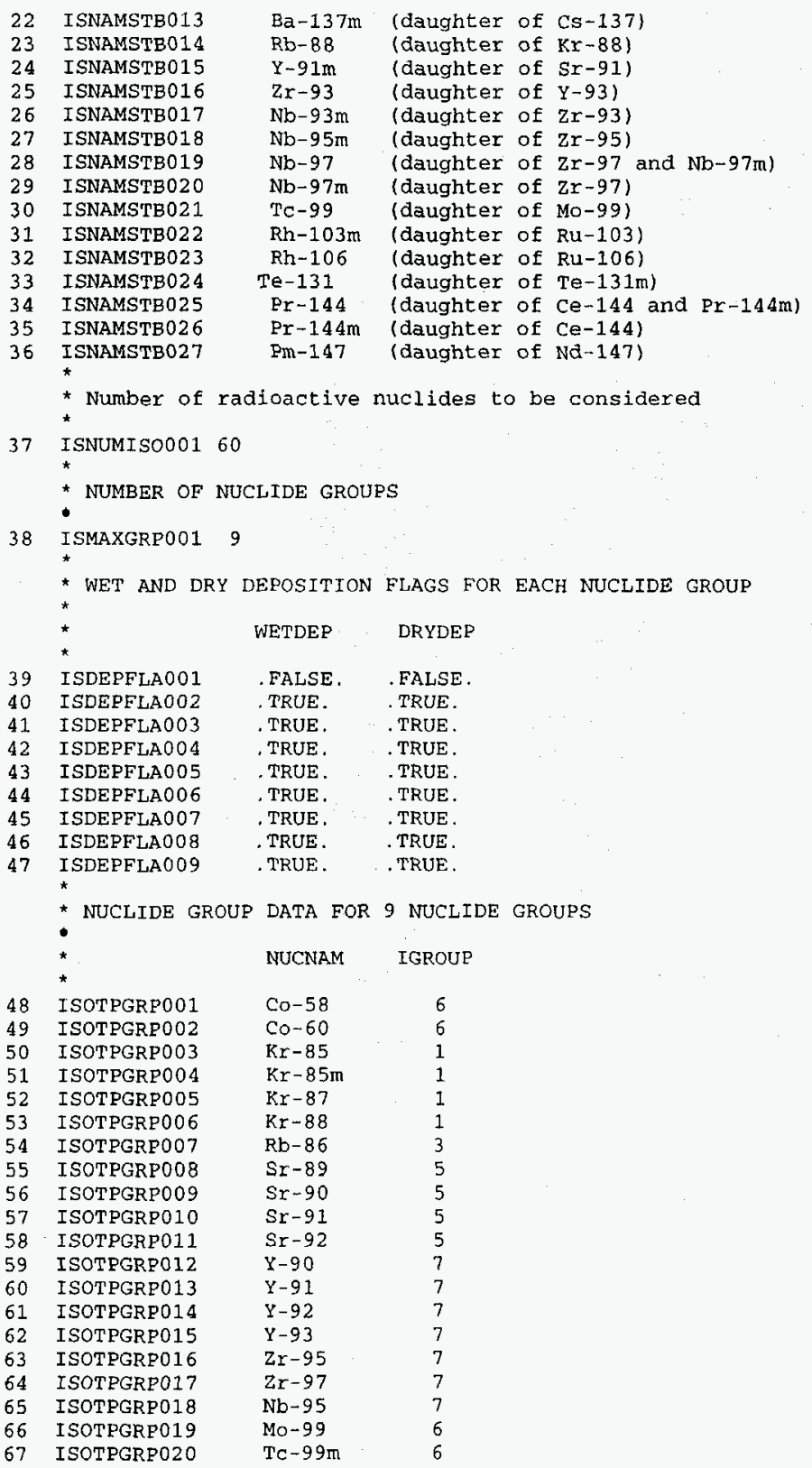


68 ISOTPGRP021 Ru-103

Ru-105

ISOTPGRP023 $3 \mathrm{Ru}-106$

ISOTPGRP024 Rh-105

ISOTPGRP025 $\quad \mathrm{Sb}-127$

$\begin{array}{ll}\text { ISOTPGRPO26 } & \text { Sb-129 } \\ \text { ISOTPGRPO27 } & \mathrm{Te}-127\end{array}$

ISOTPGRPO28 $\mathrm{Te}-127 \mathrm{~m}$

ISOTPGRPO29 Te-129

ISOTRGPO Te-129

ISOTPGR 31 Te-131m

ISOTPRP 033

ISOTPGRD 34

ISOTPGRP035 I-133

ISOTPGRP036 I-134

ISOTPGRP037 I-135

ISOTPGRP038 Xe-1.33

ISOTPGRP039 Xe-135

ISOTPGRPO4O Cs-134

ISOTPGRP041 Cs-136

ISOTPGRP042 Cs-137

ISOTPGRPO43 Ba-139

ISOTPGRPO44 Ba-140

ISOTPGPO45

ISOTPGRPO 7 La-141

ISOTPGROA8 La-142

ISOTPGRPO49 Ce-143

ISOTPGRP050 $\mathrm{Ce}-144$

ISOTPGRP051 Pr-143

ISOTPGRP052 Nd-147

$\mathrm{Np}-239$

ISOTPGRP0 54

ISOTPReO5S 239

ISOTPGRP056 Pu-240

ISOTPGRPO57 Pu-241

ISOTARP Am-241

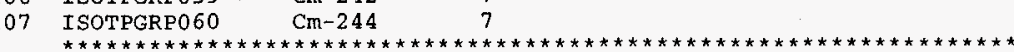

* WET DEPOSITION DATA BLOCK, LOADED BY INPWET, STORED IN/WETCON/

* Washout coefficient number one, linear factor

108 WDCWASH1001 9.5E-5 (JON HELTON AFTER JONES, 1986)

* WASHOUT COEFFICIENT NUMBER TWO, EXPONENTTAL FACTOR

109 WDCWASH2001 0.8 (JON HELTON AFTER JONES, 1986)

* DRY DEPOSITION DATA BLOCK, LOADED BY INPDRY, STORED IN /DRYCON/

* NUMBer of particle size GRoups

110 DDNPSGRPO01 1

* DEPOSITION VELOCity OF EACH PARTICLE SIZE GROUP (M/S)

111 DDVDEPOSO01 0.01 (VALUE SELECTED BY S. ACHARYA, NRC)

* DISPERSION PaRAMETER DATA BLOCK, LOADED By INPDIS, STORED IN /DISPY/, /DISPz/ 
\# of distances in plume-size tables--which can be used as an alternative to the power-law model:

* (to utilize the power-law model, set NUM DIST to zero or delete the following data card)

$112 \mathrm{~N}$

50

* A-stability

114 A-STB/DIS02

115 A-STB/DIS03

116 A-STB/DISO4

118 A-STB/DISO6

119 A-STB/DISO7

120 A-STB/DISO8

121 A-STB/DISO

122 A-STB/DISIO

123 A-STB/DIS11

125 A-STB/DIS13

126 A-STB/DIS14

127 A-STB/DIS15

128 A-STB/DIS16

129 A-STB/DIS17

130 A-STB/DIS18

131 A-STB/DIS19

132 A-STB/DIS20

133 A-STB/DIS21

134 A-STB/DIS22

135 A-STB/DIS23

$\begin{array}{lll}136 & \text { A-STB/DIS24 } \\ + & 137 & \text { A-STB/DIS25 } \\ & 138 & \text { A-STB/DIS26 }\end{array}$

139 A-STB/DIS27

140 A-STB/DIS28

141 A-STB/DIS29

142 A-STB/DIS30

143 A-STB/DIS31

144 A-STB/DIS32

145 A-STB/DIS33

146 A-STB/DIS34

147 A-STB/DIS35

148 A-STB/DIS36

140 A-STB/DIS37

151 A-STB DIS39

152 A-STB/DIS4O

153 A-STB/DIS41

154 A-STB/DIS42

155 A-STB/DIS43

156 A-STB/DIS44

157 A-STB/DIS45

158 A-STB/DIS46

159 A-STB/DIS47

160 A-STB/DIS48

$\begin{array}{ll}161 & \text { A-STB/DIS49 } \\ 162 & \text { A-STB/DIS50 }\end{array}$

* B-stability

$\begin{array}{ll}163 & \text { B-STB/DISO1 } \\ 164 & \text { B-STB/DISO2 }\end{array}$

164 B-STB/DISO2
Distance (m) Sigma-y (m)

$1.000 \mathrm{E}+00 \quad 3.6580 \mathrm{E}-01$

$1.400 \mathrm{E}+00 \quad 4.9569 \mathrm{E}-01$

$\begin{array}{ll}2.000 \mathrm{E}+00 & 6.8408 \mathrm{E}-01 \\ 3.000 \mathrm{E}+00 & 9.8658 \mathrm{E}-01\end{array}$

$\begin{array}{ll}3.000 E+00 & 9.8658 \mathrm{E}-01 \\ 4.000 \mathrm{E}+00 & 1.2793 \mathrm{E}+00\end{array}$

$\begin{array}{ll}4.000 \mathrm{E}+00 & 1.2793 \mathrm{E}+00 \\ 5.000 \mathrm{E}+00 & 1.5649 \mathrm{E}+00\end{array}$

$\begin{array}{ll}5.000 \mathrm{E}+00 & 1.5649 \mathrm{E}+00 \\ 6.000 \mathrm{E}+00 & 1.8450 \mathrm{E}+00\end{array}$

$8.000 \mathrm{E}+00 \quad 2.3923 \mathrm{E}+00$

..000E+01 2.9265E+00

3. $3726 \mathrm{E}+01$

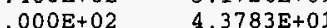

$.000 \mathrm{E}+02 \quad 6.3144 \mathrm{E}+01$

$4.000 \mathrm{E}+02 \quad 8.1877 \mathrm{E}+01$

$5.000 \mathrm{E}+02 \quad 1.0016 \mathrm{E}+02$

$6.000 E+02 \quad 1.1808 E+02$

$8.000 \mathrm{E}+02 \quad 1.5312 \mathrm{E}+02$

$1.000 \mathrm{E}+03 \quad 1.8730 \mathrm{E}+02$

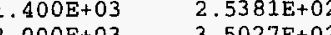

$2.000 \mathrm{E}+03 \quad 3.5027 \mathrm{E}+02$

$3.000 \mathrm{E}+03 \quad 5.0516 \mathrm{E}+02$

$5.000 \mathrm{E}+03 \quad 8.0128 \mathrm{E}+02$

$6.000 \mathrm{E}+03-9.4470 \mathrm{E}+02$

$8.000 \mathrm{E}+03 \quad 1.2250 \mathrm{E}+03$

$1.000 \mathrm{E}+04 \quad 1.4985 \mathrm{E}+03$

$1.400 \mathrm{E}+04 \quad 2.0305 \mathrm{E}+03$

$2.000 \mathrm{E}+04 \quad 2.8022 \mathrm{E}+03$

$3.000 \mathrm{E}+04 \quad 4.0414 \mathrm{E}+03$

$4.000 \mathrm{E}+04 \quad 5.2404 \mathrm{E}+03$

$5.000 \mathrm{E}+04 \quad 6.4104 \mathrm{E}+03$

6.000B+04

$1.000 \mathrm{0}-1.1988 \mathrm{E}+04$

$1.400 \mathrm{E}+05$

$2.000 \mathrm{E}+05$

$3.2332 \mathrm{E}+04$

4.000E+05 4. $1924 \mathrm{E}+04$

$5.000 \mathrm{E}+05 \quad 5.1284 \mathrm{E}+04$

$6.000 \mathrm{E}+05 \quad 6.0463 \mathrm{E}+04$

$8.000 \mathrm{E}+05 \quad 7.8401 \mathrm{E}+04$

$1.000 \mathrm{E}+06 \quad 9.5906 \mathrm{E}+04$

$1.400 \mathrm{E}+06 \quad 1.2996 \mathrm{E}+05$

$2.000 \mathrm{E}+06 \quad 1.7935 \mathrm{E}+05$

$2.5866 \mathrm{E}+05$

3.3540E+05

$6.000 \mathrm{E}+06 \quad 4.83728+05$

$.000 \mathrm{E}+06 \quad 6.2723 \mathrm{E}+05$

Distance $(\mathrm{m})$

$1.400 \mathrm{E}+00$

$2.000 \mathrm{E}+00$
Sigma-z (m)

$2.5000 \mathrm{E}-04$ Tadmor/Gur $(0.5-5 \mathrm{~km})$ $5.1105 \mathrm{E}-04$ Tadmor/Gur $(0.5-5 \mathrm{~km})$ $1.0905 \mathrm{E}-03$ Tadmor/Gur $(0.5-5 \mathrm{~km})$ $2.5812 \mathrm{E}-03$ Tadmor/Gur $(0.5-5 \mathrm{~km}$ $4.7568 \mathrm{E}-03$ Tadmor/Gur $(0.5-5 \mathrm{~km})$ $7.6428 \mathrm{E}-03$ Tadmor/Gur $(0.5-5 \mathrm{~km})$ $1.1259 \mathrm{E}-02$ Tadmor/Gur $(0.5-5 \mathrm{~km})$ $3.3338 \mathrm{E}-02$ Tadmor/Gur $(0.5-5 \mathrm{~km})$ $4.4457 \mathrm{E}+00$ Tadmor $/ \mathrm{Gur}(0.5-5 \mathrm{~km})$ $9.0879 \mathrm{E}+00$ Tadmor/Gur $(0.5-5 \mathrm{~km})$ $1.9392 \mathrm{E}+01$ Tadmor/Gur $(0.5-5 \mathrm{~km})$ $4.5901 \mathrm{E}+01$ Tadmor/Gur $(0.5-5 \mathrm{~km})$ 8.4590E+01 Tadmor/Gur $(0.5-5 \mathrm{~km})$ $1.3591 \mathrm{E}+02$ Tadmor/Gur $(0.5-5 \mathrm{~km})$ $2.0022 \mathrm{E}+02$ Tadmor/Gur $(0.5-5 \mathrm{~km})$ $3.6898 \mathrm{E}+02$ Tadmor/Gur $(0.5-5 \mathrm{~km})$ $5.9284 \mathrm{E}+02$ Tadmor/Gur $(0.5-5 \mathrm{~km})$ $1.2119 \mathrm{E}+03$ Tadmor/Gur $(0.5-5 \mathrm{~km})$ $\begin{array}{lll}2.5860 \mathrm{E}+03 & \text { Tadmor/Gur } & (0.5-5 \mathrm{~km}) \\ 6.1210 \mathrm{E}+03 & \text { Tadmor/Gur } & (0.5-5 \mathrm{~km})\end{array}$ $1.1280 \mathrm{E}+04$ Tadmor/Gur $(0.5-5 \mathrm{~km})$ $1.8124 \mathrm{E}+04$ Tadmor/Gur $(0.5-5 \mathrm{~km})$ $2.6700 \mathrm{E}+04$ Tadmor/Gur $(0.5-5 \mathrm{~km})$ $4.9205 \mathrm{E}+04$ Tadmor/Gur $(0.5-5 \mathrm{~km})$ $7.9057 \mathrm{E}+04$ Tadmor/Gur $(0.5-5 \mathrm{~km})$ $1.6161 \mathrm{E}+05$ Tadmor/Gur $(0.5-5 \mathrm{~km})$ $3.4485 \mathrm{E}+05$ Tadmor/Gur $(0.5-5 \mathrm{~km})$ $8.1625 \mathrm{E}+05$ Tadmor/Gur $(0.5-5 \mathrm{~km})$ $1.5042 \mathrm{E}+06$ Tadmor/Gur $(0.5-5 \mathrm{~km})$ $2.4169 \mathrm{E}+06$ Tadmor $/$ Gur $(0.5-5 \mathrm{~km})$ $3.5605 \mathrm{E}+06$ Tadmor/Gur $(0.5-5 \mathrm{~km})$ $1.0542 \mathrm{E}+07$ Tadmor $(\mathrm{Gur})(0.5-5 \mathrm{~km})$ $2.1551 \mathrm{E}+07$ Tadmor/Gur $(0.5-5 \mathrm{~km})$ $4.5986 \mathrm{E}+07$ Tadmor/Gur $(0.5-5 \mathrm{~km})$ $1.0885 \mathrm{E}+08$ Tadmor/Gur $(0.5-5 \mathrm{~km})$ $2.0059 \mathrm{E}+08$ Tadmor/Gur $(0.5-5 \mathrm{~km})$ $3.2229 \mathrm{E}+08$ Tadmor/Gur $(0.5-5 \mathrm{~km})$ $4.7480 \mathrm{E}+08$ Tadmor/Gur $(0.5-5 \mathrm{~km})$ $8.7500 \mathrm{E}+08$ Tadmor/Gur $(0.5-5 \mathrm{~km})$ $1.4059 \mathrm{E}+09$ Tadmor/Gur $(0.5-5 \mathrm{~km})$ $2.8738 \mathrm{E}+09$ Tadmor/Gur $(0.5-5 \mathrm{~km})$ $6.1324 \mathrm{E}+09$ Tadmor/Gur $(0.5-5 \mathrm{~km})$ $1.4515 \mathrm{E}+10$ Tadmor/Gur $(0.5-5 \mathrm{~km})$ $2.6750 \mathrm{E}+10$ Tadmor/Gur $(0.5-5 \mathrm{~km}$ ) $6.3316 \mathrm{E}+10$ Tadmor $/ \mathrm{Gur}(0.5-5 \mathrm{~km})$ $1.1668 \mathrm{E}+11$ Tadmor/Gur $(0.5-5 \mathrm{~km})$ $1.8747 \mathrm{E}+11$ Tadmor/Gur $10.5-5 \mathrm{~km})$

Sigma-z (m)

$1.9000 \mathrm{E}-03$ Tadmor/Gur $(0.5-5 \mathrm{~km})$ $\begin{array}{lll}3.2574 \mathrm{E}-03 & \text { Tadmor/Gur } & (0.5-5 \mathrm{~km}) \\ 5.7681 \mathrm{E}-03 & \text { Tadmor/Gur } & (0.5-5 \mathrm{~km})\end{array}$

$\begin{array}{llll}\text { Sigma-y (m) } & \text { Sigma-z (m) } & & \\ 2.7510 \mathrm{E}-01 & 1.9000 \mathrm{E}-03 & \text { Tadmor } / \text { Gur } & (0.5-5 \mathrm{~km}) \\ 3.7279 \mathrm{E}-01 & 3.2574 \mathrm{E}-03 & \text { Tadmor } / \text { Gur } & (0.5-5 \mathrm{~km}) \\ 5.1446 \mathrm{E}-01 & 5.7681 \mathrm{E}-03 & \text { Tadmor } / \text { Gur } & (0.5-5 \mathrm{~km})\end{array}$




\begin{tabular}{|c|c|c|c|c|c|c|c|}
\hline 166 & B-STB / DISO 4 & $3.000 \mathrm{E}+00$ & $7.4196 \mathrm{E}-01$ & $1.1045 \mathrm{E}-02$ & Tadmor/Gur & $10.5-5$ & $\mathrm{~km}$ ) \\
\hline 167 & B-STB/DISO5 & $4.000 \mathrm{E}+00$ & $9.6208 \mathrm{E}-01$ & $1.7511 \mathrm{E}-02$ & Tadmor/Gur & $10.5-5$ & $\mathrm{~km}$ ) \\
\hline 168 & B-STB /DISO6 & $5.000 E+00$ & $1.1769 \mathrm{E}+00$ & $2.5036 \mathrm{E}-02$ & Tadmor/Gur & $10.5-5$ & $\mathrm{~km}$ ) \\
\hline 169 & B-STB/DIS07 & $6.000 \mathrm{E}+00$ & $1.3875 \mathrm{E}+00$ & $3.3530 \mathrm{E}-02$ & Tadmor/Gur & $10.5-5$ & $\mathrm{~km})$ \\
\hline 170 & B-STB/DIS08 & $8.000 E+00$ & $1.7992 \mathrm{E}+00$ & $5.3161 \mathrm{E}-02$ & Tadmor/Gur & $(0.5-5$ & $\mathrm{km})$ \\
\hline 171 & B-STB /DIS09 & $1.000 \mathrm{E}+01$ & $2.2009 E+00$ & $7.6007 \mathrm{E}-02$ & Tadmor/Gur & $(0.5-5$ & $\mathrm{km}$ ) \\
\hline 172 & B-STB/DIS10 & $1.000 E+02$ & $1.7607 \mathrm{E}+01$ & $3.0406 E+00$ & Tadmor/Gur & $10.5-5$ & $\mathrm{~km})$ \\
\hline 173 & B-STB/DIS11 & $1.400 E+02$ & $2.3859 \mathrm{E}+01$ & $5.2127 \mathrm{E}+00$ & Tadmor/Gur & $(0.5-5$ & $\mathrm{km}$ ) \\
\hline 174 & B-STB $/ D I S 12$ & 2. $000 \mathrm{E}+02$ & $3.2927 \mathrm{E}+01$ & $9.2307 E+00$ & Tadmor/Gur & $(0.5-5$ & $\mathrm{km}$ ) \\
\hline 175 & B-STB/DIS13 & $3.000 \mathrm{E}+02$ & $4.7487 \mathrm{E}+01$ & $1.7675 \mathrm{E}+01$ & Tadmor/Gur & $(0.5-5$ & $\mathrm{km}$ ) \\
\hline 176 & B-STB/DIS14 & $4.000 E+02$ & $6.1576 \mathrm{E}+01$ & $2.8023 \mathrm{E}+01$ & Tadmor/Gur & $10.5-5$ & $\mathrm{~km}$ ) \\
\hline 177 & B-STB/DIS15 & $5.000 E+02$ & $7.5323 \mathrm{E}+01$ & $4.0066 \mathrm{E}+01$ & Tadmor/Gur & $10.5-5$ & $\mathrm{~km}$ ) \\
\hline 178 & B-STB/DIS16 & $6.000 \mathrm{E}+02$ & $8.8805 E+01$ & $5.3657 \mathrm{E}+01$ & Tadmor/Gur & $10.5-5$ & $\mathrm{~km})$ \\
\hline 179 & B-STB/DIS17 & $8.000 E+02$ & $1.1515 \mathrm{E}+02$ & $8.5073 \mathrm{E}+01$ & Tadmor/Gur & $(0.5-5$ & $\mathrm{km}$ ) \\
\hline 180 & B-STB/DIS18 & $1.000 \mathrm{E}+03$ & $1.4086 \mathrm{E}+02$ & $1.2163 \mathrm{E}+02$ & Tadmor/Gur & $0.5-5$ & $\mathrm{~km}$ ) \\
\hline 181 & B-STB/DIS19 & $1.400 \mathrm{E}+03$ & $1.9088 E+02$ & $2.0853 E+02$ & Tadmor/Gur & $(0.5-5$ & $\mathrm{km}$ ) \\
\hline 182 & B-STB/DIS20 & $2.000 E+03$ & $2.6342 \mathrm{E}+02$ & $3.6926 \mathrm{E}+02$ & Tadmor/Gur & $(0.5-5$ & $\mathrm{km})$ \\
\hline 183 & B-STB / DIS21 & $3.000 \mathrm{E}+03$ & $3.7991 \mathrm{E}+02$ & $7.0705 \mathrm{E}+02$ & Tadmor/Gur & $(0.5-5$ & $\mathrm{km}$ ) \\
\hline 184 & B-STB/DIS22 & $4.000 E+03$ & $4.9262 \mathrm{E}+02$ & $1.1210 E+03$ & Tadmor/Gur & $10.5-5$ & $\mathrm{~km})$ \\
\hline 185 & $\mathrm{~B}-\mathrm{STB} / \mathrm{DIS} 23$ & $5.000 \mathrm{E}+03$ & $6.0260 \mathrm{E}+02$ & $1.6028 \mathrm{E}+03$ & Tadmor/Gur & $(0.5-5$ & $\mathrm{km}$ ) \\
\hline 186 & B-STB/DIS24 & $6.000 \mathrm{E}+03$ & $7.1046 \mathrm{E}+02$ & 2. $1465 E+03$ & Tadmor/Gur & $10.5-5$ & $\mathrm{~km}$ ) \\
\hline 187 & B-STB/DIS25 & $8.000 \mathrm{E}+03$ & $9.2124 \mathrm{E}+02$ & $3.4033 \mathrm{E}+03$ & Tadmor/Gur & $10.5-5$ & $\mathrm{~km}$ ) \\
\hline 188 & B-STB/DIS26 & $1.000 E+04$ & $1.1269 \mathrm{E}+03$ & $4.8658 \mathrm{E}+03$ & Tadmor/Gur & $10.5-5$ & $\mathrm{~km})$ \\
\hline 189 & B-STB / DIS27 & $1.400 \mathrm{E}+04$ & $1.5271 \mathrm{E}+03$ & $8.3419 \mathrm{E}+03$ & Tadmor/Gur & $10.5-5$ & $\mathrm{~km}$ ) \\
\hline 190 & B-STB $/$ DIS2 8 & $2.000 \mathrm{E}+04$ & $2.1074 E+03$ & $1.4772 \mathrm{E}+04$ & Tadmor/Gur & $10.5-5$ & $\mathrm{~km}$ ) \\
\hline 191 & B-STB/DIS29 & $3.000 E+04$ & $3.0393 \mathrm{E}+03$ & $2.8285 \mathrm{E}+04$ & Tadmor/Gur & $10.5-5$ & $\mathrm{~km}$ ) \\
\hline 192 & B-STB / DIS30 & $4.000 \mathrm{E}+04$ & $3.9410 \mathrm{E}+03$ & $4.4845 \mathrm{E}+04$ & Tadmor/Gur & $10.5-5$ & $\mathrm{~km})$ \\
\hline 193 & B-STB/DIS31 & $5.000 E+04$ & $4.8209 \mathrm{E}+03$ & $6.4117 \mathrm{E}+04$ & Tadmor/Gur & $0.5-5$ & $\mathrm{~km}$ ) \\
\hline 194 & B-STB / DIS32 & $6.000 \mathrm{E}+04$ & $5.6838 \mathrm{E}+03$ & $8.5868 \mathrm{E}+04$ & Tadmor/Gur & $10.5-5$ & $\mathrm{~km}$ ) \\
\hline 195 & B-STB $/$ DIS33 & $8.000 \mathrm{E}+04$ & $7.3701 \mathrm{E}+03$ & $1.3614 \mathrm{E}+05$ & Tadmor/Gur & $10.5-5$ & $\mathrm{~km})$ \\
\hline 196 & $\mathrm{~B}-\mathrm{STB} / \mathrm{DIS} 34$ & $1.000 \mathrm{E}+05$ & $9.0155 \mathrm{E}+03$ & $1.9465 E+05$ & Tadmor/Gur & $(0.5-5$ & $\mathrm{km})$ \\
\hline 197 & $\mathrm{~B}-\mathrm{STB} / \mathrm{DIS} 35$ & $1.400 \mathrm{E}+05$ & $1.2217 \mathrm{E}+04$ & $3.3371 E+05$ & Tadmor/Gur & $10.5-5$ & $\mathrm{~km}$ ) \\
\hline 198 & B-STB /DIS36 & $2.000 E+05$ & $1.6860 \mathrm{E}+04$ & $5.9093 \mathrm{E}+05$ & Tadmor/Gur & $10.5-5$ & $\mathrm{~km})$ \\
\hline 199 & B-STB/DIS37 & $3.000 \mathrm{E}+05$ & $2.4315 \mathrm{E}+04$ & $1.1315 \mathrm{E}+06$ & Tadmor/Gur & $10.5-5$ & $\mathrm{~km})$ \\
\hline 200 & $\mathrm{~B}-\mathrm{STB} / \mathrm{DIS} 38$ & $4.000 E+05$ & $3.1529 \mathrm{E}+04$ & $1.7940 \mathrm{E}+06$ & Tadmor/Gur & $10.5-5$ & $\mathrm{~km})$ \\
\hline 201 & $\mathrm{~B}-\mathrm{STB} / \mathrm{DIS} 39$ & $5.000 E+05$ & $3.8568 \mathrm{E}+04$ & $2.5649 \mathrm{E}+06$ & Tadmor/Gur & $0.5-5$ & $\mathrm{~km})$ \\
\hline 202 & $\mathrm{~B}-\mathrm{STB} / \mathrm{DIS40}$ & $6.000 \mathrm{E}+05$ & $4.5471 \mathrm{E}+04$ & $3.4350 \mathrm{E}+06$ & Tadmor/Gur & $10.5-5$ & $\mathrm{~km}$ ) \\
\hline 203 & $\mathrm{~B}-\mathrm{STB} / \mathrm{DIS} 41$ & $8.000 \mathrm{E}+05$ & $5.8962 E+04$ & $5.4462 \mathrm{E}+06$ & Tadmor/Gur & $10.5-5$ & $\mathrm{~km})$ \\
\hline 204 & B-STB $/$ DIS 42 & $1.000 \mathrm{E}+06$ & $7.2126 \mathrm{E}+04$ & $7.7867 \mathrm{E}+06$ & Tadmor/Gur & $10.5-5$ & $\mathrm{~km})$ \\
\hline 205 & B-STB/DIS43 & 1.400 & $9.7737 \mathrm{E}+04$ & $1.3350 E+07$ & Tadmor/Gur & $(0.5-5$ & $\mathrm{km})$ \\
\hline 206 & B-STB/DIS44 & $2.000 \mathrm{E}+06$ & $\begin{array}{l}1.3488 \mathrm{E}+05 \\
1\end{array}$ & $2.3639 \mathrm{E}+07$ & Tadmor/Gur & $0.5-5$ & $\mathrm{~km}$ ) \\
\hline 207 & B-STB / DIS 45 & $3.000 \mathrm{E}+06$ & $1.9453 \mathrm{E}+05$ & $4.5264 \mathrm{E}+07$ & r/Gur & $10.5-5$ & $\mathrm{~km}$ ) \\
\hline 208 & B-STB/DIS46 6 & $4.000 \mathrm{E}+06$ & $2.5224 E+05$ & $7.1765 \mathrm{E}+07$ & Tadmor/Gur & $10.5-5$ & $\mathrm{~km}$ ) \\
\hline 209 & B-STB /DIS47 & 5.00 & $3.0855 \mathrm{E}+05$ & $1.0261 \mathrm{E}+08$ & Tadmor/Gur & $0.5-5$ & $\mathrm{~km}$ ) \\
\hline 210 & B-STB / DIS48 & $6.000 E+06$ & $3.6378 \mathrm{E}+05$ & $1.3741 \mathrm{E}+08$ & Tadmor/Gur & $10.5-5$ & $\mathrm{~km}$ ) \\
\hline 211 & B-STB /DIS 49 & $8.000 \mathrm{E}+06$ & $4.7171 \mathrm{E}+05$ & $2.1787 E+08$ & Tadmor/Gur & $10.5-5$ & $\mathrm{~km}$ ) \\
\hline 212 & B-STB/DIS5O & $1.000 \mathrm{E}+07$ & $5.7702 \mathrm{E}+05$ & $3.1150 \mathrm{E}+08$ & Tadmor/Gur & $10.5-5$ & $\mathrm{~km}$ ) \\
\hline & * c-stability & Distance $(\mathrm{m})$ & Sigma-y (m) & Sigma-z (m) & & & \\
\hline 213 & C-STB/DISO1 & $1.000 \mathrm{E}+00$ & $2.0890 \mathrm{E}-01$ & $2.0000 E-01$ & Tadmor/Gur & & $\mathrm{km})$ \\
\hline 214 & C-STB/DIS02 & $1.400 E+00$ & $2.8308 \mathrm{E}-01$ & $2.6660 \mathrm{E}-01$ & Tadmo & 10.5 & $\mathrm{~km}$ ? \\
\hline 215 & C-STB/DIS03 & 2.000 & $3.9066 \mathrm{E}-01$ & $3.6158 \mathrm{E}-01$ & Tadmor/Gur & $10.5-5$ & $\mathrm{~km}$ ) \\
\hline 216 & C-STB/DISO4 & $3.000 \mathrm{E}+00$ & $5.6341 \mathrm{E}-01$ & $5.1125 \mathrm{E}-01$ & Tadmor/Gur & $0.5-5$ & $\mathrm{~km})$ \\
\hline 217 & C-STB/DISO5 & $4.000 E+00$ & $7,3056 \mathrm{E}-01$ & $6.5369 \mathrm{E}-01$ & Tadmor/Gur & $10.5-5$ & $\mathrm{~km}$ ) \\
\hline 218 & C-STB/DIS06 & $5.000 \mathrm{E}+00$ & $8.9367 \mathrm{E}-01$ & $7.9097 \mathrm{E}-01$ & Tadmor/Gur & & $\mathrm{km}$ ) \\
\hline 219 & C-STB/DISO 7 & $6.000 \mathrm{E}+00$ & $1.0536 \mathrm{E}+00$ & 9.24 & Tadmor/Gur & $10.5-5$ & \\
\hline 220 & C-STB/DISO 8 & $8.000 \mathrm{E}+00$ & $1.3662 \mathrm{E}+00$ & $1.1818 E+00$ & Tadmor/Gur & $10.5-5$ & $\mathrm{~km})$ \\
\hline 221 & C-STB/DISO9 & $1.000 \mathrm{E}+01$ & $1.6712 \mathrm{E}+00$ & $1.4300 \mathrm{E}+00$ & Tadmor/Gur & $10.5-5$ & $\mathrm{~km})$ \\
\hline 222 & C-STB/DIS10 & $1.000 E+02$ & $\begin{array}{l}1.3370 E+01 \\
1.337\end{array}$ & $1.0224 \mathrm{E}+01$ & Tadmor/Gur & $10.5-5$ & $\mathrm{~km})$ \\
\hline 223 & C-STB & & & $1.3629 \mathrm{E}+01$ & Tadmor/Gur & & \\
\hline 224 & C-STB/DIS12 & $2.000 E+02$ & & & Tadmor/Gur & & \\
\hline 224 & C-STB/DIS13 & $\begin{array}{l}2.000 E+02 \\
3.000 E+02\end{array}$ & $3.6060 E+01$ & $2.6136 \mathrm{E}+01$ & Tadmor/Gur & $10.5-5$ & $\mathrm{~km}$ ) \\
\hline
\end{tabular}


226 C-STB/DIS14 227 C-STB/DIS15 C-STB/DIS17 C31 C-STB/DIS19 232 C-STB/DIS 20 233 C-STB/DIS21 234 C-STB/DIS22 235 C-STB/DIS 23 236 C-STB/DTS2 4 237 C-STB/DIS25 238 C-STB/DIS26 239 C-STB/DIS27 240 C-STB/DIS 28 241 C-STB/DIS29 242 C-STB/DIS30 243 C-SIB/DIS31 244 C-STB/DIS32 245 C-STB/DIS33 247 -STB/DIS35 248 C-STB/DIS 36 249 C-STB/DIS37 250 C-STB/DIS38 251 C-STB/DIS39 252 C-STB/DIS40 253 C-STB/DIS41 254 C-STB/DIS42 255 C-STB/DIS43 258 C-STB/DIS46 259 C-STB/DIS47 260 C-ST

262 C-STB/DIS50

$$
\text { - D-stability }
$$

263 D-STB/DIS01

264 D-STB/DIS02

266 D-STB/DISO4

269 D-STB/DISO7

270 D-STB/DIS08

275 D-STB/DIS13

276 D-STB/DIS14

277 D-STB/DIS15

278 D-STB/DIS16

279 D-STB/DISI7

282 DDTB/DIST

D-STB/DIS22

285 D-STB/DIS23
$4.000 \mathrm{E}+0$

$5.000 \mathrm{E}+02$

$6.000 \mathrm{E}+02$

$.000 \mathrm{E}+03$

$400 E+03$

$2.000 \mathrm{E}+03$

. $000 \mathrm{E}+03$

$4.000 \mathrm{E}+03$

$5.000 E+03$

$5.000 \mathrm{E}+03$
$6.000 \mathrm{E}+03$

8.000E+03

$.000 \mathrm{E}+04$

$.400 \mathrm{E}+04$

$2.000 \mathrm{E}+04$

3. $000 \mathrm{E}+04$

$4.000 \mathrm{E}+04$

5.000E+04

.

$1.000 \mathrm{E}+05$

$1.400 \mathrm{E}+05$

$2.000 \mathrm{E}+05$

$3.000 \mathrm{E}+05$

$4.000 E+05$

$.000 \mathrm{E}+05$

$6.000 \mathrm{E}+05$

$8.000 \mathrm{E}+05$

1. $000 \mathrm{E}+06$

1. $400 \mathrm{E}+06$

$2.000 \mathrm{E}+06$

$4.000 \mathrm{E}+06$

$5.000 \mathrm{E}+06$

$6.000 \mathrm{E}+06$

$8.000 \mathrm{E}+06$

$1.000 \mathrm{E}+07$

Distance (m)

$1.000 \mathrm{E}+00$

$1.400 E+00$

2. $.000 \mathrm{E}+00$

$3.000 \mathrm{E}+00$

$4.000 \mathrm{E}+00$
$5.000 \mathrm{E}+00$

.

$1.000 E+00$

. $000 \mathrm{E}+01$

1. $000 \mathrm{E}+02$

1. $400 \mathrm{E}+02$

$2.000 \mathrm{E}+02$

$3.000 \mathrm{E}+02$

$4.000 \mathrm{E}+02$

. $000 \mathrm{E}+02$

$.000 \mathrm{E}+02$

$8.000 \mathrm{E}+02$

1. $000 \mathrm{E}+03$

2. $400 \mathrm{E}+03$

$2.000 \mathrm{E}+03$

$4.000 \mathrm{E}+03$

$5.000 \mathrm{E}+03$
4. $6758 \mathrm{E}+01$ 5. $7198 \mathrm{E}+01$

$6.7435 \mathrm{E}+01$

1. $0696 \mathrm{E}+02$

$1.4495 \mathrm{E}+02$

$2.0003 \mathrm{E}+0$

$2.8849 \mathrm{E}+02$

3.7408E+02

4. $5759 \mathrm{E}+02$

$5.3949 \mathrm{E}+02$

$6.9955 \mathrm{E}+02$

8. $5573 \mathrm{E}+02$

1. $1596 \mathrm{E}+03$

1. $6003 \mathrm{E}+03$

$2.3080 \mathrm{E}+03$

3. $6927 \mathrm{E}+03$

3. $3608 \mathrm{E}+03$

5. $5965 \mathrm{E}+03$

$6.8460 \mathrm{E}+03$

$9.2770 \mathrm{E}+03$

$1.2803 \mathrm{E}+04$

1. $8464 \mathrm{E}+04$

2. $3942 \mathrm{E}+04$

$2.9287 \mathrm{E}+04$

$3.4529 \mathrm{E}+04$

$4.4773 \mathrm{E}+04$

7. $4218 \mathrm{E}+04$

$9154 \mathrm{E}+05$

$3430 \mathrm{E}+05$

2. $7624 \mathrm{E}+05$

$3.5819 \mathrm{E}+05$
$4.3817 \mathrm{E}+05$

Sigma-y (m)

1. $4740 \mathrm{E}-01$

1. $9974 \mathrm{E}-01$

$2.7565 \mathrm{E}-01$
$3.9754 \mathrm{E}-01$

5. $15454 \mathrm{E}-01$

5. $1549 \mathrm{E}-0.1$

7. $4344 \mathrm{E}-01$

$9.6400 \mathrm{E}-01$

$1.1792 \mathrm{E}+00$

9. $4340 \mathrm{E}+00$

1. $2784 \mathrm{E}+01$

$1.7642 \mathrm{E}+01$

2. $5444 \mathrm{E}+01$

3. $2993 \mathrm{E}+01$

4. $0359 \mathrm{E}+01$

$4.7582 \mathrm{E}+01$

7. $5474 \mathrm{E}+01$

$1.0227 \mathrm{E}+02$

1. $4114 \mathrm{E}+0$

2. $0356 \mathrm{E}+02$

$3.2288 \mathrm{E}+02$
$3.3417 \mathrm{E}+01$ Tadmor/Gur $(0.5-5 \mathrm{~km})$ $4.0435 \mathrm{E}+01$ Tadmor/Gur $(0.5-5 \mathrm{~km}$ ) $6.0414 \mathrm{E}+01$ Tadmor $/ \mathrm{Gur}(0.5-5 \mathrm{~km})$ $7.3102 \mathrm{E}+01$ Tadmor/Gur $(0.5-5 \mathrm{~km})$ $9.7447 \mathrm{E}+01$ Tadmor/Gur $(0.5-5 \mathrm{~km})$ $1.3216 \mathrm{E}+02$ Tadmor/Gur $(0.5-5 \mathrm{~km})$ $1.8687 \mathrm{E}+02$ Tadmor/Gur $(0.5-5 \mathrm{~km})$ $2.3893 \mathrm{E}+02$ Tadmor/Gur $(0.5-5 \mathrm{~km})$ $2.8911 \mathrm{E}+02$ Tadmor/Gur $(0.5-5 \mathrm{~km})$ $3.3784 \mathrm{E}+02$ Tadmor/Gur $(0.5-5 \mathrm{~km})$ $4.3196 \mathrm{E}+02$ Tadmor/Gur $(0.5-5 \mathrm{~km})$ $5.2267 \mathrm{E}+02$ Tadmor/Gur $(0.5-5 \mathrm{~km})$ $6.9673 \mathrm{E}+02$ Tadmor/Gur $(0.5-5 \mathrm{~km})$ $9.4493 \mathrm{E}+02$ Tadmor/Gur $(0.5-5 \mathrm{~km})$ $1.3361 \mathrm{E}+03$ Tadmor/Gur $(0.5-5 \mathrm{~km})$ $1.7083 \mathrm{E}+03$ Tadmor/Gur $(0.5-5 \mathrm{~km})$ $3.0155 \mathrm{E}+03$ Tadmor/Gur $(0.5-5 \mathrm{~km})$ $3.0884 \mathrm{E}+03$ Tadmor/Gur $(0.5-5 \mathrm{~km})$ $3.7371 \mathrm{E}+03$ Tadmor/Gur $(0.5-5 \mathrm{~km})$ $4.9816 \mathrm{E}+03$ Tadmor/Gur $(0.5-5 \mathrm{~km})$ $6.7562 \mathrm{E}+03$ Tadmor/Gur $(0.5-5 \mathrm{~km}$ ) $9.5529 \mathrm{E}+03$ Tadmor/Gur $10.5-5 \mathrm{~km})$ $1.2214 \mathrm{E}+04$ Tadmor/Gur $(0.5-5 \mathrm{~km})$ $1.4780 \mathrm{E}+04$ Tadmor/Gur $(0.5-5 \mathrm{~km})$ $1.7270 \mathrm{E}+04$ Tadmor/Gur $(0.5-5 \mathrm{~km})$ $2.2082 \mathrm{E}+04$ Tadmor/Gur $(0.5-5 \mathrm{~km})$ $2.6720 \mathrm{E}+04$ Tadmor $/ \mathrm{Gur}(0.5-5 \mathrm{~km})$ $3.5306 \mathrm{E}+04$ Tadmor/Gur $(0.5-5 \mathrm{~km})$ $6.8302 \mathrm{E}+04$ Tadmor/Gur $(0.5-5 \mathrm{~km})$ $8.7331 \mathrm{E}+04$ Tadmor $/ \mathrm{Gur}(0.5-5 \mathrm{~km})$ $1.0567 \mathrm{E}+05$ Tadmor/Gur $(0.5-5 \mathrm{~km})$ $1.2348 \mathrm{E}+05$ Tadmor/Gur $(0.5-5 \mathrm{~km})$ $1.5788 \mathrm{E}+05$ Tadmor/Gur $(0.5-5 \mathrm{~km})$ $1.9104 \mathrm{E}+05$ Tadmor/Gur $(0.5-5 \mathrm{~km})$

Sigma-z (m)
$3.0000 \mathrm{E}-01$ Tadmor/Gur $(0.5-5 \mathrm{~km})$ $3.7374 \mathrm{E}-01$ Tadmor/Gur $(0.5-5 \mathrm{~km})$ $4.7180 \mathrm{E}-01$ Tadmor/Gur $(0.5-5 \mathrm{~km})$ $7.4197 \mathrm{E}-01$ Tadmor/Gur $(0.5-5 \mathrm{~km})$ 8.5840E-01 Tadmor/Gur $(0.5-5 \mathrm{~km})$ $9.6696 \mathrm{E}-01$ Tadmor $/ \mathrm{Gur}(0.55 \mathrm{~km})$ $1.1669 \mathrm{E}+00$ Tadmor/Gur $(0.5-5 \mathrm{~km})$ $1.3500 \mathrm{E}+00$ Tadmor/Gur $(0.5-5 \mathrm{~km})$ $6.0746 \mathrm{E}+00$ Tadmor/Gur $(0.5-5 \mathrm{~km})$ $7.5678 \mathrm{E}+00$ Tadmor/Gur $(0.5-5 \mathrm{~km})$ $9.5533 \mathrm{E}+00$ Tadmor/Gur $(0.5-5 \mathrm{~km})$ $1.2450 \mathrm{E}+01$ Tadmor/Gur $(0.5-5 \mathrm{~km})$ $1.5024 \mathrm{E}+01$ Tadmor/Gur $(0.5-5 \mathrm{~km})$ $1.7382 \mathrm{E}+01$ Tadmor/Gur $(0.5-5 \mathrm{~km})$ $1.9580 \mathrm{E}+01$ Tadmor/Gur $(0.5-5 \mathrm{~km})$ $2.3628 \mathrm{E}+01$ Tadmor/Gur $(0.5-5 \mathrm{~km})$ $3.4054 \mathrm{E} 01$ Tadmor/Gur $(0.55 \mathrm{~km})$ $4.2989 \mathrm{E}+01$ Tadmor $/ \mathrm{Gur}(0.5-5 \mathrm{~km})$ $5.6024 \mathrm{E}+01$ Tadmor/Gur $(0.5-5 \mathrm{~km})$ $6.7606 \mathrm{E}+01$ Tadmor/Gur $(0.5-5 \mathrm{~km})$ $7.8215 \mathrm{E}+01$ Tadmor/Gur $10.5-5 \mathrm{~km}$ ) 


\begin{tabular}{|c|c|c|c|c|c|c|c|}
\hline 286 & D-STB /DIS 24 & $6.000 \mathrm{E}+03$ & $3.8067 \mathrm{E}+02$ & $8.8107 E+01$ & Tadmor/Gur & $(0.5-5$ & $\mathrm{km})$ \\
\hline 287 & $\mathrm{D}-\mathrm{STB} / \mathrm{DIS} 25$ & $8.000 \mathrm{E}+03$ & $4.9360 \mathrm{E}+02$ & $1.0632 \mathrm{E}+02$ & Tadmor/Gux & $10.5-5$ & $\mathrm{~km})$ \\
\hline 288 & D-STB/DIS26 6 & $1.000 \mathrm{E}+04$ & $6.0381 E+02$ & $1.2300 \mathrm{E}+02$ & Tađmor/Gur & . 5-5 & $\mathrm{km})$ \\
\hline 289 & D-STB/DIS27 & $1.400 \mathrm{E}+04$ & $8.1821 \mathrm{E}+02$ & $1.5324 \mathrm{E}+02$ & Tadmor/Gur & $5-5$ & $\mathrm{~km})$ \\
\hline 290 & D-STB/DIS2 8 & $2.000 E+04$ & $1.1292 \mathrm{E}+03$ & $1.9344 \mathrm{E}+02$ & Tadmor/Gur & $10.5-5$ & $\mathrm{~km})$ \\
\hline 291 & D-STB/DIS29 & $3.000 \mathrm{E}+04$ & $1.6285 \mathrm{E}+03$ & $2.5210 \mathrm{E}+02$ & Tadmor/Gur & $10.5-5$ & $\mathrm{~km})$ \\
\hline 292 & D-STB/DIS30 & $4.000 \mathrm{E}+04$ & $2.1116 \mathrm{E}+03$ & $3.0422 \mathrm{E}+02$ & Tadmor/Gur & $10.5-5$ & $\mathrm{~km}$ ) \\
\hline 293 & D-STB/DIS31 & $5.000 \mathrm{E}+04$ & $2.5831 E+03$ & $3.5196 \mathrm{E}+02$ & Tadmor/Gur & $10.5-5$ & $\mathrm{~km}$ ) \\
\hline 294 & D-STB/DIS32 & $6.000 \mathrm{E}+04$ & $3.0454 \mathrm{E}+03$ & $3.9647 \mathrm{E}+02$ & Tadmor/Gur & & $\mathrm{km})$ \\
\hline 295 & D-STB/DIS33 & $8.000 \mathrm{E}+04$ & $3.9489 \mathrm{E}+03$ & $4.7843 E+02$ & Tadmor/Gur & & $\mathrm{km})$ \\
\hline 296 & D-STB/DIS34 & $1.000 \mathrm{E}+05$ & $4.8306 \mathrm{E}+03$ & $5.5350 \mathrm{E}+02$ & Tadmor/Gur & $10.5-5$ & $\mathrm{~km})$ \\
\hline 297 & D-STB/DIS35 & $1.400 E+05$ & $6.5458 \mathrm{E}+03$ & $6.8956 \mathrm{E}+02$ & Tadmor/Gur & $10.5-5$ & $\mathrm{~km})$ \\
\hline 298 & D-STB/DIS3 6 & $2.000 \mathrm{E}+05$ & $9.0335 E+03$ & $8.7047 E+02$ & Tadmor/Gur & $10.5-5$ & $\mathrm{~km})$ \\
\hline 299 & D-STB / DIS37 & $3.000 \mathrm{E}+05$ & $1.3028 \mathrm{E}+04$ & 1. $1344 \mathrm{E}+03$ & Tadmor/Gur & $10.5-5$ & $\mathrm{~km})$ \\
\hline 300 & D-STB /DIS38 & $4.000 \mathrm{E}+05$ & 1. $6893 \mathrm{E}+04$ & $1.3689 \mathrm{E}+03$ & Tadmor/Gur & $10.5-5$ & $\mathrm{~km})$ \\
\hline 301 & D-STB/DIS39 & $5.000 \mathrm{E}+05$ & $2.0665 E+04$ & $1.5838 \mathrm{E}+03$ & Tadmor/Gur & $10.5-5$ & $\mathrm{~km})$ \\
\hline 302 & D-STB/DIS 40 & $6.000 \mathrm{E}+05$ & $2.4364 \mathrm{E}+04$ & $1.7841 \mathrm{E}+03$ & Tadmor/Gur & $10.5-5$ & $\mathrm{~km})$ \\
\hline 303 & D-STB/DIS4I & $8.000 \mathrm{E}+05$ & $3.1592 E+04$ & $2.1529 \mathrm{E}+03$ & Tadmor/Gur & $10.5-5$ & $\mathrm{~km})$ \\
\hline 304 & D-STB/DIS42 & $1.000 \mathrm{E}+06$ & $3.8645 E+04$ & $2.4907 E+03$ & Tadmor/Gur & $10.5-5$ & $\mathrm{~km})$ \\
\hline 305 & D-STB/DIS43 & $1.400 \mathrm{E}+06$ & $5.2368 \mathrm{E}+04$ & $3.1029 \mathrm{E}+03$ & Tadmor/Gur & $10.5-5$ & $\mathrm{~km}$ ) \\
\hline 306 & D-STB /DIS 44 & $2.000 E+06$ & $7.2270 E+04$ & $3: 9170 E+03$ & Tadmor/Gur & $(0.5-5$ & $\mathrm{km})$ \\
\hline 307 & D-STB / DIS45 & $3.000 \mathrm{E}+06$ & $1.0423 E+05$ & $5.1048 \mathrm{E}+03$ & Tadmor/Gur & $(0.5-5$ & $\mathrm{km})$ \\
\hline 308 & D-STB/DIS4 6 & $4.000 \mathrm{E}+06$ & $1.3515 \mathrm{E}+05$ & $6.1601 \mathrm{E}+03$ & Tadmor/Gur & $(0.5-5$ & $\mathrm{km})$ \\
\hline 309 & D-STB / DIS 47 & $5.000 E+06$ & $1.6532 \mathrm{E}+05$ & $7.1267 E+03$ & Tadmor/Gur & $(0.5-5$ & $\mathrm{km})$ \\
\hline 310 & D-STB/DIS48 & $6.000 E+06$ & 1. $9492 \mathrm{E}+05$ & $8.0280 \mathrm{E}+03$ & Tadmor/Gur & $10.5-5$ & $\mathrm{~km})$ \\
\hline 311. & D-STB/DIS 49 & $8.000 E+06$ & $2.5274 \mathrm{E}+05$ & $9.6877 \mathrm{E}+03$ & Tadmor/Gur & $10.5-5$ & $\mathrm{~km})$ \\
\hline 312 & D-STB/DIS50 & $1.000 E+07$ & $3.0917 \mathrm{E}+05$ & $1.1208 \mathrm{E}+04$ & Tadmor/Gur & $(0.5-5$ & $\mathrm{km})$ \\
\hline & * E-stability & Distance $(\mathrm{m})$ & Sigma-y (m) & Sigma-z (m) & & & \\
\hline 313 & E-STB/DISO1 & $1.000 \mathrm{E}+00$ & $1.0460 \mathrm{E}-01$ & 4.0000E-01 & Tadmor/Gur & $10.5-5$ & $\mathrm{~km})$ \\
\hline 314 & $\mathrm{E}-\mathrm{STB} / \mathrm{DISO2}$ & $1.400 \mathrm{E}+00$ & $1.4174 \mathrm{E}-01$ & $4.8983 \mathrm{E}-01$ & Tadmor/Gur & $10.5-5$ & $\mathrm{~km})$ \\
\hline 315 & E-STB/DIS03 & $2.000 E+00$ & $1.9561 \mathrm{E}-01$ & $6.0717 \mathrm{E}-01$ & Tadmor/Gur & $10.5-5$ & $\mathrm{~km})$ \\
\hline 316 & E-STB/DIS0 4 & $3.000 E+00$ & $2.8211 \mathrm{E}-01$ & $7.7506 E-01$ & Tadmor/Gur & $10.5-5$ & $\mathrm{~km})$ \\
\hline 317 & E-STB/DIS05 & 4. $000 \mathrm{E}+00$ & $3.6581 \mathrm{E}-01$ & $9.2164 \mathrm{E}-01$ & Tadmor/Gur & $10.5-5$ & $\mathrm{~km})$ \\
\hline 318 & E-STB/DIS06 & $5.000 \mathrm{E}+00$ & $4.4748 \mathrm{E}-01$ & $1.0542 \mathrm{E}+00$ & Tadmor/Gur & $10.5-5$ & $\mathrm{~km})$ \\
\hline 319 & E-STB /DIS07 & $6.000 \mathrm{E}+00$ & $5.2757 \mathrm{E}-01$ & $1.1765 \mathrm{E}+00$ & Tadmor/Gur & $(0.5-5$ & $\mathrm{km})$ \\
\hline 320 & E-STB/DIS08 & $8.000 \mathrm{E}+00$ & $6.8409 \mathrm{E}-01$ & $1.3990 \mathrm{E}+00$ & Tadmor/Gur & $(0.5-5$ & $\mathrm{km})$ \\
\hline 321 & E-STB/DISO9 & $1.000 \mathrm{E}+01$ & $8.3682 \mathrm{E}-01$ & $1.6001 \mathrm{E}+00$ & Tadmor/Gur & $(0.5-5$ & $\mathrm{km})$ \\
\hline 322 & E-STB/DISIO & $1.000 E+02$ & $6.6947 \mathrm{E}+00$ & $6.4012 \mathrm{E}+00$ & Tadmor/Gur & $(0.5-5$ & $\mathrm{km})$ \\
\hline 323 & E-STB/DISII & $1.400 \mathrm{E}+02$ & $9.0719 E+00$ & 7. $8387 \mathrm{E}+00$ & Tadmor/Gur & $(0.5-5$ & $\mathrm{km})$ \\
\hline 324 & E-STB/DIS12 & $2.000 \mathrm{E}+02$ & $1.2520 \mathrm{E}+01$ & $9.7165 \mathrm{E}+00$ & Tadmor/Gur & $10.5-5$ & $\mathrm{~km})$ \\
\hline 325 & E-STB / DIS 13 & $3.000 \mathrm{E}+02$ & $1.8056 \mathrm{E}+01$ & 1. $2403 \mathrm{E}+01$ & Tadnor/Gur & $10.5-5$ & $\mathrm{~km})$ \\
\hline 326 & E-STB / DIS14 & $4.000 E+02$ & $2.3413 \mathrm{E}+01$ & $1.4749 E+01$ & Tadmor/Gur & $10.5-5$ & $\mathrm{~km})$ \\
\hline 327 & E-STB/DIS15 & $5.000 \mathrm{E}+02$ & $2.8640 \mathrm{E}+01$ & $1.6870 \mathrm{E}+01$ & Tadmor/Gur & $10.5-5$ & $\mathrm{~km})$ \\
\hline 328 & E-STB/DIS16 & $6.000 E+02$ & $3.3766 \mathrm{E}+01$ & $1.8827 E+01$ & Tadmor/Gur & $10.5-5$ & $\mathrm{~km})$ \\
\hline 329 & E-STB/DIS17 & $8.000 \mathrm{E}+02$ & $4.3784 E+01$ & $2.2388 \mathrm{E}+01$ & Tadmor/Gur & $(0.5-5$ & $\mathrm{km})$ \\
\hline 330 & E-STB/DIS18 & $1.000 \mathrm{E}+03$ & $5.3559 \mathrm{E}+01$ & 2. $5607 \mathrm{E}+01$ & Tadmor/Gur & $(0.5-5$ & $\mathrm{km})$ \\
\hline 331 & E-STB/DIS19 & $1.400 \mathrm{E}+03$ & $7.2577 \mathrm{E}+01$ & $3.1358 \mathrm{E}+01$ & Tadmor/Gur & $(0.5-5$ & $\mathrm{km})$ \\
\hline 332 & $E-S T B / D I S 20$ & $2.000 \mathrm{E}+03$ & $1.0016 \mathrm{E}+02$ & $3.8870 \mathrm{E}+01$ & Tadmor/Gur & $(0.5-5$ & $\mathrm{km})$ \\
\hline 333 & E-STB/DIS21 & $3.000 E+03$ & $1.4445 \mathrm{E}+02$ & $4.9617 \mathrm{E}+01$ & Tadmor/Gur & $10.5-5$ & $\mathrm{~km})$ \\
\hline 334 & E-STB/DIS22 & $4.000 E+03$ & $1.8731 \mathrm{E}+02$ & $5.9001 E+01$ & Tadmor/Gur & $10.5-5$ & $\mathrm{~km})$ \\
\hline 335 & $E-S T B / D I S 23$ & $5.000 \mathrm{E}+03$ & $2.2912 \mathrm{E}+02$ & $6.7485 E+01$ & Tadmor/Gur & $10.5-5$ & $\mathrm{~km})$ \\
\hline 336 & E-STB/DIS24 & $6.000 E+03$ & $2.7013 \mathrm{E}+02$ & $7.5316 \mathrm{E}+01$ & Tadmor/Gur & $10.5-5$ & $\mathrm{~km})$ \\
\hline 337 & E-STB/DIS25 & $8.000 \mathrm{E}+03$ & $3.5028 \mathrm{E}+02$ & $8.9559 \mathrm{E}+01$ & Tadmor/Gur & $(0.5-5$ & $\mathrm{km})$ \\
\hline 338 & E-STB/DIS26 & $1.000 \mathrm{E}+04$ & $4.2848 \mathrm{E}+02$ & $1.0244 \mathrm{E}+02$ & Tadmor/Gur & $10.5-5$ & $\mathrm{~km})$ \\
\hline 339 & $E-S T B / D I S 27$ & $1.400 \mathrm{E}+04$ & $5.8063 E+02$ & 1. $2544 \mathrm{E}+02$ & Tadmor/Gur & $10.5-5$ & $\mathrm{~km})$ \\
\hline 340 & $E-S T B / D I S 28$ & $2.000 \mathrm{E}+04$ & $8.0129 E+02$ & $1.5549 \mathrm{E}+02$ & Tadmor/Gur & $10.5-5$ & $\mathrm{~km})$ \\
\hline 341 & E-STB/DIS29 & & $1.1556 \mathrm{E}+03$ & $1.9849 \mathrm{E}+02$ & Tadmor/Gur & & \\
\hline 342 & E-STB/DIS30 & $4.000 \mathrm{E}+04$ & $1.4985 \mathrm{E}+03$ & $2.3603 \mathrm{E}+02$ & Tadmor/Gur & $(0.5-5$ & $\mathrm{km})$ \\
\hline 343 & E-STB/DIS31 & $5.000 \mathrm{E}+04$ & $1.8330 \mathrm{E}+03$ & $2.6997 \mathrm{E}+02$ & Tadmor/Gur & $10.5-5$ & $\mathrm{~km})$ \\
\hline 344 & E-STB /DIS32 & $6.000 E+04$ & $2.1611 E+03$ & $3.0129 \mathrm{E}+02$ & Tadmor/Gur & $(0.5-5$ & $\mathrm{km})$ \\
\hline 345 & E-STB/DIS33 & $8.000 \mathrm{E}+04$ & $2.8023 E+03$ & $3.5827 E+02$ & Tadmor/Gur & $10.5-5$ & $\mathrm{~km})$ \\
\hline
\end{tabular}




\begin{tabular}{|c|c|c|c|c|c|c|c|}
\hline 346 & E-STB/DIS34 & $1.000 \mathrm{E}+05$ & $3.4279 E+03$ & $4.0979 E+02$ & Tadmor/Gur & $(0.5-5$ & $\mathrm{km})$ \\
\hline 347 & E-STB/DIS35 & $1.400 \mathrm{E}+05$ & $4.6452 E+03$ & $5.0182 E+02$ & Tadmor/Gur & $(0.5-5$ & $\mathrm{km})$ \\
\hline 348 & E-STB/DIS36 & $2.000 E+05$ & $6.4105 E+03$ & $6.2203 \mathrm{E}+02$ & Tadmor/Gur & $(0.5-5$ & $\mathrm{km})$ \\
\hline 349 & E-STB/DIS37 & $3.000 \mathrm{E}+05$ & $9.2453 E+03$ & $7.9403 E+02$ & Tadmor/Gur & $(0.5-5$ & $\mathrm{km})$ \\
\hline 350 & E-STB/DIS38 & $4.000 E+05$ & $1.1988 \mathrm{E}+04$ & $9.4419 E+02$ & Tadmor/Gur & $(0.5-5$ & $\mathrm{km})$ \\
\hline 351 & E-STB/DIS39 & $5.000 \mathrm{E}+05$ & $1.4665 E+04$ & $1.0800 E+03$ & Tadmor/Gur & $(0.5-5$ & $\mathrm{km}$ ) \\
\hline 352 & E-STB/DIS40 & $6.000 \mathrm{E}+05$ & $1.7289 E+04$ & $1.2053 \mathrm{E}+03$ & Tadmor/Gur & $(0.5-5$ & $\mathrm{km}$ ) \\
\hline 353 & E-STB/DIS41 & $8.000 \mathrm{E}+05$ & $2.2419 E+04$ & $1.4332 \mathrm{E}+03$ & Tadmor/Gur & $(0.5-5$ & $\mathrm{km}$ ) \\
\hline 354 & E-STB/DIS42 & $1.000 \mathrm{E}+06$ & $2.7424 E+04$ & $1.6393 \mathrm{E}+03$ & Tadmor/Gur & $(0.5-5$ & $\mathrm{km}$ ) \\
\hline 355 & E-STB/DIS43 & $1.400 \mathrm{E}+06$ & $3.7162 E+04$ & $2.0074 E+03$ & Tadmor/Gur & $(0.5-5$ & $\mathrm{km})$ \\
\hline 356 & E-STB/DIS44 & $2.000 \mathrm{E}+06$ & $5.1285 E+04$ & $2.4883 E+03$ & Tadmor/Gur & $(0.5-5$ & $\mathrm{km})$ \\
\hline 357 & E-STB/DIS45 & $3.000 \mathrm{E}+06$ & $7.3964 \mathrm{E}+04$ & $3.1764 \mathrm{E}+03$ & Tadmor/Gur & $10.5-5$ & $\mathrm{~km})$ \\
\hline 358 & E-STB/DIS46 6 & $4.000 \mathrm{E}+06$ & $9.5907 \mathrm{E}+04$ & $3.7771 E+03$ & Tadmor/Gur & $(0.5-5$ & $\mathrm{km})$ \\
\hline 359 & E-STB/DIS47 & $5.000 \mathrm{E}+06$ & $1.1732 \mathrm{E}+05$ & $4.3203 E+03$ & Tadmor/Gur & $(0.5-5$ & $\mathrm{km}$ ) \\
\hline 360 & E-STB/DIS4 8 & $6.000 \mathrm{E}+06$ & $1.3832 \mathrm{E}+05$ & $4.8215 \mathrm{E}+03$ & Tadmor/Gur & $(0.5-5$ & $\mathrm{km}$ ) \\
\hline 361 & E-STB/DIS49 & $8.000 E+06$ & $1.7935 \mathrm{E}+05$ & $5.7334 E+03$ & Tadmor/Gur & $10.5-5$ & $\mathrm{~km}$ ) \\
\hline \multirow[t]{2}{*}{362} & E-STB/DIS50 & $1.000 \mathrm{E}+07$ & $2.1940 \mathrm{E}+05$ & $6.5578 E+03$ & Tadrior/Gur & $(0.5-5$ & $\mathrm{km} /$ \\
\hline & * F-stability & Distance 1 & Sigma-y (m) & Sigma-z (m) & & & \\
\hline 363 & F-STB/DIS01 & $1.000 \mathrm{E}+00$ & $7.2200 \mathrm{E}-02$ & $2.0000 \mathrm{E}-01$ & Tadmor/Gur & $10.5-5$ & $\mathrm{~km}$ ) \\
\hline 364 & $\mathrm{~F}-\mathrm{STB} / \mathrm{DISO} 2$ & $1.400 \mathrm{E}+00$ & $9.7838 E-02$ & $2.4491 \mathrm{E}-01$ & Tadmor/Gur & $10.5-5$ & $\mathrm{~km})$ \\
\hline 365 & F-STB/DISO3 & $2.000 \mathrm{E}+00$ & $1.3502 \mathrm{E}-01$ & $3.0356 \mathrm{E}-01$ & Tadmor/Gur & $(0.5-5$ & $\mathrm{km}$ ) \\
\hline 366 & $\mathrm{~F}-\mathrm{STB} / \mathrm{DISO} 4$ & $3.000 E+00$ & $1.9473 \mathrm{E}-01$ & $3.8749 \mathrm{E}-01$ & Tadmor/Gur & $10.5-5$ & $\mathrm{~km}$ ) \\
\hline 367 & F-STB/DIS05 & 4. $.000 \mathrm{E}+00$ & $2.5250 E-01$ & $4.6076 \mathrm{E}-01$ & Tadmor/Gur & $(0.5-5$ & $\mathrm{km})$ \\
\hline 368 & F-STB/DISO6 6 & $5.000 \mathrm{E}+00$ & $3.0887 \mathrm{E}-01$ & $5.2700 \mathrm{E}-01$ & Tadmor/Gur & $(0.5-5$ & $\mathrm{km})$ \\
\hline 369 & F-STB/DIS07 & $6.000 E+00$ & $3.6415 \mathrm{E}-01$ & $5.8814 \mathrm{E}-01$ & Tadmor/Gur & $10.5-5$ & $\mathrm{~km})$ \\
\hline 370 & F-STB/DISO 8 & $8.000 \mathrm{E}+00$ & 4. $7219 E-01$ & $6.9934 \mathrm{E}-01$ & Tadmor/Gur & & $\mathrm{km})$ \\
\hline 371 & F-STB/DISO9 & $1.000 \mathrm{E}+01$ & $5.7761 \mathrm{E}-01$ & $7.9989 \mathrm{E}-01$ & Tadmor/Gur & $(0.5-5$ & $\mathrm{km}$ ) \\
\hline 372 & F-STB/DIS10 & $1.000 E+02$ & $4.6210 E+00$ & $3.1991 \mathrm{E}+00$ & Tadmor/Gur & $10.5-5$ & $\mathrm{~km})$ \\
\hline 373 & F-STB/DIS11 & $1.400 E+02$ & $6.2619 E+00$ & $3.9174 \mathrm{E}+00$ & Tadmor/Gur & $(0.5-5$ & $\mathrm{km})$ \\
\hline 374 & F-STB/DIS12 & $2.000 \mathrm{E}+02$ & $8.6417 E+00$ & $4.8557 E+00$ & Tadmor/Gur & $(0.5-5$ & $\mathrm{km})$ \\
\hline 375 & F-STB/DIS 13 & $3.000 \mathrm{E}+02$ & $1.2463 \mathrm{E}+01$ & $6.1981 E+00$ & Tadmor/Gur & $10.5-5$ & $\mathrm{~km})$ \\
\hline 376 & F-STB/DIS14 & $4.000 \mathrm{E}+02$ & $1.6161 \mathrm{E}+01$ & $7.3700 E+00$ & Tadmor/Gur & $(0.5-5$ & $\mathrm{km}$ ) \\
\hline 377 & F-STB/DIS15 & $5.000 \mathrm{E}+02$ & $1.9769 E+01$ & $8.4297 \mathrm{E}+00$ & Tadmor/Gur & $10.5-5$ & $\mathrm{~km})$ \\
\hline 378 & F-STB/DIS16 & $6.000 \mathrm{E}+02$ & $2.3307 E+01$ & $9.4076 \mathrm{E}+00$ & Tadmor/Gur & $(0.5-5$ & $\mathrm{km})$ \\
\hline 379 & F-STB/DIS17 & $8.000 \mathrm{E}+02$ & $3.0222 \mathrm{E}+01$ & $1.1186 \mathrm{E}+01$ & Tadmor/Gur & $(0.5-5$ & $\mathrm{km})$ \\
\hline 380 & F-STB/DIS18 & $1.000 E+03$ & $3.6969 E+01$ & $1.2795 E+01$ & Tadmor/Gur & $10.5-5$ & $\mathrm{~km}$ ) \\
\hline 381 & F-STB/DIS19 & $1.400 \mathrm{E}+03$ & $5.0096 E+01$ & $1.5667 E+01$ & Tadmor/Gur & $10.5-5$ & $\mathrm{~km})$ \\
\hline 382 & F-STB/DIS20 & $2.000 \mathrm{E}+03$ & $6.9135 \mathrm{E}+01$ & $1.9420 E+01$ & Tadmor/Gur & $(0.5-5$ & $\mathrm{km})$ \\
\hline 383 & F-STB/DIS21 & $3.000 \mathrm{E}+03$ & $9.9707 E+01$ & $2.4789 \mathrm{E}+01$ & Tadmor/Gur & $10.5-5$ & $\mathrm{~km})$ \\
\hline 384 & F-STB/DIS 22 & $4.000 E+03$ & 1. $2929 \mathrm{E}+02$ & $2.9476 \mathrm{E}+01$. & Tadmor/Gur & $(0.5-5$ & $\mathrm{km})$ \\
\hline 385 & F-STB/DIS23 & $5.000 \mathrm{E}+03$ & $1.5815 E+02$ & $3.3714 \mathrm{E}+01$ & Tadmor/Gur & $(0.5-5$ & $\mathrm{km})$ \\
\hline 386 & F-STB/DIS24 & 6.00 & 1.8646 & $E+01$ & Tadmor/Gur & $10.5-5$ & \\
\hline 387 & F-STB/DIS25 & $8.000 E+03$ & $2.4178 \mathrm{E}+02$ & $4.4739 \mathrm{E}+01$ & Tadmor/Gur & $(0.5-5$ & $\mathrm{km}$ ) \\
\hline 388 & F-STB/DIS26 & $1.000 E+04$ & $2.9576 E+02$ & $5.1172 E+01$ & Tadmor/Gur & $(0.5-5$ & $\mathrm{km})$ \\
\hline 389 & F-STB/DIS & $1.400 \mathrm{E}+04$ & $4.0078 \mathrm{E}+02$ & $6.2661 \mathrm{E}+01$ & Tadmor/Gur & $(0.5-5$ & $\mathrm{km})$ \\
\hline 390 & F-STB/DIS28 & $2.000 \mathrm{E}+$ & $5.5309 E+02$ & $7.7669 \mathrm{E}+01$ & Tadmor/Gur & $10.5-5$ & $\mathrm{~km}$ ) \\
\hline 391 & F-STB/DIS2 9 & $3.000 \mathrm{E}+04$ & $7.9767 E+02$ & $9.9142 E+01$ & Tadmor/Gur & $(0.5-5$ & $\mathrm{km}$ ) \\
\hline 392 & F-STB/DIS30 & $4.000 E+04$ & $1.0343 \mathrm{E}+03$ & $1.1789 \mathrm{E}+02$ & Tadmor/Gur & $10.5-5$ & $\mathrm{~km})$ \\
\hline 393 & F-STB/DIS31 & $5.000 E+04$ & $1.2653 \mathrm{E}+03$ & $1.3484 \mathrm{E}+02$ & Tadmor/Gur & $(0,5-5$ & $\mathrm{km})$ \\
\hline 394 & F-STB/DIS32 & $6.000 \mathrm{E}+04$ & 1. $4917 E+03$ & $1.5048 \mathrm{E}+02$ & Tadmor/Gur & $10.5-5$ & $\mathrm{~km})$ \\
\hline 395 & F-STB/DIS33 & $8.000 E+04$ & $1.9343 E+03$ & $1.7893 \mathrm{E}+02$ & Tadmor/Gur & $(0.5-5$ & \\
\hline 396 & F-STB/DIS34 & $1.000 \mathrm{E}+05$ & $2.3661 E+03$ & $2.0466 \mathrm{E}+02$ & Tadmor/Gur & $10.5-5$ & $\mathrm{~km}$ ) \\
\hline 397 & F-STB/DIS35 & $1.400 E+05$ & $3.2063 E+03$ & $2.5061 E+02$ & Tadmor/Gur & $(0.5-5$ & $\mathrm{km})$ \\
\hline 398 & F-STB/DIS36 & $2.000 E+05$ & 4.4248 & 3.10 & Tadmor/Gur & $(0.5-5$ & $\mathrm{km})$ \\
\hline 399 & F-STB/DIS 37 & $3.000 \mathrm{E}+05$ & $6.3815 \mathrm{E}+03$ & $3.9651 E+02$ & Tadmor/Gur & $(0.5-5$ & \\
\hline 400 & F-STB/DIS38 & $4.000 E+05$ & $8.2748 E+03$ & $4.7149 \mathrm{E}+02$ & Tadmor/Gur & $(0.5-5$ & $\mathrm{km})$ \\
\hline 401 & F-STB/DIS39 & & $1.0122 E+04$ & $5.3927 E+02$ & Tadmor/Gur & $(0.5-5$ & $\mathrm{km}$ ) \\
\hline 402 & F-STB /DIS 40 & $6.000 E+05$ & $1.1934 \mathrm{E}$ & 6.018 & Tadmor/Gur & & $\mathrm{km})$ \\
\hline 403 & F-STB/DIS41 & $8.000 E+05$ & 1. $5475 E+04$ & $7.1563 \mathrm{E}+02$ & Tadmor/Gur & $(0.5-5$ & $\mathrm{km})$ \\
\hline 404 & F-STB/DIS 42 & $1.000 \mathrm{E}+06$ & $1.8929 E+04$ & $8.1852 \mathrm{E}+02$ & Tadmor/Gur & $(0.5-5$ & $\mathrm{km})$ \\
\hline & & $1.400 \mathrm{E}+06$ & $2.5651 E+04$ & $1.0023 E+03$ & Tadmor/Gur & $(0.5-5$ & $\mathrm{km})$ \\
\hline
\end{tabular}




$\begin{array}{lllllll}406 & \text { F-STB/DIS44 } & 2.000 \mathrm{E}+06 & 3.5400 \mathrm{E}+04 & 1.2424 \mathrm{E}+03 & \text { Tadmor/Gur } & (0.5-5 \mathrm{~km}) \\ 407 & \text { F-STB/DIS45 } & 3.000 \mathrm{E}+06 & 5.1053 \mathrm{E}+04 & 1.5858 \mathrm{E}+03 & \text { Tadmor/Gur } & (0.5-5 \mathrm{~km}) \\ 408 & \text { F-STB/DIS46 } & 4.000 \mathrm{E}+06 & 6.6200 \mathrm{E}+04 & 1.8857 \mathrm{E}+03 & \text { Tadmor } / \mathrm{Gur} & (0.5-5 \mathrm{~km}) \\ 409 & \text { F-STB/DIS47 } & 5.000 \mathrm{E}+06 & 8.0980 \mathrm{E}+04 & 2.1568 \mathrm{E}+03 & \text { Tadmor } / \mathrm{Gur} & (0.5-5 \mathrm{~km}) \\ 410 & \text { F-STB/DIS48 } & 6.000 \mathrm{E}+06 & 9.5474 \mathrm{E}+04 & 2.4070 \mathrm{E}+03 & \text { Tadmor/Gur } & 10.5-5 \mathrm{~km}) \\ 411 & \text { F-STB/DIS49 } & 8.000 \mathrm{E}+06 & 1.2380 \mathrm{E}+05 & 2.8621 \mathrm{E}+03 & \text { Tadmor } / \text { Gur } & (0.5-5 \mathrm{~km}) \\ 412 & \text { F-STB/DIS50 } & 1.000 \mathrm{E}+07 & 1.5144 \mathrm{E}+05 & 3.2736 \mathrm{E}+03 & \text { Tadmor/Gur } & (0.5-5 \mathrm{~km})\end{array}$

412 F-STB/DIS50

* linear scaling factor for sigma-y function, normally 1

413 DPYSCALEOO1 1

* Linear scaling factor for Sigma-z " Function,

* $(21 / 20) * * 0.2$ FROM CRAC2 WE HAVE $(10 \mathrm{CM} / 3 \mathrm{CM}) * * 0.2=1.27$

414 DPZSCALE001 1.27

* tTIME base fOR EXPANSion Factor (SECONDS)

415 PMTIMBASO01 600. (10 MINUTES)

* BREAK POINT FOR FORMULA CHANGE (SECONDS)

416 PMBRKPNT001 3600. (1 HOUR)

* exponential expansion factor number 1

417 PMXPFAC1001 0.2

* EXPONEntial EXPANSion FActor NUMBer 2

418 PMXPFAC2001

0.25

* PLUME RISE DATA BLOCK, LOADED BY INPLRS, STORED IN /PLUMRS/

* SCALING FACTOR FOR THE CRITICAL WIND SPEED FOR ENTRAINMENT OF A BOUYANT PLUME (USED BY FUNCTION CAUGHT)

419 PRSCLCRW001 1

* SCALING FACtor FOR THE A-D STABILITY PLUME RISE FORMUla

(USED BY FUNCTION PLMRIS)

420 PRSCLADP001 1.

* SCALING Factor For the E-F STABILITY PLUME RISE FORMULA

* (USED BY FUNCTION PLMRIS)

421 PRSCLEFP001 1

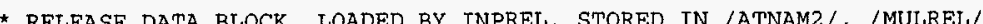

422 RDATNAM2001 'SECOND DRAFT 1150, WORST CASE SOURCE TERM FOR EARLY FATALITIES'

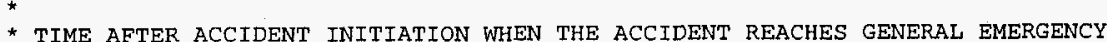
CONDITIONS (AS DEFINED IN NUREG-0654), OR WHEN PLANT PERSONNEL CAN RELIABLY * PREDICT tHAT GENERAL EMERGENCY CONDITIONS WILL BE ATTAINED

423 RDOALARMO01 1300. 


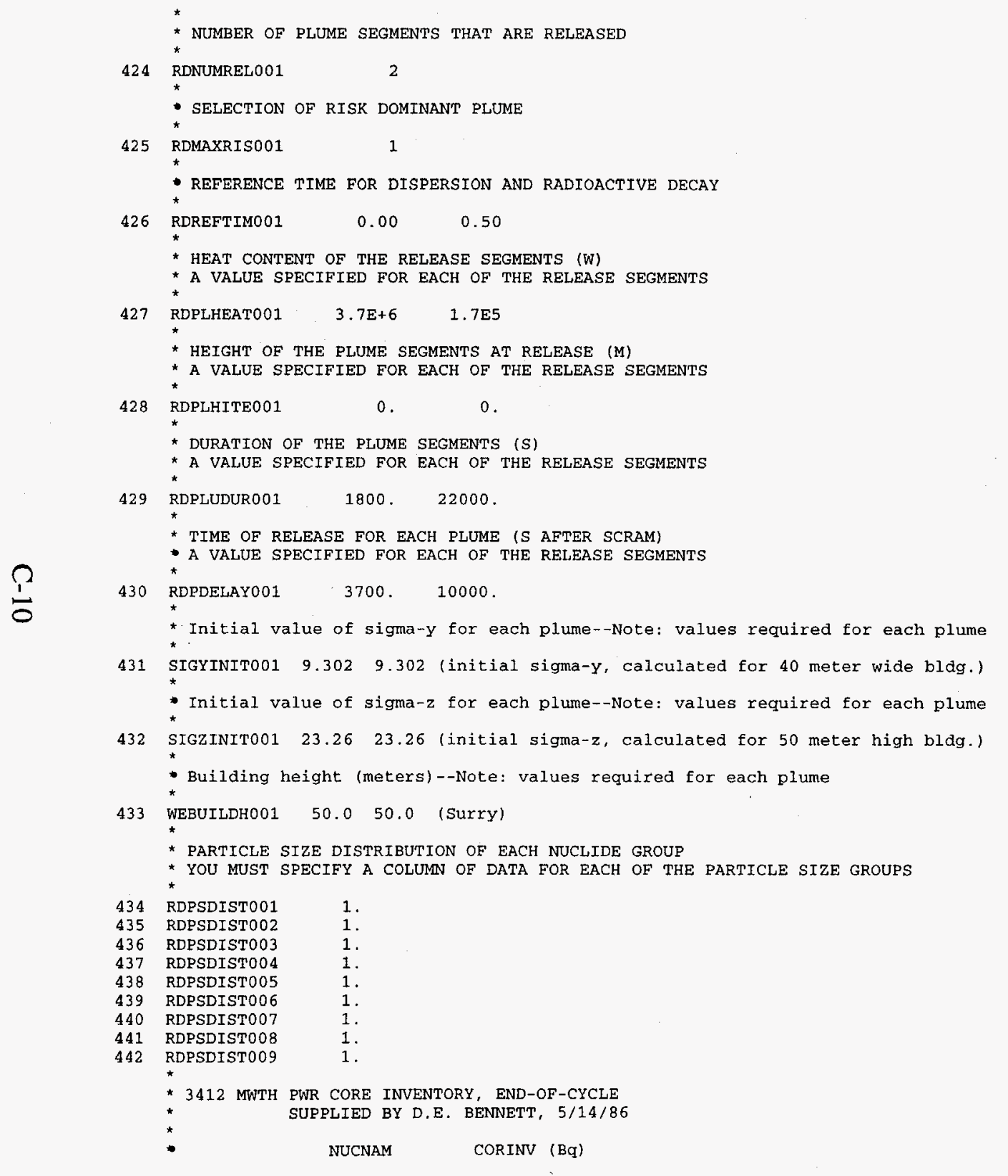




\begin{tabular}{|c|c|c|c|}
\hline 443 & RDCORINV001 & Co-58 & $3.223 E+16$ \\
\hline 444 & RDCORINV002 & $\mathrm{Co}-60$ & $2.465 \mathrm{E}+16$ \\
\hline 445 & RDCORINV003 & $K r-85$ & $2.475 \mathrm{E}+16$ \\
\hline 446 & RDCORINV004 & $\mathrm{Kr}-85 \mathrm{~m}$ & $1.159 \mathrm{E}+18$ \\
\hline 447 & RDCORINV005 & $\mathrm{Kr}-87$ & $2.118 \mathrm{E}+18$ \\
\hline 448 & RDCORINV006 & $\mathrm{Kr}-88$ & $2.864 \mathrm{E}+18$ \\
\hline 449 & RDCORINV007 & $\mathrm{Rb}-86$ & $1.888 \mathrm{E}+15$ \\
\hline 450 & RDCORINV008 & $S r-89$ & $3.590 E+18$ \\
\hline 451 & RDCORINV009 & $\mathrm{Sr}-90$ & $1.938 E+17$ \\
\hline 452 & RDCORINV010 & Sr-91 & $4.616 \mathrm{E}+18$ \\
\hline 453 & RDCORINVO11 & $5 r-92$ & $4.803 E+18$ \\
\hline 454 & RDCORINV012 & $\mathrm{Y}-90$ & $2.079 E+17$ \\
\hline 455 & RDCORINV013 & $Y-91$ & $4.374 E+18$ \\
\hline 456 & RDCORINV014 & $Y-92$ & $4.821 E+18$ \\
\hline 457 & RDCORINV015 & $X-93$ & $5.454 \mathrm{E}+18$ \\
\hline 458 & RDCORINV016 & $2 x-95$ & $5.526 \mathrm{E}+18$ \\
\hline 459 & RDCORINV017 & $\mathrm{Zr}-97$ & $5.759 E+18$ \\
\hline 460 & RDCORINV018 & $\mathrm{Nb}-95$ & $5.224 E+18$ \\
\hline 461 & RDCORINVO19 & Mo-99 & $6.098 E+18$ \\
\hline 462 & RDCORINV020 & Tc-99m & $5.263 E+18$ \\
\hline 463 & RDCORINV021 & $\mathrm{Ru}-103$ & $4.542 \mathrm{E}+18$ \\
\hline 464 & RDCORINV022 & $R u-105$ & $2.954 \mathrm{E}+18$ \\
\hline 465 & RDCORINV 023 & $\mathrm{Ru}-106$ & $1.032 \mathrm{E}+18$ \\
\hline 466 & RDCORINV024 & $\mathrm{Rh}-105$ & $2.046 E+18$ \\
\hline 467 & RDCORINV025 & $\mathrm{Sb}-127$ & $2.787 E+17$ \\
\hline 468 & RDCORINV026 & Sb-129 & $9.872 E+17$ \\
\hline 469 & RDCORINV027 & $\mathrm{Te}-127$ & $2.692 E+17$ \\
\hline 470 & RDCORINV028 & $\mathrm{Te}-127 \mathrm{~m}$ & $3.564 \mathrm{E}+16$ \\
\hline 471 & RDCORINV029 & $\mathrm{Te}-129$ & $9.267 E+17$ \\
\hline 472 & RDCORINV030 & $\mathrm{Te}-129 \mathrm{~m}$ & $2.443 \mathrm{E}+17$ \\
\hline 473 & RDCORINV031 & $\mathrm{Te}-131 \mathrm{~m}$ & $4.680 E+17$ \\
\hline 474 & RDCORINV032 & $\mathrm{Te}-132$ & $4.658 E+18$ \\
\hline 475 & RDCORINV0 33 & $I-131$ & $3.206 E+18$ \\
\hline 476 & RDCORINV034 & $\mathrm{I}-132$ & $4.725 E+18$ \\
\hline 477 & RDCORINV035 & I -133 & $6.779 \mathrm{E}+18$ \\
\hline 478 & RDCORINV036 & $I-134$ & $7.440 \mathrm{E}+18$ \\
\hline 479 & RDCORINV037 & $I-135$ & $6.392 \mathrm{E}+18$ \\
\hline 480 & RDCORINV038 & $x e-133$ & $6.782 \mathrm{E}+18$ \\
\hline 481 & RDCORINV039 & $\mathrm{Xe}-135$ & 1. $273 E+18$ \\
\hline 482 & RDCORINV 040 & $\mathrm{Cs}-134$ & 4. $324 E+17$ \\
\hline 483 & RDCORINV0 41 & Cs -136 & $1.316 \mathrm{E}+17$ \\
\hline 484 & RDCORINV 042 & Cs -137 & $2.417 E+17$ \\
\hline 485 & RDCORINV0 43 & $\mathrm{Ba}-139$ & $6.282 \mathrm{E}+18$ \\
\hline 486 & RDCORINV0 44 & $\mathrm{Ba}-140$ & $6.216 \mathrm{E}+18$ \\
\hline 4.87 & RDCORINV045 & $\mathrm{La}-140$ & $6.352 E+18$ \\
\hline 488 & RDCORINV 046 & La-141 & $5.826 E+18$ \\
\hline 489 & RDCORINV 047 & $\mathrm{La}-142$ & $5.616 \mathrm{E}+18$ \\
\hline 490 & RDCORINV0 48 & $\mathrm{Ce}-141$ & $5.651 E+18$ \\
\hline 491 & RDCORINV0 9 & $\mathrm{Ce}-143$ & $5.494 \mathrm{E}+18$ \\
\hline 492 & RDCORINV050 & $\mathrm{Ce}-144$ & $3.405 E+18$ \\
\hline 493 & RDCORINV051 & $\operatorname{Pr}-143$ & $5.395 \mathrm{E}+18$ \\
\hline 494 & RDCORINV052 & Nd-147 & $2,412 E+18$ \\
\hline 495 & RDCORINV053 & Np-239 & $6.464 \mathrm{E}+19$ \\
\hline 496 & RDCORINV054 & $\mathrm{Pu}-238$ & $3.664 \mathrm{E}+1.5$ \\
\hline 497 & RDCORINV055 & $\mathrm{Pu}-239$ & $8.263 E+14$ \\
\hline 498 & RDCORINV056 & $\mathrm{Pu}-240$ & $1.042 E+15$ \\
\hline 499 & RDCORINV057 & $P u-241$ & $1.755 E+17$ \\
\hline 500 & RDCORINV058 & $\mathrm{Am}-241$ & 1. $159 \mathrm{E}+14$ \\
\hline 501 & RDCORINV059 & $\mathrm{Cm}-242$ & $4.436 E+16$ \\
\hline 502 & RDCORINV060 & $\mathrm{Cm}-244$ & $2.596 E+15$ \\
\hline
\end{tabular}


* SCALING FACTOR TO ADJUST THE CORE INVENTORY FOR POWER LEVEL

503 RDCORSCA001 $0.715 *$ SURRY

*

504 RDAPLFRCO01 PARENT (apply rel fracs the same as prior versions)

* RELEASE FRACTIONS FOR ISOTOPE GROUPS IN RELEASE

ISOTOPE GROUPS:

* $\quad$ xE/KR I $\mathrm{CS}$ TE $\mathrm{SR}$ RU LA $\mathrm{CE}$ BA

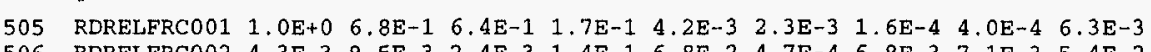

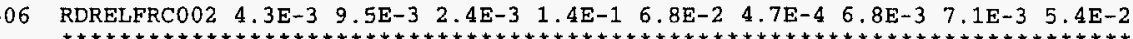

* OUTPUT CONTROL DATA BLOCK, LOADED BY INPOPT, STORED IN /STOPME/, /ATMOPT/

* FLag to indicate that this is the last program in the series to be RUN

507 OCENDAT1001 . FALSE. (SET THIS VALUE TO .TRUE. TO SKIP EARLY AND CHRONC)

508 OCIDEBUG001 0

* NAME of the nUCLIDE to BE LISTEd ON the dispersion Listings

509 OCNUCOUTO01 Cs-137

* NumO

510 TYPEONUMBER

INDREL INDRAD

512 TYPEOOUTO02 11090

* METEOROLOGICAL SAMPLING DATA BLOCK

* METEOROLOgICAL SAMPLing OPTION CODE:

* METCOD = 1, USER SPECIFIED DAY AND houR IN THE YEAR (FROM MET FILE),

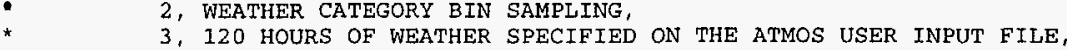

4 , CONSTANT MET (BOUNDARY WEATHER USED FROM THE START),

513 M1METCODO01 2

5. STRATIFIED RANDOM SAMPLES FOR EACH DAY OF THE YEAR.

* LAST spatial interval for measured weather

514 M2LIMSPAOO1 25

* Boundary weather mixing LAyer height

515 M2BNDMXHO01 1000. (METERS)

* Boundary weather Stability Class INDEX

516 M2IBDSTBO01 4 (D-STABILITY)

* boundary weather rain rate

517 M2BNDRANO01 5. (MM/HR) 


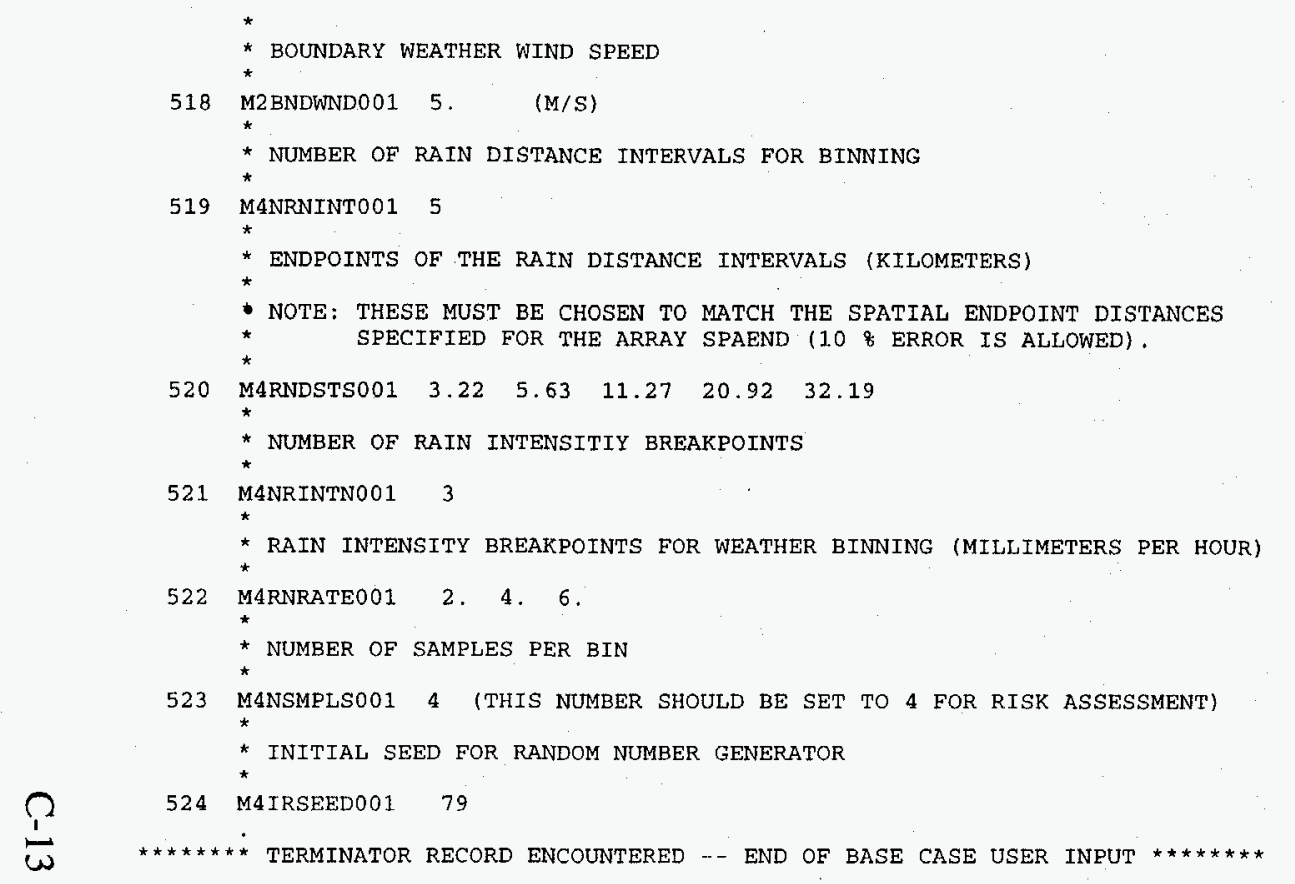

USER INPUT PROCESSING SUMMARY - BASE CASE

NUMBER OF RECORDS READ

NUMBER OF BLANK OR COMMENT RECORDS R

NUMBER OF TERMINATOR RECORDS

NUMBER OF PROCESSED RECORDS DUPLICATED

NUMBER OF PROCESSED RECORDS SORTED

Decay Chain \# Ba-139

Decay Chain \# Ba-140 La-140

Fraction of Ba-140 going to La-140 in this chain $=1.000000$

Decay Chain \# Ce-143 Pr-143

Fraction of Ce-143 going to $\mathrm{Pr}-143$ in this chain $=1.000000$

Decay Chain \# Ce-1.44

Decay Chain \# $\mathrm{Cm}-242$ Pu-238

Fraction of $\mathrm{Cm}-242$ going to Pu-238 in this chain $=1.000000$

Decay Chain \# Cm-244 Pu-240

Fraction of $\mathrm{Cm}-244$ going to $\mathrm{Pu}-240$ in this chain $=1.000000$ 
Decay Chain \# Co-58

Decay Chain \# Co-60

Decay Chain \# Cs-134

Decay Chain \# Cs-136

Decay Chain \# Cs -137

Decay Chain \# I-133 Xe-133

Fraction of $\mathrm{I}-133$ going to Xe-133 in this chain $=0.971000$

Decay Chain \# I-134

Decay Chain \# I-135 Xe-135

Fraction of $\mathrm{I}-135$ going to $\mathrm{Xe}-135$ in this chain $=0.846000$

Decay Chain \# $\mathrm{Kr}-85 \mathrm{~m} \quad \mathrm{Kr}-85$

Fraction of $\mathrm{Kr}-85 \mathrm{~m}$ going to $\mathrm{Kr}-85$ in this chain $=0.211000$

Decay Chain \# $\mathrm{kr}-87$

Decay Chain \# $\mathrm{Kr}-88$

Decay Chain \# La-141 Ce-141

Fraction of La-141 going to Ce-141 in this chain $=1.000000$

Decay chain \# La-142

Decay Chain \# Mo-99 TC-99m

Fraction of Mo-99 going to $\mathrm{Tc}-99 \mathrm{~m}$ in this chain $=0.876000$

Decay Chain \# Nd-147

Decay Chain \# Np-239 Pu-239

Fraction of $\mathrm{NP}-239$ going to $\mathrm{Pu}-239$ in this chain $=1.000000$

Decay Chain \# Pu-241 Am-241

Fraction of $\mathrm{Pu}-241$ going to $\mathrm{Am}-241$ in this chain $=1.000000$

Decay Chain \# Rb-86

Decay Chain \# Ru-10

Decay Chain \# Ru-105 Rh-105

Fraction of Ru-105 going to Rh-105 in this chain $=1.000000$

Decay Chain \# Ru-106

Decay Chain \# Sb-127 Te-127

Fraction of $\mathrm{Sb}-127$ going to $\mathrm{Te}-127$ in this chain $=0.824000$

Decay Chain \# Sb-127 Te-127m $\quad \mathrm{Te}-127$

Fraction of $\mathrm{Sb}-127$ going to $\mathrm{Te}-127 \mathrm{~m}$ in this chain $=0.176000$
Fraction of $\mathrm{Sb}-127$ going to $\mathrm{Te}-127$ in this chain $=0.171776$

Fraction of $\mathrm{Te}-127 \mathrm{~m}$ going to $\mathrm{Te}-127$ in this chain $=0.976000$

Decay Chain \# Sb-129 Te-129

Fraction of Sb-129 going to Te-129 in this chain $=0.775000$ 
Decay Chain \# Sb-129 Te-129m Te-129

Fraction of $\mathrm{Sb}-129$ going to $\mathrm{Te}-129 \mathrm{~m}$ in this chain $=0.225000$ Fraction of $\mathrm{Te}-129 \mathrm{~m}$ going to $\mathrm{Te}-129$ in this chain $=0.650000$

Decay Chain \# Sr-89

Decay Chain \# Sr-90 Y-90

Fraction of $5 r-90$ going to $Y-90$ in this chain $=1.000000$

Decay Chain \# Sr-91 Y-91

Fraction of $5 \mathrm{r}-91$ going to $\mathrm{Y}-91$ in this chain $=0.422000$

Decay Chain \# $\mathrm{Sr}-92 \quad \mathrm{Y}-92$

Fraction of $\mathrm{Sr}-92$ going to $\mathrm{Y}-92$ in this chain $=1.000000$

Decay Chain \# Te-131m I-131

Fraction of Te-131m going to $I-131$ in this chain $=0.778000$

Decay Chain \# Te-132 I-132

Fraction of Te-132 going to I-132 in this chain $=1.000000$

Decay Chain \# Y-93

Decay Chain \# Zr-95 Nb-95

Fraction of $\mathrm{Zr-95}$ going to $\mathrm{Nb}-95$ in this chain $=0.993000$

Decay Chain \# Zr-97

Using new table-lookup scheme for sigma-y/sigma-z

\begin{tabular}{|c|c|c|}
\hline & & ALL PLUMES \\
\hline Co-58 & $5.30 \mathrm{E}+13$ & $1.08 \mathrm{E}+13$ \\
\hline Co- 60 & $4.05 E+13$ & $8.28 \mathrm{E}+12$ \\
\hline $\mathrm{Kr}-85$ & 1. $77 \mathrm{E}+16$ & $7.61 \mathrm{E}+13$ \\
\hline Kr-85m & $7.07 \mathrm{E}+17$ & $1.45 \mathrm{E}+15$ \\
\hline $\mathrm{Kr}-87$ & $8.65 E+17$ & $2.71 \mathrm{E}+14$ \\
\hline $\mathrm{Kr}-88$ & 1. $59 \mathrm{E}+18$ & $2.12 \mathrm{E}+15$ \\
\hline $\mathrm{Rb}-86$ & $8.63 E+14$ & $3.21 E+12$ \\
\hline $5 r-89$ & $1.08 E+16$ & $1.74 E+17$ \\
\hline $\mathrm{Sr}-90$ & $5.82 E+14$ & $9.42 \mathrm{E}+15$ \\
\hline $5 x-91$ & 1. $29 \mathrm{E}+16$ & 1. $47 \mathrm{E}+17$ \\
\hline $5 x-92$ & 1. $11 \mathrm{E}+16$ & $5.25 \mathrm{E}+16$ \\
\hline$y-90$ & $3.00 \mathrm{E}+13$ & $1.53 \mathrm{E}+15$ \\
\hline $\mathrm{Y}-91$ & $5.03 \mathrm{E}+14$ & $2.14 \mathrm{E}+16$ \\
\hline$Y-92$ & $2.75 E+15$ & $7.93 E+16$ \\
\hline $\mathrm{Y}-93$ & $5.81 \mathrm{E}+14$ & $1.78 \mathrm{E}+16$ \\
\hline $\mathrm{zr}-95$ & $6.32 \mathrm{E}+14$ & $2.68 \mathrm{E}+16$ \\
\hline$z x-97$ & $6.32 \mathrm{E}+14$ & $2.20 \mathrm{E}+16$ \\
\hline $\mathrm{Nb}-95$ & $5.98 \mathrm{E}+14$ & $2.54 \mathrm{E}+16$ \\
\hline Mo-99 & $9.92 \mathrm{E}+15$ & $1.93 E+15$ \\
\hline Tc-99m & $8.66 \mathrm{E}+15$ & $1.75 E+15$ \\
\hline $\mathrm{Ru}-103$ & $7.46 \mathrm{E}+15$ & 1. $52 \mathrm{E}+15$ \\
\hline $\mathrm{Ru}-105$ & $4.14 \mathrm{E}+15$ & $3.99 \mathrm{E}+14$ \\
\hline $\mathrm{Ru}-106$ & 1. $70 E+15$ & $3.47 \mathrm{E}+14$ \\
\hline $\mathrm{Rh}-105$ & $3.39 \mathrm{E}+15$ & $6.83 E+14$ \\
\hline$S b-127$ & $3.36 \mathrm{E}+16$ & $2.67 \mathrm{E}+16$ \\
\hline$S b-129$ & 1. $02 \mathrm{E}+17$ & $3.88 \mathrm{E}+16$ \\
\hline $\mathrm{Te}-127$ & $27 \mathrm{E}+16$ & $2.66 \mathrm{E}+16$ \\
\hline $\mathrm{Te}-127 \mathrm{~m}$ & 4. $33 E+15$ & $3.57 E+15$ \\
\hline
\end{tabular}




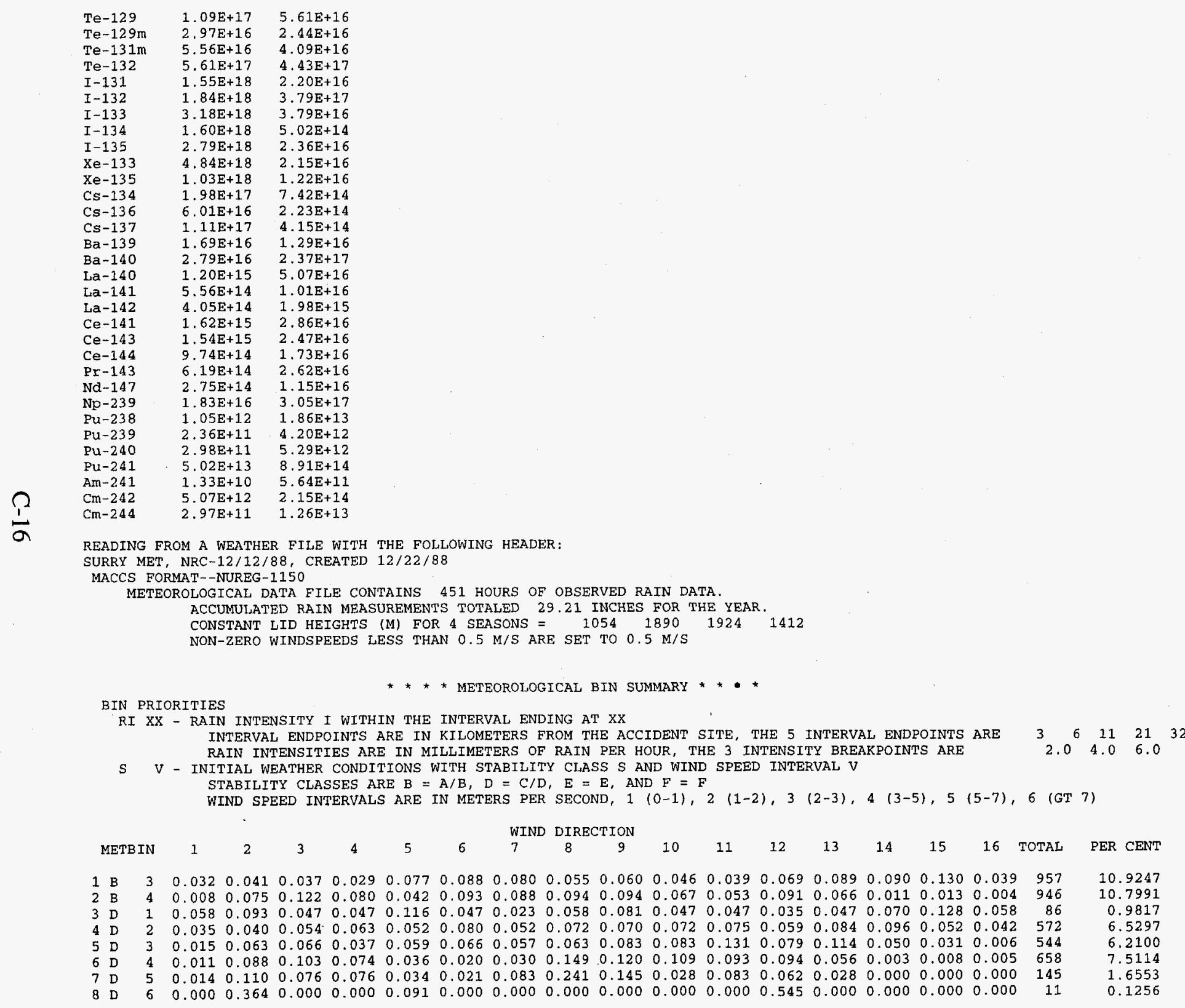




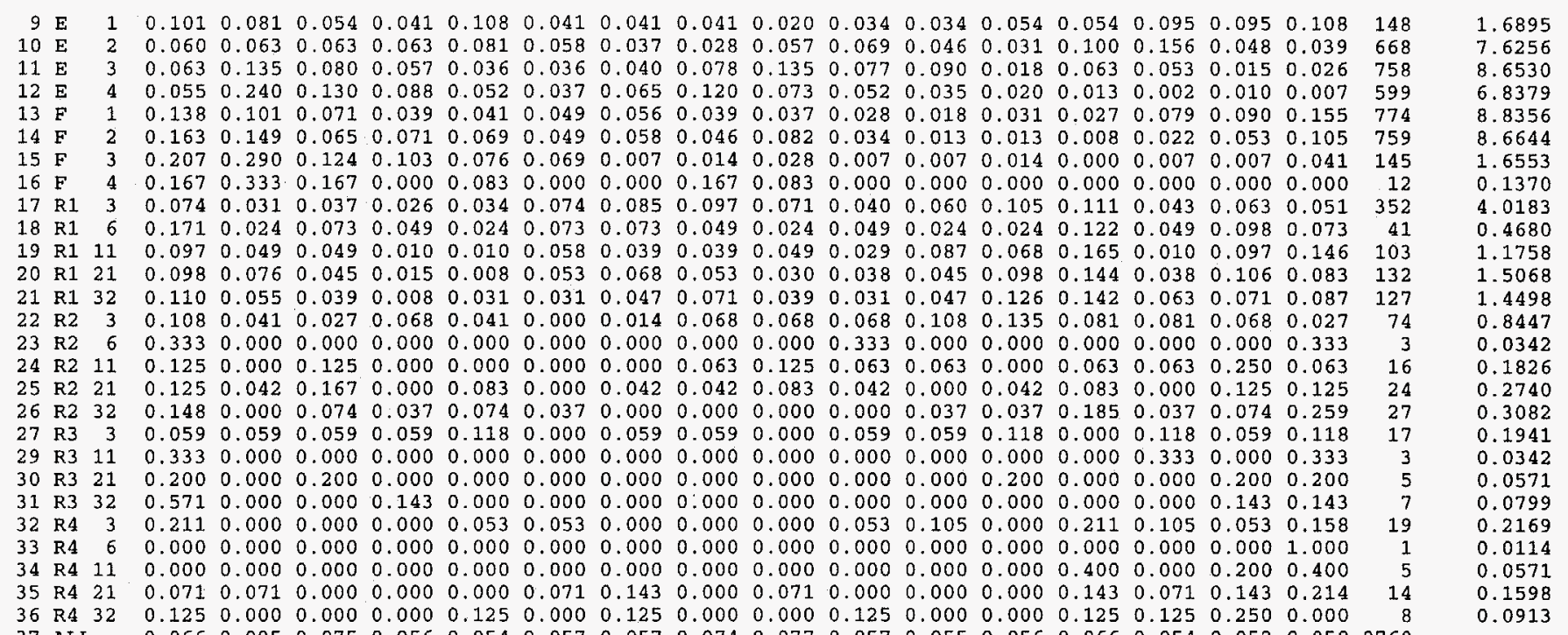

\begin{tabular}{|c|c|c|c|c|c|c|c|c|c|c|c|c|c|c|c|c|c|c|c|}
\hline \multicolumn{2}{|c|}{ METBIN } & \multirow{2}{*}{$\begin{array}{l}1 \\
31\end{array}$} & \multirow{2}{*}{$\begin{array}{l}2 \\
39\end{array}$} & \multirow{2}{*}{$\begin{array}{l}3 \\
35\end{array}$} & \multirow{2}{*}{$\begin{array}{l}4 \\
28\end{array}$} & \multirow{2}{*}{$\begin{array}{l}5 \\
74\end{array}$} & \multirow{2}{*}{$\begin{array}{l}6 \\
84\end{array}$} & \multirow{2}{*}{$\begin{array}{c}\text { WIND } \\
7 \\
77\end{array}$} & \multicolumn{2}{|c|}{ DIRECTION } & 10 & 11 & 12 & 13 & 14 & 15 & \multicolumn{2}{|c|}{16 TOTAL } & PER CENT \\
\hline $1 \mathrm{~B}$ & B & & & & & & & & 53 & 57 & 44 & 37 & 66 & 85 & 86 & 124 & 37 & 957 & 10.9247 \\
\hline $2 B$ & $B$ & 8 & 71 & 115 & 76 & 40 & 88 & 83 & 89 & 89 & 63 & 50 & 86 & 62 & 10 & 12 & 4 & 94 & 10.7991 \\
\hline $3 \mathrm{D}$ & D & 5 & 8 & 4 & 4 & 10 & 4 & 2 & 5 & 7 & 4 & 4 & 3 & 4 & 6 & 11 & 5 & & 0.9817 \\
\hline $4 \mathrm{D}$ & D & 20 & 23 & 31 & 36 & 30 & 46 & 30 & 41 & 40 & 41 & 43 & 34 & 48 & 55 & 30 & 24 & & 6.5297 \\
\hline $5 \mathrm{D}$ & $\mathrm{D}$ & 8 & 34 & 36 & & & & & 34 & 45 & & & & & & & 3 & & \\
\hline $6 \mathrm{D}$ & D & 7 & 58 & 68 & 49 & 24 & 13 & 20 & 98 & 79 & 72 & 61 & 62 & 37 & 2 & 5 & 3 & 65 & 7.5114 \\
\hline $7 \mathrm{D}$ & D & 2 & 16 & 11 & 11 & 5 & 3 & 12 & 35 & 21 & 4 & 12 & & & 0 & 0 & 0 & & 1.6553 \\
\hline $8 \mathrm{D}$ & $\mathrm{D}$ & 0 & 4 & & 0 & 1 & 0 & & & 0 & & & & & 0 & 0 & 0 & & 0.1256 \\
\hline $9 \mathrm{E}$ & E & 15 & 12 & 8 & 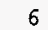 & 16 & 6 & 6 & & 3 & 5 & 5 & & & 14 & & 16 & & \\
\hline $10 \mathrm{E}$ & E & 40 & 42 & 42 & 42 & 54 & 39 & 25 & 19 & 38 & 46 & 31 & 2 & 67 & 104 & 3 & 26 & & 7.6256 \\
\hline & $E$ & & 102 & 61 & & & & 3 & 5 & 102 & & & & & 40 & 1 & 20 & & 8.6530 \\
\hline & & 3 & 14 & 7 & 5 & & & 39 & 7 & 44 & & & & & & 6 & 4 & & 6.8379 \\
\hline & $F$ & 107 & 7 & 55 & 3 & 3 & & 43 & 3 & 29 & 22 & 1 & & 21 & 6] & 70 & 120 & & 8.835 \\
\hline $14 \mathrm{~F}$ & $F$ & 124 & 113 & 49 & 54 & 52 & 3 & 44 & 3 & 62 & 26 & 10 & 1 & & 1 . & 40 & 80 & & \\
\hline $5 \mathrm{~F}$ & $F$ & 30 & 42 & 18 & 15 & 11 & 10 & & & 4 & 1 & & & & & 1 & 6 & 14 & 1.6553 \\
\hline $\mathrm{F}$ & $F$ & 2 & 4 & 2 & 0 & 1 & 0 & 0 & 2 & 1 & 0 & 0 & 0 & c & 0 & 0 & 0 & & 0.1370 \\
\hline & $\mathrm{R} 1$ & 26 & 11 & 13 & 9 & 12 & 26 & 30 & 34 & 25 & 14 & 21 & 37 & 39 & 15 & 22 & 18 & 35 & 4.0183 \\
\hline & $R 16$ & 7 & 1 & & & 1 & 3 & 3 & 3 & 1 & 2 & 1 & 1 & & & 4 & 3 & & 0.468 \\
\hline $19 \mathrm{R}$ & $R 111$ & 10 & 5 & 5 & 1 & 1 & 6 & 4 & 4 & 5 & 3 & 9 & 7 & 17 & 1 & 10 & 15 & 103 & 1.1758 \\
\hline
\end{tabular}




\begin{tabular}{|c|c|c|c|c|c|c|c|c|c|c|c|c|c|c|c|c|c|c|c|}
\hline $20 \mathrm{R} 1$ & 21 & 13 & 10 & 6 & 2 & 1 & 7 & 9 & 7 & 4 & 5 & 6 & 13 & 19 & 5 & 14 & 11 & 132 & 1.5068 \\
\hline $21 \mathrm{R} 1$ & 32 & 14 & 7 & 5 & 1 & 4 & 4 & 6 & 9 & 5 & 4 & 6 & 16 & 18 & 8 & 9 & 11 & 127 & 1.4498 \\
\hline $22 \mathrm{R} 2$ & 3 & 8 & 3 & 2 & 5 & 3 & 0 & 1 & 5 & 5 & 5 & 8 & 10 & 6 & 6 & 5 & 2 & 74 & 0.8447 \\
\hline 23 R2 & 6 & 1 & 0 & 0 & 0 & 0 & 0 & 0 & 0 & 0 & 1 & 0 & 0 & 0 & 0 & 0 & 1 & 3 & 0.0342 \\
\hline $24 \mathrm{R} 2$ & 11 & 2 & 0 & 2 & 0 & 0 & 0 & 0 & 1 & 2 & 1 & 1 & 0 & 1 & 1 & 4 & 1. & 16 & 0.1826 \\
\hline $\begin{array}{ll}25 & \mathrm{R} 2\end{array}$ & 21 & 3 & 1 & 4 & 0 & 2 & 0 & 1 & 1 & 2 & 1 & 0 & 1 & 2 & 0 & 3 & 3 & 24 & 0.2740 \\
\hline $26 \mathrm{R} 2$ & 32 & 4 & 0 & 2 & 1 & 2 & 1 & 0 & 0 & 0 & 0 & 1 & 1 & 5 & 1 & 2 & 7 & 27 & 0.3082 \\
\hline 27.83 & 3 & 1 & 1 & 1 & 1. & 2 & 0 & 1 & 1 & 0 & 1 & 1 & 2 & 0 & 2 & 1 & 2 & 17 & 0.1941 \\
\hline $28 \mathrm{R} 3$ & 6 & 0 & 0 & 0 & 0 & 0 & 0 & 0 & 0 & 0 & 0 & 0 & 0 & 0 & 0 & 0 & 0 & 0 & 0.0000 \\
\hline $\begin{array}{ll}29 & \mathrm{R} 3\end{array}$ & 11 & 1 & 0 & 0 & 0 & 0 & 0 & 0 & 0 & 0 & 0 & 0 & 0 & 0 & 1 & 0 & 1 & 3 & 0.0342 \\
\hline $30 \mathrm{R} 3$ & 21 & 1 & 0 & 1 & 0 & 0 & 0 & 0 & 0 & 0 & 0 & 0 & 1 & 0 & 0 & 1 & 1 & 5 & 0.0571 \\
\hline $\begin{array}{lll}31 & \text { R3 }\end{array}$ & 32 & 4 & 0 & 0 & 1 & 0 & 0 & 0 & 0 & 0 & 0 & 0 & 0 & 0 & 0 & 1 & 1 & 7 & 0.0799 \\
\hline $32 \mathrm{R} 4$ & 3 & 4 & 0 & 0 & 0 & 1 & 1 & 0 & 0 & 0 & 1 & 2 & 0 & 4 & 2 & 1 & 3 & 19 & 0.2169 \\
\hline $33 \mathrm{R4}$ & 6 & 0 & 0 & 0 & 0 & 0 & 0 & 0 & 0 & 0 & 0 & 0 & 0 & 0 & 0 & 0 & 1 & 1 & 0.0114 \\
\hline $\begin{array}{ll}34 & R 4\end{array}$ & 11 & 0 & 0 & 0 & 0 & 0 & 0 & 0 & 0. & 0 & 0 & 0 & 0 & 2 & 0 & 1 & 2 & 5 & 0.0571 \\
\hline $35 \mathrm{R} 4$ & 21 & 1 & 1 & 0 & 0 & 0 & 1 & 2 & 0 & 1 & 0 & 0 & 0 & 2 & 1 & 2 & 3 & 14 & 0.1598 \\
\hline $36 \mathrm{R4}$ & 32 & 1 & 0 & 0 & 0 & 1 & 0 & 1 & 0 & 0 & 1 & 0 & 0 & 1 & 1 & 2 & 0 & 8 & 0.0913 \\
\hline
\end{tabular}

$* * *$ * SUMMARIES $* * * *$

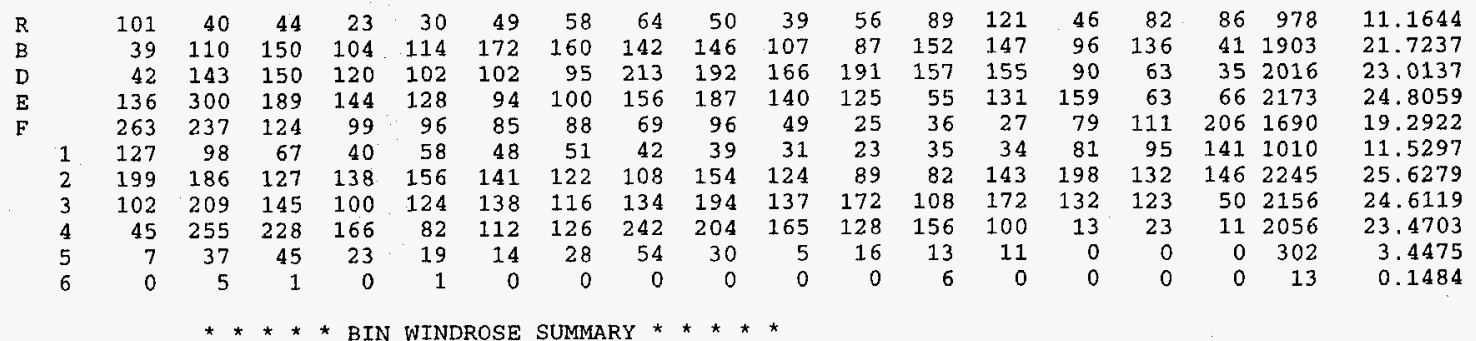

BIN

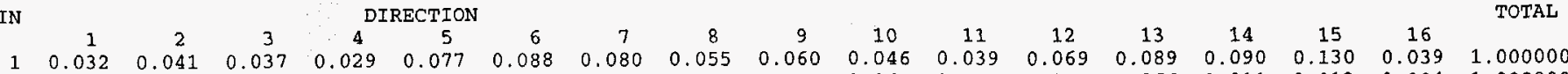

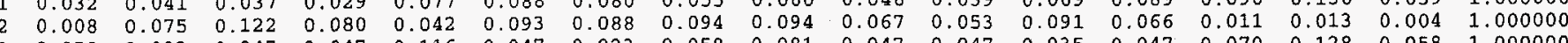
$\begin{array}{llllllllllllllllll}3 & 0.058 & 0.093 & 0.047 & 0.047 & 0.116 & 0.047 & 0.023 & 0.058 & 0.081 & 0.047 & 0.047 & 0.035 & 0.047 & 0.070 & 0.128 & 0.058 & 1.000000\end{array}$ $\begin{array}{lllllllllllllllllll}4 & 0.035 & 0.040 & 0.054 & 0.063 & 0.052 & 0.080 & 0.052 & 0.072 & 0.070 & 0.072 & 0.075 & 0.059 & 0.084 & 0.096 & 0.052 & 0.042 & 1.000000 \\ 5 & 0.015 & 0.063 & 0.066 & 0.037 & 0.059 & 0.065 & 0.057 & 0.063 & 0.083 & 0.083 & 0.131 & 0.079 & 0.114 & 0.050 & 0.031 & 0.006 & 1.000000\end{array}$ \begin{tabular}{lllllllllllllllllll}
5 & 0.015 & 0.063 & 0.066 & 0.037 & 0.059 & 0.066 & 0.057 & 0.063 & 0.083 & 0.083 & 0.131 & 0.079 & 0.114 & 0.050 & 0.031 & 0.005 & 1.0000000 \\
\hline & 0.011 & 0.088 & 0.103 & 0.074 & 0.036 & 0.020 & 0.030 & 0.149 & 0.120 & 0.109 & 0.093 & 0.094 & 0.056 & 0.003 & 0.008 & 0.005 & 1.000000
\end{tabular} $\begin{array}{llllllllllllllllllll}7 & 0.014 & 0.110 & 0.076 & 0.076 & 0.034 & 0.021 & 0.083 & 0.241 & 0.145 & 0.028 & 0.083 & 0.062 & 0.028 & 0.000 & 0.000 & 0.000 & 1.000000\end{array}$ $\begin{array}{llllllllllllllllll}0.000 & 0.364 & 0.000 & 0.000 & 0.091 & 0.000 & 0.000 & 0.000 & 0.000 & 0.000 & 0.000 & 0.545 & 0.000 & 0.000 & 0.000 & 0.000 & 1.000000\end{array}$ $9 \begin{array}{lllllllllllllllllll}9 & 0.101 & 0.081 & 0.054 & 0.041 & 0.108 & 0.041 & 0.041 & 0.041 & 0.020 & 0.034 & 0.034 & 0.054 & 0.054 & 0.095 & 0.095 & 0.108 & 1.000000\end{array}$ $10 \begin{array}{lllllllllllllllll}10.060 & 0.063 & 0.063 & 0.063 & 0.081 & 0.058 & 0.037 & 0.028 & 0.057 & 0.069 & 0.046 & 0.031 & 0.100 & 0.156 & 0.048 & 0.039 & 1.000000\end{array}$ $\begin{array}{llllllllllllllllll}11 & 0.063 & 0.135 & 0.080 & 0.057 & 0.036 & 0.036 & 0.040 & 0.078 & 0.135 & 0.077 & 0.090 & 0.018 & 0.063 & 0.053 & 0.015 & 0.026 & 1.000000\end{array}$

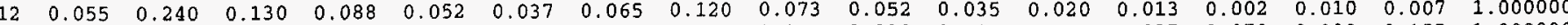
$\begin{array}{llllllllllllllllll}13 & 0.138 & 0.101 & 0.071 & 0.039 & 0.041 & 0.049 & 0.056 & 0.039 & 0.037 & 0.028 & 0.018 & 0.031 & 0.027 & 0.079 & 0.090 & 0.155 & 1.000000\end{array}$ $\begin{array}{lllllllllllllllllll}14 & 0.163 & 0.149 & 0.065 & 0.071 & 0.069 & 0.049 & 0.058 & 0.046 & 0.082 & 0.034 & 0.013 & 0.013 & 0.008 & 0.022 & 0.053 & 0.105 & 1.000000\end{array}$ $\begin{array}{llllllllllllllllll}15 & 0.207 & 0.290 & 0.124 & 0.103 & 0.076 & 0.069 & 0.007 & 0.014 & 0.028 & 0.007 & 0.007 & 0.014 & 0.000 & 0.007 & 0.007 & 0.041 & 1.000000\end{array}$

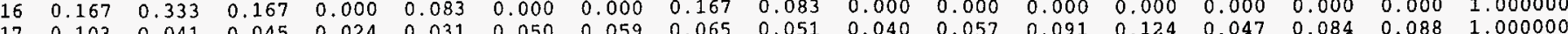

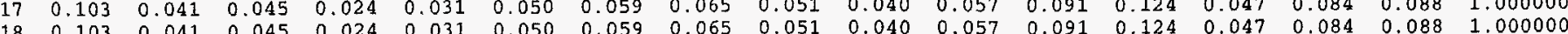
$\begin{array}{lllllllllllllllllll}18 & 0.103 & 0.041 & 0.045 & 0.024 & 0.031 & 0.050 & 0.059 & 0.065 & 0.051 & 0.040 & 0.057 & 0.091 & 0.124 & 0.047 & 0.084 & 0.088 & 1.0000000\end{array}$ $\begin{array}{llllllllllllllllll}19 & 0.103 & 0.041 & 0.045 & 0.024 & 0.031 & 0.050 & 0.059 & 0.065 & 0.051 & 0.040 & 0.057 & 0.091 & 0.124 & 0.047 & 0.084 & 0.088 & 1.00000000\end{array}$ $\begin{array}{lllllllllllllllllll}21 & 0.103 & 0.041 & 0.045 & 0.024 & 0.031 & 0.050 & 0.059 & 0.065 & 0.051 & 0.040 & 0.057 & 0.091 & 0.124 & 0.047 & 0.084 & 0.088 & 1.000000\end{array}$ $\begin{array}{llllllllllllllllll}22 & 0.103 & 0.041 & 0.045 & 0.024 & 0.031 & 0.050 & 0.059 & 0.065 & 0.051 & 0.040 & 0.057 & 0.091 & 0.124 & 0.047 & 0.084 & 0.088 & 1.000000\end{array}$

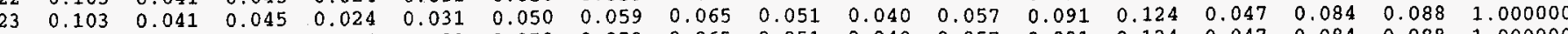
$\begin{array}{lllllllllllllllllll}24 & 0.103 & 0.041 & 0.045 & 0.024 & 0.031 & 0.050 & 0.059 & 0.065 & 0.051 & 0.040 & 0.057 & 0.091 & 0.124 & 0.047 & 0.084 & 0.088 & 1.000000\end{array}$ $\begin{array}{lllllllllllllllllll}25 & 0.103 & 0.041 & 0.045 & 0.024 & 0.031 & 0.050 & 0.059 & 0.065 & 0.051 & 0.040 & 0.057 & 0.091 & 0.124 & 0.047 & 0.084 & 0.088 & 1.000000\end{array}$ 


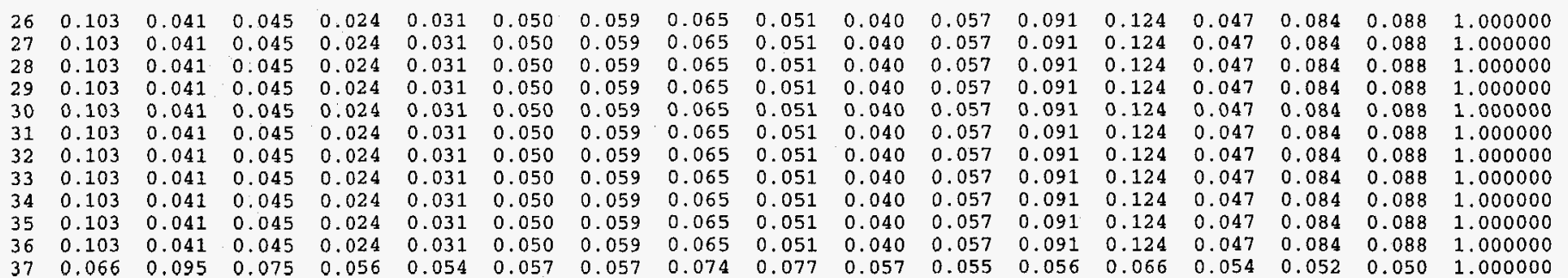

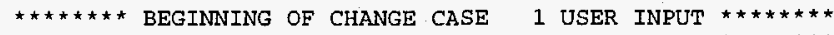

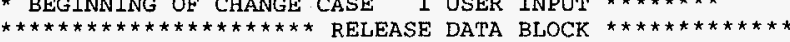

* SOURCE TERM NUMBER 2 OF 2

525 RDATNAM2001 'RELEASE FRACTIONS OF SOURCE TERM 1 REDUCED BY A FACTOR OF TEN

$$
\text { RECORD NUMBER. } 525 \text { REPLACES RECORD NUMBER } 422 \text { * }
$$

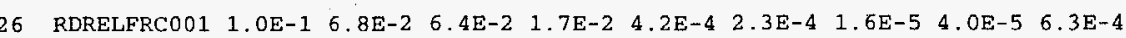

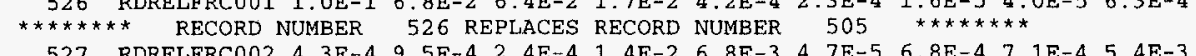

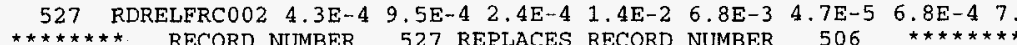

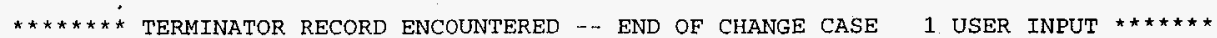

USER INPUT PROCESSING SUMMARY - CHANGE CASE 1

NUMBER OF RECORDS CHANGED $=$

NUMBER OF RECORDS ADDED

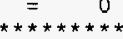

\begin{tabular}{|c|c|c|}
\hline RELEASED & INVENTORY OF & ALL PLUMES \\
\hline Co-58 & $5.30 \mathrm{E}+12$ & $1.08 \mathrm{E}+12$ \\
\hline Co- 60 & $4.05 E+12$ & 8. $28 E+11$ \\
\hline $\mathrm{Kr}-85$ & $1.77 E+15$ & $7.61 E+12$ \\
\hline $\mathrm{Kr}-85 \mathrm{~m}$ & $7.07 E+16$ & $1.45 E+14$ \\
\hline $\mathrm{Kr}-87$ & $8.65 E+16$ & $2.71 E+13$ \\
\hline $\mathrm{Kr}-88$ & $1.59 \mathrm{E}+17$ & $2.12 E+14$ \\
\hline $\mathrm{Rb}-86$ & $8.63 E+13$ & 3. $21 E+11$ \\
\hline $5 x-89$ & $1.08 \mathrm{E}+15$ & $1.74 \mathrm{E}+16$ \\
\hline$S r-90$ & $5.82 E+13$ & $9.42 \mathrm{E}+14$ \\
\hline $\mathrm{Sr}-91$ & $1.29 E+15$ & $1.47 \mathrm{E}+16$ \\
\hline $5 r-92$ & 1. $11 \mathrm{E}+15$ & $5.25 E+15$ \\
\hline $\mathrm{Y}-90$ & $3.00 \mathrm{E}+12$ & $1.53 \mathrm{E}+14$ \\
\hline$Y-91$ & $5.03 E+13$ & 2. $14 \mathrm{E}+15$ \\
\hline$Y-92$ & $2.75 E+14$ & $7.93 \mathrm{E}+15$ \\
\hline$Y-93$ & $5.81 \mathrm{E}+13$ & 1. $78 \mathrm{E}+15$ \\
\hline $\mathrm{zr}-95$ & $6.32 \mathrm{E}+13$ & $2.68 \mathrm{E}+15$ \\
\hline $\mathrm{Zr}-97$ & $6.32 \mathrm{E}+13$ & $2.20 E+15$ \\
\hline $\mathrm{Nb}-95$ & $5.98 E+13$ & $2.54 E+15$ \\
\hline Mo-99 & $9.92 \mathrm{E}+14$ & $1.93 E+14$ \\
\hline$T c-99 m$ & $8.66 \mathrm{E}+14$ & $1.75 \mathrm{E}+14$ \\
\hline$R u-103$ & $7.46 \mathrm{E}+14$ & 1. $52 \mathrm{E}+14$ \\
\hline Ru-105 & $4.14 \mathrm{E}+14$ & $3.99 \mathrm{E}+13$ \\
\hline $\mathrm{Ru}-106$ & $1.70 \mathrm{E}+14$ & $3.47 \mathrm{E}+13$ \\
\hline $\mathrm{Rh}-105$ & $3.39 E+14$ & $6.83 E+13$ \\
\hline
\end{tabular}




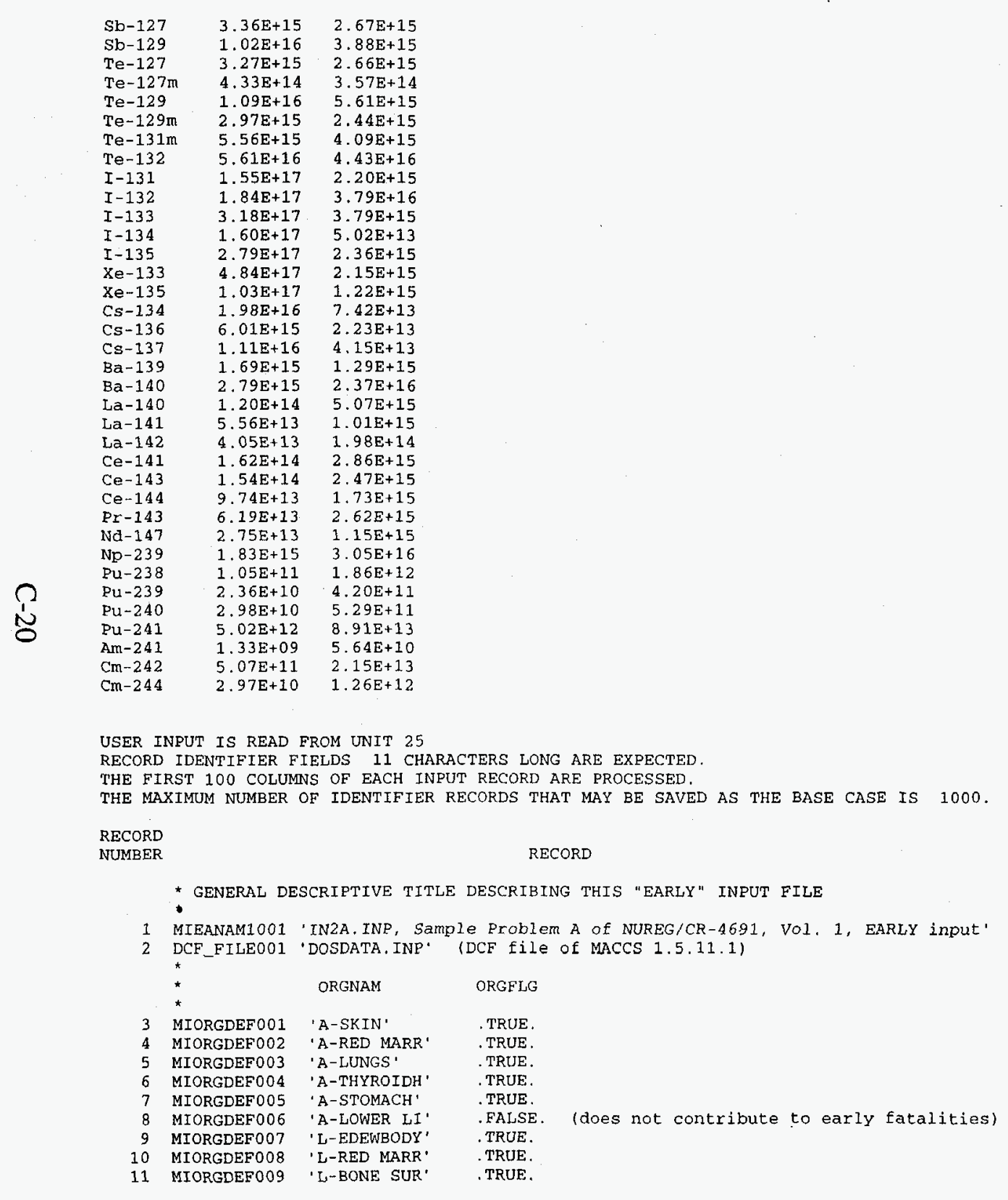


12 MIORGDEFO10 'L-BREAST' .TRUE.

13 MIORGDEF011 'L-LUNGS'

MTORGDEFO12 'L-THYROID'

MIORGDEFO13 'L-LOWER LI'

MIORGDEF014 'L-BLAD WAL'

17 MIORGDEF015 'LLLIVER' .FALSE

* FLag to indicate that this Is the last pRogram IN the SERIES to be RUN

19 MIENDAT2001 .FALSE. (SET THIS VALUE TO .TRUE. TO SKIP CHRONC)

* DISPERSion model OPtTON CODE: 1 * Straight LINE

* $\quad 3$ * WIND-SHIFT WITH ROTATION

20 MIIPLUME001 2

* NUMBer of FINE GRID SUBdivisions USEd by the MOdeL

1 MINUMFINOO1:7 (3, 5 OR 7 ALLOWED)

* LEVEL OF DEBUG OUTPUT REQUIRED, , NORMAL RUNS SHOULD SPECIFY ZERO

22 MIIPRINTO01,0

* LOGICAL FLAG SIGNIFYING THAT THE BREAKDOWN OF RISK BY WEATHER CATEGORY * BIN ARE TO BE PRESENTED tO SHOW THEIR RELATIVE CONTRIBUTION TO THE MEAN

$$
\text { * } \quad \text { RISBIN }
$$

23

MIRISCATO01 .FALSE

* FLAG INDICATING IF WIND-ROSES FROM ATMOS ARE TO BE OVERRIDDEN

24 MIOVRRIDOOI FALSE. (USE THE WIND ROSE CALCULATED FOR EACH WEATHER BIN)

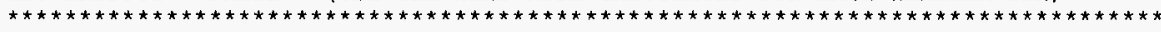
* POPULATION DISTRIBUTION DATA BLOCK, LOADED BY INPOPU, STORED IN /POPDAT/

25 PDPOPFLG001 FILE

*PDPOPFLGOO1 UNIFOR

*PDIBEGINO01 1 (SPATIAL INTERVAL AT WHICH POPULATION BEGINS)

* PDPOPDENO01 50. (POPULATION DENSITY (PEOPLE PER SQUARE KILOMETER)

* SHIElding and EXPOSURE factors, LOADED BY INDFAC, STORED IN /EADEAC/

* THREE VALUES OF EACH PROTECTION FACTOR ARE SUPPLIED,

ONE FOR EACH TYPE OF ACTIVTY.

* activity type:

1 - EVACUEES WHILE MOVING

- NORMAL ACTIVITY IN SHELTERING AND EVACUATION ZONE

3 - SHELTERED ACTIVITY

CLOUd SHIELDING FACTOR
SITE
GG PB SEQ SUR ZION
SHELTERING $\quad 0.7 \quad 0.5 \quad 0.65 \quad 0.6 \quad 0.5$
EVACUEES NORMAL SHELTER 
26 SECSFACT001 $1 . \quad 0.750 .6 *$ SURRY SHELTERING VALUE

* PROTECTION FACTOR FOR INHALATION

27 SEPROTIN001 $1 . \quad 0.41 \quad 0.33$ * VALUES FOR NORMAL ACTIVITY AND

* BREATHING RATE (CUBIC METERS PER SECOND)

SEBRRATE001 $2.66 \mathrm{E}-4 \quad 2.66 \mathrm{E}-4 \quad 2.66 \mathrm{E}-4$

* SKIN PROTECTION FACTOR

29 SESKPFACO01 $1.0 \quad 0.41$

* GROUND SHIELding FActor

* SITE GG PB SEQ SUR ZION

$30 \begin{array}{llllll}* & \text { SEGSHFACO01 } & 0.5 & 0.33 & 0.2 & \text { * VALUE FOR NORMAL ACTIVITY SELECTED BY }\end{array}$

* RESUSPENSION INHALATION MODEL CONCENTRATION COEFFICIENT (/METER)

* $\quad$ RESCON $=1$. E-4 IS APPROPRIATE FOR MECHANICAL RESUSPENSION BY VEHICLES.

RESHAF $=2.11$ DAYS CAUSES 1.E-4 TO DECAY IN ONE WEEK TO 1. E-5, THE VALUE

31 SERESCON001 1.E-4 (RESUSPENSION IS TURNED ON)

* RESUSPENSION CONCENTRATION COEFFICIENT HALF-LIFE (SEC)

32 SERESHAF001 $1.82 \mathrm{E} 5 \quad(2.11$ DAYS $)$

* EVACUATION ZONE DATA BLOCK, LOADED BY EVNETW, STORED IN /NETWOR/, /EOPTIO/

* SPECIFIC DESCRIPTION OF THE EMERGENCY RESPONSE SCENARIO BEING USED

33 EZEANAM2001 'EVACUATION WITHIN 10 MILES. RELOCATION MODELS APPLY ELSEWHERE'

* THE TYPE OF WEIGHTING TO BE APPLIED TO THE EMERGENCY RESPONSE SCENARIOS

* YOU MUST SUPPLY A VALUE OF 'TIME' OR 'PEOPLE'

34 EZWTNAME001 'PEOPLE'

* Weighting fRACTION APPLICABLE to this SCENARIO

35 EZWTFRAC001 0.95

* LAST RING IN THE MOVEMENT zONE

36 EZLASMOVOO1 15 (EVACUEES DISAPPEAR AFTER TRAVELING TO 20 MILES)

* Flag defining the time at which evacuees "enter" the destination element

*TRAVELPOINT 'CENTERPOINT' (new option implemented at MACCS2 v. 1.11f)

37 TRAVELPOINT 'BOUNDARY' (functionality derived from MACCS circa 1984)

* RADial eVAcuation SPEed (M/S) 


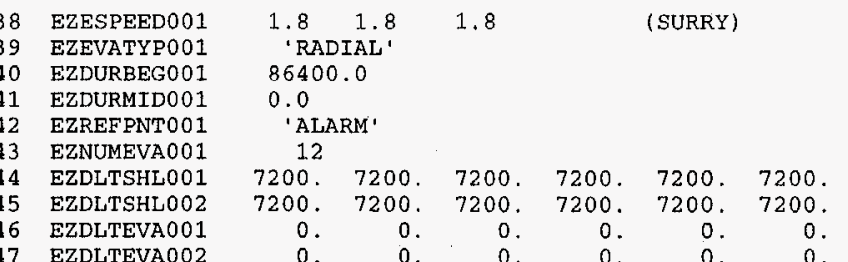

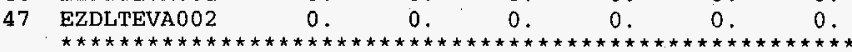

the

STORED IN /INPSRZ /, /RELOCA/

* DURATion of the emergency phase (SECONDS fRom PLUMe aRRIVAL)

48 SRENDEMPO01 604800. (ONE WEEK)

* critical organ for relocation decisions

49 SRCRIORG001 'L-EDEWBODY.

- hot SPOT RELOCATION TIME (SECONDS FROM PLUME ARRIVAL)

50 SRTIMHOTO01 43200. (ONE-HALF DAY)

* NoRmal RELOCATION tIME (SECONDS from plume arRival)

51 SRTIMNRM001 86400. (ONE DAY)

* HOT SPOT RELOCATION DOSE CRITERION THRESHOLD (SIEVERTS)

52 SRDOSHOTO01 0.5 (50 REM DOSE TO WHOLE BODY IN 1 WEEK TRIGGERS RELOCATTON) * NORMAL RELOCATION DOSE CRITERION THRESHOLd (SIEVERTS)

53 SRDOSNRMO0 10.25 (25 REM DOSE TO WHOLE BODY IN 1 WEEK TRIGGERS RELOCATION)

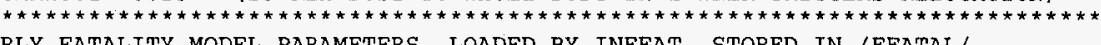
- EARLY FATALT MODEL PARAMETERS, LOADED BY INEFAT, STORED IN /EFATAL/

* Number of early fatality effects

54 EFNUMEFA001 2
*
EFFACA EFFACB EFFTHR
55 EFATAGRPOO1 'A-RED MARR'
$\begin{array}{rrr}3.8 & 5.0 & 1.5 \\ 10.0 & 7.0 & 5.0\end{array}$

* EARLY INJURY MODEL PARAMETERS, LOADED BY INEINJ, STORED IN /EINJUR/

- number of early injury efFects

57 EINUMEINOOI 7

$$
\text { * EINAME }
$$

ORGNAM EISUSC EITHRE EIFACA EIFACB

58 EINJUGRP001 'PRODROMAL VOMIT' 'A-STOMACH' 1

59 EINJUGRPO02 'DIARRHEA'

A-STOMACH'

A-SKTN'

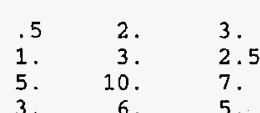


$\begin{array}{llllllrl}62 & \text { EINJUGRP005 'TRANSEPIDERMAL' } & \text { 'A-SKIN' } & 1 . & 10 . & 20 . & 5 . \\ 63 & \text { EINJUGRP006 } & \text { 'THYROIDITIS' } & \text { 'A-THYROIDH' } & 1 . & 40 . & 240 . & 2\end{array}$

64 EINJUGRP007 'HYPOTHYROIDISM' 'A-THYROIDH' 1.24 .260 .21 .3

* ACUTE EXPOSURE CANCER PARAMETERS, LOADED BY INACAN STORED IN /ACANCR/.

- NUMBer of acuTe EXPOSURE CANCER EFFECTS

65 LCNUMACA001 7

* THRESHOLd DOSE FOR APPLYING THE DOSE DEPENDENT REDUCTION FACTOR

6 LCDDTHRE001 0.2 (LOWEST DOSE FOR WHICH DDREFA WILL BE APPLIED)

* DOSE THRESHOLD FOR LINEAR DOSE RESPONSE (SV)

67 LCACTHRE001 0.0 (LINEAR-QUADRATIC MODEL IS NOT BEING USED)

* aCName orgnam aCSUSC DOSEFA DOSEFB CFRISK CIRISK DDREFA

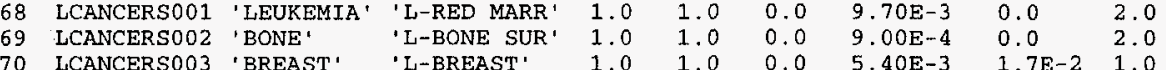

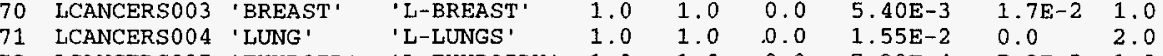

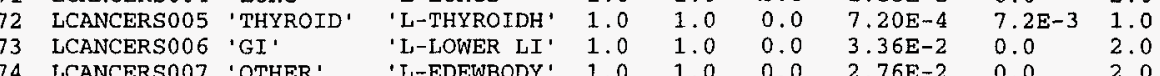

L

* TOTAL NUMBER OF A GIVEN EFFECT (LATENT CANCER, EARLY DEATH, EARLY INUURY)

* NUMBER of DESiRed RESULtSS of this type

75 TYPEINUMBER 28

77 TYPE1OUTO02 'ERL FAT/TOTAL'

TYPE1OUTO03

TYPE1OUTO04 'ERL INJ/DIARRHEA'

TYPE1OUT005 'ERL INJ/THYROIDITIS'

TYPEIOUT006 'ERL TNJ/THYOTHYROIDISM

3 TYPEIOUTO08 'ERL INJ/TRANSEPIDERMAL

TYPE10UT009

TYPE1OUT009 ' 'CAN FAT/TOTAL'

6 TYPEIOUT011 'CAN FAT/THYROID'

87 TYPE1OUT012 'CAN FAT/BREAST'

8 TYPE1OUT013 'CAN FAT/GI'

89 TYPE1OUT014 'CAN FAT/LEUKEMIA

TYPE1OUT016

TYPE1OUT017

TYPE1OUT018

'CAN FAT/OTHER'

TYPE1OUTO20 'ERL FAT/TOTAL

TYPE10UT021

'ERI INJ/PRODROMAL VOMIT'

TYPE1OUT023

INJ/DIARRHEA

TYPE1OUT025

'ERL INJ/HYPOTHYROIDISM

102 TYPE1OUT027

ERL INJ/TRANSEPIDERMAL

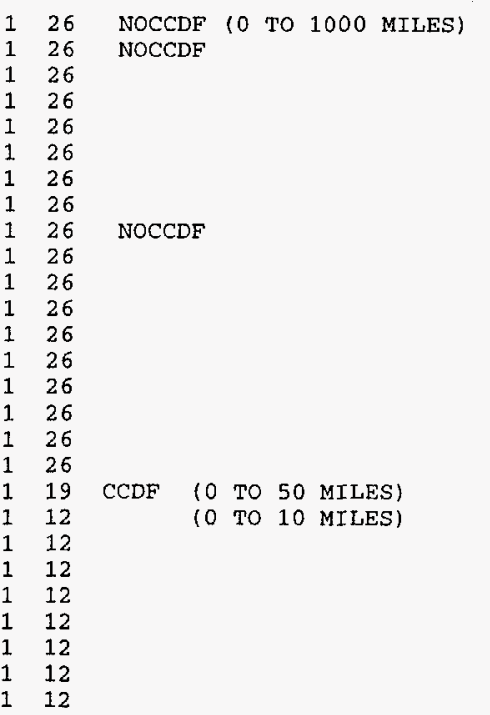


TYPE1OUT028 'CAN FAT/TOTAL

112

* RESULT 2 OPTIONS BLOCK, LOADED BY INOUT2, STORED IN / INOUT $2 /$

* FURTHEST Distance at WHICH a GIVEN RISK of EARLY DEATH IS EXCEEDED.

* NUMBER OF DESIRED RESULTS OF THIS TYPE

104

YYPE2NUMBER 1

* FATALITY RISK THRESHOLD

TYPE2OUTO01 0

* RESULT 3 OPTIONS BLOCK, LOADED BY TNOUT 3, STORED IN / INOUT 3 /

* NUMBER OF PEOPLE WHOSE DOSE tO a GIVEN ORGAN EXCEEDS a GIVEN THRESHOLD.

* NUMBER OF DESIRED RESULTS OF THIS TYPE

106 TYPE3NUMBER 3

* oRgan name dose threshold (SV)

07 TYPE3OUT001

TYPE3OUT001 'A-RED MARR

TYPE3OUT003

A-LUNEWBODY,

1.5
5.0

* RESULT 4 OPTIONS BLOCK, LOADED BY INOUT4, STORED IN / INOUT4/

* 360 DEGREe aVERAGE RISK OF a gIVEN EFFECT at a gIVEN DISTANCE.

POSSIBLE TYPES OF EFFECTS ARE:

* 'ERL FAT/TOTAL,

'ERL INJ/INJURY NAME'

CAN FAT/CANCE

* NUMBER of DESIRED RESULTS of this type

110 TYPE4NUMBER 5

* RADial indeX type of efFect

111 TYPE4OUT001 1 'ERL FAT/TOTAL'

112 TYPE4OUT002 'ERL FAT/TOTAL'

113 TYPE4OUT003 33 'ERL FAT/TOTAL'

114 TYPE4OUT004 4 ERL FAT/TOTAL'

TYPE4OUT005 'BRL FAT/TOTA

- total population dose to a given organ between two distances.

* Number of desired results of this type

116 TYPE5NUMBER 3
ORGA
I1DIS5
I2DIS5
117 TYPE5OUT001 'L-EDEWBODY'
119 NOCCDF $(0-50$ MTLES)

118 TYPE5OUTO02 'L-EDEWBODY'

TYPE5OUT003 'L-EDEWBODY' $1 \quad 26$ NOCCDF (0-1000 MILES) 
- RESUlt 6 OPTIONS BLOCK, LOADED BY INOUT6, STORED IN /INOUT6/

- CENTERltNe dose to an ORgan VS Dist by pathWay, pathway names are as follows:

- pathway name

* PATHAY NAME:
'CLD' - CLOUDSHINE
'GRD' - GROUNDSHINE

'INH ACU' - "ACUTE DOSE EOUIVALENT" FROM DIRECT INHALATION OF THE CLOUD

"INH LIF' - "LIFETIME DOSE COMMITMENT" FROM DIRECT INHALATION OF THE CLOUD

"RES ACU' - "ACUTE DOSE EQUIVALENT" FROM RESUSPENSION INHALATION

- 'RES LIF' - "LIFETIME DOSE COMMITMENT" FROM RESUSPENSION INHALATION

* 'TOT ACU' - "ACUTE DOSE EQUIVALENT" FROM ALL PATHWAYS

- 'TOT LIF" - "LIFETIME DOSE COMMITMENT" FROM ALL PATHWAYS

* NuMBer of DESIRED RESULTS of this TYPE

120 TYPE 6 NUMBER

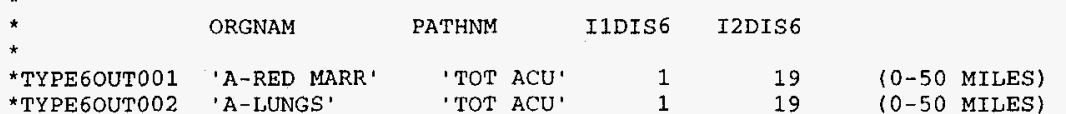

'TYPE6OUT002 'A-LUNGS' 'TOT ACU' 11019 (0-50 MILES)

*TYPE6OUT003 'L-EDEWBODY' 'TOT LIF' $\quad 1 \quad 26 \quad(0-1000$ MILES)

* RESULT 7 OPTIONS BLOCK, LOADED BY INOUT7, STORED IN / INOUT7/

* CENTERLINE RISK OF A GIVEn EFFectr vs DISTANCE

* number of desired results of this type

121 TYPE7NUMBER

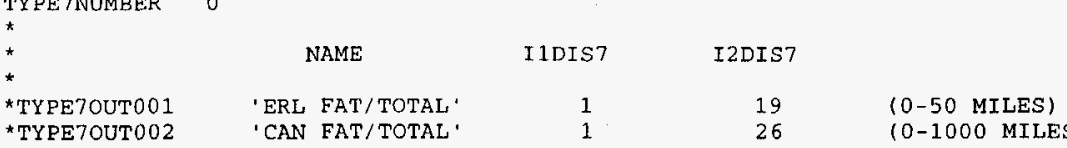

*TYPE7OUT002 'CAN FAT/TOTAL' 1 2 16 (0-1000 MILES)

* Result 8 options block, loAded by inout8, stored in / INOUT8/

* population WEIGHTED FataltTy RISK BETWEEN 2 DISTANCES

* NUMBER of DESiREd RESULts of this tyPE

122 TYPEBNUMBER 2

* NAME I1Dis8 I2DIS8

123 TYPE8OUTO01 'ERL FAT/TOTAL' 1 NOCCDF (0-EXCL ZONE + 1 MI)

124 TYPE8OUT002 'CAN FAT/TOTAL' 1 12 NOCCDF $10-10$ MILES

$\star * \star * \star * \star * * * * * \star * * *$

* RESUlt a options block, LOAded by inOUTa, StOREd IN / INOUTA/

* peak dose to a given organ

* NUMA

125 TYPEANUMBER

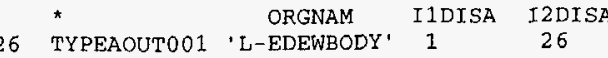

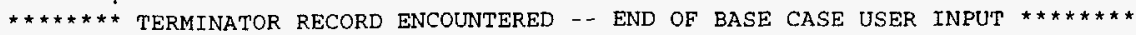


USER INPUT PROCESSTNG SUMMARY - BASE CASE

NUMBER OF RECORDS READ

NUMER OF BLANK OR COMMENT RECORDS READ

NUMBER OF TERMINATOR RECORDS

NUMBER OF PROCESSED RECORDS DUPLICATED NUMBER OF PROCESSED RECORDS SORTED

The list of defined organs is as follows (A- is ACUTE and L- is LIFETIME) :

A-SKIN

A-RED MARR
A-LUNGS

A-THYROIDH

A-STOMACH

L-EDEWBODY

L-RED MARR

L-BONE SUR

L-BREAST

L-LUNGS

L-THYROID

L-LOWER LI

L-BLAD WAL

READING FROM A DOSE CONVERSION FILE WITH THE FOLLOWING HEADER:

作

Seven new organs added with MACCS version 1.5.11.1

USING THE FOLLOWING SITE DATA FILE:

MACCS SITE DATA FILE FOR SURRY (JLS, 11/10/88)

SECPOP POP DISTRIBUTION FROM 1980 CENSUS DATA ALTERED USING 0-10 MI NRC DATA

26 SPATIAL INTERVALS

16 WIND DIRECTIONS

7 CROP CATEGORIES

2 WATERSHEDS

59 ECONOMIC REGIONS

SPATIAL DISTANC

$0.16 \quad 0.52$

$5.63 \quad 8.05$

$\begin{array}{lr}48.28 & 64.37 \\ 4.67 & 1609.34\end{array}$

$\begin{array}{cc}804.67 & 1609.34 \\ \text { POPULATION } & \end{array}$

$\begin{array}{rrrrrr}1.21 & 1.61 & 2.13 & 3.22 & 4.02 & 4.83 \\ 11.27 & 16.09 & 20.92 & 25.75 & 32.19 & 463.23\end{array}$

$\begin{array}{rrrrrr}11.27 & 16.09 & 20.92 & 25.75 & 32.19 & 40.23 \\ 80.47 & 112.65 & 160.93 & 241.14 & 321.87 & 563.27\end{array}$

$\begin{array}{cr}0 . & 0 \\ 6 . & 25\end{array}$

$\begin{array}{rrrrrr}0 & 0 . & 0 & 0 . & 4 . & 5 \\ 3341 & 7107 & 2173 & 0 & 1305 & 474\end{array}$

$\begin{array}{rrrrrr}2487346 . & 104331 . & 0 & & & \\ 2252 & 0 . & 1 . & 2 . & 9 . & 13\end{array}$

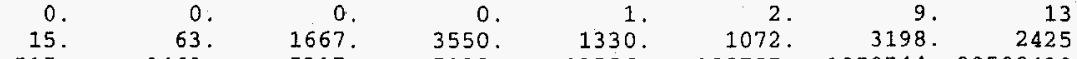

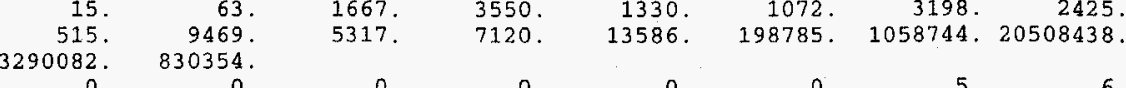

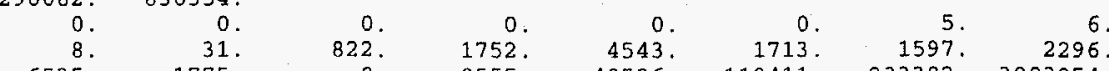

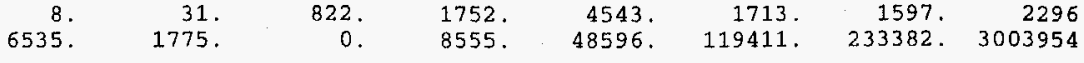




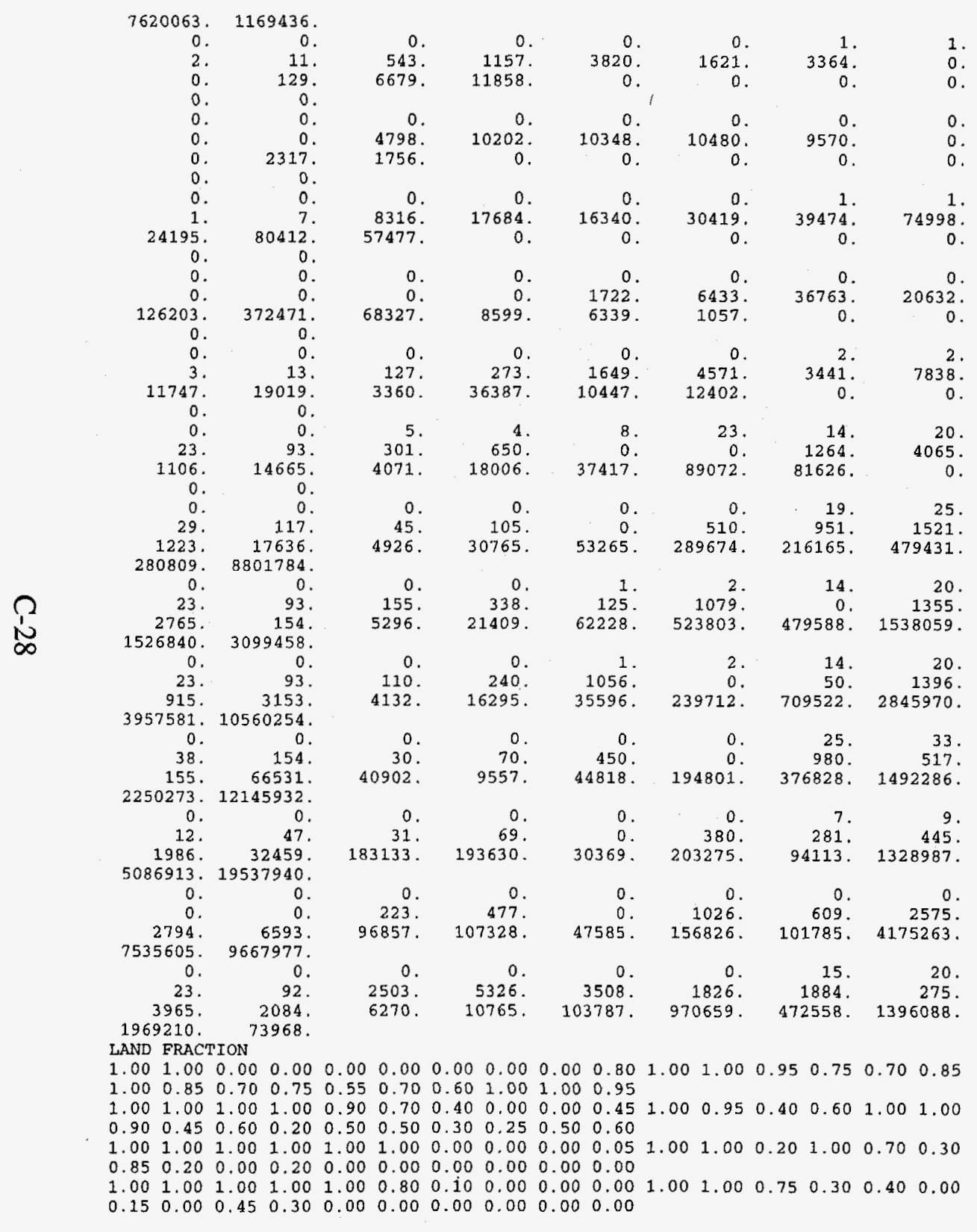


$\begin{array}{llllllllllllllll}1.00 & 1.00 & 1.00 & 1.00 & 1.00 & 0.60 & 0.00 & 0.00 & 0.00 & 0.00 & 0.95 & 1.00 & 1.00 & 0.70 & 0.40 & 0.10\end{array}$ $\begin{array}{lllllllllll}0.00 & 0.50 & 0.00 & 0.00 & 0.00 & 0.00 & 0.00 & 0.00 & 0.00 & 0.00\end{array}$

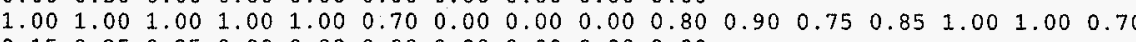

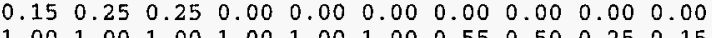

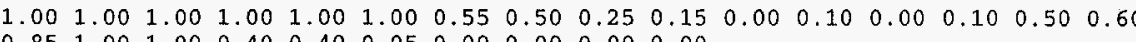

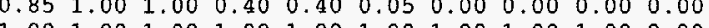

1.001 .001 .001 .001 .001 .00 1.001 .001 .001 .001 .001 .001 .801 .400 .000 .001 .20

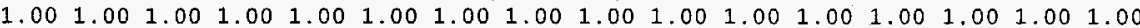

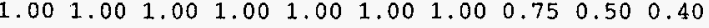

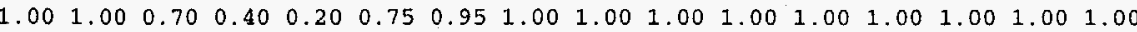

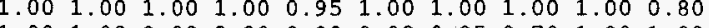

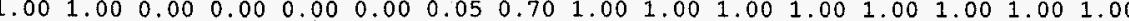

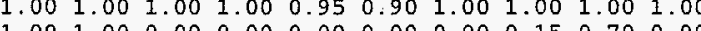

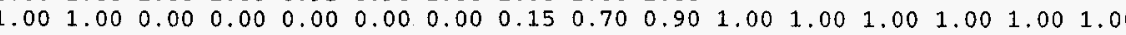
1.001 .001 .001 .001 .001 .001 .001 .001 .001 .00

$\begin{array}{llllllllllllllllll}1.00 & 1.00 & 0.00 & 0.00 & 0.00 & 0.00 & 0.00 & 0.00 & 0.20 & 0.50 & 0.40 & 0.30 & 0.25 & 0.75 & 0.80 & 0.85\end{array}$

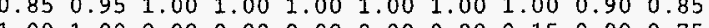

0.800 .850 .951 .001 .001 .00 1.001 .00

$1.001 .00 \quad 0.000 .000 .00 \quad 0.001 .001 .000 .000 .901 .001 .0011 .001 .0011 .001 .00$ $1.001 .001 .00 \quad 0.95 \quad 0.80 \quad 0.851 .001 .000 .550 .95$ REGION INDEX

(4) $4044444444444444444444418 \quad 728301917$

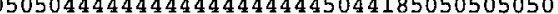
444444444444445050504444444445044504444505050505050 44444444444445050505044444444444450445050505050505050 4444444444445050504444444444444444444450505050505050

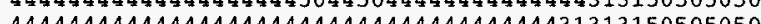

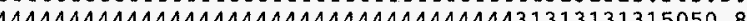

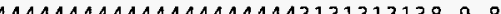
444444444444444444444444444444444444444431313138 g 1 4444505050504444444444444444444444444444444444314040 4444505050505044444444444444444444444444444444461511 44445050505050504444444444444444444444444444444463347 4444505050505044444444444444444444444444444444365320 4444505050505050504444444444444444444444441818363053 WATERSHED INDEX

$\begin{array}{llllllllllllllllllllllllll}1 & 1 & 2 & 2 & 2 & 2 & 2 & 2 & 2 & 1 & 1 & 1 & 1 & 1 & 1 & 1 & 1 & 1 & 1 & 1 & 1 & 1 & 1 & 1 & 1 & 1\end{array}$ $\begin{array}{lllllllllllllllllllllllllll}1 & 1 & 1 & 1 & 1 & 1 & 2 & 2 & 2 & 2 & 1 & 1 & 2 & 1 & 1 & 1 & 1 & 1 & 1 & 2 & 2 & 2 & 2 & 2 & 2 & 1\end{array}$ $\begin{array}{llllllllllllllllllllllllllll}1 & 1 & 1 & 1 & 1 & 1 & 2 & 2 & 2 & 2 & 1 & 1 & 2 & 1 & 1 & 2 & 1 & 2 & 2 & 2 & 2 & 2 & 2 & 2 & 2 & 2\end{array}$ $\begin{array}{lllllllllllllllllllllllllllllll}1 & 1 & 1 & 1 & 1 & 1 & 2 & 2 & 2 & 2 & 1 & 1 & 1 & 1 & 2 & 2 & 2 & 2 & 2 & 2 & 2 & 2 & 2 & 2 & 2 & 2\end{array}$

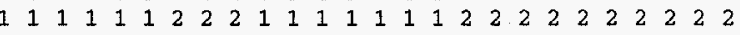
$\begin{array}{llllllllllllllllllllllllll}1 & 1 & 1 & 1 & 1 & 1 & 1 & 2 & 2 & 2 & 2 & 2 & 2 & 2 & 2 & 1 & 1 & 1 & 1 & 2 & 2 & 2 & 2 & 2 & 2 & 2 \\ 1 & 1 & 1 & 1 & 1 & 1 & 1 & 1 & 1 & 1 & 2 & 2 & 1 & 1 & 1 & 1 & 1 & 1 & 1 & 1 & 1 & 2 & 2 & 2 & 2 & 2\end{array}$ $\begin{array}{llllllllllllllllllllllllll}1 & 1 & 1 & 1 & 1 & 1 & 1 & 1 & 1 & 1 & 1 & 1 & 1 & 1 & 1 & 1 & 1 & 1 & 1 & 1 & 1 & 1 & 2 & 2 & 2 & 2\end{array}$ $\begin{array}{llllllllllllllllllllllllll}1 & 1 & 1 & 1 & 1 & 1 & 1 & 1 & 1 & 1 & 1 & 1 & 1 & 1 & 1 & 1 & 1 & 1 & 1 & 1 & 1 & 1 & 1 & 1 & 2 & 2\end{array}$

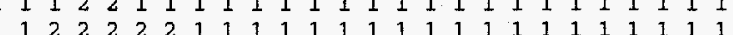
$\begin{array}{lllllllllllllllllllllllll}1 & 2 & 2 & 2 & 2 & 2 & 1 & 1 & 1 & 1 & 1 & 1 & 1 & 1 & 1 & 1 & 1 & 1 & 1 & 1 & 1 & 1 & 1 & 1\end{array}$ $\begin{array}{llllllllllllllllllllllllll}1 & 1 & 2 & 2 & 2 & 2 & 2 & 2 & 2 & 2 & 1 & 1 & 1 & 1 & 1 & 1 & 1 & 1 & 1 & 1 & 1 & 1 & 1 & 1 & 1 & 1\end{array}$ $\begin{array}{llllllllllllllllllllllllll}1 & 1 & 2 & 2 & 2 & 2 & 2 & 2 & 1 & 1 & 1 & 1 & 1 & 1 & 1 & 1 & 1 & 1 & 1 & 1 & 1 & 1 & 1 & 1 & 1 & 1 \\ 1 & 1 & 2 & 2 & 2 & 2 & 2 & 2 & 2 & 1 & 1 & 1 & 1 & 1 & 1 & 1 & 1 & 1 & 1 & 1 & 1 & 1 & 1 & 1 & 1 & 1\end{array}$

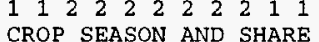

1 PASTURE

2 STORED FORAGE

90. 270. 0.41

3 GRAINS

150. $240 . \quad 0.13$




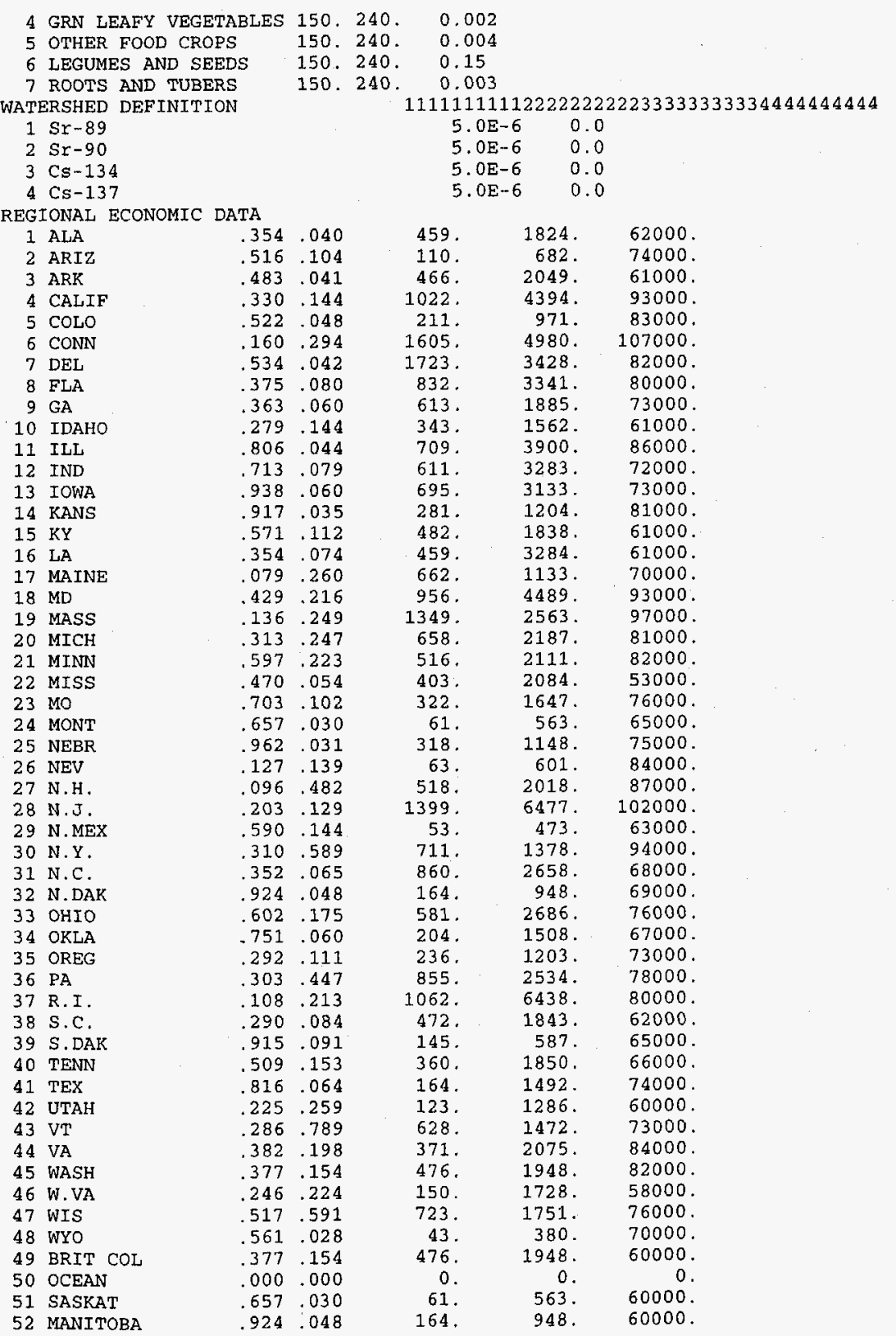




$\begin{array}{lllrrr}53 \text { ONTARIO } & .597 .223 & 516 . & 2111 . & 60000 . \\ 54 \text { QUEBEC } & .310 .589 & 711 . & 1378 . & 60000 \\ 55 \text { NOVA SCOT } & .079 .260 & 662 . & 1133 . & 60000 \\ 56 \text { BAJA CAL } & .330 .144 & 1022 . & 4394 . & 10000 \\ 57 \text { SONORA } & .516 .104 & 110 . & 682 . & 10000 . \\ 58 \text { CHIHUAHUA } & .590 .144 & 53 . & 473 . & 10000 . \\ 59 \text { COAHUILA } & .816 .064 & 164 . & 1492 . & 10000 \\ \text { END } & & & & & \end{array}$

POPULATION

$\gg$ The Record Identifier TYPEBNUMBER was not found:

$\rightarrow$ Type $B$ results not being generated

$\star * \star * * * * *$ BEGINNING OF CHANGE CASE 1 USER INPUT $* * * * * * * *$

* EMERGENCY RESPONSE SCENARIO NUMBER 2

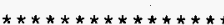

* eVAcuation zone Data BLOCK, LOADED BY EVNETW, STORED IN /NETWOR/, /EOPTIO/

* SPECTFIC DESCRIPTION OF THE EMERGENCY RESPONSE SCENARIO BEING USED

127 EZEANAM2001 'NO EVACUATION, RELOCATION MODELS APPLY EVERYWHERE'

* WEIGHTING FRACTION APPLICABLE TO THIS SCENARIO

128 EZWTFRAC001 0.05

* Last RING IN the movement zONe

129 EZLASMOV001 O (A ZERO TURNS OFF THE EVACUATION MODEL)

$3 \sin _{\star \star * * * * * * *}$

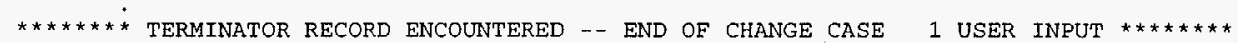

USER INPUT PROCESSING SUMMARY - CHANGE CASE 1

$\begin{array}{ll}\text { NUMBER OF RECORDS CHANGED } & =3 \\ \text { NUMBER OF RECORDS ADDED } & =0\end{array}$

NO EVACUATION REQUESTED

USER INPUT IS READ FROM UNIT 26

RECORD IDENTIFIER FIELDS 11 CHARACTERS LONG ARE EXPECTED.

THE MAXIMUM NUMBER OF IDENTIFIER RECORDS THAT MAY BE SAVED AS THE BASE CASE IS 1000.

RECORD

RECORD

NUMBER

- General Descriptive title describing this "Chronc" INPUT fIle

1 CHCHNAME001 'IN3A_N. INP, Sample Problem A, "New" COMIDA2-Based Food Model.

* EMERGENCY RESPONSE COST DATA BLOCK

* DAILY COSt FOR a PERSON WHO IS EVACUATEd (DOLLARS/PERSON-DAY) 
2 CHEVACSTOO 27.00 (INCLUDES FOOD AND HOUSING COSTS BUT NOT LOST INCOME) * DAily cost for a person who is RELocated (DOLLARs/PERSON-DAY)

3 CHRELCSTO01 27.00 (INCLUDES FOOD AND HOUSING COSTS BUT NOT LOST INCOME)

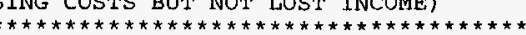
* LONG TERM PROTECTIVE ACTION DATA BLOCK

Duration of the intermediate phase period--at version 1.11C TMIPND is no longer processed. The new input variable DUR_INTPHAS is the period's * duration, not the time after plume arrival at which the period ends.

4 DUR_INTPHAS 0.0 (in seconds) (no intermediate phase)

- LONG-TERM PHASE DOSE PROJECTION PERTOD, THE DURATION OF THE EXPOSURE * PERIOD OVER WHICH THE LONG-TERM DOSE CRITERION IS EVALUATED (SECONDS)

5 CHTMPACTO01 1.58E8 (5 YEARS)

* Dose criterion for intermediate phase relocation (Sv)

6 CHDSCRTIOO1 1.0E5 (NO INTERMEDIATE PHASE RELOCATION)

- DOSE CRITERION FOR LONG-TERM PHASE RELOCATION (Sv)

7 CHDSCRLT001 0.04

* CRITiCal oRgan Name For LONG-TERM actions

8 CHCRTOCRO01 'L-EDEWBODY'

* Long Term Exposure Period Previously permanently set to:

- one million years $=3.15$ E13 seconds

MACCS2 allowable range is $3.15 E 7$ to $1 . E 10$

9 CHEXPTIMOO

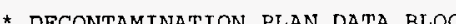

* NumBer of LEVELS of DEContamination

10 CHLVLDECOO1

* DECONTAMINATION TIMES CORRESPONDING TO THE LVLDEC LEVELS OF DECONTAMTNATION

- (SECONDS)

11 CHTIMDEC001 5.184E6 $1.0368 E 7 \quad(60,120$ DAYS $)$

* DOSE REDUCTION FACTORS CORRESPONDING TO THE LVLDEC LEVELS OF DECONTAMINATION

12 CHDSRFCTOOI 3

15

* COST OF FARM DECONTAMINATION PER FARMLAND UNIT AREA (DOLUARS/HECTARE)

* For the VARIOUS leEvels of DEContamination

13 CHCDFRM0001 $562.5 \quad 1250$

* Cost of nonfarm DECONTAMINATION PER RESIDENT PERSON (DOLLARS/PERSON)

* for the various levels of decontamination

14 CHCDNFRM001 3000. 8000 
* Fraction of non-Farm decontamination cost due to labor

* for the various decontamination levels

16 CHFRNFDLO01 7

* FRACTION OF time WORKERS IN FARM AREAS SPEND IN CONTAMINATED aREAS

* For the VARIOUS DECONTAMINATION LEVELS

17 CHTFWKF0001 $\quad .10 \quad .33$

* FRACTION OF TIME WORKERS IN NON-FARM AREAS SPEND IN CONTAMINATED AREAS * FOR the VARIOUS DECONTAMINATION LEVELS

18

CHTFWKNF001 $\quad .33 \quad .33$

* average cost of Decontamination labor (DOLlars/man-year)

19 CHDLBCSTO01 35000.

* INTERDICTION COST DATA BLOCK

* DEPRECIATION (DETERIORATION) RATE DURING INTERDICTION PERIOD (PER YEAR)

20 CHDPRATE001 20 (VALUE OBTAINED FROM WASH-1400, APPENDIX 6)

* INVESTMENT INCOME RETURN (DISCOUNT RATE) DURING INTERDICTION PERIOD (PER YEAR) * THIS VALUE SHOULD BE DERIVED AS a REAL RETURN RATE ADJUSTED FOR INFLATION

21 CHDSRATE001 .12 (VALUE OBTAINED FROM WASH-1400, APPENDIX 6)

- population Relocation COSt (Dollars/PERSON):

* ALTERNATIVE hOUSING, MOVING COSTS, AND LOST INCOME FOR PEOPLE IN

* AREAS WHICH REQUIRE DECONTAMINATION, INTERDICTION, OR CONDEMNATION

22 CHPOPCSTO01 5000. * GROUNDSHINE WEATHERING DEFINITION DATA BLOCK

* NUMBer of terms in the groundshine WEATHERING RELATtonship (EITHer 1 Or 2)

23 CHNGWTRMO01 2

* GROUNDSHINE WEATHERING COEFFICIENTS

24 *
CHGWCOEFO01 0.5
(JON HELTON)

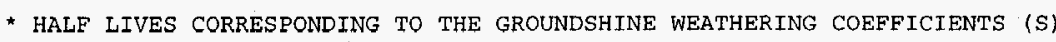

25 CHTGWHLF001 1.6E7 2.8E9 (JON HELTON)

* RESUSPENSION WEATHERING DEFINITION DATA BLOCK

* NUMBer of terms in the resuspension weathering RELATIONShip

26 CHNRWTRM001 3 
- RESUSPENSION CONCENTRATION COEFFICIENTS (/ METER)

* RELATIONSHIP BETWEEN GROUND CONCENTRATION AND INSTANTANEOUS aIR CONC.

27 CHRWCOEF001 1.0E-5 $1.0 \mathrm{E}-7 \quad 1.0 \mathrm{E}-9$ (VALUES HERE SELECTED BY JON HELTON)

* HALF-LIVES CORRESPONDING TO THE RESUSPENSION CONCENTRATION COEFFICIENTS (S)

28 CHTRWHLFO01 $1.6 \mathrm{E} 7 \quad 1.6 \mathrm{E} 8 \quad 1.6 \mathrm{E} 9 \quad$ (6 MONTHS, 5 YEARS, 50 YEARS)

* SITE REGION DESCRIPTION DATA BLOCK

- Fraction of aRea that is Land in the region

29 CHFRACLD001 0.95 (ROUGH GUESS VALUE, SITE FILE OVERRIDES THIS VALUE)

* FRACTION OF LAND DEVOTED TO FARMing IN THE REgION

30 CHFRCFrm001 0.382 (VIRGINIA STATE VALUE, SITE FILE OVERRIDES THIS VALUE)

- average value of annual farm production in the region (Dollars/hectare)

* (CASH RECEIPTS From FARMing plus VALUE OF hOME CONSUMPTION)/(LAND IN FARMS)

31 CHFRMPRDO01 371.0 (VIRGINIA STATE VALUE, SITE FILE OVERRIDES THIS VALUE)

* FRACTION OF FARM PRODUCTION RESULTING FROM DAIRY PRODUCTION IN THE REGION

- (VALUE OF MILK PRODUCED)/(CASH RECEIPTS FROM FARMING PLUS HOME CONSUMPTION)

32 CHDPFRCT001 0.198 (VIRGINIA STATE VALUE, SITE FILE OVERRIDES THIS VALUE)

* VAlue of farm wealth (DOLlars/hectare)

* (AVERAge VAlue per hectare of farm land and buildings to 100 miles)

33 CHVALWF0001 2613. * SURRY

- fraction of farm weatsh in improvements for the region

34 CHFRFIMOOO1 $0.25 \cdot$ SURRY

* NON-FARM WEALTh, PROPERTY AND IMPROVEMENTS FOR THE REGION (DOLlARS/PERSON)

- THE VALUE OF ALI RESIDENTIAL, BUSTNESS, AND PUBLIC ASSETS WHICH WOULD BE

35 CHVALWNF001 84000. SURRY

* FRACTION OF NON-FARM WEALTH IN IMPROVEMENTS FOR THE REGION

36 CHFRNFIMO01 0.8

37 CHFDPATH001 'NEW'

* name of the Comida2 binary output file

38 BIN_FILE001 'C:\COMIDA2A \SAMP_A.BIN' (revised data file of 8/12/95)

* Dose limits triggering first year crop disposal of the separate

* milk and non-milk components of the diet, corresponding in purpose,

- For NUREG-1150 calculations, the maximum allowable ground concentrations for

* production of milk and non-milk crops contaminated by an accident occurring

* in the growing season were derived based on an assumed maximum allowable

* dose of 5 rem effective or 15 rem thyroid, per the 1982 FDA guidance that's 
* reprinted in the 1992 EPA PAG Manual. For purposes of comparison against * the prior results, it is being assumed, for simplicity, that milk and

* non-milk crops contribute equally to the first year dose. Thus, the 5 ren effective dose limit used in NUREG-1150 is equally split between milk and

non-milk crops, with 2.5 rem allowed for each. Similarly, the 15 rem

thyroid limit is split into 7.5 and $7.5 \mathrm{rem}$ for the milk and non-milk

Dos

- Annual dose limits for the subsequent year's (i.e., after the first year)

* interdiction of BOTH the milk and non-milk (combined) components of the diet

Note: the long-term food critera, GCMAXR, used for NUREG-1150 wre based on an ingestion dose integrated from zero to infinity. It is not possible to

translate those parameter values into corresponding annual dose limits, as is

required by the COMIDA2-based food model. The "total" dose limits used in

NUREG-1150 for "root uptake", 0.5 rem effective and 1.5 rem thyroid, are used

here as annual dose limits for interaiction

41 DOSELONG001 0 effective thyroid (doses in sieverts)

* NUMBER OF nUCLIDES IN THE WATER INGESTION PATHWAY MODEI

CHNUMWPI001 4

* TABLE OF NUCLIDE DEFINITIONS IN THE WATER INGESTION PATHWAY MODEI

* if a site data file is Defined, the data defining the watershed ingestion

* FACTOR IS SUPERSEDED BY THE CORRESPONDING DATA IN T'HE SITE DATA FILE

* initial anNual ingestion factor

* $\begin{array}{llll}\text { * WATER } & \text { WASHOFF } \\ * & \text { NUCLIDE } & \text { FRACTION } & \\ & & \end{array}$

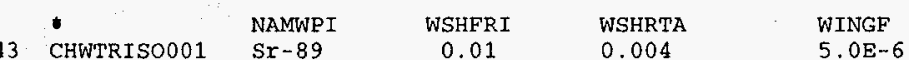

$\begin{array}{llllll}44 & \text { CHWTRISO002 } & \text { Sr-89 } & 0.01 & 0.004 & 5.0 \mathrm{E}-6 \\ \text { Sr-90 } & 0.01 & 0.004 & 5.0 \mathrm{E}-6\end{array}$

45 CHWTRISO003 Cs-134 $0.005 \quad 0.001$ 5.0E-6

46 CHWTRISO004 Cs-137 $0.005 \quad 0.001 \quad 5.0 \mathrm{E}-6$

* SPECIAL OPTIONS DATA BLOCK

- DETAILED PRINT OPTION CONTROL SWITCHES, LOOK AT THE CODE BEFORE TURNING ON!!

47 CHKSWTCHOO1 O

* DEFINE THE TYPE 9 RESULT

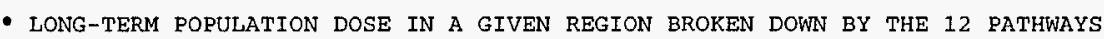

- number of results of this type that are being REQUested

* FOR EACH RESULT YoU REQUEST, THE CODE WILI PRODUCE A SET OF 12

48
*
* oranam
ORGNAM INNER OUTER 
TYPE9OUT001 'L-EDEWBODY' $1226 \quad$ (0-1000 MILES)

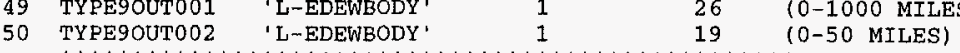

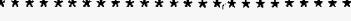

* ECONOMIC COST RESULTS IN A REGION BROKEN DOWN BY 12 TYPES OF COSTS

* NUMBER OF RESULTS OF THIS TYPE THAT ARE BEING REQUESTED

* FOR EACH RESULT YOU REQUEST, THE CODE WILL PRODUCE A SET OF 12

51 TYPIONUMBER 2 (UP TO 10 ALLOWED)

\begin{tabular}{|c|c|c|c|}
\hline * & INNER & OUTER & \\
\hline $\begin{array}{l}\text { TYP10OUT001 } \\
\text { TYP100UT002 }\end{array}$ & 1 & 26 & (0-1000 MILES) \\
\hline TYP100UT002 & 1 & 19 & (0-50 MILES) \\
\hline
\end{tabular}

$\begin{array}{llccc}52 & \text { TYP100UT001 } & 1 & 26 & (0-1000 \text { MILES } \\ 53 & \text { TYP100UT002 } & 1 & 19 & (0-50 \text { MILES) }\end{array}$

DEFTNE A FLAG THAT CONTROLS THE PRODUCTION OF THE

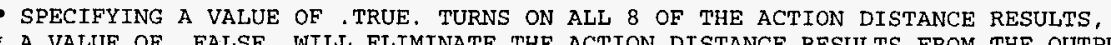

* AP11FLAG19

TRUE.

- IMPACTED AREA/POPULATION RESULTS IN A REGION BROKEN DOWN BY 6 TYPES OF IMPACTS

* number of REsUlts of this type that are berng ReQuested

* FOR EACH RESULT YOU REOUEST THE CODE WILL PRODUCE A SET OF 8

55 TYP12NUMBER 2 (UP TO 10 ALLOWED)

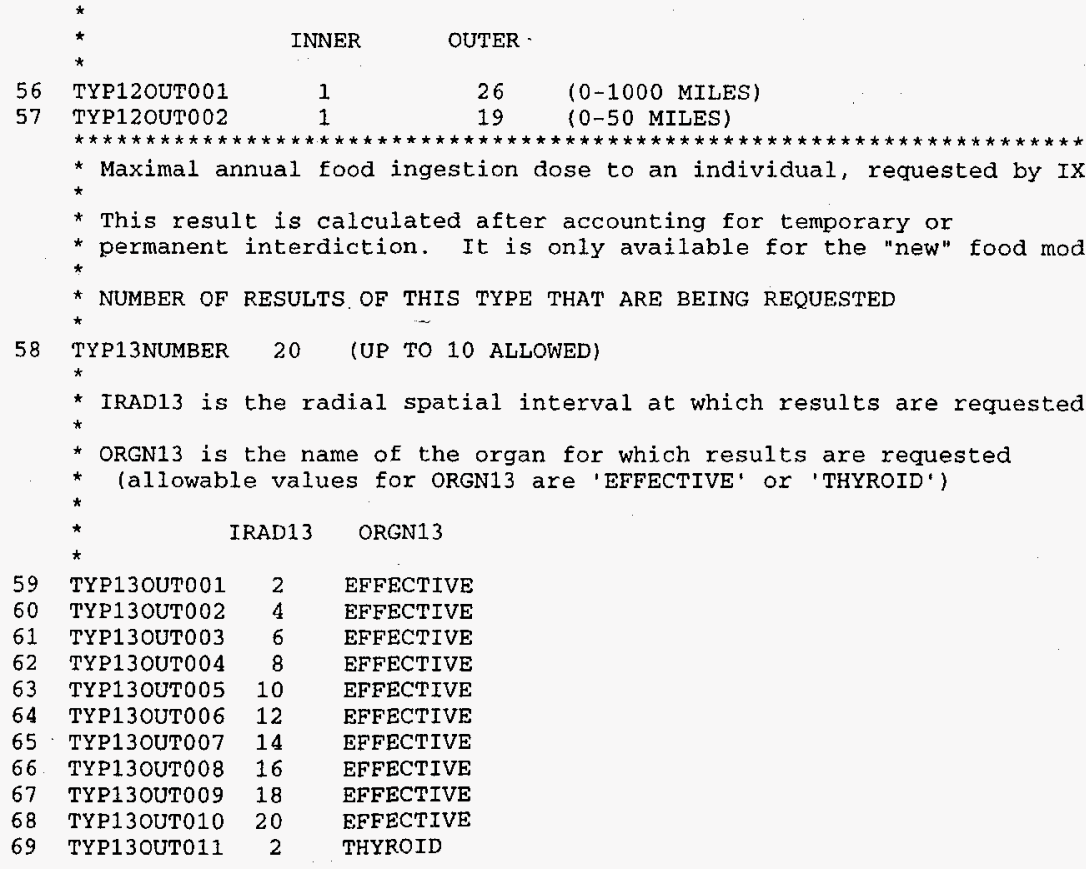




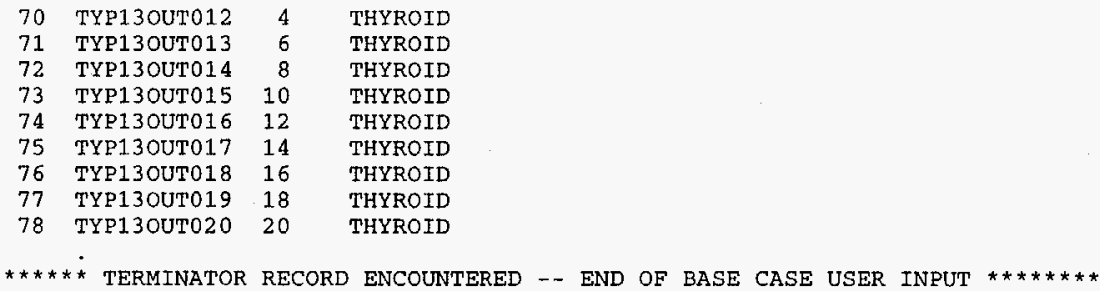

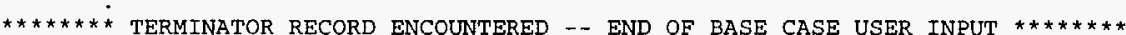

USER INPUT PROCESSING SUMMARY - BASE CASE

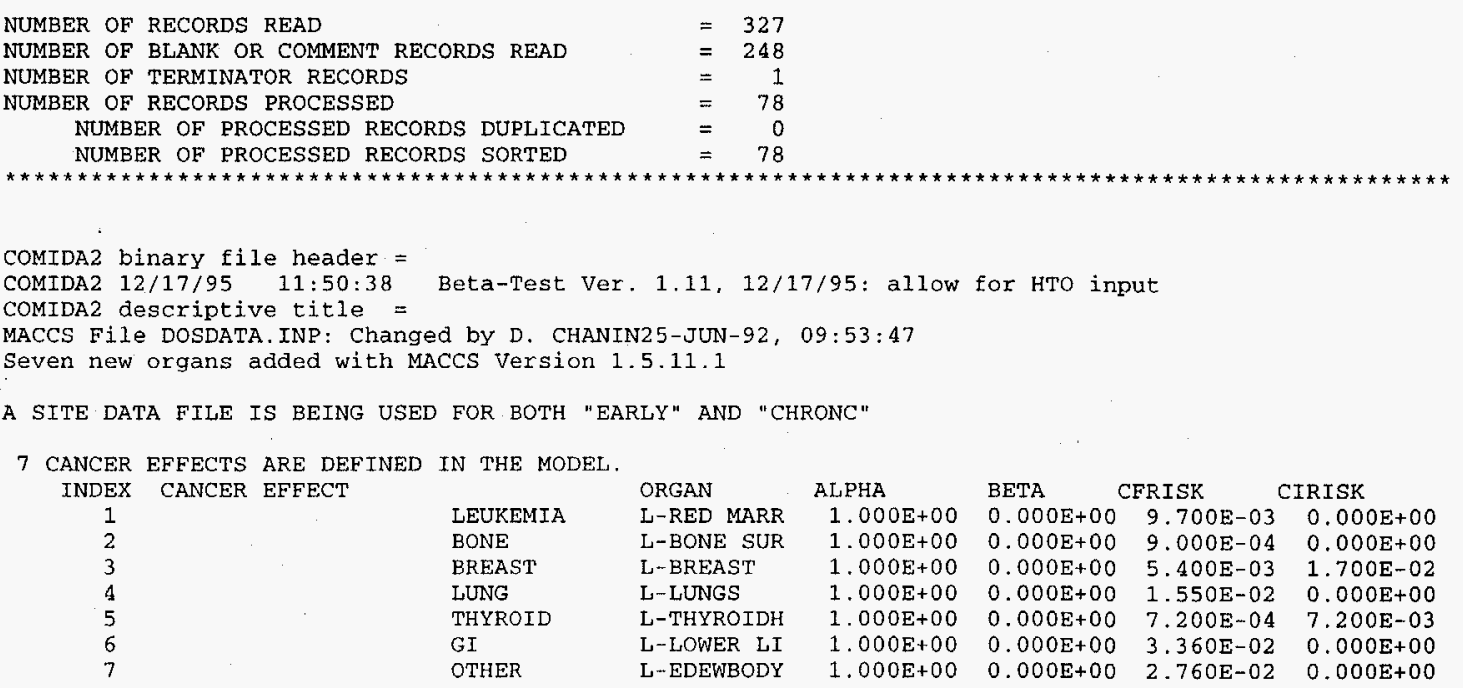

TIME OF HOTSPOT. REIOCATION IS $4.3200 \mathrm{E}+04$.
TIME OF NORMAL RETURN IS $8.640 \mathrm{E}+04$ AND THE EMERGENCY PHASE ENDS AT $6.048 \mathrm{E}+05$.

GROUNDSHINE SHIELDING FACTOR $=0.330$

RESUSPENSION PROTECTION FACTOR $=0.410$

BREATHING RATE (CUBIC $\mathrm{M} / \mathrm{S}$ ) $=2.660 \mathrm{E}-04$

DISPERSION MODEL FLAG IS 2

WINDROSE PROBABILITIES BY WIND DIRECTION AND MET BIN NUMBER

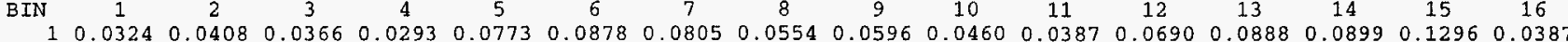
$\begin{array}{lllllllllllllllll}2 & 0.0085 & 0.0751 & 0.1216 & 0.0803 & 0.0423 & 0.0930 & 0.0877 & 0.0941 & 0.0941 & 0.0666 & 0.0529 & 0.0909 & 0.0655 & 0.0106 & 0.0127 & 0.0042\end{array}$ $\begin{array}{lllllllllllllllll}3 & 0.0581 & 0.0930 & 0.0465 & 0.0465 & 0.1163 & 0.0465 & 0.0233 & 0.0581 & 0.0814 & 0.0465 & 0.0465 & 0.0349 & 0.0465 & 0.0698 & 0.1279 & 0.0581\end{array}$ $\begin{array}{lllllllllllllllll}4 & 0.0350 & 0.0402 & 0.0542 & 0.0629 & 0.0524 & 0.0804 & 0.0524 & 0.0717 & 0.069 & 0.0717 & 0.0752 & 0.0594 & 0.0839 & 0.0962 & 0.0524 & 0.0420\end{array}$

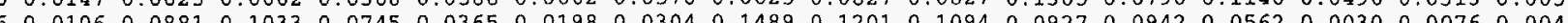


$\begin{array}{llllllllllllllllll}7 & 0.0138 & 0.1103 & 0.0759 & 0.0759 & 0.0345 & 0.0207 & 0.0828 & 0.2414 & 0.1448 & 0.0276 & 0.0828 & 0.0621 & 0.0276 & 0.0000 & 0.0000 & 0.0000\end{array}$

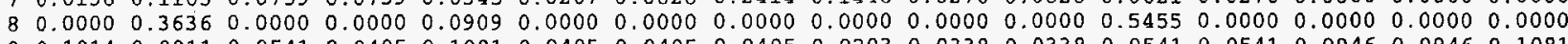
$\begin{array}{lllllllllllllllll}9 & 0.1014 & 0.0811 & 0.0541 & 0.0405 & 0.1081 & 0.0405 & 0.0405 & 0.0405 & 0.0203 & 0.0338 & 0.0338 & 0.0541 & 0.0541 & 0.0946 & 0.0946 & 0.1081\end{array}$ $\begin{array}{lllllllllllllllllll}11 & 0.0633 & 0.0629 & 0.0629 & 0.0629 & 0.0808 & 0.0584 & 0.0374 & 0.0284 & 0.0569 & 0.0689 & 0.0464 & 0.0314 & 0.1003 & 0.1557 & 0.0479 & 0.0389\end{array}$ $\begin{array}{llllllllllllllllll}12 & 0.0551 & 0.2404 & 0.1302 & 0.0885 & 0.0518 & 0.0367 & 0.0651 & 0.1202 & 0.0735 & 0.0518 & 0.0351 & 0.0200 & 0.0134 & 0.0017 & 0.0100 & 0.0067\end{array}$ $\begin{array}{llllllllllllllllll}13 & 0.1382 & 0.1008 & 0.0711 & 0.0388 & 0.0413 & 0.0491 & 0.0556 & 0.0388 & 0.0375 & 0.0284 & 0.0181 & 0.0310 & 0.0271 & 0.0788 & 0.0904 & 0.1550\end{array}$ $\begin{array}{llllllllllllllllll}14 & 0.1634 & 0.1489 & 0.0646 & 0.0711 & 0.0685 & 0.0487 & 0.0580 & 0.0461 & 0.0817 & 0.0343 & 0.0132 & 0.0132 & 0.0079 & 0.0224 & 0.0527 & 0.1054\end{array}$ $\begin{array}{llllllllllllllllll}15 & 0.2069 & 0.2897 & 0.1241 & 0.1034 & 0.0759 & 0.0690 & 0.0069 & 0.0138 & 0.0276 & 0.0069 & 0.0069 & 0.0138 & 0.0000 & 0.0069 & 0.0069 & 0.0414\end{array}$ $\begin{array}{llllllllllllllllllll}16 & 0.1667 & 0.3333 & 0.1667 & 0.0000 & 0.0833 & 0.0000 & 0.0000 & 0.1667 & 0.0833 & 0.0000 & 0.0000 & 0.0000 & 0.0000 & 0.0000 & 0.0000 & 0.0000\end{array}$ $\begin{array}{llllllllllllllllll}17 & 0.1033 & 0.0409 & 0.0450 & 0.0235 & 0.0307 & 0.0501 & 0.0593 & 0.0654 & 0.0511 & 0.0399 & 0.0573 & 0.0910 & 0.1237 & 0.0470 & 0.0838 & 0.0879\end{array}$

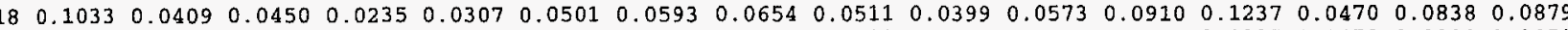

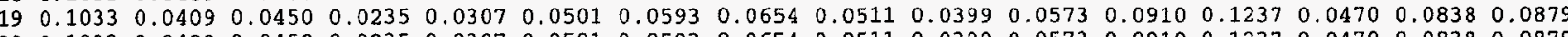

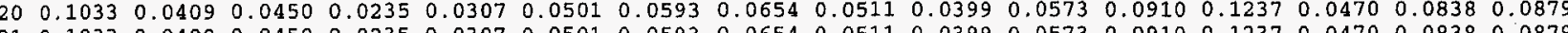

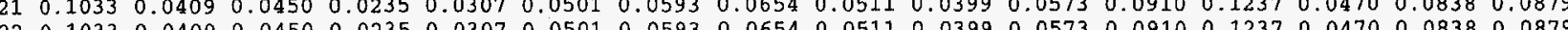

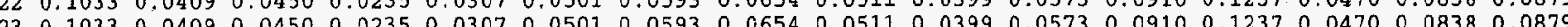

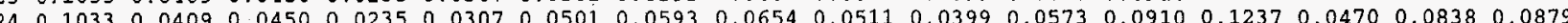
$\begin{array}{lllllllllllllllllll}25 & 0.1033 & 0.0409 & 0.0450 & 0.0235 & 0.0307 & 0.0501 & 0.0593 & 0.0654 & 0.0511 & 0.0399 & 0.0573 & 0.0910 & 0.1237 & 0.0470 & 0.0838 & 0.0879\end{array}$ $\begin{array}{llllllllllllllllll}26 & 0.1033 & 0.0409 & 0.0450 & 0.0235 & 0.0307 & 0.0501 & 0.0593 & 0.0654 & 0.0511 & 0.0399 & 0.0573 & 0.0910 & 0.1237 & 0.0470 & 0.0838 & 0.0879\end{array}$ $\begin{array}{llllllllllllllllll}27 & 0.1033 & 0.0409 & 0.0450 & 0.0235 & 0.0307 & 0.0501 & 0.0593 & 0.0654 & 0.0511 & 0.0399 & 0.0573 & 0.0910 & 0.1237 & 0.0470 & 0.0838 & 0.0879\end{array}$ $\begin{array}{llllllllllllllllll}28 & 0.1033 & 0.0409 & 0.0450 & 0.0235 & 0.0307 & 0.0501 & 0.0593 & 0.0654 & 0.0511 & 0.0399 & 0.0573 & 0.0910 & 0.1237 & 0.0470 & 0.0838 & 0.0879\end{array}$ $\begin{array}{llllllllllllllllll}29 & 0.1033 & 0.0409 & 0.0450 & 0.0235 & 0.0307 & 0.0501 & 0.0593 & 0.0654 & 0.0511 & 0.0399 & 0.0573 & 0.0910 & 0.1237 & 0.0470 & 0.0838 & 0.0879\end{array}$ $\begin{array}{lllllllllllllllll}30 & 0.1033 & 0.0409 & 0.0450 & 0.0235 & 0.0307 & 0.0501 & 0.0593 & 0.0654 & 0.0511 & 0.0399 & 0.0573 & 0.0910 & 0.1237 & 0.0470 & 0.0838 & 0.0879\end{array}$ $\begin{array}{llllllllllllllllll}31 & 0.1033 & 0.0409 & 0.0450 & 0.0235 & 0.0307 & 0.0501 & 0.0593 & 0.0654 & 0.0511 & 0.0399 & 0.0573 & 0.0910 & 0.1237 & 0.0470 & 0.0838 & 0.0879\end{array}$

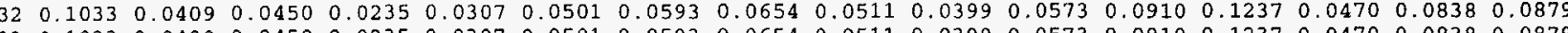

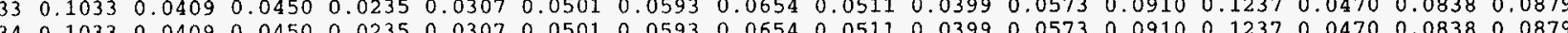

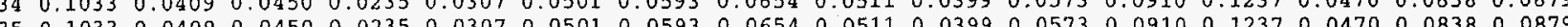

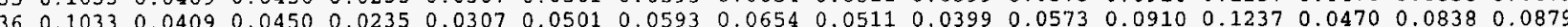
$\begin{array}{lllllllllllllllllll}37 & 0.0563 & 0.0947 & 0.0750 & 0.0559 & 0.0537 & 0.0573 & 0.0572 & 0.0735 & 0.0756 & 0.0572 & 0.0553 & 0.0558 & 0.0663 & 0.0537 & 0.0519 & 0.0495\end{array}$ $\begin{array}{llllllllllllllllll}38 & 0.0000 & 0.0000 & 0.0000 & 0.0000 & 0.0000 & 0.0000 & 0.0000 & 0.0000 & 0.0000 & 0.0000 & 0.0000 & 0.0000 & 0.0000 & 0.0000 & 0.0000 & 0.0000\end{array}$ $\begin{array}{lllllllllllllllll}39 & 0.0000 & 0.0000 & 0.0000 & 0.0000 & 0.0000 & 0.0000 & 0.0000 & 0.0000 & 0.0000 & 0.0000 & 0.0000 & 0.0000 & 0.0000 & 0.0000 & 0.0000 & 0.0000\end{array}$ $\begin{array}{lllllllllllllllllllll}40 & 0.0000 & 0.0000 & 0.0000 & 0.0000 & 0.0000 & 0.0000 & 0.0000 & 0.0000 & 0.0000 & 0.0000 & 0.0000 & 0.0000 & 0.0000 & 0.0000 & 0.0000 & 0.0000\end{array}$

$\begin{array}{lllllllllllllllllllllll}41 & 0.0000 & 0.0000 & 0.0000 & 0.0000 & 0.0000 & 0.0000 & 0.0000 & 0.0000 & 0.0000 & 0.0000 & 0.0000 & 0.0000 & 0.0000 & 0.0000 & 0.0000 & 0.0000\end{array}$

rocessing a Site Data File with Header: MACCS SITE DATA FILE FOR SURRY (JLS, $11 / 10 / 88$ )
SECPOP POP DISTRIBUTION FROM 1980 CENSUS DATA ALTERED USING 0-10 MI NRC DATA

THIS PROGRAM CURRENTLY ALLOWS THE GENERATION OF UP TO 394 RESULTS

YOU HAVE REQUESTED 68 RESULTS FROM "EARLY" COMPOSED OF:

$$
\begin{array}{rlll}
28 & \text { RESULTS OF TYPE } & 1 \\
1 & \text { RESULTS OF } & \text { TYPE } & 2 \\
3 & \text { RESULTS OF } & \text { TYPE } & 3 \\
5 & \text { RESULTS OF } & \text { TYPE } & 4 \\
3 & \text { RESULTS OF } & \text { TYPE } & 5 \\
0 & \text { RESULTS OF } & \text { TYPE } & 6 \\
0 & \text { RESULTS OF } & \text { TYPE } & 7 \\
2 & \text { RESULTS OF } & \text { TYPE } & 8 \\
26 & \text { RESULTS OF } & \text { TYPE } & \text { A } \\
0 & \text { RESULTS OF } & \text { TYPE } & \text { B }
\end{array}
$$

YOU HAVE REQUESTED 104 RESULTS FROM "CHRONC" COMPOSED OF:

34 RESULTS OF TYPE 9

34 RESULTS OF TYPE 9

8 RESULTS OF TYPE 1

16 RESULTS OF TYPE 12

20 RESULTS OF TYPE 13 


\begin{tabular}{|c|c|c|c|c|}
\hline${ }^{T R \perp A L}{ }_{1}$ & $\begin{array}{c}\text { DAY } \\
153\end{array}$ & Hovo & & PRBMET \\
\hline 2 & 157 & 10 & 1 & $2.73 \mathrm{E}-02$ \\
\hline 3 & 163 & 14 & 21 & 3. $62 \mathrm{E}-03$ \\
\hline $\begin{array}{l}4 \\
5\end{array}$ & 166 & 7 & 9 & 4. $22 \mathrm{E}-03$ \\
\hline $\begin{array}{l}5 \\
6\end{array}$ & $\begin{array}{l}167 \\
168\end{array}$ & $\begin{array}{r}17 \\
4\end{array}$ & $\begin{array}{r}2 \\
12\end{array}$ & 2.70E-02 \\
\hline & $\begin{array}{l}168 \\
171\end{array}$ & 7 & 6 & $\begin{array}{l}1.71 \mathrm{E}-02 \\
1.88 \mathrm{E}-02\end{array}$ \\
\hline & 171 & 13 & 36 & $2.28 \mathrm{E}-04$ \\
\hline 9 & 171 & 15 & 35 & $4.00 \mathrm{E}-04$ \\
\hline 10 & 171 & 16 & 32 & $5.42 \mathrm{E}-04$ \\
\hline 11 & 179 & 24 & 31 & $2.00 \mathrm{E}-04$ \\
\hline 12 & 180 & 2 & 30 & 1. $43 \mathrm{E}-04$ \\
\hline $\begin{array}{l}13 \\
14\end{array}$ & $\begin{array}{l}180 \\
181\end{array}$ & $\begin{array}{l}23 \\
15 \\
15\end{array}$ & $\begin{array}{l}10 \\
25\end{array}$ & $\begin{array}{l}1.91 \mathrm{E}-02 \\
6 \\
655 \mathrm{E}-0\end{array}$ \\
\hline $\begin{array}{l}14 \\
15\end{array}$ & $\begin{array}{l}181 \\
188\end{array}$ & $\begin{array}{r}15 \\
8\end{array}$ & $\begin{array}{r}25 \\
4\end{array}$ & $\begin{array}{l}6.85 \mathrm{E}-04 \\
1.63 \mathrm{E}-02\end{array}$ \\
\hline 16 & 193 & 3 & 18 & $1.17 \mathrm{E}-03$ \\
\hline 17 & 195 & 22 & 15 & $4.14 \mathrm{E}-03$ \\
\hline 18 & 200 & 14 & 36 & $2.28 \mathrm{E}-04$ \\
\hline $\begin{array}{l}19 \\
20\end{array}$ & 201 & $\begin{array}{l}21 \\
6\end{array}$ & 34 & $1.43 \mathrm{E}-04$ \\
\hline 21 & 203 & 8 & $\begin{array}{l}31 \\
31\end{array}$ & \\
\hline 22 & 203 & 9 & 31 & $\begin{array}{l}2.00 \mathrm{E}-04 \\
2.00 \mathrm{E}-04\end{array}$ \\
\hline 23 & 203 & 10 & 30 & $1.43 \mathrm{E}-04$ \\
\hline 24 & 203 & 11 & 29 & $1.14 \mathrm{E}-04$ \\
\hline 25 & 204 & 9 & 36 & $2.28 E-04$ \\
\hline $\begin{array}{l}26 \\
27\end{array}$ & $\begin{array}{l}204 \\
204\end{array}$ & 11 & $\begin{array}{l}35 \\
34\end{array}$ & 4.00E-04 \\
\hline 28 & $\begin{array}{l}204 \\
204\end{array}$ & 14 & $\begin{array}{l}34 \\
32\end{array}$ & $\begin{array}{l}1.43 \mathrm{E}-04 \\
5,42 \mathrm{E}-4\end{array}$ \\
\hline 29 & 205 & $\begin{array}{l}19 \\
24\end{array}$ & 11 & $2.16 \mathrm{E}-02$ \\
\hline 30 & 215 & 18 & 3 & 2. $45 \mathrm{E}-03$ \\
\hline 31 & 220 & $21^{5}$ & 5 & $1.55 \mathrm{E}-02$ \\
\hline $\begin{array}{l}32 \\
33\end{array}$ & $\begin{array}{l}222 \\
224 \\
\end{array}$ & $\begin{array}{r}21 \\
4\end{array}$ & $\begin{array}{l}16 \\
27\end{array}$ & $\begin{array}{l}3.42 E-04 \\
4\end{array}$ \\
\hline $\begin{array}{l}35 \\
34\end{array}$ & $\begin{array}{l}224 \\
228\end{array}$ & $\begin{array}{l}4 \\
5\end{array}$ & 13 & $2.21 \mathrm{E}-02$ \\
\hline 35 & 229 & 24 & 7 & $4.14 \mathrm{E}-03$ \\
\hline 36 & 231 & 2 & 20 & $3.77 \mathrm{E}-03$ \\
\hline 37 & 232 & 14 & 30 & $1.43 \mathrm{E}-04$ \\
\hline 38 & 232 & 16 & 22 & $2.11 \mathrm{E}-03$ \\
\hline 39 & 232 & 22 & 35 & $4.00 \mathrm{E}-04$ \\
\hline & $\begin{array}{l}232 \\
232\end{array}$ & 年 & ${ }^{34}$ & $1.43 \mathrm{E}-04$ \\
\hline 41 & $\begin{array}{l}235 \\
240\end{array}$ & $\begin{array}{r}19 \\
3\end{array}$ & 14 & 2.17E-02 \\
\hline 作 & 244 & 21 & 19 & $2.94 \mathrm{E}-03$ \\
\hline 44 & 248 & 20 & 9 & 4.22E-03 \\
\hline & 250 & 22 & 26 & $7.71 \mathrm{E}-04$ \\
\hline 46 & 251 & 2 & 25 & $6.85 \mathrm{E}-04$ \\
\hline 47 & 253 & 2 & 13 & 2.21E-02 \\
\hline 48 & 263 & 16 & 17 & $1.00 \mathrm{E}-02$ \\
\hline 49 & 268 & 8 & 4 & $1.63 \mathrm{E}-02$ \\
\hline 50 & 270 & 3 & 14 & $2.17 \mathrm{E}-02$ \\
\hline TRIAL & DAY & HOUR & BIN & PRBMET \\
\hline 51 & 271 & 20 & 10 & 1.91E-02 \\
\hline 52 & 272 & 10 & 1 & $2.73 \mathrm{E}-02$ \\
\hline 53 & $\begin{array}{l}282 \\
286\end{array}$ & 6 & $\begin{array}{l}18 \\
24\end{array}$ & $4.57 \mathrm{E}-04$ \\
\hline 55 & $\begin{array}{l}286 \\
288\end{array}$ & $\begin{array}{l}22 \\
10\end{array}$ & $\begin{array}{r}24 \\
2\end{array}$ & $2.70 \mathrm{E}-02$ \\
\hline 56 & 299 & 10 & 32 & $5.42 E-04$ \\
\hline
\end{tabular}




\begin{tabular}{|c|c|c|c|c|}
\hline & & & & \\
\hline 57 & 299 & 11 & 27 & $4.85 E-04$ \\
\hline $\begin{array}{l}58 \\
59\end{array}$ & $\begin{array}{l}\begin{array}{l}301 \\
301\end{array} \\
3\end{array}$ & $\begin{array}{r}2 \\
15\end{array}$ & $\begin{array}{r}11 \\
5\end{array}$ & $\begin{array}{l}2.16 \mathrm{E}-02 \\
1.55 \mathrm{E}-02\end{array}$ \\
\hline 60 & 307 & 5 & 6 & $1.88 \mathrm{E}-02$ \\
\hline 61 & 309 & 11 & 21 & $3.62 \mathrm{E}-03$ \\
\hline 62 & $\begin{array}{l}309 \\
309\end{array}$ & 14 & 19 & 2.94E-03 \\
\hline $\begin{array}{l}63 \\
64\end{array}$ & $\begin{array}{l}309 \\
371\end{array}$ & $\begin{array}{l}20 \\
18\end{array}$ & $\begin{array}{l}9 \\
3\end{array}$ & $\begin{array}{l}4.22 \mathrm{E}-03 \\
2.45 \mathrm{E}-0\end{array}$ \\
\hline $\begin{array}{l}64 \\
65\end{array}$ & $\begin{array}{l}311 \\
315\end{array}$ & 21 & 20 & $\begin{array}{l}2.45 \mathrm{E}-03 \\
3.77 \mathrm{E}-03\end{array}$ \\
\hline 66 & 319 & 5 & 10 & $\begin{array}{l}3.77 \mathrm{E}-03 \\
1.91 \mathrm{E}-02\end{array}$ \\
\hline 67 & 321 & 9 & 4 & $\begin{array}{l}.91 \mathrm{E}-02 \\
1.63 \mathrm{E}-02\end{array}$ \\
\hline & 330 & 9 & 19 & $2.94 \mathrm{E}-03$ \\
\hline $\begin{array}{l}69 \\
70\end{array}$ & $\begin{array}{l}330 \\
320\end{array}$ & 10 & 18 & $1.17 E-03$ \\
\hline 71 & $\begin{array}{l}\begin{array}{l}330 \\
335\end{array} \\
335\end{array}$ & $\begin{array}{l}18 \\
23\end{array}$ & $\begin{array}{r}36 \\
8\end{array}$ & $2.28 \mathrm{E}-04$ \\
\hline $\begin{array}{l}1 \\
7\end{array}$ & $\begin{array}{l}335 \\
335\end{array}$ & 24 & $\begin{array}{l}8 \\
8\end{array}$ & $\begin{array}{l}3.14 \mathrm{E}-04 \\
3\end{array}$ \\
\hline $\begin{array}{l}73 \\
74\end{array}$ & 336 & 7 & 22 & $2.11 \mathrm{E}-03$ \\
\hline $\begin{array}{l}74 \\
75\end{array}$ & 336 & 21 & 23 & 1. $14 \mathrm{E}-04$ \\
\hline $\begin{array}{r}75 \\
76 \\
7\end{array}$ & $\begin{array}{l}342 \\
346\end{array}$ & $\begin{array}{l}10 \\
13\end{array}$ & $\begin{array}{l}3 \\
1\end{array}$ & $\begin{array}{l}2.45 \mathrm{E}-03 \\
2.73 \mathrm{E}-02\end{array}$ \\
\hline 77 & 347 & 22 & 11 & $2.16 \mathrm{E}-02$ \\
\hline $\begin{array}{l}78 \\
79\end{array}$ & 348 & 9 & 9 & $\begin{array}{l}4.22 \mathrm{E}-03 \\
\end{array}$ \\
\hline $\begin{array}{l}79 \\
80\end{array}$ & $\begin{array}{l}357 \\
358\end{array}$ & 12 & $\begin{array}{l}11 \\
22\end{array}$ & $\begin{array}{l}1.00 \mathrm{E}-02 \\
2.11 \mathrm{E}-02\end{array}$ \\
\hline 81 & 358 & 14 & 27 & $\begin{array}{l}2.11 \mathrm{E}-03 \\
4.85 \mathrm{E}-04\end{array}$ \\
\hline 82 & 358 & 17 & 32 & $5.42 \mathrm{E}-04$ \\
\hline $\begin{array}{l}83 \\
84\end{array}$ & $\begin{array}{r}365 \\
8\end{array}$ & $\begin{array}{ll}16 \\
16\end{array}$ & 3 & $2.45 \mathrm{E}-03$ \\
\hline 84 & $\begin{array}{l}8 \\
9\end{array}$ & $\begin{array}{r}16 \\
6\end{array}$ & $\begin{array}{r}5 \\
15\end{array}$ & 1. $55 \mathrm{E}-02$ \\
\hline $\begin{array}{l}86 \\
87\end{array}$ & 12 & 23 & 12 & $1.71 \mathrm{E}-02$ \\
\hline $\begin{array}{l}87 \\
88\end{array}$ & 12 & 24 & 16 & $3.42 \mathrm{E}-04$ \\
\hline $\begin{array}{l}88 \\
89\end{array}$ & $\begin{array}{l}13 \\
15\end{array}$ & $\begin{array}{c}3 \\
10\end{array}$ & 14 & $\begin{array}{l}2.17 E-02 \\
2.70 E-02\end{array}$ \\
\hline 90 & $\begin{array}{l}15 \\
23\end{array}$ & 9 & $\begin{array}{l}2 \\
7\end{array}$ & $\begin{array}{l}2.70 \mathrm{E}-02 \\
4.14 \mathrm{E}-03\end{array}$ \\
\hline 91 & 25 & 6 & 20 & $\begin{array}{l}4.14 \mathrm{E}-03 \\
3.77 \mathrm{E}-03\end{array}$ \\
\hline 92 & 25 & 10 & 18 & $1.17 \mathrm{E}-03$ \\
\hline 93 & 29 & 14 & 17 & $1.00 \mathrm{E}-02$ \\
\hline 94 & 32 & 5 & 13 & $2.21 E-02$ \\
\hline 95 & 37 & 18 & 8 & $3.14 \mathrm{E}-04$ \\
\hline 96 & 47 & 11 & 4 & $\begin{array}{l}1.63 \mathrm{E}-02 \\
3\end{array}$ \\
\hline 98 & 49 & $\begin{array}{l}2 \\
10\end{array}$ & $\begin{array}{l}16 \\
26\end{array}$ & $\begin{array}{l}3.42 \mathrm{E}-04 \\
7.71 \mathrm{E}-04\end{array}$ \\
\hline $\begin{array}{l}30 \\
99\end{array}$ & 50 & 18 & $\begin{array}{l}20 \\
24\end{array}$ & $4.57 \mathrm{E}-04$ \\
\hline 100 & 51 & 19 & 5 & $1.55 \mathrm{E}-02$ \\
\hline TRIAL & DAY & HOUR & BIN & PRBMET \\
\hline 101 & 53 & 1 & 5 & $1.88 \mathrm{E}-02$ \\
\hline 102 & 53 & 13 & 21 & $3.62 \mathrm{E}-03$ \\
\hline 103 & 57 & 22 & 17 & $1.00 \mathrm{E}-02$ \\
\hline 104 & 59 & 20 & 13 & 2.21E-02 \\
\hline 105 & $\begin{array}{l}65 \\
69 \\
69\end{array}$ & ${ }_{2}$ & 12 & $\begin{array}{l}4.14 \mathrm{E}-03 \\
1.71 \mathrm{~F}\end{array}$ \\
\hline 106 & 69 & 24 & 25 & $\begin{array}{l}1.71 E-02 \\
6.85 E-2\end{array}$ \\
\hline 108 & 12 & & 25 & $6.85 E-04$ \\
\hline 108 & 72 & 4 & 23 & $\begin{array}{l}0.830-04 \\
1\end{array}$ \\
\hline 110 & 72 & 14 & 20 & $377 \mathrm{E}-0$ \\
\hline 111 & 74 & 20 & 23 & 1. $14 \mathrm{E}-04$ \\
\hline 112 & 78 & & 8 & $3.14 \mathrm{E}-04$ \\
\hline 113 & 83 & 17 & 6 & $1.88 \mathrm{E}-02$ \\
\hline 114 & 84 & 19 & 10 & $1.91 \mathrm{E}-02$ \\
\hline 115 & 85 & 2 & 14 & $2.17 \mathrm{E}-02$ \\
\hline
\end{tabular}




$\begin{array}{rrrrr}116 & 87 & 21 & 15 & 4.14 \mathrm{E}-03 \\ 117 & 101 & 4 & 16 & 3.42 \mathrm{E}-04 \\ 118 & 107 & 14 & 19 & 2.94 \mathrm{E}-03 \\ 119 & 107 & 20 & 21 & 3.62 \mathrm{E}-03 \\ 120 & 112 & 22 & 7 & 4.14 \mathrm{E}-03 \\ 121 & 115 & 13 & 2 & 2.70 \mathrm{E}-02 \\ 122 & 122 & 19 & 11 & 2.16 \mathrm{E}-02 \\ 123 & 124 & 23 & 15 & 4.14 \mathrm{E}-03 \\ 124 & 125 & 6 & 12 & 1.71 \mathrm{E}-02 \\ 125 & 127 & 18 & 26 & 7.71 \mathrm{E}-04 \\ 126 & 133 & 24 & 24 & 4.57 \mathrm{E}-04 \\ 127 & 134 & 1 & 22 & 2.11 \mathrm{E}-03 \\ 128 & 139 & 22 & 35 & 4.00 \mathrm{E}-04 \\ 129 & 139 & 23 & 34 & 1.43 \mathrm{E}-04 \\ 130 & 139 & 24 & 33 & 1.14 \mathrm{E}-04 \\ 131 & 140 & 2 & 30 & 1.43 \mathrm{E}-04 \\ 132 & 140 & 3 & 29 & 1.14 \mathrm{E}-04 \\ 133 & 140 & 7 & 27 & 4.85 \mathrm{E}-04 \\ 134 & 140 & 19 & 29 & 1.14 \mathrm{E}-04 \\ 135 & 151 & 9 & 1 & 2.73 \mathrm{E}-02\end{array}$

DATE AND TIME OF RUN = MACCS2 09/26/96 08:40:08 Version 1.12 , Last Modified $9 / 25 / 96$ by D. Chanin

"ATMOS" DESCRIPTION = IN1A. INP, Sample Problem A--Using Table-Lookup Sigmas, ATMOS input

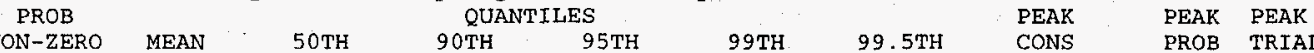

Source Term 1: Plume 1, at $4.8-5.6 \mathrm{~km}$ $\mathrm{Cs}-137$ Center Air Conc. Cs-137 Ground Air Conc. (Bq-s/m3) $\mathrm{Cs}-137$ Center Ground Cone. ( $\mathrm{Bq} / \mathrm{m} 2)$ Total Center Ground Conc. $(\mathrm{Bg} / \mathrm{m} 2)$ Ground-Level Dilution, $\mathrm{X} / \mathrm{Q}(\mathrm{s} / \mathrm{m} 3)$ Cs-137 Adjusted Source, $Q$ (B)

Plume Sigma-y (m)

Plume Height $(m)$

Plume Arrival Time (s) $\begin{array}{llllllllll}1.0000 & 4.07 \mathrm{E}+11 & 2.40 \mathrm{E}+11 & 1.07 \mathrm{E}+12 & 1.49 \mathrm{E}+12 & 2.09 \mathrm{E}+12 & 2.16 \mathrm{E}+12 & 2.35 \mathrm{E}+12 & 7.71 \mathrm{E}-04 & 45 \\ 1.0000 & 3.63 \mathrm{E}+11 & 2.16 \mathrm{E}+11 & 1.02 \mathrm{E}+12 & 1.10 \mathrm{E}+12 & 1.30 \mathrm{E}+12 & 1.41 \mathrm{E}+12 & 1.72 \mathrm{E}+12 & 7.71 \mathrm{E}-04 & 45 \\ 0.9872 & 3.89 \mathrm{E}+09 & 2.31 \mathrm{E}+09 & 1.03 \mathrm{E}+12 & 1.12 \mathrm{E}+10 & 1.35 \mathrm{E}+10 & 1.47 \mathrm{E}+10 & 3.04 \mathrm{E}+10 & 1.14 \mathrm{E}-04 & 130\end{array}$ $\begin{array}{llllllllll}0.9872 & 3.89 \mathrm{E}+09 & 2.31 \mathrm{E}+09 & 1.03 \mathrm{E}+10 & 1.12 \mathrm{E}+10 & 1.35 \mathrm{E}+10 & 1.47 \mathrm{E}+10 & 3.04 \mathrm{E}+10 & 1.14 \mathrm{E}-04 & 130 \\ 0.9872 & 3.69 \mathrm{E}+11 & 2.37 \mathrm{E}+11 & 9.30 \mathrm{E}+11 & 1.07 \mathrm{E}+12 & 1.31 \mathrm{E}+12 & 1.43 \mathrm{E}+12 & 2.90 \mathrm{E}+12 & 1.14 \mathrm{E}-04 & 130\end{array}$

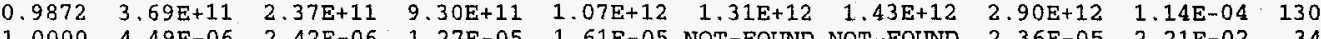
$\begin{array}{lllllllll}1.000 & 4.49 \mathrm{E}-06 & 2.42 \mathrm{E}-06 & 1.27 \mathrm{E}-05 & 1.61 \mathrm{E}-05 & \text { NOT-FOUND NOT-FOUND } & 2.36 \mathrm{E}-05 & 2.21 \mathrm{E}-02 & 34\end{array}$ $1.00004 .21 \mathrm{~B}+163.81 \mathrm{~B}+16$ 1.03 $1.00002 .66 \mathrm{E}+02 \quad 1.09 \mathrm{E}+02 \quad 7.33 \mathrm{E}+02 \quad 8.06 \mathrm{E}+02$ NOT-FOUND NOT-FOUND $2.40 \mathrm{E}+03 \quad 1.06 \mathrm{E}-02 \quad 28$ $\begin{array}{lllllllllll}0.4148 & 1.24 \mathrm{E}+02 & 0.00 \mathrm{E}+00 & 7.06 \mathrm{E}+02 & 8.84 \mathrm{E}+02 & 1.02 \mathrm{E}+03 & 1.04 \mathrm{E}+03 & 1.11 \mathrm{E}+03 & 2.00 \mathrm{E}-04 & 21\end{array}$ $\begin{array}{llllllllll}1.0000 & 6.86 \mathrm{E}+03 & 6.06 \mathrm{E}+03 & 9.90 \mathrm{E}+03 & 1.08 \mathrm{E}+04 & 1.30 \mathrm{E}+04 & 1.41 \mathrm{E}+04 & 1.42 \mathrm{E}+04 & 4.94 \mathrm{E}-03 & 36\end{array}$ Source Term 1: Plume 1 , at $5.6-8.1 \mathrm{~km}$
$\mathrm{Cs}-137 \quad$ Center Air Conc. (Bq-s/m3) $\mathrm{Cs}-137$ Ground Air Conc. (Bq-s/m3) $\mathrm{Cs}-137$ Center Ground Conc. (Bq/m2) Total Center Ground Conc. (Bq/m2) Ground-Level Dilution, $x / Q$ ( $\mathrm{s} / \mathrm{m} 3$ ) Cs-137 Adjusted Source, $Q(B q)$ Plume Sigma-y (m)

Plume sigma-z (m)

plume Arrival Time (s)

$\begin{array}{rrlllrrrrr}1.0000 & 2.51 \mathrm{E}+11 & 1.37 \mathrm{E}+11 & 6.76 \mathrm{E}+11 & 9.86 \mathrm{E}+11 & \text { NOT-FOUND } & \text { NOT-FOUND } & 1.26 \mathrm{E}+12 & 2.21 \mathrm{E}-02 & 47 \\ 1.0000 & 2.46 \mathrm{E}+11 & 1.25 \mathrm{E}+11 & 7.73 \mathrm{E}+11 & 9.66 \mathrm{E}+11 & \text { NOT-FOUND } & \text { NOT-FOUND } & 1.12 \mathrm{E}+12 & 2.21 \mathrm{E}-02 & 47 \\ 0.9975 & 2.61 \mathrm{E}+09 & 1.40 \mathrm{E}+09 & 7.76 \mathrm{E}+09 & 9.72 \mathrm{E}+09 & 1.14 \mathrm{E}+10 & 1.21 \mathrm{E}+10 & 1.63 \mathrm{E}+10 & 1.43 \mathrm{E}-04 & 129 \\ 0.9975 & 2.39 \mathrm{E}+11 & 1.40 \mathrm{E}+11 & 6.81 \mathrm{E}+11 & 7.33 \mathrm{E}+11 & 8.27 \mathrm{E}+11 & 8.70 \mathrm{E}+11 & 1.49 \mathrm{E}+12 & 1.43 \mathrm{E}-04 & 129 \\ 1.0000 & 3.37 \mathrm{E}-06 & 1.46 \mathrm{E}-06 & 9.63 \mathrm{E}-06 & 1.26 \mathrm{E}-05 & \text { NOT-FOUND } & \text { NOT-FOUND } & 1.73 \mathrm{E}-05 & 2.21 \mathrm{E}-02 & 34 \\ 1.0000 & 8.83 \mathrm{E}+16 & 8.60 \mathrm{E}+16 & 1.02 \mathrm{E}+17 & 1.04 \mathrm{E}+17 & 1.07 \mathrm{E}+17 & 1.09 \mathrm{E}+17 & 1.11 \mathrm{E}+17 & 2.45 \mathrm{E}-03 & 75 \\ 1.0000 & 6.34 \mathrm{E}+02 & 5.16 \mathrm{E}+02 & \text { NOT-FOUND } & \text { NOT-FOUND } & \text { NOT-FOUND NOT-FOUND } & 1.33 \mathrm{E}+03 & 1.77 \mathrm{E}-01 & 2 \\ 1.0000 & 2.96 \mathrm{E}+02 & 1.51 \mathrm{E}+02 & 7.60 \mathrm{E}+02 & 8.57 \mathrm{E}+02 & 1.31 \mathrm{E}+03 & 1.71 \mathrm{E}+03 & 1.82 \mathrm{E}+03 & 4.22 \mathrm{E}-03 & 4 \\ 0.4148 & 1.27 \mathrm{E}+02 & 0.00 \mathrm{E}+00 & 7.33 \mathrm{E}+02 & 8.96 \mathrm{E}+02 & 1.02 \mathrm{E}+03 & 1.04 \mathrm{E}+03 & 1.11 \mathrm{E}+03 & 2.00 \mathrm{E}-04 & 21 \\ 1.0000 & 7.78 \mathrm{E}+03 & 6.78 \mathrm{E}+03 & 1.10 \mathrm{E}+04 & 1.20 \mathrm{E}+04 & 1.49 \mathrm{E}+04 & 1.63 \mathrm{E}+04 & 1.69 \mathrm{E}+04 & 3.77 \mathrm{E}-03 & 36\end{array}$

DATE AND TIME OF RUN = MACCS2 09/26/96 08:40:08 Version 1.12, Last Modified 9/25/96 by D. Chanin "ATMOS" DESCRIPTION = IN1A.INP, Sample Problem A--Using Table-Lookup Sigmas, ATMOS input

"CHRONC" DESCRIPTION = IN3A_N.INP, Sample Problem A, "New" COMIDA2-Based Food Model

SOURCE TERM 1 OF 2 :

SECOND DRAFT 1150, WORST CASE SOURCE TERM FOR EARLY FATALITIES

OVERALL RESULTS OBTAINED BY COMBINING 2 EMERGENCY RESPONSE COHORTS FROM "EARLY" WITH THE WEIGH'TING FRACTIONS BELOW APPLIED TO THEM: 
COHORT 1 = EVACUATION WITHIN 10 MILES, RELOCATION MODELS APPLY ELSEWHERE

AND THEN MERGING THE 2 RESULTS ABOVE WITH THE SINGLE SET OF RESULTS FROM "CHRONC" DESCRIBED BELOW:

COHORT 3 = IN3A_N. INP, Sample Problem A, "New" COMIDA2-Based Food Model

RESULTS WHICH ARE PRODUCED ONLY BY "EARLY" OR ONLY BY "CHRONC" ARE PRESENTED IN LATER SECTIONS.

09/26/96 08:40:08 PAGE 1

HEALTH EFFECTS CASES ERL FAT/TOTAL
ERL INJ/PRODROMAL VOMIT

ERL INJ/DIARRHEA
ERL INJ/PNEUMONITIS

ERL INJ/PNEUMONITIS

ERL INJ/THYROIDITIS
ERL INJ/HYPOTTHYROIDISM

ERL INJ/SKIN ERYTHEMA
ERL INU/TRANSEPIDERMAL

CAN FAT/TOTAL

CAN FAT/LUNG

CAN FAT/BREAS

CAN FAT/GI

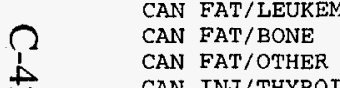

CAN INJ/THYROID

CAN INJ/BREAST

CAN FAT/TOTAL
ERL FAT/TOTAL

ERL INJ/PRODROMAL VOMIT

ERL INJ/DIARRHEA

ERL INJ/PNEUMONITIS

ERL INJ/THYROIDITIS
ERL INJ/HYPOTHYROIDISM

ERL INJ/HYPOTHYROIDISM

ERL INJ/SKIN ERYTHEMA
ERL INJ/TRANSEPIDERMAL

ERL INJ/TRANSE

PROB
NON-ZERO MEAN $50 T H$

$\begin{array}{cc}\text { QUANTILES } \\ 90 \mathrm{TH} & 95 \mathrm{TH}\end{array}$

$99 \mathrm{TH}$

$99.5 \mathrm{TH}$

PEAK

PEAK PEAK

$\begin{array}{lllllllllll}0-1609 \mathrm{~km} & 0.6836 & 1.34 \mathrm{E}+01 & 2.38 \mathrm{E}-01 & 2.77 \mathrm{E}+01 & 4.76 \mathrm{E}+01 & 1.57 \mathrm{E}+02 & 3.35 \mathrm{E}+02 & 3.18 \mathrm{E}+03 & 2.85 \mathrm{E}-04 & 85\end{array}$ $\begin{array}{lllllllllll}0-1609 \mathrm{~km} & 0.8218 & 8.84 \mathrm{E}+01 & 5.58 \mathrm{E}+00 & 1.57 \mathrm{E}+02 & 3.12 \mathrm{E}+02 & 1.58 \mathrm{E}+03 & 2.51 \mathrm{E}+03 & 8.03 \mathrm{E}+03 & 2.85 \mathrm{E}-04 & 85\end{array}$ $\begin{array}{lllllllllll}0-1609 \mathrm{~km} & 0.7422 & 4.00 \mathrm{E}+01 & 1.34 \mathrm{E}+00 & 7.58 \mathrm{E}+01 & 1.41 \mathrm{E}+02 & 6.54 \mathrm{E}+02 & 1.23 \mathrm{E}+03 & 5.19 \mathrm{E}+03 & 2.85 \mathrm{E}-04 & 85\end{array}$ $\begin{array}{lllllllllll}0-1609 \mathrm{~km} & 0.5941 & 5.65 \mathrm{E}+00 & 6.33 \mathrm{E}-03 & 1.14 \mathrm{E}+01 & 2.63 \mathrm{E}+01 & 9.41 \mathrm{E}+01 & 1.26 \mathrm{E}+02 & 8.76 \mathrm{E}+02 & 2.85 \mathrm{E}-04 & 85\end{array}$ $\begin{array}{llll} & \end{array}$ $\begin{array}{llllllll} & \end{array}$ $\begin{array}{lllllllllll}0-1609 \mathrm{~km} & 0.9967 & 5.02 \mathrm{E}+03 & 1.04 \mathrm{E}+03 & 1.11 \mathrm{E}+04 & 1.99 \mathrm{E}+04 & 7.07 \mathrm{E}+04 & 1.05 \mathrm{E}+05 & 1.80 \mathrm{E}+05 & 7.13 \mathrm{E}-04 & 11\end{array}$ $\begin{array}{lllllllllll}0-1609 \mathrm{~km} & 0.9321 & 1.38 \mathrm{E}+03 & 1.52 \mathrm{E}+02 & 3.67 \mathrm{E}+03 & 6.81 \mathrm{E}+03 & 1.88 \mathrm{E}+04 & 2.40 \mathrm{E}+04 & 4.22 \mathrm{E}+04 & 7.71 \mathrm{E}-04 & 29\end{array}$ $\begin{array}{lllllllllll}0-1609 \mathrm{~km} & 1.0000 & 1.13 \mathrm{E}+04 & 6.85 \mathrm{E}+03 & 2.59 \mathrm{E}+04 & 3.56 \mathrm{E}+04 & 6.07 \mathrm{E}+04 & 7.15 \mathrm{E}+04 & 1.01 \mathrm{E}+05 & 1.11 \mathrm{E}-03 & 135\end{array}$

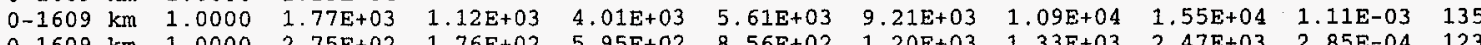
$\begin{array}{lllllllllll}0-1609 \mathrm{~km} & 1.0000 & 2.75 \mathrm{E}+02 & 1.76 \mathrm{E}+02 & 5.95 \mathrm{E}+02 & 8.56 \mathrm{E}+02 & 1.20 \mathrm{E}+03 & 1.33 \mathrm{E}+03 & 2.47 \mathrm{E}+03 & 2.85 \mathrm{E}-04 & 123\end{array}$ $\begin{array}{lllllllllll}0-1609 \mathrm{~km} & 1.0000 & 1.22 \mathrm{E}+03 & 5.63 \mathrm{E}+02 & 2.86 \mathrm{E}+03 & 4.23 \mathrm{E}+03 & 8.10 \mathrm{E}+03 & 9.51 \mathrm{E}+03 & 1.23 \mathrm{E}+04 & 1.11 \mathrm{E}-03 & 135 \\ 0-1509 \mathrm{~km} & 1.0000 & 3.25 \mathrm{E}+03 & 1.89 \mathrm{E}+03 & 7.64 \mathrm{E}+03 & 1.04 \mathrm{E}+04 & 1.68 \mathrm{E}+04 & 2.05 \mathrm{E}+04 & 2.93 \mathrm{E}+04 & 1.11 \mathrm{E}-03 & 135\end{array}$

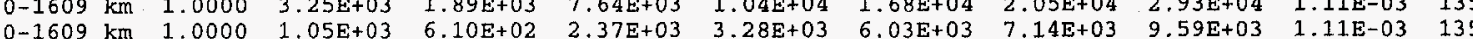

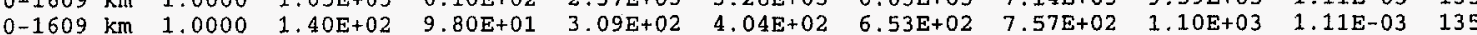
$\begin{array}{lllllllllll}0-1609 \mathrm{~km} & 1.0000 & 3.57 \mathrm{E}+03 & 2.24 \mathrm{E}+03 & 7.98 \mathrm{E}+03 & 1.10 \mathrm{E}+04 & 2.10 \mathrm{E}+04 & 2.35 \mathrm{E}+04 & 3.15 \mathrm{E}+04 & 1.11 \mathrm{E}-03 & 135\end{array}$ $\begin{array}{lllllllllll}0-1609 \mathrm{~km} & 1.0000 & 3.57 \mathrm{E}+03 & 2.24 \mathrm{E}+03 & 7.95 \mathrm{E}+03 & 1.10 \mathrm{E}+04 & 2.10 \mathrm{E}+04 & 2.35 \mathrm{E}+04 & 3.15 \mathrm{E}+04 & 1.11 \mathrm{E}-03 & 135 \\ 0-1609 \mathrm{~km} & 1.0000 & 2.75 \mathrm{E}+03 & 1.76 \mathrm{E}+03 & 5.95 \mathrm{E}+03 & 8.56 \mathrm{E}+03 & 1.20 \mathrm{E}+04 & 1.33 \mathrm{E}+04 & 2.47 \mathrm{E}+04 & 2.85 \mathrm{E}-04 & 123\end{array}$ $\begin{array}{llllllllllll}0-1609 \mathrm{~km} & 1.0000 & 3.85 \mathrm{E}+03 & 2.19 \mathrm{E}+03 & 9.10 \mathrm{E}+03 & 1.30 \mathrm{E}+04 & 2.50 \mathrm{E}+04 & 3.04 \mathrm{E}+04 & 3.89 \mathrm{E}+04 & 1.11 \mathrm{E}-03 & 135\end{array}$ $\begin{array}{lllllllllll}0-80.5 \mathrm{~km} & 1.0000 & 1.72 \mathrm{E}+03 & 9.55 \mathrm{E}+02 & 4.07 \mathrm{E}+03 & 5.93 \mathrm{E}+03 & 9.56 \mathrm{E}+03 & 1.11 \mathrm{E}+04 & 2.07 \mathrm{E}+04 & 2.85 \mathrm{E}-04 & 123\end{array}$

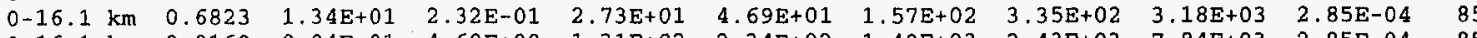
$\begin{array}{lllllllllll}0-16.1 \mathrm{~km} & 0.8169 & 8.04 \mathrm{E}+01 & 4.60 \mathrm{E}+00 & 1.31 \mathrm{E}+02 & 2.34 \mathrm{E}+02 & 1.49 \mathrm{E}+03 & 2.43 \mathrm{E}+03 & 7.84 \mathrm{E}+03 & 2.85 \mathrm{E}-04 & 85\end{array}$

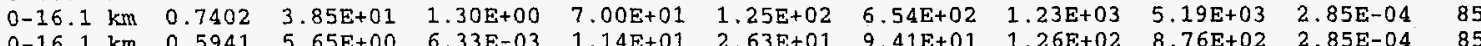
$\begin{array}{lllllllllll}0-16.1 \mathrm{~km} & 0.5941 & 5.65 \mathrm{E}+00 & 6.33 \mathrm{E}-03 & 1.14 \mathrm{E}+01 & 2.63 \mathrm{E}+01 & 9.41 \mathrm{E}+01 & 1.26 \mathrm{E}+02 & 8.76 \mathrm{E}+02 & 2.85 \mathrm{E}-04 & 85\end{array}$ $\begin{array}{lllllllllll}0-16.1 \mathrm{~km} & 0.5735 & 9.94 \mathrm{E}+00 & 2.88 \mathrm{E}-02 & 1.87 \mathrm{E}+01 & 4.50 \mathrm{E}+01 & 1.85 \mathrm{E}+02 & 2.62 \mathrm{E}+02 & 1.23 \mathrm{E}+03 & 2.85 \mathrm{E}-04 & 85\end{array}$ $\begin{array}{lllllllllll}0-16.1 \mathrm{~km} & 0.9515 & 1.65 \mathrm{E}+02 & 2.35 \mathrm{E}+01 & 2.77 \mathrm{E}+02 & 8.13 \mathrm{E}+02 & 2.88 \mathrm{E}+03 & 3.68 \mathrm{E}+03 & 9.09 \mathrm{E}+03 & 5.71 \mathrm{E}-04 & 123 \\ 0-16.1 \mathrm{~km} & 0.9941 & 1.69 \mathrm{E}+03 & 3.26 \mathrm{E}+02 & 4.78 \mathrm{E}+03 & 9.41 \mathrm{E}+03 & 2.03 \mathrm{E}+04 & 2.09 \mathrm{E}+04 & 2.60 \mathrm{E}+04 & 2.29 \mathrm{E}-05 & 9\end{array}$

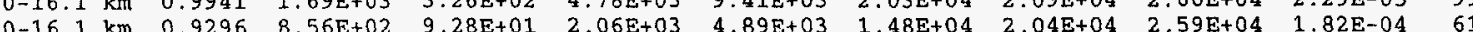

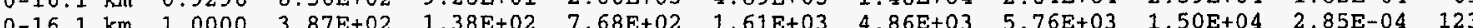

EARLY FATALITY DISTANCE $(\mathrm{km})$
ERL FAT/TOTAL RISK $>0.000$

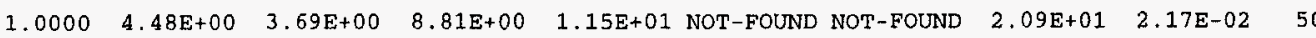

$09 / 26 / 96 \quad 08: 40: 08$ PAGE 2

POPULATION EXCEEDING DOSE EARLY dOSE A-RED MARR > $1.50 \mathrm{SV}$ EARLY dOSE A-LUNGS $>5.00 \mathrm{SV}$

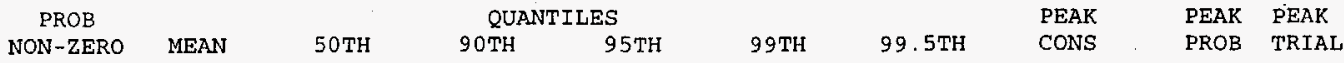
AVERAGE INDIVIDUAL RISK ERL, FAT/TOTAL ERL FATIOTAL

ERL FAT/TOTAL

$\begin{array}{llllllllllll}0-0.2 \mathrm{~km} & 1.0000 & 1.76 \mathrm{E}-01 & 1.41 \mathrm{E}-01 & 2.10 \mathrm{E}-01 & 2.19 \mathrm{E}-01 & 2.39 \mathrm{E}-01 & 2.48 \mathrm{E}-01 & 2.94 \mathrm{E}-01 & 2.28 \mathrm{E}-04 & 18\end{array}$

ERL FAT/TOTAL

$\begin{array}{llllllllllll}0.2-0.5 \mathrm{~km} & 1.0000 & 6.71 \mathrm{E}-02 & 6.59 \mathrm{E}-02 & 1.02 \mathrm{E}-01 & 1.08 \mathrm{E}-01 & 1.25 \mathrm{E}-01 & 1.33 \mathrm{E}-01 & 1.61 \mathrm{E}-01 & 5.42 \mathrm{E}-04 & 82 \\ 0.5-1.2 \mathrm{~km} & 0.8572 & 3.76 \mathrm{E}-02 & 4.35 \mathrm{E}-02 & 5.44 \mathrm{E}-02 & 5.65 \mathrm{E}-02 & 6.18 \mathrm{E}-02 & 6.43 \mathrm{E}-02 & 9.40 \mathrm{E}-02 & 5.42 \mathrm{E}-04 & 28\end{array}$ $\begin{array}{lllllllllll}1.2-1.6 \mathrm{~km} & 0.8032 & 2.71 \mathrm{E}-02 & 3.11 \mathrm{E}-02 & 5.16 \mathrm{E}-02 & 5.40 \mathrm{E}-02 & 6.00 \mathrm{E}-02 & 6.27 \mathrm{E}-02 & 6.39 \mathrm{E}-02 & 3.77 \mathrm{E}-03 & 36\end{array}$

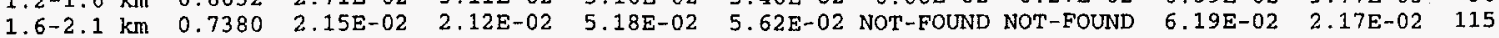


POPULATION DOSE (SV) I-EDEWBODY TOT LI $\begin{array}{lll}\text { L-EDEWBODY } & \text { TOT LIF } \\ \text { L-EDEWBODY } & \text { TOT LIF }\end{array}$

$\begin{array}{lllllllllll}0-16.1 \mathrm{~km} & 1.0000 & 7.13 \mathrm{E}+03 & 3.01 \mathrm{E}+03 & 1.40 \mathrm{E}+04 & 2.70 \mathrm{E}+04 & 8.68 \mathrm{E}+04 & 1.13 \mathrm{E}+05 & 2.56 \mathrm{E}+05 & 2.85 \mathrm{E}-04 & 123 \\ 0-80.5 \mathrm{~km} & 1.0000 & 3.54 \mathrm{E}+04 & 1.99 \mathrm{E}+04 & 8.82 \mathrm{E}+04 & 1.18 \mathrm{E}+05 & 1.96 \mathrm{E}+05 & 2.19 \mathrm{E}+05 & 3.70 \mathrm{E}+05 & 2.05 \mathrm{E}-04 & 123\end{array}$ POPULATION WEIGHTED RISK

ERL FAT/TOTAL

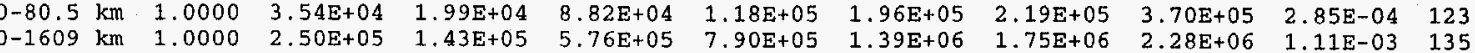

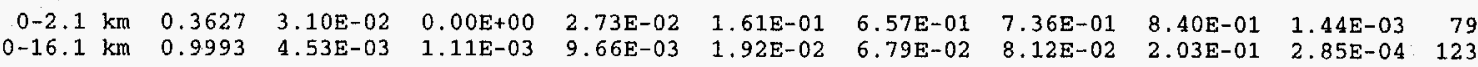

PEAK DOSE FOUND ON SPATIAL, GRTD (SV

L-EDEWBODY

L-EDEWBODY

L-EDEWBODY

L-EDEWBODY

L-EDEWBODY

L-EDEWBODY

L-EDEWBODY

L-EDEWBODY

L-EDEWBODY

L-EDENBODY

L-EDENBODY

L-EDENBODY

L-EDEWBODY

L-EDEWBODY

L-EDEWBODY

L-EDEWBODY

L-EDEWBODY

L-EDEWBODY

L-EDEWBODY

L-EDEWBODY

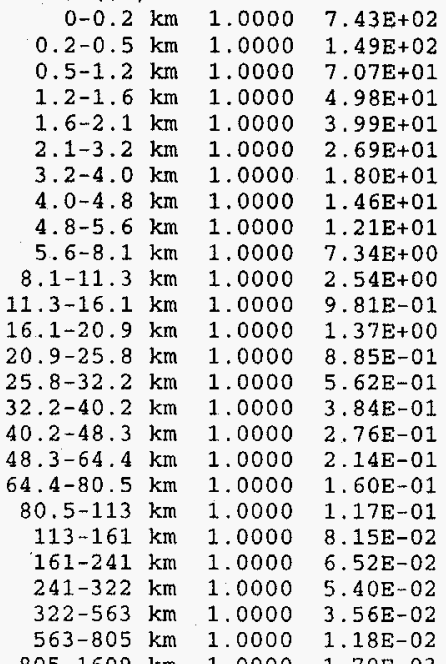

$6.01 \mathrm{E}+02$

$\begin{array}{lllllll}.01 \mathrm{E}+02 & 1.33 \mathrm{E}+03 & 1.58 \mathrm{E}+03 & \text { NOT-FOUND NOT-FOUND } & 1.93 \mathrm{E}+03 & 2.21 \mathrm{E}-02 \\ .06 \mathrm{E}+02 & 3.31 \mathrm{E}+02 & 3.96 \mathrm{E}+02 & 5.91 \mathrm{E}+02 & 6.94 \mathrm{E}+02 & 7.35 \mathrm{E}+02 & 4.14 \mathrm{E}-03\end{array}$

$3.76 \mathrm{E}+01 \quad 1.06 \mathrm{E}+02$

$\begin{array}{ll}1.80 \mathrm{E}+01 & 7.05 \mathrm{E}+01 \\ 8 & 0.01\end{array}$

$1.23 \mathrm{E}+02$ NOT-FOUND NOT-FOUND

$1.18 \mathrm{E}+02 \quad 4.14 \mathrm{E}-03$

$.53 \mathrm{E}+02 \quad 2.14 \mathrm{E}-03$

$.48 \mathrm{E}+02 \quad 2.21 \mathrm{E}-02$

$\begin{array}{rrr}.11 \mathrm{E}+02 & 1.43 \mathrm{E}-04 & 94\end{array}$

$\begin{array}{rrrrrrrr}.54 \mathrm{E}+00 & 6.34 \mathrm{E}+01 & 7.22 \mathrm{E}+01 & 7.90 \mathrm{E}+01 & 8.21 \mathrm{E}+01 & 1.11 \mathrm{E}+02 & 1.43 \mathrm{E}-04 & 129 \\ .53 \mathrm{E}+00 & 4.38 \mathrm{E}+01 & 7.41 \mathrm{E}+01 & \text { NOT-FOUND NOT-FOUND } & 8.87 \mathrm{E}+01 & 2.17 \mathrm{E}-02\end{array}$

$\begin{array}{lllllll}.76 \mathrm{E}+00 & 3.72 \mathrm{E}+01 & 6.21 \mathrm{E}+01 & \text { NOT-FOUND NOT-FOUND } & 8.82 \mathrm{E}+01 & 2.17 \mathrm{E}-02\end{array}$

$\begin{array}{lllll}3.72 \mathrm{E}+01 & 6.21 \mathrm{E}+01 & \text { NOT-FOUND NOT-FOUND } & 8.82 \mathrm{E}+01 & 2.17 \mathrm{E}-02 \\ 2.35 \mathrm{E}+01 & 2.95 \mathrm{E}+01 & \text { NOT-FOUND NOT-FOUND } & 4.37 \mathrm{E}+01 & 2.17 \mathrm{E}-02\end{array}$

$8.14 \mathrm{E}-01 \quad 8.30 \mathrm{E}+00$

. $47 \mathrm{E}+00 \quad 4.53 \mathrm{E}+00 \quad 5.08 \mathrm{E}+00 \quad 1.62 \mathrm{E}+01 \quad 3.14 \mathrm{E}-03$

3.95E+00 NOT-FOUND NOT-FOUND $6.04 \mathrm{E}+00 \quad 2.17 \mathrm{E}-02$

$\begin{array}{llllll}7.116 \mathrm{E}-01 & 1.86 \mathrm{E}+00 & 2.68 \mathrm{E}+00 & \text { NOT-FOUND NOT-FOUND } & 3.33 \mathrm{E}+00 & 1.17 \mathrm{E}-02 \\ 4.85 \mathrm{E}-01 & 9.07 \mathrm{E}-01 & 1.16 \mathrm{E}+00 & \text { NOT-FOUND NOT-FOUND } & 1.85 \mathrm{E}+00 & 1.91 \mathrm{E}-02 \\ 3.202 & \end{array}$

$4.85 \mathrm{E}-01 \quad 9.07 \mathrm{E}-01 \quad 1.16 \mathrm{E}+00$ NOT-FOUND NOT-FOUND

$1.07 \mathrm{E}+00 \quad 1.71 \mathrm{E}-02$

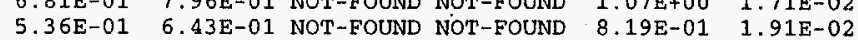

4.06E-01 5.04E-01 NOT-FOUND NOT-FOTND

$2.69 \mathrm{E}-01 \quad 3.17 \mathrm{E}-01$ NOT-FOUND NOT-FOUND

$6.25 \mathrm{E}-01 \quad 1.91 \mathrm{E}-02$

3.17E-01 NOT-FOUND NOT-FOUND $3.89 \mathrm{E}-01 \quad 1.71 \mathrm{E}-02$

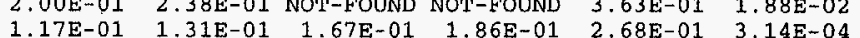

$\begin{array}{llllll}1.17 \mathrm{E}-01 & 1.31 \mathrm{E}-01 & 1.67 \mathrm{E}-01 & 1.86 \mathrm{E}-01 & 2.68 \mathrm{E}-01 & 3.14 \mathrm{E}-04 \\ 9.00 \mathrm{E}-02 & 1.00 \mathrm{E}-01 & 1.13 \mathrm{E}-01 & 1.20 \mathrm{E}-01 & 1.22 \mathrm{E}-01 & 3.77 \mathrm{E}-03\end{array}$

$1.00 \mathrm{E}-01 \quad 1.13 \mathrm{E}-01 \quad 1.20 \mathrm{E}-01$

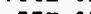

$3.12 \mathrm{E}-02$

$8.96 \mathrm{E}-02$
$7.12 \mathrm{E}-02$

$563-805 \mathrm{~km} \quad 1.0000$

$1.70 \mathrm{E}-03$

$1.03 E-01$ NOT-FOUND NOT-FOUND

$\begin{array}{ll}1.12 \mathrm{E}-01 & 2.70 \mathrm{E}-02 \\ 1.03 \mathrm{E}-01 & 2.73 \mathrm{E}-02\end{array}$

$\begin{array}{rrr}3.22 \mathrm{E}-02 & 4.07 \mathrm{E}-02 & 4.51 \mathrm{E}-02 \\ 7.12 \mathrm{E}-03 & 8.87 \mathrm{E}-03 & 9.75 \mathrm{E}-03\end{array}$

$1.67 \mathrm{E}-02 \quad 2.45 \mathrm{E}-03$
$2.54 \mathrm{E}-02$

$2.45 \mathrm{E}-03 \quad 64$ 
DATE AND TIME OF RUN = MACCS2 09/26/96 08:40:08 Version 1.12, Last Modified 9/25/96 by D. Chanin

"ATMOS" DESCRIPTION = IN1A. INP, Sample Problem A--Using Table-Lookup Sigmas, ATMOS input

SOURCE TERM 1 OF 2

SECOND DRAFT 1150, WORST CASE SOURCE TERM FOR EARLY FATALITIES

RESULTS FOR A SINGLE EMERGENCY RESPONSE COHORT WITHOUT ANY WEIGHTING FRACTIONS BEING APPLIED

COHORT 1 = EVACUATION WITHIN 10 MILES, RELOCATION MODELS APPLY ELSEWHERE



ERL FAT/TOTAL RISK $>0.000$

$\begin{array}{llllll}1.0000 & 4.32 \mathrm{E}+00 & 3.59 \mathrm{E}+00 & 8.81 \mathrm{E}+00 & 1.15 \mathrm{E}+01 & \text { NOT-FOUND NOT-FOUND } \\ 2.09 \mathrm{E}+01 & 2.17 \mathrm{E}-02 & 50\end{array}$

POPULATION EXCEEDING DOSE EARLY dOSe A-RED MARR > 1.50 EARLY dose A-LUNGS > 5.00 SV

$\begin{array}{llllllllll}0.4947 & 9.66 \mathrm{E}+01 & 0.00 \mathrm{E}+00 & 1.17 \mathrm{E}+02 & 2.31 \mathrm{E}+02 & 2.54 \mathrm{E}+03 & 3.68 \mathrm{E}+03 & 1.09 \mathrm{E}+04 & 2.85 \mathrm{E}-05 & 87\end{array}$ $\begin{array}{llllllllll}0.4015 & 4.47 \mathrm{E}+01 & 0.00 \mathrm{E}+00 & 6.29 \mathrm{E}+01 & 1.18 \mathrm{E}+02 & 7.03 \mathrm{E}+02 & 1.94 \mathrm{E}+03 & 8.32 \mathrm{E}+03 & 2.85 \mathrm{E}-04 & 8\end{array}$ AVERAGE INDIVIDUAL RISK ERL FAT/ TOTAL

ERL FAT / TOTAL

ERL FAT/TOTAL

$\begin{array}{llllllllll}1.0000 & 7.53 \mathrm{E}+04 & 2.92 \mathrm{E}+04 & 2.18 \mathrm{E}+05 & 3.19 \mathrm{E}+05 & 5.46 \mathrm{E}+05 & 6.43 \mathrm{E}+05 & 7.47 \mathrm{E}+05 & 4.06 \mathrm{E}-05 & 108\end{array}$

ERL FAT/TOTAL

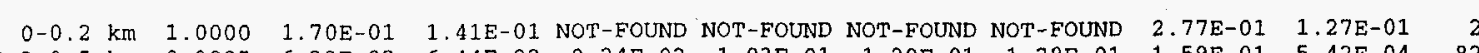
$\begin{array}{llllllllllll}0.2-0.5 \mathrm{~km} & 0.9885 & 6.33 \mathrm{E}-02 & 6.44 \mathrm{E}-02 & 9.24 \mathrm{E}-02 & 1.03 \mathrm{E}-01 & 1.20 \mathrm{E}-01 & 1.28 \mathrm{E}-01 & 1.59 \mathrm{E}-01 & 5.42 \mathrm{E}-04 & 82 & \\ 0.5-1.2 \mathrm{~km} & 0.6964 & 3.62 \mathrm{E}-02 & 3.92 \mathrm{E}-02 & 5.36 \mathrm{E}-02 & 5.55 \mathrm{E}-02 & 6.03 \mathrm{E}-02 & 6.25 \mathrm{E}-02 & 9.19 \mathrm{E}-02 & 5.42 \mathrm{E}-04 & 28\end{array}$ $\begin{array}{lllllllll}0.5-1.2 & \mathrm{~km} & 0.6964 & 3.61 \mathrm{E}-02 & 3.92 \mathrm{E}-02 & 5.36 \mathrm{E}-02 & 5.56 \mathrm{E}-02 & 6.03 \mathrm{E}-02 & 6.2 \mathrm{E}-02 \\ 1.2-1.6 \mathrm{~km} & 0.6902 & 2.64 \mathrm{E}-02 & 3.02 \mathrm{E}-02 & \text { NOT-FOUND NOT-FOUND NOT-FOUND NOT-FOUND } & 6.25 \mathrm{E}-02 & 1.21 \mathrm{E}-01 & 20\end{array}$

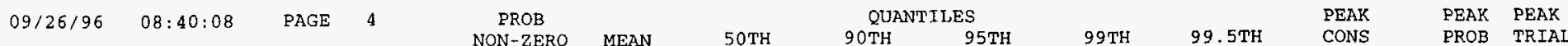

AVERAGE INDIVIDUAL RISK ERL FAT/TOTAL

$\begin{array}{lllllllll}1.6-2.1 \mathrm{~km} & 0.6573 & 2.12 \mathrm{E}-02 & 2.06 \mathrm{E}-02 & 6.00 \mathrm{E}-02 & \text { NOT-FOUND NOT-FOUND NOT-FOUND } & 6.25 \mathrm{E}-02 & 9.35 \mathrm{E}-02 & 20\end{array}$ 
POPULATION DOSE (SV)

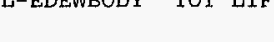
ERL FAT/TOTAL $\begin{array}{lllllllllll}0-16.1 \mathrm{~km} & 0.9074 & 5.44 \mathrm{E}+03 & 8.19 \mathrm{E}+02 & 1.12 \mathrm{E}+04 & 2.54 \mathrm{E}+04 & 8.81 \mathrm{E}+04 & 1.15 \mathrm{E}+05 & 2.65 \mathrm{E}+05 & 2.85 \mathrm{E}-04 & 123\end{array}$

$\begin{array}{lllllllllll}0-80.5 \mathrm{~km} & 1.0000 & 1.77 \mathrm{E}+04 & 8.73 \mathrm{E}+03 & 4.48 \mathrm{E}+04 & 6.55 \mathrm{E}+04 & 1.08 \mathrm{E}+05 & 1.37 \mathrm{E}+05 & 3.27 \mathrm{E}+05 & 2.85 \mathrm{E}-04 & 123 \\ 0-1609 \mathrm{~km} & 1.0000 & 3.03 \mathrm{E}+04 & 2.14 \mathrm{E}+04 & 6.16 \mathrm{E}+04 & 7.63 \mathrm{E}+04 & 1.17 \mathrm{E}+05 & 1.44 \mathrm{E}+05 & 3.27 \mathrm{E}+05 & 2.85 \mathrm{E}-04 & 123\end{array}$

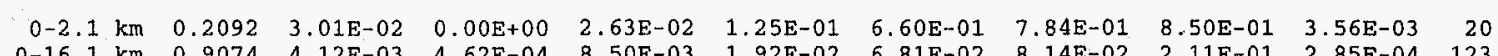

PEAK DOSE FOUND ON SPATIAL GRID (SV)

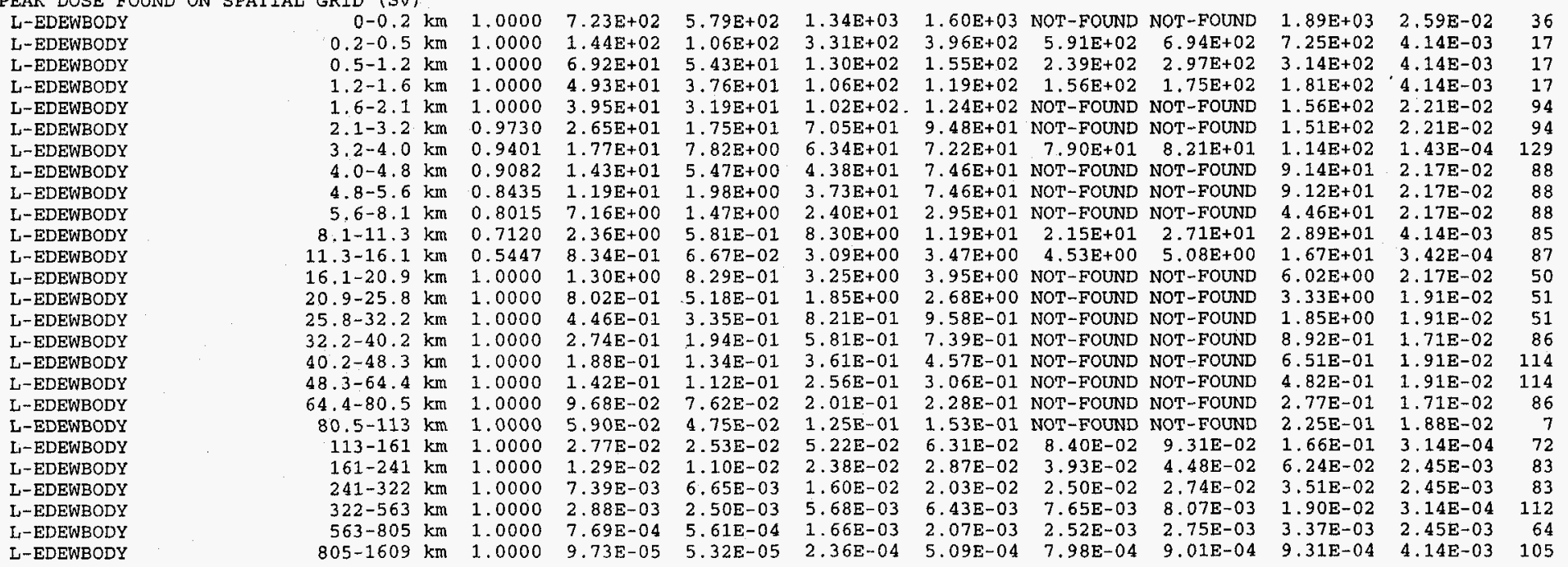

DATE AND TIME OF RUN = MACCS2 09/26/96 08:40:08 Version 1.12 , Last Modified $9 / 25 / 96$ by $D$. Chanin

"ATMOS". DESCRIPTION = IN1A. INP, Sample Problem A--Using Table-Lookup Sigmas, ATMOS input

SOURCE TERM 1 OF 2 :
SECOND DRAFT 1150 , WORST CASE SOURCE TERM FOR EARLY FATALITIES

RESULTS FOR A SINGLE EMERGENCY RESPONSE COHORT WITHOUT ANY WEIGHTING FRACTIONS BEING APPLIED

COHORT 2 = NO EVACUATION, RELOCATION MODELS APPLY EVERYWHERE

$09 / 26 / 96 \quad 08: 40: 08 \quad$ PAGE

HEALTH EFFECTS CASES ERL INJ TORODROMAL VOMIT ERL INJ/DIARRHEA

ERL INJ/PNEUMONITIS

ERL INJ/THYROIDITIS

ERL INJ/HYPOTHYROIDISM

ERL INJ/SKIN ERYTHEMA

PROB

QUANTILES
$90 \mathrm{TH}$

$99 \mathrm{TH}$

$99.5 \mathrm{TH}$

PEAK $\quad$ PEAK PEAK $\begin{array}{lllllllllll}0-1609 \mathrm{~km} & 0.7404 & 7.14 \mathrm{E}+01 & 8.59 \mathrm{E}+00 & 1.87 \mathrm{E}+02 & 4.31 \mathrm{E}+02 & 9.60 \mathrm{E}+02 & 1.09 \mathrm{E}+03 & 3.64 \mathrm{E}+03 & 2.00 \mathrm{E}-05 & 128 \\ 0-1609 \mathrm{~km} & 0.5934 & 4.06 \mathrm{E}+00 & 8.81 \mathrm{E}-02 & 1.42 \mathrm{E}+01 & 2.22 \mathrm{E}+01 & 3.19 \mathrm{E}+01 & 3.38 \mathrm{E}+01 & 4.15 \mathrm{E}+02 & 7.15 \mathrm{E}-06 & 129\end{array}$

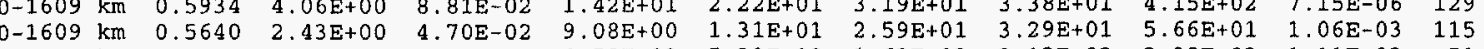
$\begin{array}{lllllllllll}0-1609 \mathrm{~km} & 0.9325 & 1.53 \mathrm{E}+02 & 3.13 \mathrm{E}+01 & 4.58 \mathrm{E}+02 & 7.33 \mathrm{E}+02 & 1.63 \mathrm{E}+03 & 2.12 \mathrm{E}+03 & 2.98 \mathrm{E}+03 & 1.11 \mathrm{E}-03 & 51\end{array}$ $\begin{array}{rrlllllllll}0-1609 \mathrm{~km} & 0.9555 & 5.97 \mathrm{E}+03 & 8.94 \mathrm{E}+02 & 1.41 \mathrm{E}+04 & 2.70 \mathrm{E}+04 & 8.02 \mathrm{E}+04 & 1.09 \mathrm{E}+05 & 1.81 \mathrm{E}+05 & 7.13 \mathrm{E}-04 & 114 \\ 0-1609 \mathrm{~km} & 0.8175 & 1.64 \mathrm{E}+03 & 1.42 \mathrm{E}+02 & 5.23 \mathrm{E}+03 & 7.65 \mathrm{E}+03 & 2.14 \mathrm{E}+04 & 2.73 \mathrm{E}+04 & 5.20 \mathrm{E}+04 & 7.71 \mathrm{E}-04 & 29\end{array}$ 
CAN FAT/TOTAL

CAN FAT/LUNG

CAN FAT/THYROID
CAN FAT/BREAST

CAN FAT/GI

CAN FAT/LEUKEMIA

CAN FAT/BONE

CAN FAT/OTHER

CAN INJ/THYROID

CAN INJ/BREAST

CAN FAT/TOTAL

ERL FAT/TOTAL

ERL INJ/PRODROMAL VOMIT

ERL INJ/DIARRHEA

ERL INJ/PNEUMONITIS

ERL INJ/THYROIDITIS

ERL. INJ/HYPOTHYROIDIS

ERT INT/SKINANTDERM

CAN FAT/TOTAL

EARLY FATALITY DISTANCE (km) ERL FAT/TOTAL RISK > 0.000

POPULATION EXCEEDING DOSE EARLY dose A-RED MARR > 1.50 $.00 \mathrm{~Sv}$

SV

VERAGE INDIVIDUAL RISK

\section{ERL FAT/TOTAL}

ERL FAT/TOTAI

09/26/96 08:40:08 PAGE

AVERAGE INDIVIDUAL RISK ERL FAT/TOTAL

POPULATION DOSE (SV) LIF $\begin{array}{ll}\text { L-EDEWBODY } & \text { TOT LIF } \\ \text { L-EDEWBODY } & \text { TOT LIF }\end{array}$

POPULATION WEIGHTED RISK

ERL FAT/TOTAL PEAK DOSE FOUND ON SPATIAL GRID (SV)

L-EDEWBODY

L-EDEWBODY

L-EDEWBODY

L-EDEWBODY

L-EDEWBODY

U-EDEWBODY

L-EDEWBODY

I-

L-EDEWBODY

L-EDEWBODY

$\begin{array}{llllllllllll}0-1609 \mathrm{~km} & 1.0000 & 1.58 \mathrm{E}+03 & 1.16 \mathrm{E}+03 & 3.05 \mathrm{E}+03 & 3.74 \mathrm{E}+03 & 5.43 \mathrm{E}+03 & 5.94 \mathrm{E}+03 & 9.00 \mathrm{E}+03 & 1.11 \mathrm{E}-03 & 51\end{array}$

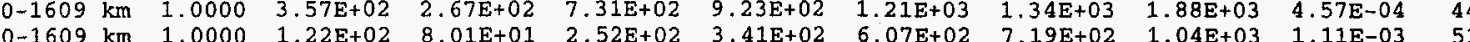

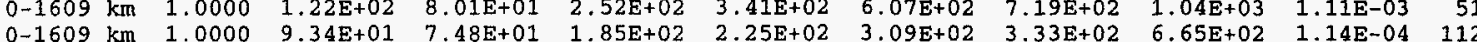

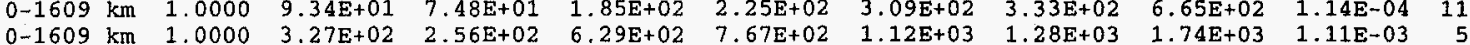

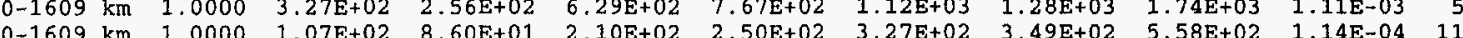

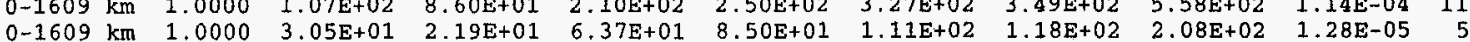
$\begin{array}{lllllllllll}0-1609 \mathrm{~km} & 1.0000 & 5.44 \mathrm{E}+02 & 3.93 \mathrm{E}+02 & 1.06 \mathrm{E}+03 & 1.38 \mathrm{E}+03 & 2.19 \mathrm{E}+03 & 2.42 \mathrm{E}+03 & 3.86 \mathrm{E}+03 & 1.11 \mathrm{E}-03 & 51\end{array}$

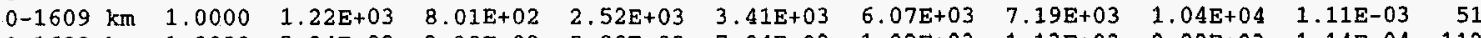

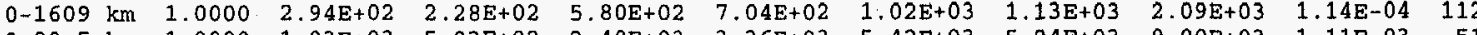

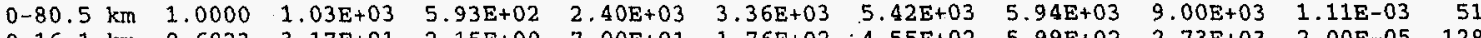

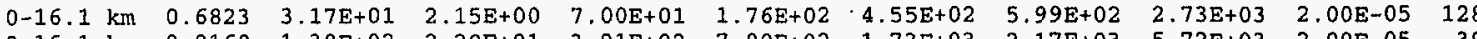

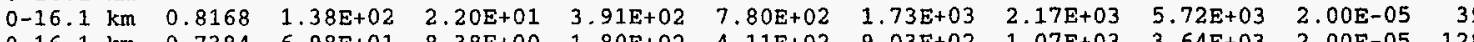

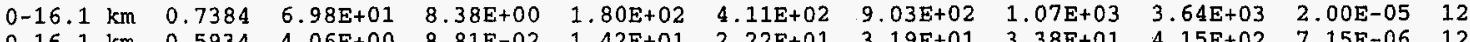

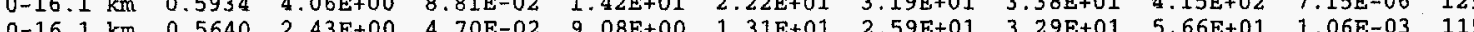

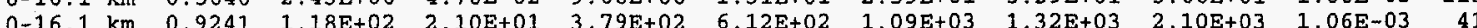
$\begin{array}{llllllllllll}0-16.1 & \mathrm{~km} & 0.9529 & 2.65 \mathrm{E}+03 & 5.22 \mathrm{E}+02 & 8.66 \mathrm{E}+03 & 1.29 \mathrm{E}+04 & 2.18 \mathrm{E}+04 & 2.38 \mathrm{E}+04 & 3.98 \mathrm{E}+04 & 5.03 \mathrm{E}-04 & 103\end{array}$

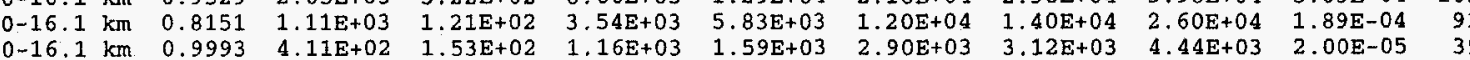

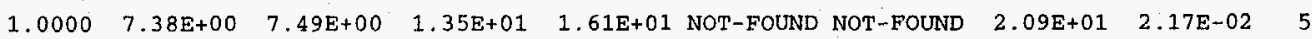

$\begin{array}{llllllllll}0.6836 & 1.94 \mathrm{E}+02 & 1.52 \mathrm{E}+01 & 4.81 \mathrm{E}+02 & 1.16 \mathrm{E}+03 & 2.85 \mathrm{E}+03 & 3.49 \mathrm{E}+03 & 1.11 \mathrm{E}+04 & 2.00 \mathrm{E}-05 & 128\end{array}$ $\begin{array}{llllllllll}0.5934 & 4.05 \mathrm{E}+01 & 1.57 \mathrm{E}+00 & 5.60 \mathrm{E}+01 & 2.62 \mathrm{E}+02 & 5.65 \mathrm{E}+02 & 1.01 \mathrm{E}+03 & 1.19 \mathrm{E}+03 & 3.86 \mathrm{E}-05 & 125\end{array}$

$\begin{array}{lllllllllll}0-0.2 \mathrm{~km} & 1.0000 & 2.88 \mathrm{E}-01 & 2.69 \mathrm{E}-01 & 3.92 \mathrm{E}-01 & 4.46 \mathrm{E}-01 & 5.24 \mathrm{E}-01 & 5.41 \mathrm{E}-01 & 6.24 \mathrm{E}-01 & 2.28 \mathrm{E}-04 & 18\end{array}$

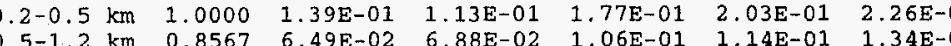

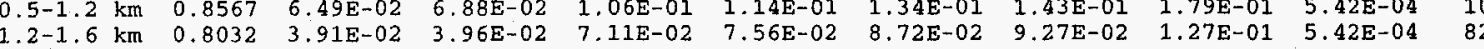$$
\text { PROB }
$$

$50 \mathrm{TH}$

QUANTILES
$90 \mathrm{TH}$

$99 \mathrm{TH}$

$99.5 \mathrm{TH}$ PEAK

$\begin{array}{ll}\text { PEAK } & \text { PEAK } \\ \text { PROB } & \text { TRIAL }\end{array}$

$\begin{array}{lllllllllll}1.6-2.1 \mathrm{~km} & 0.7380 & 2.79 \mathrm{E}-02 & 3.03 \mathrm{E}-02 & 5.14 \mathrm{E}-02 & 5.96 \mathrm{E}-02 & 7.74 \mathrm{E}-02 & 8.38 \mathrm{E}-02 & 2.08 \mathrm{E}-01 & 5.42 \mathrm{E}-04 & 28\end{array}$

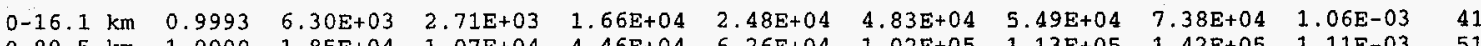

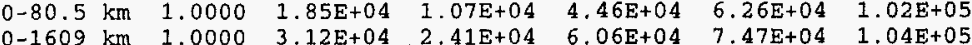
$\begin{array}{rllllllllll}0-2.1 \mathrm{~km} & 0.3627 & 4.68 \mathrm{E}-02 & 0.00 \mathrm{E}+00 & 4.53 \mathrm{E}-02 & 4.30 \mathrm{E}-01 & 6.85 \mathrm{E}-01 & 7.17 \mathrm{E}-01 & 8.50 \mathrm{E}-01 & 8.32 \mathrm{E}-05 & 28 \\ 0-16.1 \mathrm{~km} & 0.9993 & 5.59 \mathrm{E}-03 & 2.07 \mathrm{E}-03 & 1.41 \mathrm{E}-02 & 2.10 \mathrm{E}-02 & 3.41 \mathrm{E}-02 & 3.88 \mathrm{E}-02 & 6.05 \mathrm{E}-02 & 2.00 \mathrm{E}-05 & 39\end{array}$ $\begin{array}{llllllllll}0-0.2 \mathrm{~km} & 1.0000 & 1.11 \mathrm{E}+03 & 9.48 \mathrm{E}+02 & 1.99 \mathrm{E}+03 & 2.24 \mathrm{E}+03 & \text { NOT-FOUND NOT-FOUND } & 2.57 \mathrm{E}+03 & 2.21 \mathrm{E}-02 & 47\end{array}$ $\begin{array}{lllllllll} & \end{array}$ $\begin{array}{lllllllll} & \end{array}$ $\begin{array}{llllllllll}2.1-3.2 \mathrm{~km} & 1.0000 & 3.16 \mathrm{E}+01 & 2.52 \mathrm{E}+01 & 7.38 \mathrm{E}+01 & 8.37 \mathrm{E}+01 & \text { NOT-FOUND NOT-FOUND } & 9.72 \mathrm{E}+01 & 2.21 \mathrm{E}-02 & 34\end{array}$

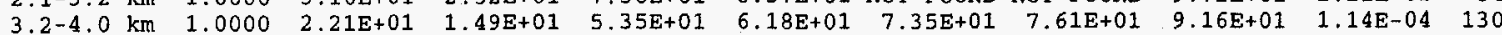
$\begin{array}{llllllllll}4.0-4.8 \mathrm{~km} & 1.0000 & 1.74 \mathrm{E}+01 & 1.19 \mathrm{E}+01 & 3.92 \mathrm{E}+01 & 4.99 \mathrm{E}+01 & \text { NOT-FOUND NOT-FOUND } & 6.75 \mathrm{E}+01 & 2.21 \mathrm{E}-02 & 34\end{array}$ $\begin{array}{lllllllllll}4.8-5.6 \mathrm{~km} & 1.0000 & 1.35 E+01 & 9.08 \mathrm{E}+00 & 3.83 \mathrm{E}+01 & 4.94 \mathrm{E}+01 & 5.23 \mathrm{E}+01 & 5.33 \mathrm{E}+01 & 5.62 \mathrm{E}+01 & 7.71 \mathrm{E}-04 & 45\end{array}$

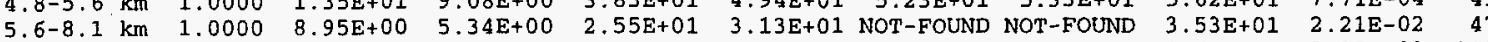

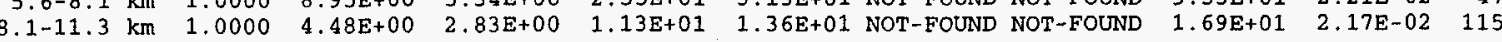




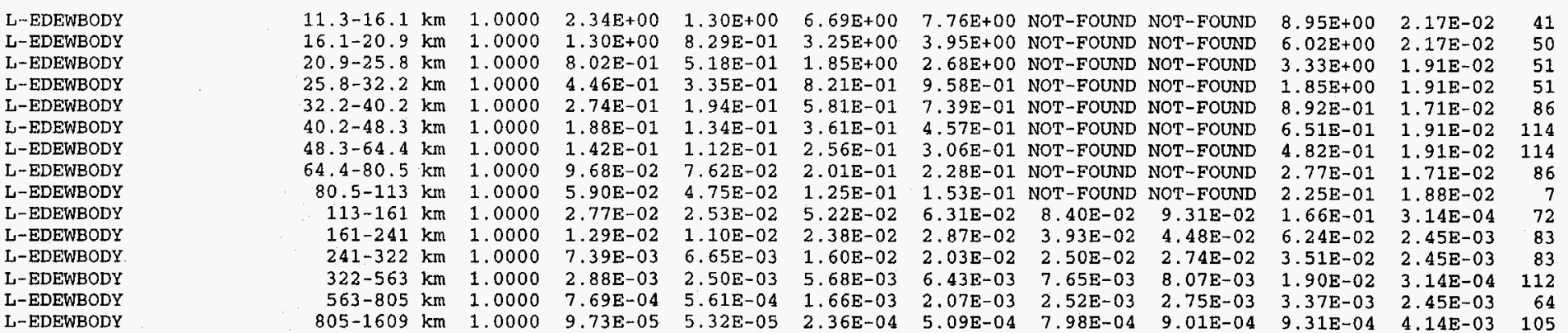

DATE AND TIME OF RUN $=$ MACCS2 09/26/95 08:40:08 Version 1.12, Last Modified 9/25/96 by D. Chanin

"ATMOS" DESCRIPTION = IN1A. INP, Sample Problem A--Using Table-Lookup Sigmas, ATMOS input

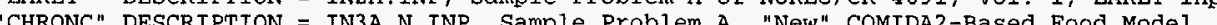

SOURCE TERM 1 OF 2 :

SECOND DRAFT 1150, WORST CASE SOURCE TERM FOR EARLY FATALITIES

RESULTS FROM THE "CHRONC" MODULE ALONE

COHORT $3=$ IN3A_N. INP, Sample Problem A, "New" COMIDA2-Based Food Model

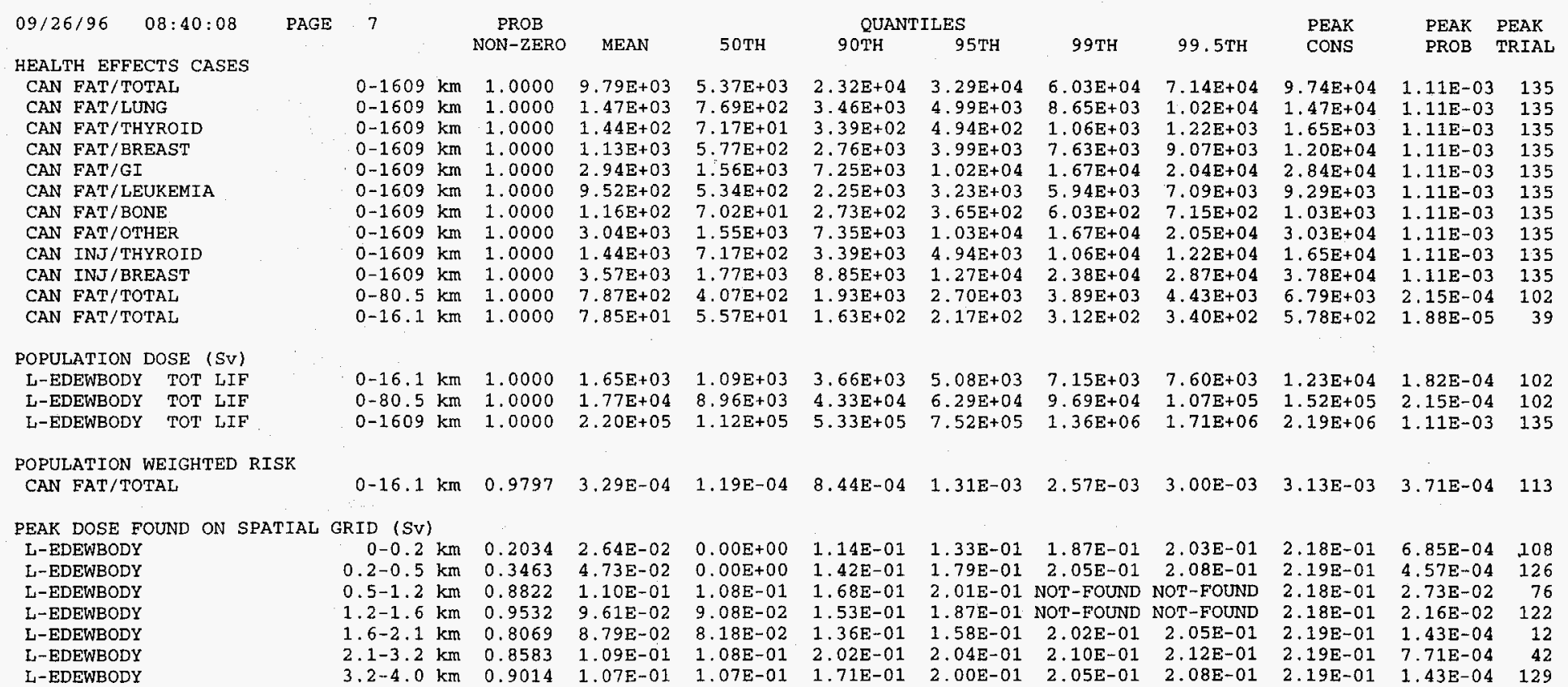


L-EDEWBODY

L-EDEWBODY

L-EDENDODY

L-EDENBDY

L-EDEWBODY

L-EDEWBODY

L-EDEWBODY

L-EDEWBODY

L-EDEWBODY

L-EDEWBODY

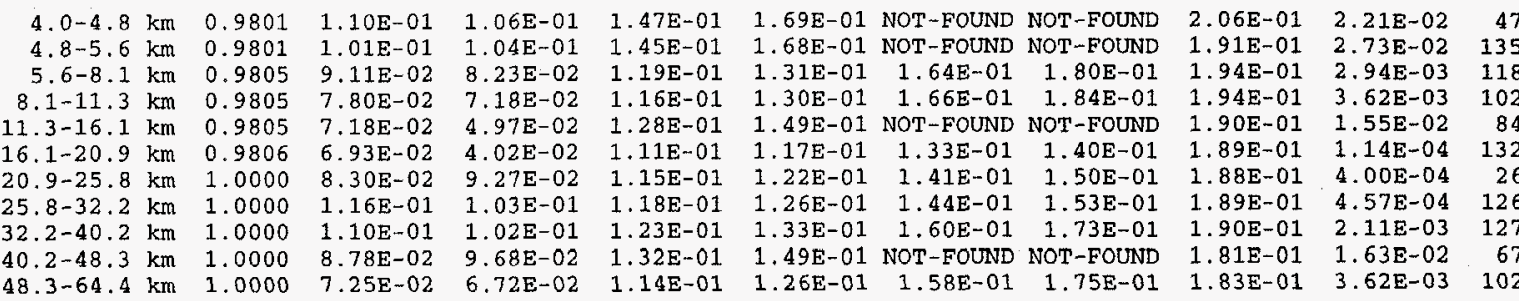

$09 / 26 / 96$

08:40:08 PAGE 8

PROB
NON-ZERO MEAN $50 \mathrm{TH}$

QUANTILES
90TH $95 \mathrm{TH}$

$99 \mathrm{TH}$

$99.5 \mathrm{TH}$

PEAK

PEAK PEAK

PEAK DOSE FOUND ON SPATIAL GRID (SV)

L-EDEWBODY

L-EDEWBODY

L-EDEWBODY

L-EDEWBODY

L-EDEWBODY

L-EDEWBODY

$\begin{array}{lll}8.4-50.5 & 1.0000 \quad 6.31 \mathrm{E}-02\end{array}$

$.75 \mathrm{E}-02 \quad 1.08 \mathrm{E}-01$

$1.33 E-01$

$1.45 E-01 \quad 1.75 E-01$

1. $17 \mathrm{E}-03$

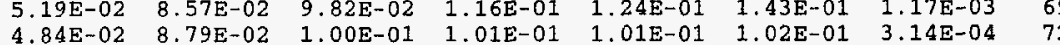

$\begin{array}{lllllllllll}113-161 \mathrm{~km} & 1.0000 & 5.38 \mathrm{E}-02 & 4.84 \mathrm{E}-02 & 8.79 \mathrm{E}-02 & 1.00 \mathrm{E}-01 & 1.01 \mathrm{E}-01 & 1.01 \mathrm{E}-01 & 1.02 \mathrm{E}-01 & 3.14 \mathrm{E}-04 & 72 \\ 161-241 \mathrm{~km} & 1.0000 & 5.23 \mathrm{E}-02 & 5.20 \mathrm{E}-02 & 7.36 \mathrm{E}-02 & 7.79 \mathrm{E}-02 & 8.91 \mathrm{E}-02 & 9.43 \mathrm{E}-02 & 9.66 \mathrm{E}-02 & 3.77 \mathrm{E}-03 & 65 \\ \end{array}$

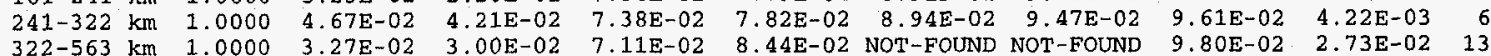

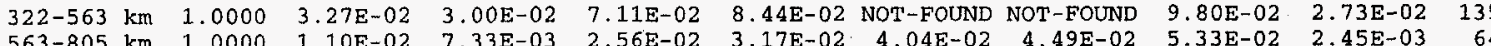

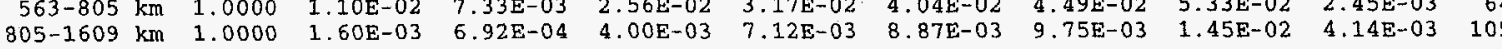

L-EDEWBODY POP. DOSE (SV)

TOTAL LONG-TERM PATHWAYS DOSE $0-1609 \mathrm{~km}$

LONG-TERM DIRECT EXPOSURE PATHWAYS

TOTAL INGESTION PATHWAYS DOSE

LONG-TERM GROUNDSHINE DOSE

INGESTION DOSE

POP.-DEPENDENT DECONTAMINATION DOSE

FARM-DEPENDENT DECONTAMINATION DOSE

INGESTION OF GRAINS

INGESTION OF LEAF VEG

INGESTION OF ROOT CROPS

INGESTION OF FRUITS

INGESTION OF LEGUMES

INGESTION OF BEEF

INGESTTION OF POULTRY

INGESTION OF POULTRY

$1.0000 \quad 2.208+05$

$\begin{array}{llllllll} & & & & \end{array}$

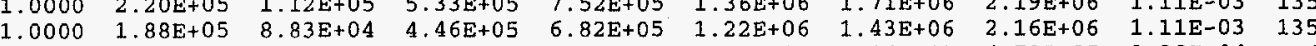

$\begin{array}{llllllllll}1.0000 & 2.89 \mathrm{E}+04 & 1.04 \mathrm{E}+04 & 7.36 \mathrm{E}+04 & 1.21 \mathrm{E}+05 & 2.66 \mathrm{E}+05 & 3.14 \mathrm{E}+05 & 4.71 \mathrm{E}+05 & 2.28 \mathrm{E}-04 & 4\end{array}$

$\begin{array}{rlllllllll}1.0000 & 1.83 \mathrm{E}+05 & 8.50 \mathrm{E}+04 & 4.43 \mathrm{E}+05 & 6.82 \mathrm{E}+05 & 1.22 \mathrm{E}+06 & 1.42 \mathrm{E}+06 & 2.13 \mathrm{E}+06 & 1.11 \mathrm{E}-03 & 135 \\ 1.0000 & 4.54 \mathrm{E}+03 & 2.95 \mathrm{E}+03 & 9.92 \mathrm{E}+03 & 1.38 \mathrm{E}+04 & 2.29 \mathrm{E}+04 & 2.56 \mathrm{E}+04 & 3.60 \mathrm{E}+04 & 6.85 \mathrm{E}-04 & 15\end{array}$

$\begin{array}{llllllllll}1.0000 & 4.54 \mathrm{E}+03 & 2.95 \mathrm{E}+03 & 9.92 \mathrm{E}+03 & 1.38 \mathrm{E}+04 & 2.29 \mathrm{E}+04 & 2.56 \mathrm{E}+04 & 3.60 \mathrm{E}+04 & 6.85 \mathrm{E}-04 & 15 \\ 1.0000 & 1.77 \mathrm{E}+03 & 1.31 \mathrm{E}+03 & 3.47 \mathrm{E}+03 & 4.60 \mathrm{E}+03 & 6.41 \mathrm{E}+03 & 7.14 \mathrm{E}+03 & 1.23 \mathrm{E}+04 & 1.88 \mathrm{E}-05 & 39\end{array}$

$\begin{array}{llllllllll}1.0000 & 3.14 \mathrm{E}+03 & 1.66 \mathrm{E}+03 & 7.69 \mathrm{E}+03 & 1.07 \mathrm{E}+04 & 1.91 \mathrm{E}+04 & 2.13 \mathrm{E}+04 & 4.32 \mathrm{E}+04 & 2.15 \mathrm{E}-04 & 102\end{array}$

$\begin{array}{rlllllllll}1.0000 & 3.14 \mathrm{E}+03 & 1.66 \mathrm{E}+03 & 7.69 \mathrm{E}+03 & 1.07 \mathrm{E}+04 & 1.91 \mathrm{E}+04 & 2.13 \mathrm{E}+04 & 4.32 \mathrm{E}+04 & 2.15 \mathrm{E}-04 & 102 \\ 1.0000 & 1.01 \mathrm{E}+02 & 6.82 \mathrm{E}+01 & 2.25 \mathrm{E}+02 & 2.68 \mathrm{E}+02 & 3.36 \mathrm{E}+02 & 3.59 \mathrm{E}+02 & 4.69 \mathrm{E}+02 & 3.14 \mathrm{E}-04 & 83\end{array}$

$\begin{array}{lllllllll}1.0000 & 2.05 \mathrm{E}+03 & 3.63 \mathrm{E}+02 & 6.47 \mathrm{E}+03 & 1.03 \mathrm{E}+04 & 2.22 \mathrm{E}+04 & 2.84 \mathrm{E}+04 & 4.00 \mathrm{E}+04 & 2.43 \mathrm{E}-03\end{array}$

$\begin{array}{lllllllll}1.0000 & 2.05 \mathrm{E}+03 & 3.63 \mathrm{E}+02 & 6.47 \mathrm{E}+03 & 1.03 \mathrm{E}+04 & 2.22 \mathrm{E}+04 & 2.84 \mathrm{E}+04 & 4.00 \mathrm{E}+04 & 2.43 \mathrm{E}-03\end{array}$

$\begin{array}{lllllllll}1.0000 & 1.37 \mathrm{E}+03 & 2.37 \mathrm{E}+02 & 3.82 \mathrm{E}+03 & 7.26 \mathrm{E}+03 & 1.57 \mathrm{E}+04 & 2.09 \mathrm{E}+04 & 3.39 \mathrm{E}+04 & 2.28 \mathrm{E}-04\end{array}$

$\begin{array}{lllllllll}1.0000 & 1.93 \mathrm{E}+03 & 2.60 \mathrm{E}+02 & 5.29 \mathrm{E}+03 & 1.05 \mathrm{E}+04 & 2.38 \mathrm{E}+04 & 3.05 \mathrm{E}+04 & 4.94 \mathrm{E}+04 & 2.28 \mathrm{E}-04\end{array}$

$\begin{array}{lllllllll}1.0000 & 1.35 \mathrm{E}+03 & 3.96 \mathrm{E}+02 & 3.48 \mathrm{E}+03 & 6.42 \mathrm{E}+03 & 1.33 \mathrm{E}+04 & 1.67 \mathrm{E}+04 & 2.93 \mathrm{E}+04 & 2.28 \mathrm{E}-04\end{array}$

$\begin{array}{lllllllll}1.0000 & 9.60 \mathrm{E}+03 & 2.62 \mathrm{E}+03 & 2.31 \mathrm{E}+04 & 4.40 \mathrm{E}+04 & 1.10 \mathrm{E}+05 & 1.42 \mathrm{E}+05 & 2.35 \mathrm{E}+05 & 2.28 \mathrm{E}-04 \\ 1.0000 & 6.76 \mathrm{E}+03 & 2.30 \mathrm{E}+03 & 1.59 \mathrm{E}+04 & 2.90 \mathrm{E}+04 & 6.44 \mathrm{E}+04 & 8.71 \mathrm{E}+04 & 1.29 \mathrm{E}+05 & 2.28 \mathrm{E}-04\end{array}$

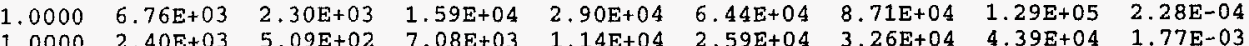

$\begin{array}{lllllllll}1.0000 & 7.39 \mathrm{E}+02 & 3.24 \mathrm{E}+02 & 1.99 \mathrm{E}+03 & 2.57 \mathrm{E}+03 & 5.40 \mathrm{E}+03 & 6.73 \mathrm{E}+03 & 8.33 \mathrm{E}+03 & 2.43 \mathrm{E}-03\end{array}$

L-EDEWBODY POP. DOSE (SV) $0-80.5 \mathrm{~km}$

TOTAL LONG-TERM PATHWAYS DOSE

LONG-TERM DIRECT EXPOSURE PATHWAYS

TOTAL, INGESTION PATHWAYS DOSE

LONG-TERM GROUNDSHINE DOSE

LONG-TERM RESUSPENSION

POP - DEPENTION DOSE

FARM-DEPENDENT DECONTAMTNATION DOSE

INGESTION OF GRAINS

INGESTION OF LEAF VEG

INGESTION OF ROOT CROPS
INGESTION OF FRUITS

INGESTION OF FRUITS

INGESTION OF BEEF

INGESTION OF MILK

INGESTION OF POULTRY

INGESTION OF OTHER MEAT CROPS

$\begin{array}{rllllllllr}1.0000 & 1.77 \mathrm{E}+04 & 8.96 \mathrm{E}+03 & 4.33 \mathrm{E}+04 & 6.29 \mathrm{E}+04 & 9.69 \mathrm{E}+04 & 1.07 \mathrm{E}+05 & 1.52 \mathrm{E}+05 & 2.15 \mathrm{E}-04 & 102 \\ 1.0000 & 1.33 \mathrm{E}+04 & 5.97 \mathrm{E}+03 & 3.48 \mathrm{E}+04 & 5.30 \mathrm{E}+04 & 7.47 \mathrm{E}+04 & 7.88 \mathrm{E}+04 & 1.06 \mathrm{E}+05 & 2.15 \mathrm{E}-04 & 102 \\ 1.0000 & 1.87 \mathrm{E}+03 & 1.45 \mathrm{E}+03 & 3.16 \mathrm{E}+03 & 4.05 \mathrm{E}+03 & 6.41 \mathrm{E}+03 & 7.22 \mathrm{E}+03 & 1.26 \mathrm{E}+04 & 3.22 \mathrm{E}-05 & 107 \\ 1.0000 & 1.24 \mathrm{E}+04 & 5.40 \mathrm{E}+03 & 3.34 \mathrm{E}+04 & 5.10 \mathrm{E}+04 & 7.47 \mathrm{E}+04 & 7.88 \mathrm{E}+04 & 1.05 \mathrm{E}+05 & 2.15 \mathrm{E}-04 & 102 \\ 1.0000 & 9.30 \mathrm{E}+02 & 4.16 \mathrm{E}+02 & 2.42 \mathrm{E}+03 & 3.83 \mathrm{E}+03 & 6.47 \mathrm{E}+03 & 7.21 \mathrm{E}+03 & 1.26 \mathrm{E}+04 & 5.84 \mathrm{E}-06 & 109 \\ 1.0000 & 1.18 \mathrm{E}+03 & 8.16 \mathrm{E}+02 & 2.34 \mathrm{E}+03 & 3.05 \mathrm{E}+03 & 5.71 \mathrm{E}+03 & 6.92 \mathrm{E}+03 & 1.22 \mathrm{E}+04 & 1.88 \mathrm{E}-05 & 39 \\ 1.0000 & 2.41 \mathrm{E}+03 & 1.00 \mathrm{E}+03 & 6.59 \mathrm{E}+03 & 1.01 \mathrm{E}+04 & 1.83 \mathrm{E}+04 & 2.10 \mathrm{E}+04 & 4.32 \mathrm{E}+04 & 2.15 \mathrm{E}-04 & 102 \\ 1.0000 & 5.79 \mathrm{E}+01 & 5.25 \mathrm{E}+01 & 1.03 \mathrm{E}+02 & 1.08 \mathrm{E}+02 & 1.21 \mathrm{E}+02 & 1.27 \mathrm{E}+02 & 1.56 \mathrm{E}+02 & 2.16 \mathrm{E}-04 & 110 \\ 1.0000 & 1.26 \mathrm{E}+02 & 1.02 \mathrm{E}+02 & 2.53 \mathrm{E}+02 & 3.24 \mathrm{2}+02 & 5.11 \mathrm{E}+02 & 5.43 \mathrm{E}+02 & 6.18 \mathrm{E}+02 & 1.14 \mathrm{E}-03 & 50 \\ 1.0000 & 1.26 \mathrm{E}+02 & 1.02 \mathrm{E}+02 & 2.53 \mathrm{E}+02 & 3.24 \mathrm{E}+02 & 5.11 \mathrm{E}+02 & 5.43 \mathrm{E}+02 & 6.18 \mathrm{E}+02 & 1.14 \mathrm{E}-03 & 50 \\ 1.0000 & 7.31 \mathrm{E}+01 & 6.30 \mathrm{E}+01 & 1.25 \mathrm{E}+02 & 1.46 \mathrm{E}+02 & 2.04 \mathrm{E}+02 & 2.15 \mathrm{E}+02 & 3.56 \mathrm{E}+02 & 1.41 \mathrm{E}-05 & 109 \\ 1.0000 & 6.26 \mathrm{E}+01 & 5.53 \mathrm{E}+01 & 1.03 \mathrm{E}+02 & 1.08 \mathrm{E}+02 & 1.18 \mathrm{E}+02 & 1.23 \mathrm{E}+02 & 1.78 \mathrm{E}+02 & 1.04 \mathrm{E}-05 & 109 \\ 1.0000 & 1.25 \mathrm{E}+02 & 1.05 \mathrm{E}+02 & 2.27 \mathrm{E}+02 & 2.67 \mathrm{E}+02 & 3.45 \mathrm{E}+02 & 3.76 \mathrm{E}+02 & 6.62 \mathrm{E}+02 & 1.41 \mathrm{E}-05 & 109 \\ 1.0000 & 1.01 \mathrm{E}+02 & 6.33 \mathrm{E}+01 & 2.11 \mathrm{E}+02 & 3.48 \mathrm{E}+02 & 5.36 \mathrm{E}+02 & 5.61 \mathrm{E}+02 & 7.10 \mathrm{E}+02 & 3.92 \mathrm{E}-05 & 107 \\ 1.0000 & 1.32 \mathrm{E}+02 & 9.69 \mathrm{E}+01 & 2.72 \mathrm{E}+02 & 3.55 \mathrm{E}+02 & 5.16 \mathrm{E}+02 & 5.36 \mathrm{E}+02 & 7.08 \mathrm{E}+02 & 5.37 \mathrm{E}-06 & 109 \\ 1.0000 & 1.84 \mathrm{E}+01 & 1.05 \mathrm{E}+01 & 4.70 \mathrm{E}+01 & 6.75 \mathrm{E}+01 & 1.05 \mathrm{E}+02 & 1.10 \mathrm{E}+02 & 1.19 \mathrm{E}+02 & 1.14 \mathrm{E}-03 & 50 \\ 1.0000 & 2.62 \mathrm{E}+01 & 1.39 \mathrm{E}+01 & 6.94 \mathrm{E}+01 & 8.58 \mathrm{E}+01 & 1.16 \mathrm{E}+02 & 1.28 \mathrm{E}+02 & 1.80 \mathrm{E}+02 & 4.00 \mathrm{E}-04 & 78\end{array}$


ECONOMIC COST MEASURES (\$) $0-1609 \mathrm{~km}$ TOTAL ECONOMIC COSTS
POP, -DEPENDENT COSTS

FARM-DEPENDENT COSTS

POP.-DEPENDENT DECONTAMINATION COST

FARM-DEPENDENT DECONTAMINATION COST

$\begin{array}{lllllllll}.0000 & 1.18 \mathrm{E}+10 & 6.46 \mathrm{E}+09 & 2.33 \mathrm{E}+10 & 3.54 \mathrm{E}+10 & 6.48 \mathrm{E}+10 & 9.38 \mathrm{E}+10 & 2.69 \mathrm{E}+11 & 1.11 \mathrm{E}-03\end{array}$ $\begin{array}{lllllllll}.0000 & 1.07 \mathrm{E}+10 & 5.45 \mathrm{E}+09 & 2.15 \mathrm{E}+10 & 3.26 \mathrm{E}+10 & 6.35 \mathrm{E}+10 & 8.23 \mathrm{E}+10 & 2.68 \mathrm{E}+11 & 1.11 \mathrm{E}-03\end{array}$ $+09 \quad 4.00 \mathrm{E}-04$

$\begin{array}{llllllllll}1.0000 & 2.71 \mathrm{E}+09 & 1.32 \mathrm{E}+09 & 5.97 \mathrm{E}+09 & 9.02 \mathrm{E}+09 & 1.77 \mathrm{E}+10 & 2.50 \mathrm{E}+10 & 6.60 \mathrm{E}+10 & 1.11 \mathrm{E}-03 & 76\end{array}$

$09 / 26 / 96 \quad 08: 40: 08 \quad P A G E \quad 9$

pros

ECONOMIC COST MEASURES (\$) $0-1609 \mathrm{~km}$

POP. -DEPENDENT INTERDICTION COST

FARM-DEPENDENT INARDCTION COST

FARM-DEPENDENT CONDEMNATION COST

EMERGENCY PHASE COST

INTERMEDIATE PHASE COST

MILK DISPOSAL COST
CROP DISPOSAL COST

1.0

MEAN

QUANTILES

99TH $99.5 \mathrm{TH} \quad$ PEAK

PEAK PEAK

ECONOMIC COST MEASURES (\$)

TOTAL ECONOMIC COSTS

POP. -DEPENDENT COSTS

POP. -DEPENDENT DECONTAMINATION COST

FARM-DEPENDENT DECONTAMINATION COST

POP. -DEPENDENT INTERDICTION COST

FARM-DEPENDENT INTERDICTION COST

POP. -DEPENDENT CONDEMNATION COST
FARM-DEPENDENT CONDEMNATION COST

EMERGENCY PHA.SE COST

INTERMEDIATE PHASE COS

MILK DISPOSAL COST

MAXIMUM LONG-TERM ACTION DISTANCE (km)

FARM-DEPENDENT DECONTAMINATION DIST

FARM-DEPENDENT DECONTAMINATION DIST,
POP.-DEPENDENT DECONTAMINATION DIST

FARM-DEPENDENT INTERDICTION DIST.

POP. -DEPENDENT INTERDICTION DIST

FARM-DEPENDENT CONDEMNATION DIST.
ROP. -DEPENDENT CONDEMNATION DIST.

MILK DISPOSAL DIST
CROP DISPOSAL DIST

$\begin{array}{lllllll}.0000 & 7.31 E+09 & 3.51 E+09 & 1.56 E+10 & 2.59 E+10 & 4.41 E+10 & 7.2\end{array}$

$\begin{array}{llllllllll}.9121 & 6.35 \mathrm{E}+08 & 1.26 \mathrm{E}+08 & 1.50 \mathrm{E}+09 & 3.33 \mathrm{E}+09 & 6.70 \mathrm{E}+09 & 7.80 \mathrm{E}+09 & 4.44 \mathrm{E}+10 & 5.71 \mathrm{E}-05\end{array}$

$\begin{array}{llllllllll}1.0000 & 1.45 \mathrm{E}+07 & 1.18 \mathrm{E}+07 & 2.90 \mathrm{E}+07 & 3.38 \mathrm{E}+07 & 4.68 \mathrm{E}+07 & 5.25 \mathrm{E}+07 & 7.91 \mathrm{E}+07 & 1.45 \mathrm{E}-04\end{array}$

$\begin{array}{lllllllll}0.0000 & 0.00 \mathrm{E}+00 & 0.00 \mathrm{E}+00 & 0.00 \mathrm{E}+00 & 0.00 \mathrm{E}+00 & 0.00 \mathrm{E}+00 & 0.00 \mathrm{E}+00 & 0.00 \mathrm{E}+00 & 0.00 \mathrm{E}+00\end{array}$

$\begin{array}{lllllllll}1.0000 & 3.38 \mathrm{E}+07 & 4.87 \mathrm{E}+06 & 9.63 \mathrm{E}+07 & 1.84 \mathrm{E}+08 & 2.98 \mathrm{E}+08 & 3.71 \mathrm{E}+08 & 1.91 \mathrm{E}+09 & 4.00 \mathrm{E}-04 \\ 1.0000 & 4.12 \mathrm{E}+08 & 1.42 \mathrm{E}+08 & 1.23 \mathrm{E}+09 & 1.89 \mathrm{E}+09 & 2.14 \mathrm{E}+09 & 2.21 \mathrm{E}+09 & 2.60 \mathrm{E}+09 & 1.43 \mathrm{E}-04\end{array}$

$\begin{array}{llllllllll}1.0000 & 4.30 \mathrm{E}+09 & 1.87 \mathrm{E}+09 & 1.12 \mathrm{E}+10 & 1.69 \mathrm{E}+10 & 2.61 \mathrm{E}+10 & 3.01 \mathrm{E}+10 & 5.72 \mathrm{E}+10 & 2.15 \mathrm{E}-04 & 102\end{array}$

$\begin{array}{llllllllll}1.0000 & 1.35 \mathrm{E}+08 & 1.14 \mathrm{E}+08 & 2.32 \mathrm{E}+08 & 2.84 \mathrm{E}+08 & 3.22 \mathrm{E}+08 & 3.33 \mathrm{E}+08 & 3.57 \mathrm{E}+08 & 1.26 \mathrm{E}-03 & 52 \\ 1.0000 & 1.02 \mathrm{E}+09 & 3.71 \mathrm{E}+08 & 2.68 \mathrm{E}+09 & 5.06 \mathrm{E}+09 & 6.45 \mathrm{E}+09 & 7.05 \mathrm{E}+09 & 7.34 \mathrm{E}+09 & 2.37 \mathrm{E}-03 & 55\end{array}$

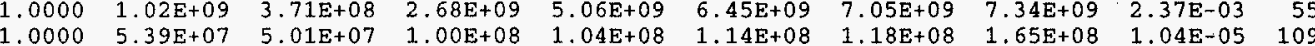

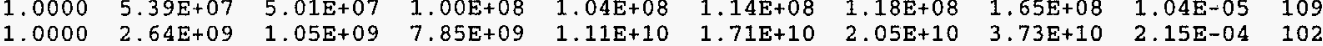

$\begin{array}{llllllllll}1.0000 & 4.20 \mathrm{E}+07 & 3.23 \mathrm{E}+07 & 9.12 \mathrm{E}+07 & 1.05 \mathrm{E}+08 & 1.22 \mathrm{E}+08 & 1.30 \mathrm{E}+08 & 1.49 \mathrm{E}+08 & 1.17 \mathrm{E}-03 & 49\end{array}$

$\begin{array}{lllllllllll}0.9121 & 6.35 \mathrm{E}+08 & 1.26 \mathrm{E}+08 & 1.50 \mathrm{E}+09 & 3.33 \mathrm{E}+09 & 6.70 \mathrm{E}+09 & 7.80 \mathrm{E}+09 & 4.44 \mathrm{E}+10 & 5.71 \mathrm{E}-05 & 75\end{array}$

$\begin{array}{lllllllllll}1.0000 & 1.45 \mathrm{E}+07 & 1.18 \mathrm{E}+07 & 2.90 \mathrm{E}+07 & 3.38 \mathrm{E}+07 & 4.68 \mathrm{E}+07 & 5.25 \mathrm{E}+07 & 7.91 \mathrm{E}+07 & 1.45 \mathrm{E}-04 & 92 \\ 1.0000 & 5.94 \mathrm{E}+06 & 2.40 \mathrm{E}+06 & 1.24 \mathrm{E}+07 & 3.08 \mathrm{E}+07 & 6.16 \mathrm{E}+07 & 8.41 \mathrm{E}+07 & 1.11 \mathrm{E}+08 & 1.11 \mathrm{E}-03 & 86\end{array}$

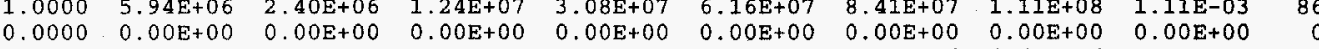

$\begin{array}{llllllllll}1.0000 & 1.33 \mathrm{E}+06 & 9.85 \mathrm{E}+05 & 2.57 \mathrm{E}+06 & 3.05 \mathrm{E}+06 & 3.29 \mathrm{E}+06 & 3.40 \mathrm{E}+06 & 4.61 \mathrm{E}+06 & 9.57 \mathrm{E}-06 & 24\end{array}$

$\begin{array}{lllllllll}1.0000 & 2.00 \mathrm{E}+02 & 1.78 \mathrm{E}+02 & 3.77 \mathrm{E}+02 & 4.69 \mathrm{E}+02 & \text { NOT-FOUND NOT-FOUND } & 5.63 \mathrm{E}+02 & 4.07 \mathrm{E}-02 \\ 1.0000 & 2.10 \mathrm{E}+02 & 1.96 \mathrm{E}+02 & 3.88 \mathrm{E}+02 & 4.79 \mathrm{E}+02 & \text { NOT-FOUND NOT-FOUND } & 5.63 \mathrm{E}+02 & 4.36 \mathrm{E}-02\end{array}$

$\begin{array}{lllllll}1.0000 & 2.99 \mathrm{E}+02 & 2.30 \mathrm{E}+02 & 6.90 \mathrm{E}+02 & \text { NOT-FOUND NOT-FOUND NOT-FOUND } & 8.05 \mathrm{E}+02 & 9.36 \mathrm{E}-02 \\ 1.0000 & 2.10 \mathrm{E}+02 & 1.96 \mathrm{E}+02 & 3.88 \mathrm{E}+02 & 4.79 \mathrm{E}+02 & 02\end{array}$

$\begin{array}{lllllllll}1.0000 & 2.10 \mathrm{E}+02 & 1.96 \mathrm{E}+02 & 3.88 \mathrm{E}+02 & 4.79 \mathrm{E}+02 & \text { NOT-FOUND NOT-FOUND } & 5.63 \mathrm{E}+02 & 4.36 \mathrm{E}-02\end{array}$

$\begin{array}{llllllllll}1.0000 & 2.79 \mathrm{E}+01 & 2.81 \mathrm{E}+01 & 3.67 \mathrm{E}+01 & 4.03 \mathrm{E}+01 & 4.99 \mathrm{E}+01 & 5.54 \mathrm{E}+01 & 8.05 \mathrm{E}+01 & 1.08 \mathrm{E}-03\end{array}$

$\begin{array}{llllllllll}0.9121 & 1.81 \mathrm{E}+01 & 2.06 \mathrm{E}+01 & 3.13 \mathrm{E}+01 & 3.42 \mathrm{E}+01 & 4.19 \mathrm{E}+01 & 4.57 \mathrm{E}+01 & 6.44 \mathrm{E}+01 & 2.45 \mathrm{E}-03\end{array}$

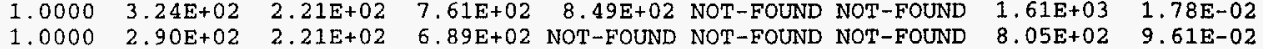

AFFECTED AREA/POPULATION $0-1609 \mathrm{~km}$ FARM DECONTAMINATION (HECTARES) POP. DECONTAMINATION (INDIVIDUAI )

FARM INTERDICTION (HECTARES)

DARM COMDEMTATTON (INECTIDUS)

POR. CONDEMNATION (INDIVIDUALS)

MILK DISPOSAL AREA (HECTIRES)

CROP DISPOSAL AREA (HECTARES)

$\begin{array}{lllllllll}1.0000 & 3.19 \mathrm{E}+05 & 1.70 \mathrm{E}+05 & 7.65 \mathrm{E}+05 & 9.25 \mathrm{E}+05 & 2.05 \mathrm{E}+06 & 2.12 \mathrm{E}+06 & 2.29 \mathrm{E}+06 & 9.70 \mathrm{E}-04\end{array}$

$\begin{array}{lllllllll}1.0000 & 6.62 \mathrm{E}+05 & 3.04 \mathrm{E}+05 & 1.40 \mathrm{E}+06 & 2.62 \mathrm{E}+06 & 4.69 \mathrm{E}+06 & 7.29 \mathrm{E}+06 & 2.19 \mathrm{E}+07 & 1.11 \mathrm{E}-03\end{array}$

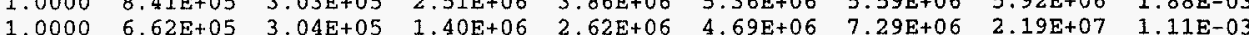

$1.0000 \quad 6.98 \mathrm{E}+03 \quad 5.99 \mathrm{E}+03 \quad 1.28 \mathrm{E}+04 \quad 1.53 \mathrm{E}+04 \quad 2.35 \mathrm{E}+04 \quad 2.82 \mathrm{E}+04 \quad 3.81 \mathrm{E}+04 \quad 1.45 \mathrm{E}-04$

$\begin{array}{lllllllll}0.9121 & 7.19 \mathrm{E}+03 & 1.33 \mathrm{E}+03 & 1.60 \mathrm{E}+04 & 3.34 \mathrm{E}+04 & 9.36 \mathrm{E}+04 & 1.07 \mathrm{E}+05 & 4.99 \mathrm{E}+05 & 5.71 \mathrm{E}-05\end{array}$

$\begin{array}{lllllllll}1.0000 & 1.20 \mathrm{E}+06 & 2.71 \mathrm{E}+05 & 3.75 \mathrm{E}+06 & 5.36 \mathrm{E}+06 & 9.34 \mathrm{E}+06 & 2.18 \mathrm{E}+07 & 3.69 \mathrm{E}+07 & 2.28 \mathrm{E}-04\end{array}$

$\begin{array}{lllllllll}1.0000 & 8.09 \mathrm{E}+05 & 2.78 \mathrm{E}+05 & 2.49 \mathrm{E}+06 & 3.86 \mathrm{E}+06 & 5.36 \mathrm{E}+06 & 5.59 \mathrm{E}+06 & 5.92 \mathrm{E}+06 & 1.88 \mathrm{E}-03\end{array}$

AFFECTED AREA/POPULATION $0-80.5 \mathrm{~km}$

FARM DECONTAMINATION (HECTARES)

POP. DECONTAMINATION (INDIVIDUALS)

1.0000
1.0000
1.0000

$\begin{array}{llllllllll}1.0000 & 5.73 \mathrm{E}+04 & 4.57 \mathrm{E}+04 & 1.04 \mathrm{E}+05 & 1.10 \mathrm{E}+05 & 1.27 \mathrm{E}+05 & 1.35 \mathrm{E}+05 & 2.22 \mathrm{E}+05 & 1.04 \mathrm{E}-05 & 109\end{array}$

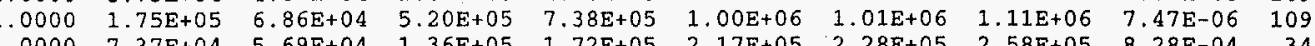

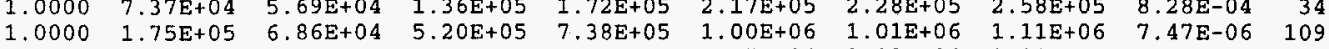
POP. INTERDICTION (INDIVIDUALS) 
POP. CONDEMNATION (INDIVIDUALS) MILK DISPOSAL AREA (HECTARES

$09 / 26 / 96 \quad 08: 40: 08 \quad$ PAGE 10 $\begin{array}{llllllllll}0.9121 & 7.19 \mathrm{E}+03 & 1.33 \mathrm{E}+03 & 1.60 \mathrm{E}+04 & 3.34 \mathrm{E}+04 & 9.36 \mathrm{E}+04 & 1.07 \mathrm{E}+05 & 4.99 \mathrm{E}+05 & 5.71 \mathrm{E}-05 & 75\end{array}$

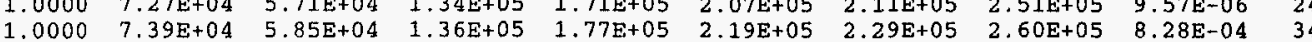

MAXIMUM ANNUAL FOOD DOSE (EFFECTIVE)

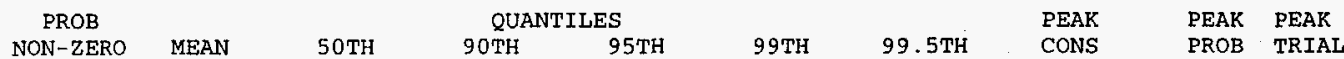

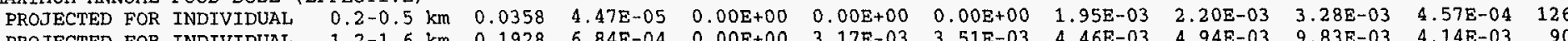

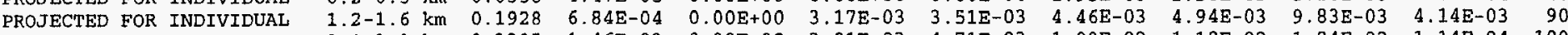

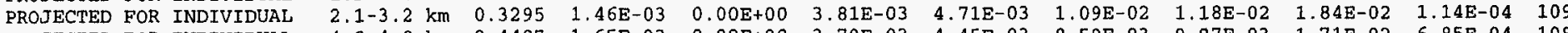

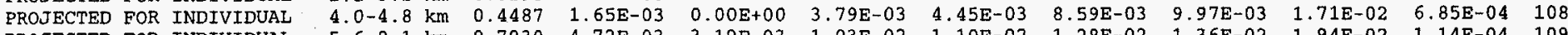

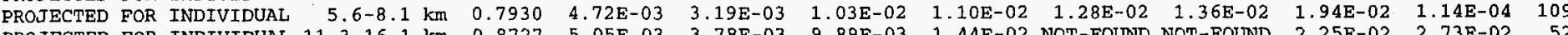

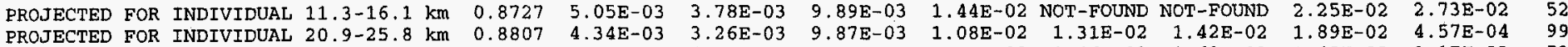

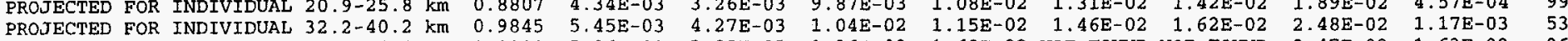

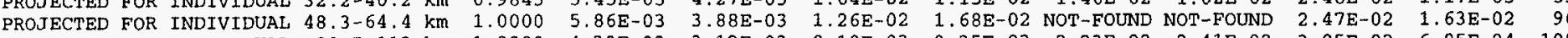

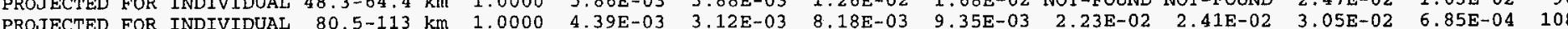

MAXIMUM ANNUAL FOOD DOSE (THYROID)

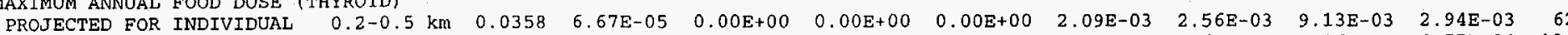

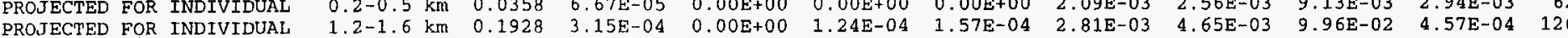

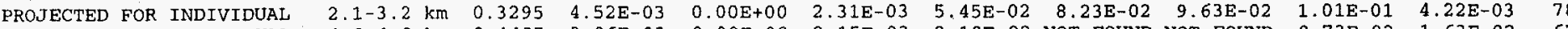

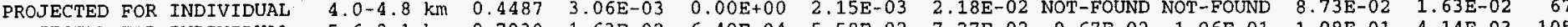

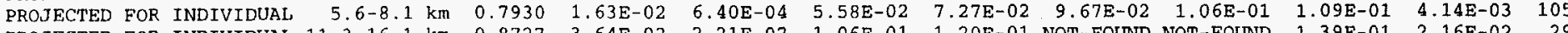

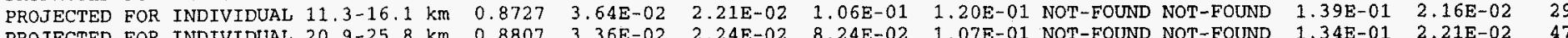

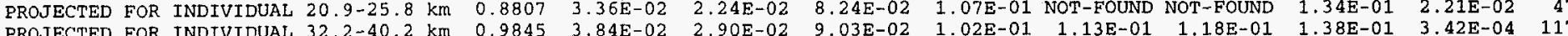

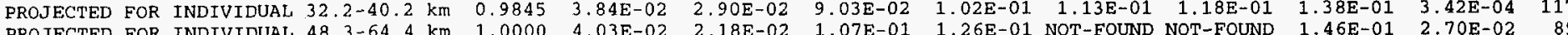
PROJECTED FOR

SOURCE TERM 1 OF 2 :

SECOND DRAFT 1150, WORST CASE SOURCE TERM FOR EARLYY FATALITIES

RESULT NAME = HEALTH EFFECTS CASES

$$
\text { CAN FAT/TOTAL }
$$

PEOPLE FRACTION $=$

0.9500

$0-80.5 \mathrm{~km}$

\begin{tabular}{|c|c|c|c|}
\hline \multicolumn{2}{|c|}{ OVERALL } & \multicolumn{2}{|c|}{ EMER, RESP, \# 1} \\
\hline $\mathrm{x}$ & $\mathrm{PROB}>=\mathrm{X}$ & $\mathrm{x}$ & PROB $>=$ \\
\hline $00 \mathrm{E}-04$ & $1.00 \mathrm{E}+00$ & 1.00E-04 & $1.00 \mathrm{E}+00$ \\
\hline OOE -04 & $1.00 \mathrm{E}+00$ & $2.00 E-04$ & $1.00 E+00$ \\
\hline $00 \mathrm{E}-04$ & $1.00 \mathrm{E}+00$ & $3.00 E-04$ & $1.00 \mathrm{E}+00$ \\
\hline $00 \mathrm{E}-04$ & $1.00 \mathrm{E}+00$ & 5. $00 \mathrm{E}-04$ & $1.00 \mathrm{E}+00$ \\
\hline OOE -04 & $1.00 \mathrm{E}+00$ & $7.00 \mathrm{E}-04$ & $1.00 \mathrm{E}+00$ \\
\hline OOE -03 & $1.00 E+00$ & $1.00 \mathrm{E}-03$ & 1. $00 \mathrm{E}+00$ \\
\hline $00 E-03$ & $1.00 \mathrm{E}+00$ & $2.00 \mathrm{E}-03$ & $1.00 \mathrm{E}+00$ \\
\hline $00 E-03$ & $1.00 \mathrm{E}$ & $3.00 \mathrm{E}-03$ & $1.00 \mathrm{E}+00$ \\
\hline $00 \mathrm{E}-03$ & $1.00 \mathrm{E}+00$ & 5. $00 \mathrm{E}-03$ & $1.00 \mathrm{E}+00$ \\
\hline $00 E-03$ & $1.00 \mathrm{E}+00$ & $7.00 \mathrm{E}-03$ & $1.00 \mathrm{E}+00$ \\
\hline $00 \mathrm{E}-02$ & $1.00 \mathrm{E}+00$ & $1.00 \mathrm{E}-02$ & $1.00 \mathrm{E}+00$ \\
\hline $00 \mathrm{E}-02$ & 1. $00 \mathrm{E}+00$ & $2.00 E-02$ & $1.00 \mathrm{E}+00$ \\
\hline & $1.00 \mathrm{E}+00$ & $3.00 \mathrm{E}-02$ & $1.00 \mathrm{E}+00$ \\
\hline $.00 E-02$ & $1.00 \mathrm{E}+00$ & $5.00 \mathrm{E}-02$ & $1.00 \mathrm{E}+00$ \\
\hline & $1.00 \mathrm{E}+00$ & $7.00 \mathrm{E}-02$ & $1.00 \mathrm{E}+00$ \\
\hline & & & \\
\hline
\end{tabular}

$$
0.0500
$$

EMER, RESP. \# 2 $\begin{array}{cc}X & x \\ X & \text { PROB }>=X\end{array}$ $1.00 \mathrm{E}-04 \quad 1.00 \mathrm{E}+00$ $2.00 \mathrm{E}-04 \quad 1.00 \mathrm{E}+00$ $3.00 \mathrm{E}-04 \quad 1.00 \mathrm{E}+00$ $5.00 \mathrm{E}-04 \quad 1.00 \mathrm{E}+00$ $7.00 \mathrm{E}-04 \quad 1.00 \mathrm{E}+0$ $1.00 \mathrm{E}-03 \quad 1.00 \mathrm{E}+0$ $2.00 \mathrm{E}-03 \quad 1.00 \mathrm{E}+00$ $5.00 \mathrm{E}-03 \quad 1.00 \mathrm{E}+00$ $7.00 \mathrm{E}-03 \quad 1.00 \mathrm{E}+00$ $1.00 \mathrm{E}-02 \quad 1.00 \mathrm{E}+00$ $2.00 \mathrm{E}-02 \quad 1.00 \mathrm{E}+00$ $3.00 \mathrm{E}-02 \quad 1.00 \mathrm{E}+00$ $5.00 \mathrm{E}-02 \quad 1.00 \mathrm{E}+00$ $7.00 \mathrm{E}-02 \quad 1.00 \mathrm{E}+00$ $1.00 \mathrm{E}-01 \quad 1.00 \mathrm{E}+00$
CHRONC RESULTS

$\begin{array}{cr}\mathrm{X} & \text { PROB }>=\mathrm{X} \\ 1.00 \mathrm{E}-04 & 1.00 \mathrm{E}+00 \\ 2.00 \mathrm{E}-04 & 1.00 \mathrm{E}+00 \\ 3.00 \mathrm{E}-04 & 1.00 \mathrm{E}+00 \\ 5.00 \mathrm{E}-04 & 1.00 \mathrm{E}+00 \\ 7.00 \mathrm{E}-04 & 1.00 \mathrm{E}+00 \\ 1.00 \mathrm{E}-03 & 1.00 \mathrm{E}+00 \\ 2.00 \mathrm{E}-03 & 1.00 \mathrm{E}+00 \\ 3.00 \mathrm{E}-03 & 1.00 \mathrm{E}+00 \\ 5.00 \mathrm{E}-03 & 1.00 \mathrm{E}+00 \\ 7.00 \mathrm{E}-03 & 1.00 \mathrm{E}+00 \\ 1.00 \mathrm{E}-02 & 1.00 \mathrm{E}+00 \\ 2.00 \mathrm{E}-02 & 1.00 \mathrm{E}+00 \\ 3.00 \mathrm{E}-02 & 1.00 \mathrm{E}+00 \\ 5.00 \mathrm{E}-02 & 1.00 \mathrm{E}+00 \\ 7.00 \mathrm{E}-02 & 1.00 \mathrm{E}+00 \\ 1.00 \mathrm{E}-01 & 1.00 \mathrm{E}+00\end{array}$




\begin{tabular}{|c|c|c|c|c|c|c|c|}
\hline $.00 \mathrm{E}-01$ & $1.00 E+00$ & $2.00 E-01$ & $1.00 \mathrm{E}+00$ & $2.00 \mathrm{E}-01$ & $1.00 \mathrm{E}+00$ & $2.00 \mathrm{E}-01$ & $1.00 \mathrm{E}+00$ \\
\hline & $1.00 \mathrm{E}+00$ & $3.00 \mathrm{E}-01$ & $1.00 E+00$ & $3.00 \mathrm{E}-01$ & $1.00 \mathrm{E}+00$ & $3.00 \mathrm{E}-01$ & $1.00 \mathrm{E}+00$ \\
\hline 5.00E-01 & $1.00 \mathrm{E}+00$ & $5.00 E-01$ & $1.00 \mathrm{E}+00$ & $5.00 E-01$ & $1.00 \mathrm{E}+00$ & $5.00 \mathrm{E}-01$ & $1.00 \mathrm{E}+00$ \\
\hline $.00 \mathrm{E}-01$ & $1.00 \mathrm{E}+00$ & $7.00 \mathrm{E}-01$ & $1.00 \mathrm{E}+00$ & $7.00 \mathrm{E}-01$ & $1.00 \mathrm{E}+00$ & $7.00 \mathrm{E}-01$ & $1.00 \mathrm{E}+00$ \\
\hline $1.00 E+00$ & $1.00 \mathrm{E}+00$ & $1.00 \mathrm{E}+00$ & $1.00 \mathrm{E}+00$ & 1. $.00 E+00$ & 1. $.00 \mathrm{E}+00$ & $1.00 \mathrm{E}+00$ & $1.00 \mathrm{E}+00$ \\
\hline $2.00 \mathrm{E}+00$ & $1.00 \mathrm{E}+00$ & $2.00 \mathrm{E}+00$ & $1.00 \mathrm{E}+00$ & $2.00 \mathrm{E}+00$ & $1.00 \mathrm{E}+00$ & $2.00 \mathrm{E}+00$ & $1.00 \mathrm{E}+00$ \\
\hline 3. $00 \mathrm{E}+00$ & $1.00 \mathrm{E}+00$ & 3. $00 \mathrm{E}+00$ & $1.00 \mathrm{E}+00$ & $3.00 \mathrm{E}+00$ & $1.00 \mathrm{E}+00$ & $3.00 \mathrm{E}+00$ & $1.00 \mathrm{E}+00$ \\
\hline $5.00 \mathrm{E}+00$ & $1.00 E+00$ & $5.00 \mathrm{E}+00$ & $1.00 \mathrm{E}+00$ & $5.00 E+00$ & $1.00 \mathrm{E}+00$ & $5.00 \mathrm{E}+00$ & 1. $.00 \mathrm{E}+00$ \\
\hline $7.00 E+00$ & $1.00 E+00$ & $7.00 E+00$ & $1.00 \mathrm{E}+00$ & $7.00 \mathrm{E}+00$ & 1. $.00 \mathrm{E}+00$ & $7.00 \mathrm{E}+00$ & $1.00 E+00$ \\
\hline $1.00 \mathrm{E}+01$ & $1.00 \mathrm{E}+00$ & $1.00 \mathrm{E}+01$ & $1.00 \mathrm{E}+00$ & $1.00 \mathrm{E}+01$ & $1.00 \mathrm{E}+00$ & $1.00 \mathrm{E}+01$ & $1.00 \mathrm{E}+00$ \\
\hline $2.00 \mathrm{E}+01$ & $1.00 \mathrm{E}+00$ & $2.00 E+01$ & $9.99 \mathrm{E}-01$ & $2.00 \mathrm{E}+01$ & 1. $00 \mathrm{E}+00$ & $2.00 \mathrm{E}+01$ & $1.00 \mathrm{E}+00$ \\
\hline $3.00 \mathrm{E}+01$ & $1.00 \mathrm{E}+00$ & $3.00 E+01$ & $9.99 \mathrm{E}-01$ & $3.00 \mathrm{E}+01$ & $1.00 \mathrm{E}+00$ & $3.00 \mathbf{E}+01$ & $1.00 \mathrm{E}+00$ \\
\hline $5.00 \mathrm{E}+01$ & $1.00 \mathrm{E}+00$ & $5.00 \mathrm{E}+01$ & $9.90 \mathrm{E}-01$ & $5.00 \mathrm{E}+01$ & 1. $.00 \mathrm{E}+00$ & $5.00 \mathrm{E}+01$ & 1. $.00 \mathrm{E}+00$ \\
\hline & & & & & & & \\
\hline $1.00 E+02$ & $9.99 \mathrm{E}-01$ & $1.00 \mathrm{E}+02$ & $9.43 E-01$ & $1.00 \mathrm{E}+02$ & $9.91 \mathrm{E}-01$ & $1.00 \mathrm{E}+02$ & $9.97 \mathrm{E}-01$ \\
\hline $2.00 \mathrm{E}+02$ & $9.92 \mathrm{E}-01$ & $2.00 \mathrm{E}+02$ & $7.98 \mathrm{E}-01$ & $2.00 \mathrm{E}+02$ & $8.87 E-01$ & $2.00 E+02$ & $8.60 \mathrm{E}-01$ \\
\hline $3.00 E+02$ & $9.57 \mathrm{E}-0$ & 3.0 & & $E+02$ & 7.5 & $3.00 E+02$ & $6.40 \mathrm{E}-01$ \\
\hline & 8.00 & .00 & 4.44 & 5.0 & 5.7 & $5.00 E+02$ & $3 E-01$ \\
\hline+02 & 6.321 & 7.00 & 3.2 & & & 7.0 & \\
\hline $1.00 \mathrm{E}+03$ & $4.83 \mathrm{E}-01$ & $1.00 E+03$ & $2.42 \mathrm{E}-01$ & 1. $00 \mathrm{E}+03$ & $3.03 \mathrm{E}-01$ & $1.00 \mathrm{E}+03$ & $2.40 \mathrm{E}-01$ \\
\hline $2.00 \mathrm{E}+03$ & 2.361 & 2.00 & $1.34 \mathrm{E}-$ & 2.0 & 1.4 & $2.00 \mathrm{E}+03$ & \\
\hline $3.00 E+03$ & $1.54 \mathrm{E}-0$ & $3.00 \mathrm{E}$ & $5.48 \mathrm{E}$ & 3.0 & $6.61 \mathrm{E}$ & $3.00 \mathrm{E}+03$ & $8 E-02$ \\
\hline 5.00 & 7.47 & 5.0 & 1.7 & $E+03$ & $1.86 \mathrm{~F}$ & $5.00 \mathrm{E}+03$ & $2.64 \mathrm{E}-03$ \\
\hline $7.00 \mathrm{E}+03$ & $3.39 \mathrm{E}-$ & 7.00 & 1.0 & & & $6.79 \mathrm{E}$ & $2.15 \mathrm{E}-04$ \\
\hline $1.00 \mathrm{E}+04$ & $8.40 \mathrm{E}-$ & 1.00 & $3.27 \mathrm{E}-\mathrm{C}$ & $9.00 \mathrm{E}+03$ & $1.11 \mathrm{E}-03$ & N.D. & N.I \\
\hline $2.00 \mathrm{E}+04$ & $2.85 E-$ & $1.90 E+04$ & $2.85 E-04$ & N.D. & N.D. & N.D. & N.D. \\
\hline 2.07 & $285 \mathrm{E}$ & N.D. & N.D. & N.D. & N.D & N.D. & N.D. \\
\hline & & & & & & & \\
\hline & N.D. & N.D. & & & & N.D & \\
\hline & & & & & & & \\
\hline
\end{tabular}

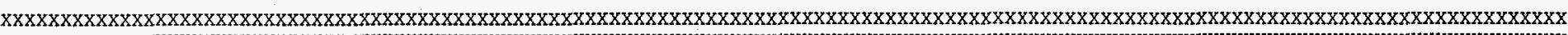

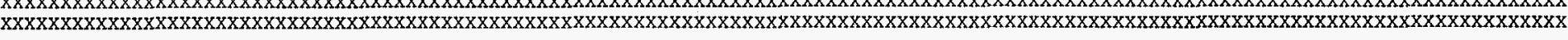

DATE AND TIME OF RUN = MACCS2 09/26/96 08:40:08 Version 1.12 , Last Modified 9/25/96 by D. Chanin

"ATMOS" DESCRIPTION = IN1A. INP, Sample Problem A--Using Table-Lookup Sigmas, ATMOS input
"EARLY" DESCRIPTION = IN2A. INP, Sample Problem A of NUREG/CR-4691, Vol. 1, EARLY input

"EARLY" DESCRIPTION = IN2A. INP, Sample Problem A of NUREG/CR-4691, Vol. 1, EARLY inDut

SOURCE TERM 2 OF 2 :

RELEASE FRACTIONS OF SOURCE TERM 1 REDUCED BY A FACTOR OF TEN

OVERALL RESULTS OBTAINED BY COMBINING 2 EMERGENCY RESPONSE COHORTS FROM "EARLY" WITH THE WEIGHTING FRACTIONS BELOW APPLIED TO THEM: FRACTION OF THE PEOPLE

COHORT 1 = EVACUATION WITHIN 10 MILES, RELOCATION MODELS APPLY ELSEWHERE

COHORT 2 = NO EVACUATION, RELOCATION MODELS APPLY EVERYWHERE

AND THEN MERGING THE 2 RESULTS ABOVE WITH THE SINGLE SET OF RESULTS FROM "CHRONC" DESCRIBED BELOW:

COHORT 3 = IN3A_N.INP, Sample Problem A, "NeW" COMIDA2-Based Food Mode1

RESULTS WHICH ARE PRODUCED ONLY BY "EARLY" OR ONLY BY "CHRONC" ARE PRESENTED IN LATER SECTIONS.
$09 / 26 / 96 \quad 08: 40: 08$
PAGE 12
PROB
$50 \mathrm{TH}$
QUANTILES
90TH $95 \mathrm{TH}$
99TH
99.5TH PEAK
$\begin{array}{ll}\text { PEAK } & \text { PEAK } \\ \text { PROB } & \text { TRIAL }\end{array}$
HEALTH EFFECTS CASES

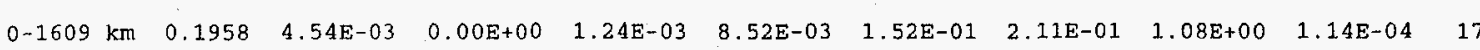


ERL INJ/PRODROMAL VOMIT

ERL INJ / PNEUMONITIS

ERL

DRL

CAN FAT/TOTAL

CAN FAT/THYROID

CAN FAT/BREAST

CAN FAT/GI

CAN FAT/LEUKEMIA

CAN FAT/BONE

CAN FAT/OTHER

CAN INJ/THYROID

CAN INJ/BREAST

CAN FAT/TOTAL

ERL INJ/PRODROMAL VOMI

ERL INJ/DIARRHEA

ERL INJ/PNEUMONITIS

ERL INJ/THYROIDITIS

ERL INJ/SKIN ERYTHEMA

ERL INJ/TRANSEPIDERMA

CAN FAT/TOTAL

EARLY FATALITY DISTANCE $(\mathrm{km})$

ERL FAT/TOTAL RISK $>0.000$ ERL INJ/DIARRHEA

ERL INJ/THYROIDITIS

$\begin{array}{lllllllllll}0-1609 \mathrm{~km} & 0.4849 & 1.48 \mathrm{E}-01 & 0.00 \mathrm{E}+00 & 3.20 \mathrm{E}-01 & 8.49 \mathrm{E}-01 & 2.94 \mathrm{E}+00 & 3.66 \mathrm{E}+00 & 6.90 \mathrm{E}+00 & 8.28 \mathrm{E}-04 & 94\end{array}$ $\begin{array}{lllllllllll}0-1609 \mathrm{~km} & 0.3026 & 3.57 \mathrm{E}-02 & 0.00 \mathrm{E}+00 & 3.81 \mathrm{E}-02 & 1.31 \mathrm{E}-01 & 1.06 \mathrm{E}+00 & 1.31 \mathrm{E}+00 & 2.68 \mathrm{E}+00 & 1.14 \mathrm{E}-04 & 17\end{array}$ $0-1609 \mathrm{~km} 0.0872 \quad 6.48 \mathrm{E}-03 \quad 0.00 \mathrm{E}+000.0 \mathrm{E}$ $0.1609 \mathrm{~km} \quad 0.6486 \quad 7.68 \mathrm{E}+00 \quad 1.12 \mathrm{E}-01 \quad 1.37 \mathrm{E}+01 \quad 2.73 \mathrm{E}+01 \quad 1.48 \mathrm{E}+02 \quad 2.16 \mathrm{E}+02 \quad 6.39 \mathrm{E}+02 \quad 2.85 \mathrm{E}-04 \quad 85$

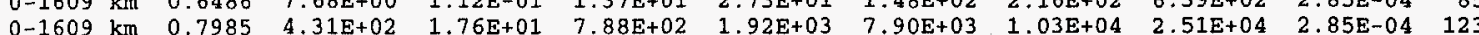

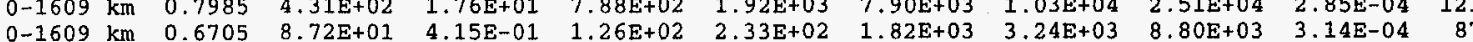
$\begin{array}{lllllllllll}0-1609 \mathrm{~km} & 1.0000 & 1.95 \mathrm{E}+03 & 1.32 \mathrm{E}+03 & 4.13 \mathrm{E}+03 & 5.45 \mathrm{E}+03 & 9.04 \mathrm{E}+03 & 1.06 \mathrm{E}+04 & 1.95 \mathrm{E}+04 & 1.14 \mathrm{E}-04 & 112\end{array}$

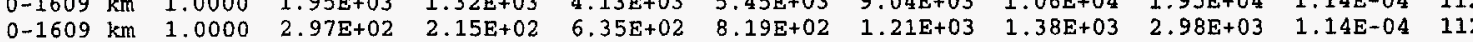
$\begin{array}{lllllllllll}0-1609 \mathrm{~km} & 1.0000 & 3.86 \mathrm{E}+01 & 2.74 \mathrm{E}+01 & 7.88 \mathrm{E}+01 & 1.04 \mathrm{E}+02 & 1.68 \mathrm{E}+02 & 2.02 \mathrm{E}+02 & 3.58 \mathrm{E}+02 & 1.14 \mathrm{E}-04 & 112\end{array}$

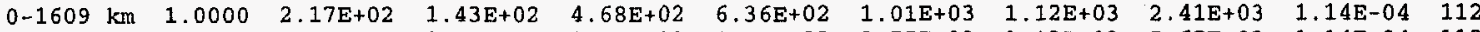

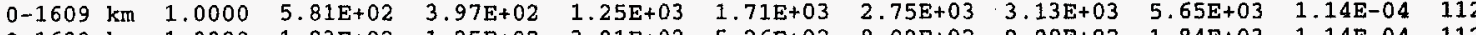

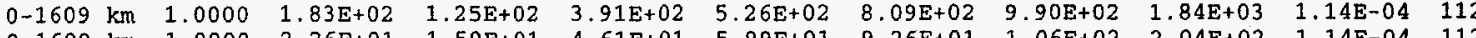

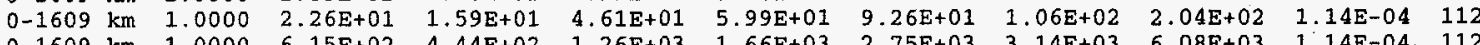

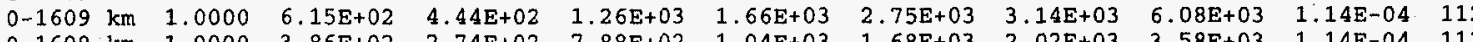
$\begin{array}{lllllllllll}0-1609 \mathrm{~km} & 1.0000 & 3.86 \mathrm{E}+02 & 2.74 \mathrm{E}+02 & 7.88 \mathrm{E}+02 & 1.04 \mathrm{E}+03 & 1.68 \mathrm{E}+03 & 2.02 \mathrm{E}+03 & 3.58 \mathrm{E}+03 & 1.14 \mathrm{E}-04 & 112\end{array}$

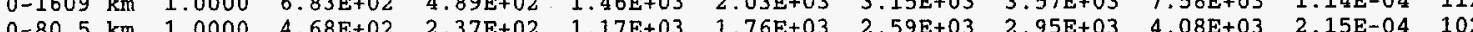

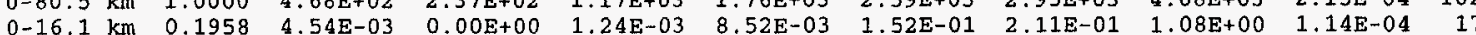

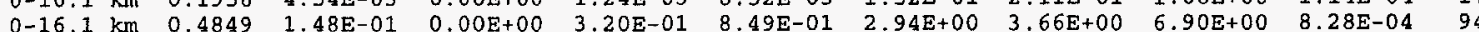

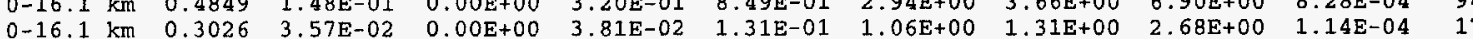
$\begin{array}{llllllllllll}0-16.1 \mathrm{~km} & 0.0312 & 3.92 \mathrm{E}-04 & 0.00 \mathrm{E}+00 & 0.00 \mathrm{E}+00 & 0.00 \mathrm{E}+00 & 1.18 \mathrm{E}-02 & 1.72 \mathrm{E}-02 & 4.25 \mathrm{E}-01 & 1.14 \mathrm{E}-04 & 17\end{array}$ $\begin{array}{lllllllllll}0-16.1 \mathrm{~km} & 0.0872 & 6.48 \mathrm{E}-03 & 0.00 \mathrm{E}+00 & 0.00 \mathrm{E}+00 & 3.12 \mathrm{E}-02 & 1.39 \mathrm{E}-01 & 1.85 \mathrm{E}-01 & 1.03 \mathrm{E}+00 & 8.28 \mathrm{E}-04 & 94\end{array}$ $\begin{array}{lllllllllll}0-16.1 \mathrm{~km} & 0.6474 & 7.59 \mathrm{E}+00 & 1.09 \mathrm{E}-01 & 1.34 \mathrm{E}+01 & 2.72 \mathrm{E}+01 & 1.48 \mathrm{E}+02 & 2.16 \mathrm{E}+02 & 6.39 \mathrm{E}+02 & 2.85 \mathrm{E}-04 & 85\end{array}$

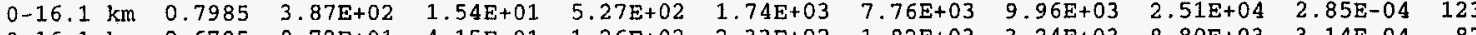

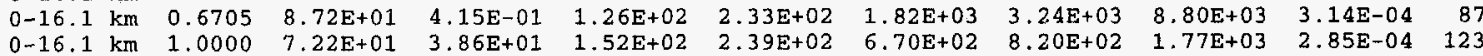

$0.9998 \quad 7.61 \mathrm{E}-01 \quad 5.28 \mathrm{E}-01 \quad 2.03 \mathrm{E}+00 \quad 2.44 \mathrm{E}+00 \quad$ NOT-FOUND NOT-FOUND $\quad 3.22 \mathrm{E}+00 \quad 2.25 \mathrm{E}-02$

$09 / 26 / 96 \quad 08: 40: 08 \quad$ PAGE 13

POPULATION EXCEEDING DOSE EARLY dOSE A-LUNGS $>5.00$ SV EARLY dOSe L-EDEWBODY >

AVERAGE INDIVIDUAL RISK

ERL FAT/TOTAL

ERL FAT TOTAL

ERL FAT/TOTAL

ERL FAI/TOTAL

POPULATION DOSE (SV)

L-EDEWBODY TOT LIF

L-EDEWBODY TOT LIF

$$
61 \mathrm{E}-01 \quad 5.28 \mathrm{E}
$$

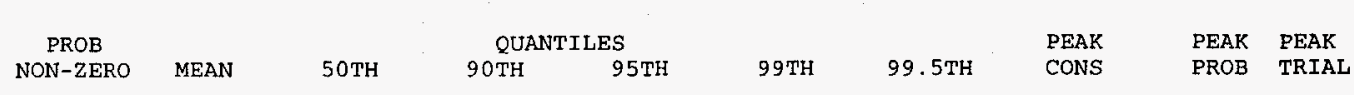
$\begin{array}{llllllllll}0.1958 & 8.72 \mathrm{E}-02 & 0.00 \mathrm{E}+00 & 8.39 \mathrm{E}-02 & 2.71 \mathrm{E}-01 & 2.52 \mathrm{E}+00 & 3.41 \mathrm{E}+00 & 7.20 \mathrm{E}+00 & 8.48 \mathrm{E}-04 & 94 \\ 0.0312 & 1.00 \mathrm{E}-02 & 0.00 \mathrm{E}+00 & 0.00 \mathrm{E}+00 & 0.00 \mathrm{E}+00 & 5.77 \mathrm{E}-01 & 7.56 \mathrm{E}-01 & 2.83 \mathrm{E}+00 & 1.49 \mathrm{E}-04 & 17 \\ 0.9999 & 6.74 \mathrm{E}+03 & 2.00 \mathrm{E}+03 & 1.56 \mathrm{E}+04 & 2.74 \mathrm{E}+04 & 8.65 \mathrm{E}+04 & 1.04 \mathrm{E}+05 & 1.78 \mathrm{E}+05 & 5.71 \mathrm{E}-05 & 75\end{array}$

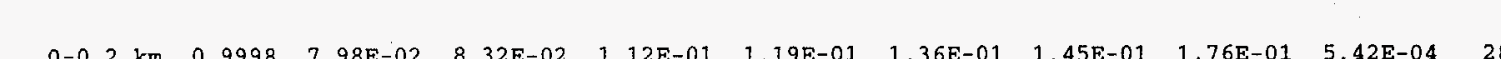
$\begin{array}{rllllllllll}0-0.2 \mathrm{~km} & 0.9998 & 7.98 \mathrm{E}-02 & 8.32 \mathrm{E}-02 & 1.12 \mathrm{E}-01 & 1.19 \mathrm{E}-01 & 1.36 \mathrm{E}-01 & 1.45 \mathrm{E}-01 & 1.76 \mathrm{E}-01 & 5.42 \mathrm{E}-04 & 28 \\ 0.2-0.5 \mathrm{~km} & 0.7557 & 6.76 \mathrm{E}-03 & 1.80 \mathrm{E}-03 & 3.01 \mathrm{E}-02 & 3.16 \mathrm{E}-02 & 3.55 \mathrm{E}-02 & 3.73 \mathrm{E}-02 & 4.41 \mathrm{E}-02 & 4.85 \mathrm{E}-04 & 57\end{array}$

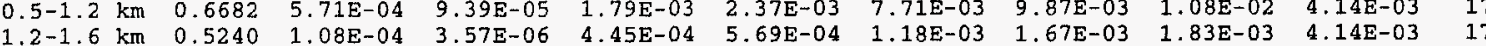
$\begin{array}{llllllllll}1.6-2.1 \mathrm{~km} & 0.3664 & 3.65 \mathrm{E}-05 & 0.00 \mathrm{E}+00 & 1.28 \mathrm{E}-04 & 2.34 \mathrm{E}-04 & \text { NOT-FOUND NOT-FOUND } & 5.86 \mathrm{E}-04 & 1.00 \mathrm{E}-02 & 79\end{array}$

$\begin{array}{rrrrrrrrrrr}0-16.1 \mathrm{~km} & 1.0000 & 1.50 \mathrm{E}+03 & 7.91 \mathrm{E}+02 & 3.20 \mathrm{E}+03 & 4.81 \mathrm{E}+03 & 1.09 \mathrm{E}+04 & 1.38 \mathrm{E}+04 & 3.10 \mathrm{E}+04 & 2.85 \mathrm{E}-04 & 85 \\ 0-80.5 \mathrm{~km} & 1.0000 & 1.04 \mathrm{E}+04 & 5.22 \mathrm{E}+03 & 2.81 \mathrm{E}+04 & 3.92 \mathrm{E}+04 & 6.02 \mathrm{E}+04 & 6.81 \mathrm{E}+04 & 9.24 \mathrm{E}+04 & 2.15 \mathrm{E}-04 & 102\end{array}$ $\begin{array}{lllllllllll}0-1609 \mathrm{~km} & 1.0000 & 4.41 \mathrm{E}+04 & 3.18 \mathrm{E}+04 & 9.90 \mathrm{E}+04 & 1.22 \mathrm{E}+05 & 1.94 \mathrm{E}+05 & 2.20 \mathrm{E}+05 & 4.41 \mathrm{E}+05 & 1.14 \mathrm{E}-04 & 112\end{array}$

POPULATION WEIGHTED RISK ERL FAT/TOTAL
CAN FAT/TOTAL

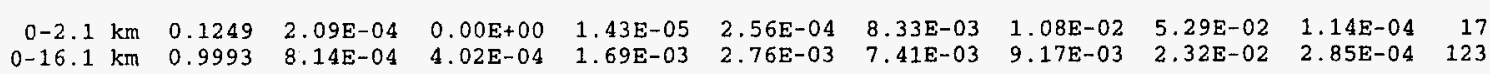
PEAK DOSE FOUND ON SPATIAL GRID (SV)

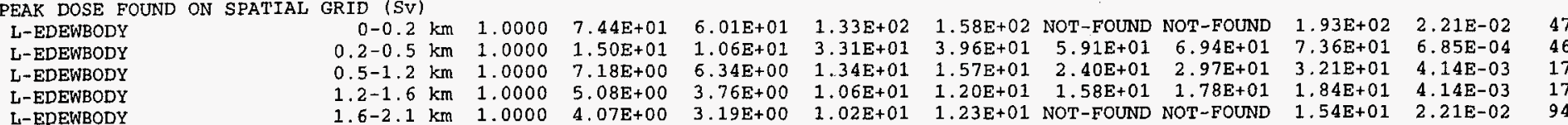




\begin{tabular}{|c|c|c|c|c|c|c|c|c|c|c|c|c|}
\hline L-EDEWBODY & $2.1-3.2$ & & 1.0000 & $2.76 \mathrm{E}+00$ & $1.80 E+00$ & $7.05 E+00$ & $9.48 \mathrm{E}+00$ & NOT-FOUND & NOT-FOUND & $1.48 \mathrm{E}+01$ & $2.21 \mathrm{E}-02$ & \\
\hline L-EDEWBODY & $3.2-4.0$ & $\mathrm{~km}$ & 1.0000 & $1.86 \mathrm{E}+00$ & $8.63 E-01$ & $6.34 \mathrm{E}+00$ & $7.22 \mathrm{E}+00$ & $7.90 \mathrm{E}+00$ & $8.21 \mathrm{E}+00$ & 1.11E+01 & $.43 \mathrm{E}-04$ & 129 \\
\hline L-EDEWBODY & $4.0-4.8$ & $\mathrm{~km}$ & 1.0000 & $1.51 \mathrm{E}+00$ & $6.83 \mathrm{E}-01$ & $4.38 E+00$ & $7.41 \mathrm{E}+00$ & NOT-FOUND & NOT-FOUND & $8.88 \mathrm{E}+00$ & $.17 \mathrm{E}-02$ & 88 \\
\hline L-EDEWBODY & $5.6-8.1$ & $\mathrm{~km}$ & 1.0000 & $8.02 \mathrm{E}-01$ & $2.58 \mathrm{E}-01$ & $2.35 \mathrm{E}+00$ & $2.95 \mathrm{E}+00$ & NOT-FOUND & NOT-FOUND & $4.39 \mathrm{E}+00$ & 2. $17 \mathrm{E}-02$ & \\
\hline L-EDEWBODY & $8.1-11.3$ & $\mathrm{~km}$ & 1.0000 & $3.44 \mathrm{E}-01$ & $1.64 \mathrm{E}-01$ & $9.45 \mathrm{E}-01$ & $1.25 \mathrm{E}+00$ & $2.17 \mathrm{E}+00$ & $2.79 \mathrm{E}+00$ & $2.99 \mathrm{E}+00$ & $4.14 E-03$ & \\
\hline L-EDENBODY & $16.1-20.9$ & $\mathrm{~km}$ & 1.0000 & $2.47 \mathrm{E}-01$ & $2.17 \mathrm{E}-01$ & $4.00 \mathrm{E}-01$ & $4.75 \mathrm{E}-01$ & NOT-FOUND & NOT-FOUND & $7.47 \mathrm{E}-01$ & $2.17 \mathrm{E}-02$ & \\
\hline L-EDEWBODY & $20.9-25.8$ & $\mathrm{~km}$ & 1.0000 & $1.90 \mathrm{E}-01$ & 1. $70 \mathrm{E}-01$ & 3. $44 \mathrm{E}-01$ & $3.88 \mathrm{E}-01$ & NOT-FOUND & NOT-FOUND & $4.61 \mathrm{E}-01$ & $91 \mathrm{E}-02$ & \\
\hline L-EDEWBODY & $25.8-32.2$ & $\mathrm{~km}$ & 1.0000 & $1.33 \mathrm{E}-01$ & $1.10 \mathrm{E}-01$ & $2.28 \mathrm{E}-01$ & 2. $88 \mathrm{E}-01$ & $3.20 \mathrm{E}-01$ & $3.30 \mathrm{E}-01$ & $3.80 \mathrm{E}-01$ & & \\
\hline L-EDEWBODY & $32.2-40.2$ & $\mathrm{~km}$ & & & & & & & & & & \\
\hline L-EDEWBODY & $40.2-48.3$ & $\mathrm{~km}$ & 1.0000 & $8.36 \Xi-02$ & $7.79 \mathrm{E}-02$ & $1.22 \mathrm{E}-01$ & $1.38 \mathrm{E}-01$ & $1.84 \mathrm{E}-01$ & $2.23 \mathrm{E}-01$ & $2.56 E-01$ & $3.77 \mathrm{E}-03$ & 110 \\
\hline L-EDEWBODY & $113-161$ & $\mathrm{~km}$ & & $3.25 \mathrm{E}-02$ & & $7.01 \mathrm{E}$ & & NOT- & FOUND & $9.76 \mathrm{E}-02$ & $1.63 \mathrm{E}-02$ & \\
\hline L-EDEWBODY & $161-241$ & $\mathrm{~km}$ & 1. & 1.6 & & & & & $E-02$ & & & \\
\hline L-EDEWBODY & $241-322$ & $\mathrm{~km}$ & 1.0000 & $9.72 E-03$ & $7.57 \mathrm{E}-03$ & $2.17 E-02$ & 2. $61 \mathrm{E}-02$ & 3. $62 \mathrm{E}-02$ & $4.08 \mathrm{E}-02$ & 4. $61 \mathrm{E}-02$ & $2.45 \mathrm{E}-03$ & 83 \\
\hline L-EDEWBODY & $322-563$ & $\mathrm{~km}$ & 1.0000 & $4.14 \mathrm{E}-03$ & $3.17 \mathrm{E}-03$ & $9.91 \mathrm{E}-03$ & $1.09 \mathrm{E}-02$ & $1.32 \mathrm{E}-02$ & $1.43 \mathrm{E}-02$ & $2.17 \mathrm{E}-02$ & $3.14 \mathrm{E}-04$ & 112 \\
\hline & & & & & & & & & & & & \\
\hline & & & & & & & & $8.87 \mathrm{E}$ & & & & \\
\hline
\end{tabular}

DATE AND TIME OF RUN = MACCS2 09/26/96 08:40:08 Version 1.12, Last Modified 9/25/96 by D. Chanin

"EARLY" DESCRIPTION $=$ IN1A. INP, Sample Problem A--USing Table-Lookup Sigmas, ATMOS input
DESCRIPTION $=$ IN2A. INP, Sample Problem A of NUREG/CR-4691, Vo1. 1, EARLY input

SOURCE TERM 2 OF $2:$
RELEASE FRACTIONS OF SOURCE TERM 1 REDUCED BY A FACTOR OF TEN

RESULTS FOR A SINGLE EMERGENCY RESPONSE COHORT WITHOUT ANY WEIGHTING FRACTIONS BEING APPLIED

COHORT 1 = EVACUATION WITHIN 10 MILES, RELOCATION MODELS APPLY ELSEWHERE

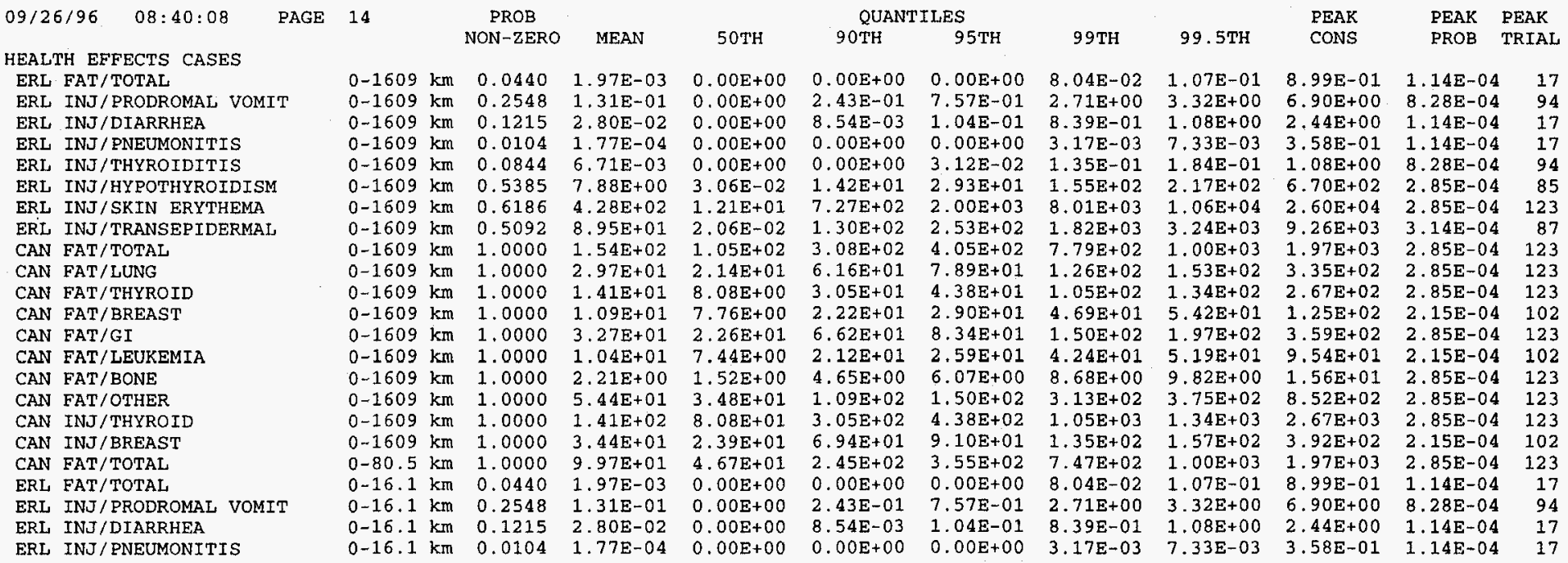


ERL INJ/THYROIDITIS

ERL INJ/HYYOTHYROIDISM ERL INJ/SKIN ERYTHEMA

CAN FAT/TOTAL EARLY FATALITY DISTANCE $(\mathrm{km})$
ERL FAT/TOTAL RISK $>0.000$

POPULATION EXCEEDING DOSE EARLY dOSe A-RED MARR > $1.50 \mathrm{SV}$

EARLY dose A-LUNGS > $5.00 \mathrm{~S}$ EARLY dose L-EDEWBODY >

AVERAGE INDIVIDUAL RISK

ERL FAT/TOTAL

ERL FAT/TOTAL

ERL FAT/TOTAL $\begin{array}{lllllllllll}0-16.1 \mathrm{~km} & 0.0844 & 6.71 \mathrm{E}-03 & 0.00 \mathrm{E}+00 & 0.00 \mathrm{E}+00 & 3.12 \mathrm{E}-02 & 1.35 \mathrm{E}-01 & 1.84 \mathrm{E}-01 & 1.08 \mathrm{E}+00 & 8.28 \mathrm{E}-04 & 94\end{array}$

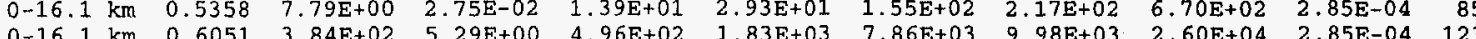

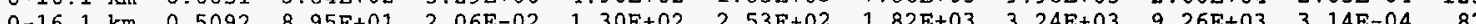
$\begin{array}{lllllllllll}0-16.1 \mathrm{~km} & 0.9074 & 2.93 \mathrm{E}+01 & 3.74 \mathrm{E}+00 & 5.09 \mathrm{E}+01 & 1.41 \mathrm{E}+02 & 4.81 \mathrm{E}+02 & 5.82 \mathrm{E}+02 & 1.61 \mathrm{E}+03 & 2.85 \mathrm{E}-04 & 123\end{array}$

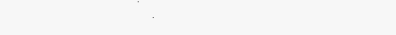

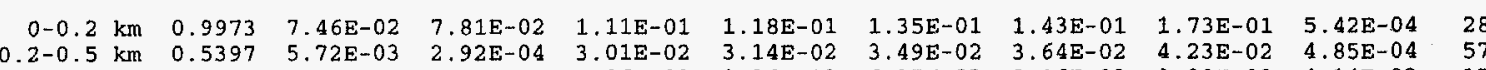
$\begin{array}{llllllllllll}0.2-0.5 \mathrm{~km} & 0.5397 & 5.72 \mathrm{E}-03 & 2.92 \mathrm{E}-04 & 3.01 \mathrm{E}-02 & 3.14 \mathrm{E}-02 & 3.49 \mathrm{E}-02 & 3.64 \mathrm{E}-02 & 4.23 \mathrm{E}-02 & 4.85 \mathrm{E}-04 & 57 \\ 0.5-1.2 \mathrm{~km} & 0.3217 & 3.33 \mathrm{E}-04 & 0.00 \mathrm{E}+00 & 1.06 \mathrm{E}-03 & 1.36 \mathrm{E}-03 & 6.35 \mathrm{E}-03 & 9.16 \mathrm{E}-03 & 9.99 \mathrm{E}-03 & 4.14 \mathrm{E}-03 & 17\end{array}$

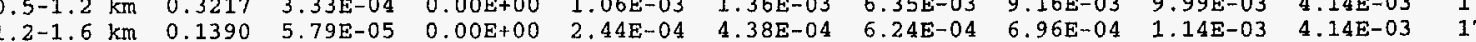

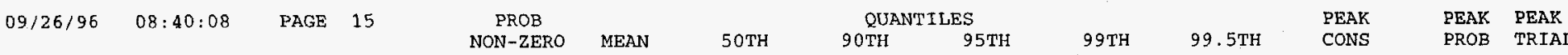
AVERAGE INDIVIDUAL RISK ERL FAT/TOTAL

$\begin{array}{lllllll}0.9973 & 7.13 \mathrm{E}-01 & 5.28 \mathrm{E}-01 & 2.03 \mathrm{E}+00 & 2.44 \mathrm{E}+00 & \text { NOT-FOUND NOT-FOUND } \quad 3.22 \mathrm{E}+00 \quad 2.25 \mathrm{E}-02 & 94\end{array}$

$\begin{array}{llllllllll}0.0440 & 6.38 \mathrm{E}-02 & 0.00 \mathrm{E}+00 & 0.00 \mathrm{E}+00 & 0.00 \mathrm{E}+00 & 2.50 \mathrm{E}+00 & 3.21 \mathrm{E}+00 & 7.14 \mathrm{E}+00 & 8.48 \mathrm{E}-04 & 94\end{array}$ 1.14E+00 $8.48 \mathrm{E}-04 \quad 94$ $\begin{array}{llllllllll}0.0104 & 8.32 \mathrm{E}-03 & 0.00 \mathrm{E}+00 & 0.00 \mathrm{E}+00 & 0.00 \mathrm{E}+00 & 7.03 \mathrm{E}-01 & 7.62 \mathrm{E}-01 & 2.71 \mathrm{E}+00 & 3.77 \mathrm{E}-04 & 17 \\ 0.9418 & 6.66 \mathrm{E}+03 & 1.91 \mathrm{E}+03 & 1.51 \mathrm{E}+04 & 2.78 \mathrm{E}+04 & 8.65 \mathrm{E}+04 & 1.04 \mathrm{E}+05 & 1.78 \mathrm{E}+05 & 5.71 \mathrm{E}-05 & 75\end{array}$

POPULATION DOSE (SV) L-EDEWBODY TOT LIF L-EDEWBODY TOT LIF

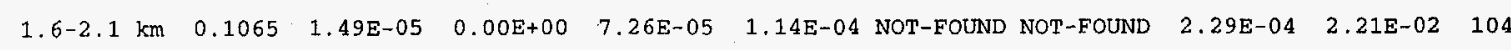

$\begin{array}{lllllllllll}0-16.1 \mathrm{~km} & 0.9074 & 5.44 \mathrm{E}+02 & 8.19 \mathrm{E}+01 & 1.12 \mathrm{E}+03 & 2.54 \mathrm{E}+03 & 8.81 \mathrm{E}+03 & 1.15 \mathrm{E}+04 & 2.65 \mathrm{E}+04 & 2.85 \mathrm{E}-04 & 123\end{array}$

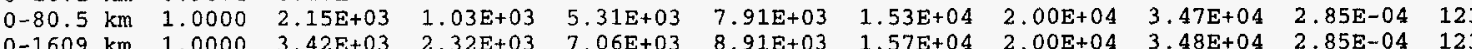

POPULATION WEIGHTED RISK ERL FAT/TOTAL

$\begin{array}{rrrrrrrrrrr}0-2.1 \mathrm{~km} & 0.0440 & 9.69 \mathrm{E}-05 & 0.00 \mathrm{E}+00 & 0.00 \mathrm{E}+00 & 0.00 \mathrm{E}+00 & 3.55 \mathrm{E}-03 & 5.16 \mathrm{E}-03 & 4.49 \mathrm{E}-02 & 1.14 \mathrm{E}-04 & 17 \\ 0-16.1 \mathrm{~km} & 0.9074 & 3.99 \mathrm{E}-04 & 5.16 \mathrm{E}-05 & 6.84 \mathrm{E}-04 & 1.75 \mathrm{E}-03 & 6.68 \mathrm{E}-03 & 8.23 \mathrm{E}-03 & 2.19 \mathrm{E}-02 & 2.85 \mathrm{E}-04 & 123\end{array}$

PEAK DOSE FOUND ON SPATIAL GRID (SV)

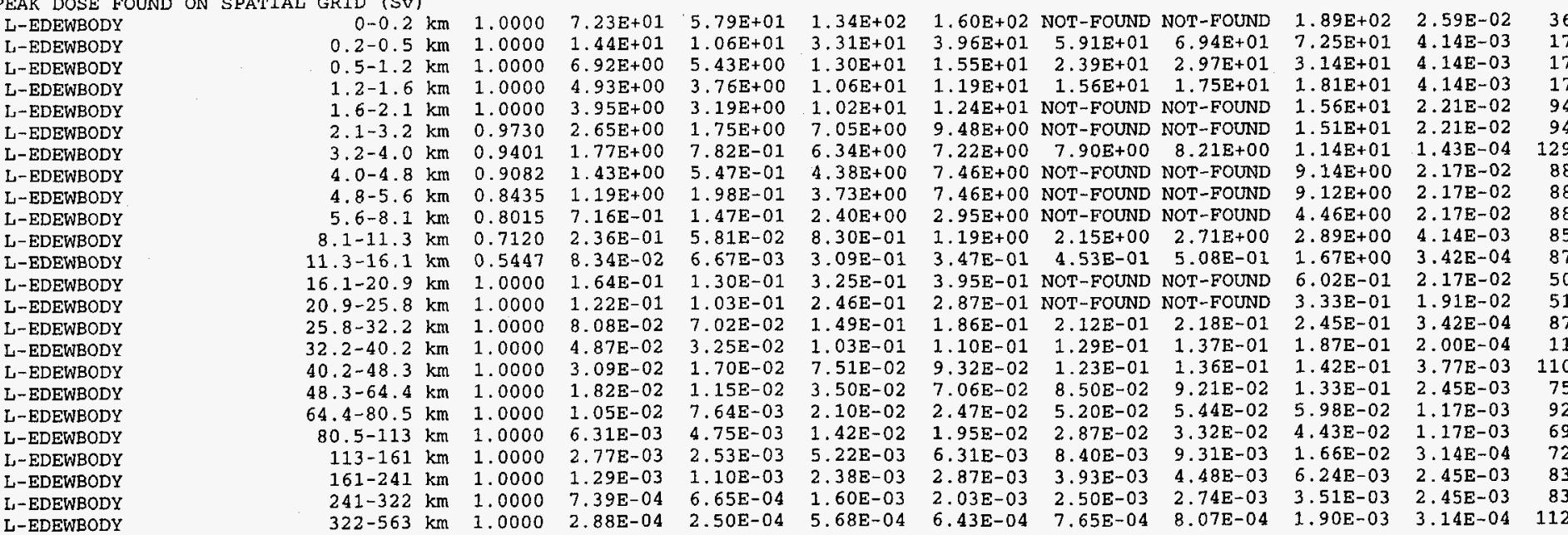


DATE AND TIME OF RUN = MACCS2 09/26/96 08:40:08 Version 1.12, Last Modified $9 / 25 / 96$ by D. Chanin

"ATMOS" DESCRIPTION $=$ IN1A. INP, Sample Problem A--Using Table-Lookup Sigmas, ATMOS input

"EARLY" DESCRIPTION = IN2A. INP, Sample Problem A of NUREG/CR-4691, Vol. 1, EARLY input

SOURCE TERM 2 OF 2 :

REIEASE FRACTIONS OF SOURCE TERM 1 REDUCED BY A FACTOR OF TEN

RESULTS FOR A SINGLE EMERGENCY RESPONSE COHORT WITHOUT ANY WETGHTING FRACTIONS BEING APPLIED

COHORT 2 = NO EVACUATION, RELOCATION MODELS APPLY EVERYWHERE

\begin{tabular}{|c|c|c|c|c|c|c|c|c|c|c|c|}
\hline $08: 40: 08$ & 16 & PROB & & & & LLES & & & PEAK & PEAK & EAK \\
\hline & & NON-ZERO & MEAN & $50 \mathrm{TH}$ & $90 \mathrm{TH}$ & $95 \mathrm{TH}$ & $99 \mathrm{TH}$ & $99.5 \mathrm{TH}$ & CONS & & \\
\hline EALTH EEFECTS CASES & & & & & & & & & & & \\
\hline ERL FAT/TOTAL & $0-1609 \mathrm{k}$ & 0.1958 & $5.34 E-02$ & $0.00 E+00$ & $2.35 E-02$ & $1.52 E-01$ & $2.10 \mathrm{E}+00$ & $2.38 \mathrm{E}+00$ & $6.57 E+00$ & $5.84 \mathrm{E}-06$ & 130 \\
\hline ERL INJ/PRODROMAL VOMIT & $0-1609 k$ & 0.4849 & $4.70 E-01$ & $0.00 E+00$ & $1.26 E+00$ & $2.78 \mathrm{E}+00$ & $6.34 \mathrm{E}+00$ & $7.56 \mathrm{E}+00$ & $4.80 E+01$ & $7.15 \mathrm{E}-06$ & 129 \\
\hline ERL INJ/DIARRHEA & $0-1609 \mathrm{k}$ & 0.3026 & $1.81 \mathrm{E}-01$ & $0.00 \mathrm{E}+00$ & $3.90 \mathrm{E}-01$ & $1.13 \mathrm{E}+00$ & $3.41 \mathrm{E}+00$ & $3.91 E+00$ & 1. $16 \mathrm{E}+01$ & $5.84 E-06$ & 130 \\
\hline ERL INJ/PNEUMONITIS & $0-1609 \mathrm{k}$ & 0.0303 & $4.49 \mathrm{E}-03$ & $0.00 \mathrm{E}+00$ & $0.00 \mathrm{E}+00$ & $0.00 \mathrm{E}+00$ & $2.21 \mathrm{E}-01$ & $2.75 \mathrm{E}-01$ & $1.90 \mathrm{E}+00$ & $3.50 \mathrm{E}-05$ & 46 \\
\hline ERL INJ/THYROIDITIS & $0-1609 \mathrm{k}$ & 0.0355 & $2.27 E-03$ & $0.00 \mathrm{E}+00$ & $0.00 \mathrm{E}+00$ & $0.00 \mathrm{E}+00$ & $1.05 \mathrm{E}-01$ & 1. $22 \mathrm{E}-01$ & $4.72 \mathrm{E}-01$ & $3.50 \mathrm{E}-05$ & 46 \\
\hline ERL INJ/HYPOTHYROIDISM & $0-1609 \mathrm{k}$ & 0.6262 & $3.81 \mathrm{E}+00$ & $1.50 \mathrm{E}-01$ & $9.53 E+00$ & $2.32 \mathrm{E}+01$ & $5.23 E+01$ & $6.70 E+01$ & $1.01 E+02$ & $1.06 \mathrm{E}-03$ & 41 \\
\hline ERL INJ/SKIN ERYTHEMA & $0-1609 k$ & 0.7347 & $4.88 E+02$ & 1. $53 E+01$ & $1.37 \mathrm{E}+03$ & $3.07 E+03$ & $7.09 \mathrm{E}+03$ & $1.06 E+04$ & $1.74 E+04$ & $1.06 \mathrm{E}-03$ & 50 \\
\hline ERL INJ/TRANSEPIDERMAL & $0-1609 \mathrm{k}$ & 0.5891 & $4.49 E+01$ & $5.26 \mathrm{E}-01$ & $8.66 \mathrm{E}+01$ & $2.24 \mathrm{E}+02$ & $8.22 \mathrm{E}+02$ & $1.04 \mathrm{E}+03$ & $2.31 E+03$ & $1.06 \mathrm{E}-03$ & 115 \\
\hline CAN FAT/TOTAL & $0-1609 \mathrm{k}$ & 1.0000 & $1.69 \mathrm{E}+02$ & $1.23 E+02$ & $3.30 \mathrm{E}+02$ & $4.21 \mathrm{E}+02$ & $6.50 \mathrm{E}+02$ & $7.29 \mathrm{E}+02$ & $1.11 \mathrm{E}+03$ & $2.15 \mathrm{E}-04$ & 102 \\
\hline CAN FAT/LUNG & $0-1609 \mathrm{k}$ & 1.0000 & $3.57 E+01$ & $2.66 E+01$ & $7.27 \mathrm{E}+01$ & $8.84 \mathrm{E}+01$ & $1.11 E+02$ & $1.18 \mathrm{E}+02$ & $2.30 E+02$ & $1.28 \mathrm{E}-05$ & 56 \\
\hline CAN FAT/THYROID & $0-1609 \mathrm{k}$ & 1.0000 & 1. $32 \mathrm{E}+01$ & $8.45 E+00$ & $2.62 \mathrm{E}+01$ & $3.64 \mathrm{E}+01$ & $7.26 \mathrm{E}+01$ & $8.03 E+01$ & $1.07 \mathrm{E}+02$ & $1.11 \mathrm{E}-03$ & 51 \\
\hline CAN FAT/BREAST & $0-1609 k$ & 1.0000 & 1. $22 E+01$ & $9.13 E+00$ & $2.45 E+01$ & $3.15 \mathrm{E}+01$ & $4.60 E+01$ & $5.34 \mathrm{E}+01$ & 1. $26 \mathrm{E}+02$ & $2.15 E-04$ & 102 \\
\hline CAN FAT/GI & $0-1609 \mathrm{k}$ & 1.0000 & $3.63 E+01$ & $2,73 E+01$ & $7.40 \mathrm{E}+01$ & $9.48 \mathrm{E}+01$ & $1.32 \mathrm{E}+02$ & $1.50 E+02$ & $3.01 \mathrm{E}+02$ & $2.15 \mathrm{E}-04$ & 102 \\
\hline CAN FAT/LEUKEMIA & $0-1609 k$ & 1.0000 & $1.19 \mathrm{E}+01$ & $9.04 \mathrm{E}+00$ & $2.38 \mathrm{E}+01$ & 3. $04 \mathrm{E}+01$ & $4.03 E+01$ & $4.56 \mathrm{E}+01$ & $9.71 \mathrm{E}+01$ & $2.15 \mathrm{E}-04$ & 102 \\
\hline CAN FAT/BONE & $0-1609 \mathrm{k}$ & 1.00 & $2.98 E+00$ & $2.17 \mathrm{E}+00$ & $6.08 \mathrm{E}+00$ & $7.80 E+00$ & $1.26 E+01$ & $1.53 E+01$ & $2.83 E+01$ & $1.28 \mathrm{E}-05$ & 56 \\
\hline CAN FAT/OTHER & $0-1609 \mathrm{k}$ & 1.0000 & $5.68 \mathrm{E}+01$ & $4.11 E+01$ & $1.13 \mathrm{E}+02$ & $1.50 E+02$ & $2.35 E+02$ & $2.67 \mathrm{E}+02$ & $3.56 \mathrm{E}+02$ & $2.15 \mathrm{E}-04$ & 102 \\
\hline CAN INJ/THYROID & $0-1609 \mathrm{k}$ & 1.00 & 1. $32 \mathrm{E}+02$ & $8.45 E+01$ & $2.62 \mathrm{E}+02$ & $3.64 E+02$ & $7.26 \mathrm{E}+02$ & $8.03 E+0.2$ & $1.07 E+03$ & $1.11 \mathrm{E}-03$ & 51 \\
\hline CAN INJ/BREAST & $0-1609 k$ & 1.0000 & $3.83 \mathrm{E}+01$ & & & $1.02 \mathrm{E}+02$ & $1.38 \mathrm{E}+02$ & $1.57 \mathrm{E}+02$ & $3.98 E+02$ & $2.15 \mathrm{E}-04$ & 102 \\
\hline CAN FAT/TOTAL & $0-80.5 \mathrm{k}$ & 1.0000 & $1.14 \mathrm{E}+02$ & $6.50 \mathrm{E}+01$ & $2.73 E+02$ & $3.93 \mathrm{E}+02$ & $6.50 \mathrm{E}+02$ & $7.29 \mathrm{E}+02$ & $1 E+03$ & $2.15 E-04$ & 102 \\
\hline ERL FAT/TOTAL & $0-16.1 \mathrm{k}$ & 0.1958 & $5.34 E-02$ & $0.00 E+00$ & $2.35 \mathrm{E}-02$ & $1.52 \mathrm{E}-01$ & $2.10 \mathrm{E}+00$ & $2.38 \mathrm{E}+00$ & $6.57 E+00$ & $5.84 E-06$ & 130 \\
\hline ERI INJ/PRODROMAL VOMIT & $0-16.1 \mathrm{k}$ & 0.48 & 4. $70 \mathrm{E}-01$ & $0.00 \mathrm{E}+00$ & $1.26 \mathrm{E}+00$ & $2.78 \mathrm{E}+00$ & $6.34 \mathrm{E}-$ & $7.56 \mathrm{E}$ & 01 & $7.15 \mathrm{E}-06$ & 129 \\
\hline ERL INJ/DIARR & $0-16.1 \mathrm{k}$ & 0.30 & & & $3.90 E-01$ & $1.13 \mathrm{E}+00$ & $3.41 \mathrm{E}$ & $3.91 \mathrm{E}+00$ & $1.16 \mathrm{E}+01$ & $5.84 \mathrm{E}-06$ & 130 \\
\hline ERL INJ/PNEUMONITIS & $0-16.1 \mathrm{k}$ & 0.030 & $4.49 \mathrm{E}-03$ & 0.001 & 0.00 & $0.00 \mathrm{E}+$ & $2.21 E-01$ & $2.75 \mathrm{E}-01$ & $1.90 \mathrm{E}+00$ & $3.50 \mathrm{E}-05$ & 46 \\
\hline ERL INJ/TTYYROIDITIS & $0-16.1 \mathrm{k}$ & 0.0355 & $2.27 \mathrm{E}-03$ & $0.00 \mathrm{E}+00$ & $0.00 \mathrm{E}+00$ & $0.00 E+00$ & $1.05 \mathrm{E}-01$ & $1.22 E-01$ & 4. $72 \mathrm{E}-01$ & E- 05 & 46 \\
\hline ERL INJ/HYPOTHYROIDISM & $0-16.1 k$ & 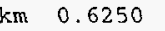 & $3.72 E+00$ & $1.47 \mathrm{E}-01$ & & $2.32 E+01$ & $4.98 \mathrm{E}$ & $6.18 \mathrm{E}+01$ & $1.01 E+02$ & $1.06 \mathrm{E}-03$ & 41 \\
\hline ERL INJ/SKIN E & $0-16.1 \mathrm{k}$ & $\mathrm{km}$ & 4.45 & & & 3.0 & & & & & 36 \\
\hline ERL INJ/TRANSEPIDERMAL & $0-16.1 \mathrm{k}$ & 0.58 & $4.49-10$ & & & $2.24 \mathrm{E}$ & $8.22 \mathrm{E}$ & 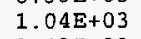 & $2.31 \mathrm{E}+03$ & $1.06 \mathrm{E}-03$ & 115 \\
\hline CAN FAT/TOTAL & $0-16.1 \mathrm{k}$ & 0.9993 & $4.39 \mathrm{E}+01$ & $1.84 \mathrm{E}+01$ & $1.16 \mathrm{E}+02$ & $1.59 \mathrm{E}+02$ & $2.91 \mathrm{E}+02$ & $3.43 \mathrm{E}+02$ & $4.84 \mathrm{E}+02$ & $1.06 \mathrm{E}-03$ & 41 \\
\hline EARLY FATALITY & & & & & & & & & & & \\
\hline ERL FAT/TOTAL RISK $>0.000$ & & 0.9998 & $1.68 \mathrm{E}+00$ & $1.40 E+00$ & $3.19 \mathrm{E}+00$ & $3.37 \mathrm{E}+00$ & $3.84 \mathrm{E}+00$ & $4.06 \mathrm{E}+00$ & $5.63 \mathrm{E}+00$ & $3.71 \mathrm{E}-04$ & 40 \\
\hline ON EY & & & & & & & & & & & \\
\hline EARLY dose A-RED M? & Sv & 0.1958 & $5.31 E-01$ & $0.00 \mathrm{E}+00$ & 1. $48 \mathrm{E}+00$ & $4.23 \mathrm{E}+$ & 8.65 & 1.0 & $3.27 \mathrm{E}+01$ & $1 \mathrm{E}-06$ & 130 \\
\hline EARLY dose A-LUNGS $>5.00 \mathrm{SV}$ & & 0.0303 & $4.26 \mathrm{E}-02$ & & $0.00 \mathrm{E}+00$ & $0.00 \mathrm{E}+00$ & $2.13 E+00$ & & & $1.49 \mathrm{E}-04$ & 17 \\
\hline EARLY dose L-EDEWBODY $>5.000$ & $0 \mathrm{E}-02$ & 0.9990 & $8.34 E+03$ & $3.14 E+03$ & $2.02 E+04$ & $3.37 \mathrm{E}+04$ & $8.61 \mathrm{E}+04$ & $1.04 E+05$ & $1.94 \mathrm{E}+05$ & $5.71 \mathrm{E}-05$ & 75 \\
\hline AVERAG & & & & & & & & & & & \\
\hline ERL FAT/TOTAL & & $\mathrm{km}$ & & & & & NOT-FOUND & NOT-FOUND & & & 59 \\
\hline ERL FAT/TOTAL & & $\mathrm{km}$ & & 2.13 & $5.91 \mathrm{E}$ & & & 8.6 & & & 28 \\
\hline ERL FAT/TOTAL & $.5-1.2 \mathrm{k}$ & $\mathrm{km}$ & 5.09 & $1.89 \mathrm{E}$ & & & & & & & 8 \\
\hline ERL FAT/TOTAL & $.2-1.6 \mathrm{k}$ & 0.5240 & $1.05 E-03$ & $7.80 E-05$ & $2.72 E-03$ & $3.99 E-03$ & $1.17 \mathrm{E}-02$ & $1.37 \mathrm{E}-02$ & $2.79 \mathrm{E}-02$ & $4.85 \mathrm{E}-04$ & 33 \\
\hline
\end{tabular}


$\begin{array}{llllllllllll}09 / 26 / 96 & 08: 40: 08 & \text { PAGE } & 17 & \text { PROB } & & & \text { QUANTILES } & & \text { PEAK } & \text { PEAK } & \text { PEAK } \\ \text { NON-ZERO } & \text { MEAN } & 50 \mathrm{TH} & 90 \mathrm{TH} & 95 \mathrm{TH} & 99 \mathrm{TH} & 99.5 \mathrm{TH} & \text { CONS } & \text { PROB } & \text { TRIAL }\end{array}$ AVERAGE INDIVIDUAL RISK
ERL FAT/TOTAL

POPULATION DOSE (SV) L-EDEWBODY TOT LIF TOT LIF L-EDEWBODY TOT LIF POPULATION WEIGHTED RISK ERL FAT/TOTAL $\begin{array}{rlllllllllr}0-16.1 \mathrm{~km} & 0.9993 & 7.72 \mathrm{E}+02 & 3.26 \mathrm{E}+02 & 2.10 \mathrm{E}+03 & 2.78 \mathrm{E}+03 & 4.85 \mathrm{E}+03 & 5.49 \mathrm{E}+03 & 7.99 \mathrm{E}+03 & 1.06 \mathrm{E}-03 & 41\end{array}$ $\begin{array}{lllllllllll}0-80.5 \mathrm{~km} & 1.0000 & 2.38 \mathrm{E}+03 & 1.28 \mathrm{E}+03 & 5.64 \mathrm{E}+03 & 8.35 \mathrm{E}+03 & 1.25 \mathrm{E}+04 & 1.41 \mathrm{E}+04 & 2.58 \mathrm{E}+04 & 2.15 \mathrm{E}-04 & 102 \\ 0-1609 \mathrm{~km} & 1.0000 & 3.65 \mathrm{E}+03 & 2.73 \mathrm{E}+03 & 7.19 \mathrm{E}+03 & 9.40 \mathrm{E}+03 & 1.28 \mathrm{E}+04 & 1.44 \mathrm{E}+04 & 2.58 \mathrm{E}+04 & 2.15 \mathrm{E}-04 & 102\end{array}$

$\begin{array}{lllllllllll}0-2.1 \mathrm{~km} & 0.1249 & 2.33 \mathrm{E}-03 & 0.00 \mathrm{E}+00 & 2.99 \mathrm{E}-04 & 3.61 \mathrm{E}-03 & 1.05 \mathrm{E}-01 & 1.19 \mathrm{E}-01 & 3.19 \mathrm{E}-01 & 2.48 \mathrm{E}-05 & 33\end{array}$

PEAK DOSE FOUND ON SPATIAL GRID (SV)

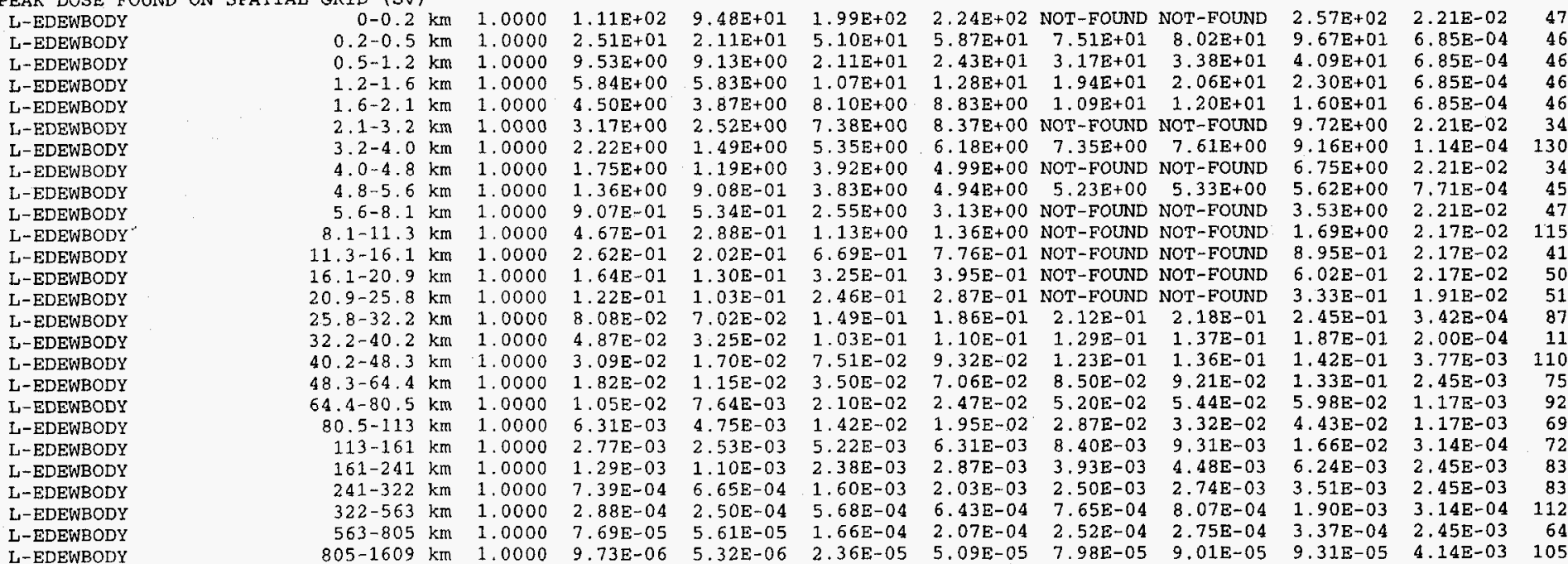

L-EDEWBODY

DATE AND TIME OF RUN $=$ MACCS2 09/26/96 08:40:08 Vexsion 1.12, Last Modified 9/25/96 by D. Chanin

"ATMOS" DESCRIPTION = IN1A.INP, Sample Problem A--Using Table-Lookup Sigmas, ATMOS input

EARLY DESCRIPTION = IN2A.INR, Sample Problem A OL NUREG/CR-4691, Vol. 1, EARLY input

"CHRONC" DESCRIPTION = IN3A_N. INP, Sample Problem A, "New" COMIDA2-Based Food Mode1

SOURCE TERM 2 OF 2 :

RELEASE FRACTIONS OF SOURCE TERM 1 REDUCED BY A FACTOR OF TEN

RESULTS FROM THE "CHRONC" MODULE ALONE

COHORT 3 = IN3A_N. INP, Sample Problem A, "New" COMIDA2-Based Food Mode1
$09 / 26 / 96 \quad 08: 40: 08$
PAGE 18
PROB
$50 \mathrm{TH}$
QUANTILES
$90 \mathrm{TH}$
$95 \mathrm{TH}$
$99 \mathrm{TH}$
$99.5 \mathrm{TH}$
PEAK
CONS
PEAK PEAK

HEALTH EFFECTS CASES 
CAN FAT/TOTAL CAN FAT/LUNG

CAN FAT/THYROID

CAN FAT/GI

CAN FAT/LEUKEMIA

CAN FAT/BONE

CAN FAT/OTHER

CAN INJ/BREAST

CAN FAT/TOTA

POPULATION DOSE (SV)

L-EDEWBODY TOT LIF
L-EDEWBODY

POPULATION WEIGHTED RISK

CAN FAT/TOTAL

I-EDEWBODY

L-EDEWBODY

L-EDEWBODY

L-EDEWBODY

L-EDEWBODY

L-EDEWBODY

I-EDEWBODY

L-EDEWBODY

L-EDEWBODY

L-EDENBODY

L-EDEWBODY

L-EDEWBODY

L-

L-EDEWBODY

L-EDEWBODY $\begin{array}{lll}\text { L-EDEWBODY TOT LIF } & \text { T-EDEWBODY } \\ \text { TOT LIF }\end{array}$

PEAK DOSE FOUND ON SPATIAL GRID (SV)

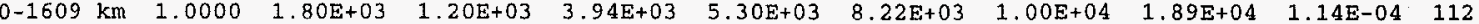

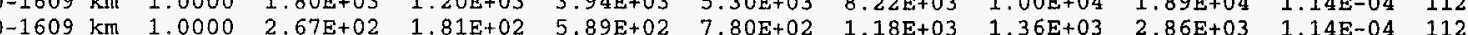

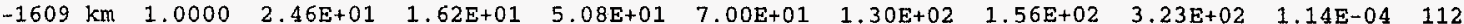

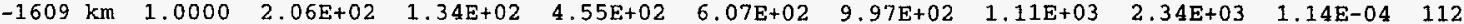
$\begin{array}{lllllllllll}0-1609 \mathrm{~km} & 1.0000 & 5.48 \mathrm{E}+02 & 3.69 \mathrm{E}+02 & 1.24 \mathrm{E}+03 & 1.70 \mathrm{E}+03 & 2.58 \mathrm{E}+03 & 2.98 \mathrm{E}+03 & 5.48 \mathrm{E}+03 & 1.14 \mathrm{E}-04 & 112\end{array}$

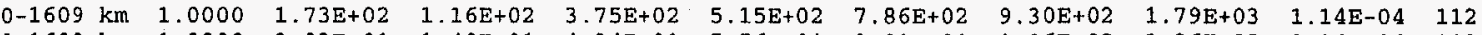
$\begin{array}{lllllllllll}0-1609 \mathrm{~km} & 1.0000 & 2.03 \mathrm{E}+01 & 1.40 \mathrm{E}+01 & 4.24 \mathrm{E}+01 & 5.56 \mathrm{E}+01 & 9.01 \mathrm{E}+01 & 1.06 \mathrm{E}+02 & 1.96 \mathrm{E}+02 & 1.14 \mathrm{E}-04 & 112\end{array}$

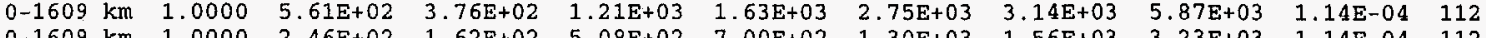

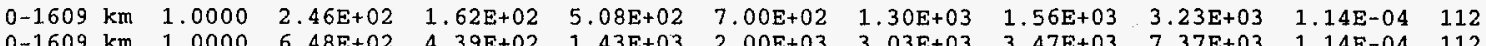

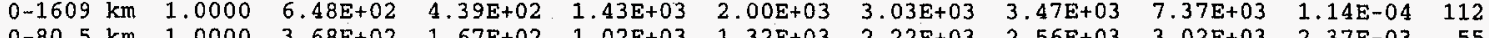
$\begin{array}{lllllllllll}0-80.5 \mathrm{~km} & 1.0000 & 3.68 \mathrm{E}+02 & 1.67 \mathrm{E}+02 & 1.02 \mathrm{E}+03 & 1.32 \mathrm{E}+03 & 2.22 \mathrm{E}+03 & 2.56 \mathrm{E}+03 & 3.02 \mathrm{E}+03 & 2.37 \mathrm{E}-03 & 55 \\ 0-16.1 \mathrm{~km} & 1.0000 & 4.21 \mathrm{E}+01 & 2.55 \mathrm{E}+01 & 9.50 \mathrm{E}+01 & 1.25 \mathrm{E}+02 & 2.09 \mathrm{E}+02 & 2.24 \mathrm{E}+02 & 3.39 \mathrm{E}+02 & 7.15 \mathrm{E}-06 & 37\end{array}$

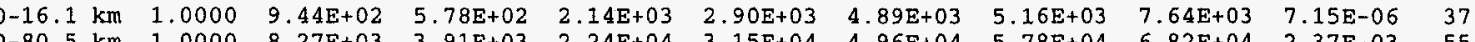
$\begin{array}{lllllllllll}0-1609 \mathrm{~km} & 1.0000 & 4.06 \mathrm{E}+04 & 2.82 \mathrm{E}+04 & 9.31 \mathrm{E}+04 & 1.18 \mathrm{E}+05 & 1.87 \mathrm{E}+05 & 2.78 \mathrm{E}+04 & 6.82 \mathrm{E}+04 & 2.37 \mathrm{E}+03 & 55 \\ & & & & & & \end{array}$

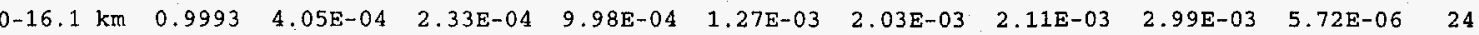

$\begin{array}{lllllllllll}0-0.2 \mathrm{~km} & 0.4675 & 7.17 \mathrm{E}-02 & 0.00 \mathrm{E}+00 & 2.00 \mathrm{E}-01 & 2.02 \mathrm{E}-01 & 2.07 \mathrm{E}-01 & 2.09 \mathrm{E}-01 & 2.18 \mathrm{E}-01 & 2.28 \mathrm{E}-04 & 18\end{array}$

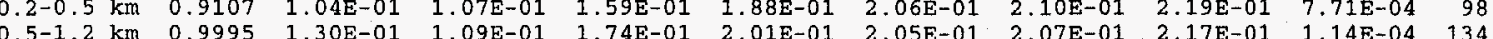
$1.2-1.6 \mathrm{~km} 0.9995 \quad 1.30 \mathrm{E}-01 \quad 1.09 \mathrm{E}-01 \quad 1.35 \mathrm{E}-01 \quad 2.01 \mathrm{E}-01 \quad 2.05 \mathrm{E}-01$ 2.07E \begin{tabular}{llllllll}
$1.6-2.1 \mathrm{~km}$ & 0.9801 & 9 & $49 \mathrm{E}-02$ & 9 & $.82 \mathrm{E}-02$ & $1.32 \mathrm{E}-01$ & 1 \\
\hline
\end{tabular}

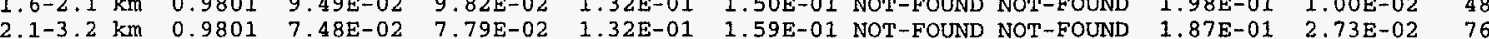
$\begin{array}{llllllllll}3.2-4.0 \mathrm{~km} & 0.9806 & 6.52 \mathrm{E}-02 & 6.00 \mathrm{E}-02 & 1.09 \mathrm{E}-01 & 1.34 \mathrm{E}-01 & \text { NOT-FOUND NOT-FOUND } & 1.88 \mathrm{E}-01 & 1.55 \mathrm{E}-02 & 59\end{array}$ $\begin{array}{lllllllllll}4.0-4.8 \mathrm{~km} & 0.9806 & 5.93 \mathrm{E}-02 & 4.74 \mathrm{E}-02 & 1.02 \mathrm{E}-01 & 1.13 \mathrm{E}-01 & 1.41 \mathrm{E}-01 & 1.55 \mathrm{E}-01 & 1.90 \mathrm{E}-01 & 1.17 \mathrm{E}-03 & 92\end{array}$

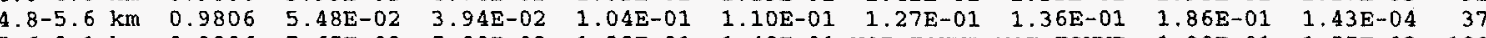

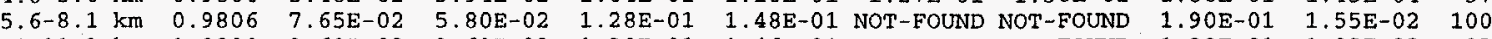
$\begin{array}{llllllllll}8.1-11.3 \mathrm{~km} & 1.0000 & 9.69 \mathrm{E}-02 & 9.61 \mathrm{E}-02 & 1.30 \mathrm{E}-01 & 1.46 \mathrm{E}-01 & \text { NOT-FOUND NOT-FOUND } & 1.90 \mathrm{E}-01 & 1.00 \mathrm{E}-02 & 93\end{array}$

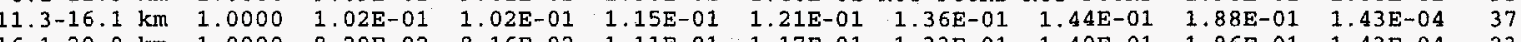

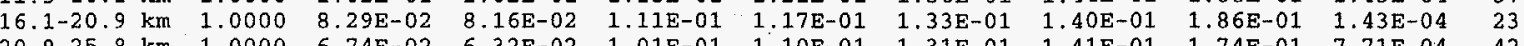

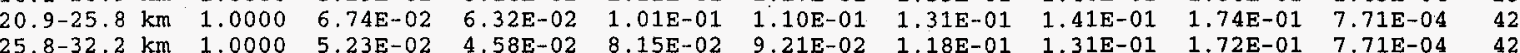
$\begin{array}{lllllllllll}25.8-32.2 \mathrm{~km} & 1.0000 & 5.23 \mathrm{E}-02 & 4.58 \mathrm{E}-02 & 8.15 \mathrm{E}-02 & 9.21 \mathrm{E}-02 & 1.18 \mathrm{E}-01 & 1.31 \mathrm{E}-01 & 1.72 \mathrm{E}-01 & 7.71 \mathrm{E}-04 & 42 \\ 32.2-40.2 \mathrm{~km} & 1.0000 & 4.87 \mathrm{E}-02 & 4.07 \mathrm{E}-02 & 7.22 \mathrm{E}-02 & 7.55 \mathrm{E}-02 & 8.38 \mathrm{E}-02 & 8.77 \mathrm{E}-02 & 1.27 \mathrm{E}-01 & 2.28 \mathrm{E}-04 & 25\end{array}$ $\begin{array}{lllllllll} & \end{array}$

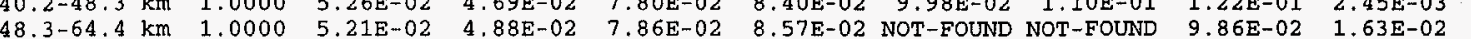

$09 / 26 / 96 \quad 08: 40 ; 08 \quad$ PAGE

(1) PROB NON-ZERO L-EDEWBODY L-EDEWBODY L-EDEWBODY L-EDEWBODY

L-DDEMODY

L-EDEWBODY

$64.4-80.5 \mathrm{~km} 1.0000$ $\begin{array}{rrr}80.5-113 & \mathrm{~km} & 1.0000 \\ 113-161 \mathrm{~km} & 1.0000\end{array}$

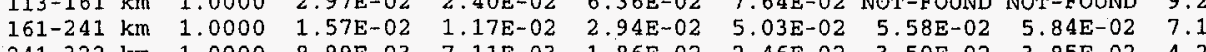
$\begin{array}{lllllllllll}322-563 \mathrm{~km} & 1.0000 & 3.85 \mathrm{E}-03 & 3.13 \mathrm{E}-03 & 8.11 \mathrm{E}-03 & 9.68 \mathrm{E}-03 & 1.23 \mathrm{E}-02 & 1.35 \mathrm{E}-02 & 1.98 \mathrm{E}-02 & 3.14 \mathrm{E}-04 & 112\end{array}$ $\begin{array}{llllllllll}5 & & \end{array}$

L-EDEWBODY POP. DOSE (SV) TOTAL LONG-TERM PATHWAYS DOSE $0-1609 \mathrm{~km}$ LONG-TERM DIRECT EXPOSURE PATHWAYS TOTAL INGESTION PATHWAYS DOSE LONG-TERM GROUNDSHINE DOSE

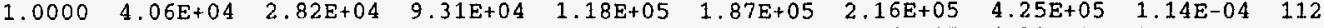
$\begin{array}{llllllllll}1.0000 & 3.22 \mathrm{E}+04 & 2.21 \mathrm{E}+04 & 6.60 \mathrm{E}+04 & 9.10 \mathrm{E}+04 & 1.81 \mathrm{E}+05 & 2.16 \mathrm{E}+05 & 4.21 \mathrm{E}+05 & 1.14 \mathrm{E}-04 & 112\end{array}$

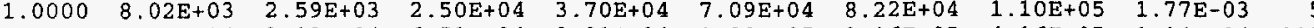
$\begin{array}{llllllllll}1.0000 & 3.15 \mathrm{E}+04 & 2.13 \mathrm{E}+04 & 6.53 \mathrm{E}+04 & 9.01 \mathrm{E}+04 & 1.80 \mathrm{E}+05 & 2.16 \mathrm{E}+05 & 4.16 \mathrm{E}+05 & 1.14 \mathrm{E}-04 & 112 \\ 1.0000 & 7.11 \mathrm{E}+02 & 5.03 \mathrm{E}+02 & 1.50 \mathrm{E}+03 & 2.04 \mathrm{E}+03 & 2.67 \mathrm{E}+03 & 2.99 \mathrm{E}+03 & 5.31 \mathrm{E}+03 & 1.14 \mathrm{E}-04 & 112\end{array}$ 
WATER INGESTION DOSE

POP. -DEPENDENT DECONTAMTNATION DOSE NTAMINATION DOSE

INGESTION OF ROOT CROPS

INGESTION OF FRUITS

INGESTION OF MILK

INGESTION OF POULTRY

INGESTION OF OTHER MEAT CROPS

-EDEWBODY POP, DOSE (SV)

$0-80.5 \mathrm{k}$

LONG-TERM DIRECT EXPOSURE PATHWAYS

TOTAL INGESTION PATHWAYS DOSE

LONG-TERM GROUNDSHINE DOSE

WATER INGESTION DOSE

FARM-DEPENDENT DECONTAMINATION DOSE

INGESTION OF GRAINS

INGESTION OF LEAF VEG

INGESTION OF ROOT CROPS

INGESTION OF FRUITS

INGESTION OF LEGUMES

INGESTION OF MILK INCESTION OF TEAT VEC

INGESTION OF LEGUMES

INGESTION OF BEEF

POP. -DEPENDENT DECONTAMINATION DOSE

INGESTION OF BEE

$\begin{array}{lllllllll}1.0000 & 1.77 \mathrm{E}+02 & 1.31 \mathrm{E}+02 & 3.47 \mathrm{E}+02 & 4.60 \mathrm{E}+02 & 6.41 \mathrm{E}+02 & 7.14 \mathrm{E}+02 & 1.23 \mathrm{E}+03 & 1.88 \mathrm{E}-05\end{array}$ $\begin{array}{lllllllll}1.0000 & 3.65 \mathrm{E}+02 & 1.79 \mathrm{E}+02 & 8.29 \mathrm{E}+02 & 1.44 \mathrm{E}+03 & 2.95 \mathrm{E}+03 & 3.63 \mathrm{E}+03 & 1.05 \mathrm{E}+04 & 5.71 \mathrm{E}-05\end{array}$ $\begin{array}{lllllllll}1.0000 & 1.01 \mathrm{E}+01 & 8.96 \mathrm{E}+00 & 2.06 \mathrm{E}+01 & 2.55 \mathrm{E}+01 & 3.22 \mathrm{E}+01 & 3.37 \mathrm{E}+01 & 4.42 \mathrm{E}+01 & 8.56 \mathrm{E}-05\end{array}$ $1.00005 .85 \mathrm{E}+026.16 \mathrm{E}+01 \quad 1.68 \mathrm{E}+03 \quad 2.59 \mathrm{E}+03 \quad 7.95 \mathrm{E}+03 \quad 1.03 \mathrm{E}+04 \quad 1.15 \mathrm{E}+04 \quad 1.88 \mathrm{E}-03$

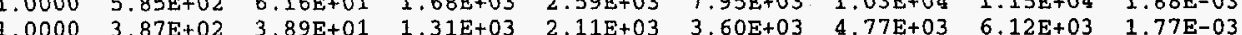

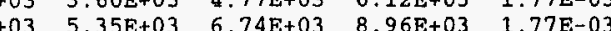
$\begin{array}{lllllllll}1.0000 & 3.55 \mathrm{E}+02 & 6.67 \mathrm{E}+01 & 1.16 \mathrm{E}+03 & 1.75 \mathrm{E}+03 & 2.83 \mathrm{E}+03 & 3.51 \mathrm{E}+03 & 5.24 \mathrm{E}+03 & 1.77 \mathrm{E}-03\end{array}$ $\begin{array}{lllllllll}1.0000 & 2.66 \mathrm{E}+03 & 8.96 \mathrm{E}+02 & 7.89 \mathrm{E}+03 & 1.17 \mathrm{E}+04 & 2.10 \mathrm{E}+04 & 2.94 \mathrm{E}+04 & 3.95 \mathrm{E}+04 & 2.28 \mathrm{E}-04\end{array}$ $\begin{array}{lllllllll}1.0000 & 2.01 \mathrm{E}+03 & 7.58 \mathrm{E}+02 & 5.79 \mathrm{E}+03 & 9.18 \mathrm{E}+03 & 1.69 \mathrm{E}+04 & 2.17 \mathrm{E}+04 & 3.41 \mathrm{E}+04 & 2.28 \mathrm{E}-04\end{array}$

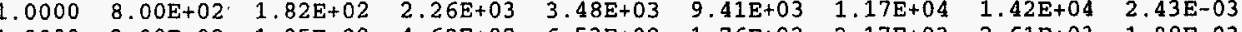
$\begin{array}{lllllllll}1.0000 & 2.08 \mathrm{E}+02 & 1.05 \mathrm{E}+02 & 4.69 \mathrm{E}+02 & 6.53 \mathrm{E}+02 & 1.76 \mathrm{E}+03 & 2.17 \mathrm{E}+03 & 2.61 \mathrm{E}+03 & 1.88 \mathrm{E}-03\end{array}$

$\begin{array}{lllllllll}1.0000 & 8.27 \mathrm{E}+03 & 3.91 \mathrm{E}+03 & 2.24 \mathrm{E}+04 & 3.15 \mathrm{E}+04 & 4.96 \mathrm{E}+04 & 5.78 \mathrm{E}+04 & 6.82 \mathrm{E}+04 & 2.37 \mathrm{E}-03\end{array}$

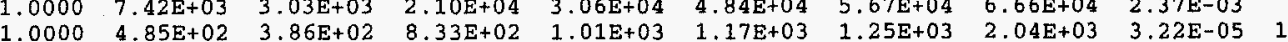

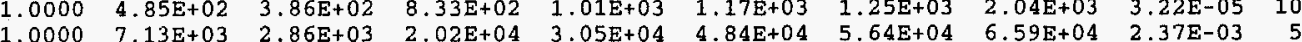
$\begin{array}{lllllllll}1.0000 & 2.82 \mathrm{E}+02 & 1.36 \mathrm{E}+02 & 6.31 \mathrm{E}+02 & 1.10 \mathrm{E}+03 & 2.10 \mathrm{E}+03 & 2.30 \mathrm{E}+03 & 5.11 \mathrm{E}+03 & 3.64 \mathrm{E}-04\end{array}$

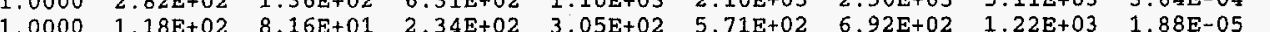
$\begin{array}{lllllllll}0.9999 & 3.58 \mathrm{E}+02 & 1.64 \mathrm{E}+02 & 8.22 \mathrm{E}+02 & 1.44 \mathrm{E}+03 & 2.95 \mathrm{E}+03 & 3.63 \mathrm{E}+03 & 1.05 \mathrm{E}+04 & 5.71 \mathrm{E}-05\end{array}$ $\begin{array}{lllllllll}1.0000 & 9.60 \mathrm{E}+00 & 8.35 \mathrm{E}+00 & 1.88 \mathrm{E}+01 & 2.33 \mathrm{E}+02 & 3.15 \mathrm{E}+01 & 3.31 \mathrm{E}+01 & 4.42 \mathrm{E}+01 & 8.56 \mathrm{E}-05 \\ 1.0000 & 4.59 \mathrm{E}+01 & 2.59 \mathrm{E}+01 & 8.33 \mathrm{E}+01 & 1.72 \mathrm{E}+02 & 3.21 \mathrm{E}+02 & 3.61 \mathrm{E}+02 & \mathbf{4 . 6 4 \mathrm { E } + 0 2} & 1.14 \mathrm{E}-03\end{array}$ $\begin{array}{lllllllll}1.0000 & 4.59 \mathrm{E}+01 & 2.59 \mathrm{E}+01 & 8.33 \mathrm{E}+01 & 1.72 \mathrm{E}+02 & 3.21 \mathrm{E}+02 & 3.61 \mathrm{E}+02 & 4.64 \mathrm{E}+02 & 1.14 \mathrm{E}-03\end{array}$ $\begin{array}{lllllllll}1.0000 & 2.32 \mathrm{E}+01 & 1.61 \mathrm{E}+01 & 4.90 \mathrm{E}+01 & 6.03 \mathrm{E}+01 & 9.58 \mathrm{E}+01 & 1.01 \mathrm{E}+02 & 1.05 \mathrm{E}+02 & 1.14 \mathrm{E}-03\end{array}$ $\begin{array}{lllllllll}1.0000 & 2.79 \mathrm{E}+01 & 2.03 \mathrm{E}+01 & 6.18 \mathrm{E}+01 & 8.27 \mathrm{E}+01 & 1.13 \mathrm{E}+02 & 1.22 \mathrm{E}+02 & 1.45 \mathrm{E}+02 & 1.14 \mathrm{E}-03\end{array}$

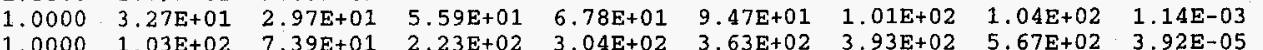

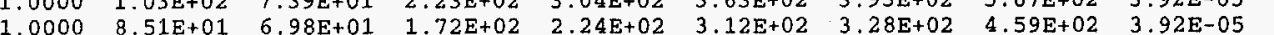

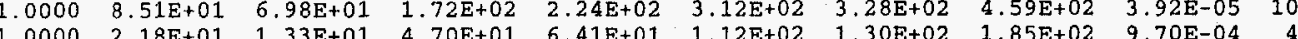

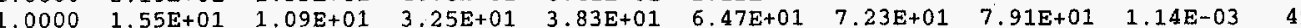
ECONOMIC COST MEASURES (\$) $\quad 0-1609$ TOTAL ECONOMIC COSTS POP. -DEPENDENT COSTS

TAMINATION COST FARM-DEPENDENT DECONTAMINATION COST

$\begin{array}{llllllllll}1.0000 & 1.46 \mathrm{E}+09 & 8.07 \mathrm{E}+08 & 3.22 \mathrm{E}+09 & 5.96 \mathrm{E}+09 & 8.64 \mathrm{E}+09 & 9.63 \mathrm{E}+09 & 1.50 \mathrm{E}+10 & 2.15 \mathrm{E}-04 & 102\end{array}$ $\begin{array}{llllllllll}1.0000 & 1.24 \mathrm{E}+09 & 5.87 \mathrm{E}+08 & 2.95 \mathrm{E}+09 & 5.85 \mathrm{E}+09 & 8.60 \mathrm{E}+09 & 9.61 \mathrm{E}+09 & 1.49 \mathrm{E}+10 & 2.15 \mathrm{E}-04 & 102\end{array}$ $\begin{array}{lllllllll}1.0000 & 2.24 \mathrm{E}+08 & 7.05 \mathrm{E}+07 & 6.89 \mathrm{E}+08 & 9.57 \mathrm{E}+08 & 2.05 \mathrm{E}+09 & 2.28 \mathrm{E}+09 & 2.64 \mathrm{E}+09 & 1.88 \mathrm{E}-03\end{array}$ $\begin{array}{llllllllll}1.0000 & 3.33 \mathrm{E}+08 & 1.36 \mathrm{E}+08 & 7.66 \mathrm{E}+08 & 1.48 \mathrm{E}+09 & 2.53 \mathrm{E}+09 & 2.89 \mathrm{E}+09 & 5.28 \mathrm{E}+09 & 2.15 \mathrm{E}-04 & 102 \\ 1.0000 & 2.38 \mathrm{E}+07 & 1.82 \mathrm{E}+07 & 5.16 \mathrm{E}+07 & 5.99 \mathrm{E}+07 & \mathbf{7 . 9 6 \mathrm { E } + 0 7} & \mathbf{8 . 8 0 \mathrm { E } + 0 7} & 1.18 \mathrm{E}+08 & 2.46 \mathrm{E}-04 & 68\end{array}$

$09 / 26 / 96 \quad 08: 40: 08$

ECONOMIC COST MEASURES (\$) $0-1609 \mathrm{~km}$ POP. -DEPENDENT INTERDICTION COST FARM-DEPENDENT INTERDICTION COST POP. -DEPENDENT CONDEMNATION COST FARM-DEPENDENT CONDEMNATION COST EMERGENCY PHASE COST

INTERMEDIATE PHASE COST

MILK DISPOSAL COST

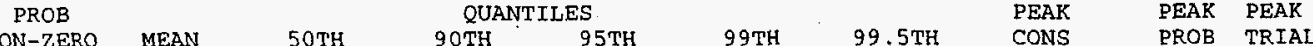
$\begin{array}{llllllllll}1.0000 & 8.71 \mathrm{E}+08 & 4.00 \mathrm{E}+08 & 2.34 \mathrm{E}+09 & 4.07 \mathrm{E}+09 & 6.09 \mathrm{E}+09 & 6.76 \mathrm{E}+09 & 9.95 \mathrm{E}+09 & 2.23 \mathrm{E}-04 & 110\end{array}$ $\begin{array}{lllllllll}1.0000 & 9.80 \mathrm{E}+07 & 2.19 \mathrm{E}+07 & 3.16 \mathrm{E}+08 & 4.70 \mathrm{E}+08 & 1.02 \mathrm{E}+09 & 1.08 \mathrm{E}+09 & 1.17 \mathrm{E}+09 & 1.88 \mathrm{E}-03\end{array}$ $\begin{array}{llllllllll}0.6064 & 3.24 \mathrm{E}+07 & 5.08 \mathrm{E}+05 & 4.66 \mathrm{E}+07 & 2.18 \mathrm{E}+08 & 4.97 \mathrm{E}+08 & 7.61 \mathrm{E}+08 & 2.71 \mathrm{E}+09 & 1.14 \mathrm{E}-05 & 18\end{array}$ $\begin{array}{llllllllll}1.0000 & 1.34 \mathrm{E}+06 & 1.09 \mathrm{E}+06 & 3.12 \mathrm{E}+06 & 3.54 \mathrm{E}+06 & 4.76 \mathrm{E}+06 & 5.33 \mathrm{E}+06 & 1.06 \mathrm{E}+07 & 5.04 \mathrm{E}-05 & 42 \\ 0.8625 & 8.59 \mathrm{E}+05 & 1.48 \mathrm{E}+05 & 2.29 \mathrm{E}+06 & 3.54 \mathrm{E}+06 & 9.90 \mathrm{E}+06 & 1.10 \mathrm{E}+07 & 2.12 \mathrm{E}+07 & \mathbf{5 . 7 1 E - 0 5} & 75\end{array}$ $\begin{array}{lllllllllr}0.8625 & 8.59 \mathrm{E}+05 & 1.48 \mathrm{E}+05 & 2.29 \mathrm{E}+06 & 3.54 \mathrm{E}+06 & 9.90 \mathrm{E}+06 & 1.10 \mathrm{E}+07 & 2.12 \mathrm{E}+07 & 5.71 \mathrm{E}-05 & 75 \\ 0.0000 & 0.00 \mathrm{E}+00 & 0.00 \mathrm{E}+00 & 0.00 \mathrm{E}+00 & 0.00 \mathrm{E}+00 & 0.00 \mathrm{E}+00 & 0.00 \mathrm{E}+00 & 0.00 \mathrm{E}+00 & 0.00 \mathrm{E}+00 & 0\end{array}$ $\begin{array}{llllllllll}0.0000 & 0.00 \mathrm{E}+00 & 0.00 \mathrm{E}+00 & 0.00 \mathrm{E}+00 & 0.00 \mathrm{E}+00 & 0.00 \mathrm{E}+00 & 0.00 \mathrm{E}+00 & 0.00 \mathrm{E}+00 & 0.00 \mathrm{E}+00 & \\ 1.0000 & 7.92 \mathrm{E}+06 & 8.43 \mathrm{E}+05 & 2.05 \mathrm{E}+07 & 3.44 \mathrm{E}+07 & 1.10 \mathrm{E}+08 & 1.21 \mathrm{E}+08 & 1.59 \mathrm{E}+08 & 6.85 \mathrm{E}-04 & 15\end{array}$ $\begin{array}{lllllllll}1.0000 & 9.25 \mathrm{E}+07 & 1.36 \mathrm{E}+07 & 2.93 \mathrm{E}+08 & 4.63 \mathrm{E}+08 & 9.12 \mathrm{E}+08 & 1.13 \mathrm{E}+09 & 1.74 \mathrm{E}+09 & 8.85 \mathrm{E}-04\end{array}$

ECONOMIC COST MEASURES (\$) $0-80.5 \mathrm{~km}$ TOTAL ECONOMIC COSTS TOTAL ECONOMIC COSTS
POP. -DEPENDENT COSTS FARM-DEPENDEN COSTS

POP -DEPENDENT DECONTAMINATION COST FAR -DEPENDENT DECONTAMINATION COST POP. DEPENDENT INTERDICTION COST FARM-DEPENDENT INTERDICTION COST

$\begin{array}{llllllllll}1.0000 & 1.22 \mathrm{E}+09 & 5.58 \mathrm{E}+08 & 2.82 \mathrm{E}+09 & 5.73 \mathrm{E}+09 & 8.60 \mathrm{E}+09 & 9.62 \mathrm{E}+09 & 1.50 \mathrm{E}+10 & 2.15 \mathrm{E}-04 & 102\end{array}$

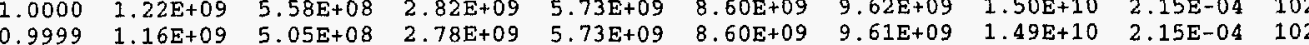
$\begin{array}{llllllllll}1.0000 & 5.75 \mathrm{E}+07 & 5.37 \mathrm{E}+07 & 9.89 \mathrm{E}+07 & 1.06 \mathrm{E}+08 & 1.22 \mathrm{E}+08 & 1.613 \mathrm{E}+08 & 1.74 \mathrm{E}+08 & 1.85 \mathrm{E}-04 & 3.02\end{array}$

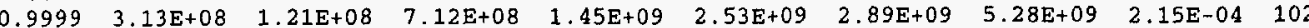
$\begin{array}{llllllllll}1.0000 & 1.91 \mathrm{E}+07 & 1.58 \mathrm{E}+07 & 3.48 \mathrm{E}+07 & 3.99 \mathrm{E}+07 & 5.13 \mathrm{E}+07 & 5.35 \mathrm{E}+07 & 6.46 \mathrm{E}+07 & 2.16 \mathrm{E}-04 & 110\end{array}$ $\begin{array}{llllllllll}0.9999 & 8.16 \mathrm{E}+08 & 3.51 \mathrm{E}+08 & 2.23 \mathrm{E}+09 & 4.00 \mathrm{E}+09 & 6.09 \mathrm{E}+09 & 6.76 \mathrm{E}+09 & 9.95 \mathrm{E}+09 & 2.23 \mathrm{E}-04 & 110 \\ 1.0000 & 2.33 \mathrm{E}+07 & 1.72 \mathrm{E}+07 & 4.88 \mathrm{E}+07 & 6.28 \mathrm{E}+07 & 7.68 \mathrm{E}+07 & 8.06 \mathrm{E}+07 & 9.00 \mathrm{E}+07 & 1.06 \mathrm{E}-03 & 135\end{array}$ 
POP. -DEPENDENT CONDEMNATION COST FARM-DEPENDTNT CONDEMNATTON COST EMERGENCY PHASE COST

MTTERMEDIATE PHASE COST

MILK DISPOSAL COST
CROP DISPOSAL COST

MAXIMUM LONG-TERM ACTION DISTANCE (km) FARM-DEPENDENT DECONTAMINATION DIST. POP.-DEPENDENT DECONTAMINATION DI FARM-DEPENDENT INTERDICTION DIST POP. DBPENDEN INBR POP. -DEPENDENT CONDEMNATION DIST CROP DISPOSAL DIST.

AFFECTED AREA/POPULATION $0-1609 \mathrm{~km}$ FARM DECONTAMINATION (HECTARES) POP. DECONTAMINATION (INDIVIDUALS) FARM INTERDICTION (HECTARES) POP. INTERDICTION (INDIVIDUALS) FARM CONDEMNATION (HECTARES) POP. CONDEMNATION (INDIVIDUALS) MILK DISPOSAL AREA (HECTARES)

AFFECTED AREA/POPULATION $0-80.5 \mathrm{~km}$ FARM DECONTAMINATION (HECTARES) POP. DECONTAMINATION (INDIVIDUALS) FARM INTERDICTION (HECTARES)

Un POP. INTERDICTION (INDIVIDUALS) FARM CONDEMINATION (HECTARES) POP. CONDEMNATION (INDIVIDUALS) MILK DISPOSAL AREA (HECTARES)
CROP DISPOSAL AREA (HECTARES)

$\begin{array}{lllllllll}0.6064 & 3.24 \mathrm{E}+07 & 5.08 \mathrm{E}+05 & 4.66 \mathrm{E}+07 & 2.18 \mathrm{E}+08 & 4.97 \mathrm{E}+08 & 7.61 \mathrm{E}+08 & 2.71 \mathrm{E}+09 & 1.14 \mathrm{E}-05\end{array}$ $1.0000 \quad 1.34 E+06 \quad 1.09 E+06 \quad 3.12 E+06 \quad 3.54 E+06 \quad 4.76 E+06 \quad 5.33 E+06 \quad 1.06 E+07 \quad 5.04 E-05$ $\begin{array}{llllllllll}0.8625 & 8.59 \mathrm{E}+05 & 1.48 \mathrm{E}+05 & 2.29 \mathrm{E}+06 & 3.54 \mathrm{E}+06 & 9.90 \mathrm{E}+06 & 1.10 \mathrm{E}+07 & 2.12 \mathrm{E}+07 & 5.71 \mathrm{E}-05\end{array}$ $\begin{array}{lllllllll}0.0000 & 0.00 \mathrm{E}+00 & 0.00 \mathrm{E}+00 & 0.00 \mathrm{E}+00 & 0.00 \mathrm{E}+00 & 0.00 \mathrm{E}+00 & 0.00 \mathrm{E}+00 & 0.00 \mathrm{E}+00 & 0.00 \mathrm{E}+00\end{array}$ $\begin{array}{lllllllll}1.0000 & 8.17 \mathrm{E}+05 & 7.13 \mathrm{E}+05 & 1.73 \mathrm{E}+06 & 2.08 \mathrm{E}+06 & 2.38 \mathrm{E}+06 & 2.52 \mathrm{E}+06 & 2.87 \mathrm{E}+06 & 1.06 \mathrm{E}-03\end{array}$ $\begin{array}{lllllllll}1.0000 & 1.30 \mathrm{E}+07 & 1.04 \mathrm{E}+07 & 2.59 \mathrm{E}+07 & 3.27 \mathrm{E}+07 & 4.66 \mathrm{E}+07 & 5.10 \mathrm{E}+07 & 5.48 \mathrm{E}+07 & 1.26 \mathrm{E}-03\end{array}$

$1.0000 \quad 6.83 E+01 \quad 5.958+01 \quad 1.148+02 \quad 1.348+02$ NOT-FOUND NOT-FOUND $1.618+02 \quad 2.268-02$ $1.00006 .83 E+01 \quad 5.95 E+01 \quad 1.14 E+02 \quad 1.34 E+02$ NOT-FOUND NOT-FOUND $1.618+02 \quad 2.26 \mathrm{E}-02$ $\begin{array}{llllllll}1.0000 & 6.87 \mathrm{E}+01 & 6.14 \mathrm{E}+01 & 1.14 \mathrm{E}+02 & 1.35 \mathrm{E}+02 & \text { NOT-FOUND NOT-FOUND } & 1.61 \mathrm{E}+02 & 2.42 \mathrm{E}-02\end{array}$ $\begin{array}{llllll}1.0000 & 1.38 \mathrm{E}+02 & 9.89 \mathrm{E}+01 . & 3.29 \mathrm{E}+02 & 4.05 \mathrm{E}+02 & \mathrm{~N}\end{array}$ $1.00000 \quad 8.63 \mathrm{E}+00 \quad 9.42 \mathrm{E}+00 \quad 1.24 \mathrm{E}+01 \quad 1.37 \mathrm{E}+01 \quad 1.72 \mathrm{E}+01 \quad 1.89 \mathrm{E}+01 \quad 3.22 \mathrm{E}+012.42 \mathrm{E}-02$ $0.6064 \quad 4.74 E+00 \quad 5.43 E+001.27 E+01 \quad 1.37 E+01 \quad 1.46 E+01 \quad 1.60 E+012.58 E+0121.44 E-04$ $1.0000 \quad 1.67 \mathrm{E}+02 \quad 1.01 \mathrm{E}+02$ NOT-FOUND NOT-FOUND NOT-FOUND NOT-FOUND $5.63 \mathrm{E}+02$ 1.44E-04

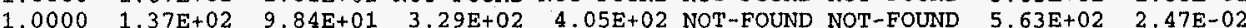

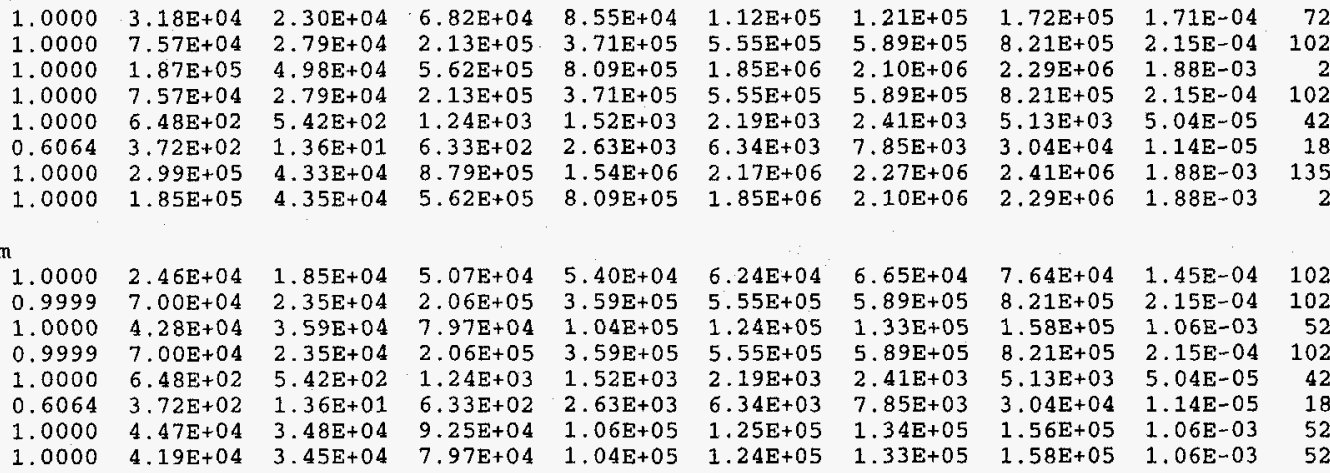

09/26/96 08:40:08 PAGE 21 PROB NON-ZERO MEAN

$50 \mathrm{TH}$ QUANTILES
90 $95 \mathrm{TH} \quad 95 \mathrm{TH}$

$99 \mathrm{TH}$

$99.5 \mathrm{TH}$

PEAK PEAK PEAK

MAXIMUM ANNUAL FOOD DOSE (EFFECTIVE) PROJECTED FOR INDTVIDUAL $\quad 0.2-0.5 \cdot \mathrm{kn}$ \begin{tabular}{ll}
$0.2-0.5 \cdot \mathrm{km}$ & 0.4165 \\
$1.2-1.6$ & 0.6819 \\
\hline
\end{tabular} $1.63 \mathrm{E}-02$ PROB TRIAL PROJECTED INDIVIDUAL $2.1-3.2 \mathrm{~km} \quad 0.7828$ $\begin{array}{llll}\text { PROJECTED FOR INDIVIDUAL } & 4.0-4.8 \mathrm{~km} & 0.8553 \\ \text { PROJEC } & 5.6-8.1 \mathrm{~km} & 0.8920\end{array}$ PROJECTED FOR INDIVIDUAL $11.3-16.1 \mathrm{~km} \quad 0.9809$ PROJECTED FOR INDIVIDUAL $20.9-25.8 \mathrm{~km} 1.0000$ PROJECTED FOR INDIVIDUAL $48.3-64.4 \mathrm{~km} \quad 1.0000$

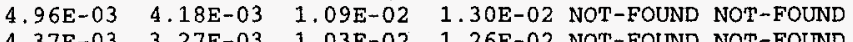
$4.37 \mathrm{E}-03 \quad 3.27 \mathrm{E}-03$ 1.03E-02 $1.26 \mathrm{E}-02$ NOT-FOUND NOT-FOUND $\begin{array}{llllll}7.35 \mathrm{E}-03 & 5.76 \mathrm{E}-03 & 1.22 \mathrm{E}-02 & 1.40 \mathrm{E}-02 & 1.89 \mathrm{E}-02 & 2.54 \mathrm{E}-02\end{array}$ $4.95 \mathrm{E}-03 \quad 3.56 \mathrm{E}-03 \quad 1.07 \mathrm{E}-02 \quad 1.36 \mathrm{E}-02$ NOT-FOUND NOT-FOUND $\begin{array}{llll}4.89 \mathrm{E}-03 & 3.23 \mathrm{E}-03 & 9.39 \mathrm{E}-03 & 1.37 \mathrm{E}-02 \\ & \text { NOT-FOUND NOT-FOUND }\end{array}$ PROTECTED FOR INDTVIDUAL $80.5-113 \mathrm{~km} \quad 1.0000 \quad 5.90 \mathrm{E}-03 \quad 3.98 \mathrm{E}-03 \quad 1.32 \mathrm{E}-02 \quad 1.88 \mathrm{E}-02$ NOT-FOUND NOT-FOUND

MAXIMUM ANNUAL FOOD DOSE (THYROID)

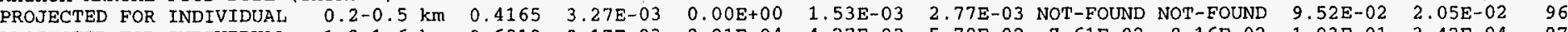

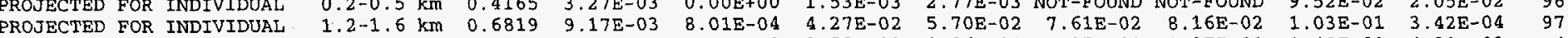

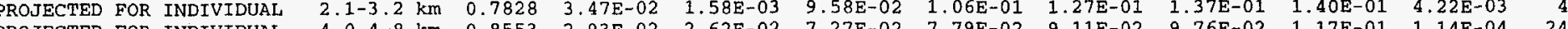

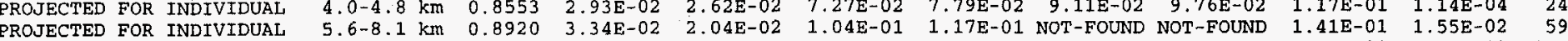

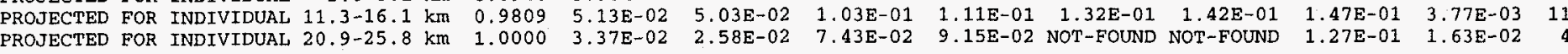




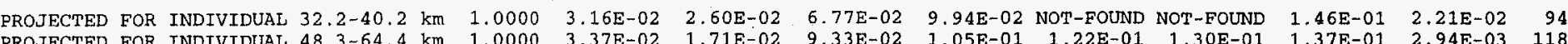

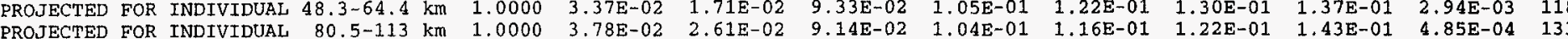

MACCS2 $09 / 26 / 9608: 40: 08$ Version 1.12 , Last Modified $9 / 25 / 96$ by D. Chanin PAGE 22

SOURCE TERM 2 OF 2

RELEASE FRACTIONS OF SOURCE TERM 1 REDUCED BY A FACTOR OF TEN RESULT NAME = HEALTH EFFECTS CASES

PEOPLE FRACTION =

0.9500

$0-80.5 \mathrm{~km}$

OVERALL

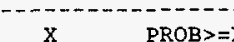

..00E-04 $1.00 \mathrm{E}+00$

$2.00 \mathrm{E}-04 \quad 1.00 \mathrm{E}+00$

.00E-04 1.00E+00

$5.00 \mathrm{E}-04 \quad 1.00 \mathrm{E}+00$

$\begin{array}{ll}.00 \mathrm{E}-04 & 1.00 \mathrm{E}+00\end{array}$

$1.00 \mathrm{E}-03 \quad 1.00 \mathrm{E}+00$

$1.00 E-03 \quad 1.00 E+00$

5.00E-03 1.00E+00

1.00E-03 $1.00 \mathrm{E}+00$

$1.00 \mathrm{E}-02 \quad 1.00 \mathrm{E}+00$

$2.00 \mathrm{E}-02 \quad 1.00 \mathrm{E}+00$

$3.00 \mathrm{E}-02 \quad 1.00 \mathrm{E}+00$

$5.00 \mathrm{E}-02 \quad 1.00 \mathrm{E}+00$

$7.00 \mathrm{E}-02 \quad 1.00 \mathrm{E}+00$

$1.00 \mathrm{E}-01 \quad 1.00 \mathrm{E}+00$

$2.00 \mathrm{E}-011.00 \mathrm{E}+00$

3.00E-01 1.00E+00

$5.00 \mathrm{E}-01 \quad 1.00 \mathrm{E}+00$

$1.00 \mathrm{E}+001.00 \mathrm{E}+00$

$1.00 \mathrm{E}+00 \quad 1.00 \mathrm{E}+00$

.

$5.00 \mathrm{E}+00 \quad 1.00 \mathrm{E}+00$

$7.00 \mathrm{E}+00 \quad 1.00 \mathrm{E}+00$

$1.00 E+01 \quad 1.00 E+00$

$.00 \mathrm{E}+01 \quad 9.99 \mathrm{E}-0$

$3.00 \mathrm{E}+01 \quad 9.99 \mathrm{E}-01$

$5.00 \mathrm{E}+01 \quad 9.96 \mathrm{E}-01$

$7.00 \mathrm{E}+01 \quad 9.78 \mathrm{E}-0$

$1.00 \mathrm{E}+02 \quad 9.15 \mathrm{E}-01$

$.00 \mathrm{E}+02 \quad 5.99 \mathrm{E}-01$

$3.00 \mathrm{E}+02$ 3.91E-01

.

$7.00 \mathrm{E}+02 \quad 1.78 \mathrm{E}-01$

$1.00 \mathrm{E}+03 \quad 1.29 \mathrm{E}-01$

.00E 03 (. 58E-03

$4.08 \mathrm{E}+03 \quad 2.15 \mathrm{E}-04$

N.D. N.D.

N.D. N.D.

$$
0.0500
$$

\begin{tabular}{c} 
EMER. RESP. $\# 1$ \\
\hline $\mathrm{x} \quad$ PROB $>=\mathrm{x}$
\end{tabular}

$1.00 \mathrm{E}-05 \cdot 1.00 \mathrm{E}+00$

$2.00 \mathrm{E}-05 \quad 1.00 \mathrm{E}+00$

$3.00 \mathrm{E}-05 \quad 1.00 \mathrm{E}+00$

$5.00 \mathrm{E}-05 \quad 1.00 \mathrm{E}+00$

$7.00 \mathrm{E}-05 \quad 1.00 \mathrm{E}+00$

$1.00 \mathrm{E}-04 \quad 1.00 \mathrm{E}+00$

$3.00 \mathrm{E}-041.00 \mathrm{E}+00$

$5.00 \mathrm{E}-04 \quad 1.00 \mathrm{E}+00$

$7.00 \mathrm{E}-04 \quad 1.00 \mathrm{E}+00$

$1.00 \mathrm{E}-03 \quad 1.00 \mathrm{E}+00$

$2.00 \mathrm{E}-03 \quad 1.00 \mathrm{E}+00$

$3.00 \mathrm{E}-03 \quad 1.00 \mathrm{E}+00$

$5.00 \mathrm{E}-03 \quad 1.00 \mathrm{E}+00$

$7.00 \mathrm{E}-03 \quad 1.00 \mathrm{E}+00$

$1.00 \mathrm{E}-02 \quad 1.00 \mathrm{E}+00$

$2.00 \mathrm{E}-02 \cdot 1.00 \mathrm{E}+00$

$3.00 \mathrm{E}-02 \quad 1.00 \mathrm{E}+00$

$5.00 \mathrm{E}-021.00 \mathrm{E}+00$

$1.00 \mathrm{E}-01 \quad 1.00 \mathrm{E}+00$

$2.00 \mathrm{E}-01 \quad 1.00 \mathrm{E}+00$

$3.00 \mathrm{E}-01 \quad 1.00 \mathrm{E}+00$

$5.00 \mathrm{E}-01 \quad 1.00 \mathrm{E}+00$

$7.00 \mathrm{E}-01 \quad 1.00 \mathrm{E}+00$

$1.00 \mathrm{E}+00 \quad 1.00 \mathrm{E}+00$

$2.00 \mathrm{E}+00 \quad 9.99 \mathrm{E}-01$

$3.00 \mathrm{E}+00 \quad 9.99 \mathrm{E}-01$

$5.00 \mathrm{E}+00 \quad 9.95 \mathrm{E}-01$

$7.00 \mathrm{E}+00 \quad 9.81 \mathrm{E}-01$

$1.00 \mathrm{E}+01 \quad 9.57 \mathrm{E}-01$

$2.00 \mathrm{E}+01 \quad 8.27 \mathrm{E}-01$

$5.00 \mathrm{E}+01$ $6.97 \mathrm{E}-01$

$7.00 \mathrm{E}+01 \quad 3.57 \mathrm{E}-01$

$1.00 \mathrm{E}+02 \quad 2.44 \mathrm{E}-01$

$2.00 \mathrm{E}+021.42 \mathrm{E}-01$

$3.00 \mathrm{E}+02 \quad 7.02 \mathrm{E}-02$

$5.00 \mathrm{E}+02 \quad 2.50 \mathrm{E}-02$

$7.00 \mathrm{E}+02 \quad 1.16 \mathrm{E}-02$

$1.00 \mathrm{E}+03 \quad 5.11 \mathrm{E}-03$

N.D. N.D.

N.D.

N.D.

\begin{tabular}{|c|c|}
\hline & \\
\hline $00 \mathrm{E}-0$ & \\
\hline & $1.00 \mathrm{E}+0$ \\
\hline-0 & $1.00 \mathrm{E}+0$ \\
\hline & \\
\hline 00E-05 & \\
\hline $00 E-04$ & $1.00 \mathrm{E}+00$ \\
\hline & $1.00 E+00$ \\
\hline & \\
\hline & \\
\hline $00 E-04$ & $1.00 E+0$ \\
\hline $00 \mathrm{E}-03$ & $1.00 \mathrm{E}+0$ \\
\hline & \\
\hline & \\
\hline & 1.8 \\
\hline $00 E-03$ & 1.0 \\
\hline & 1.0 \\
\hline & \\
\hline OOE- & 1.0 \\
\hline $0 \mathrm{E}$ & $E+0$ \\
\hline OOE- & 1.0 \\
\hline & \\
\hline OE- 01 & \\
\hline OOE-01 & 1.0 \\
\hline & 1.0 \\
\hline S & \\
\hline & \\
\hline $0 E+00$ & \\
\hline & \\
\hline & \\
\hline & \\
\hline E+ & \\
\hline & \\
\hline & \\
\hline $\mathrm{E}+$ & $6.23 E-01$ \\
\hline & \\
\hline & \\
\hline & \\
\hline & \\
\hline & \\
\hline & 7.3 \\
\hline & \\
\hline $.11 \mathrm{E}+$ & $2.15 \mathrm{E}$ \\
\hline & \\
\hline
\end{tabular}

CHRONC RESULTS

$\begin{array}{cc}x & \text { PROB }>=\mathrm{X} \\ \mathrm{x} & \mathrm{x}\end{array}$

$1.00 \mathrm{E}-04 \quad 1.00 \mathrm{E}+00$

$2.00 \mathrm{E}-04 \quad 1.00 \mathrm{E}+00$

3. $00 \mathrm{E}-04 \quad 1.00 \mathrm{E}+00$

5. $00 \mathrm{E}-04 \quad 1.00 \mathrm{E}+00$

$1.00 \mathrm{E}-04 \quad 1.00 \mathrm{E}+00$

1.00E-03 $1.00 \mathrm{E}+00$

3.00E-03 $1.00 \mathrm{E}+00$

$5.00 \mathrm{E}-03 \quad 1.00 \mathrm{E}+00$

7.00E-03 $1.00 \mathrm{E}+00$

$1.00 \mathrm{E}-02 \quad 1.00 \mathrm{E}+00$

$2.00 \mathrm{E}-02 \quad 1.00 \mathrm{E}+00$

$3.00 \mathrm{E}-02 \quad 1.00 \mathrm{E}+00$

5. $00 \mathrm{E}-02 \quad 1.00 \mathrm{E}+00$

7.00E-02 $1.00 \mathrm{E}+00$

$1.00 \mathrm{E}-01 \quad 1.00 \mathrm{E}+00$

2. $00 \mathrm{E}-01 \quad 1.00 \mathrm{E}+00$

$3.00 \mathrm{E}-01 \quad 1.00 \mathrm{E}+00$

$7.00 \mathrm{E}-01 \quad 1.00 \mathrm{E}+00$

$1.00 \mathrm{E}+00$ 1.00E+00

2. $00 \mathrm{E}+00 \quad 1.00 \mathrm{E}+00$

3. $00 \mathrm{E}+00 \quad 1.00 \mathrm{E}+00$

$5.00 \mathrm{E}+00 \quad 1.00 \mathrm{E}+00$

$7.00 \mathrm{E}+00 \quad 1.00 \mathrm{E}+00$

1. $00 \mathrm{E}+01 \quad 9.99 \mathrm{E}-01$

$2.00 \mathrm{E}+01 \quad 9.99 \mathrm{E}-01$

3. $00 \mathrm{E}+01 \quad 9.98 \mathrm{E}-01$

5. $00 \mathrm{E}+01 \quad 9.82 \mathrm{E}-01$

$\begin{array}{ll}7.00 \mathrm{E}+01 & 9.29 \mathrm{E}-01 \\ 1.00 \mathrm{E}+02 & 8.17 \mathrm{E}-01\end{array}$

2.00E+02 $0.17 \mathrm{E}-01$

$2.00 \mathrm{E}+02$ 4.22E-01

$5.00 \mathrm{E}+02 \quad 3.09 \mathrm{E}-01$

$7.00 \mathrm{E}+02$ 1. $1.0 \mathrm{E}-01$

$1.00 \mathrm{E}+03 \quad 1.06 \mathrm{E}-01$

$2.00 \mathrm{E}+03 \quad 1.64 \mathrm{E}-02$

$3.00 \mathrm{E}+03 \quad 2.37 \mathrm{E}-03$

$3.02 \mathrm{E}+03 \quad 2.37 \mathrm{E}-03$

$\begin{array}{ll}\text { N.D. } & \text { N.D. } \\ \text { N.D. } & \text { N.D. }\end{array}$

N.D. N.D.

N.D. N.D. 


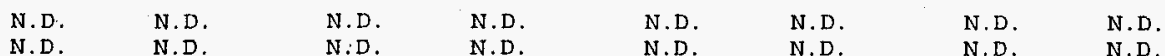

$\begin{array}{llllll}\text { N.D. N.D. } & \text { N.D. } & \text { N.D. } & \text { N.D. } & \text { N.D. } & \text { N.D. } \\ \text { N.D. N.D } & \text { N.D. } & \text { N.D. } & \text { N.D. } & \text { N.D. } & \text { N.D. }\end{array}$

Successful completion of MACCS2 was achieved!
This job required a total of 2708.588 CPU seconds

Input processing required $10.209 \mathrm{CPU}$ seconds

$\begin{array}{lr}\text { Simulation required } & 2579.520 \mathrm{CPU} \text { seconds } \\ \text { output processing required } & 118.859 \mathrm{CPU} \text { seconds }\end{array}$ 


\section{Appendix D: Glossary of Input File Variables}

This appendix is a glossary of the input variable names for the MACCS2 code. The name of the user input file in which each variable belongs is noted. The location in the user's guide where the variable is defined is given by section number. Each variable name is also briefly described.

\begin{tabular}{|c|c|c|c|}
\hline $\begin{array}{l}\text { VARIABLE } \\
\text { NAME }\end{array}$ & $\begin{array}{l}\text { INPUT } \\
\text { FILE }\end{array}$ & SECT. & DESCRIPTION \\
\hline ACNAME & EARLY & 6.9.3 & Latent Cancer Effect \\
\hline ACSUSC & EARLY & 6.9 .3 & Population Susceptible to Cancer \\
\hline ACTHRE & EARLY & 6.9 .3 & Linear Dose-Response Threshold \\
\hline APLFRC & ATMOS & 5.11 & Method of Applying Release Fractions \\
\hline ATNAM1 & ATMOS & 5.2 & Title Describing the ATMOS Assumptions \\
\hline ATNAM2 & ATMOS & 5.11 & Title Describing the Source Term \\
\hline BIN_FILE & CHRONC & 7.10 .2 & Name of COMIDA2 Ingestion Data File \\
\hline BNDMXH & ATMOS & 5.14 & Boundary Weather Mixing Layer Height \\
\hline BNDRAN & ATMOS & 5.14 & Boundary Weather Rain Rate \\
\hline BNDWND & ATMOS & 5.14 & Boundary Weather Wind Speed \\
\hline BRKPNT & ATMOS & 5.8 & Breakpoint Time for Plume Meander \\
\hline BRRATE & EARLY & 6.5 & Breathing Rate \\
\hline BUILDH & ATMOS & 5.10 & Reactor Building Height \\
\hline CDFRM & CHRONC & 7.5 & Farmland Decontamination Cost \\
\hline CDNFRM & CHRONC & 7.5 & Nonfarm Decontamination Cost \\
\hline CFRISK & EARLY & 6.9 .3 & Lifetime Cancer Fatality Risk Factors \\
\hline CHNAME & CHRONC & 7.2 & CHRONC Problem Identification \\
\hline CIRISK & EARLY & 6.9 .3 & Lifetime Cancer Injury Risk Factors \\
\hline CORINV & ATMOS & 5.11 & Core Inventory by Radionuclide \\
\hline CORSCA & ATMOS & 5.11 & Linear Scaling Factor on Core Inventory \\
\hline COUPLD & CHRONC & 7.11 .3 & Coupling Flag for Food Actions \\
\hline CRIORG & EARLY & 6.6 .8 & Critical Organ for EARLY Phase \\
\hline CRTOCR & CHRONC & 7.4 & Critical Organ for CHRONC Phase \\
\hline CSFACT & EARLY & 6.5 & Cloudshine Shielding Factor \\
\hline CTCOEF & CHRONC & 7.11 .1 & Growing Season Transfer Coefficient \\
\hline CTHALF & CHRONC & 7.11 .1 & Growing Season Weathering Half-Life \\
\hline CWASH1 & ATMOS & 5.5 & Linear Coefficient for Washout \\
\hline CWASH2 & ATMOS & 5.5 & Exponential Term for Washout \\
\hline CYSIGA & ATMOS & 5.7 & Linear Coefficient for $\sigma_{y}$ \\
\hline CYSIGB & ATMOS & 5.7 & Exponential Term for $\sigma_{y}$ \\
\hline CZSIGA & ATMOS & 5.7 & Linear Coefficient for $\sigma_{z}$ \\
\hline CZSIGB & ATMOS & 5.7 & Exponential Term for $\sigma_{z}$ \\
\hline DCF_FILE & EARLY & 6.2 .1 & Name of Dose Conversion Factor File \\
\hline DCYPBH & CHRONC & 7.11 .1 & Decay/Processing Retention Meat to Humans \\
\hline DCYPCB & CHRONC & 7.11 .1 & Decay/Processing Retention Crops to Meat \\
\hline
\end{tabular}




\begin{tabular}{|c|c|c|c|}
\hline DCYPCH & CHRONC & 7.11.1 & Decay/Processing Retention Crops to Humans \\
\hline DCYPCM & CHRONC & 7.11 .1 & Decay/Processing Retention Crops to Milk \\
\hline DCYPMH & CHRONC & 7.11 .1 & Decay/Processing Retention Milk to Humans \\
\hline DDREFA & EARLY & 6.9 .3 & Dose-Dependent Reduction Factor \\
\hline DDTHRE & EARLY & 6.9 .3 & Threshold for Applying Dose-Dependent Reduction Factor \\
\hline DISTANCE & ATMOS & 7.5 .2 & Downwind Distance for $\sigma_{\mathrm{y}}$ and $\sigma_{\mathrm{z}}$ Parameters \\
\hline DLBCST & CHRONC & 7.5 & Decontamination Worker Labor Cost \\
\hline DLTEVA & EARLY & 6.6 .8 & Delay to Evacuation for Each Ring \\
\hline DLTSHL & EARLY & 6.6 .8 & Delay to Take Shelter for Each Ring \\
\hline DOSEFA & EARLY & 6.9 .3 & Cancer Dose-Response Alpha Factors \\
\hline DOSEFB & EARLY & 6.9 .3 & Cancer Dose-Response Beta Factors \\
\hline DOSELONG & CHRONC & 7.10 .2 & COMIDA2 Model Long-Term Dose Limit \\
\hline DOSEMILK & CHRONC & 7.10 .2 & COMIDA2 Model First-Year Milk Dose Limit \\
\hline DOSEOTHR & CHRONC & 7.10 .2 & COMDA2 Model First-Year Nonmilk Dose Limit \\
\hline DOSHOT & EARLY & 6.6 .8 & Normal Relocation Dose Threshold \\
\hline DOSNRM & EARLY & 6.6 .8 & Hot-Spot Relocation Dose Threshold \\
\hline DOSTH3 & EARLY & 6.13 & Dose Threshold for Result 3 \\
\hline DPFRCT & CHRONC & 7.9 & Farm Production Dairy Fraction \\
\hline DPRATE & CHRONC & 7.6 & Property Depreciation Rate \\
\hline DRYDEP & ATMOS & 5.4 & Dry Deposition Flag \\
\hline DSCRLT & CHRONC & 7.4 & Long-Term Phase Dose Criterion \\
\hline DSCRTI & CHRONC & 7.4 & Intermediate-Phase Dose Criterion \\
\hline DSRATE & CHRONC & 7.6 & Societal Discount Rate for Property \\
\hline DSRFCT & CHRONC & 7.5 & Decontamination Factors \\
\hline DURBEG & EARLY & 6.6 .8 & Duration of Beginning of Evacuation Phase \\
\hline DUR_INTPHAS & S CHRONC & 7.4 & Duration of Intermediate-Phase Period \\
\hline DURMID & EARLY & 6.6 .8 & Duration of Middle of Evacuation Phase \\
\hline EANAM1 & EARLY & 6.2 .2 & Text Describing the EARLY Assumptions \\
\hline EANAM2 & EARLY & 6.6 .8 & Text Describing the Emergency Response \\
\hline EFFACA & EARLY & 6.7 & Hazard Function Fatal Alpha Factors \\
\hline EFFACB & EARLY & 6.8 & Hazard Function Fatal Beta Factors \\
\hline EIFACA & EARLY & 6.8 & Hazard Function Injury Alpha Factors \\
\hline EIFACB & EARLY & 6.8 & Hazard Function Injury Beta Factors \\
\hline EINAME & EARLY & 6.8 & Early Injury Effect Names \\
\hline EISUSC & EARLY & 6.8 & Susceptible Population Fraction \\
\hline EITHRE & EARLY & 6.8 & Early Injury Dose Threshold \\
\hline EFFTHR & EARLY & 6.7 & Threshold Dose to Target Organ \\
\hline ENDAT1 & ATMOS & 5.12 & Control Flag Indicating Only ATMOS Is to be Run \\
\hline ENDAT2 & EARLY & 6.2 .2 & $\begin{array}{l}\text { Control Flag Indicating Only ATMOS and EARLY Are } \\
\text { to be Run }\end{array}$ \\
\hline ENDEMP & EARLY & 6.6 .8 & Duration of the Early Phase \\
\hline ESPEED & EARLY & 6.6 & Radial Evacuation Speed Array \\
\hline EVACST & CHRONC & 7.3 & Emergency-Phase Cost of Evacuation/Relocation \\
\hline EVATYP & EARLY & 6.6 .8 & Type of Evacuation \\
\hline EXPTIM & CHRONC & 7.4 & Maximum Exposure Time for CHRONC Calculations \\
\hline
\end{tabular}




\begin{tabular}{|c|c|c|c|}
\hline FDPATH & CHRONC & 7.10 .2 & COMIDA2 vs. MACCS Food Model Switch \\
\hline FDPATH & CHRONC & 7.11 & COMIDA2 vs. MACCS Food Model Switch \\
\hline FLAG11 & CHRONC & 7.16 & Flag to Request Maximum Distance Results \\
\hline FPLSCH & CHRONC & 7.11 .1 & Losses in Processing Crops for Human Consumption \\
\hline FRACLD & CHRONC & 7.9 & Fraction of Site Area that is Land \\
\hline FRCFRM & CHRONC & 7.9 & Fraction of Site Land Used for Farming \\
\hline FRCTCB & CHRONC & 7.11 .1 & Fraction of Crop Consumed by Meat Animals \\
\hline FRCTCH & CHRONC & 7.11 .1 & Fraction of Crop Consumed by Humans \\
\hline FRCTCM & CHRONC & 7.11 .1 & Fraction of Crop Consumed by Milk Animals \\
\hline FRCTFL & CHRONC & 7.11 .2 & Fraction of Farmland Used for Each Crop \\
\hline FRFDL & CHRONC & 7.5 & Farm Labor Cost Fraction \\
\hline FRFIM & CHRONC & 7.9 & Farm Wealth Improvements Fraction \\
\hline FRMPRD & CHRONC & 7.9 & Average Annual Farm Production Value \\
\hline FRNFDL & CHRONC & 7.5 & Nonfarm Labor Cost Fraction \\
\hline FRNFIM & CHRONC & 7.9 & Nonfarm Wealth Improvements Fraction \\
\hline GCMAXR & CHRONC & 7.11 .3 & $\begin{array}{l}\text { Protective Action Guides for Long-Term Uptake to Milk or } \\
\text { Crops }\end{array}$ \\
\hline GSHFAC & EARLY & 6.5 & Groundshine Shielding Factor Table \\
\hline GWCOEF & CHRONC & 7.7 & Long-Term Groundshine Coefficients \\
\hline HRMXHT & ATMOS & 5.17 & Mixing Layer Heights \\
\hline HRRAIN & ATMOS & 5.17 & Rain Rates \\
\hline HRWNDV & ATMOS & 5.17 & Wind Speeds \\
\hline IIDIS1 & EARLY & 6.11 & Inner Spatial Interval for Result 1 \\
\hline I1DIS4 & EARLY & 6.14 & Spatial Interval for Result 4 \\
\hline I1DIS5 & EARLY & 6.15 & Inner Spatial Interval for Result 5 \\
\hline I1DIS6 & EARLY & 6.16 & Inner Spatial Interval for Result 6 \\
\hline I1DIS7 & EARLY & 6.17 & Inner Spatial Interval for Result 7 \\
\hline I1DIS8 & EARLY & 6.18 & Inner Spatial Interval for Result 8 \\
\hline IIDISA & EARLY & 6.19 & Inner Spatial Interval for Result A \\
\hline I1DS10 & CHRONC & 7.15 & Inner Spatial Interval for Result 10 \\
\hline I1DS12 & CHRONC & 7.17 & Inner Spatial Interval for Result 12 \\
\hline I2DISI & EARLY & 6.11 & Outer Spatial Interval for Result 1 \\
\hline I2DIS5 & EARLY & 6.15 & Outer Spatial Interval for Result 5 \\
\hline I2DIS6 & EARLY & 6.16 & Outer Spatial Interval for Result 6 \\
\hline I2DIS7 & EARLY & 6.17 & Outer Spatial Interval for Result 7 \\
\hline I2DIS8 & EARLY & 6.18 & Outer Spatial Interval for Result 8 \\
\hline I2DISA & EARLY & 6.19 & Outer Spatial Interval for Result A \\
\hline I2DS10 & CHRONC & 7.15 & Outer Spatial Interval for Result 10 \\
\hline I2DS12 & CHRONC & 7.17 & Outer Spatial Interval for Result 12 \\
\hline IANG_B & EARLY & 6.20 & Angular Index for Type B Results \\
\hline IBDSTB & ATMOS & 5.14 & Boundary Weather Stability Class \\
\hline IBEGIN & EARLY & 6.3 & Interval at Which Population Begins \\
\hline IDEBUG & ATMOS & 5.12 & Debug Switch for Dispersion Print \\
\hline IDIREC & EARLY & 6.6 .8 & Array of Exit Directions for Network Evacuation \\
\hline IGROUP & ATMOS & 5.4 & Radionuclide Groups \\
\hline
\end{tabular}




\begin{tabular}{|c|c|c|c|}
\hline IHRDIR & ATMOS & 5.17 & Wind Directions \\
\hline IHRSTB & ATMOS & 5.17 & Stability Classes \\
\hline INDRAD & ATMOS & 5.18 & Distance Index for ATMOS CCDFs \\
\hline INDREL & ATMOS & 5.18 & Plume Index for ATMOS CCDFs \\
\hline INDXBN & ATMOS & 5.16 & Index to Bin Number Used for Met Sample \\
\hline INWGHT & ATMOS & 5.16 & Number of Samples to Take from the Bin \\
\hline IORG13 & CHRONC & 7.18 & Organ Index for Maximum Food Dose Result \\
\hline IPLUME & EARLY & 6.2 .2 & Plume Model Dispersion Code \\
\hline IPRINT & EARLY & 6.2 .2 & Quantity of Early Debug Output \\
\hline IRAD13 & CHRONC & 7.18 & Distance Index for Maximum Food Dose Result \\
\hline IRAD_B & EARLY & 6.20 & Inner Radial Boundary for Type B Results \\
\hline IRSEED & ATMOS & 5.16 & Seed for Random Number Generator \\
\hline ISTRDY & ATMOS & 5.15 & Starting Day of the Accident \\
\hline ISTRHR & ATMOS & 5.15 & Starting Hour of the Accident \\
\hline IX1DS9 & CHRONC & 7.14 & Inner Spatial Interval for Result 9 \\
\hline IX2DS9 & CHRONC & 7.14 & Outer Spatial Interval for Result 9 \\
\hline KSWTCH & CHRONC & 7.13 & Diagnostic Output Option Switch \\
\hline LASMOV & EARLY & 6.6 .8 & Last Evacuation Movement Interval \\
\hline LIMSPA & ATMOS & 5.14 & Last Spatial Interval for Recorded Weather \\
\hline LVLDEC & CHRONC & 7.5 & Number of Decontamination Strategies \\
\hline MAXGRP & ATMOS & 5.4 & Number of Radionuclide Groups \\
\hline MAXRIS & ATMOS & 5.11 & Selection of Risk Dominant Plume \\
\hline METCOD & ATMOS & 5.13 & Meteorological Sampling Option Code \\
\hline NAMCRP & CHRONC & 7.11 .1 & Names of the Crop Categories \\
\hline NAMCRP & CHRONC & 7.11 .2 & Names of the Crop Categories \\
\hline NAME & EARLY & 6.11 & Result Type 1 Health Effect Name \\
\hline NAME & EARLY & 6.13 & Result Type 3 Organ Name \\
\hline NAME & EARLY & 6.14 & Result Type 4 Health Effect Name \\
\hline NAME & EARLY & 6.15 & Result Type 5 Organ Name \\
\hline NAME & EARLY & 6.17 & Result Type 7 Health Effect Name \\
\hline NAME & EARLY & 6.18 & Result Type 8 Health Effect Name \\
\hline NAME & EARLY & 6.19 & Result Type A Organ Name \\
\hline NAME & EARLY & 6.20 & Result Type B Organ Name \\
\hline NAMIPI & CHRONC & 7.11 .1 & Names of Food Ingestion Radionuclides \\
\hline NAMIPI & CHRONC & 7.11 .3 & Names of Food Ingestion Radionuclides \\
\hline NAMSTB & ATMOS & 5.4 & List of Pseudostable Nuclides \\
\hline NAMWPI & CHRONC & 7.12 & Names of Water Ingestion Radionuclides \\
\hline NFICRP & CHRONC & 7.11 .1 & Number of Crop Categories \\
\hline NFIISO & CHRONC & 7.11.1 & Number of Radionuclides for Food Ingestion \\
\hline NGWTRM & CHRONC & 7.7 & Number of Terms in Groundshine Weathering Equation \\
\hline NPSGRP & ATMOS & 5.6 & Number of Particle Size Groups \\
\hline NRINTN & ATMOS & 5.16 & Number of Rain Intensity Breakpoints \\
\hline NRNINT & ATMOS & 5.16 & Number of Rain Distance Intervals \\
\hline NRWTRM & CHRONC & 7.8 & Number of Terms in Resuspension Weathering Equation \\
\hline NSBINS & ATMOS & 5.16 & Number of Weather Bins to Sample \\
\hline
\end{tabular}




\begin{tabular}{|c|c|c|c|}
\hline NSMPLS & ATMOS & 5.16 & Number of Weather Samples per Bin \\
\hline NTTRM & CHRONC & 7.11 .1 & Number of Terms in Growing-Season Food Model \\
\hline NUCNAM & ATMOS & 5.4 & Radionuclide Names \\
\hline NUCOUT & ATMOS & 5.12 & Radionuclide Used in Dispersion Print \\
\hline NUMO & ATMOS & 5.18 & Number of Requests for ATMOS CCDFs \\
\hline NUM1 & EARLY & 6.11 & Number of Health Effect Results \\
\hline NUM2 & EARLY & 6.12 & Number of Early Fatality Radius Results \\
\hline NUM3 & EARLY & 6.13 & Number of Early Dose Threshold Results \\
\hline NUM4 & EARLY & 6.14 & Number of Avg. Individual Risk Results \\
\hline NUM5 & EARLY & 6.15 & Number of Population Dose Results \\
\hline NUM6 & EARLY & 6.16 & Number of Centerline Dose/Distance Results \\
\hline NUM7 & EARLY & 6.17 & Number of Centerline Risk/Distance Results \\
\hline NUM8 & EARLY & 6.18 & Number of Population-Weighted Risk Results \\
\hline NUM12 & CHRONC & 7.17 & Number of Affected Area Results \\
\hline NUM13 & CHRONC & 7.18 & Number of Ingestion Dose Results \\
\hline NUMA & EARLY & 6.19 & Number of Peak Dose at a Distance Results \\
\hline NUMACA & EARLY & 6.9 .3 & Number of Latent Cancer Effects \\
\hline NUMB & EARLY & 6.20 & Peak Dose at an $(r, \theta)$ Location \\
\hline NUMEFA & EARLY & 6.7 & Number of Early Fatality Effects \\
\hline NUMEN & EARLY & 6.8 & Number of Early Injury Effects \\
\hline NUMEVA & EARLY & 6.6 .8 & Outer Boundary of Evacuation/Shelter Region \\
\hline NUMFIN & EARLY & 6.2 .2 & Number of Fine Grid Subdivisions \\
\hline NUMISO & ATMOS & 5.4 & Number of Defined Radionuclides \\
\hline NUMRAD & ATMOS & 5.3 & Number of Radial Spatial Intervals \\
\hline NUMREL & ATMOS & 5.11 & Number of Released Plume Segments \\
\hline NUMSTB & ATMOS & 5.4 & Number of Defined Pseudostable Radionuclides \\
\hline NUMWPI & CHRONC & 7.12 & Number of Radionuclides for Water Ingestion \\
\hline NUM_DST & ATMOS & 5.7 .2 & Number of Distances Defined in Dispersion Table \\
\hline NXUM9 & CHRONC & 7.14 & Number of Population Dose Results \\
\hline NXUM10 & CHRONC & 7.15 & Number of Economic Block Results \\
\hline OALARM & ATMOS & 5.11 & Off-Site Alarm Time \\
\hline ORGFLG & EARLY & 6.4 & Doses to be Calculated for Specified Organ \\
\hline ORGNAM & EARLY & 6.4 & Defined Organ Names \\
\hline ORGNAM & EARLY & 6.7 & Early Fatality Target Organs \\
\hline ORGNAM & EARLY & 6.8 & Early Injury Target Organs \\
\hline ORGNAM & EARLY & 6.9 .3 & Latent Cancer Target Organs \\
\hline ORGNAM & EARLY & 6.16 & Organ Name of the Centerline Doses \\
\hline ORGNAM & CHRONC & 7.14 & Organ Name for Population Dose Results \\
\hline ORGN13 & CHRONC & 7.18 & Organ Name for Type 13 Results \\
\hline OVRRID & EARLY & 6.2 .2 & Wind Rose Probability Override \\
\hline PATHNM & EARLY & 6.16 & Pathway Code for Result 6 \\
\hline PDELAY & ATMOS & 5.11 & Plume Release Times \\
\hline PLHEAT & ATMOS & 5.11 & Plume Heat Contents \\
\hline PLHITE & ATMOS & 5.11 & Plume Release Heights \\
\hline PLUDUR & ATMOS & 5.11 & Plume Segment Durations \\
\hline
\end{tabular}




\begin{tabular}{|c|c|c|c|}
\hline POPCST & CHRONC & 7.6 & Per Capita Cost of Long-Term Relocation \\
\hline POPDEN & EARLY & 6.3 & Average Regional Population Density \\
\hline POPFLG & EARLY & 6.3 & Population Distribution Flag \\
\hline PROTIN & EARLY & 6.5 & Inhalation Protection Factor \\
\hline PSCMLK & CHRONC & 7.11 .3 & Growing-Season Protective Action Criteria for Milk \\
\hline PSCOTH & CHRONC & 7.11 .3 & Growing-Season Protective Action Criteria for Crops \\
\hline PSDIST & ATMOS & 5.11 & Particle Size Distribution by Group \\
\hline QROOT & CHRONC & 7.11 .3 & Decay Rate for Long-Term Food Dose \\
\hline REFPNT & EARLY & 6.6 .8 & Reference Time Point (ARRIVAL or SCRAM) \\
\hline REFTIM & ATMOS & 5.11 & Plume Reference Time Point \\
\hline RELCST & CHRONC & 7.3 & Relocation Cost per Person-Day \\
\hline RELFRC & ATMOS & 5.11 & Release Fractions of the Source Term \\
\hline RESCON & EARLY & 6.5 & Resuspension Concentration Coefficient \\
\hline RESHAF & EARLY & 6.5 & Resuspension Concentration Half-Life \\
\hline RISCAT & EARLY & 6.2 .2 & Risk by Weather-Category Flag \\
\hline RISTHR & EARLY & 6.12 & Risk Threshold for Fatality Radius \\
\hline RNDSTS & ATMOS & 5.16 & Rain Distances Used for Binning \\
\hline RNRATE & ATMOS & 5.16 & Rain Intensities Used for Binning \\
\hline RWCOEF & CHRONC & 7.8 & Long-Term Resuspension Factor Coefficients \\
\hline SCLADP & ATMOS & 5.9 & Scaling Factor for A-D Plume Rise \\
\hline SCLCRW & ATMOS & 5.9 & Scaling Factor for Critical Wind Speed \\
\hline SCLEFP & ATMOS & 5.9 & Scaling Factor for E-F Plume Rise \\
\hline SIGMA_Y & ATMOS & 5.7 .2 & Gaussian Crosswind Dispersion Parameter $\left(\sigma_{\mathrm{y}}\right)$ \\
\hline SIGMA_Z & ATMOS & 5.7 .2 & Gaussian Vertical Dispersion Parameter $\left(\sigma_{z}\right)$ \\
\hline SIGYINIT & ATMOS & 5.10 & Initial Value of $\sigma_{\mathrm{y}}$ \\
\hline SIGZINIT & ATMOS & 5.10 & Initial Value of $\sigma_{z}$ \\
\hline SKPFAC & EARLY & 6.5 & Skin Protection Factors \\
\hline SPAEND & ATMOS & 5.3 & Endpoints of Spatial Intervals \\
\hline TCROOT & CHRONC & 7.11 .1 & Transfer Factor Long-Term Root Uptake \\
\hline TFBF & CHRONC & 7.11 .1 & Transfer Factor Animal to Meat \\
\hline TFMILK & CHRONC & 7.11 .1 & Transfer Factor Animal to Milk \\
\hline TFWKF & CHRONC & 7.5 & Farm Worker's Work Fraction \\
\hline TFWKNF & CHRONC & 7.5 & Nonfarm Workers Work Fraction \\
\hline TGSBEG & CHRONC & 7.11 .2 & Growing Season Start Time for Each Crop \\
\hline TGSEND & CHRONC & 7.11 .2 & Growing Season End Time for Each Crop \\
\hline TGWHLF & CHRONC & 7.7 & Groundshine Weathering Half-Lives \\
\hline TIMBAS & ATMOS & 5.8 & Time Base for Plume Expansion Factor \\
\hline TIMDEC & CHRONC & 7.5 & Decontamination Times \\
\hline TIMHOT & EARLY & 6.6 .8 & Hot Spot Relocation Time \\
\hline TIMNRM & EARLY & 6.6 .8 & Normal Relocation Time \\
\hline TMIPND & CHRONC & 7.4 & Time Intermediate Phase Ends \\
\hline TMPACT & CHRONC & 7.4 & Time Action Period Ends \\
\hline TRAVELPONNT & T EARLY & 6.6 .8 & Evacuee Movement Option \\
\hline TRWHLF & CHRONC & 7.8 & Resuspension Weathering Half-Lives \\
\hline VALWF & CHRONC & 7.9 & Value of Farm Wealth (per Hectare) \\
\hline
\end{tabular}




$\begin{array}{llll}\text { VALWNF } & \text { CHRONC } & 7.9 & \text { Value of Nonfarm Wealth (per Capita) } \\ \text { VDEPOS } & \text { ATMOS } & 5.6 & \text { Dry Deposition Velocities } \\ \text { WETDEP } & \text { ATMOS } & 5.4 & \text { Wet Deposition Flag } \\ \text { WINGF } & \text { CHRONC } & 7.12 & \text { Water Transfer Fraction to Humans } \\ \text { WINROS } & \text { EARLY } & 6.2 .2 & \text { Wind Rose Probabilities } \\ \text { WSHFRI } & \text { CHRONC } & 7.12 & \text { Initial Water Washoff Fraction } \\ \text { WSHRTA } & \text { CHRONC } & 7.12 & \text { Annual Water Washoff Fraction } \\ \text { WTFRAC } & \text { EARLY } & 6.6 .8 & \text { Weighting Fraction for Combined Results } \\ \text { WTNAME } & \text { EARLY } & 6.6 .8 & \text { Type of Weighting (PEOPLE, TIME, or SUMPOP) } \\ \text { XPFAC1 } & \text { ATMOS } & 5.8 & \text { Base Time for Meander Expansion Factor } \\ \text { XPFAC2 } & \text { ATMOS } & 5.8 & \text { Breakpoint for Expansion Factor Model } \\ \text { YSCALE } & \text { ATMOS } & 5.7 .3 & \text { Scale Factor for Horizontal Dispersion } \\ \text { ZSCALE } & \text { ATMOS } & 5.7 .3 & \text { Scale Factor for Vertical Dispersion }\end{array}$


THIS PAGE INTENTIONALLY LEFT BLANK 


\section{Distribution List}

\section{J. Grupa}

Netherlands Energy Research

Foundation, ECN

Westerduinweg 3

P.O. Box 1

NL-1755 LE Petten

The NETHERLANDS

Dr. J. Stuznik

Josef Stefan Inst.

Jamova 39

P.O. Box 100

1001 Ljubljan

Slovenia

Aldo Fanfarillo

ENEL SpA - ATN

Italian Electricity Company

Nuclear Energy Division

Viale Regina Margherita, 137

I-00198 Rome

ITALY

J. Enrique Garcia-Ramirez

Comision Nacional de Seguridad

Nuclear \& Salvaguardias (CNSNS)

Dr. Barragan No. 779

1 Er. Piso

Col. V. Narvarte

03020 Mexico City, D.F.

MEXICO

Toshimitsu Homma

Nuclear Power Engineering Corporation

Institute of Nuclear Safety

Safety Analysis Division III

3-17-1 Toranomon, Minato-ku

Tokyo 105 JAPAN

H.F.B. Minnaar

Atomic Energy Corporation of South

Africa, Ltd.

P.O. Box 582

Pretoria 0001

SOUTH AFRICA
Eduardo Gallego

Universidad Politecnica de Madrid (UPM)

Escuela Tecnica Superior de Ingenieros

Industriales

Catedra de Tecnologia Nuclear

Calle Jose Gutierrez Abascal, 2

E-28006 Madrid

SPAIN

Juraj Duran

Vskumny Ustaav Jadrovych Elektrarni

(VUJE)

Nuclear Power Plants Research Institute

Okruzna 5

91864 Trnava

SLOVAK REPUBLIC

Richard Davis

Brookhaven National Laboratory

Bldg. 130, Advanced Technology

32 Lewis Road

P.O. Box 5000

Upton, New York 11973-5000

Laszlo Koblinger

KFKI Atomic Energy Research Institute

(AEKI)

P.O. Box 49

H-1525 Budapest

HUNGARY

John Kollas

Institute of Nuclear Technology \&

Radiation

Protection

N.C.S.R. "Demokritos"

15310 Aghia Paraskevi

P.O. Box 60228

Athens

GREECE

Kamiar Jamili

DP-62-FTN

Department of Energy

Washington, D.C. 20585 
Stefano Monti

Ente per le Nuove Tecnologie,

L'Energia e L'Ambiente

(ENEA - ERG/FISS/FIRE)

Dept. of Energy

Nuclear Fission Energy Division

Via Martiri di Monte Sole, 4

40129 Bologna

ITALY

Vinod Mubayi

Brookhaven National Laboratory

Bldg. 130, Advanced Technology

Department

32 Lewis Road

P.O. Box 5000

Upton, NY 11973-5000

M. Arturo Reyes-Sanchez

Comision Nacional de Seguridad

Nuclear \& Salvaguardias (CNSNS)

Dr. Barragan No. 779

1 Er. Piso

Co. V. Narvarte

03020 Mexico City D. F.

MEXICO

ULF Tveten

Head, Environment Physics Section

Institutt for Energiteknikk (IFE)

Postboks 40

N-2007 Kjeller

NORWAY

Oak Ridge National Laboratory(2)

Attn: Steve Fisher

Sherrel Greene

MS-8057

P.O. Box 2009

Oak Ridge, TN 37831

Westinghouse Savannah River Co. (2)

Attn: Kevin O'Kula

Jackie East

Safety Technology Section

1991 S. Centennial Ave. Bldg. 1

Aiken, SC 29803
EG\&G Idaho, Inc.(2)

Attn: Art Rood

Mike Abbott

MS-2110

P.O. Box 1625

Idaho Falls, ID 83415

Sarbes Acharya

Department of Energy

NS-1/FORS

Washington, DC 20585

Robert Bari

Brookhaven National Laboratory

Bldg. 197C, Advanced Technology

29 Cornell Ave.

P.O. Box 5000

Upton, NY 11973-5000

Romney Duffey

Brookhaven National Laboratory

Bldg 197C, Advanced Technology

29 Cornell Avenue

P.O. Box 5000

Upton, New York 11973-5000

Albert Hanson

Brookhaven National Laboratory

Bldg. 130, Advanced Technology

32 Lewis Rd.

P.O. Box 5000

Upton, NY 11973-5000

Christiana Lui

T10E50

RES/DST/PRAB

U.S. Nuclear Regulatory Commission

Washington, DC 20555

Mr. Steve Fisher

MS-8057

Oak Ridge National Laboratory

P.O. Box 2009

Oak Ridge, TN 37831

Lev Neymotin

Brookhaven National Laboratory

Building 130

Upton, NY 11973 
Mr. Vern Peterson

EG\&G Rocky Flats, Inc.

Rocky Flats Plant

P.O. Box 464

Bldg. T886B

Golden, CO 80402-0464

Ms. Jackie East

Safety Technology Section

Westinghouse Savannah River Company

1991 S. Centennial Avenue, Bldg. 1

Aiken, SC 29803

Mr. Mike Abbott

MS-2110

EG\&G Idaho, Inc.

P.O. Box 1625

Idaho Falls, ID 83415

Mr. Joe Shinn

Mailstop L-453

Lawrence Livermore National Laboratory

P.O. Box 808

Livermore, CA 94551-9900

Mr. Anthony Savino

Westinghouse Hanford Company

P.O. Box 1970 (MSIN H4-64)

2355 Stevens Drive

Richland, WA 99352

Mr. Pat McClure

Mailstop K-556

Los Alamos National Laboratory

P.O. Box 1663

Los Alamos, NM 87545

Prof. Eric Haskin

Prof. Ken Summa

Department of Chemical and Nuclear Eng.

Farris Engineering Center

University of New Mexico

Albuquerque, NM 87131-1341

Jeff Latkowski

Lawrence Livermore National Laboratory

L-481 P.O. Box 808

Livermore, CA 94550
Mr. Dennis Strenge

Mailstop K3-54

Pacific Northwest Laboratories

P.O. Box 999

Richland, WA 99352

tel. 509-375-6944

Roy Karimi

SAIC

20201 Century Blvd

3rd Floor

Germantown, Maryland 20874

John Randall

MS T10 E50

RES/DST/PRAB

U.S. Nuclear Regulatory Commission

Washington, DC 20555

Internal

MS0405 D. D. Carlson, 12333

MS1175 L. A. Miller, 9364

MS0748 F. T. Harper, 641320 copies

MS0748 M. L. Young, 641320 copies

MS0748 J. J. Gregory, 6413

MS0748 T. D. Brown, 6413

MS0748 L-J. Shyr, 6413

MS0767 B. A. Boughton, 5514

MS0767 D. B. Clauss, 5514

MS1146 J. H. Soloio, 9364

MS1175 J. L. Powell, 9364

MS0718 J. L. Sprung, 6641

MS9018 Central Technical Files, 8940-2

MS0899 Technical Library, 4414

MS0619 Review \& Approval Desk, 12690 for DOE/OSTI 